DESK INDEX FORCHHEIMER - THERAPEUSIS 


\section{,}




$$
\text { kxertest }
$$




\section{Digitized by the Internet Archive in 2007 with funding from Microsoft Corporation}




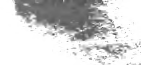




\section{THERAPEUSIS OF INTERNAL DISEASES}


$$
\text { - }
$$$$
=
$$ 


\title{
THERAPEUSIS OF
}

\section{INTERNAL DISEASES}

\author{
EDITED BY
}

FREDERICK FORCHHEIMER, M.D., Sc.D. (Harv.)

Professor of Medicine, Ohio-Miami Medical College, Lniversity of Cincinnati

GENERAL I N D E X

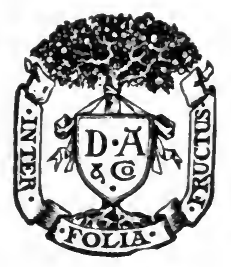

NEW YORK AND LONDON

D. APPLETON AND COMPANY 1913

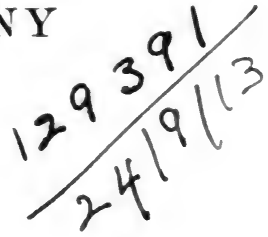


Copyright, 1913, BY

D. APPLETON AND COMPANY

Printed in the United States of America 


\section{GENERAL INDEX}

Aaron bandage, iii. 248.

АввE, Dr. Ronert, method of using radium tubes suggested by, i. 464; operation by, for relief of trigeminal neuralgia, i. 354; report by, of sarcoma of the eyelid successfully treated with racium, i. 486.

Abdonen, massage of, i. 323.

Abdominal binder, use of, for relief of constipation associated with visceroptosis and intestinal atony, iv. 566.

Abdominal massage. See Massage.

Abdominal supporter, use of, in prolapse of liver, iii. 289 ; in visceroptosis, iii. 247.

Abdominal viscera, effect of massage upon, i. 297.

Abdominal walls, measures for increasing tone of, in prolapse of liver, iii. 289.

Ablutions, cold, to chest daily, in bronchitis due to chronic myocardial insufficiency, iii. 738 .

ABortion, inducement of, in eclampsia of pregnancy, iv. 83.

Abortive treatment of syphilis, ii. 376 .

Abram, inhalation of chloroform recommended by, for relief of dyspnea due to pressure by a mediastinal tumor, iii. 503.

Abrams, double current method of colonic flushing suggested by, in intestinal autointoxication, ii. 674 ; on use of purgatives in treatment of intestinal autointoxication, ii. 671 .

Abscess, accompanying acute peritonsillitis, iii. 43; cerebral, see Cerebral abscess; of colon, mesentery, and liver, electrotherapeutics for relief of, i. 405; extradural, operative measures for relief of, iv. 422 ; of infiltration, iv. 173 ; of liver, iii. 290; of liver, as complication of influenza, ii. 190; of lungs, see Pulmonary abscess; perinephritic, complicating tuberculous kidney, iv. 11s; periurethral, complicating acute gonorrhea, iv. 148; prostatic, as cause of painful urination in acute urethral gonorrhea, iv. 141; prostatic, complicating acute gonorrhea, iv. 149 ; pretracheal, as a complication of diphtheria, ii. 279; pulmonary, see Pulmonary abseess; retropharyngeal, see Retropharyngeal abscess; of spleen, operation for, in malaria, iv. 728; tuberculous, as a complication of tuberculosis, ii. 509; of urethral glands, complicating acute gonorrhea, iv. 148 .

Abscesses, as a cause of pain in bladder, iv. 123; radiant light and heat in treatment of, i. 450 .

"Abscess fixation," in treatment of pneumonia, ii. 246.

Absorption, of exudates in pleuritis, iii. 457; of fibrin in pleuritis, iii. 458; of fluid in pleuritis, iii. 458; of gases in pleuritis, iii. 459.

“Accelerated reaction," i. 134.

AcCelerator heart fibers, iii. 538.

Accessorius spasm, iv. 655.

Acetanilid, use of, in migraine, iv. 592 ; objections to, in influenza, ii. 187; to reduce temperature in acute cerebral meningitis, iv. 372 ; for relief of headache in neurasthenia, iv. 566; for relief of headache due to sunstroke, iv. 679 ; for relief of neuralgic headache, iv. 599; for relief of pain in acute rheumatism, ii. 300 ; for relief of pain in neuralgia, iv. 339 ; for relief of pain in neuritis, iv. 286 ; for relief of pain in pyelitis, iv. $8 s$; for relief of pain in Rocky Mountain fever, ii. 565.

Acetanilid, alcoholic solution of, applied by tampon in atrophic rhinitis, iii. 332.

Acetate of ammonia. See Ammonium acetate.

Acetate of potassium. See Potassium acetate.

Acetate of sodium. See Sodium acetate. 
Acetates, use of, as diuretics in acute nephritis, iv. 58 .

Acetone Bodies, tests for, ii. 732.

Acetozone, destruction of cholera germs by, iv. 829 .

Acetphenetidin. See Phenacetin.

Acetyl atoxyl. See Arsazetin

Acetylisalicylic acid. See Aspirin.

Achalme, report by, of organism found in acute rheumatism, ii. 293.

Achilles jerk. See Tendon reflex.

Achondroplasia, ii. 812 .

ACHYlia GASTRICA AND sUBACIDITY, iii. 144.

Acidosis, Diaberic. See Diabetic acidosis.

AcIDs, overindulgence in, a cause of gastric hyperacidity, iii. 77 .

Acids, use of, to change reaction of urine in tuberculous kidney, iv. 117.

ACland, tabulation by, of dates for appearance of eruptions and complications following vaccination, ii. 80 .

AcNe, radiant light and heat in treatment of, i. 459; vaccine treatment for, i. 145; X-ray therapy for relief of, i. 529.

ACNe, indurated, treatment of, by electricity conjoined with X-ray, i. 406.

ACNE, KELOID, radium therapy for relief of, i. 475; X-ray therapy for relief of, i. 531 .

ACNE, RosACEA, radium in treatment of, i. 474 ; X-ray treatment of, i. 530 .

Acne vulgaris, radium in treatment of, i. 475 .

Aconite, use of, in active congestion of lungs, iii. 406; in acute nasopharyngitis, iii. 31 ; in acute peritonsillitis, iii. 43 ; in acute tonsillitis, iii. 41 ; in cerebral hemorrhage, iv. 391; to control epistaxis, iii. 336; effect produced by, on pulmonary circulation, iii. 405; as an expectorant in acute bronchitis, iii. 360 ; in gonorrheal epididymitis, iv. 151; in headache from ear disease, iv. 603; in migraine, iv. 593; in pneumonia with abnormally high blood pressure, ii. 255 ; for relief of cardiac palpitation in neurasthenia, iv. 568; in stomatitis catarrhalis, iii. 10.

Aconite root, tincture of, use of, in acute laryngitis, iii. 338 .

Aconitin, poisoning by, i. 764; poisonous action of, i. 765 ; poisonous dose of, $\mathrm{i}$.
765; properties of, i. 765 ; treatment of poisoning by, i. 766 .

Aconitin, use of, for relief of pain in neuralgia, iv. 340 ; in tetanus, ii. 348 .

ACquired hydrocephalus. See Hydrocephalus.

Acromegaly, relation between hyperactivity of the hypophysis and, i. 102; therapeutic use of the hypophysis for relief of, i. 107; views regarding cause of, iv. 442.

Actinium, discovery of, i. 460.

Actinomyces bovis, ii. 359.

Actinomycosis, ii. 359 ; association of empyema with, iii. 489; organism causing, ii. 359 ; treatment of, ii. 360 ; vaccine treatment for relief of, i. 147.

Activity, return to, in neurasthenia and psychasthenia, iv. 552.

Actual cautery. See Cautery.

"ACUTE sinus infection," complications due to, iii. 333.

Adams-Stokes syndrome. See Heart block.

Addison's DIsEAse, iii. 882; circulatory disturbances in, iii. 885; diet in, iii. 884; gastrointestinal symptoms in, iii. 884; nervous symptoms in, iii. 885 ; results of Koch's tuberculin in treatment of, iii. 883 ; results of organotherapy for, iii. 882 ; suprarenal insufficiency in, i. 88,96 ; transplantation for relief of, iii. 883.

Addison's pill, iii. 286.

Adenectomy, for adenoid vegetations, iii. 37 ; for hypertrophy of pharyngeal tonsil, iii. 36 ; technique of, iii. 36 .

Adenitis, accompanying glandular fever, ii. 576; complicating acute gonorrhea, iv. 149.

Adenitis, Cervical, as a complication of diphtheria, ii. 277 ; as a complication of scarlet fever, ii. 131.

Adenitis, tuberculous, effect of radiotherapy upon, iii. 848; radiant heat and light in treatment of, i. 452; X-ray therapy in treatment of, i. 344; X-ray therapy and electricity conjoined in treatment of, i. 401.

Adenoid vegetations, iii. 33, 37.

Adenolds, association of, with enuresis, iv. 130 ; removal of, a factor in prevention of acute rheumatism, ii. 296; removal 
of, necessary, in chronic rhinitis, iii. 328 ; removal of, as prophylactic measure against bronchitis, iii. 352; removal of, as prophylactic measure against tuberculosis of mediastinal lymph nodes, iii. 506.

Adenolds, Drseased, associated with chronic nasopharyngitis, iii. 32, 33; removal of, iii. $33,36,37$.

Adnesions, as cause of chronic dilatation of stomach, iii. 179.

Adhesive plaster. See Plaster.

Adrondack Cottage Sanatorium, plan of admission to, ii. 471 .

Adnephrin. See Epinephrin.

Adolescence, prophylaxis against the neuroses in, iv. 522.

Adonis vernalis, fluid extract of, use of, combined with sodium bromid for relief of restlessness in neurasthenia, iv. 567.

Adrenal gland, extract of, use of, in Addison's disease, iii. 882 .

Adrenalin, use of, in acute dilatation of stomach, iii. 174, 175; in acute nasopharyngitis, iii. 32 ; in acute rhinitis, iii. 327 ; in acute suppurative inflammation of nasal sinuses, iii. 333 ; in acute uvulitis, iii. 35 ; in asthenic form of measles, ii. 153; in carcinomatous stenosis of esophagus, iii. 55 ; in cardiac toxemias of pneumonia, ii. 252 ; in collapse due to thoracentesis, iii. 482 ; contraindicated in acute pulmonary edema, iv. 55; for control of gastric hemorrhage, iii. 113; for control of hemorrhage in tuberculosis, ii. 500; for control of hemorrhage in typhoid fever, ii. 20 ; in endemic hematuria, iv. 784; in hematuria, iv. 4 ; in hemophilia, iii. 867,870 ; in hypertrophy of pharyngeal tonsil, iii. 35 ; by hypodermic injection in bronchial asthma, iii. 387 ; by hypodermic injection in cardiac weakness of scarlet fever, ii. 127 ; by instillation in acute rhinitis of young children, iii. 328 ; by intramuscular injection in collapse associated with renal colic, iv. 106; by intravenous injection a cause of experimental pulmonary edema, iv. 55; by intravenous injection in post-hemorrhagic anemia, iii. 820 ; for lavage of stomach in gastric hemorrhage, ii. 155; necessity for caution in, for acute rhinitis, iii. 328 ; in neurasthenia with vasomotor relaxation, iv. 65.9 ; objections to, for pulmonary odema occurring during thoracentesis, iii. 480; in paralytic ileus, iii. 238 ; in pulmonary edema with low blood pressure, iii. 410; for relief of dyspnea in chronic pulmonary emphyserna, iii. 435; in septicopyemia, ii. 291; as spray during adenectomy, iii. 36; as spray in adenoid vegetations, iii. 38; as spray in bronchial asthma, iii. 386; as spray, for "colds" accompanying tuberculosis, ii. 508 ; as spray in edematous laryngitis, iii. 341 ; as spray in hay fever, iii. 391; as spray in hemorrhagic form of measles, iii. 155; as spray in otitis media complicating scarlet fever, ii. 131; to stimulate absorption of pleural exudate, iii. 482; on tampon for control of epistaxis, iii. 335 ; in tonsillectomy, iii. 45 ; in Vincent's angina for relief of pain in swallowing, iii. 65; see also Epinephrin.

Adrenalin and eucain hydrochlorate, use of, as spray for nasal passages, iii. 334 .

Adrenalin ointment, use of, in hypertrophy of pharyngeal tonsil, iii. 35 .

Adrenals, disease of, iii. 882 ; relation between over-function of, and arteriosclerosis, iii. 757 .

Adrenin. See Epinephrin.

Adrian, isolation of influenza bacillus by, ii. 190.

Adrin. See Epinephrin.

Adsorptios, i. 22.

Adults, prophylaxis against the neuroses in, iv. 523.

Aerophagia, associated with cardiac reflex neuroses, iii. 779; treatment of, iii. 793 .

Aerotherapy, employment of, in chronic pulmonary emphysema, iii. 434.

Asculus hippocastanum. See Horsechestnut.

Esculus pavia. See Buckeye.

Afebrile pxedmonia. See Pneumonia, afebrile form of.

Afferent heart fibers, iii. 539.

Affusion, cold, use of, in delirium tremens, ii. 606 .

African millipedes, poisonous, iv. 656.

African poison test, iv. 695.

Agar-agar, use of, in ehronic constipation, iii. 235; in constipation in arteriosclerosis, iii. $75 \mathrm{~s}$; in constipation due to 
cardiac insufficiency, iii. 684; in constipation due to chronic myocardial insufficiency, iii. 740 ; in constipation accompanying tuberculosis, iii. 505.

Age metabolism, i. 605.

Agglutination reaction, value of, in diagnosis between typhoid and paratyphoid infections causing bacillary dysentery, ii. 642 .

AgGlutinins, action of, i. 131.

Agminate folliculitis, X-ray therapy for relief of, i. 533.

Agramonte, on use of liquids in yellow fever, iv. 856.

Agraphia, motor, iv. 440.

Agrostemma githago. See Corn cockle.

Ainhum, chiggers as a factor in causation of, iv. 690; removal of toe ior, iv. 691 .

AIR, artificial introduction of, into stomach, iii. 779; humidity of, i. 251.

Air, sterile, intrapleural injection of, to stimulate absorption of exudate, iii. 482.

Air bath. See Bath.

AIR GUlPING, in angina pectoris, iii. 770, 771 ; in reflex neuroses of gastrointestinal disturbances, iii. 779 .

Aitken, on mortality from small-pox before and after vaccination, ii. 93 .

Akatama, iv. 863.

AKer laMpong, iv. 694.

Albers-Schönberg, experiments by, on exposure to secondary radiation in deep X-ray therapy, i. 507; summary by, of action of X-ray on uterine fibroids, 555 .

Albolene, liquid, use of, in chronic constipation, iii. 235.

Albumin in sputum, method of examining for, in chronic bronchitis, iii. 710 .

Albuminolysins, nature of, i. 132.

Albuminuria, significance of, as functional kidney test, iv. 23.

Albumoses, use of, as nutrient enemata in gastric ulcer, iii. 118.

AlcOHоL, action of, in diabetes, ii. 744, 757; action of, in obesity, ii. 805; as a cause of acute hemorrhagic superior polioencephalitis, iv. 406; as a cause of arteriosclerosis, iii. 755 ; as a cause of chronic gastritis, iii. 158; as a cause of chronic myocardial insufficiency, iii. 707, 729; as a cause of chronic rhinitis, iii. 328; as a cause of gastric hyperacidity, iii. 76 ; as a cause of leukoplakia, iii. 25; as a cause of reflex cardiac neuroses, iii. 786; contraindications to use of, in chancre of lips, mouth, or tongue, ii. 425; contraindications to use of, in gout, ii. 698; contraindications to use of, in intestinal autointoxication, ii. 677; contraindications to use of, in nephritis, iv. 44; contraindications to use of, in syphilis, ii. 369; danger of using, in acute infections with risk of nephritis, iv. 57 ; of doubtful value in acute vasomotor paralysis, iii. 753 ; as indirect cause of occupation neuroses, iii. 660,661 ; influence of, on obesity, iii. 706; moderate use of, in chronic gonorrheal urethritis, iv. 153; moderate use of, in chronic interstitial nephritis, iv. 68; moderate use of, in spastic spinal paralysis, iv. 270; objections to, in nutrient enemata, for gastric ulcer, iii. 118 ; oxidation of, in body, ii. 783 ; prohibition of, during acute laryngitis, iii. 340 ; prohibition of, in acute urethral gonorrhea, iv. 138; prohibition of, in aneurysm of cerebral arteries, iv. 457; prohibition of, in asthma, iii. 381; prohibition of, in cardiac insufficiency, iii. 687; prohibition of, in cerebral hyperemia, iv. 385 ; prohibition of, in chronic "catarrhal jaundice, iii. 263; prohibition of, during chronic laryngitis, iii. 340; prohibition of, in chronic myocardial insufficiency due to obesity, iii. 727; prohibition of, in chronic myocardial insufficiency due to overstimulation, iii. 728 ; prohibition of, in chronic ophthalmoplegia, iv. 510; prohibition of, in chronic pharyngitis, iii. 33; prohibition of, in cirrhosis of liver, iii. 280; prohibition of, in exophthalmic goiter, iii. 897; prohibition of, in multiple neuritis due to alcoholism, iv. 322 ; prohibition of, in nephrolithiasis, iv. 109 ; prohibition of, in palpitation of heart in neurasthenia, iv. 567; prohibition of, in progressive paralysis of the insane, iv. 493; prohibition of, in pyelitis, iv. 88; prohibition of, for relief of tachycardia, iii. 787; restriction of, in angina pectoris, iii. 770 ; restriction of, in cardiac insufficiency, iii. 687; restriction of, in cholecystitis and cholelithiasis, iii. 271; restriction of, in 
chronic myocardial insufficiency due to alcohol, iii. 728; restriction of, in pernicious anemia, iii. 826 ; sponging with, to control night sweats, iv. 117; tolerince for fats increased by, ii. 738; use of, ats food, ii. 602; vertigo due to use of,iv. 624 .

Alcohol, therapeutic use of, in acute bronchitis, iii. 35.t; in Addison's disease, iii. 884 ; in ascites, iii. 287 ; in asthenic type of measles, ii. 152 ; in bacillary dysentery, ii. 227 ; in barbel cholera, ii. 644 ; in bronchopneumonia, iii. 420 ; in cerebrospinal meningitis, ii. 216 ; in chronic bronchitis, iii. 378 ; by deep injection for relief of pain in laryngeal tuberculosis, iii. 347 ; in delirium tremens, ii. 603, 607; in diphtheria, ii. 276; in gastric hyperacidity, iii. 90 ; in influenza, ii. 90 ; locally in echinococeus disease, iii. 256; locally, in small-pox, ii. 49 ; in malignant endocarditis, iii. 746 ; in night sweats of tuberculosis, ii. 505; as oral antiseptic in prevention of bronchopneumonia, iii. 413; in Pasteur treatment of rabies, ii. 339 ; in pharyngitis, iii. 40; in pneumonia, ii. 229, 230, 255; in pneumonia of alcoholics, ii. 258; in pneumonia complicating influenza, ii. 192; for relief of insomnia due to cerebral anemia, iii. 383; in Rocky Mountain fever, ii. 566; in scarlet fever, ii. 127; in septicopyemia, ii. 290; in smallpox, ii. 47; as stimulant in cerebral anemia, iv. 382 ; as stimulant in cerebral thrombosis, iv. 399; as stimulant in pneumothorax with empyema, iii. 493; as stimulant in snake poisoning, iv. 683; in toxemia complicating acute diffuse peritonitis, iii. 318 ; in treatment of alcoholism, ii. 598; in tuberculosis, ii. 504; in tuberculosis of larynx, 346; in typhoid fever, ii. 14, 23, 26.

Alcohol compresses. See Compresses.

Alcohol injections, for cervico-oceipital neuralgia, iv. 356; for herpes zoster, iv. 360 ; for intercostal neuralgia, iv. 357; for neuritis of external cutaneous nerve, iv. 293; for neuritis of third, fourth, and sixth nerves, iv. 308 ; for relief of spasticity and over-action of muscles after cerebral palsies, iv. 416; for spasmodic movements in tic, iv. 654, 659; use of, contraindicated in sciatica, iv. 300 .
Alcohol injections for trigeminal neuralgia, iv. 341 ; at cranial foramina, iv. 346 ; at foramen ovale, iv. 348 ; at foramen rotundum, iv. 351; at infraorbital foramen, iv. 344; at mental foramen, iv. 346; of palatine nerve, iv. 346; solution used for, iv. 343 ; at superficial foramina, iv. 344 ; of supraorbital branch, iv. 344; technique of deep injection, iv. 347 ; technique of superficial injection, iv. $343,344,345$.

Alconolic beverages, as aids to digestion in achylia gastrica, iii. 148; as aids to digestion in depressive disorders of gastric secretion, iii. 148; contraindicated in acute infections with risk of nephritis, iv. 57 ; contraindicated in chronic interstitial nephritis, iv. 65 ; cont raindicated in cirrhosis of liver, iii. 280; objections to, in ehronic gastritis, iii. 148; objections to, in ehronic progressive bulbar paralysis, iv. 251; objections to, in chronic sclerosis, iii. 758; prohibition of, in brain tumor, iv. 447; prohibition of, in prophylaxis of eholera, iv. 829 ; for relief of insomnia in neurasthenia, iv. 564; useless in seasickness, iv. 625 .

Alcoholic stimulants. See Stimulants.

Alcoholics, pneumonia in, ii. 258.

Alconolism, ii. 587; acute form of, ii. 588; acute hallucinosis due to, ii. 608; atropin and strychnin treatment of, ii. 595, 596; causation of, ii. 590; in causation of brain syphilis, iv. 479 ; in causation of occupation pareses, iv. 332 ; chronic form of, ii. 590; delirium tremens due to, ii. 601; dipsomania a form of, ii. 591; headache a symptom of, iv. 610; hydrotherapy for relief of, i. 22s; hygienic measures in treatment of, ii. 599; hypnotic suggestion in treatment of, ii. 600 ; intestinal autointoxication associated with, ii. 683; Keeley gold cure for, ii. 600; Lambert's method of treatment for, ii. 597; McBride's method of treatment for, ii. 595; periodic form of, ii. 591; prophylaxis against, ii 592; Town's specific treatment for, ii. 597 ; treatment of, in general, ii. 593; treatment of multiple neuritis due to, iv. 322 ; treatment of, in a sanatorium, ii. 594; types of, 591 ; varieties of, iii. 587; wood alcohol poisoning in relation to, ii. 609 . 
Alexander's modification of Curling's BANDAGE, for use in acute gonorrhea as preventive of epididymitis, iv. 150.

Alexin, i. 128.

Alimentary diseases, hydrotherapy for relief of, i 241.

Alimentary glycosuria. See Glycosuria.

Alimentary hypersecretion, iii. 101.

Alimentary tract, effect of epinephrin upon, i. 91.

Alimentation, normal serum as means of, i. 173.

Alkalies, use of, in acute endocarditis, iii. 745 ; in acute or intermittent gastric hypersecretion, iii. 102 ; in acute peritonsillitis due to rheumatism, iii. 43 ; in acute urethral gonorrhea, iv. 139; in carcinoma of stomach, iii. 165 ; to change reaction of urine in tuberculous kidney, iv. 117; in continuous gastric hypersecretion, iii. 106; in diabetes before surgical operations, ii. 769 ; in diabetic acidosis, ii. 756; in diabetic coma, ii. 757; as expectorants in chronic bronchitis, iii. 372 ; in gastric hyperacidity, iii. 93,100 ; in gastric hyperacidity with hypermotility, iii. 101; in gastric lavage, iii. 180; in gastritis acida, iii. 101; with iodids in syphilitic gastritis, iii. 167; in non-bleeding gastric ulcer, iii. 133; in secretory and motor gastric neuroses, iii. 188.

Alkaline baths. See Baths.

Alkaline diluents, use of, in acute cystitis, iv. 120 .

Alkaline disinfectants, value of, in disinfection for tuberculosis, ii. 452.

Alkaline diuretics, use of, in acute nephritis with edema, iv. 62 ; in chronic parenchymatous nephritis, iv. 65 ; in nephritis, iv. 49 .

Alkaline drinks, use of, in acute bronchitis, iii. 354 ; in acute phosphorus poisoning, ii. 628 .

Alkaline expectorant mixture, for use in chronic bronchitis, iii. 373 .

Alkaline mineral waters, use of, in acute articular rheumatism, ii. 298; in chronic gout, ii. 703; in intestinal autointoxication, ii. 677 ; in pneumonia of diabetics, ii. 259.

Alkaline powders, use of, in acute gastritis, iii. 155 ; in after-treatment of acute gastritis, iii. 157.
Alkaline salt solution, intravenous or rectal injection of, in blackwater fever, iv. 741 .

Alkaline salts, use of, in pyelitis, with acid urine, iv. 90; for relief of anuria due to nephrolithiasis, iv. 107.

Alkaline solutions, irrigation with, in acute peritonsillitis, iii. 43 ; local use of, in atrophic rhinitis, iii. 331; use of, to allay itching from stings or bites, iv. 686 ; for lavage in gastric atony, iii. 172.

Alkaline sprays, use of, during attack of hay fever, iii. 390; in local treatment of pharyngeal cough, ii. 495.

Alkaline steam inhalations, use of, in acute laryngitis, iii. 338 ; in edematous laryngitis, iii. 341 ; in membranous laryngitis, iii. 343 ; in spasmodic laryngitis, iii. 342 .

Alkaline substances, use of, in onyalai, iv. 861.

Alkaline treatment, use of, in acute rheumatism, ii. 301.

Alkaline wash, use of, in laryngeal tuberculosis, ii. 493.

Alkaline waters, action of, i. 236; classification of, i. 233; use of, in acute urethral gonorrhea, iv. 139; use of, in after-treatment of acute gastritis, iii. 157 ; use of, in diet for gastric hyperacidity, 91; use of, in gastric lavage for hyperacidity, iii. 98 ; use of, in gastritis acida, iii. 101; use of, in nephrolithiasis, iv. 109 .

Alkaline-saline waters, inhalation of, in bronchitis due to chronic myocardial insufficiency, iii. 738 .

AlKalinity of URINe, as a cause of enuresis, iv. 128.

Alkaloidal poisons, antidote for, i. 672.

Alkaloids, solanaceous, use of, in treatment of morphinism, ii. 620 .

"All or Nothing" LAW of Bowditch, iii. 533.

Allbutt, Sir Clifford, method of using salt-free diet in cirrhosis of liver described by, iii. 286 ; on value of mountain resort in neurasthenia, iv. 556 .

"Allergie," definition of, i. 132.

Allison, Nathaniel, method of operation for cerebral palsies of children introduced by, iv. 416 .

Allorrhythmias, iii. 564; classes of, iii. 564 ; of extracardiac origin, iii. 564; 
due to extrasystoles, iii. 566; due to faulty conduction of impulses, iii. 566; of intra-cardiac origin, iii. 565; psychogenic, iii. 565; reflex, iii. 565); youthful type of, iii. 56.5.

Almateina, use of, for diarrhea in pellagra, ii. 662 .

Aloes, use of, in chronic constipation, iii. 233 ; in chronic intestinal obstruction, iii. 242 ; in nephritis, iv. 33 .

Aloin, use of, in chronic constipation, iii. 233 ; to correct constipation in tuberculous kidney, iv. 117.

Aloin, belladonna, and strychnin, pills of, as a laxative after hemorrhage in pulmonary tuberculosis, ii. 501.

Alopecia, as a result of X-ray therapy, i. 516.

Alopecia, syphilitic, local treatment of, ii. 428.

Alpha RAYs, i. 462 ; penetrative power of, i. 465; use of, in emanation of radium, i. 466 .

Alphazone, destruction of cholera germs by, iv. 829 .

Alteratives, use of, in ozena, iii. 336.

Alternating currents, explanation of term in electrotherapeutics, i. 365.

Altitude, benefit from, in secondary anemias of certain kinds, iii. 810; contraindicated in pernicious anemia, iii. 825; effect of, in cardiac insufficiency, iii. 680 ; influence of, in chronic parenchymatous nephritis, iv. 30; influence of, in convalescence from beri-beri, iv. 855; influence of, in neurasthenia, iv. 556 ; relation of, to causation of beriberi, iv. 845 ; relation of, to climate, $\mathrm{i}$. 255.

Alum, external use of, in small-pox, ii. 49; external use of, in varicella, ii. 112; use of, as gargle in acute nasopharyngitis, iii. 31 ; use of, as gargle in acute tonsillitis, iii. 42 ; use of, as gargle, in acute uvulitis, iii. 35; use of, as gargle, with carbolic acid, in chronic pharyngitis, iii. 33 ; use of, as gargle, with potassium chlorate, in chronic pharyngitis, iii. 33; use of, as spray in chronic laryngitis, iii. 340 .

Aluminium glycerin paste. See Escalin.

Aluminium preparations, use of, in gastric hyperacidity, iii. 97.
Aluminium silicate. Sce Kaolin.

Alumnol, intralaryngeal application of, in acute laryngitis, iii. 340; use of, in chronic nasopharyngitis, 33 ; use of, as spray in chronic laryngitis, ii. 340 .

Alypin, use of, as anesthetic in chronic rhinitis, iii. 329 ; in treatment of bite by rabid animal, ii. 336 .

Amanita muscakia, ii. 648.

Amanita phalloides, ii. 648; poisoning by, ii. 649 .

Amanitotoxin, ii. 649.

Amaurosis, tabetic, iv. 199.

Амвосертонs, nature of, i. 129, 131.

Aмeв E, concerned in intestinal amebiasis, iv. 703; mode of entrance and exit of, iv. 705; resistance of, to destructive agencies, iv. 705; species of, iv. 704.

Amebiasis, iv. 703.

Amebiasis, hepatic, iv. 710; after-treatment of, iv. 711; exploratory puncture in diagnosis of, iv. 711 ; prophylaxis against, iv. 710 ; treatment of, iv. 711.

Amebiasis, intestinal, iv. 703; distribution of, and of species concerned in, iv. 703; drug treatment of, by mouth, iv. 708; general nature of, iv. 707; hygienic treatment of, iv. 708; local treatment of, by enemata, iv. 709; mode of entrance and exit of parasites in, iv. 705 ; pathogenicity of species concerned in, iv. 704; resistance of amebre to destructive agencies in, iv. 705; rôle of symbiotic bacteria in, iv. 706 ; species of ameba present in, iv. 704; treatment of, iv. 708.

AmenorrheA, hydrotherapy for relief of, i. 211 ; as symptom of tuberculosis, ii. 507.

America, introduction of vaccination into, ii. 61 .

Ammonia, aromatic spirits of. See Aromatic spirits of ammonia.

Ammonia, poisoning by, i. 745 .

Ammonia, inhalation of, for prevention of collapse in snake poisoning, iv. 683; inhalation of, for relief of cerebral anemia, iv. 382; inhalation of, for relief of syncope due to cerebral anemia, iv. 39s; use of, internally, to control epistaxis, iii. 336 .

Ammonia water, local use of, in itching complicating diabetes mellitus, ii. 764 ; 
use of, for relief of local edema in snake bite, iv. 682 .

Ammonium acetate, use of, in nephritis, iv. 49.

Ammonium carbonate, use of, in chronic pulmonary emphysema, iii. 438; as expectorant, in acute bronchitis, iii. 362 .

Ammonium chlorid, use of, in bronchitis complicating influenza, ii. 192; in bronchopneumonia complicating influenza, ii. 189; in chronic bronchitis, iii. 372 ; in chronic pulmonary emphysema, iii. 438; in lobar pneumonia, to keep urine alkaline, ii. 284; in lozenges in acute laryngitis, iii. 339; in pulmonary tuberculosis as expectorant, ii. 498; to remove bronchial secretion in acute bronchitis, iii. 365 .

Ammonium iodid, use of, in syphilis, ii. 409.

Amperage, explanation of term, i. 364.

Amplitude of PUlse, as indicator of effective digitalis action, iii. 580 .

Amputation, question of, in diabetic gangrene, ii. 770.

Amusements, as a factor in treatment of tuberculosis, ii. 488 .

Amyl nitrite, use of, in angina pectoris, iii. 768; for cardiac failure in beri-beri, iv. 854 ; in cardiac insufficiency, iii. 606, 610; in chronic interstitial nephritis, iv. 71; during paroxysm of asthma, iii. 385 ; in paroxysmal hemoglobinuria, iv. 7; in pulmonary tuberculosis to control hemorrhage, ii. 500; in strophanthus poisoning, iv. 695; in tetanus, ii. 348 .

Amylene hydrate, use of, for relief of insomnia in neurasthenia, iv. 565 .

Amyloid degeneration, due to phosphorus poisoning, ii. 630 .

Amyloid diseases of KIDNEY, iv. 81 ; kidney of pregnancy, iv. 82 .

Amylum, use of, as substitute for sugar in nutrient enemata, iii. 119.

Amytropic LATERAL sclerosis, 258; treatment of, iv. 259.

AmyXorrhea gastricA, iii. 100.

Anadon compress, use of, in hemophilia, iii. 867 .

Anakhre. See Goundoo.

Anal fissure See Fissure of anus.

Analgesia, in diagnosis of tabes, iv. 188.

Analgesics, use of, in migraine, iv. 592; for relief of late pain in herpes zoster, iv.
359 ; for relief of neuralgias accompanying chlorosis, iii. 824; in trigeminal neuritis, iv. 309.

Analysis, Gastric, value of, in diagnosis of carcinoma of stomach, iii. 163.

ANAPHYLAXIS, associated with gastrointestinal intoxication, ii. 682 ; as explanation of affinity between the skin and putrefactive substances, ii. 682; following antitoxin treatment of diphtheria, ii. 271 .

ANASARCA, GENERAL, accompanying cardiac insufficiency, iii. 672 ; elimination of fluid through skin for relief of, iii. 678; methods of diuresis for relief of, iii. 676; removal of edema in, iii. 674 ; removal of fluid by intestines for relief of, iii. 678 ; technique in puncture of skin for relief of, iii. 676 ; treatment of, in severe cases of chronic myocardial insufficiency, iii. 720 .

Anchylostoma duodenale, iv. 791.

Anemia, associated with intestinal autointoxication, 680; as cause of neuralgia, iv. 336 ; as complication of acute rheumatism, ii. 303 ; following acute infectious myocarditis, 751; headache as a symptom of, iv. 611; hydrotherapy for relief of, i. 206; influence of climate upon, i. 280; mechanotherapy for relief of, i. 348 ; as a predisposing cause of rachitis, ii. 814; Spa treatment for, i. 239 ; as a symptom of acute nephritis, 63 ; as a symptom of tuberculosis, ii. 507 ; treatment of, when associated with chronic myocardial insufficiency, 741.

Anemia of brain. See Cerebral anemia.

Anemia, Cerebral. See Cerebral anemia. Anemia, infantile splenic. See Infantile splenic anemia.

Anemia, PERnicious, iii. 824; antiseptic treatment for, iii. 837; arsenic in, iii. 826; blood examination in, iii. 824; cholesterin in, iii. 835; constipation accompanying, iii. 837; diet in, iii. 825; gastrointestinal symptoms in, iii. 837; glycerin for, iii. 836; hemolysin treatment of, iii. 834; hemorrhages during, iii. 837; hydrochloric acid for, iii. 826; organotherapy for, iii. 836; oxygen inhalations for, iii. 826; Pappenheim's classification of, iii. 805, 824; plasmotherapy for, iii. 833; pyorrhea alveolaris 
associated with, iii. 22 ; serum therapy for, iii. 832; symptoms of, iii. 824; transfusion of blood in, iii. 829; treatment of, in general, iii. 825; treatment of special symptoms in, iii. 837; X-ray treatment of, iii. 826.

Anemia, post-hemorrhagic, iii. 808; transfusion of blood by subcutaneous method employed for, iii. 831.

Anemias, iii. 802: chlorosis, iii. 811 ; general introduction to subject, iii. 802; Hodgkin's disease, see Pseudoleukemia; leukemia, iii. 844; Naegeli's classification, iii. 806; Pappenheim's classification, iii. 805; pernicious anemia, iii. 824 ; pseudoleukemia, iii. 854 ; secondary anemias, iii. 808.

Anemias, cryptogenetic, iii. 807.

Anemias, phanerogenetic, iii. 808.

Anemias, primary myelogenetic, iii. 807.

Anemias, Secondary, iii. 805,808 ; posthemorrhagic, iii. 808; toxic, iii. 809; treatment of, iii. 809.

Anemias, secondary myelogenetic, iii. 807.

Anemias, toxic, iii. 809.

Anesthesia, in diagnosis of tabes, iv. 188; of tongue, iii. 3.

Anesthesia, general, danger of, in diabetic patients, ii. 769; edema of lungs sometimes caused by, iii. 407 ; precautions regarding, in chronic myocardial insufficiency, iii. 742 ; use of, contraindicated in thoracentesis, iii. 474; use of, in tonsillectomy, iii. 45.

Anesthesia, local, advantages of, in diabetes mellitus, ii. 770; in tonsillectomy, iii. 45.

Anesthesia, nitrous-oxid-oxygen, advantages of, in diabetes mellitus, ii. 770 .

Anesthesin, use of, for relief of pain in laryngeal tuberculosis, iii. 347.

Anesthetics, local, action of, enhanced by use of epinephrin, i. 99 ; use of, by injection into nerve in sciatica, iv. 300 .

ANEurysm, iii. 763 ; as a cause of compression bulbar paralysis, iv. 501; associated with angina pectoris vera, iii. 768 ; dietetic treatment of, iii. 765; gymnastics contraindicated in treatment of, iii. 765 ; medicinal treatment of, iii. 765 ; spontaneous cure of, iii. 763 ; surgical treatment of, iii. 766 .
ANeurysm of ascending arch of aOrTa, as a cause of cerebral embolism, iv. 400 .

Aneurysm of ceremral artery, iv. 456; differential diagnosis of, iv. 457; etiology of, iv. 456; introduction to subject of, iv. 456 ; pathology of, iv. 456 ; prognosis of, iv. 457 ; symptoms of, iv. 456 ; treatment of, iv. 457.

Angina ludovici, iii. 30.

Angina pectoris iii. 767; blood pressure raised by, iii. 555; elinical conditions associated with, iii. 767,768 ; diet in iii. 770; Nauheim baths for relief of, iii. 624 ; treatment of attack of, iii. 768 ; treatment of, in interim, iii. 769 ; value of nitrites in, iii. 768 .

Angina pectoris nervosa, in cardiac neurasthenia, iii. 778 .

ANGINA PECTORIS VASOMOTORIA, iii. 768.

Angina pectoris vera, iii. 767.

Angina, syphilitic, local treatment of, ii. 426.

Angina Vincent's. See Vincent's angina. Angiomata, electrolysis in treatment of, i. 397 ; radium in treatment of, i. 476 .

ANilin DYes, food poisoning due to, ii. 634 .

Animal boDY, disposition of poisons by, $i$. 666.

Animal extracts, use of, in acquired spinal progressive muscular atrophy, iv. 248; in asthenic bulbar paralysis, iv. 256 ; in muscular dystrophy, iv. 264.

Animal organisms, food poisoning due to, ii. 634 .

Animal poisons, i. 674.

Animals, as carriers of infection in trypanosomiasis districts, iv. 749; laws controlling tuberculosis in, in Denmark, ii. 442; relation of, to spread of plague, iv. 818 .

Anisated liquor ammonii, use of, as an expectorant in pneumonia of children, ii. 257.

Anodynes, use of, in acute urethral gonorrhea, iv. 141; to relieve spasm in acute laryngitis, iii. 340 .

Anopheles mosquito, manner of destroying, iv. 730; protection of individual from, iv. 734; transmission of malaria by, iv. 729 .

Anorexia, due to ehlorosis, iii. 822 ; as a symptom of Addison's disease, iii. 884; as a symptom of neurasthenia, iv. 566; as a symptom of tuberculosis, ii. 504 . 
Anosmia, symptoms of, in diagnosis of brain tumor in frontal lobe, iv. 439.

ANTAGONISM BETWEEN RADIANT LIGHT AND HEAT AND Röntgen RAY, i. 447.

Anterior CruRal Nerve, neuritis of, iv. 293.

Anthelmintics, use of, for relief of trichiniasis, iii. 254; in tricocephalus dispar, iii. 255 .

Anthrax, ii. 330; bacteriology of, ii. 330 ; immunization against, ii. 331; mortality of, affected by serum treatment of, ii. 332 ; prophylaxis of, ii. 330 ; serum treatment of, ii. 331 ; sources of infection in, ii. 330 ; transmission of, ii. 330 .

Antiaggressins, i. 148.

Antianthrax serum, ii. 331 ; combined method of using, ii. 331; mortality of anthrax affected by use of, ii. 332 ; production of, ii. 331 ; technique employed in use of, ii. 332 ; value of, as an immune serum, i. 167.

Antibodies, action of, i. 131; method of stimulating formation of, i. 138; presence of, in acquired immunity, i. 130.

Anticholera serum, i. 167.

Anti-colon bacillus serum, i. 166 .

Anticyclones, i. 254.

Antidiphtheritic serum, use of, in membranous pharyngitis, iii. 38 ; in membranous rhinitis, iii. 329 ; in ozena, iii. 337 ; in treatment of whooping-cough, ii. 179 .

Antidotes, administration of, in treatment of poison, i. 671 ; for alkaloidal poisons, i. 672; for corrosive poisons, i. 671 .

Antidysenteric serum, i. 165.

Antifebrin, use of, for relief of lancinating pains in tabes, iv. 196.

Antiferment treatment, i. 171.

Antifermentative remedies, use of, in gastric atony, iii. 171.

Antigens, forms of, for injection, i. 138; nature of, i. 130.

Antigonococcus serum, i. 165.

Anti-influenza serum, i. 162, ii. $194, ' 213$.

Antileproi, use of, in leprosy, iv. 815.

Antimeningitic serum, discovery of, i. 161; dose of, ii. 207; frequency of administration of, ii. 207; indications for use of, ii. 206 ; preparation of, i. 162 ; summary of results from use of, ii. 211 ; technique employed in use of, ii. 204; use of, in cerebrospinal meningitis, ii. 202; use of, in chronic and resistant cases, ii. 209; use of, in prolonged cases, ii. 208; use of, in relapses, ii. 209.

Antimeningococcus serum. See Antimeningitic serum.

Antimonial compounds, use of, for trypanosomiasis, iv. 747.

Antimonial poisoning, i. 688; dose causing, i. 691; poisonous action of antimony, i. 689 ; post-mortem appearances in, i. 691 ; preparations causing, i. 689 ; treatment of, i. 691 .

Antimonial wine. See Antimony, wine of.

Antimony, poisoning by. See Antimonial poisoning.

Antimony, use of, as expectorant in acute bronchitis, iii. 360 ; as expectorant in chronic bronchitis, iii. 373 ; in tropical relapsing fever, iv. 760 .

Antimony and ipecac, use of in spasmodic laryngitis, iii. 342 .

Antimony sodiotartrate, use of, in human trypanosomiasis, iv. 747.

Antimony, wine of, use of, in spasmodic laryngitis, iii. 342 .

Antineuralgic drugs, use of, for relief of pudendo-hemorrhoidal neuralgia, iv. 357.

Antineuralgics, use of, in irritative gastric neuroses, iii. 188; in neuralgic headache, iv. 596 .

Antiphlogistic preparations, use of, in spasmodic laryngitis, iii. 342.

Antiplague serum, i. 167.

Antipneumococcus serum, dose of, in pneumonia, i. 165, ii. 240; preparation of, i. 164; results from use of, in treatment of pneumonia, ii. 239, 240; use of, in eye practice, i. 165 ; use of, in pneumonia, i. 165, ii. 238; use of, in prophylaxis against pneumonia, ii. 240.

Antipyretics, use of, in acute hemorrhagic encephalitis, iv. 405; use of, contraindicated in acute diffuse peritonitis, iii. 318 ; to control fever in shima mushi, iv. 692 ; in tuberculosis of mediastinal lymph glands, iii. 508 .

Antipyrin, use of, in acute endocarditis, iii. 745; in acute gout, ii. 703 ; in acute polymyositis, ii. 685 ; in acute rheumatism, ii. 300 ; in apoplectic form of vestibular vertigo, iv. 629 ; in asthenic 
form of measles, ii. 154; to control sweating in malignant endocarditis, iii. 747; to deplete circulation in cerebral hyperemia, iv. 386 ; in diabetie neuralgia and neuritis, ii. 766 ; by hypodermic injection for relief of museular pain in Weil's disease, ii. 573; in laryngismus stridulus, complieating rachitis, ii. 820 ; in measles, ii. 150, 151, 152, 153; in migraire, iv. 592 ; in mumps, ii. 170 ; in neuralgia, complieating acute rheumatism, ii. 304 ; to reduce temperature in acute cerebral meningitis, iv. 372 ; to reduce temperature in dengue, iv. 859 ; for relief of headache, due to cerebral thrombosis, iv. 399; for relief of headache in chronic interstitial nephritis, iv. 72 ; for relief of headache in pachymeningitis interna hemorrhagica, iv. 364 ; for relief of insomnia due to brain tumor, iv. 447; for relief of muscular pain in trichiniasis, iii. 254; for relief of pain in dengue, iv. 859 ; for relief of pain in neuralgia, iv. 339 ; for relief of pain in neuritis, iv. 286; for relief of syringomyelia, iv. 278; in Rocky Mountain fever, ii. 565; in scarlet fever, ii. 126; in small-pox, ii. 47 ; in tetany, iii. 910 ; in varicella, ii. 111; in whooping-cough, ii. 181.

Antipyrin and cocain, loeal use of, in acute nasopharyngitis, iii. 32 .

Antirabic serum, ii. 339 .

Antisepsis, intestinal. See Intestinal antisepsis.

Antiseptic alkaline solutions, use of, in adenoid vegetations, iii. 38 ; in ozena, iii. 336 .

Antiseptic remedies, use of, in gastric atony, iii. 171.

Antiseptic solutions, use of, in gastric lavage, iii. 180; in lavage for chronic gastritis, iii. 161; in lavage for gastric atony, iii. 172; in leprosy, iv. 816.

Antiseptic treatment, for pernicicus anemia, iii. 837 .

Antiseptics, use of, in pulmonary abscess and gangrene, iii. 440.

Antiseptic, intestinal, use of, in chronic enteritis, iii. 200; in enterocolitis complicating measles, ii. 160 ; in intestinal autointoxication, ii. 674 ; with iron in chlorosis, iii. 820; in peristaltic unrest, iii. 250 ; in trichiniasis, iii. 254 ; unsatis- factory in chronie stage of baeillary dysentery, ii. 328.

Antiseptic, urinary, use of, in acute urethral gonorrhea, iv. 139; in pyelitis, iv. 89, 90.

Antisklerosin, use of, in arteriosclerosis, iii. 762.

Antispasmodics, use of, to prevent recurrence of spasmodic laryngitis, iii. 342.

Antistaphylococcus serum, i. 166.

Antistreptococcic serum, preparation of, i. 163; results of, in general, i. 163; use of, for control of hemorrhage in hemorrhagic form of measles, ii. 155; use of, in scarlet fever complicated by sepsis, ii. 128 .

Antisyphilitic treatment, for cerebral thrombosis due to syphilitic endarteritis, iv. 399 ; in chronic myelitis, iv. 230; in chronic ophthalmoplegia, iv. 510; in compression bulbar paralysis, iv. 502; in epilepsies of cerebrospinal syphilis, iv. 638; in hereditary eerebellar ataxia, iv. 514; in hydrocephalus with syphilitic antecedents, iv. 464; indications for in acute myelitis, iv. 226, 228; indications for, in brain tumor, iv. 609; indications for, in tabes, iv. 193; in progressive paralysis of the insane, iv. 492; in pseudobulbar paralysis, iv. 504; question of, in aneurysm, iii. 766; in spinal syphilis, iv. 273.

Antithyroid serum, i. 169.

Antitoxic sera, i. 154: diphtheria antitoxin, i. 154; graminol, i. 161 ; meat poisoning antitoxin, i. 161; polantin, i. 160; snake venom antitoxin, i. 159 ; tetanus antitoxin, i. 158.

Antitoxic serum, use of, in meat poisoning due to Baeillus botulinus, ii. 639 ; use of, in whooping-cough, ii. 183.

Antitoxin, of diphtheria, see Diphtheria; of meat poisoning, i. 161; of snake venom, i. 159; of tetanus, see Tetanus; use of, to control epistaxis, iii. 336; use of, in membranous laryngitis, iii. 342 .

Antitoxin treatment of diphtheria. See Diphtheria.

Antituberculosis serum, i. 168.

Antituberculosis societies, work done by, in prevention of tuberculosis, ii. 451 . 
Antituberculosis work, ii. 440 ; in Denmark, ii. 442; in the District of Columbia, ii., 444; in England, ii. 440; in France, ii. 442 ; in Germany, ii. 441 ; in Ireland, ii. 441; in Massachusetts, ii. 445; in Michigan, ii. 446; in New Jersey, ii. 446; in New York City, ii., 446; in Pennsylvania, ii. 446; in Scotland, ii. 440 ; by societies, ii. 451 ; in the United States, ii. 443.

Antityphoid serum, i. 166.

Antity Phold vaccination, ii. 3 ; objects of, ii. 6 ; results of, ii. 4 ; use of sera in, ii. 7 ; technique of, ii. 6 ; use of, in typhoid carriers, ii. 6 .

Antivaccination agitation, ii. 104.

Antivenin, i. 159.

Antizeists, ii. 657.

Antral disease, radiant heat and light in treatment of, i. 452.

Antrum of Highmore, method of irrigating, iii. 334 .

ANuria, iv. 3 ; accompanying acute nephritis, iv. 62; Fischer's method of relieving, iv. 838, 856; measures for prevention of, in plague, iv. 827 .

Aortic insufficiency, use of digitalis in, iii. 589, 733.

Aortitis, ACUte, iii. 762.

Apenta water, use of, in nephritis, iv. 32 .

ApE-Pox, ii. 56.

Apex beat of heart, iii. 532, 542.

Aphasia, due to brain syphilis, iv. 475; significance of, in traumatic hematoma, iv. 365 ; as symptom in cerebral palsies of children, iv. 410,413 ; as symptom of tuberculous meningitis, iv. 375.

Aphasia, motor, as localizing symptom of tumor in left cerebral hemisphere, iv. 440.

Aphasia, optic, as localizing symptom of tumor in left cerebral hemisphere, iv. 440.

Aрhth ж, Bednar's. See Bednar's aphthæ.

Aphth e, Confluent, iii. 9.

Apocynum, use of, in cirrhosis of liver, iii. 286.

Apomorphin, use of, in acute alcoholism, ii. 589 ; in chronic alcoholism, ii. 595; to control cough due to chronic bronchitis associated with cardiac insufficiency, iii. 731 ; in dipsomania, ii. 601 ; as an expectorant in acute bronchitis, iii. 360 , by hypodermic injection as emetic in acute bronchitis, iii. 365 ; by hypodermic injection in plastic bronchitis, iii. 392; to induce emesis in acute gastritis, iii. 154 ; in muscarin poisoning, ii. 648 ; in plastic bronchitis, iii. 391 .

Apparatus, for assisting deep breathing in chronic serous pleural effusion, iii. 484 .

Apparatus, compression. See Compression apparatus.

Apparatus employed in electrotherapeutics, i. 367: coils and transformers, i. 367; high frequency apparatus, i. 368 ; interrupters, i. 368 ; motor dynamo, static machines, i. 367; Tesla machine, i. 368 ; wall plates, i. 369 .

Apparatus employed in light therapy, i. 441: colored screens, i. 441; Finsen lamp, i. 441; Görl lamps, i. 443; high candle power incandescent lights, i. 442; light bath cabinets, i. 442; marine searchlights, i. 441; minim light, i. 441.

Apparatus, Meara's. See Meara's apparatus.

Appendicitis, acute, iii. 213; diet in, iii. 215; hydrotherapy for relief of, i. 213; indications for operation in mild cases of, iii. 213; indications for operation in severe cases of, iii. 217 ; medical treatment of, in mild cases, iii. 213; medical treatment of severe cases of, iii. 217; treatment of, in pregnancy, iii. 220; treatment of, in typhoid fever, iii. 219.

Appendicitis, Chronic, as cause of gastric disease, iii. 180; electricity conjoined with X-ray in treatment of, i. 405 ; influence of, upon gastric disturbances, iii. 71,72 ; radiant light and heat in treatment of, i. 453.

Appendicitis, Chronic Catarrhal, association of, with habitual constipation a cause of autointoxication, ii. 678 .

APPENDICITIS IN GENERAL, iii. 212; classification of, iii. 212; considerations affecting operation in, iii. 213.

Appendicostomy with irrigation, in ulcerative colitis due to Balantidium coli, iv. 762.

APpetrte, influence of, upon digestion, i. 625; influence of exercise upon, i. 333. “Appetite Juice," i. 625, 626, 627. 
Applications, cold, use of, in acute attacks of hydrocephalus, iv. 465; in acute bronchitis, iii. 357 ; in acute hemorrhagic inferior polioencephalitis, iv. 408; in atriplicism, iv. 693; in cerebral anemia, iv. 382 ; in cerebral hyperemia, iv. 385 ; in climatic buboes, iv. 864; contraindicated in hemorrhagic form of ulcerative colitis, iii. 212 ; in pleurisy, iii. 466,468 ; to reduce high temperature in pneumonia, ii. 232 ; for relief of cardiac palpitation in neurasthenia, iv. 568 ; for relief of headache in acute cerebral meningitis, iv. 372 ; for relief of headache in acute nephritis, iv. 63 ; for relief of headache in cerebral abscess, iv. 423; for relief of headache due to cerebral thrombosis, iv. 399; for relief of pain in acute localized peritonitis, iii. 310 ; in renal colic, iv. 103; contraindicated in rheumatic or idiopathic cases of facial paralysis, iv. 311 ; contraindicated in sciatica, iv. 299; in edema of glottis in acute nephritis, iv. 63 ; in endocarditis complicating pneumonia, ii. 261 ; in exophthalmic goiter, iii. 898; in glandular fever, ii. 576 ; to head, i. 198; to head in acute hemorrhagic encephalitis, iv. 405; to head for relief of headache due to brain tumor, iv. 448; to head and spine for relief of insomnia due to chronic cerebral hypermia, iv. 388 ; in laryngeal complications of measles, ii. 157; for laryngo-tracheal cough in measles, ii. 152 ; in measles, ii. 150 , 154; in pachymeningitis interna hemorrhagica, iv. 364 ; in pericarditis, iii. 517.

Applications, hot, use of, in acute diarrhea of older people, iii. 195; in acute enteritis, iii. 194; in acute fibrinous pleurisy, iii. 466 ; in acute intestinal obstruction, iii. 241 ; in acute laryngitis, of doubtful benefit, iii. 338; in acute nasopharyngitis, iii. 31 ; in anuria, iv. 3 ; in bronchial asthma, iii. 389 ; in early stage of mediastinitis, to check suppuration, iii. 504; in enteritis of infancy, iii. 203; in gallstone colic, iii. 268; in gastric hyperacidity, iii. 100 ; to lower extremities in cerebral anemia, iv. 387 ; in membranous enteritis, iii. 208; in paralytic ileus, iii. 238; in pericarditis, iii. 520 ; for relief of brachial neuritis accompanying diabetes mellitus, ii. 766; for relicf of headache in acute nephritis, iv. 6.3 ; for relief of meteorism in acute diffuse peritonitis, iii. 318; for relief of muscular cramps, iv. 689 ; for relief of pain in acute localized peritonitis, iii. 311; for relief of pain in bladder accompanying tuberculous kidney, 117; for relief of pain following bite of scorpions, 685; for relief of painful urination in acute urethral gonorrhea, 142 ; in spastic constipation, iii. 231 ; in transit of gallstones, iii. 273.

Applications, hot stimulating, use of, for relief of lancinating pains in tabes, iv. 197.

Applications, hydrotherapeutic. See Hydrotherapeutic applications.

Applications, intralaryngeal, use of, in acute laryngitis, iii. 340 ; in chronic laryngitis, iii. 340 .

Applications, pneumotherapeutic. See Pneumotherapeutic application

Applications, Priessnitz. See Priessnitz' applications.

Applications, warm, in multiple neuritis, iv. 326 ; in severe attacks of appendicitis, iii. 218.

Aran-Duchenne type of progressive MUSCULAR ATROPHY, iv. 245.

Areteus, the Cappadocian, description of diabetes mellitus by, ii. 717 .

Argentine, trial of antianthrax sera in, ii. 332 .

Argentum nitrate. See Silver nitrate.

Argyll-Robertson pupil, in diagnosis of tabes, iv. 187; presence of, in progres. sive paralysis of the insane, iv. 490; presence of, in spinal syphilis, iv. 271, 273.

Argyrol, intralaryngeal application of, in acute laryngitis, iii. 340 ; intralaryngeal application of, in chronic laryngitis, iii. 341 ; irrigation of bladder with, in acute cystitis, iv. 120; irrigation of colon with, in operative treatment of ulcerative colitis, iii. 212; rectal injections of, in chronic mucous colitis, iii. 207; rules for injection of, in acute urethral gonorrhea, iv. 145; use of, in acute atrophic rhinitis, iii. 332 ; use of, in acute urethral gonorrhea, iv. 144; use of, in 
chronic atrophic rhinitis, iii. 343; use of, in chronic nasopharyngitis, iii. 33; use of, in chronic pharyngitis, iii. 33; use of, in chronic rhinitis, iii. 329; use of, in laryngeal tuberculosis, iii. 347; use of, in papular or tubercular tertiary lesions of syphilis, ii. 426; use of, .in pellagra, ii. 662; use of, in phlegmonous pharyngitis, iii. 40; use of, in suppositories for rectal chancre, ii. 424 .

Aristol, use of, as dusting powder in gangrenous chancre,-ii. 423; as dusting powder on mucous patches of genitalia, ii. 427 ; by insufflation after cauterization of nasal mucous membrane in chronic rhinitis, iii. 329 ; by insufflation in membranous rhinitis, iii. 330 ; by insufflation in syphilis of upper respiratory tract, iii. 349 ; as ointment, in herpes of lips, iii. 6 .

Armstrong, William, results of radioactivity summarized by, i. 472 .

ARNeTH's NEUTROPHILIC BLOOD PICTURE, as guide to dosage of tuberculin, ii. 520 .

Arnold, report by, on use of electricity to reduce blood pressure, iv. 48

Aromatic expectorants. See Expectorants, aromatic.

Aromatic inhalations. See Inhalations, aromatic.

Aromatic spirits of ammonia, use of, in bronchopneumonia complicating influenza, ii. 192; as a cardiac stimulant in strophanthus poisoning, iv. 694; for edema of glottis in acute nephritis, iv. 63; for flatulency associated with gastric atony, iii. 172 ; to relieve chill in first stage of pneumonia, ii. 254; to relieve faintness during thoracentesis, iii. 478; in scarlet fever, ii. 128.

Aromatic substances, use of, in depressive disorders of gastric secretion, iii. 151.

Aron, experiments by, on effects of tropical heat upon white men, iv. 669; experiments by, on regulation of animal heat, iv. 675 .

Aron And Hocson, on causation of beriberi, iv. 850 .

Aronson's antistreptococcic serum, i. 163; use of, in scarlet fever, ii. 128.

Arrhenal, use of, in pernicious anemia, iii. 828.

Arrhythmia, theory of, iii. 536 .
Arrhythmias, iii. 564; presence of, in cardiac insufficiency, iii. 774.

Arrowroot, as article of invalid diet, i. 649.

Arsacetin, use of, in diabetes insipidus, ii. 777 ; in pellagra, ii. 661 .

Arsazetin, use of, in pernicious anemia, iii. 828,829 ; in pseudo-leukemia, iii. 854 .

Arsenic, in causation of nephritis, iv. 28; effect of, upon kidney, iv. 14; occurrence of, in nature, i. 674; poisoning by, see Arsenical poisoning; presence of, in food, a possible cause of beri-beri, iv. 848 .

Arsenic, use of, in Addison's disease, iii. 884; in bronchial asthma, iii. 383 ; in chlorosis, iii. 821 ; in chorea minor, iv. 663 ; in chronic malaria, iv. 728 ; in chronic myelitis, iv. 230 ; in convalescence from influenza, ii. 196; in convalescence from pneumonia, ii. 262 ; in epilepsy, iv. 648 ; in exophthalmic goiter, iii. 901; in funicular myelitis, iv. 232; in hay fever, iii. 390 ; by hypodermic injection in leukemia, iii. 847 ; by hypodermic injection in pernicious anemia, iii. 827 ; in intermittent hemoglobinuria, iv. 7; in kala-azar, iv. 754; in kubisagari, iv. 861 ; in leprosy, iv. 816 ; in leukemia, iii. 847 ; in neuralgia, iv. 337 ; in neuralgia due to anemia, iv. 336 ; in neuralgic headache, iv. 598; in neurasthenia, iv. 558; in occupation pareses, iv. 332 ; in palliative treatment of mediastinal tumors, iii. 501; in pellagra, ii. 659 ; in pernicious anemia, iii. 827 ; in polycythemia with splenomegaly. iii. 878; in prevention of scarlet fever, ii. 123; in pseudoleukemia, iii. 854; in rachitis, ii. 818; for relief of leukemic headache, iv. 611; as substitute for quinin, iv. 726; in syphilis, ii. 411 ; in syringomyelia, iv. 279 ; in tabes, iv. 213 ; in treatment of anemia during tuberculosis, ii. 507; in treatment of diabetes mellitus, ii. 759 ; in treatment of enuresis, iv. 133; in treatment of morphinism, ii. 620; in tuberculosis of mediastinal lymph nodes, iii. 507; in verruga peruviana, iv. 864 .

Arsenic, Fowler's solution of. See Fowler's solution.

Arsenic, organic preparations of, use of, in pernicious anemia, iii. 827 ; in syphilis, 
ii. 412 ; in tetanus, ii. 350 ; in tropical relapsing fever, iv. 759 ; in trypanosomiasis, iv. 745).

Arsenic trioxid and atoxyl, use of, in treatment of pellagra, ii. 659 .

Arsenical, polsoning, i. 674; dose of arsenic required for, i. 685 ; due to arsenic eating, i. 686; due to use of arsenobenzol in syphilis, ii. 414; histological changes due to, i. 684; hydrotherapy for chronic form of, i. 228; post-mortem appearances in, i. 682 ; preparations causing, 677 ; symptoms of, i. 678 ; treatment of, i. 686 ; treatment of multiple neurosis due to, iv. 323 ; unusual forms of, i. 688.

Arsenical preparations, use of, in destruction of filaria bancrofti, iv. 787 ; in frambosia tropica, iv. 765 .

Arsenical waters, action of, i. 236; classification of, i. 234 .

Arsenious acid, use of, in syringomyelia, iv. 279.

Arsenobenzol, aetion of, ii. 414 ; comparison of, with mercury in syphilis, ii. 413; dangers attending use of, ii. 414; history of, ii. 411 ; iv. 481 ; methods of using, in syphilis, ii. 415.

Arsenobenzol, use of, in acute myelitis, iv. 226 ; in apoplectic form of vestibular vertigo with syphilitic origin, iv. 628 ; in brain syphilis, iv. 481,484 ; in chronic myocardial insufficiency due to syphilis, iii. 730 ; contraindicated in disease of middle ear, iv. 315 ; contraindicated in optic atrophy, iv. 307 ; contraindicated in optic neuritis, iv. 305 ; in epilepsies of cerebrospinal syphilis, iv. 638; in frambœsia tropica, iv. 764; in hereditary syphilis, ii. 433 ; by hypodermic injection in destruction of filaria bancrofti, iv. 788; indications for, in tabes, iv. 212 ; by intramuscular injection in syphilis, ii. 415 ; by intravenous injection in destruction of filaria bancrofti, iv. 788; by intravenous injection, in spinal syphilis, iv. 274 ; by intravenous injection, in syphilis, ii. 415; by intravenous injection, in tabes, iv. 194; in kala-azar, ii. 660 ; in paralysis of bladder, iv. 123; in pellagra, ii. 661 ; in pernicious anemia, iii. 829 ; in pregnaney, ii. 433 ; in progressive paralysis of the insane, iv. 492; as substi- tute for atoxyl in trypanosomiasis, iv. 746 ; in syphilis, ii. 412 ; in syphilis, contraindicated, ii. 414 ; in syphilis of liver, iii. 26i); in syphilis of pancreas, iii. 302; in syphilis of upper respiratory tract, iii. 345 ; in syphilitic arthritis, ii. 306 ; in tabes not contraindicated by impending amaurosis, iv. 199; in tropical relapsing fever, iv. 759 .

Arsenophenylglycin, use of, as substitute for atoxyl in trypanosomiasis, iv. 746 .

Arsenophenylglycochol, use of, in hydrophobia, ii. 343.

Arteries, diskases of, iii. 753: AdamsStokes syndrome, iii. 772; aneurysm, iii. 763 ; angina pectoris, iii. 767 ; arteritis, iii. 762; arterioselerosis, iii. 753 ; heart block, iii. 772 .

Arteriosclerosis, iii. 554; association of, with cerebral hyperemia, iv. 385; ehronic myocardial insufficiency due to, iii. 707; digitalis in treatment of, iii. 589 ; effect of occupation upon, iii. 757 ; etiological considerations in, iii. 754; as a factor in causation of cerebral thrombosis, iv. 395; hydrotherapy for relief of, i. 208, iii. 760 ; influence of climate upon, i. 282 , iii. 760 ; mechanotherapy for relief of, i. 311; medicinal treatment of, iii. 761 ; phleboselerosis associated with, iii. 762; as predisposing eause of neuralgia, iv. 336; rise of blood pressure in, iii. 754 ; treatment of, in general, iii. 757 .

Arteriosclerotic vertigoes; iv. 624.

Arteritss, iii. 762; acnte, iii. 762; chronic, iii. 762 ; syphilitic, iii. 763 .

Arthritis, as a complication of scarlet fever, ii. 132 ; connection of, with intestinal autointoxication, ii. 680 .

Arthritis, ATrophic, ii. 707; treatment of, ii. 710 .

Arthritis, Chronic gouty, ii. 710 .

ARthritis DEForMaNs, hydrotherapy for relief of, i. 226; massage and gymnestics for relief of, i. 327 .

ARthritis DEFORMANS AND CHRONiC RHEUMATISM, ii. 707; atrophic arthritis, ii. 707; chronic gouty arthritis, ii. 710; diet in treatment of, ii. 711; drugs in treatment of, ii. 713; hydrotherapy for, ii. 712 ; hygienic measures in treatment of, ii. 711 ; hypertrophic arthritis, ii. 
708; infectious arthritis, ii. 709; spondylitis, ii. 710; static ailments, ii. 709; Still's disease, ii. 710; surgical treatment of, ii. 714 ; therapy in, ii. 710 ; toxemic arthritis, ii. 709.

Arthritis, GONococcic, ii. 306; Bier's method of treatment for, ii. 307; electrotherapy for relief of, i. 414; hydrotherapy for relief of, i. 210; treatment of, in general, ii. 307.

Arthritis, gonorrheal. See Arthritis, gonococcic.

Arthritis, hypertrophic, ii. 708; treatment of, 710 .

Arthritis, infectious, ii. 709; surgical treatment of, ii. 712 ; treatment of, in general, ii. 710

Arthritis, rheumatord. See Rheumatoid arthritis.

Arthritis, syphilitic, ii. 306.

Arthritis, toxemic. See Arthritis, infectious.

Arthritis, tuberculous, electricity conjoined with X-ray in treatment of, $\mathrm{i}$. 403 ; radiant light and heat in treatment of, i. 452.

Arthropathies, in tabes, iv. 191; treatment of, in hemophilia, iii. 870.

Arthropons, treatment of bites and stings by, iv. 685 .

Arthrosia podagra. See Gout.

Articular rheumatism, acute, hydrotherapy for relief of, i. 227 ; serumtherapy for relief of, i. 164 .

Articular rheumatism, chronic, application of hydrotherapy to relief of, i. 208 .

Artificial feeding. See Feeding.

Artificial pneumothorax. See Pneumothorax.

Artificial respiration, employment of, in asphyxia of newborn from paralysis of diaphragm, iv. 290; in bronchial asthma, iii. 389 ; in curare poisoning, iv. 694 ; in fish poisoning, iv. 684 ; in Landry's paralysis, iv. 329 ; in poisoning from strychnos and allied plants, iv. 696 ; in pulmonary edema, iii. 409 ; as remedy for poisons causing heart failure, i. 672 ; in strophanthus poisoning, iv. 695 .

Arylarsonates, use of, in pernicious anemia, iii. 828 .
Asafetida, use of, in peristaltic unrest, iii. 250.

Ascariasis, iii. 250; prophylaxis against, iii. 251 ; treatment of, iii. 251.

Ascaris lumbricoides, iii. 250.

Ascites, accompanying cirrhosis of liver, iii. 283.

AsEPsis, of eatheterism, iv. 157.

Ashburn and Craig, investigations by, on transmission of yellow fever, iv. 858 .

Asiatic cholera, iv. 827; action of toxins in, iv. 831 ; communal prophylaxis against, iv. 328; convalescence from, iv. 838; diet in, iv. 838; Fischer's method of relieving anuria in, iv. 838; hydrotherapy for, i. 218; individual prophylaxis against, iv. 828; mortality from under Rogers' treatment for, iv. 833; mortality from under serum treatment for, iv. 832; mortality from, with and without inoculation, iv. 830; nature of toxins in, 831; prophylactic inoculation against, i. 218; prophylaxis against, in general, iv. 827; Rogers' treatment for, iv. 833; serum therapy for, iv. 832; stamping out of epidemics of, iv. 830; transmission of, iv. 827 ; treatment of, iv. 832 ; uremia as a complication of, iv. 836 .

Aspergillus flavescens, as cause of pellagra, ii. 658 .

Aspergillus fumigatus, as cause of pellagra, ii. 658.

Aspiration, in acute nephritis for relief of edema, iv. 62; of bladder for relief of urethral stricture, iv. 172; of body cavities in uremia with edema, iv. 54; danger of introducing infected material in, during unconsciousness, iii. 439; in diagnosis of empyema accompanying tuberculosis, ii. 507; in echinococcus disease, iii. 256; as exploratory measure in mediastinitis, iii. 504; for relief of pleural effusion accompanying tuberculosis, ii. 507; for relief of pneumothorax accompanying tuberculosis, ii. 507; of serous cavities in beri-beri, iv. 854; in serous effusion from cardiac insufficiency, iii. 672; useless in empyema, iii. 485.

Aspiration pneumonia. See Pneumonia. Aspirin, use of, in acute gout, ii. 703; in acute laryngitis, iii. 338 ; in acute rheu- 
matism, ii. 300; in acute scarlatinal arthritis, ii. 305; in bronchial asthma, iii. 388 ; in chorea minor, iv. 662 ; in "colds" during tubereulosis, ii. 505; in diabetes mellitus, ii. 760; in herpess zoster, iv. 359; in influenza, ii. 187; in Landry's paralysis, iv. 329; in measles, ii. 151; for neuralgia associated with rheumatism, ii. 304 , iv. 337 ; in optic neuritis without apparent cause, iv. 306 ; in pneumonia, ii. 226 ; in polymyositis, ii. 685 ; to reduce temperature in bronchopneumonia, iii. 418 ; to reduce temperature in pleurisy, iii. 468; to reduce temperature and pain in acute tonsillitis, iii. 41 ; to reduce temperature in tuberculosis of larynx, iii. 346 ; for relief of headache due to cerebral thrombosis, iv. 399; for relief of headache in neurasthenia, iv. 566; for relief of headache in pachymeningitis interna hemorrhagica, iv. 364; for relief of insomnia due to brain tumor, iv. 447 ; for relief of myositis, iv. 605; for relief of pain in acute localized peritonitis, iii. 311 ; for relief of pain and irritability in acute inflammation of nasal sinuses, iii. 334; for relief of pain in Malta fever, iv. 839 ; for relief of pain in polymyositis, iv. 330 ; in rheumatic cases of facial paralysis, iv. 311; in Rocky Mountain fever, ii. 564; in septicopyemia, ii. 291; in Weil's disease, ii. 572.

Aspirin combined with aconite, use of, for nasal and frontal sinus headaches, iv. 602.

Aspirin combined with Dover's powder, use of, in acute laryngitis, iii. 338.

Aspirin and quinin, use of, combined with Dover's powder in abortive treatment of acute rhinitis, iii. 327 .

Association experiment, employment of, in psychotherapy, iv. 579.

Asthma, BRONCHIAL, iii. 379 ; drugs in treatment of, iii. 382 ; epinephrin for relief of, i. 99 ; frequency of, among coal miners, iii. 425; hydrotherapy for relief of, i. 204; influence of climate upon, iii. 380; influence of environment upon, iii. 379 ; pulmonary emphysema due to, iii. 433; sudden death following injection of antitoxin for relief of, i. 135; treatment of, between attacks, iii. 379 ; treatment of, during attack, iii. 385; vaceine therapy for, i. 147.

Astuma, виonchitic, iii. 379.

Astuma, carbiac, iii. 407; due to myocardial insufficiency, iii. 737 .

Astima, hay. Sée Hay fever.

A.thim, nenvous, iii. 379.

Astuma, pertic, iii. 381.

Astuma, toxic, iii. 379.

Astringents, use of, in acute diarrhea of old persons, iii. 195; contraindieated in leukoplakia, iii. 26; in diarrhea of pellagra, ii. 662 ; in enterocolitis complicating measles, ii. 160; in hemorrhagic form of measles, ii. 154, 155; by injections, in chronic gonorrheal urethritis, iv. 155; locally in nasal and frontal sinus headache, iv. 603; in stomatitis of pellagra, iii. 662; unsatisfactory in chronic stage of bacillary dysentery, ii. 328 .

Asymmetry of FACE, presence of, in traumatic hematoma, iv. 366 .

Ataxia, appearance of, in tabes, iv. 189; massage and gymnastics in treatment of, i. 316.

Ataxia, Cerebellar, hereditary, see Hereditary cerebellar ataxia; as a symptom of thrombosis, iv. 396 ; as a symptom of tumor in cerebellopontine recess, iv. 444.

Ataxia, Friedreich's. See Friedreich's ataxia.

Ataxia, Frontal, as symptom of brain tumor in frontal lobe, iv. 439.

Atelectasis, method of combating, in bronchopneumonia of young children, iii. 419.

Atherosclerosis. See Arteriosclerosis.

Athetosis, as a symptom of cerebral diplegia type of cerebral palsies of children, iv. 411.

Athletics, competitive, as cause of cardiac insufficiency, iii. 700.

Athyrea and hypothyrea, iii. 906; general considerations regarding, iii. 906; iodothyrin in treatment of, iii. 907; thyroid gland in treatment of, iii. 906, 907; thyroidin in treatment of, iii. 907 ; transplantation for relief of, iii. 907 .

Atmosphere, barometric pressure of, i. 257; composition of, i. 257; dust contained in, i. 258, 274; humidity of, i. 250; impurities contained in, i. 258, 274; relation of altitude to, i. 255. 
Atmospheric Dust, influence of, upon health, i. 274 ; nature of, i. 258.

Atmospheric humidity, as a climatic factor, i. 250 ; determination of, i. 252 ; physiological influence of, i. 263.

Atmospheric impurities, influence of, on health, i. 274 ; nature of, i. 258.

Atony of sphincter, as a cause of enuresis, iv. 128.

Atony of stomach. See Gastric atony.

Atophan, use of, in acute gout, ii. 702.

Atoxyl, use of, in frambosia tropica, iv. 765 ; by hypodermic injection, necessary n pellagra, ii. 660; by hypodermic injection, suggested in verruga peruviana, iv. 864; by intramuscular injection, in kala-azar, iv. 754 ; by intravenous injection, in destruction of filaria bancrofti, iv. 788 ; in pellagra, ii. 659 ; in pellagra followed by atrophy of optic nerve, ii. 660 ; in pernicious anemia, iii. 828 ; in syphilis, ii. 412 ; in trypanosomiasis, iv. 740 .

Atoxyl and thioglycholic acid, use of, in treatment of hydrophobia, ii. 343 .

Atoxyl derivatives, use of, in trypanosomiasis as substitute for atoxyl, iv. 746.

Atriplex littoralis, iv. 693.

Atriplicism, iv. 693.

Aтrophy, of cerebellum. See Cerebellum; massage and gymnastics in treatment of, i. 314 ; of muscles, in anterior poliomyelitis, iv. 235; of optic nerve, see Optic atrophy; presence of, in tabes, iv. 191; of skin, due to X-ray therapy, i. 516 .

Atropin, use of, in acute opium poisoning, ii. 615 ; as antidote to physostigma venenosum, iv. 696; in bronchial asthma, iii. 383 ; in cardiac failure, during pneumonia, ii. 193; in cardiac toxemia of pneumonia, ii. 251; in cholera infantum, iii. 204; in chronic dilatation of stomach, iii. 180; in chronic opium poisoning, ii. 620 ; in "colds" accompanying tuberculosis, ii. 508; in collapse due to thoracentesis, iii. 482 ; in continuous gastric hypersecretion, iii. 106; contraindicated for edema of glottis in acute nephritis, iv. 63 ; to control night sweats, ii. 505; iv. 117 ; in diabetes mellitus, ii. 759; in gastric hyperacidity, iii. 91 ; in heart block, iii. 772 ; by hypo- dermic injection in acute gastritis, iii. 156; by hypodermic injection in gastric hypersecretion, iii. 102; by hypodermic injection in membranous enteritis, iii. 208; by hypodermic injection in multiple neuritis, iv. 326 ; by hypodermic injection in renal colic, iv. 105; by instillation in care of eyes during small-pox, ii. 50; in muscarin poisoning, ii. 648; in non-bleeding gastric ulcer, iii. 134; in paralytic ileus, iii. 238; in poisoning by amanita phalloides, ii. 649; in progressive bulbar paralysis to relieve dribbling of saliva, iv. 499; in ptyalism in mumps, ii. 171; in pulmonary edema, iii. 409 ; in pulmonary edema complicating pneumonia, ii. 259; in pulmonary edema due to injection of pilocarpin, iii. 408; for relief of acute pulmonary edema in nephritis, iv. 55; for relief of dyspnea in chronic pulmonary emphysema, iii. 438 ; for relief of vomiting in neurasthenia, iii. 567; as a respiratory stimulant in Landry's paralysis, iv. 329 ; in spastic constipation, iii. 231; as spray in hay fever, iii. 391 ; in syphilitic iritis, ii. 429 ; in tetanus, ii. 348 ; in treatment. of enuresis, iv. 133; in whooping-cough sometimes followed by disagreeable symptoms, ii. 175.

Atropin combined with anodynes, use of, for relief of dyspnea in progressive paralysis, iv. 500 .

Atropin and morphin, use of, in preparation for thoracentesis, iii. 478; in renal colic, iv. 105; in strangulation of intestines, iii. 240.

Atropin and strychnin, use of, in alcoholism, ii. 596; to counteract effects of pilocarpin, iv. 317.

Atropin, strychnin, and digitalis, use of, in delirium tremens, ii. 604.

Atropin poisoning, i. 752; poisonous dose of atropin in, i. 753; post-mortem appearances in, i. 754; symptoms of, i. 752; treatment of, i. 753 .

Atwater AND Benedict, value of alcohol in sparing fats and carbohydrates demonstrated by, ii. 558 .

Arwood, factors given by, for calculating dietaries for children, i. 605.

Audaut, oil of turpentine recommended by, in phosphorus poisoning, ii. 628 . 
AUdRY, administration of mercury by rectum advocated by, ii. 387 .

Aural vertigo, iv. 314, 629; treatment of, iv. 315 .

Auricular extrasystoles, iii. 568.

Auriculoventricular extrasystoles, iii. 569.

Auriculoventricular heart Block, iii. 566.

Auscultation, in diagnosis of brain tumor, iv. 444

Austen, on recognition of tsetse fly, iv. 750.

Austria, introduction of vaccination into, ii. 60 .

Autogenous vaccines, nature of, i. 140; use of, in staphylococcus infections, i. 145.

Autohypnosis, i. 567.

Autolnfection, by oxyuris vermicularis, iii. 252.

Autoinoculation, generalized vaccinia from, ii. 82; theory of, i. 139.

Autoinoculation in tuberculosis, ii. 525 ; control of, ii. 526 ; exercise in relation to, ii. 527; rest in relation to, ii. $528,529$.

Autointoxication, intestinal. See Intestinal autointoxication.

Autointoxications, as a cause of chronic myelitis, iv. 229.

Autolyzed PNeumococci. See Pneumococci.

Automassage, use of, in constipation, iii. 228.

Autoserotherapy, i. 170 , iii. 483 ; in tuberculous peritonitis, iii. 320 .

Autosuggestion, association of, with hysteria, i. 560 .

Autotoxic methemoglobinemia, iii. 879.

A UXILIARY AR'icles of Diet, i. 603.

Avulsion method, of nerve cutting for relief of trigeminal neuralgia, iv. 341 .

BabÉS AND Zambolovice, bacillus isolated by, in stomatitis gangrenosa, iii. 17 .

BABINSKI SIGN, presence of, in acute hemorrhagic superior polioencephalitis, iv. 406; in cerebral diplegia type of the cerebral palsies of children, iv. 410 ; in diagnosis of Friedreich's ataxia, iv. 216; in diagnosis of hereditary cerebellar ataxia, iv. 217; in multiple sclerosis, iv.
280; in spastic spinal paralysis, iv. 268; in tramatic hematoma, iv. 366.

Bacelli's method, of treating tetanus, ii. 349.

Bacillin-emulsion, Koch's, see Koch; use of, in tuberculosis of mediastinal lymph glands, iii. 507.

Bacillin-emulsion and broth filtrate, use of, in tuberculosis of mediastinal lymph glands, iii. 507.

Bacillus anthracis, meat poisoning caused by, ii. 643 .

Bacillus botulinus, meat poisoning due to, ii. 638 ; symptoms of, ii. 639 ; treatment of, ii. 639 .

Bacillus breslaviensis, meat poisoning due to, ii. 641.

Bacillus BUlgaricus, intestinal putrefaction treated by cultures of, ii. 671 .

Bacillus enteritidis, meat poisoning due to, ii. 640 ; treatment of, ii. 641 .

Bacillus gangosa, iv. 864.

Bacillus of influenza, discovery of, ii. 184; endocarditis caused by, ii. 193; meningitis caused by, ii. 193; pure culture of, obtained from an appendiceal abscess, ii. 190.

Bacillus paratyphosus, epidemics of fish poisoning due to, ii. 644 ; meat poisoning caused by, ii. 642 .

Bacillus PERfRingens infeCtion, vaccine therapy in, i. 147.

BaCillus PEstis, iv. 816.

Bacillus piscicidus agilis, epidemic among fish due to, ii. 644 .

BACILLUS PNEUMONIE, meat poisoning caused by, ii. 643 .

Bacillus proteus vUlgaris, epidemic among fish caused by, ii. 644; meat poisoning caused by, ii. 642 .

BACILLUS PSEUDO-DIPHTHERITICUS INFECTION, vaccine therapy for, i. 147 .

Bacillus pyocaneus infection, vaccine therapy for, i. 147.

Bacillus salmonicida, epidemic among trout due to, ii. 644 .

Bacillus scarlatine, ii. 114.

Bacillus suisepticus, meat poisoning due to, ii. 642 .

BACKACHE, accompanying acute nephritis, iv. 63.

BACON, use of, in diabetes mellitus, ii. 744 . BACTERIA, contained in mouth, iii. 1. 
Bacteria, diseases due to, iv. 809; Asiatic cholera, iv. 827; leprosy, iv. 809; Malta fever, iv. 839; plague, iv. 816.

BACTERIAL CLUMPS, as a cause of cerebral embolism, iv. 400.

BACTERIAL Disintegration OF FOOD, intestinal intoxication due to abnormal products of, ii. 667; intestinal intoxication due to normal products of, ii. 667 .

BACTERIAL EXTRACTs, immunization with, i. 148.

Bacterial growths, extracts from, use of, in leprosy, iv. 814.

BACTERIAL LIFE, effects of X-ray upon, $i$. 525.

Bacterial poisons, i. 674.

BACTERIAL PRODUCTS, intoxication from a possible cause of beri-beri, iv. 849 .

Bacterial products, treatment with, i. 173; principles of, i. 173; prodigiosus toxins in treatment of tumors, i. 174; pyocyanase, i. 173; streptococcus toxins in treatment of tumors, i. 174.

BACTERIAL TOXINS, influence of, in production of arteriosclerosis, iii. 756 .

Bacterial vaccines, action of, i. $139 ;$ varieties of, i. 140.

BACTERIAL virus, use of, in destruction of rats conveying plague, iv. 825 .

Bactericidal sera, i. 161; antianthrax serum, i. 167; anticholera serum, i. 671; anticolon-bacillus serum, i. 166; antidysenteric serum, i. 165; antigonococcus serum, i. 165; antiinfluenza serum, i. 162; antimeningococcus serum, i. 161; antiplague serum, i. 167; antipneumococcus serum, i. 164; antistaphylococcus serum, i. 166; antistreptococcus serum, i. 163; antityphoid serum, i. 166.

Bacterins. See Bacterial vaccines.

BACTERIOLOGICAL EXAMINATION, in serous meningitis, iv. 469.

BACTERIOLYTIC IMMUNITY, discovery of, $i$. 127.

BACterioria, as a cause of enuresis, iv. 128.

BAELz, diuretic mixture recommended by, for use in beri-beri, iv. 852 .

BAELz's DISEASE. See Inflammation of labial glands.

Baillie's pill, iii. 286.

Balanitis, as a cause of enuresis, iv. 130.
BALANOPOSTHITIS, complicating acute gonorrhea, iv. 149.

Balanotherapeutics, value of, in tabes, iv. 201.

Balantidium coli, diseases due to, iv. 760 .

BALFOUR, new species of spirochete described by, iv. 758 .

BALlinger, leukodescent lamp recommended by, in treatment of acute uvulitis, iii. 35 .

Balneology, i. 229; action of mineral waters in, i. 235 ; in alimentary diseases, i. 241; in blood diseases, i. 239; in cardiac diseases, i. 240; choice of Spa for, i. 237; classification of mineral waters for, i. 232; composition of mineral waters for, i. 231; indications for Spa for, i. 237; introduction to, i. 229 ; principles of, i. 229 ; in renal and bladder disorders, i. 241 ; in respiratory diseases, i. 239 ; in rheumatism, i. 241; special applications of, i. 238; in tardy convalescence, i. 238; temperature of mineral waters, i. 231; virtues of Spas for, i. 230.

Balneotherapy, employment of, in exophthalmic goiter, iii. 898; in neurasthenia and psychasthenia, iv. 557.

Balsam of Peru, use of, in atrophic rhinitis, iii. 331 ; in leukoplakia, iii. 26 ; to relieve itching, in varicella, ii. 113.

Balsamics, inhalation of, for relief of gangrene of lung complicating pneumonia, ii. 261; use of, in acute urethral gonorrhea, iv. $139,140$.

BAmBURGer, description by, of paroxysm of asthma, iii. 385 .

Bandage, abdominal, employment of, in movable spleen, iii. 887; suspensory, see Suspensory bandage; use of, for relief of intercostal neuralgia, iv. 357 .

Bandaging of extremities, for relief of cerebral anemia, iv. 382 ; for relief of phlebitis complicating typhoid fever, ii. 28 .

Banks' bougie, use of, in urethral stricture, iv. 170 .

BANNERMANN, method of precaution instituted by, in preparation of plague vaccine, iv. 824 .

BANTI's DISEASE, iii. 889; results of operation for, iii. 890; X-ray therapy for relief of, i. 550 . 
Barbel cholera. See Cholera

BARBzzanox, mothod employed by, for use of salicylates in beri-beri, iv. 853 .

BARDSWELL, scheme devised by, to supply variety of diet in tuberculosis, ii. 547.

BARLEY WATER, Use of, as article of invalid dictary, i. 645; as food in cholera infantum, iii. 205; to relieve cough in chronic bronchitis, iii. 374 .

Barometric pressure, as climatic factor, i. 257; physiological influence of reduction in, i. 268.

BArucir, method of giving general bath employed by, i. 194; "neurovascular training" devised by, i. 224.

BASAL TUMORS, iv. 441.

BASE OF BRAIN, tumors at. See Basal tumors.

Basedow's DISEASE. See Exophthalmie goiter.

Basham's mixture, use of, in nephritis complieating influenza, ii. 194; in nephritis complicating scarlet fever, ii. 134.

Basic fuchsin, use of, as application to ulcers in frambosia tropica, iv. 765.

Basic triphenylmethane dyes, use of, in trypanosomiasis, iv. 747.

Basilar syphilitic meningitis. See Meningitis.

BAss, on advantages of local reduetion of temperature in acute localized peritonitis, iii. 311.

BASSETT-SмITH, report by, on use of vaccine therapy in Malta fever, iv. 839.

"Bath, air," use of, to reduce temperature in pneumonia, ii. 231.

Bath, alcohol sponge, use of, to relieve night sweats in tubereulosis, ii. 505.

Bath, arsenic, use of, in pellagra, ii. 662 .

Bath, cold sponge, therapeutic use of, in bacillary dysentery, ii. 324 ; in measles, ii. 149 ; in meat poisoning, ii. 639 ; in pneumonia, ii. 233; in Rocky Mountain fever, ii. 565; in scarlet fever, ii. 125; in typhoid fever, ii. 18; in Weil's disease, ii. 572.

Bath, continuous, therapeutic uses of, i. 199.

Bath, continuous warm, use of, in treatment of bedsores accompanying acute myelitis, iv. 227.

Bath, creosote vapor, method of, in bronchiectasis, iii. 395.
Bath, fan, therapeutic use of, in typhoid fever, ii. 16.

Bath, full tub, use of, in neurasthenia, iv. 547.

Bath, half. See Half bath.

Bath, hot air, use of, in cardiac insufficiency, iii. 678; in nephritis complicating scarlet fever, ii. 133, 134; as preventive of acute bronchitis, iii. 352 ; for relief of anuria in nephrolithiasis, iii. 107; in treatment of morphinism, ii. 624 .

Bath, hot foot, use of, to relieve "colds" accompanying tubereulosis, ii. 568.

Bath, mustard, use of, in cholera infantum, iii. 204.

Bath, mustard foot, use of, in acute rhinitis, iii. 327; in bronchial asthma, iii. 389.

Bath, neutral, use of, to relieve nervous symptoms in intestinal autointoxication, ii. 673.

Bath, permanent, use of, in arthritis deformans, ii. 713 .

Bath, salt sponge, use of, to reduce temperature in tuberculosis of mediastinal lymph glands, iii. 507.

Bath, sitz. See Sitz bath.

Bath, soap, use of, as general tonic in atrophic rhinitis, iii. 330 .

Bath, sponge, use of, in chronic pneumonia, iii. 423.

Bath sulphur, use of, in pellagra, ii. 662 .

Bath, vapor, therapeutic use of, for elimination of toxic substances in intestinal autointoxication, ii. 672 ; in nephritis complicating scarlet fever, ii. 133, 134.

Bathing, importanee of, in nephritis, iv. 32.

BATHMOTROPIC INFLUENCES, on cardiac muscle, iii. 537.

Baths, advantages from early use of, i. 185; benefit of cold in, i. 189; benefit from heat in, i. 192; extremes of temperature applied by means of, i. 200; general, i. 194; localized, i. 200; therapeutic uses of, in general, i. 185; value of, in cardiac insufficiency, iii. 618; value, of, in cardiae neurasthenia, iii. 777, 778; value of, in typhoid fever, $i$. 218.

Baths, alkaline, use of, in eopaibal poisoning, iv. 141; for relief of itching in acute nephritis, iv. 63. 
Baths, alternate cold and hot, use of, in yellow fever, iv. 855 .

Baths, bran, use of, to relieve itching in carcinoma of pancreas, iii. 305 .

Baths, carbon dioxid, use of, in after-treatment of acute infectious myocarditis, iii. 751 ; in cardiac insufficiency, iii. 615 ; in chronic myocarditis, iii. 724, 734; contraindications to, in aneurysm, iii. 766; contraindications to, in chronic myocardial insufficiency, iii. 717, 721, 731; indications and contraindications for, iii. 622 ; in late stages of acute endocarditis, iii. 746; in neurasthenia and psychasthenia, iv. 557; physiological effects of, iii. 621; in syringomyelia, iv. 278; see also Nauheim baths.

Baths, cold full, use of, in bronchial asthma, iii. 381 ; in hay fever, iii. 390 ; in tetany, iii. 910 .

Baths, cold plunge, use of, in chronic bronchitis, iii. 367 ; contraindicated in arteriosclerosis, iii. 761 .

Baths, cold sponge, use of, in acute rhinitis, iii. 326 ; in chronic bronchitis, iii. 367 ; in hyperpyrexial fever, iv. 869 ; as protection against bronchitis, iii. 351 ; to reduce temperature in epidemic dropsy, iv. 860 ; to reduce temperature in plague, iv. 827 ; to reduce temperature in polymyositis, iv. 330; to reduce temperature in reaction stage of Asiatic cholera, iv. 836; over region of larynx as prophylactic measure against spasmodic laryngitis, iii. 342 .

Baths, cold tub, use of, in cerebral hyperemia, iv. 385; contraindicated in acute nephritis, iv. 58; contraindicated in chronic parenchymatous nephritis, iv. 29,32 ; contraindicated in diabetes mellitus, ii. 762 ; contraindicated in influenza, ii. 188; in erysipelas, ii. 284 ; in intestinal autoinfection, ii. 677 ; in measles, ii. 149 ; in pellagra, ii. 662 ; in pneumonia, ii. 232 ; to reduce temperature in acute cerebral meningitis, iv. 372 ; to reduce temperature in bronchopneumonia, iii. 418 ; to reduce temperature in dengue, iv. 859 ; in reduction of obesity, ii. 808; in scarlet fever, ii. 126; in septicopyemia, ii. 290; in small-pox, ii. 47; in tuberculosis of kidney, iv. 115; in typhoid fever, ii. 16.
Baths, compressed air, use of, in bronchial asthma, iii. 381.

Baths containing iron, use of, in chlorosis, iii. 820 .

Baths, cool full, use of, in hyperpyrexial fever, iv. 869; for relief of headache due to brain tumor, iv. 448 .

Baths, cool sponge, use of, in acute diffuse peritonitis, iii. 318; contraindicated in cerebrospinal meningitis, ii. 215 ; in infantile diarrhea, ii. 329 ; to reduce temperature in pleurisy, iii. 468.

Baths, effervescent saline, use of, in bronchial asthma, iii. 382 .

Baths, electric light, therapeutic use of, in arthritis deformans, ii. 713.

Baths, general, i. 194; ablution in, i. 194; cold plunge, i. 199; cold rub, i. 196; cold sponge, i. 196; continuous i. 199; drip sheet, i. 195; effervescent, i. 200; full, i. 198; graduated, i. 198; half, i. 195; hammock, i. 199; Neptune's girdle, i. 198; of radiant light and heat, i. 454,456 ; of radioactive waters, $i$. 467,473 ; sheet, i. 195; warm full, i. 199.

Baths, hot salt, use of, in tuberculosis of mediastinal lymph glands, iii. 506 .

Baths, hot sponge, use of, in pneumonia, ii. 231, 298.

Baths, hot tub, use of, in acute alcoholism, ii. 589 ; in after-treatment of morphinism, ii. 624; in arthritis deformans, ii. 712 ; in asthenic form of measles, ii. 154; in bronchopneumonia, iii. 418; in cardiac insufficiency, iii. 678; in cerebrospinal meningitis, ii. 215; in chlorosis, iii. 814 ; in chronic opium poisoning, ii. 619 ; contraindicated in cerebral hyperemia, iv. 385; contraindicated in chronic cerebral hyperemia, iv. 387 ; contraindicated in marantic sinus thrombosis, iv. 427 ; contraindicated in multiple sclerosis, iv. 281 ; in measles, ii. 150 ; in migraine, iv. 594; in myositis, iv. 605; in nephritis complicating scarlet fever, ii. 133; objections to, for relief of headache in neurasthenia, iv. 566; in pellagra, ii. 662; in pneumonia, ii. 233; for relief of renal colic, iv. 101; in Rocky Mountain fever, ii. 566; in spasmodic laryngitis, iii. 342 ; in spinal syphilis, iv. 274 ; in wood alcohol poisoning, ii. 610 . 
Baths, hydroelectric, use of, in cardiac insufficiency, iii. 634; indications for, iii. 635); physiological action of, iii. 634 .

Baths, iced, use of, in insolation, iv. 678.

Baths, incandescent light, use of, in chronic bronchitis, iii. 378 .

Baths, localized, i. 200.

Baths, medicated, use of, in leprosy, iv. 814; in pellagra, ii. 662 ; of no value in treatment of nephritis, iv. 32 .

Baths, mercurial, use of, in hereditary syphilis, ii. 433; in syphilis, ii. 389.

Baths, modified carbonated, use of, for relief of cardiac palpitation in neurasthenia, iv. 568 .

Baths, Nauheim. See Nauheim baths.

Baths, oxygen, iii. 634.

Baths, prolonged, hot tub, use of, for relief of spasticity in spinal syphilis, ii. 274 .

Baths, prolonged lukewarm, use of, in amytropic lateral sclerosis, iv. 259.

Baths, prolonged warm tub, use of, for relief of spasticity in syringomyelia, ii. 279 ; in spastic spinal paralysis, ii. 270 .

Baths, radium. See Radium baths.

Baths, salt, use of, in arthritis deformans, iii. 712,713 ; in chronic bronchitis, iii. 367 ; as protection against bronchitis, iii. 351 ; in rachitis, ii. 816.

Bath, tepid full, use of, for relief of cardiac palpitation in neurasthenia, iv. 568.

Bath, tepid sponge, use of, in erysipelas, ii. 284 ; in infantile diarrhea, ii. 239 ; in neurasthenia, iv. 547.

Baths, tepid tub, use of, in amytropic lateral sclerosis, iv. 259; in infantile diarrhea, ii. 239; in measles, ii. 149; in varicella, ii. 111.

Baths, tonic, use of, in multiple sclerosis, iv. 281 ; in tabes, iv. 200.

Baths, Turkish. See Turkish bath.

Baths, warm full, in arteriosclerosis, iii. 760.

Baths, warm salt, use of, locally in arthritis deformans, ii. 712, 713.

Bath, warm shower, value of, in arteriosclerosis, iii. 760 .

Bath, warm sponge, use of, in neurasthenia, iv. 547 ; to relieve insomnia in cardiac insufficiency, iii. 66 .

Baths, warm tub, use of, in anterior poliomyelitis, iv. 238, 240; in arthritis deformans, ii. 713 ; for attacks of excitement in progressive paralysis of the insane, iv. 493; in cerebrospinal meningitis, ii. 215; in chronic myelitis, iv. 230; in delirium tremens, ii. 602 ; in diabetes mellitus, ii. 762; in nephritis complicating, ii. 133; in neuritis, iv. 288; in paralysis following meningitis, ii. 216 ; for relief of insomnia in chorea minor, iv. 663; for relief of lancinating pains in tabes, iv. 196, 200; for relief of pain in sciatica, iv. 299; in scarlet fever, ii. 125; in small-pox, ii. 47; in syphilis, ii. 365.

Batrachia, treatment of poisoning due to, iv. 683.

Baumann, thyroidin first discovered by, iii. 907.

Bavaria, introduction of vaccination into, ii. 61 .

BEARD, on neurasthenia, iv. 525.

Beck's bismuth paste, injection of, in treatment of fistulæ complicating tuberculosis, ii. 509.

BED, arrangement of, in bacillary dysentery, ii. 324.

BEDBugs, as carriers of Indian relapsing fever, iv. 759 .

Bedding, care of, in tuberculosis, ii. 467.

BeDNAR's APHTHE, iii. 10; treatment of, iii. 11.

Bedsores, precautions against, in acute myelitis, iv. 224; precautions against, in tabes, iv. 199; in progressive paralysis of the insane, iv. 494; treatment of, in acute myelitis, iv. 227; treatment of, in typhoid fever, ii. 10; treatment of, in typhus fever, ii. 38 .

"Bedsores, acute," danger of, in cerebral hemorrhage, iv. 392.

Beef juice, use of, for anemia accompanying rachitis, ii. 818; as article of invalid dietary, i. 647.

Beef pulp, as article of invalid dictary, i. 647.

Beef tea, as article of invalid dietary, i. 646.

Beef and pancreas infusion, use of, in diabetes mellitus, i. 761 .

BEER, as a cause of chronic myocardial insufficiency, iii. 729 ; prohibition or restriction of, in cardiac insufficiency, iii. 687.

BeEr heart, iii. 707. 
BELChing, induction of, for relief of tachyeardia, iii. 787.

BELGIUM, introduction of vaccination into, ii. 60 .

Belladonna, use of, in acute bronchitis, iii. 362 ; in acute enteritis, iii. 194; in acute myelitis, iv. 226; in bronchial asthma, iii. 383 ; in cardiac toxemias of pneumonia, ii. 251 ; in chronic dilatation of stomach, iii. 180; in colon bacilluria, iv. 128 ; in enterocolitis complicating measles, ii. 161; for enuresis, iv. 122, 133; in epilepsy, iv. 648; in exophthalmic goiter, iii. 901; in gastric hyperacidity, iii. $92,95,100$; by inhalation in acute bronchitis, iii. 363; iodin combined with, to lessen ill effects of, ii. 406; to lessen irritability of bladder in tuberculous kidney, iv. 117; in membranous enteritis, iii. 208; mixture containing, in vesical crises of tabes, iv. 197; in peristaltic unrest, iii. 250; in prevention of scarlet fever, ii. 123; in rectal suppositories for acute or intermittent gastric hypersecretion, iii. 102; in rectal suppositories for colitis, iii. 205; for relief of "colds" accompanying tuberculosis, ii. 508; for relief of cough in measles, ii. 153; for relief of headache from cardiac disease, iv. 603 ; for relief of profuse expectoration in chronic bronchitis, iii. 376; for relief of sciatic pain in diabetes, ii. 766; for relief of vesical disturbance in tabes, iv. 198; in spastic constipation, iii. 231; symptoms due to, in whooping-cough, ii. 175 .

Belladonna, hyoscyamus, and xanthoxylum, use of, in treatment of alcoholism, ii. 598 ; in treatment of morphinism, ii. 621.

Belladonna fomentations, use of, in adenitis accompanying glandular fever, ii. 576.

Belladonna ointment, use of, in mumps, ii. $170,171$.

Bell's PALSY. See Neuritis of seventh nerve.

Belt, abdominal, use of, in gastroptosis, iii. 182.

BenEKe, Nauheim baths first recommended by, iii. 616 .

Benign Growths of skin, value of X-ray therapy in treatment of, i. 536.
Benign tumors of brain, iv. 433; varieties of, iv. 434.

BENNETT, method of treating trigeminal neuralgia by osmic acid injection introduced by, iv. 342 .

Bennett, Hughes, leukemia first described by, iii. 844 .

Benzene, use of, as substitute for male fern, iv. 779 .

Benzidin dyes, use of, in trypanosomiasis, iv. 747.

Benzin, injections of, to destroy larvæ in myiasis, iv. 689 .

Benzoic acid, use of, in destruction of filaria bancrofti, iv. 787; in pyelitis with alkaline urine, iv. 90.

Benzoic acid combined with camphor, use of, in pneumonia of young children, iv. 254.

Benzoin, compound tincture of, use of, in bronchorrhea complicating pneumonia, ii. 261 ; in herpes of lips, iii. 6 ; in inflammation of larynx during small-pox, ii. 51 ; by inhalation in acute bronchitis, iii. 358; for relief of cough in pneumonia, ii. 257; for relief of gangrene of lung complicating pneumonia, ii. 261 ; in respiratory type of influenza, ii. 189.

Benzoin, compound tincture of, and paregoric, use of, by inhalation in spasmodic laryngitis, iii. 342 .

Benzoinated steam, inhalations of, in chronic atrophic laryngitis, iii. 343.

Benzosol, use of, in chronic enteritis, iii. 200; in chronic mucous colitis, iii. 206; in enlargement of lymphatic glands complicating measles, ii. 162; in tuberculosis of mediastinal lymph nodes; iii. 507.

Benzoylchlorid combined with nastin, use of, in leprosy, iv. 815.

Berghausen, rules suggested by, for prevention of tetanus, ii. 350 .

BERI-BERI, iv. 844; arsenic in food a possible cause of, iv. 848; combination of factors as cause of, iv. 849 ; conditions influencing, iv. 845 ; definition of, iv. 844; diet in, iv. 853; diet badly balanced a predisposing cause of, iv. 847; etiology of, iv. 845; infected food a possible cause of, iv. 848; infected places a possible cause of, iv. 849; infection theory of, iv. 849; intoxication from bacterial infection a possible cause of, iv. 849; 
nitrogen starvation a possible cause of, iv. 846 ; prophylaxis against, iv. 850); surgical measures in, iv. 8.54; treatment of, iv. 852 .

BerLin, mortality from small-pox in, before and after vaccination, ii. 99.

BERNHEIM, views held by, on hypnosis, iv. 570.

Bernheim's method of hypnotization, i. 566.

Beta eucain, use of, as spray in adenoid vegetations, iii. 38 .

BETA RAYs, i. 462; penetration of, i. 465; use of, in eancer of buccal mucous membrane, i. 484.

Betanaphthol, use of, as anthelmintic in hookworm disease, iv. 793; as antiseptic in intestinal autointoxication, ii. 675; in chronic enteritis, iii. 200; to create intestinal antisepsis, in chlorosis, iii. 815; in diarrhea complicating septicemia, ii. 291 ; in enterocolitis, complicating measles, ii. 161; in hookworm disease, iv. 793; in typhoid fever, ii. 261.

Betanaphthol ointment, use of, in crawcraw, iv. 692.

BETA-OXYBUTYRIC ACID, relation of, to diabetes mellitus, ii. 731 ; test for, in urine, ii. 733 .

BEverages, as auxiliary articles of diet, $\mathrm{i}$. 603.

Beverages, alcoholic. See Alcoholic beverages.

Beverages containing caffein. See Caffein-containing beverages.

Bichlorid of mercury. See Mercury bichlorid.

Bier's method of artificial hyperemia, i. 449; in arthritis deformans, ii. 712; in erysipelas, ii. 285; in gonococcus arthritis, ii. 307 ; for relief of boils in diabetes mellitus, ii. 765 ; for relief of muscular atrophy due to beri-beri, iv. 854; for relief of trophic disturbances in syringomyelia, iv. 279.

Biermer, use of lime-water in plastic bronchitis suggested by, iii. 392 .

Biggs; Dr. Herman, antituberculosis work done by, ii. 448 .

Bilateral empyema. See Empyema.

Bile passages, diseases of, iii. 258.

Bile salts, therapeutic use of, in tumors of pancreas, iii. 305 .
BILARY-IIFATIC DISEASE, hydrotherapy for relief of, i. 212 .

Bhaous peneumonia. Sce Pneumonia.

Gillings, FraNk, on administration of mercury in syphilitic disease of liver, iii. 26.5; on operation for gallstones, iii. 276; on value of salvarsan in syphilitic disease of liver, iii. 265.

Binswater, baths of graduated temperature recommended by, in epilepsy, i. 222.

Binz, on effect of alcohol on temperature of body, ii. 230 .

Biological efFects of the X-RAy, i. 508.

Bismuth, use of, in amyxorrhea gastrica, iii. 101; in Bednar's aphthe, iii. 11; in chronic enteritis of molerate severity, iii. 199, 200; in chronic mucous colitis, iii. 206; in cirrhosis of liver, iii. 281; in continuous gastric hypersecretion, iii. 106; to control diarrhea of Addison's disease, iii. 885; to control diarrhea in plague, iv. 827; to control diarrhea in tuberculosis of intestines, ii. 505 ; to control diarrhea in tubereulous kidney, iv. 117; to control gastric hemorrhage, iii. 113 ; to control infantile diarrhea, ii. 329 ; in enteritis of infancy, iii. 203; in enterocolitis complicating measles, ii. 160; in gastric hyperacidity, iii. 92 ; in intestinal amebiasis, iv. 709; in gastrointestinal type of influenza, ii. 190; in non-bleeding gastric ulcer, iii. 134; in stomatitis herpetica, iii. 10; on tampon for control of epistaxis, iii. 335; in ulcerative colitis, iii. 211; in Vincent's angina, iii. 65.

Bismuth and opium, use of, for relief of pain in meat posioning from $\mathrm{B}$. enteritidis, ii. 641.

Bismuth and sodium bicarbonate, use of, for relief of vomiting in acute gastritis, iii. 155.

Bismuth, sodium bicarbonate, and nux vomica, use of, in delirium tremens, ii. 602.

Bismuth preparations, use of, in acute enteritis, iii. 194.

Bismuth subcarbonate, use of, in typhoid fever, ii. 26.

Bismuth subgallate, use of, in enterocolitis complicating measles, ii. 161.

Bismuth subnitrate, poisoning by, i. 721. 
Bismuth subnitrate, use of, as antiseptic in intestinal autoinoculation, ii. 675; in chronic stage of bacillary dysentery, ii. 328 ; to control diarrhea complicating septicopyemia, ii. 291; to control diarrhea in pellagra, ii. 662; as dusting powder in diabetic vulvitis, ii. 765; as dusting powder in tropical phagedenic ulcer, iv. 868 .

Bitter waters, i. 233.

Bitters, use of, in after-treatment of acute gastritis, iii. 157; in carcinoma of stomach, iii. 165; in chronic myocardial insufficiency, for relief of gastric intestinal symptoms, iii. 739 ; in depressive disorders of gastric secretion, iii. 150; in gastric atony, iii. 172; in secretory and motor gastric neuroses, iii. 188; in solution, in gastric lavage, iii. 172, 180; in tabes, iv. 213.

"Black measles," ii. 154.

Black wash, use of, in chancre of vagina or uterine neck, ii. 425; in exulcerated chancre, ii. 423; as gargle, in chancre of mouth, lips, or tongue, ii. 425; in secondary syphilis of mucosæ and mucocutaneous surfaces, ii. 426.

Blackwater Fever, iv. 738; definition of, iv. 738; preventive measures in, iv. 741; remedial measures in, iv. 740 ; relation of, to malaria, iv. 738,742 ; symptoms of, iv. 739 ; theories of causation of, iv. 739 .

BlADDER, aspiration of, see Aspiration; distinction between atony of, and paralysis of, iv. 124; disturbance of, in spinal syphilis, iv. 271, 273; galvanization of, for relief of vesical disturbances in tabes, iv. 198; impairment in function of, in tabes, iv. 190; importance of attention to, in acute myelitis, iv. 225; importance of attention to, in chronic myelitis, iv. 231 ; irrigation of, in chronic cystitis, iv. 120 ; irrigation of, in cystitis complicating acute myelitis, iv. 227; irrigation of, in tabes, iv. 198; irritability of, a cause of enuresis, iv. 129; measures for relieving distress from implication of, in tuberculous kidney, iv. 117; prevention of catheteral infection of, iv. 120; prevention of infection of, by tubercle bacillus, iv. 119; prevention of spontaneous pyogenic infection of, iv. 119 ; treatment of functional neuroses of, iv. 121.

Bladder, Diseases of, hydrotherapy for relief of, i. 209; Spa treatment for relief of, i. 241.

Bladder, Functional Neuroses of, iv. 121 ; treatment of enuresis in, iv. 121; treatment of paralysis of bladder in, iv. 123 ; treatment of reflex neuroses in, iv. 122.

Bladder, Paralysis of, iv. 124.

Bladder, Reflex neuroses of, iv. 122.

Bladder worm. See Echinococcus.

Blanket pack. See Pack.

Blastomycosis, X-ray therapy for relief of, i. 536.

Blaud's pills, use of, in chlorosis, iii. 817 ; in convalescence from acute endocarditis, iii. 746; in hookworm disease, iv. 794; in neurasthenia, iv. 558; for relief of anemia in acute nephritis, iv. 63.

BLeEders, vaccination contraindicated in case of, ii. 74 .

Bleeding. See Venesection.

Blepharospasm, iv. 655.

Buindness, due to wood alcohol poisoning, ii. 610 .

Blister, fly, use of, in syphilitic iritis, ii. 429.

Blisters, use of, contraindicated in active congestion of the lungs, iii. 406; over mastoid in aural vertigo, iv. 316 ; in pericarditis, iii. 518; for relief of intercostal neuralgia, iv. 357 ; for relief of late pain in herpes zoster, iv. 359; for relief of pain in sciatica, iv. 299; for relief of pain in spinal syphilis, iv. 274; in rheumatic cases of facial paralysis, iv. 311.

BLood, action of radium upon, in leukemia, iii. 853; changes in, due to rachitis, ii. 818; coagulants of, see Coagulants; effect of cold baths upon, i. 190; effect of hot baths upon, i. 192; effect of massage upon, i. 294; effect of X-rays upon, i. 517.

Blood, defibrinated, use of, by transfusion in pernicious anemia, iii. 831; by transfusion and intravenous injection of, in treatment of poisons, i. 672 .

Blood, lavage of. See Lavage.

Blood, transfusion of. See Transfusion.

Blood, withdrawal of. See Venesection. 
Blood niseases, balneology for relief of, $i$. 239 ; causing cyanosis, iii. 877 ; concerned in causation of acute myelitis, iv. 221 .

Blood examination, in chlorosis, iii. 811; in pernicious anemia, iii. 824 ; in polycythemia with splenomegaly, iii. 878 .

Blood-Forming ongans, effect of X-rays upon, i. 518.

Bloon pressure, iii. 550; clinical rletermination of, iii. 556; effect of amyl nitrite upon, iii. 609; effect of camphor upon, iii. 614 ; effect of erythrol tetranitrate upon, iii. 609 ; effect of meats upon, iv. 43; effect of nitroglycerin upon, iii. 606; effect of potassium and sodium nitrite upon, iił 607; effect of tobacco smoking upon, iii. 783, 784; factors influencing, iii. 5.52; increase of, in chronic interstitial nephritis, iv. 67 ; increase of, by extract of pituitary gland, iv. 51 ; increase of, by extract of posterior lobe of hypophysis, i. 109; increase of, in nephritis, iv. 18; influence of high, in causation of arterial sclerosis, iii. 757 ; lateral, iii. 550; lowering of, in pleurisy, iii. 556; maximal, iii. 553; minimal, iii. 553; normal values in, iii. 558 ; not increased by digitalis, iv. 50; pathological considerations lowering, iii. 555; pathological conditions raising, iii. 554; physical variations in, iii. 554; pulse pressure due to, iii. 553 ; relation of maximal and minimal to chronic myocardial insufficiency, iii. 695; terminal, iii. 551; tests for myocardial insufficiency dependent upon, iii. 694; value of determining, iii. 558; velocity pressure and, iii. 550 .

Blood vessels, effects of epinephrin upon, i. 91 .

BLoODY vomit, measures for relief of, in yellow fever, iv. 856 .

Blue book on vaccination, ii. 59.

Blue mass, use of, in biliousness, iii. 260; for constipation in acute bronchitis, iii. 354 ; in peritonsillitis, iii. 43.

Blundin, Dr. Emil, on infection by filaria loa, iv. 790 .

BoAs, formula for nutrient enema recommended by, iii. 119.

Bodenhamer's ReCtal IRRIGators, ii. 674.

BoDy, loss of heat from, iv. 674; position of, see Posture.
BODY WEIGHT, average, at different ages, ii. 782 ; secretion of sexual glands in relation to, ii. 786 .

Bo(iHEAN, apparatus for compressing thorax devised by, iii. 435.

Вонвмг, mortality from small-pox in, before and after vaceination, ii. 99.

Bons, accompanying diabetes mellitus, ii. 765 ; electrotherapeutics for relief of, i. 404.

Borx, experiments by, on cause of atrophic cirrhosis of liver, ii. 679.

Bolus alba. See Kaolin.

BONE AND PERIOSTEAl heAdaches, iv. 603.

BONE MARRow, effect of X-rays upon, $\mathrm{i}$. 519 ; therapeutic use of, iii. 836 .

BoNes, SYPHILITIC LEsioNs OF, local treatment of, ii. 430.

BONNIER's SYNDROME, iv. 628.

Воокs, for patient's use in psychotherapy, iv. 551 .

Boos, poisoning from aisorption of magnesium sulphate demonstrated by, iii. 317.

Boracic acid, use of, as dusting powder in herpes zoster, iv. 359 ; effects of, upon metabolism, i. 714; for irrigation of bladder in acute cystitis, iv. 120; for irrigation of bladder in cystitis complicating acute myelitis, iv. 227.

Boracic acid ointment, use of, in oriental sore, iv. 756 .

Borax, use of, to relieve itching in chickenpox, ii. 112.

Borax and boric acid, animal experiments concerning, i. 713; effects of, on digestion, i. 712 ; effects of, on mucous membranes, i. 713 ; poisoning by, i. 710 ; preservative properties of, i. 719; use of, for irrigation, in acute peritonsillitis, iii. 43.

Borax and camphor water, use of, to relieve itching in hay fever, iii. 391 .

Borax ointment, use of, in non-bleeding gastric ulcer, iii. 133.

Boric acid, use of, in acute glossit is, iii. 24; in chronic glossitis, iii. 25; in destruction of filaria bancrofti, iv. 787; as dusting powder in craw-craw, iv. 692; in gastric lavage for chronic gastritis, iii. 161; in hygiene of mouth, iii. 2, 3; for injections in chancre with phimosis, ii. 423 ; for irrigation of abscess cavity 
in cervical glands in diphtheria, ii. 278; for irrigation of bladder in endemic hematuria, iv. 785; for irrigation of colon in colitis, iii. 205; as mouth wash in stomatitis herpetica, iii. 10; as nasal douche in membranous rhinitis, iii. 330; in prophylaxis of symptomatic parotitis, iii. 28; in stomatitis catarrhalis, iii. 7; in stomatitis gangrenosa, iii. 18; in stomatitis hypermycetica, iii. 13; in stomatitis ulcerosa chronica, iii. 16; in varicella, ii. 113; on wet compresses for inflamed chancre, ii. 422.

Boric acid and sodium bicarbonate, use of, as douche in chronic nasopharyngitis, iii. 32 .

Boric acid mouth wash, use of, in measles, ii. 148; in mumps, ii. 176.

Bosworth's method, of treating nose in acute rhinitis, iii. 327 .

BotTINI's GaLVANOCAUTERY, use of, in retention due to chronic prostatitis, iv. 168.

BouCHARD, autointoxication attributed by, to dilatation of stomach, ii. 678 .

Bouchard's method, for reduction of obesity, ii. 794 .

Bougies, esophageal, passage of, in cicatricial stenosis of esophagus, iii. 49 ; in diverticula of esophagus, iii. 62 ; in idiopathic dilatation of esophagus, iii. 58 ; in Vincent's angina, iii. 65.

Bouillaud, potassium iodid first employed by, iii. 765 .

Bouillon filtrate, Denys. See Denys.

Bouveret, essentials for success in treatment of the neuroses emphasized by, iv. 531 .

BowDITCH, paracentesis in diseases of the pleura advocated by, iii. 457 .

BoweLs, care of, in acute diffuse peritonitis, iii. 317 ; care of, in acute localized peritonitis, iii. 312; care of, in chronic enteritis, iii. 198; care of, in pyelitis, iv. 89; care of, in typhoid fever, ii. 25; elimination through, in nephritis, iv. 32; elimination through, in uremia, iv. 53 ; evacuation of, in acute gastritis, iii. 155; evacuation of, in acute infectious myocarditis, iii. 750; evacuation of, in cardiac insufficiency, iii. 572; evacuation of, in chronic bronchitis, iii. 372 ; evacuation of, in gastric atony, iii. 172; importance of attention to, in acute myelitis, iv. 225; importance of elimination through, in pregnancy, iv. 82.

BOWMAN'S THEORY, of renal secretion, iv. 11. "Box system," employment of, in scarlet fever, ii. 122.

Brachial neuralgia. See Neuralgia.

Brachial neuritis. See Neuritis.

Brachial plexus, neuritis of. See Neuritis.

BradDON's THEORY, as to causation of beri-beri, iv. 848 .

Bradycardia, as a complication of pneumonia, ii. 260; paroxysmal, iii. 787.

-BraID, pioneer in hypnotism in America, i. 563.

Brain, anemia of. See Cerebral anemia.

Brain, circulatory diseases of, iv. 381: cerebral anemia, iv. 381 ; cerebral hemorrhage, iv. 388; hyperemia of brain, iv. 383.

Brain, hemorrhage in. See Cerebral hemorrhage.

Brain, hyperemia of. See Hyperemia.

Brain, Parasites of, iv. 459; diagnosis of, iv. 460 ; introduction to subject of, iv. 459 ; prognosis in, iv. 460 ; prophylaxis in, iv. 460 ; symptoms of, iv. 459; treatment of, iv. 461.

Brain, softening of. See Cerebral softening.

Brain, syphilitic diseases of, iv. 471; basilar meningitis, iv. 473; diagnosis of, iv. 476; etiology of, iv. 472; introduction to subject of, iv. 471; mortality from, iv. 477; pathological anatomy of, iv. 471 ; prognosis in, iv. 477 ; progressive paralysis of the insane, iv. 489; prophylaxis against, iv. 478; salvarsan treatment of, iv. 479, 481; symptoms of, iv. 473; vascular type of, iv. 474 .

Brain, tumors of, iv. 432; as cause of compression bulbar paralysis, iv. 501; definition of, iv. 432; determination of depth of tumor, iv. 445; diagnosis of, iv. 436; differential diagnosis of, iv. 436 ; etiology of, iv. 433 ; focal symptoms of, iv. 435; general symptoms of, iv. 434; headache as symptom of, iv. 608; hygienic treatment of, iv. 447; introduction of, iv. 432; localization of tumor, iv. 444; operability of, iv. 449; 
operation for, iv. 449; prognosis in, iv. 446; prophylaxis against, iv. 446; regional diagnosis of, iv. 437; situation of, iv. 434; special symptoms of, iv. 435; symptomatic treatment of, iv. 447 ; symptoms of, iv. 434; treatment of, in general, iv. 447 ; trephining operation for, with extirpation, iv. 451; varieties of, iv. 432.

Brain abscess. See Cerebral abscess.

Brain Coverings, diseases of, iv. 362 ; acute inflammation of soft ccrebral membranes, iv. 367 ; diseases of dura mater, iv. 362 ; introduction to subject of, iv. 362 .

Brain puncture, employment of, in acute attacks of hydrocephalus, iv. 465; in hydrocephalus, iv. 465 ; in pachymeningitis hemorrhagica, iv. 364 ; in tuberculous meningitis, iv. 377 .

Brain syphilis. See Brain, syphilitic diseases of.

Bramwell, Dr. Miles, statistics published by, on results of hypnotic suggestion on alcoholism, ii. 600 .

Bran, use of, to stimulate intestinal peristalsis in chronic constipation, iii. 235.

Bran baths. See Baths, bran.

BRAND, use of hydrotherapy in treatment of general infections introduced by, ii. 16.

Brand system of bathing, value of, in pneumonia, i. 221 ; value of, in typhoid fever, i. 219 .

Brandy, use of, in acute diarrheas of the aged, iii. 195; in acute gastritis, iii. 157; as cardiac stimulant in blackwater fever, iv. 741; as cardiac stimulant in curare poisoning, iv. 694; as cardiac stimulant in malarial fever, iv. 727; as cardiac stimulant in strophanthus poisoning, iv. 695 ; in syncope due to cerebral thrombosis, iv. 398 .

Branting, physiology of medical gymnastics investigated by, i. 298.

BRAUER, introduction of operation called cardiolysis by, iii. 508; "open method" of producing artificial pneumothorax in tuberculosis devised by, ii. 534; operation devised by, for relief of adhesive pericarditis, iii. 528; technique of operation for relief of adhesive pericarditis, iii. 529 .
Brauer apparatus, for use of differential air pressure, iii. $446,447$.

Brauer's positive pressure device, use of, in traumatic hemothorax, iii. 490.

Brazil, infantile cachexia in. See Infantile cachexia.

BrEAD, digestibility of, i. 630; over-indulgence in, a cause of gastric hyperacidity, iii. 79; use of, in diabetes mellitus, ii. 746.

BrEad MAKING, i. 623.

BREAD PUDDING as article of invalid dietary, i. 651 .

Breast-FEedixg, importance of, in prophylaxis against rachitis, ii. 815; importance of, in prophylaxis against tuberculosis, ii. 456.

Breath, offensive, iii. 3.

Breathing exercises, i. 304; use of, in bronchial asthma, iii. 380,385 ; in bronchiectasis, iii. 393,394 ; in chronic bronchitis, iii. 369 .

Brehmer, hygienic-dietetic treatment tuberculosis inaugurated by, ii. 477 .

Broadbent, Sir William, use of cold effusion in delirium tremens recommended by, ii. 606 .

Bromid of ammonium, use of, in treatment of morphinism, ii. 620 .

Bromid of potassium. See Potassium bromid.

Bromid of sodium. See Sodium bromid.

Bromid of strontium. See Strontium bromid.

BRomid INTOXICATION, from continuous use of bromids in epilepsy, iv. 648 .

Bromids, use of, in acute myelitis, iv. 226; with analgesics in post-infectious headache, iv. 612 ; for attacks of excitement in progressive paralysis of the insane, iv. 493; in aural vertigo, iv. 316 ; in bronchial asthma, iii. 384 ; in cardiac neurasthenia, iii. 778; for control of convulsions in anterior myelitis, iv. 238 ; for control of convulsions in rachitis, ii. 819 ; in delirium tremens, ii. 606 ; in diabetes insipidus, ii. 775 ; in the epilepsies, iv. 647; in epilepsy due to cerebral palsies of children, iv. 413; for epileptiform attacks in acute stage of cerebral palsies of children, iv. 412; in exophthalmic goiter, iii. 901; before intubation in diphtheria, ii. 275; in 
lathyrism, iv. 694; in migraine, iv. 593; in mumps, ii. 170; in neurasthenia and psychasthenia, iv. 558, 565; objections to, for relief of insomnia due to cerebral anemia, iv. 383 ; in pericarditis, iii. 520; with quinin to obviate unpleasant effects, iv. 726; by rectum, in cerebrospinal meningitis, ii. 215 ; by rectum, in gastric hyperacidity, iii. 95 ; by rectum in hydrophobia, ii. 342 ; for relief of cardiac palpitation in neurasthenia, iv. 568 ; for relief of headache in acute cerebral meningitis, iv. 372 ; for relief of headache due to brain tumor, iv. 447; for relief of insomnia in acute rheumatism, ii. 302 ; for relief of insomnia in cardiac insufficiency, iii. 664; for relief of insomnia due to chronic cerebral hyperemia, iv. 387; for relief of insomnia in dengue, iv. 860; for relief of insomnia in neurasthenia, iv. 565; for relief of insomnia in neuritis, iv. 286; for relief of insomnia due to sunstroke, iv. 679; for relief of pain in Rocky Mountain fever, ii. 564; for relief of restlessness in acute ophthalmoplegia, iv. 508; for relief of restlessness in neurasthenia, iv. 567; for relief of spasmodic croup in measles, ii. 153; for relief of tinnitus and vertigo, iv. 317 ; in scarlet fever, ii. 127 ; in septicopyemia, ii. 291 ; in spasm of esophagus, iii. 58; in strophanthus poisoning, iv. 695 ; in tetany, iii. 910 ; tuberculosis, ii. 507; in typhoid fever, ii. 26.

Bromin, poisoning by, i. 733 .

Bromoform, use of, in whooping-cough, ii. 181 ; method of administering in whooping-cough, ii. 182.

Brompton Hospital, formula for expectorant mixture in use at, iii. 373.

Bromural, use of, to induce sleep in acute bronchitis, iii. 362 .

BronCHI, DISEASES OF, iii. 350: acute bronchitis, iii. 350 ; bronchial asthma, iii. 379 ; bronchiectasis, iii. 392 ; bronchoadenitis, iii. 398; hay fever, iii. 389 ; plastic bronchitis, iii. 391 .

Bronchial asthma. See Asthma.

Bronchial muscles, effect of epinephrin upon, i. 92.

Bronchiectasis, iii. 392; as a complication of influenza, ii. 194; specific treatment of, iii. 393; surgical treatment of, iii. 394; symptomatic treatment of, iii. 395.

Bronchitic asthma. See Asthma.

Bronchitis, ACUTE, iii. 350; complicating influenza, ii. 189; complicating measles, ii. 153; complicating Rocky Mountain fever, ii. 565; complicating tuberculosis, ii. 507; complicating whooping-cough, ii. 175; definition of, iii. 350 ; hydrotherapy for relief of, iii. 205; internal medication in, iii. 359; local treatment of, iii. 356; prophylaxis against, iii. 351 ; specific treatment of, iii. 354 ; symptomatic treatment of, iii. 355 ; therapy for, in general, iii. 350 ; treatment of, when complicating whooping-cough, ii. 181; treatment of, when due to chronic myocardial insufficiency, iii. 788 .

Bronchitis, Chronic, iii. 366; climatic treatment of, iii. 366 ; clothing in treatment of, iii. 368 ; contagion in, iii. 369 ; diet in, iii. 370 ; environment in treatment of, iii. 366; hydrotherapy for, iii. 369 ; incandescent light baths for, iii. 378; myocardial insufficiency associated with, iii. 710; physical exercises in treatment of, iii. 369 ; postural treatment of, iii. 376 ; serum therapy for, iii. 374 ; treatment of, in general, iii. 370 ; treatment of myocardial insufficiency associated with, iii. 731 .

Bronchitis, Plastic, iii. 391.

Bronchitis kettle, use of, in acute bronchitis, iii. 353.

Bronchoadenitis, iii. 398 .

BRoNCHOPNEUMONIA, iii. 412; circulatory collapse in, iii. 420 ; as a complication of diphtheria, ii. 278; as a complication of influenza, ii. 191; as a complication of measles, iii. 158; as a complication of whooping-cough, ii. 175; complications in, iii. 421; convalescence from, iii. 421; cough in, iii. 421 ; cyanosis in, iii. 419 ; dyspnea in, iii. 419; hydrotherapy for, iii. 419; hygienic measures in treatment of, iii. 416; pain in, iii. 421 ; prophylaxis against, iii. 412 ; specific treatment of, iii. 414 ; treatment of, in general, iii. 414.

BronchorRheA, as a complication of pneumonia, ii. 261.

Brosch, case of poisoning from oysters reported by, ii. 645 . 
Brouardel, early use of steam for disinfection by, ii. 579 .

Brown, Lawrason, cases of tubereulosis considered suitable for sanatorium treatment by, ii. 470 .

Brown-SÉQUARD, revival of organotherapeutics by, i. 67 .

Brucin, poisoning by, i. 751 .

Bruns' symptom, iv. 460.

Brunton, Sir Lauder, experiments by, on effect of ice on mucous membrane, iii. 518 ; use of amyl nitrite in angina pectoris introduced by, iii. 768 .

Bryson, poisoning from use of mutton reported by, ii. 638 .

Buboes, surgical treatment of, in plague, iv. 827.

Buboes, climatic. See Climatic buboes.

Buccal aCcidents, due to hydrargyrism, ii. 384 .

BUCKEYE, poisoning by, ii. 651 .

Budin-Maloney, method employed by, for giving hot baths in cholera infantum, $i$. 218.

BuERgER'S POSTERIOR URETHROSCOPE, iv. 167.

Buffalo gnat. See Simulium reptans.

Bufotalin, iv. 683.

Bufotenin, iv. 683.

Bufragin, iv. 683.

BuHL's disease, iii. 860 .

BÜLAU, method of permanent aspiration of pleural cavity devised by, iii. 485 .

Bulbar paralysis, aCUte apoplectic, iv. 500.

Bulbar paralysis, asthenic, iv. 254; causes of, iv. 254; symptoms of, iv. 255; treatment of, iv. 256.

Bulbar paralysis, ChroNic PROGRessive, iv. 249; clinical phenomena of, iv. 249; duration of, iv. 250; exciting causes, iv. 249; family form of, iv. 253; treatment of, iv. 250.

Bulbar paralysis, COMPression, iv. 501; pathology of, iv. 501; prognosis of, iv. 501; symptoms of, iv. 501; treatment of, iv. 502.

Bulbar paralysis, Progressive, iv. 496; definition of, iv. 496; differential diagnosis of, iv. 497; etiology of, iv. 496; hygienic measures in, iv. 498; medicinal treatment of, iv. 499; prognosis in, iv. 498; symptomatic treatment of, iv. 499; symptoms of, iv. 496; treatment of, iv. 498.

BULiBar paralysis WithoUt anatomical Foundation. See Bulbar paralysis, asthenic.

BulBar symptoms, in myasthenia gravis, iv. 50.5; predominance of, in acute hemorrhagic inferior polioencephalitis, iv. 407.

BULBAR TYPE OF MYASTHENiA GRAVIS, iv. 506.

Burow's solution, use of, in inflamed chancre, ii. 422 .

Burroughs and Wellcome, extract of hypophysis prepared by, for use as diuretic in nephritis, iv. 51 .

Burton, on stenosis of salivary duct in mumps, ii. 171.

Butter, in diet of achylia gastrica, iii. 148; in diet of depressive disorders of gastric secretion, iii. 148; in diet of diabetes mellitus, ii. 743 .

Butterman, experiments by, on venesection in nephritis, iv. 47.

Buttermilk, fat-poor, use of, in acidosis of children, ii. 757.

Buttermilk mixture, use of, as food in cholera infantum, iii. 205; as food in enteritis of infancy, iii. 204.

Butylchloral hydrate, use of, for relief of pain in neuralgia, iv. 340 .

CABOT, modification of Brand bath recommended by, i. 219.

Cabot, Follen, instructions given by, to patients with acute gonorrhea, iv. 142.

Cabot's operation, for resection of urethra, iv. $168,173$.

Cachexia strumipriva, effects of thyroid gland upon, i. 73 ; origin of term, i. 70.

Cacodylate of sodium. See Sodium cacodylate.

Cacodylic acid, salts of, use of, in pernicious anemia, iii. 827, 828 .

Cactus, use of, in diphtheria, ii. 277.

Cadaverin, isolation of, from B. piscicidus agilis, ii. 644 .

Caffein, use of, in acute alcoholism, ii. 589; in acute infectious myocarditis, iii. 750; in acute opium poisoning, ii. 615 ; in acute vasomotor paralysis, ii. 753 ; in asthenic form of measles, ii. 153; in 
barbel cholera, ii. 644; in bronchopneumonia complicating influenza, ii. 192; as cardiac stimulant in beri-beri, iv. 853 ; as cardiac stimulant in bronchopneumonia, iii. 421 ; as cardiac stimulant in strophanthus poisoning, iv. 695; in cardiac toxemias of pneumonia, ii. 255 ; as cardiovascular stimulant in pleurisy, iii. 469; in cerebrospinal meningitis, ii. 215; in cheese poisoning, ii. 647; in cholera infantum, iii. 205; combined with antipyrin or phenacetin, for relief of headache due to toxemia, iv. 610 ; in delirium tremens, ii. 605; in diphtheria, ii. 277; as diuretic in acute nephritis, iv. 58; as diuretic in anuria due to nephrolithiasis, iv. 107; as diuretic in chronic parenchymatous nephritis, iv. 65 ; as diuretic in nephritis, iv. 49 ; as diuretic in pleurisy, iii. 469; as diuretic in uremia, iv. 53 ; in erysipelas, ii. 284; by hypodermic injection in attacks of cardiac asthma, iii. 737 ; by hypodermic injection in methemoglobinuria, iv. 5 ; by hypodermic injection in nephritis, iv. 50; by hypodermic injection in paralytic ileus, iii. 238; by hypodermic injection in prevention of collapse from angina pectoris, iii. 769 ; by hypodermic injection in pulmonary edema, iii. 410; in influenza, ii. 187; in malignant endocarditis, iii. 747 ; in migraine, iv. 593 ; in pneumonia, ii. 235 ; in poisoning by amanita phalloides, ii. 649 ; in poisoning by wood alcohol, ii. 610 ; in pulmonary thrombosis, iii. 411; for relief of edema in acute nephritis, iv. 62 ; for relief of pain in syringomyelia, iv. 278; in scarlet fever with cardiac weakness, ii. 127 ; in septicopyemia, ii. 610 ; as stimulant in collapse associated with renal colic, iv. 106; in toxemia complicating acute diffuse peritonitis, iii. 318.

Caffein and epinephrin, use of, in Addison's disease to relieve disturbances of circulation, iii. 885 .

Caffein citrate, use of, for relief of headache in neurasthenia, iv. 566 .

Caffein-containing beverages, iii. 603.

Caffein-free coffee, use of, in cardiac insufficiency with insomnia, iii. 686 .
Caffein iodid, use of, in bronchial asthma, iii. 384.

Caffein salicylate, intramuscular injection of, in nephritis, iv. 50.

Caffein sodium benzoate, use of, by hypodermic injection, as cardiac stimulant in pleurisy, iii. 469 ; by hypodermic injection in uremia, iv. 53 ; by intramuscular injection in nephritis, iv. 50.

Caffein sodium salicylate, use of, by hypodermic injection in uremia, iv. 53.

Caffein series, use of, in cardiac insufficiency, iii. 601; beverages containing, iii. 603; derivation of, iii. 602 ; diuretic effect of, iii. 604; members of, iii. 601; physiological action of, iii. 602 ; structural formula of, iii. 601 ; therapeutic value of, iii. 605 .

Cajeput, oil of, use of, for flatulency in gastric atony, iii, 172.

Calabar bean, use of, in tetanus, ii. 348 .

"Calabar swellings," iv. 790.

Calcareous waters, action of, i. 236; classification of, 234.

Calcium, deficiency of, in causation of rachitis, ii. 813; withdrawal of excess of, from diet in treatment of arteriosclerotic vertigoes, iv. 624 .

Calcium, use of, in neuralgic headache, iv. 598.

Calcium chlorid, use of, in addition to normal saline solution in Asiatic cholera, iv. 834 ; in addition to salt bath in chronic bronchitis, iii. 367 ; to control gastric hemorrhage, iii. 112; in epidemic dropsy, iv. 860; in hematemesis during cirrhosis of liver with ascites, iii. 288; in hematuria complicating scarlet fever, ii. 134; before operation in chronic catarrhal jaundice, iii. 264; use of, with quinin to obviate quinin intolerance, iv. 726.

Calcium hypochlorite, hypodermic injection of, in solution for snake bite, iv. 682.

Calcium iodid, use of, in syphilis, ii. 409.

Calcium lactate, use of, for control of epistaxis, iii. 336; for control of hemorrhage in pulmonary tuberculosis, ii. 500 ; in hematuria, iv. 4 ; in scurvy, iii. 876 .

Calcium metabolism, in its relation to epilepsy, iv. 640. 
Calcium permanganate, use of, recommended by Rogers in Asiatic cholerie, iv. 836 .

Calcium preparations, use of, in gastric hyperacidity, iii. 95.

Calcium salts, use of, in bronehial asthma, iii. 384; in chronic enteritis, iii. 200); in chronic panceratitis as prophylactio against hemorrhage at operation, iii. 300; in hemophilia, iii. 862, 867; in tetany, iii. 910 ; in tuberculous kidney, iv. 117.

Calculi, pancreatic, iii. 302.

Calculi, renal, formation of, iv. 108; treatment to dislodge, iv. 110.

Calculus, vesical, as a cause of enuresis, iv. 130 .

Calisaya, elixir of, use of, as tonic, in stomatitis ulcerosa, iii. 15.

Calisthenic exercises, value of, in treatment of asthma, iii. 380 .

Caimette's antivenin, use of, against scorpion venom, iv. 685 ; against snake poison, iv. 682 .

Calomel, use of, in active congestion of lungs, iii. 405; in acute apoplectic bulbar paralysis, iv. 501; in acute bronchitis, iii. 354 ; in acute catarrhal jaundice, iii. 261 ; in acute diffuse peritonitis, iii. 317 ; in acute enterit is with vomiting, iii. 193, 195; in acute fibrinous pleurisy, iii. 467; in acute gastritis, iii. 155; in acute gout, ii. 703 ; in acute laryngitis, iii. 338 ; in acute myelitis, iv. 225; in acute nasopharyngitis, iii. 31 ; in acute nephritis, iv. 58; in acute ophthalmoplegia, iv. 508; in acute peritonsillitis, iii. 43 ; in acute rheumatism, ii. 298 ; in acute tonsillitis, iii. 41 ; in anterior poliomyelitis, iv. 237; in bacillary dysentery, ii. 326 ; in blackwater fever, iv. 740 ; in biliousness, iii. 258; in cardiac insufficiency with general anasarca to create diuresis, iii. 677; in cerebral hyperemia, iv. 386 ; in chronic catarrhal jaundice, iii. 264; in chronic enteritis, iii. 200; in cirrhosis of liver, iii. 282, 286; contraindicated as cathartic in nephritis, iv. 32 ; in diarrhea of septicopyemia, ii. 291; in disease of eighth nerve, iv. 316 ; as dressing for sores in frambosia tropica, iv. 765 ; as dusting powder in diabetic vulvitis, ii. 765; as dusting powder in exulcerated ehanere, ii. 423; as dusting powrer in mueons patches on genitalia, ii. 427; as dusting powder in secondary syphilitie cutancous lesions of face, ii. 425; as clusting powder in syphilitic condylomata, ii. 428; as dusting powder in uncomplicaterl chancer, ii. 422; in olematons laryngitis, iii. 341 ; in enteritis of infancy, iii. 201 ; to expel titpeworms, iv. 778; in glandular fever, ii. 576 ; in hydrocephalus, iv. 465 ; in infantile diarrher, ii. 329; in influenza, ii. 187; as insoluble injection in syphilis, ii. 396 ; in intestinal autointoxication, ii. 672 ; by insufflation in syphilis of upper respiratory tract, iii. 349 ; in meat poisoning from $\mathrm{B}$. botulinus, ii. 639 ; in meat poisoning from B. paratyphosus, ii. 642 ; in membranous laryngitis, iii. 342 ; in membranous rhinitis, iii. 330 ; in multiple neuritis, iv. 326 ; for oxyuris vermicularis, iii. 252; in phlegmonous pharyngitis, iii. 40 ; in plague, iv. 827 ; in pneumonia, ii. 227 ; for relief of constipation in neurasthenia, iv. 566; for relief of constipation in tuberculosis, ii. 504; in spasmodic laryngitis, iii. 342 ; in sprue, iv. 862 ; in stomatitis catarrhalis, iii. 7 ; in stomatitis herpetica, iii. 9 ; by sublimation in membranous, laryngitis, iii. 343 ; in syphilis, ii. 388 ; in treatment of gallstones in transit, iii. 274 ; for trichiniasis, iii. 254 ; in typhoid fever, ii. 25; in yellow fever, iv. 855; on vaginal tampons for chancre of vagina and uterine neck, ii. 425 .

Calomel and santonin, use of, in ascariasis, iii. 251.

Calomel and sodium bicarbonate, use of, in acute alcoholism, ii. 589; in chronic alcoholism, ii. 595; in delirium tremens, ii. 602; in Rocky Mountain fever, ii. 564.

Calomel ointment, use of, in chancre of integument, ii. 425; in chancre of lips, ii. 425; in exulcerated chancre, ii. 423; by inunction in prophylaxis of syphilis, ii. 373 ; in symptomatic parotitis, iii. 28 ; in syphilitic alopecia, ii. 429.

Calomel suppositories, use of, in rectal chancre, ii. 424 .

Caloric needs, in diabetes mellitus, ii. 737. 
Calory, use of term as expression of food requirement, i. 604 .

Cammidge reaction, of no value in diagnosis of pancreatic disease, iii. 298.

Camomile tea, use of, for irrigation of colon in colitis, iii. 205.

Campbell's method, of administering izal by intratracheal injection, iii. 398 .

Camphor, use of, in abortive treatment of acute rhinitis, iii. 354 ; in acute endocarditis, iii. 747; in acute infectious myocarditis, iii. 750 ; in acute opium poisoning, ii. 615 ; in acute vasomotor paralysis, iii. 753; in barbel cholera, ii. 644; in cardiac insufficiency, iii. 613; in cholera infantum, iii. 205; in collapse associated with renal colic, iv. 106; in collapse during pneumonia, ii. 327 ; to control epistaxis, iii. 336 ; in delirium tremens, ii. 605; in destruction of tsetse fly, iv. 750 ; in diphtheria, ii. 277; in erysipelas, ii. 284; by hypodermic injection, as a cardiac stimulant in bronchopneumonia, iii. 421 ; by hypodermic injection in pulmonary edema, iii. 410; by hypodermic injection in pulmonary thrombosis, iii. 411; by intratracheal injection in chronic atrophic laryngitis with involvement of trachea, iii. 343; locally, in atrophic rhinitis, iii. 331; locally, in chronic atrophic rhinitis, iii. 343 ; locally, in herpes of lips, iii. 6 ; in methemoglobinuria, iv. 6; in pneumonia, ii. 235 ; in pneumonia with cardiac failure, ii. 253; in poisoning by amanita phalloides, ii. 649; to raise blood pressure, ii. 255; in scarlet fever with cardiac weakness, ii. 127; in septicemia, ii. 291; in toxemia complicating acute diffuse peritonitis, iii. 318 ; in typhoid fever, ii. 23; with spirits of chloroform in acute catarrhal cholecystitis, iii. 267; in whooping-cough, ii. 181.

Camphor and menthol, use of, to allay burning and itching caused by larvæ or plants, iv. 686 .

Camphor, powdered, use of, as dusting powder in herpes zoster, iv. 359.

Camphor, spirits of, inhalation of, for relief of cerebral anemia, iv. 382 .

Camphor, subcutaneous injections of. See Injection.
Camphor monobromate, use of, in neurasthenia, iv. 558, 565.

Camphor oil, use of, in asthenic form of measles, ii. 154; by hypodermic injection in septicemia, ii. 291.

Camphorated oil, use of, in cardiac insufficiency, iii. 614 ; by hypodermic injection as cardiac stimulant in beriberi, iv. 854 ; by hypodermic injection for relief of edema of glottis in acute nephritis, iv. 63.

Camphorated sterile oil, use of, in pneumonia, ii. 235.

Camphoric acid, use of, to control night sweats, ii. 505, iv. 117.

Cancer of BREASt, radium treatment of, i. 484 .

Cancer of buccal mucous membrane, radium in treatment of, i. 483.

Cancer of LIP, radium treatment of, i. 484.

Cancer of tongue, radium treatment of, i. 484 .

Cancer of uterus, radium treatment for, i. 485 .

Cancer cells, action of X-ray upon, i. 537.

Cane-sugar. See Saccharose.

Cannabis indica, use of, in after-treatment of morphinism, ii. 624; in migraine, iv. 593 ; in neurasthenia, iv. 558, 565; for relief of headache in neurasthenia, iv. 566.

Canned fish, poisoning due to, ii. 645 .

Canned meat, poisoning by Bacillus enterıtidis caused by, ii. 640 .

Cannon, Dr., work by, on gastric peristalsis, iii. 68 .

Cantharides, irritation of kidneys by, iv. $14,57$.

Cantharidin, use of, dangerous in nephritis, iv. 49 .

Capps and Leurs, conclusions drawn by, as to cause of accidents following irrigation of pleural cavity, iii. 487; investigations by, on death rate from thoracentesis, iii. 418.

Carbohydrate and fat requirement, i. 610 ; injurious effects of excess of fat, $i$. 611 ; method of reckoning, for a diet of definite energy value, i. 613.

Carbohydrate equivalents in food, ii. 740.

Carbohydrate metabolism, relation between, and diabetes mellitus, ii. 720 . 
Carbohydrate tolerance, hyperactivity of the hypophysis a eause of, i. 103, 108.

Carmohymrates, classification of, as food, i. 600 ; in diet for acute nephritis, iv. 60 ; in diet of chronic parenchymatous nephritis, iv. 65; in diet for gastric hyperaeidity, iii. 81,88 ; in diet for neurasthenia, iv. 539 ; in diet for yellow fever, iv. 856 ; digestibility of, i. 629 ; extra supply of, required during convalescence from pncumonia, ii. 262; function of, ii. 782; pancreatic secretion increased by diet of, iii. 307 ; presence of, in bread, i. 630; presence of, in breakfast cereals, i. 630 ; as source of fat, ii. 783; as source of heat and energy, ii. 782 ; sugar derived from, 782 ; in treatment of acidosis, ii. 756 ; in treatment of uremia, iv. 52 ; value of, as food, i. 601.

Carbolic acid, use of, in atriplicism, iv. 693; in depressive disorders of gastric secretion, iii. 151 ; to dilute tuberculin, iii. 507; as gargle in acute nasopharyngitis, iii. 31 ; as gargle, with alum, in chronic pharyngitis, iii. 33 ; as gargle with compound antiseptic solution in acute tonsillitis, iii. 412; for irrigation of bladder in treatment of tubercular cystitis, iv. 121 ; for irrigation of pleural cavity followed by poisoning, iii. 487; objections to, for vesical irrigation in cystitis complicating acute myelitis, iv. 227; in plague, iv. 827 ; for relief of itching in varicella, ii. 112; in scarlet fever, ii. 120; in tonsillectomy, iii. 45; in treatment of abscess due to cervical adenitis complicating diphtheria, ii. 277.

Carbolic acid and water, use of, as spray in small-pox, ii. 49.

Carbolic acid lotion, use of, warm, in crawcraw, iv. 692.

Carbolic acid pills, use of, in gastric atony, iii. 72 .

Carbolic solution, use of, to destroy larvæ in myiasis, iv. 689.

Carbon dioxid baths. See Baths.

Carbon dioxid gas, use of, as a cutaneous stimulant in tabes, iv. 200.

Carbon monoxid, poisoning by, i. 735 .

Carbonic acid gas, inhalations of, during paroxysm of asthma proposed as relief for, iii. 389.
Carbuncles, treatment of, by electricity conjoined with X-ray, i. 404.

Carcinoma, as a cause of chronic intestinal obstruction, iii. 242; as cause of esophageal stenosis, iii. 48; value of X-ray therapy in, i. 540 .

Carcinoma of esophagus. See Esophagus.

Carcinoma of liver, iii. 291.

Carcinoma of lung, results of operation for, iii. 448.

Carcinoma of pancreas, iii. 303.

Carcinoma of stomach. See Stomach.

Carcinomata of brain, iv. 433.

Carcinomata, secondary mediastinal, improvement in, due to $\mathrm{X}$-ray treatment, iii. 502.

Cardamom, compound tincture of, use of, in pncumonia, for relief of gas aceumulated in stomach, ii. 260; for relief of hiccough, ii. 260 .

Cardiac asthma. See Asthma.

Cardiac beat, cause and origin of, iii. 533.

Cardiac compensation, preservation of, iii. 698; restoration of, iii. 715 .

Cardiac cycle, events of, iii. 541.

Cardiac dilatation. See Dilatation of heart.

Cardiac diseases, balneology for relief of, i. 240.

Cardiac hypertrophy, in nephritis, iv. 20 ; from overwork, a cause of heightened blood pressure, iii. 555; in pyelitis, iv. 94.

Cardiac insufficiency, iii. 571 ; absolute rest important in, iii. 571; alcohol in, iii. 687; carbon dioxid baths for relief of, see Nauheim baths; climatic influence upon, iii. 664; as a complication of chronic pulmonary emphysema, iii. 438; constipation due to, iii. 684 ; diet in treatment of, iii. 573, 683 ; digitalis for relief of, iii. 575; drugs in treatment of, iii. 575; evacuation of bowels in, iii. 572 ; general treatment of, iii. 571 ; gymnastics in treatment of, iii. 636; hydroelectric baths in, iii. 634; hydrotherapy for, iii. 615; marriage in, iii. 680; motoring as recreation in, iii. 666; Nauheim baths for, iii. 616 ; nitroglycerin and the nitrites for relief of, iii. 605 ; occupation in, iii. 678; paracentesis for relief of scrous effusion in, iii. 674 ; parturition dangerous in, iii. 681 ; pregnancy dan- 
gerous in, iii. 681 ; recreation in, iii. 682 ; relative rest in treatment of, iii. 574 ; removal of edema in, iii. 674 ; resumption of vocation in, iii. 662 ; sleep in, iii. 663; strophanthus for relief of, iii. 599; strychnin for relief of, iii. 614 ; travel in, iii. 665 ; treatment of general anasarca in, iii. 672 ; treatment of serous effusion in, iii. 673; venesection for relief of, iii. 667 .

Cardiac neurasthenia, iii. 773; angina pectoris nervosa in, iii. 778; arrhythmias associated with, iii. 774 ; diet in, iii. 776; exercise in, iii. 775, 776; medicinal treatment of, iii. 778; prophylaxis against, iii. 774; rest in, iii. 776; sanatorium treatment of, iii. 777 ; symptoms of, iii. 773 ; treatment of, iii. 775.

Cardiac neuroses, general, iii. 773; cardiac neurasthenia, iii. 773; mechanotherapy for relief of, i. 352 ; reflex, iii. 778; toxic, iii. 782; with well-defined symptoms, iii. 786 .

Cardiac neuroses, reflex, iii. 778; of gastrointestinal origin, iii. 778 ; of respiratory origin, iii. 781 ; of sexual origin, iii. 781.

Cardiac neuroses, toxic, iii. 782; due to alcohol, iii. 786; due to coffee and tea, iii. 786; due to tobacco, iii. 782 .

Cardiac neuroses With Welul-defined sYмРтомs, iii. 786; essential paroxysmal tachycardia, iii. 786; paroxysmal bradycardia, iii. 787; phrenocardia, iii. 788.

Cardiac rhythi, irregularities in, iii. 564.

Cardiac stasis, as indication for use of digitalis, iii. 587 .

Cardiac toxemias, blood pressure affected by, in pneumonia, ii. 254; radiant light and heat in treatment of, i. 455 ; treatment of, in pneumonia, ii. 246; vasomotor centers in spinal cord affected by, ii. 247.

Cardiac weakness, as a complication of scarlet fever, ii. 127.

Cardio-circulatory disturbances, as a complication of influenza, ii. 193.

Cardiolysis, employment of, in cardiac insufficiency due to concretio pericardii, iii. 730; introduction of, by Brauer, iii. 508 ; technique of, iii. 509.

Cardiospasm, iii. 56.
Cardiovascular diseases, electrotherapeutics for relief of, i. 425 .

Cardiovascular disturbances, aecompanying or following paracentesis, iii. 285.

Cardiovascular lesions, presence of, in gout, ii. 695 .

Cardiovascular stimulant. See Stimulants. Carlsbad, treatment at, benefit from, in brachial neuritis accompanying diabetes mellitus, ii. 766; benefit from, in nonbleeding gastric ulcer, iii. 134 .

Carlsbad salts, use of, in cerebral hyperemia, iv. 386 ; in constipation accompanying cirrhosis of liver, iii. 281.

Carlsbad water, use of, in treatment of nonbleeding gastric ulcer, iii. 133.

Carlson, observation by, on cardiac contraction in Limulus polyphemus, iii. 535.

Carminatives, use of, in peristaltic unrest, iii. 250.

Carnivora, treatment of bites by, iv. 684, 685.

Carotid artery, ligature of, for relief of trigeminal neuralgia not to be recommended, iv. 354.

Carpenter, W. B., anecdote of, iv. 338.

"Carriers," of cholera, iv. 828; of dysentery, ii. 320 ; of pneumonia, ii. 222 ; of typhoid fever, ii. 2, 6, 9.

Cascara preparations, use of, in acute infectious myocarditis, iii. 750; in aneurysm, iii. 765; in cardiac insufficiency, iii. 573; in chronic myocardial insufficiency, iii. 740 .

Cascara sagrada, use of, after hemorrhage in tuberculosis, ii. 501; in chronic constipation, iii. 233 ; to control constipation in tuberculous kidney, iv. 117; in nephritis, iv. 33; in neurasthenia, iv. 544 ; to promote evacuation of bowels in gastric atony, iii. 172; for relief of constipation in neurasthenia, iv. 566; for relief of constipation in tuberculosis, ii. 505 .

Casein milk. See Eiweiss milk.

Casper and Richter, phloridzin injection test developed by, iv. 25.

Cassava root, poisoning by, ii. 650 .

Castellani, experiments by, on nature of frambœesia tropica, iv. 762, 763.

Castellani and Chalmers, on treatment of epidemic dropsy, iv. 860. 
Castellor, investigations by, on treatment of typhus fever, ii. 37.

Castlebury method, of feeding diphtheria eases after intubation, ii. 276.

Castor oil, use of, in acute enteritis, iii. 193; in acute gastritis, iii. 155; in bacillary dysentery, ii. 326; in barbel cholera, ii. 644; in Bednar's aphtha, iii. 11; in chronic intestinal obstruction, iii. 242; in chronic mucous colitis, iii. 206; in diarrhea accompanying septicemia, ii. 291; in diarrhea of tuberculosis, ii. 505; in enteritis of infancy, iii. 201; in infantile diarrhea, ii. 329; in Lambert's method of treating alcoholism, ii. 598; in Lambert's method of treating morphinism, ii. 623 ; in meat poisoning due to $\mathrm{B}$; paratyphosus, ii. 642 ; in membranous enteritis, iii. 210; in muscarin poisoning, ii. 648 ; in pericarditis, iii. 515 ; in stomatitis herpetica, iii. 9 ; in tabes, iv. 198.

Castor oil and eucalyptus oil, use of, in intestinal distomiasis, iv. 782.

Castor oil and opium, use of, in ulcerative colitis, iii. 211.

Castor oil and santonin, use of, for ascariasis, iii. 251.

CAstor oll BEAN, proteid character of poisonous elements in, ii. 635.

Casts, urinary. See Urinary casts.

Catacrotic waves of the pulse, iii. 560.

Cataplasms, hot, use of, in myositis, iv. 605.

Cataract, accompanying diabetes mellitus, ii. 767.

Catarrhal conditions, influence of climate in treatment of, i. 281.

Catarrhal stomatipis. See Stomatitis catarrhalis.

Catharsis, employment of, in acute nephritis, iv. 58; necessity for caution in, for nephritis, iv. 32 ; for relief of anuria in acute nephritis, iv. 62; for relief of anuria in nephrolithiasis, iv. 107; for relief of dyspnea in chronic interstitial nephritis, iv. 72; for relief of lightning pains and crises in tabes, iv. 198.

Cathartics, use of, in acute gout, ii. 703; in anuria, iv. 3 ; in beri-beri, iv. 852 ; contraindicated in acute intestinal obstruction, iii. 240; contraindicated in acute localized peritonitis, iii. 312 ; contraindicated in spastic constipation, iii.
231 ; in enterocolitis complicating measles, ii. 160); in gastric irritability of scpticemia, ii. 291; in intestinal autointexication, of doubtful benefit, ii. 671; in reduetion of obesity, ii. 810 .

Cathartics, cholagog, use of, contraindicated in acute catarrhal jaundice, iii. 260.

Cathartics, concentrated saline, objections to use of, in gastric atony, iii. 172.

Cathartics, drastic, use of, in pachymeningitis interna hemorrhagica, iv. 364 .

Cathartics, hydragog, use of, in pleurisy, iii. 469.

Cathartics, saline, use of, in acute catarrhal jaundice, iii. 261; in acute diffuse peritonitis, iii. 317 ; in acute nasopharyngitis, iii. 31 ; in acute nephritis, iv. 58; in acute rheumatism, ii. 298; in acute rhinitis, iii. 327 ; in acute tonsillitis, iii. 41 ; in barbel cholera, ii. 644 ; in biliousness, iii. 258; after calomel, in active congestion of lungs, iii. 405; after calomel in acute bronchitis, iii. 354 ; after calomel in acute gastritis, iii. 155; after calomel in acute laryngitis, iii. 338; after calomel in acute myelitis, iv. 225; after calomel in anterior poliomyelitis, iv. 237; after calomel in plague, iv. 827; after calomel in yellow fever, iv. 855; in cerebral hyperemia, iv. 386 ; chronic catarrhal jaundice, iii. 264; in chronic constipation, iii. 234; in cirrhosis of liver, iii. 283; in constipation associated with gastric hyperacidity, iii. 94; contraindicated in aneurysm, iii. 765; contraindicated in cardiac insufficiency, iii. 685; contraindicated in chronic mucous colitis, iii. 206; contraindicated in chronic myocardial insufficiency, iii. 721; contraindicated in constipation due to chronic myocardial insufficiency, iii. 740 ; in delirium tremens, ii. 602; in diabetes mellitus, ii. 602; in influenza, ii. 187; in intestinal autointoxication, ii. 672 ; in kidney of pregnancy, iv. 82 ; in Lambert's method of treating alcoholism, ii. 579, 598; in meat poisoning due to B. botulinus, ii. 639 ; in meat poisoning due to $\mathrm{B}$. enteritidis, ii. 641 ; in meat poisoning due to $\mathrm{B}$. paratyphosus, ii. 642 ; in multiple neuritis, iv. 326 ; in 
mumps, ii. 170; in muscarin poisoning, ii. 648 ; in nephritis, iv. 32,33 ; in nephritis complicating scarlet fever, ii. 133,134 ; in optic neuritis without apparent cause, iv. 306 ; for oxyuris vermicularis, iii. 252; in phlegmonous pharyngitis, iii. 40 ; in phosphorus poisoning, ii. 628; in pleurisy, iii. 468; in pneumonia, ii. 227; in prophylaxis of cerebral hemorrhage, iv. 391; for relief of migraine, iv. 593 ; in stomatitis catarrhalis, iii. 7; in treatment of gallstones in transit, iii. 274; in uremia, iv. 53.

Cathartics, strong saline, use of, contraindicated in chronic gastritis, iii. 162.

Cathartics, vegetable, use of, in acute nephritis, iv. 58 ; in chronic gastritis, iii. 162 ; in constipation associated with gastric hyperacidity, iii. 595; contraindicated in intestinal autointoxication, ii. 672 ; in nephritis, iv. 33.

Cathelin's epidural injections, method of, iv. 134; for relief of late pain in herpes zoster, iv. 360; for relief of pain in sciatica, iv. 296, 300.

Cathelin's treatment, for enuresis, iv. 122.

Catheter, technique of irrigating urethra with, iv. 157; technique of irrigating urethra without, iv. 157.

Catheterization, asepsis in, iv. 157; indications for, in paralysis of bladder, iv. 124; necessity for, in cerebral hemorrhage, iv. 392 ; necessity for, in curare poisoning, iv. 694 ; for retention of urine in tabes, iv. 198.

CATS, importance of, in prevention of plague, iv. 822 ; katayama disease transmitted by, iv. 786 .

Caudle, as article of invalid dietary, i. 649.

Causal therapy, for tabes, iv. 193.

Caustic alkalies, poisoning by, i. 743.

Caustic, lunar, use of, in stomatitis herpetica, iii. 10.

Caustic potash, poisoning by, i. 744 .

Caustic potash, use of, in treatment of anthrax, ii. 333.

Caustic soda, as a disinfectant for tuberculosis, ii. 453; poisoning by, i. 744 .

Caustics, use of, contraindicated in leukoplakia, iii. 26.

Cauterization, of accidental syphilitic lesions, ii. 375 ; of bite by rabid animal, ii. 336 ; in chronic tonsillitis, iii. 45; of mucous patches, ii. 427; of sloughing tonsils complicating scarlet fever, ii. 130; in stomatitis gangrenosa, iii. 18; in syphilitic onychia, ii. 429.

Cauterization of bladder, objections to removal of tubercular lesions by, iv. 121 .

Cautery, actual, use of, in bite of rabid animals, ii. 336 ; in chronie myelitis, iv. 231 ; in chronic nasopharyngitis, iii. 32 , 33 ; in chronic pharyngitis, iii. 33 ; in gangrenous or phagedenic chancre, ii. 423; in laryngeal tuberculosis, ii. 493; for relief of lancinating pains in tabes, iv. 197; for relief of pain in pyelitis, iv. 88; for relief of pain in spinal syphilis, iv. 274 ; in snake bite, iv. 682 ; in syphilitic onychia, ii. 429; in ulceration of cornea in small-pox, ii. 50.

Cautery, Paquelin. See Paquelin cautery.

Celli, on prophylactic use of quinin in malaria, iv. 735 .

Cellular membranes, i. 53.

Central convolution, diagnosis of brain tumor in, iv. 437; localization symptoms of brain tumor in, 437.

Cereal breakfast foods, digestibility of, i. 630 .

Cereals, cooking of, i. 623; in diet for nephritis, iv. 42.

Cerebellar ataxia. See Ataxia.

Cerebellopontine recess, localizing symptoms of tumor in, iv. 444 .

Cerebellum, atrophy and sclerosis of, iv. 512 ; treatment of, iii. 513.

Cerebellum, diseases of, iv. 512; atrophy and sclerosis, iv. 512; hereditary cerebellar ataxia, iv. 513 .

Cerebellum, tumor in, general symptoms of, iv. 443; local symptoms of, iv. 443.

Cerebral abscess, iv. 419; diagnosis of, iv. 420; diagnosis between, and brain tumor, iv. 437; etiology of, iv. 419; headache as symptom of, iv. 609 ; introduction to subject of, iv. 419 ; microorganisms present in, iv. 419; mortality following operation for, iv. 424; mortality from, iv. 421 ; occurrence of, iv. 421 ; operation for, iv. 423 ; otitic form of, iv. 421 ; prognosis in, iv. 421 ; prophylaxis against, iv. 422 ; surgical prophylaxis against, iv. 422; symptoms of, iv. 420 ; traumatic form of, iv. 421 ; 
treatment of, iv. 423 ; varieties of, iv. 420.

Cerebleal abscess, traumatic, iv. 421.

Cerebral anemia, iv. 381 ; hydrotherapy for relief of, i. 221 ; introduction to subject of, iv. 381; prognosis in, iv. 382 ; prophylactic treatment for, iv. 381; treatment of, during attack, iv. 382.

Cerebral circulation, vertigoes due to impairment of, iv. 623.

Ceremral, conditions, blood pressure raised by, iii. 555 .

Cerebral cortex, indications of a tumor in, iv. 445 .

Cerebral diplegia type of cerebral Palsies of ChildRen, iv. 410.

Cerebral embolism, iv. 400; differential diagnosis in, iv. 400; etiology of, iv. 400 ; pathology of, 401; prognosis in, iv. 401 ; symptoms of, iv. 400 ; treatment of, iv. 402.

Cerebral hemorrhage, iv. 388 ; aftertreatment of, iv. 392 ; chronic interstitial nephritis a cause of, iv. 72 ; as a complication of whooping-cough, ii. 175; diagnosis of, iv. 390; etiology of, iv. 388 ; hydrotherapy for relief of, i. 222 ; prognosis in, iv. 390; prophylaxis against, iv. 390 ; symptoms of, iv. 389 ; treatment of, iv. 391 .

Cerebral hyperemia, active, iv. 383 ; hydrotherapy for relief of, i. 222; passive, iv. 383 .

Cerebral meningitis, acute. See Meningitis.

Cerebral palsies of children, iv. 409; cerebral diplegia type of, iv. 410 ; classification of, iv. 409; diagnosis of, iv. 411; etiology of, iv. 409; hemiplegic type of, iv. 410; prognosis in, iv. 411; prophylaxis against, iv. 412 ; surgical treatment of, iv. 413; treatment of acute stage of, iv. 412 ; treatment of, in general, iv. 412; treatment of paralytic stage of, iv. 413; cerebral embolism, iv. 400 .

Cerebral puncture, in traumatic hematoma, iv. 366 .

Cerebral softening, iv. 395.

Cerebral symptoms of poison, i. 667.

Cerebral thrombosis, iv. 395 ; differential diagnosis in, iv. 397; etiology of, iv. 395 ; pathology of, iv. 396 ; prog- nosis of, iv. 396; symptoms of, iv. 395 ; treatment of, iv. 398.

Cerbiral, venous thrombosis, as a cause of cereb'al palsies of children, iv. 410.

Crenentrs, complieating cerebral hemorrhage, iv. 392.

Chirbmoibulbar glossopharymgolahial paralysis, iv. 5(5).

Cerebrospinal, fluid, examination of, in diagnosis of tuberculous meningitis, iv. 376.

Cerebiospinal meningitis, ii. 199; comparison of therapeutic measures for relief of, ii. 200; diagnosis between, and typhus fever, ii. 39 ; definition of, ii. 199; diet in, ii. 215; etiological forms of, ii. 199; epidemic form of, ii. 200; gavage in treatment of, ii. 215; hydrotherapy for relief of, i. 221; organisms causing, ii. 200; pneumococcus form of, ii. 213; serum treatment in, ii. 202 ; specific treatment in, ii. 200; staphylococcus form of, ii. 214; streptococcus form of, ii. 213 ; symptomatic treatment of, ii. 214 ; treatment of, in general, ii. 214.

Cerebrospinal meningitis, epidemic, ii. 200 ; comparison of specific therapeutic measures in, ii. 200; diphtheria antitoxin for treatment of, ii. 201; leukocyte extract in treatment of, ii. 201; lumbar puncture in diagnosis of, ii. 204; lumbar puncture in treatment of, ii. 201 ; organisms causing, ii. 200; serum treatment for, ii. 202; urotropin treatment for, ii. 201 ; vaccine treatment of, ii. 201.

Cerium oxylate, use of, for relief of gastric crises in tabes, iv. 197.

Cervical adenitis. See Adenitis.

Cervicoöccipital neuralgia. See Neuralgia.

Chair, for use in active stage of tuberculosis, ii. 485.

Chalybeate waters, action of, i. 236 ; classification of, i. 233.

Снамот, statistics of phosphorus poisoning published by, ii. 626 .

Champagne, use of, in acute gastritis, iii. 157 ; as stimulant in cerebral anemia, iv. 382 .

Chancre, excision of, ii. 377; method employed for, ii. 378 .

Chancre, local treatment of, ii. 422; chancre of general integument, ii. 425; chancre 
of lips, mouth, or tongue, ii. 425; chancre with paraphimosis, ii. 424; chancre with phimosis, ii. 423; chancre of vagina and uterine neck, ii. 425; exulcerated chancre, ii. 422; gangrenous or phagedenic chancre, ii. 423; operative measures in gangrenous or phagedenic chancre, ii. 423 ; operative measures in urethral chancre, ii. 424; rectal chancre, ii. 424; urethral chanere, ii. 424.

Chancre of larynx, iii. 348.

Change of climate, importance of, in sprue, iv. 862 .

Change of employment, as a therapeutic measure in occupation neuroses, iv. 661 .

Change of scene, importance of, in convalescence from beri-beri, iv. 854; in convalescence from dengue, iv. 860 ; in convalescence from multiple neuritis, iv. 328; in convalescence from neuritis, iv. 289 .

Chaplin, Arnold, creosote vapor bath in bronchiectasis suggested by, iii. 395 .

Charcoal, suspensions of, use of, in alkaloidal poisoning, i. 672 .

Charcoal mixtures, use of, for flatulency in gastric atony, iii. 172.

Charcot, arthropathies present in tabes first described by, iv. 191; as pioneer in hypnotism, i. 563; use of quinin in nervous deafness and aural vertigo introduced by, iv. 317 .

Charrin, isolation of microörganisms pathogenic for fish by, ii. 644 .

Charrin and Langlois, organotherapy first employed in Addison's disease by, iii. 882.

Chart, showing blood and temperature in a case of African tick fever, iv. 758; showing comparative effect of nitroglycerin, erythrol tetranitrates, and sodium nitrite, on blood pressure, iv. 47; showing relation between rainfall, mosquitoes, and malarial fever, iv. 732; showing spleen index, iv. 714; showing temperature curve in blackwater fever, iv. 740; showing temperature curve in malarial abscess of spleen, iv. 729 .

Chaulmoogra oil, use of, in leprosy, iv. 814, 815.

Cheadle, Dr., summary by, of evidence for supposing acute rheumatism to be an infection, ii. 295.
Cheese, use of, in diabetes mellitus, ii. 743 .

Chense poisoning, ii. 647.

Chekan, use of, for relief of bronchorrhea complicating pneumonia, ii. 261.

Chelodonium, tincture of, use of, in treatment of gallstones in passing, iii. 274.

Chemical agents, as cause of acute nephritis, iv. 27.

Chemnitz, mortality in, from small-pox during epidemic in 1870, ii. 102.

Chemo-immunology, use of, in treatment of pneumonia, ii. 245.

Chetwood's galvanocautery, use of, in retention due to chronic prostatitis, iv. 168.

Chetwood's tube, use of, for rectal douche in chronic gonorrheal urethritis, iv. 165.

Chicken вRoth, as article of invalid dietary, i. 646.

Chicken Jelly, as article of invalid diet, i. 646.

Chicken pea, poisoning by. See Lathyrism.

Chicken-pox. See Varicella.

Chiggers, iv. 690; preventive measures against, iv. 691; remedial measures for, iv. 691 .

Chindhood, prophylaxis against the neuroses in, iv. 52 .

Childhood sports, as a cause of cardiac insufficiency, iii. 699.

Childoren, diabetes in, ii. 768; effect of X-ray therapy upon tissues of young, i. 523; empyema in, see Empyema; malaria in, iv. 728; protection of, against tuberculosis, ii. 458; reduction of obesity in, ii. 787 .

Chill and fever, association of, with renal colic, iv. 106.

Chills, as a symptom of cerebral abscess, iv. 420 ; as symptom of pneumonia, ii. 226 ; as symptom of typhus fever, ii. 35.

Chin-cough. See Whooping-cough.

Chinese, infrequency of typhoid among, ii. 2 ; inoculation for small-pox as practiced among, ii. 52.

Chinosol, use of, in leprosy, iv. 816; in sprue, iv. 862 .

Chipault, mortality after operation for infectious thrombosis reported by, iv 430.

Chittenden and Geiss, work done by, on effects of boracic acid on metabolism, $i$. 714. 
Chloral and morphin, use of, to relieve insomnia associated with mediastinal tumors, iii. 504.

Chloral hydrate, use of, in after-treatment of morphinism, ii. 624 ; in apoplectic form of vestibular vertigo, iv. 629 ; as an antagonist to strophanthus poisoning, iv. 695; in bronehial asthma, iii. 389; contraindieated in alcoholism, ii. 595; contraindieated in neurasthenia, iv. 559, 565 ; to eontrol motor excitement in acute hemorrhagic inferior polioencephalitis, iv. 408; to control restlessness in uremia, iv. 54 ; to control violent movement in chorea, iv. 663 ; dangerous in pericarditis, iii. 520; in delirium of pneumonia, ii. 228; in delirium tremens, iv. 606; for deodorization of sputum in pulmonary abscess or gangrene, iiji. 441; in eclampsia of pregnancy, iv. 83; in epilepsy, iv. 648; by hypodermic injection in carcinoma of stomach, iii. 166; to induce sleep in acute bronchitis, iii. 362 ; in measles, ii. 152; in migraine, iv. 593; mixture containing, for relief of vesical crises in tabes, iv. 197; in pneumonia of alcoholics, ii. 258; by rectum in acute hemorrhagic polioencephalitis with extreme moior excitement, iv. 408; by rectum in cerebral hyperemia, iv. 387 ; by rectum in cerebrospinal meningitis, ii. 215 ; by rectum to control restlessness due to uremia, iv. 54 ; by rectum in hydrophobia, ii. 342 ; by rectum in laryngismus stridulus complicating tetanus, ii. 820 ; by rectum for relief of convulsions in acute stage of cerebral palsies of children, iv. 412 ; by rectum for relief of serial convulsions due to brain tumor, iv. 448; by rectum in renal colic, iv. 106; for relief of arteriosclerotic vertigo, iv. 624; for relief of convulsions in rachitis, ii. 819 ; for relief of insomnia associated with mediastinal tumors, iii. 504; for relief of insomnia due to brain tumor, iv. 449; for relief of insomnia in dengue, iv. 860; for relief of insomnia in erysipelas, ii. 284; for relief of insomnia in multiple neuritis, iv. 327; for relief of insomnia in typhoid fever, ii. 26; in renal colic, iv. 106; to secure sleep in neuritis, iv. 286 ; in scarlet fever for prevention of nephritis, ii. 133 ; in tetany, iii. 910.

Chloral hydrate and bromids, administration of, by enemata for relief of restlessness in cerebral hemorrhage, iv. 392.

Chloralamid, use of, eontraindicated in cardiac insufficiency, iii. 664; to induce sleep in aeute bronchitis, iii. 362 ; in nervous type of influenza, ii. 191.

Chlorate of potash. See Potassium chlorate.

Chloric ether, use of, in pneumonia, to relieve accumulation of gas in stomach, ii. 260.

Chlorid of gold. See Gold chlorid.

Chlorin, poisoning by, i. 733 .

Chlorinated lime, as a disinfectant in tubereulosis, ii. $453,458$.

Chlorinated soda, use of, as disinfectant, in stomatitis gangrenosa, iii. 18; in tuberculosis, ii. 453.

Chloroform, inhalation of, in cough of tubereulosis, ii. 498; in gallstone colic, iii. 268; in laryngeal crises of tabes, iv. 197 ; in laryngismus stridulus complicating rachitis, ii. 820 ; in paroxysms of asthma, iii. 385; in paroxysms of hydrophobia, ii. 342 ; in pulmonary edema, iii. 409; for relief of convulsions from acute alcoholism, ii. 589; for relief of convulsions in acute stage of cerebral palsies of children, iv. 412 ; for relief of convulsions in anterior myelitis, iv. 238; for relief of convulsions in pregnancy, iv. 83 ; for relief of dyspnea due to pressure from a mediastinal tumor, iii. 503 ; for relief of serial convulsions due to brain tumor, iv. 448; in renal colic, iv. 104; in strophanthus poisoning, iv. 695; in tetanus, ii. 348,352 ; in tetany, iii. 910 ; in whooping-cough, ii. 180 .

Chloroform, injections of, to destroy larvæ in myiasis, iv. 689.

Chloroform, internal use of, in eholecystitis, iii. 266; in intestinal distomiasis, iv. 782.

Chloroform, local use of, in atriplicism, iv. 693.

Chloroform and creosote vapor, inhalation of, in acute rhinitis, iii. 328 .

Chloroform and menthol, inhalation of, in acute rhinitis, iii. 328 .

Chloroform anesthesia, use of, in cauterization of wounds made by rabid animals, 
ii. 336 ; in chronic myocardial insufficiency, iii. 743; contraindicated in adenectomy, iii. 36,37 ; contraindicated in retropharyngeal abscess, iii. 34; contraindicated in tonsillectomy, iii. 45; danger from, in operation for liver abscess, iv. 711; injury of kidney by, iv. 28.

Chloroform liniment, use of, for relief of pain in chest accompanying tuberculosis, ii. 506; for relief of pain in syringomyelia, ii. 278.

Chloroform post-anesthetic poisoning, association of, with autointoxication, ii. 683.

Chlorosis, iii. 811; arsenic in treatment of, iii. 821; blood examination in, iii. 811; cholesterin in treatment of, iii. 822 ; complications in, iii. 811,823 ; constipation in, iii. 823; definition of, iii. 811; diaphoresis in treatment of, iii. 814 ; diet in, iii. 813,823 ; headache due to, iv. 611 ; hydrotherapy for, i. 206, iii. 813; intestinal antisepsis in treatment of, iii. 815; iron in treatment of, iii. 812,815 ; manganese in treatment of, iii. 821; mechanotherapy for, i. 348; nervous symptoms in, iii. 823; plasmatic treatment of, iii. 821 ; plasmotherapy for, iii. 822 ; serum therapy for, iii. 822 ; symptoms of, iii. 822 ; treatment of, in general, iii. 812; treatment of special symptoms of, iii. 822 .

Chlorotone, use of, in tetanus, ii. 348; in tetanus combined with antitoxin, ii. 354 .

Chocolate, food value of, iii. 604 .

Choked DISK, iv. 305; as a symptom of brain tumor, iv. 435; as a symptom of serous meningitis, iv. 469 .

Cholagogs, use of, in chronic catarrhal jaundice, iii. 264; in chronic cholecystitis and cholelithiasis, iii. 273.

Cholecystitis, as cause of gastric disease, iii. 180; as complication of typhoid fever, ii. 28; due to influenza bacillus, ii. 190.

Cholecystitis and Cholelithiasis, iii. 265: arguments for considering chronic or recurrent gall bladder disease a medical disease, iii. 277; arguments for considering chronic or recurrent gall bladder disease a surgical disease, iii.
277; general considerations concerning, iii. 265; indications for medical treatment of, iii. 279; indications for surgical treatment of, iii. 279 ; respective indications for medical or surgical treatment of, iii. 275; treatment of acute catarrhal cholecystitis, iii. 266; treatment of chronic cholecystitis, iii. 269; treatment of gallstone colic, iii. 267; treatment of gallstones in transit, iii. 273.

Cholecystotomy, performance of, suggested in distomiasis, iv. 780 .

Cholelithiasis, association of, with chronic pancreatitis, iii. 298, 299; hydrotherapy for relief of, i. 212.

Cholera, Asiatic. See Asiatic cholera.

Cholera infantum, iii. 204; hydrotherapy for relief of, i. 218.

Cholera morbus, iii. 195.

Cholera nostras, iii. 195.

Cholesterin, therapeutic use of, in chlorosis, iii. 822 ; in pernicious anemia, iii. 835 .

Cholin, as a cause of food poisoning, ii. 635.

Chordee, treatment of, in acute gonorrhea, iv. 141.

Chorea, as a complication of acute rheumatism, ii. 304; degenerative, iv. 664; electrica, iv. 664; electrotherapy for relief of, i. 431; hydrotherapy for relief of, i. 222; infectious, see Chorea minor.

Chorea minor, iv. 662; chorea electrica, iv. 664 ; chronic form of, iv. 663 ; congenital form of, iv. 664; definition of, iv. 662 ; degenerative form of, iv. 664 ; habit choreas, iv. 664; Huntington choreas, iv. 664; imitative form of, iv. 664; as isolated phenomena, iv. 665; in pregnancy, iv. 663; treatment of, iv. 662 .

Choroid, tubercles in, a diagnostic sign in tuberculous meningitis, iv. 375 .

Choskx, investigation by, as to antiplague serum, iv. 826.

Chromic acid, local use of, in chronic rhinitis, iii. 329 ; in herpetic stomatitis, iii. 10 ; in mucous patches, ii. 427 ; in stomatitis due to hydrargyrism, ii. 385 ; in stomatitis syphilitica, iii. 21 ; in tertiary syphilitic lesions of mucous and mucocutaneous surfaces, ii. 427 ; in Vincent's angina, iii. 40. 
Chromosantonin, use of, in sprue, iv. 862.

Chromotropic influnnces, on cardiac muscle, iii. 537.

Chrysarobin, use of, in plague, iv. 816 .

Chrysomyea macellaria, iv. 688.

Chrysomyea viridula, iv. 688.

Chuckerbutty, potassium iodid first introduced into England by, iii. 765.

Chvostek's method, of employing electricity in exophthalmic goiter, iii. 898.

Chyostek's symptom in tetany complicating rachitis, ii. 819 .

Chylothorax, iii. 489 ; treatment of, iii. 490.

Cicatrices, radium in treatment of, i. 476.

Cicuta maculata. See Cicuta roots.

Cicuta roots, poisoning by, ii. 650 .

Cigarette smoking, effects of, iii. 783; prohibition of, in cardiac insufficiency, iii. 689 .

Cinchona, alkaloids of, use of, as substitutes for quinin, iv. 726 .

Cinchona bark, use of, in depressive disorders of gastric secretion, iii. 150.

Cinchona, ferrated elixir of, use of, as tonic, in stomatitis ulcerosa, iii. 15.

Cinchona, tincture of, use of, in depressive disorders of gastric secretion, iii. 150.

Cinchonin and ipecac, use of, in acute laryngitis in young children, iii. 339 .

Circulation, collapse of, in bronchopneumonia, iii. 420; disturbances of, in Addison's disease, iii. 885; influence of pleural exudates upon, iii. 460.

Circulation, disorders of, association of, with intestinal autointoxication, ii. 679; due to use of arsenobenzol in syphilis, ii. 415; hydrotherapy for relief of, i. 202 ; massage and gymnastics in treatment of, i. 301 ; in typhoid fever, ii. 23.

Circulation time, iii. 544.

Circulatory apparatus, physiological effects of massage upon, i. 294.

Circumcision, performance of, in excision of chancre, ii. 378.

Cirrhosis of liver, iii. 280; drugs in treatment of, iii. 282 ; electrotherapeutics for relief of, i. 432 ; operative measures for relief of ascites in, iii. 287; organotherapy for, iii. 286; paracentesis for relief of, iii. 284; treatment of, in developmental stage, iii. 280 ; treatment of, in terminal stage, iii. 287.
Citrate of iron and strychnin, hypodermic injection of, in atrophic rhinitis, iii. 330 .

Citrate of magnesium. See Magnesium citrate.

Citrates, use of, as diuretics in acute nephritis, iv. 58.

Citric acid, destruction of cholera germs by, iv. 829 .

Citrin ointment, use of, in syphilitic onychia, ii. 429 .

Claisse, P., formula suggested by, for use as an inhalation in acute bronchitis, iii. 3.58 .

Clark's method, for use of opium in acute diffuse peritonitis, iii. 316 .

Claviceps purpurea, ergotism due to, ii. 652 .

Clavus, hysterical, iv. 614.

Claw hand, in Friedreich's disease, iv. 216.

Cleanliness, importance of, in acute myelitis, iv. 224; importance of, in acute urethral gonorrhea, iv. 137.

Cleansing solutions. See Solutions.

Cleaves, marine searchlight adapted to therapeutic use by, i. 441.

Climate, altitude in relation to, i. 255; atmospheric humidity as a factor in, $i$. 250 ; barometric pressure a factor in, $\mathrm{i}$. 257; definition of, i. 276; dust and atmospheric impurities a factor in, i. 274; electricity a factor in, i. 257 ; factors in, i. 247; influence of, upon acute inflammation of nasal sinuses, iii. 333 ; influence of, upon acute nephritis, iv. 29 ; influence of, upon asthma, iii. 380; influence of, upon atrophic rhinitis, iii. 330 ; influence of, in bronchiectasis, iii. 393 ; influence of, upon cardiac insufficiency, iii. 665; influence of, in cholera infantum, iii. 205; influence of, upon chronic interstitial nephritis, iv. 69; influence of, upon chronic laryngitis, iii. 340 ; influence of, upon chronic parenchymatous nephritis, iv. 30, 64; influence of, upon chronic pulmonary emphysema, iii. 433; influence of, in convalescence from bronchopneumonia, iii. 421; influence of, in convalescence from neuritis, iv. 289 ; influence of, in exophthalmic goiter, iii. 898; influence of, upon neuralgia, iv. 338; influence of, upon neurasthenia and psychasthenia, iv. 556; influence 
of, upon pneumonia, i. 281; influence of, upon pulmonary disturbances associated with cardiac insufficiency, iii. 731 ; influence of, upon respiratory affections, i. 281 ; influence of, upon rheumatoid affections, i. 281; influence of, upon skin diseases, i. 282; influence of, upon tabes, iv. 213; influence of, upon tuberculosis, i. 278; influence of, upon tuberculosis of larynx, iii. 344; influence of vegetation upon, i. 258; insolation a factor in, i. 272 ; land and water as factors in, i. 279; light a factor in, i. 273; physiological adaptation of human beings to, i. 246 ; physiological influence of atmospheric humidity in, i. 263; physiological influence of diminished barometric pressure in, i. 268; psychology of, i. 275 ; reaction of human beings to, i. 246 ; soil as a factor in, i. 258 ; of Spas, i. 240 ; temperature a factor in, i: 247 ; winds a factor in, i. 253.

Chimates beneficial in toberculosis, $i$ i. 474.

Climatic buboes, iv. 864.

Climatic treatment, of acute bronchitis, iii. 351 ; of arteriosclerosis, iii. 760 ; of bronchitis due to chronic myocardial insufficiency, iii. 738; of chronic bronchitis, iii. 366; in chronic rhinitis, iii. 328 ; in convalescence from pneumonia, ii. 262 ; in tuberculosis, ii. 472 ; in tuberculosis of mediastinal lymph nodes, iii. 506.

Climatology, i. 245; application of, to treatment of disease, i. 277 ; classification of climates in, i. 276; meteorological, i. 247; physiological and medical, i. 259; physiological reaction to external temperature, i. 260 ; scope of, i. 245.

Clitoris, adherent, as a cause of enuresis, iv. 130.

Cloetta's digalen. See Digalen.

Clonorchis epidemicus, iv. 779 ; pathology of, iv. 780.

Clonorchis sinensis, iv. 779; pathology of, iv. 780 .

Clonorchis watsoni, iv. 781.

ClopatT, method employed by, for X-ray treatment of mediastinal tumors, iii. 502.

Closure time of heart, iii. 542.
Clothing, choice of, in acute rhinitis, iii. 326; choice of, in chronic bronchitis, iii. 326 ; choice of, in tuberculosis, 489; choice of, in tuberculosis of kidney, iv. 115; disinfection of, in tuberculosis, ii. 467 ; protection against plague by means of, iv. 825.

Clubfoot, following progressive neural muscular dystrophy, iv. 266; prevention of, after anterior poliomyelitis, 241.

Clysma, administration of medicines by, in acute rheumatism, ii. 299.

Coagulants of blood, use of, in hemophilia, iii. 362 ; mineral ions in, iii. 862 ; substances forming complex insoluble colloids, iii. 863.

Coagulins. 'See Precipitins.

Coal miners, frequency of asthma among, iii. 425 .

Coal tar derivatives, use of, in biliousness, iii. 259; conditions contraindicating, in pneumonia, ii. 246; conditions contraindicating, in typhoid fever, ii. 26 ; contraindicated in plague, iv. 827 ; contraindicated in pleurisy, iii. 468; dangerous as substitute for quinin, iv. 726; introduction of, into medicine, ii. 16; methemoglobinuria due to, iv. 4; precautions against methemoglobinuria from, iv. 5; for relief of headache in acute nephritis, iv. 63 ; for relief of headache in cerebral thrombosis, iv. 399 ; for relief of headache in chronic interstitial nephritis, iii. 72; for relief of headache due to high blood pressure,iv. 48 ; for relief of headache in pachymeningitis interna hemorrhagica, iv. 364; for relief of headache in typhoid fever, ii. 27; for relief of headache due to uremia, iv. 54; for relief of lancinating pains in tabes, iv. 196; for relief of neuralgic headache, iv. 598; for relief of pain in neuritis, iv. 285; for relief of pain in neuralgia, iv. 339 ; for relief of pain in pyelitis, iv. 88; for relief of sciatic pain in diabetes mellitus, ii. 766 ; in scarlet fever, ii. 27 ; symptoms due to misuse of, in whoopingcough, ii. 175.

Cocain, use of, in acute inflammation of nasal sinuses, iii. 333 ; in acute opium poisoning, ii. 615 ; as anesthetic in cauterization of bites from rabid animals, ii. 336 ; as anesthetic in cauterization 
for chronie pharyngitis, iii. 33 ; as anesthetic in ehronic rhinitis, iii. 329; as anesthetic in excision of chancre, ii. 378 ; as anesthetic in laryngeal tuberculosis, iii. 346 ; as anesthetic in operation for appendicitis, iii. 220 ; as anesthetic in operation for my cosis of tonsils, iii. 42 ; as anesthetic in searification of larynx, iii. 341 ; as anesthetic in thoracentesis, iii. 474; as anesthetic in tonsillectomy, iii. 45; in asthma, iii. 386 ; in chancre with paraphimosis, ii. 424; to control spasmodic cough in chronic progressive bulbar paralysis, iv. 253; by epidural injection for relief of pain in sciatica, iv. 296, 300; in hay fever, iii. 390 ; by hypodermic injection for relief of pain in neuritis, iv. 285; by hypodermic injection in trigeminal neuralgia, iv. 342 ; as mouth wash in stomatitis due to hydrargyrism, ii. 385; for otitis complicating scarlet fever, ii. 131; for relief of gastric irritability, in typhoid fever, ii. 25 ; for relief of pain following bite of scorpions, iv. 685; for relief of vomiting due to brain tumor, iv. 448; in small-pox accompanied with dysphagia, ii. 47; as spray in asthma, iii. 386 ; as spray to relieve painful deglutition in laryngeal tuberculosis, iii. 347 ; by subarachnoid injection in radicular sciatica, iv. 296; substitutes for, for relief of pain in multiple neuritis, iv. 285 ; in'tertiary syphilitic lesions of mucous and mucocutaneous surfaces, ii. 427 ; in Vincent's angina for relief of dysphagia, iii. 65; in whooping-cough, ii. 182.

Cocain and antipyrin, use of, in acute nasopharyngitis, iii. 32 ; on tampon, for control of epistaxis, 335.

Cocain and oxylate of cerium, use of, for rclief of gastric crises in tabes, iv. 197.

Cocain habit, danger of, from use of cocain in asthma, iii. 387; danger of, from use of cocain in hay fever, iii. 390; danger of, from use of cocain to relieve pain in neuritis, iv. 285; precautions against, in writing prescription for cocain, iii. 328 ; Town's specific treatment for, ii. 597.

Cocain ointment, use of, in herpes zoster, iv. 359 .
Cocain poisoning, i. 763; treatment of, 764.

Cocain suppositories, use of, for relief of pain of nervous character in the anus, perineum, or rectum, iv. 358 .

Cocainism. See Cocain habit.

Coccygodynis, treatment of, iv. 358 .

Cochin China diarirhea, iv. 796; parasite causing, iv. 796; preventive measures in, iv. 797; remedial measures, iv. 797.

Cocos, use of, as article of invalid dietary, i. 651 ; in diet for gastric hyperacidity, iii. 91 .

COCOA WITH MILK, as article of invalid dietary, i. 651.

Cocon Junket, as an article of invalid diet, i. 650 .

Cod liver oil, use of, in after-treatment of herpes zoster, iv. 359; in bronchial asthma, iii. 385; in bronchoadenitis, iii. 399 ; in chronic bronchitis, iii. 371 ; in convalescence from pneumonia, ii. 262 ; in endemic hematuria, iv. 785 ; in laryngeal tuberculosis, iii. 348 ; in measles complicated with enlargement of lymphatic glands, ii. 162; in rest cure for neurasthenia, iv. 558; in treatment of enuresis, iv. 133; in tuberculosis of mediastinal lymph glands, iii. 507 .

Cod liver oil and phosphorus, use of, in rachitis, ii. 817.

Cod liver oil, deodorized, use of, as spray in hay fever, iii. 391.

Codein, use of, in acute bronchitis, iii. 361 ; to allay irritation in laryngeal tuberculosis, iii. 346 ; in bronchitis complicating influenza, ii. 189; in bronchitis due to chronic myocardial insufficiency, iii. 738; in cardiac asthma due to chronic myocardial insufficiency, iii. 737; in chronic intestinal obstruction, iii. 242; in chronic opium poisoning, ii. 620; contraindicated in chronic bronchiectasis complicating influenza, ii. 195; to control cough in active stage of tuberculosis, ii. 482 ; to control cough due to chronic bronchitis associated with cardiac insufficiency, iii. 731 ; to control cough in laryngeal tuberculosis, ii. 498; to control cough in measles, ii. 153 ; in diabetes mellitus, ii. 789 ; in edematous laryngitis due to foreign body, iii. 341 ; in gastric hyperacidity, 
iii. 95,100 ; by hypodermic injection in acute peritonsillitis, iii. 431 ; by hypodermic injection in carcinoma of stomach, iii. 166; by hypodermic injection, for relief of vomiting in cerebral abscess, iv. 423 ; in laryngismus stridulus complicating rachitis, ii. 820 ; in migraine, iv. 593; in mumps, ii. 170; for relief of cervicoöccipital neuralgia, iv. 356; for relief of dyspnea due to chronic myocardial insufficiency, iii. 736; for relief of insomnia in cardiac insufficiency, iii. 664; for relief of painful erection or chordee in acute urethral gonorrhea, iv. 141 ; for relief of pain in neuralgia, iv. 340; for relief of pain in neuritis, iv. 286; for relief of pain in small-pox, ii. 47; for relief of pain and cough in bronchopneumonia, iii. 421; for relief of pain and cough in pneumonia, ii. 226; for relief of vomiting due to brain tumor, iv. 448 ; to restrain delirium in pneumonia, ii. 228; as substitute for morphin in treatment of morphin habit, ii. 618; by suppositories, to relieve pain in acute gastritis, iii. 156; in varicella, ii. 113; in whooping-cough, ii. 181.

Codein and ammonium chlorid, use of, for relief of cough in pneumonia, ii. 257.

Coefficient, of distribution, i. 19; of partition, i. 19 .

Coffee, abuse of, a cause of chronic gastritis, iii. 159; abuse of, a cause of gastric hyperacidity, iii. 76 ; as cause of reflex cardiac neuroses, iii. 786; prohibition of, in brain tumor, iv. 447.

Coffee, administration of, by rectum, in pneumonia, with marked cardiac depression, ii. 230, 249; as article of invalid dietary, i. 652 ; benefit from, during attack of angina pectoris, iii. 769; in diet for chronic parenchymatous nephritis, iv. 66; in diet for gastric hyperacidity, iii. 90; in diet for nephritis, iv. 44,59 ; to prevent collapse in snake poisoning, iv. 683 ; as stimulant in cerebral anemia, iv. 382 .

Coffee, strong, use of, in acute opium poisoning, i. 615 ; in diet of pneumonia, i. 230; in septicopyemia, i. 291.

CoHen, directions by, for use of quinin and urea hydrochlorid in pneumonia, ii. 235.
Cонnнeim, calcium salts recommended by, in chronic enteritis, iii. 200; oil treatment for gastric ulcer recommended by, iii. 137 .

Coils and transformers, i. 367.

Colchicum, poisoning by, i. 758 .

Colchicum, use of, in acute gout, ii. 702; in aural vertigo, iv. 316 .

Cold, EXPosure to, as cause of nephritis, iv. 28; as cause of neuritis of third, fourth, and sixth nerves, iv. 308; as cause of sciatica, iv. 298.

Cold, therapeutic use of, i. 189; degree of, required for stimulation, i. 191; physiological effects of, i. 189.

Cold and heat, alternation of, in treatment of cerebral anemia, iv. 382 .

COLD, WET, importance of protection against, in chronic interstitial nephritis, iv. 69.

Cold applications. See Applications, cold.

Cold sores. See Herpes of lips.

Cold storage system, risk of food poisoning from, ii. 637.

"Colds." See Nasopharyngitis, acute.

Coleman, Warren, studies of diet in typhoid fever by, ii. 13.

Coley's fluid, use of, suggested in rhinoscleroma, iii. 332 .

Coley's mixed toxins of streptococcus, effect of, on mediastinal tumors, iii. 501.

Coley's serum, use of, in leukemia, iii. 847.

Colic, mucous. See Enteritis, membranous.

Colic, Renal. See Renal colic.

Colitis, iii. 205; cases of, with colonic tenderness and diarrhea, iii. 206; chronic mucous, iii. 206; pain in bladder due to, iv. 122.

Colitis, mucomembranous, due to autointoxication associated with habitual constipation, ii. 678 .

Colitis, ulcerative, due to Balantidium coli, iv. 760; treatment of, iv. 762.

Collapse, accompanying renal colic, iv. 106 ; danger of, in attack of angina pectoris, iii. 767; stage of, in Asiatic diarrhea, iv. 833.

Collapse of lung, production of, as therapeutic measure in abscess and gangrene of lung, iii. 445.

Collapsing PUlse, iii. 560 .

Collargol, use of, in angina Ludovici, iii. 30. 
Collargol ointment, use of, in angina Ludovici, iii. 30 .

Collargolum, use of, by subcutaneous injection in typhus fever, ii. 37; in suppository form, in polymyositis, ii. 68.5; in thyroiditis acuta and struma inflammatoria, iii. 891 .

Colles-Baumes law, ii. 433.

Collodion, flexible, use of, in cervical adenitis complicating scarlet fever, ii. 131.

Colloidal state, definition of, i. 5; effect of external conditions upon, i. 28.

Colloins, i. 4 ; classification of, i. 6 ; difference between crystalloids and, i. 4; diffusibility of, i. 5, 17; discovery of, i. 4 ; effects of, upon crystalloids, i. 34; hydration capacity of, i. 33; osmotic pressure in, i. 4; saturation of, with water, i. 42.

Coloclyster, use of, in intestinal autointoxication, ii. 673.

Cologne water, local use of, in herpes of lips, iii. 6.

Colon bacilluria, as a cause of enuresis, iv. 128 .

Colon Bacillus infection, vaccine treatment in, i. 147.

Colonic flushing, employment of, in acute enteritis, iii. 195; in acute gastritis, iii. 155; in arthritis associated with intestinal autointoxication, ii. 681 ; in chronic catarrhal jaundice, iii. 263; in chronic cholecystitis, iii. 270; in chronic enteritis, iii. 200; in chronic gastritis, iii. 162; in chronic intestinal autointoxication, ii. 672 ; in colitis, iii. 205 ; in colon bacilluria, iv. 128; in constipation, iii. 230 ; contraindicated in acute catarrhal jaundice, iii. 261; in fecal impaction, iii. 239; in gastric ulcer, iii. 118; in infantile diarrhea, ii. 329 ; in intestinal amebiasis, iv. 709 ; for relief of constipation in neurasthenia, iv. 566; in septicemia, ii. 290,291 ; in severe cases of chronic enteritis, iii. 200; in spastic constipation, iii. 231 ; in treatment of gallstones in transit, iii. 274 ; in tricocephalus dispar, iii. 255; in tubcrculosis of intestines, ii. 505; in ulcerative colitis, iii. 211.

Colonic irrigation. See Colonic flushing.

Color, as property of radiant energy, i. 436.
Colored screens, use of, in light therapy, i. 442 .

Columbian spirits. See Wood alcohol.

Сома, as a symptom of cerebral thrombosis, iv. 399 .

Coma, dianetic. See Diabetic coma.

Combination methods of active immunization, i. 152.

Combined pseudosystem disease. See Myelitis, funicular.

Combined sclerosis of spinal cord. See Myelitis, funicular.

Сомву, case cited by, of mumps during pregnancy and appearance of, in child at birth, ii. 167.

Communal prophylaxis, in Asiatic cholera, iv. 828 .

Compensatory dilatation of heart, iii. 547.

Complement, i. 128, 131.

Complement-Bearing bodies. See Albuminolysins.

Complex mineralization, use of, in hemophilia, iii. 863 .

Complexes, as·a psychological mechanism, i. 585 .

Compound antiseptic powder, use of, in acute laryngitis, iii. 339 ; in acute rhinitis, iii. 327; as douche for nasopharynx, iii. 31 ; by irrigation in acute peritonsillitis, iii. 43 ; in ozena, iii. 336 .

Compound antiseptic solution, use of, as gargle in acute tonsillitis, iii. 42.

Compound cathartic pills, use of, in treatment of chronic alcoholism, ii. 598; in treatment of morphinism, ii. 621 .

Compound ipecacuanha powder, use of, to control cough in chronic bronchitis, iii. 375 .

Compound jalap powder, use of, in pleurisy, iii. 469 ; in uremia, iv. 53.

Compound liquorice powder, use of, in acute catarrhal jaundice, iii. 261; after hemorrhage in pulmonary tuberculosis, ii. 501 ; in chronic constipation, iii. 232; to correct constipation in tuberculous kidney, iv. 117.

Compound mustard liniment, use of, for relief of pain and cough in pneumonia, ii. 227 .

Compound spirits of ether, use of, in cardiac failure complicating influenza, ii. 193; in delirium of pneumonia, ii. 228 ; in pneu- 
monia with cardiac toxemia, ii. 228; for relief of gas in stomach during pneumonia, ii. 260; for relief of hiccough, ii. 260; as stimulant in cerebral anemia, iv. 382 ; as stimulant in curare poisoning, iv. 694 .

Compound spirits of lavender, use of, in pneumonia with cardiac toxemia, ii. 254.

Compress, abdominal, i. 198; combination, i. 198; wet, i. 197.

Compress, ammonia, use of, for relief of bite by poisonous arthropod, iv. 685 .

Compressed air, inhalation of, in treatment of whooping-cough, ii. 179.

Compresses, alcohol, use of, to relieve pain in acute gastritis, iii. 156.

Compresses, cold, use of, in acute bronchitis, iii. 357 ; in acute gout, ii. 702 ; in acute laryngitis, iii. 338 ; in acute tonsillitis, iii. 41 ; in adenitis accompanying glandular fever, ii. 576; in membranous laryngitis, iii. 343 ; to reduce temperature in pneumonia, ii. 233 ; for relief of cardiac symptoms in chronic progressive bulbar paralysis, iv. 253; for relief of headache in neurasthenia, iv. 565 ; for relief of night sweats in tuberculosis, ii. 505; for relief of pain in acute fibrinous pleurisy, iii. 466; for relief of pain in acute gastritis, iii. 156; for relief of pain in pulmonary tuberculosis, iii. 411.

Compresses, hot, use of, in gastric hyperacidity, iii. 100; over inflamed joints associated with acute endocarditis, iii. 746 ; for relief of headache in neurasthenia, iv. 565; for relief of pain in legs in beri-beri, iv. 853; for relief of pain in pleurisy, iii. 466,468 .

Compress, hot wet, use of, to relieve pain in brachial neuritis accompanying diabetes mellitus, ii. 766 .

Compresses, Priessnitz. See Priessnitz.

Compresses, warm wet, use of, in acute catarrhal cholecystitis, iii. 266.

Compresses, wet, use of, in gastric hyperacidity, iii. 100.

Compression apparatus, advantages of, in $\mathrm{X}$-ray treatment of mediastinal tumors, iii. 502.

Compression bulbar paralysis. See Bulbar paralysis.
Compression of head, as a means of relief in hydrocephalus, iv. 465, 466.

Compression of OUTLET, as cause of chronic dilatation of stomach, iii. 179.

ConCretio PERICARDII, chronic myocardial insufficiency and, iii. 708; treatment of, iii. 730 .

Condensation as a part of dream mechanism in psychotherapy, i. 586.

Condiments, avoidance of, in chronic parenchymatous nephritis, iv. 65; avoidance of, in pyelitis, iv. 88; irritation of kidneys by, iv. 57,59 ; objections to, in nephritis, iv. 43, 59; prohibition of, in acute urethral gonorrhea, iv. 138; use of, contraindicated in cirrhosis of liver, iii. 280.

Condiments AND sPICES, as auxiliary articles of diet, i. 603; over-indulgence in, a cause of gastric hyperacidity, iii. 77; use of, in diet for gastric hyperacidity, iii. 90.

Conducting Paths, for nerve fibers of heart, iii. 539.

Conductivity, as cardiac function, iii. 536.

Condurango, use of, in combination with calumba, for chronic enteritis, iii. 200.

Condurango preparations, use of, in carcinoma of stomach, iii. 165.

Condyloma latum, local treatment of, ii. 428.

Congenital chorea, iv. 664.

Congenital facial paralysis, iv. 314.

Congenital Heart lesions, treatment of chronic myocardial insufficiency caused by, iii. 734 .

Congenital hydrocephalus. See Hydrocephalus.

Congenital SyPhilis of Liver, iii. 265.

Conirn poisoning, i. 759; treatment of, i. 760 .

Conium, use of, in tetanus, ii. 348 .

Conjunctiva, incision of, in care of eyes during small-pox, ii. 50 .

Conjunctivitis, vaccine treatment in, i. 147.

Consciousness, Loss óf, significance of, in differential diagnosis between cerebral thrombosis and embolism, iv. 399 ; as symptom of basilar syphilitic meningitis, iv. 473; as symptom of cerebral thrombosis, iv. 396. 
Constant current, determining characteristics of, in electrotherapeutics, i. 367; explanation of term in electrotherapeutics, i. 366; use of, for paralysis aecompanying meat poisoning, ii. 640 .

Constant current, interrupted, as an electromechanical method, i. 390.

Constipation, accompanying chlorosis, iii. 823; accompanying pernicious anemia, iii. 837; accompanying tuberculosis, ii. 504; due to administration of iron in chlorosis, iii. 818; associated with intestinal autoinoculation, ii. 677 ; associated with scurvy, iii. 876 ; as a cause of enuresis, iv. 130; danger from, in aneurysm, iii. 765 ; danger from, in angina pectoris, iii. 770; danger from, in arteriosclerosis, iii. 758; diet for advanced cases of, ii. 555 ; electrotherapy for relief of, i. 424; hydrotherapy for relief of, i. 212; massage and gymnastics for relicf of, i. 212; mechanotherapy for relief of, i. 352 ; relation between infection of bladder and, iv. 119; relief of, in gonorrheal epididymitis, iv. 151; as a symptom of neurasthenia, iv. 566; treatment of, in cardiac insufficiency, iii. 684 ; treatment of, in gastric atony, iii. 171 ; treatment of, in chronic gastritis, iii. 162; treatment of, in chronic myocardial insufficiency, iii. 739 ; in tuberculous peritonitis, iii. 321.

Constipation, chronic, iii. 221; causes of, iii. 232 ; classification of, iii. 221 ; definition of, iii. 221; diet schemes for use in, iii. $225,226,227$; diet in treatment of, iii. 222; drugs in treatment of, iii. 232; electricity in treatment of, iii. 229; enterospasm, iii. 231; habitual form of, iii. 221; hydrotherapy for relief of, iii. 230; mechanotherapy for relief of, iii. 227; due to obstruction, iii. 236; operative measures in, iii. 237; spastic form of, iii. 223, 231; treatment of, by diet, iii. 224 ; vibratory massage in treatment of, iii. 230.

Constitutional causes, of multiple neuritis, iv. 283 ; of sciatica, iv. 298.

"Constitutional MaKe-UP," significance of term in psychotherany, iv. 574 .

Constitutional treatment, of syphilis, ii. 416.

Constriction of extremities, edema during pneumonia prevented by, iii. 406 .
Continuous bath. See Bath.

Continuous intermittent treatment, administration of mercury by, in syphilis, ii. 418.

Continuous treatment, administration of mercury by, in syphilis, ii. 417 .

Contracted KIDNey, presence of, in chronic myocardial insufficieney, iii. 730.

Contractility, as eardiac function, iii. 536.

Contraction wave, initiation and propagation of, iii. 540.

Contractures, treatment of, when due to beri-beri, iv. 854 ; when due to multiple sclerosis, iv. 281; when due to muscular dystrophy, iv. 264.

"Controlled autolnoculation," ii. 526.

Conus myelitis, iv. 223.

Conus tabes, iv. 186.

Convalescence, mechanotherapy in, i. 348.

Convallerin, use of, as cardiac stimulant in beri-beri, iv. 853 .

CoNveXITY OF BRAIN, syphilis of, iv. 475.

Conveyances, disinfection of, in cases of tuberculosis, ii. 454.

Convulsions, characteristics of, in parasites of brain, iv. 459; as a symptom of basilar syphilitic meningitis, iv. 473; as a symptom of brain tumor, iv. 435; as a symptom of rachitis, ii. 819 ; as a symptom of traumatic coma, iv. 365 ; treatment of, in cerebrospinal meningitis, ii. 215.

Convulsions, serial, due to brain tumor, measures for relief of, iv. 448.

Convulsive seizures, fatal, occurrence of, during irrigation of pleural cavity, iii. 487.

CoOKING, EFFects of, UPON FOOD, i. 621 ; in bread making, i. 623; upon cereals, i. 623 ; upon meats, i. 622 ; upon vegetables, i. 623.

Copaiba, use of, in acute urethral gonorrhea, iv. 140.

Copaiba resin, use of, to delay reaccumulation of fluid in cirrhosis of liver, iii. 286.

Copaibal poisoning, iv. 141.

Copper, polsoning by, i. 697; fatal dose in, i. 700; poisonous action of copper, i. 679 ; post-mortem appearances in, i. 700 ; symptoms of, i. 700 ; treatment of, i. 700 . 
Copper sulphate, use of, by instillation, in chronic gonorrheal urethritis, iv. 160; locally, in membranous rhinitis, iii. 330; locally, in oriental sore, iii. 756; locally, in scurvy, iii. 875 ; in treatment of acute phosphorus poisoning, ii. 627.

Cord PAINS, accompanying pernicious anemia, iii. 838 .

Corn, association of, with pellagra, ii. 657 .

Corn COCKLe, poisoning by, ii. 651 .

Cornea, ulceration of, in small-pox, ii. 50.

Corneal ulcers, radium in treatment of, i. 480 .

Cornet method of hydrotherapy, i. 217.

Coronary circulation, iii. 544; action of digitalis upon, iii. 586.

Coronary sclerosis, associated with angina pectoris vera, iii. 767 .

Corporations, disinfection in tuberculosis and, ii. 453.

Corpus luteum in organotherapy, i. 115; preparations of, i. 116.

Corrosive poisons, antidote for, i. 671.

Corrosive sublimate. See Mercury bichlorid.

Corset, use of, as support for prolapsed liver, iii. 289.

Corx, Dr., vaccination by, with virus from syphilitic patient, ii. 88 .

Coryza, accompanying tuberculosis, ii. 507 ; acute, see Rhinitis, acute; recurrent, iii. 326 .

Cosmolin, use of, in stomatitis hypermycetica, iii. 13.

Coto, fluid extract of, use of, to diminish hyperacidity, ii. 672 .

Cough, in bronchiectasis, iii. 395; due to bronchitis associated with chronic myocardial insufficiency, iii. 731; . control of, in bronchopneumonia, iii. 421; control of, in pericarditis, iii. 515; measures for relief of, in acute bronchitis, iii. 372, 373 ; measures for relief of, in chronic bronchitis, iii. 372, 373; measures for relief of, in pleurisy, iii. 468; in pneumonia, ii. 226, 257; significance of, during thoracentesis, iii. 477.

Cough in pulmonary tuberculosis, ii. 495; causes of, ii. 495; control of, during active stage, ii. 482; control of, in laryngeal complications, ii. 491, 498; local measures for relief of, ii. 497; prophy- lactic measures for relief of, ii. 496; sedative drugs for relief of, ii. 497; suppression of, ii. 496 ; varieties of, ii. 495 .

Councilman, studies by, on small-pox organisms, ii. 41, 104.

Counterirritants, use of, in hydrocephalus, iv. 465 .

Counterirritation, use of, in active congestion of lungs, iii. 405; in arthritis deformans, and chronic rheumatism, ii. 712 ; in brachial neuralgia, iv. 357 ; in bronchopneumonia complicating measles, ii. 159; in chronic myelitis, iv. 231 ; contraindicated in acute myelitis, iv. 225; for enlarged spleen in malaria, iv. 728 ; in Landry's paralysis, iv. 329 ; in later stages of anterior poliomyelitis, iv. 238 ; in neuralgias due to chlorosis, iii. 824 ; in neuritis, iv. 286 ; in neuritis of third, fourth, and sixth nerves, iv. 308; in phrenic neuritis, iv. 289; in reflex tenderness of scalp, iv. 600 ; for relief of cough in tuberculosis, ii. 497; for relief of endocarditis complicating pneumonia, ii. 261; for relief of lancinating pains in tabes, iv. 196; for relief of pain in chest in tuberculosis, ii. 506; for relief of pain in sciatica, iv. 299; for relief of pain in spinal syphilis, iv. 274 ; for relief of pain in syringomyelia, iv. 278; over spine in lathyrism, iv. 693; without value in cerebrospinal meningitis, ii. 215.

Cow-pox, ii. 56.

Craig, experiments by, showing interval of time between infection and beginning of symptoms in dengue, iv. 859; on prophylactic use of quinin in malaria, iv. 736.

Crameria, lozenges of, use of, in acute uvulitis, iii. 35 .

Cranial foramina, alcohol injection at, for trigeminal neuralgia, iv. 346.

Cranial nerves, diseases of, iv. 305: diseases of optic nerve, iv. 305; neuritis of eighth nerve, iv. 314; neuritis of eleventh nerve, iv. 320 ; neuritis of ninth nerve, iv. 318 ; neuritis of seventh nerve, iv. 310 ; neuritis of tenth nerve, iv. 319 ; neuritis of third, fourth, and sixth nerves, iv. 308 ; neuritis of twelfth nerve, iv. 321 ; periodical ocular paralysis, iv. 309 ; trigeminal neuritis, iv. 309.

Craniotabes, ii. 817. 
Craniotomy, indications for, in pachymeningitis interna hemorrhagiea, iv. 364.

Cranium, neuritis over, electrotherapeuties for relief of, i. 417.

Craw-craw, iv. 691; remedial measures for, iv. 692 .

Cream, as article of diet, in acute nephritis, iv. 60 ; in diabetes mellitus, ii. 743 ; in nephritis in general, iv. 41 ; in uremia, iv. 52 .

Cream of tartar, use of, in cirrhosis of liver, iii. 286.

Creatinin, elimination of, with difficulty by kidney, iv. 60; excretion of, in pneumonia, ii. 229.

Credé's ointment, use of, in dermatomyositis, iv. 330; in multiple sclerosis, iv. 281.

Creolin, irrigation of bowels with a solution of, in chronic stage of bacillary dysentery, ii. 32s; local use of, in ozena, iii. 337 ; use of, as lotion in pustular or ulcerative syphiloderm, ii. 426.

Creosotal, use of, in pneumonia, ii. 235.

Creosote, use of, in bronchiectasis with fetid expectoration, iii. 395 ; in bronchopneumonia, iii. 415; in chronic enteritis, iii. 200; in depressive disorders of gastric secretion, iii. 151; in enlargement of lymphatic glands complicating measles, ii. 162; as expectorant in bronchiectasis, iii. 396; as expectorant in chronic bronchitis, iii. 376 ; in gastric atony, iii. 172 ; by inhalation in bronchiectasis, iii. 396 ; by inhalation in whooping-cough, ii. 181; in laryngeal tuberculosis, iii. 348 ; in pneumonia, ii. 235 ; for relief of expectoration in pulmonary tuberculosis, ii. 498 ; in tuberculosis of mediastinal lymph nodes, iii. 507.

Creosote bath. See Bath.

Creosote ointment, use of, in ulcerating granuloma of pudenda, iv. 866 .

Creosote vapor, inhalations of, in plastic bronchitis, iii. 392.

Creta preparata, use of, in phosphaturia, iv. 9.

Cretinism. See Athyrea and Hypothyrea.

Crile, on artificial respiration in treatment of post-hemorrhagic anemia, iii. 810 .

Crises, presence of, in tabes, iv. 190; treatment of, iv. 197, 198.
Cnocker, lotion recommended by, for itching in diabetes mellitus, ii. 764.

"Cross-fire" method of applying radium, i. 465 ; in treatment of angiomata and nevi, i. 477; in treatment of fungating epitheliomata, i. 482; in treatment of keloids, i. 476.

Croton oil, use of, in acute nephritis with coma, iv. 58; in cerebral hemorrhage, iv. 391 ; in cerebral hyperemia, iv. 386 ; externally in hydrocephalus, iv. 465; in nephritis complicating scarlet fever, ii. 134.

Croup, diphtheritic, administration of antitoxin in, ii. 269, 270, 275.

Croup, ralse. See Laryngitis, spasmodic.

Croup, membranous. See Laryngitis, membranous.

Croup, Non-Diphtheritic, as a complication of measles, ii. 157.

Croup, spasmodic. See Laryngitis, spasmodic.

Croup kettle, use of, in acute peritonsillitis, iii. 43 ; in cough of tuberculosis, ii. 497; in laryngeal complications of diphtheria, ii. 157; in membranous pharyngitis, iii. 39 ; in spasmodic laryngitis, iii. 342 .

Croup tent, use of, in acute laryngitis, iii. 338 ; in membranous laryngitis, iii. 343.

Croupous stomatitis. See Stomatitis crouposa.

Crural neuralgia. See Neuralgia.

Cryptogenetic anemias. See Anemias.

Crystalloidal state, i. 5.

Crystalloids, i. 7; difference between colloids and, i. 4; diffusibility of, i. 4, 5, 17 ; discovery of, i. 4 ; effects of colloids upon, i. 34 ; groups of, i. 7 ; osmotic pressure in, i. 4.

Crystallose, as substitute for sugar in diabetes mellitus, ii. 750 .

Cuba, history of vaccination in, ii. 103.

Cubeb, use of, in acute urethral gonorrhea, iv. 141.

Culex fatigans, dengue transmitted by, iv. 859.

Cullen, on headache, iv. 596.

Culture, method of taking, in membranous laryngitis, iii. 343.

"Cumulative action" of X-ray, i. 512.

Cups, dry, use of, in active congestion of lung, iii. 405; in acute bronchitis, iii. 357 ; in anterior poliomyclitis, iv. 237; 
in anuria, iv. 3 ; in beri-beri, iv. 854 ; in cerebral hyperemia, iv. 386 ; in nephritis complicating scarlet fever, ii. 133; for relief of anuria in nephrolithiasis, iv. 107 ; for relief of cyanosis in acute bronchitis, iii. 363; for relief of cyanosis and dyspnea in bronchopneumonia, iii. 419; for relief of headache due to brain tumor, iv. 448; for relief of lancinating pains in tabes, iv. 197; for relief of pain in pleurisy, iii. 468; for relief of pain in pulmonary thrombosis, iii. 411; for relief of pain in sciatica, iv. 299.

Cups, wet, use of, in active congestion of lungs, iii. 406; in acute bronchitis, iii. 357 ; in cerebral hyperemia, iv. 386 ; in pachymeningitis interna hemorrhagica, iv. 364 ; in pneumonia, together with inhalations of oxygen, ii. 237; in pulmonary thrombosis, iii. 411 ; for relief of anuria in nephrolithiasis, iv. 107; for relief of cyanosis in acute bronchitis, iii. 363 ; for relief of headache due to brain tumor, iv. 448; for relief of pain in cerebral abscess, iv. 423; for relief of pain in nephritis, iv. 88; for relief of pain in pulmonary thrombosis, iii. 411; for relief of pain in sciatica, iv. 299; for relief of vomiting due to cerebellar tumor, iv. 448.

Curare, use of, in tetany, iii. 910.

Curare poisoning, iv. 694.

Curarin, iv. 694.

Curettage, employment of, in adenectomy, iii. 36 ; in chronic tonsillitis, iii. 44 ; in laryngeal tuberculosis, ii. 493; in mycosis of tonsils and fauces, iii. 42 ; to remove nasal obstruction, iii. 37 ; to remove tubercular deposits in laryngeal tuberculosis, iii. 347 .

Curettage of bladder, objections to removal of tubercular lesions by, iv. 121 .

Curie, Mme., discovery of radium by, i. 460.

Currie, use of hydrotherapy in general infections first advised by, ii. 16 .

Curschmann, cannulæ devised by, iii. 676 .

Curschmann's spirals, presence of, in bronchial asthma, iii. 379 .

Cushing, Dr. E. W., on water in diet of typhoid fever, ii. 14.

Cushing, Harvey, on operation for subdural hemorrhage from prolonged labor, iv. 367 .
Cushing decompressive operation, for relief of brain tumor, iv. 451.

Cushney, on digitalis and blood pressure, iv. 50.

Cushny, A. R., on value of heroin hydrochlorid, iii. 361.

Cuspidors, disinfection of, in tuberculosis, ii. 453.

Custard, baked, as article of invalid dietary, i. 651; soft, as invalid dietary, i. 650.

Cutaneous Disorders, associated with intestinal autointoxication, ii. 682 .

Cutaneous symptoms, due to chronic myocardial insufficiency, iii. 740.

Cutaneous trophic disturbances, in tabes, iv. 192.

Cutaneous tuberculosis, action of radium upon, i. 469.

Cyanid of gold and potassium, injection of, in tabetic amaurosis, iv. 199.

Cyanosis in blood diseases, iii. 877; in bronchopneumonia, iii. 419; enterogenous, iii. 878; measures for relief of, in acute bronchitis, iii. 363 ; in polycythemia with splenomegaly, iii. 877 .

Cyclastia scarlatinalis, ii. 115.

Cyclones, i. 254.

Cyclothymia, iv. 614.

Cyllin, use of, in sprue, iv. 862.

Cynanche Parotidea. See Mumps.

Cyprus oil, use of, in whooping-cough, ii. 181.

Cysticercus cellulose, iii. 255.

Cystectomy, performance of, to relieve pain of tubercular cystitis, iv. 121.

Cysticercus cellulos iv. 459.

Cystic growths of Brain, iv. 433.

Cystitis, iv. 119; associated with acute myelitis, iv. 227; as a cause of enuresis, iv. 129 ; as a complication of acute gonorrhea, iv. 150; as a complication of pyelitis, iv. 94; hydrotherapy for, i. 209; microörganisms present in, iv. 119; prevention of gonorrheal, iv. 119; prophylaxis against, iv. 119; treatment of acute, iv. 120; treatment of chronic, iv. 120 ; treatment of tuberculous, iv. 121.

Cystocele, as a cause of pain in bladder, iv. 123.

Cystoscopy, employment of, in diagnosis of tuberculous kidney, iv. 114.

Cysts, pancreatic, iii. 302. 
Cytorrhyctes variol. F, ii. 41.

Cytotoxic sera. See Bacterieidal sera.

DANA, condition ealled "wet brain," first deseribed by, ii. 608 .

Dancing, injury from, in eardiac insuffieiency, iii. 682 .

DArier, trachoma successfully treated with radium by, i. 480 .

Darnel, the, polsoning by, ii. 651 .

Davainea Asiatica, iv. 777.

Davainea madagascariensis, iv. 777.

Davenport and Weekes, investigation by, on inheritance of epilepsy, iv. 641.

Davidson, Dr. Mackenzie, investigations by, on value of radium therapy in ophthalmology, i. 480.

Davis, D. J., investigation by, of organisms in acute rheumatism, ii. 293.

DeAD, disposal of, in the plague, iv. 825; in small-pox, ii. 45.

Dearden, on constitutional effects of phosphorus poisoning, ii. 630 .

DEАтн, from collapse during or after thoracentesis, iii. 480; from convulsive seizures during irrigation of pleural cavity, iii. 487; from pleural exudate, 461.

DEAvER, on indications for operation in typhoid cholecystitis, iii. 275 ; on mortality following operation for acute diffuse peritonitis, iii. 313 .

Decompression, indications for, in optic neuritis, iv. 305.

DeEks aND James, on administration of quinin in tropical malaria by hypodermoclysis, iv. 722 .

Deep irradiation, uniformity of, in X-ray treatment, i. 505.

Deep structures of BODY, effects of X-rays upon, i. 417.

Deformities, accompanying rachitis, ii. 818.

Degenerative chorea. See Chorea.

Degrais, Dr., work done by, on radium, i. 460.

Dehypnotization, process of, i. 568.

"DEкоFA," use of, as substitute for coffee in eardiac insufficiency with insomnia, iii. 686 .

"Delayed Resolution," treatment of, in pneumonia, ii. 257.

Delirium states, in epilepsy, iv. 638.
Dellatum tremens, ii. 601; alcohol in causation of, ii. 607; diet in, ii. 603; drugs in, ii. 604; hypnotics in, ii. 605; mortality from, iv. 607; in multiple neuritis due to alcohol, iv. 323; treatment of, at beginning of attack, ii. 602 ; treatment of, at height of attack, ii. 603; "wet brain" in, ii. 608 .

Dementia, in progressive paralysis of the insane, iv. 490.

Dementia paralytica. See Insane, progressive paralysis of.

Dementia precox, comparison between, and hysteria, iv. 584; headache in, iv. 615.

Dementia of schizopirrenia, iv. 584.

Demulcent drinks, use of, in barbel cholera, ii. 644.

Demulcent substances, use of, to relieve cough in tuberculosis, ii. 496.

Demulcents, use of, in acute urethral gonorrhea, iv. 139.

Dengue, iv. 858; convaleseence from, iv. 860; definition of, iv. 858; diet in, iv. 860; mortality from, iv. 858 ; prognosis in, iv. 858; prophylaxis against, iv. 859 ; symptoms of, iv. 858; theories concerning etiology of, iv. 858; transmission of, iv. 859; treatment of, iv. 859.

Denmark, antituberculosis work in, ii. 442; introduction of vaceination into, ii. 60 .

Dennet, on treatment of summer diarrhea by withdrawal of sugar from food, iii. 202.

Dennet's, formula, for Eiweiss milk, iii. 203.

Denys bacillen filtrate, use of, in tuberculosis of larynx, iii. 346.

Denys bouillon filtrate, ii. 512; use of, in tuberculosis, ii. 513.

Depression, atTacks of, in progressive paralysis of the insane, iv. 493.

Dermatitis, due to X-rays, acute, i. 514; chronic, i. 515.

Dermatology, value of radiant light and heat in, i. 458; value of radioactivity in, i. 473 ; value of $\mathrm{X}$-ray therapy in, i. 526 .

Dermatomyositis. See Polymyositis, acute.

Dermoid cysts of mediastinum, iii. 500.

D'Espine, Adolphe, results of seaside residence on enlargement of bronchial glands reported by, iii. 398 . 
Desserts, use of, in diet of gastric hyperacidity, iii. 89 .

Dessner's modification of deep X-ray therapy, i. 505.

Destructive symptoms, of brain tumor, iv. 436 .

Detweiler, rest in treatment of tuberculosis advocated by, ii. 477.

Deutschmann's serum, i. 169.

Dextrose, administration of, by rectum, in acute diffuse peritonitis, iii. 317; subcutaneous injection of, in diabetic coma, ii. 758 .

Deycke AND MUCH, preparation by, of tuberculin with human brain, i. 151.

Diabetes insipidus, ii. 774; causes of, ii. 774 ; definition of, ii. 774 ; diet in, ii. 776 ; drugs in treatment of, ii. 777 ; hydrotherapy in treatment of, i. 206; organic extracts in treatment of, ii. 778 ; treatment of, ii. 775 .

Diabetes mellitus, ii. 717; acidosis in, ii. 731 ; as a cause of multiple neuritis, iv. 322 ; anesthesia dangerous in, ii. 779 ; boils as symptom of, ii. 765; brachial neuritis as symptom of, ii. 766; caloric needs of body in, ii. 737; in children, ii. 768; coma a symptom of, ii. 731; diabetophobia and, ii. 768; diet lists for use in, ii. 738; dietetic regimen in, ii. 750; drugs in treatment of, ii. 759 ; electrotherapy for relief of, i. 213; energy requirements in, ii. 724; exercise in, 763; eye affections accompanying, ii. 766; foods allowable in, ii. 738, 751; foods allowable in, under restriction, ii. 739,751 ; foods forbidden in, ii. 751 ; frequency of, ii. 718; gangrene accompanying, ii. 770; general principles in treatment of, ii. 734; glycosuria in, ii. 719; grades of severity in, ii. 735, 758; green days in treatment of, ii. 756; health resorts in treatment of, ii. 762 ; heredity in causation of, ii. 719 ; history of, ii. 717; hydrotherapy for relief of, i. 213 ; ii. 762; hyperglycemia in, ii. 722; impotence accompanying, ii. 766 ; itching as a symptom of, ii. 764; Jewish race specially liable to, ii. 719 ; marriage undesirable in, ii. 769; massage and gymnastics in, i. 327; mechanotherapy for, i. 348; mortality from, ii. 718 ; neuralgia a symptom of, ii.
766; neuritis a symptom of, ii. 766; oatmeal treatment for, ii. 753 ; operative measures for, ii. 770; opotherapy for, ii. 760; organic extracts in treatment of, ii. 760; over-feeding in causation of, ii. 719; pathogenesis of, ii. 719 ; physical examinations in, ii. 736; in pregnancy, ii. 768; prognosis of, ii. 729; prognosis of diabetic coma in, ii. 757; prophylaxis against, ii. 730; prostatic disease as a symptom of, ii. 766; sciatic pain accompanying, ii. 766; sources of sugar in the body, ii. 725; Spa treatment of, ii. 763; substitutes for sugar in diet of, ii. 749; substitutes for vegetable starch in diet for, ii. 748; sugars present in urine of, beside glucose, ii. 727; summary of dietetic treatment of, ii. 758; surgical operations in, ii. 769 ; syphilis associated with, ii. 768; temperature of atmosphere an influence in, ii. 764; tests for acetone bodies in, ii. 732 ; thyroid gland and its influence upon, ii. 767; transient glycosuria in, 726; treatment of, 734; treatment of acidosis in, 756; treatment of coma in, ii. 757; treatment of multiple neuritis due to, iv. 325 ; treatment of special symptoms in, ii. 764; tuberculosis associated with, ii. 767; vulvitis accompanying, ii. 765 ; xanthoma diabeticorum as a symptom of, ii. 765 .

Diabetic acidosis, ii. 731; treatment of, ii. 756.

Diabetic coma, ii. 731; prognosis of, ii. 758 ; treatment of, ii. 757.

Diabetic eye affections, ii. 766.

Diabetic gangrene, ii. 770.

Diabetic headaches. See Headaches.

Diabetic neuralgia, ii. 766.

Diabetic neuritis, ii. 766.

Diabetic pruritis, ii. 765 .

Diabetrc volvitis, ii. 765.

Diabetics, pneumonia of, ii. 258.

Diabetophobia, ii. 768.

DiACETIC ACID, test for, ii. 732.

Diaphoresis, use of, in chlorosis, iii. 814; dangerous, in pericarditis with effusion, iii. 522; for relief of anuria in nephrolithiasis, iv. 107; in uremia, iv. 53.

Diaphoretics, use of, in acute myelitis, iv. 225; in cerebral hyperemia, iv. 386 ; in epidemic dropsy, iv. 860; in pleurisy, iii. 470. 
DIARRHEA, accompanying tuberculosis, ii. 505; concentrated diet for, ii. 556; hydrotherapy for relief of, i. 213; treatment of, in pellagra, ii. 66i2.

DiarrньА, ACUTE, peculiarities of, in older peopl, iii. 195.

Diarkhea, Cochin China. See Cochin China.

Diarrifa, Hul. See Hill diarrhea.

DiarRILA, INFANTILE, treatment of, ii. 328.

Diarlilea, morning, iii. 250.

Diarrhea, nervous, iii. 249.

DiarkHeA, PREMonitory, in Asiatic cholerit, iv. 833.

Diarrieas, infectious, iii. 204.

Diet, in acute nephritis, iv. 59; in acute urethral gonorrhea, iv. 138; auxiliary articles of, i. 603 ; in beri-beri, iv. 853 ; as cause of enuresis, iv. 127; change of, important in epidemie dropsy, iv. 860; in chronic interstitial nephritis, iv. 68; in chronic parenchymatous nephritis, iv. 65; in ehronic progressive bulbar paralysis, iv. 251; in diabetes, ii. 738; for epilepsy, iv. 646; in kidney of pregnancy, iv. 82 ; in nephritis, iv. 39 ; in nephrolithiasis, iv. 108; principles for, in neurasthenia, iv. 536; in prophylaxis of cerebral hemorrhage, iv. 391 ; in prophylaxis of cerebral hyperemia, iv. 385; in pyelitis, iv. 87; in tabes, iv. 213; in tuberculosis of kidney, iv. 116; in uremia, iv. 52.

Dietaries for children, method of calculating, i. 605 .

Dietary, for invalid, see Invalid dietary; in obesity associated with ehronic myocardial insufficiency, iii. 725.

Differential air pressure, employment of, in tumors of lung, iii. 446.

Diffusion in living tissues, i. 16.

Digalen, use of, during attack of cardiac asthma, iii. 737 ; by intravenous injection, in chronic myocardial insufficiency, iii. 718,719 ; in pneumonia with cardiac depression, ii. 251; therapeutic value of, iii. 595 .

Digalen, Cloetta's, toxic effect of, iii. 584 .

Digestibility of Foods, i. 624.

Digestion, abnormal products of, a eause of intestinal autoinfection due to, ii. 666; effect upon, of beverages containing caffein, iii. 603; influence of appetite upon, i. 625; influence of fat-content upon, i. 627; intestinal autointoxication not due to normal products of, ii. 6666 .

Digestive JuICES AND secretions, not concerned in intestinal autointoxieation, ii. 666 .

Digipuratum, use of, as cardiac stimulant in fibrinous pleurisy, iii. 469 ; as diuretic in acute nephritis, iv. 59; diuretic effect of, iii. 597; by hypodermic injection, iii. 598; by intravenous injection, iii. 598; by intravenous injection in severe cases of chronic myocardial insufficiency, iii. 718,719 ; method of dosage for, iii. 598; method of preparing, iii. 596; physiological action of, iii. 597; preparations of, in use, iii. 596; therapeutics of, iii. 596; toxic effects of, iii. 597 .

Digital compression, use of, in aneurysm of iii. abdominal organ, iii. 767 .

Digitalen, poisoning by, i. 757.

Digitalen, therapeutic value of, iii. 595.

Digitalis, action of, on coronary circulation, iii. 586; action of, in general, iii. 577; active principles, iii. 594; blood pressure not increased by, iv. 50; continuous administration of, iii. 599 ; cumulative effect of, iii. 584; diuresis as an indication of effect of, 589 ; dosage of, iii. 593; effect produced by, on pulmonary circulation, iii. 405; history of, iii. 575; influence of, on blood pressure, iii. 578; influence of, on kidney: iii. 579; influence of, on pulse, iii. 579; limited value of, in cardiac neurasthenia, iii. 778; loss of susceptibility to, iii. 583; method of administering, iii. 592; pharmacology of, iii. 577; physiologically standardized Galenical preparations of, iii. 595; powdered leaves of, iii. 593; pulse pressure as indication of effect produced by, iii. 580; pulse rate as indication of effect produced by, iii. 581; resinoids of, unsuitable, in pneumonia, iii. 251; results of, when efficient, iii. 591; standardization of, iii. 582; substitutes for, iii. 599; tincture of, iii. 592 ; toxic action of, iii. 584 ; variations in strength of preparations, iii. 581 .

Digitalis, therapeutic use of, in acute infectious myocarditis, iii. 750 ; in acute 
myelitis, iv. 226; in acute vasomotor paralysis, iii. 753; in amyloid kidney, iv. 81 ; in angina pectoris, iii. 770 ; in aortic insufficiency, iii. 733 ; in bronchopneumonia complicating influenza, ii. 192 ; in cardiac dilatation associated with acute endocarditis, iii. 746 ; in cardiac failure complicating influenza, ii. 193; in cardiac insufficiency, iii. 575; in cardiac toxemias of pneumonia, ii. 249 ; as cardiac stimulant in after-treatment of cerebral thrombosis, iv. 398 ; as cardiac stimulant in beri-beri, iv. 853, 854; as cardiac stimulant in cerebral embolism, iv. 402 ; as cardiac stimulant in multiple neuritis, iv. 326; as cardiac stimulant in pericarditis, iii. 520; as cardiac stimulant in plague, iv. 827; as cardiac stimulant in Rocky Mountain fever, ii. 565, 566 ; in cardiovascular disturbances following paracentesis, iii. 285 ; in cerebrospinal meningitis, ii. 216; in chronic bronchitis, iii. 378 ; in chronic myocardial insufficiency, iii. 722 ; in chronic myocardial insufficiency due to chronic nephritis, iii. 730; contraindications to, iii. 588; in delirium tremens, ii. 604 ; in diphtheria, ii. 277; as diuretic in acute nephritis, iv. 59; as diuretic in chronic parenchymatous nephritis, iv. 65; as diuretic in nephritis, iv. 50; as diuretic in uremia, iv. 53; in epilepsy, iv. 648; in erysipelas, ii. 277 ; by hypodermic injection in secondary chronic gastritis, iii. 159 ; in hypostatic congestion of lungs, iii. 707; in malignant endocarditis, iii. 747 ; in mild cases of chronic myocardial insufficiency, iii. 715; in nephritis complicating scarlet fever, ii. 134 ; by rectum, iii. 594; for relief of dyspnea in acute nephritis, iv. 63 ; for relief of dyspnea in chronic interstitial nephritis, iv. 71 ; for relief of edema in acute nephritis, iv. 62; for relief of edema of glottis in acute nephritis, iv. 63 ; for relief of gastrointestinal symptoms in chronic myocardial insufficiency, ii. 738; in pleurisy, iii. 469; in scarlet fever with cardiac weakness, ii. 127; in septicemia, ii. 291; in sunstroke, iii. 679 ; in toxemia complicating acute diffuse peritonitis, iii. 318 ; in typhoid fever, ii. 23.
Digitalis and squills, use of, as a diuretic in hydrocephalus, iv. 465.

Digitalis bodies, use of, by intravenous injection, in cardiac insufficiency, iii. 577; in chronic myocardial insufficiency, iii. $718,719$.

Digitoxin, early use of, iii. 577; therapeutic value of, iii. 594; toxic effects of, iii. 584 .

Dilatation of heart, acúte, iii. 548; compensatory, iii. 546; pathological physiology of, iii. 546 ; stasis and, iii. 547.

Dilatation of stomach, aCUTE, as a complication of pneumonia, ii. 259.

Dilatation of urethra, in chronic gonorrheal urethritis, iv. 161; contraindications to employment of, iv. 163 ; limits of, iv. 163; technique of, iv. 162; in urethral stricture, iv. 168, 169.

Dilator, esophageal, iii. 49.

Dilator, rubber-bag. See Rubber-bag dilator.

Diluents, use of, in acute urethral gonorrhea, iv. 139.

Dimethyl xanthin, use of, as diuretic in acute nephritis, iv. 59 .

Dinner pill, formula for. See Formula.

Dionin, substitution of, for morphin in treatment of morphinism, ii. 618; use of, for relief of lancinating pain in tabes, iv. 196; use of, for relief of pain in angina pectoris, iii. 768; use of, for relief of pain in syringomyelia, iv. 278 .

Diphtheria, ii. 266; adenitis complicating, ii. 277 ; alcohol in, ii. 276; anaphylaxis following injection of antitoxin in, ii. 271; antitoxin treatment of, ii. 269; bacteriology of, ii. 266; bronchopneumonia complicating, ii. 278; classification of, ii. 268; complications of, ii. 276; definition of, ii. 266; diet in, ii. 276; dose of antitoxin for, ii. 267; hydrotherapy for, i. 215; immunization against, ii. 268; indications for use of antitoxin in, ii. 269; intubation for, ii. 272; irrigation of ear in, ii. 278; irrigation of nasopharynx in, ii. 271; kidney complications in, ii. 279; larynx affected in, ii. 272; local treatment for, ii. 271 ; location of membrane in, ii. 269 ; medicinal treatment of, ii. 275; mental apathy in, ii. 270 ; method of feeding after intubation in, ii. 276; mode of injecting antitoxin in, ii. 270 ; nursing in, ii. 275 ; operative 
measures for cervical adenitis complicating, ii. 277; otitis media complicating, ii. 277; physician's duty as regards infection in, ii. 267; post-diphtheritic paralysis following, ii. 278; pretracheal abscess complicating, ii. 279; prophylaxis against acute myocarditis in, iii. 749; prophylaxis in, ii. 266 ; quarantine in, ii. 267 ; site for injection of antitoxin in, ii. 370; skin affections complicating, ii. 276 ; use of sprays in, ii. 272 ; use of stimulants in, ii. 277 ; toxalbumins obtained from, ii. 635; tracheotomy in, ii. 279 ; treatment of, in general, ii. 268.

Diphtheria antitoxin, i. 154; administration of, i. 157; anaphylaxis following use of, ii. 269 ; concentration of, i. 156 ; in diphtheria, ii. 269 ; discovery of, i. 127,128 ; dose of, ii. 269; eruptions following, in diphtheria, ii. 269 ; indications for, in diphtheria, ii. 267 ; in laryngeal complications of measles, ii. 157; mode of injecting, ii. 270 ; nature of toxins concerned in, i. 154; in pneumonia, ii. 240; in post-diphtheritic paralysis, ii. 278 ; precautions in, i. 135; preparations of, i. 155 ; results of, i. 178; site for injection of, ii. 270; standardization of, i. 157; in stomatitis diphtheritica, iii. 20 ; in stomatitis gangrenosa, iii. 19; sudden death following, by intravenous injection, i. 134; in typhus fever, ii. 37; value of, i. 157 .

Diphtheritic Croup. See Croup.

Diphtheritic serum, injection of, for control of hemorrhage in hemorrhagic form of measles, ii. 155.

Diphtheritic stomatitis. See Stomatitis, diphtheritic.

Diplogonopores grandis, iv. 776; mode of infection by, iv. 777 .

Diplopia, measures for relief of, when associated with neuritis of third, fourth, and sixth nerves, iv. 309.

Dipsomania, ii. 591; association of, with intestinal autoinfection, ii. 683; treatment of, ii. 601 .

Disease, certain forms of, a predisposing cause of alcoholism, ii. 590.

Diseases of REMOTE origin, as a cause of enuresis, iv. 130.

Diseases of TOXIC origin, electrotherapeutics for relief of, i. 408.
Diseases of UNCERTAIN ETOLOGY, iv. $84 t$ akatama, iv. 8633 ; beri-beri, iv. 844 ; elimatic buboes, iv. 864; dengue, iv. 8.58 ; epidemic dropsy, iv. 860; epidemic gangrenous proctitis, iv. 866 ; gangosa, iv. 864; goundoo, iv. 868 ; hill diarrhea, iv. 862 ; hyperpyrexial fever, iv. 868 ; kubisagari, iv. 860 ; onyalai, iv. 861 ; sprue, iv. 861 ; tropical sloughing phagedena, iv. 866; ulcerating granuloma of pudenda, iv. 865; undifferentiated tropical fevers, iv. 869 ; verruga peruviana, iv. 863 ; yellow fever, iv. 855 .

Disinfectants, for use in tuberculosis, ii. 452 .

Displacement, as a part of dream mechanism, in psychotherapy, i. 587.

Distomiasis, iv. 779; hepatic, iv. 779; intestinal, iv. 780 .

District of Columbia, antituberculosis work in, ii. 444.

Diuresis, as an indicator of effective digitalis action, iii. 580; methods for increasing, in cardiac insufficiency, iii. 676 .

Diuretics, use of, in acute myelitis, iv. 225; in acute nephritis, iv. 59 ; in beri-beri, iv. 852 ; in chronic parenchymatous nephritis, iv. 65 ; in cirrhosis of liver with ascites, iii. 284; contraindicated in non-obstructive form of anuria, iv. 3 ; to delay accumulation of fluid in cirrhosis of liver, iii. 286; in nephritis, iv. 49 ; in nephritis complicating scarlet fever, ii. 132,133 ; in pericarditis with effusion, iii. 526 ; in pleurisy, iii. 469 ; in pyelitis, iv. 89 ; for relief of anuria in nephrolithiasis, iv. 107; in scarlet fever, ii. 125 ; in severe cases of myocardial insufficiency, with caution, iii. 722 ; in uremia, iv. 53 .

Diuretics, alkaline. See Alkaline diuretics.

Diuretin, use of, in acute nephritis, iv. 58; to induce diuresis in cardiac insufficiency, iii. 676 ; in nephritis, iv. 49,50 ; in nephritis complicating scarlet fever, ii. 134; not beneficial in cirrhosis of liver, iii. 286 ; in pleurisy, iii. 469 ; for relief of anuria in nephrolithiasis, iv. 107.

Dobell's solution, use of, in diphtheria, ii. 272 ; in hygiene of mouth, iii. 3 ; in laryngeal tuberculosis, ii. 493; as mouth wash in mumps, ii. 170; in ozena, iii. 336 ; for relief of "colds" accompanying 
tuberculosis, ii. 508; as spray in acute tonsillitis, iii. 442; as spray in chronic atrophic laryngitis, iii. 343 ; as spray in chronic rhinitis, iii. 329 ; as spray for cleansing larynx before local treatment in tuberculosis, iii. 347 ; as spray in syphilis of upper respiratory tract, iii. 349 ; as spray or irrigation in acute laryngitis, iii. 339; in typhoid fever, ii. 11.

DoBson AND Poole, sugar first isolated from diabetic urine by, ii. 717.

Dock AND BAss, essentials in hookworm prophylaxis given by, iv. 794 .

DoнI, case reported by, showing local effect of mercury on syphilitic lesions, ii. 421 .

Dominici AND BARcat, work done by, on histological reaction of radium, i. 468 .

Donovan's solution, use of, in pellagra, ii. 661 ; in trypanosomiasis, iv. 747.

Double current tube, use of, as rectal douche in chronic gonorrheal urethritis, iv. 165.

Douche, cold, use of, in after-treatment of morphinism, ii. 624; in bronchial asthma, iii. 381 ; in chronic bronchitis, iii. 367 ; to reduce fever in acute hemorrhagic encephalitis, iv. 405.

Douche, hot, use of, in hemorrhagic form of measles, ii. 154; in renal colic, iv. 102.

Douche, nasal, use of, in acute nasopharyngitis, iii. 31 ; in chronic nasopharyngitis, of hot compound antiseptic powder, iii. 32; in chronic pharyngitis, iii. 33 ; in membranous rhinitis, of normal salt solution, iii. 330 .

Douche, post-nasal, use of, in acute inflammation of sinuses, iii. 334 .

Douche, rectal, employment of, in chronic gonorrheal urethritis, iv. 165.

Douche, stomach, use of, in depressive disorders of gastric secretion, iii. 152.

Douche, throat, in chronic nasopharyngitis, iii. 32 ; in membranous pharyngitis, of hydrogen peroxid, iii. 39 .

Douche, tonsillar, in acute tonsillitis, iii. 41.

Douche, vaginal, use of, in diabetic vulvitis, ii. 765 .

Douches, therapeutic value of, i. 200 ; use of alternate hot and cold, for relief of headache in neurasthenia, iv. 566; use of, in neurasthenia, iv. 548; use of, in respiratory type of influenza, ii. 189.
Dover's powder, use of, in abortive treatment, for acute rhinitis, iii. 327 ; in acute laryngitis, iii. 338; in edematous laryngitis due to foreign body, iii. 241 ; in enteritis of infancy, iii. 203; for relief of cervicoöccipital neuralgia, iv. 356; for relief of "colds" in tuberculosis, ii. 508; for relief of neuralgia associated with rheumatism, iv. 337 ; for relief of pain in acute fibrinous pleurisy, iii. 467; for relief of pain in pericarditis, iii. 520 .

Dracunulus medinensis, infection with, iv. 788; treatment of, iv. 789.

Drainage, employment of, to lessen irritability of bladder in tuberculous kidney, iv. 117; in uremia with edema of extremities, iv. 53.

Dramatization, as a part of dream mechanism in psychotherapy, i. 587.

Dream analysis, employment of, in psychotherapy, iv. 578; as part of technique of psychoanalysis, i. 593.

Dream content, iv. 579 .

Dream mechanism, i. 586.

Dream states, in epilepsy, iv. 637.

"Dream-work," i. 586.

Dresser, contraction of bronchial tubes caused by electrical stimulation of vagi demonstrated by, iii. 383 .

DREYER, potassium permanganate treatment of small-pox advocated by, ii. 51 .

Drinking water. See Water.

Drinks, warm, use of, in renal colic, iv. 104. Drip sheet, use of, in neurasthenia, iv. 548 .

Drip sheet bath, i. 195.

"Dromotherapy," iv. 523.

Dromotropic INFLUENCES, iii. 537.

Drooling, measures for relief of, in chronic progressive bulbar paralysis, iv. 253.

Dropsy, epidemic. See Epidemic dropsy.

Dropsy, general. See Anasarca, general.

DrUG ERUPTIONs, diagnosis between, and vaccination, ii. 92 .

DRUG HABIT, danger of forming, in neurasthenia, iv. 565 .

Drugs, use of, acute nephritis due to, iv. 27,57 ; contraindicated to check diarrhea during stage of reaction in cholera, iv. 836; contraindicated in pyelitis, iv. 89; hemoglobinuria due to, iv. 4 ; irritation of kidney due to, iv. 27, 57; neuritis of third, fourth, and sixth nerves due to, iv. 308; effect of, on pulmo- 
nary circulation, iii. 404; excessive, a factor in causation of chronic rhinitis, iii. 328; habitual or long-continued, a cause of ehronic gastritis, iii. 159; misuse of, in whooping cough, ii. 175; objections to, for relief of insomnia in neurasthenia, iv. 564; prevention of scarlet fever by, ii. 123; vertigo due to, iv. 624 .

Dry cups. See Cups.

"Dry diet," employment of, in gastric atony, iii. 170; in pleurisy with effusion, iii. 468.

Dry heat. See Heat.

"DRY KNEE JOINT," ii. 709.

"Dry TAP," ii. 205.

Dubois' method of reëducation, i. 579 ; for treatment of neurasthenia and psychasthenia, iv. 544, 571.

Duke's disease, ii. 165.

Dulcin, as substitute for sugar in diabetes mellitus, ii. 750 .

DuNBar, theory of causation of hay fever held by, i. 160.

Dura mater, diseases of, iv. 362 : pachymeningitis externa, iv. 362 ; pachymeningitis interna hemorrhagica, iv. 362; traumatic hematoma, iv. 364.

DURA MATER, indications of tumor in, iv. 445.

DURHAM's THEORY, of causation of beri-beri, iv. 849 .

Dust, as a factor in causation of measles, ii. 147; inhalation of, injurious in chronic bronchitis, iii. 368 ; inhalation of, in trades a cause of pneumonoconiosis, iii. 423 ; injury from, in chronic pneumonia, iii. 423 ; measures for protection from, iii. 428; transmission of small-pox by, ii. 42,43 .

Dusting powder, use of, in connection with counterirritation for relief of bronchopneumonia complicating measles, ii. 159; in herpes zoster, iv. 359 ; in treatment of nasal and pharyngeal complications of measles, ii. 156; in treatment of smallpox, ii. 48,49 ; in treatment of varicella, ii. 112.

Duval and Bassete, determination by, of special causal agent in infantile summer diarrhea, ii. 316.

Dwarfism, relation between the hypophysis and, i. 103.
Dysentery, hydrotherapy for, i. 219; liver abseess associated with, iii. 290; prophylaetic inoculation against, i. 142.

DYSENTEHY, ACUTE HACILLARY, ii. 316 ; agglutination reaction in, ii. 320 ; bacteriology of, ii. 317 ; "carriers" of, ii. 320 ; classification of, ii. 316 ; definition of, ii. 316 ; diet in acute cases of, ii. 324 ; diet in ehronie cases of, ii. 328; discovery of bacillus causing, ii. 316; drugs in, ii. 326 , 329; epidemiology of, ii. 319 ; geography of, ii. 319 ; infantile diarrhea and, ii. 328 ; introduction to subject of, ii. 316 ; operative measures indicated in, ii. 328; organisms present in, ii. 317; pathology of, ii. 318; serum treatment of, ii. 321 ; sourees of infection in, ii. 319 ; transmission of, ii. 319 ; treatment of acute cases of, ii. 324 ; treatment of chronic cases of, ii. 327 ; treatment of, in general, ii. 321 ; treatment other than specified in, ii. 323 ; vaccine treatment of, ii. 322 .

DrsmenorRheA, electrotherapy for relief of, i. 419; hydrotherapy for relief of, i. 211 .

Dyspepsia, ACID, iii. 74.

Dyspepsia, Nervous, iii. 182; hydrotherapy for relief of, i. 214.

Dyspeptin, iii. 150.

Dysphagia, measures for relief of, in tuberculosis of mediastinal lymph glands, iii. 508 .

DysPNEA, accompanying acute nephritis, iv. 63 ; accompanying tuberculosis, ii. 506; in bronchopneumonia, iii. 419; in cardiac insufficiency associated with pulmonary conditions, iii. 709 ; in chronic interstitial nephritis, iv. 71; complicating chronic pulmonary emphysema, iii. 438; method of relieving, in cardiac insufficiency, iii. 571; presence of, in cardiac disease a contraindication to use of gymnastics, iii. 639; relief of, in mediastinal tumors, iii. 503; as symptom of chronic myocardial insufficiency, iii. 736; during thoracentesis, iii. 578; due to uremia, iv. 54.

Dystrophia adiposogenitalis, iii. 917; relation between hypopituitarism and, $\mathrm{i}$. 103 ; therapeutic use of the hypophysis for relief of, i. 104, 108.

Dystrophy, muscular. See Muscular dystrophy. 
EAR, influence of poisons upon, i. 668.

Ear disease headaches, iv. 603.

EAR LABYRINTH, relation between disturbance of, and vertigo, iv. 617 .

"Early reaction" of X-ray, i. 512.

Eating utensils, disinfection of, in tuberculosis, ii. 466.

Echinococcus, iii. 255; affecting brain, iv. 459.

Echinococcus cysts of pleura, iii. 493.

Echinococcus disease, iii. 255.

EClampsia, method of treatment for, employed by Stronganoff, iv. 83; thyroid gland in treatment of, i. 76 .

EClaMpsia, INFANTILE, relation of, to rickets, ii. 819.

Eczema, as a complication of vaccination, ii. 91 ; radiant light and heat in treatment of, i. 488 ; radium in treatment of, i. 474; X-ray therapy for relief of, i. 527.

EDEMA, accompanying acute nephritis, iv. 62 ; associated with acute uvulitis, iii. 35 ; associated with amyloid kidney, iv. 81; due to nephritis, iv. 15; removal of, iii. 674 ; removal of, by diuresis, iii. 676 ; removal of, by elimination of fluid through intestine, iii. 678; removal of, by elimination through skin, iii. 678 ; removal of, by skin puncture, iii. 676 .

Edema of genitalia, due to chronic myocardial insufficiency, iii. 740.

EDEMA OF GLOTTIS, as a complication of acute nephritis, iv. 63 ; as a complication of mumps, ii. 171 ; as a complication of small-pox, ii. 51 .

EDEMA OF LARYNX, as a complication of measles, ii. 158.

Edema of lungs. See Pulmonary edema.

Edema, malignant, epidemic of, apparently due to $B$. proteus vulgaris infecting sardines, ii. 644.

EDINGER's HYPOTHESIs, concerning etiology of tabes, iv. 182.

Eduuo. See Onyalai.

Edsall and Pemberton, report by, on $\mathrm{X}$-ray treatment of delayed resolution in pneumonia, ii. 258.

Education of nerve centers, after Korsakow's psychosis, ii. 609.

Education of PUBLIC, in prophylaxis against alcoholism, ii. 591 ; in prophylaxis against leprosy, iv. 812 ; in prophy- laxis against malaria, iv. 738 ; in prophylaxis against pneumonia, ii. 223.

Effervescent bath, i. 200.

Effervescing saline laxatives, use of, in acute nephritis, iv. 58.

Effusions, serous, accompanying cardiac insufficiency, iii. 672; aspiration for, iii. 672 ; localization of, iii. 673 ; management of, in severe cases of chronic myocardial insufficiency, iii. 720 ; paracentesis for, iii. 674.

EgG albumin water, as article of invalid diet, i. 648.

EGG FLIP, as article of invalid diet, i. 648.

EGG-NOG, as article of invalid diet, i. 648.

EgGs, in diet of achylia gastrica, iii. 147; in diet of chronic parenchymatous nephritis, iv. 65 ; in diet of depressive disorders of gastric secretion, iii. 147; in diet of diabetes mellitus, ii. 744; in diet of gastric hyperacidity, iii. 85; in diet for nephritis, iv. 43, 65; digestibility of, i. 628; idiosyncrasies toward, as article of food, i. 629; poisoning by, ii. 636 ; in treatment of uremia, iv. 52 ; value of, as article of food, i. 628.

EGYPT, climate of, for laryngeal tuberculosis, iii. 345 .

ЕнRLich, on use of arsenobenzol for brain syphilis, iv. $481,484,487$; work by, on leukemia, iii. 844.

" Ehrlich-Hata," use of, in destruction of filaria bancrofti, iv. 788 .

Ehrlich's theory. See Side-chain theory.

Eichнorst, formula employed by, for use of cream of tartar in cirrhosis of liver with ascites, iii. 286 ; on injurious effect of high altitudes on neurotic conditions, iv. 556.

Eighth cranial Nerve, diseases of, iv. 314.

EINHoRn, modification of von Noorden diet for membranous enteritis introduced by, iii. 209; objections by, to massage in spastic constipation, iii. 228.

Einhorn bandage, iii. 248.

Erweiss MILK, in enteritis of infancy, iii. 203.

Elaterin, use of, in uremia, iv. 53.

Elaterium, use of, following calomel, in edematous laryngitis, iii. 341 ; in nephritis, iv. 33 ; in nephritis complicating scarlet fever, ii. 134. 
Elbow bath, i. 200.

EleCtric ARC, as source of radiant energy, i. 439.

Electric heating apparatus, use of, in toxemia complicating acute diffuse peritonitis, iii. 319.

Electric light cabinet, use of, for elimination of poisons in intestinal autointoxication, ii. 672.

Electric pad, use of, in gastric hyperacidity, iii. 100; in non-bleeding gastric ulcer, iii. 133; to relieve backache in acute nephritis, iv. 63.

Electric Pains. See Lancinating pains.

Electricity, as a Climatic factor, i. 257; effects of, on living body, i. 7; electrolytic effects of, i. 369 ; mechanical effects of, i. 370; physical effects of, i. 369 ; properties of, in hydrotherapy, $i$. 193; thermic effects of, i. 369.

Electricity, Physiological efFects of, i. 370: basis of physiological effects, i. 376 ; determining characteristics of electrical currents, i. 374 ; effect of high frequency current, i. 375; effect of induced current, i. 374 ; effect of sinusoidal current, i. 375; effect of static current, i. 374, 377; independent irritability, i. 372 ; mechanical effects of, i. 376 ; muscular response to nervous irritability, i. 371 ; in tuberculosis of skin, i. 400 ; in tuberculous infections, i. 399.

Electricity, use of, in acute hemorrhagic encephalitis, iv. 406; in acute myelitis, iv. 227; in after-treatment of anterior poliomyelitis, iv. 238; in arthritis associated with intestinal autointoxication, ii. 681; arthritis deformans and chronic rheumatism, ii. 713 ; in asthenic bulbar paralysis, iv. 256; in beri-beri to prevent contractures, iv. 854; in bronchial asthma, iii. 381 ; in cardiac neurasthenia, of no value, iii. 777 ; in cardiovascular disease, i. 425; in cerebral anemia, iv. 382 ; for cerebral hemorrhage due to chronic interstitial nephritis, iv. 72 ; in chronic myelitis, iv. 230; in chronic progressive bulbar paralysis, iv. 251; contraindicated in acute intestinal obstruction, iii. 240 ; contraindicated in myasthenia gravis, iv. 506; contraindicated in recent cases of neuritis, iv. 284; in convalescence from dengue, iv. 860 ; in convales- cence from Landry's paralysis, iv. 329; in depressive gastric neuroses, iii. 188; in diabetic neuralgia and neuritis, ii. 766 ; in diseases of toxic origin, i. 408; in exophthalmic goiter, iii. 898; for gastric atony, iii. 173; in gastric hyperacidity, iii. 100; in glossopharyngeal paralysis, iv. 318; in gonorrheal infections, i. 406; in infections in general, i. 398; in inflammation of joints, i. 412; in Korsakow's psychosis, ii. 609; in mumps, ii. 172; in myotonia congenita, of no value, iv. 268 ; for neuralgic headache, iv. 598; in neurasthenia, iv. 540, 559; in neuritis, in general, i. 414, iv. 287; in neuritis of third, fourth, and sixth nerves, iv. 308; in neuritis of twelfth cranial nerve, iv. 321 ; objections to, in irritative gastric neuroses, iii. 188; for obstetric paralysis, iv. 282 ; in occupation neuroses, iv. 661 ; in occupation paresis, iv. 332 ; in ocular paralysis accompanying meat poisoning, ii. 640; in optic atrophy, iv. 307 ; for paralysis due to cerebral hemorrhage, iv. 393 ; in paralysis following cerebrospirc. meningitis, ii. 216 ; in pelvic inflarrr : tion, i. 418; in phrenic neuritis, iv. 290; in pneumogastric paralysis, iv. 820 ; in post-gonorrheal neuroses, iv. 175; to prevent collapse in snake poisoning, iv. 682 ; in progressive bulbar paralysis, iv. 499 ; in progressive neural muscular dystrophy, iv. 266; in pulmonary tuberculosis, i. 400; in pyogenic infections, i. 403 ; to reduce blood pressure, iv. 48 ; for relief of cervicoöccipital neuralgia, iv. 356; for relief of constipation, iii. 229 ; for relief of constipation in neurasthenia, iv. 566; for relief of enuresis due to atony of sphincter, iv. 128; for relief of headache in neurasthenia, iv. 566; for relief of neuralgic pain following cure of morphin habit, ii. 624; for relief of ozena, iii. 337; for relief of pain in legs in beriberi, iv. 853; for relief of pain in neuralgia, iv. 338; for relief of pain in sciatica, iv. 299; for relief of pain in spinal syphilis, iv. 274; for relief of paralysis of muscles of mastication, iv. 310 ; for relief of spasmodic movements of neck muscles, iv. 656 ; for relief of vomiting in neurasthenia, iv. 567; in rheumatic or idiopathic cases of facial paralysis, iv. 312; 
in second stage of multiple neuritis, iv. 328; in simple inflammation, i. 409; in spinal accessory paralysis, iv. 320 ; in spinal cord affections, i. 428; in spinal progressive muscular atrophy of acquired type, iv. 247; in tabes, iv. 201; in tabetic amaurosis, iv. 199; in tetany, iii. 910 ; in tuberculosis of skin, i. 400 ; in tuberculous infections, i. 399; through urethroscope for removal of foreign growths, iv. 166; in vertigo and tinnitus aurium, iv. 818.

Electrolysis, use of, in chronic rhinitis, iii. 329 ; indications for, i. 396.

Electrolytes, i. 7; capacity of tissue colloids for water reduced by, i. 38 ; comparative activity of, in living cells, i. 55; definition of, i. 7; effect of, upon gelatin and fibrin in acid solution, i. 31; effect upon, of solution in water, i. 26 ; relation between electricity and, i. 7; relation between the laws of osmotic pressure and, i. 54.

Electrolytic dissociation, pharmacological importance of, i. 26; process of, in living tissues, i. 7.

Electromechanical methods, i. 379: constant current interrupted, i. 390 ; induced current, i. 389 ; pulsatory Oudin current, i. 388; sinusoidal current, i. 388 ; static brush discharge, i. 386 ; static induced current, i. 382 ; static spark, i. 383; static spray, i. 387 ; static wave current, i. 379 ; vacuum tube wave current, i. 382.

Electrophoresis, application of iodin by, in atrophic rhinitis, iii. 330 .

Electrotherapeutics, i. 363; apparatus employed in, i. 367 ; application of electric currents for mechanical effects in, i. 364; application of electric currents for thermic effects in, i. 390; definition of, i. 363; electrolysis and indications for its use, i. 396; explanation of terms employed in, i. 363 ; methods and principles of applying electrical currents in, i. 379 ; physical effects of electricity, i. 369 ; therapeutic application of electricity, i. 397; therapeutic indications for mechanical effects of, i. 394; therapeutic indications for thermic effects of, i. 393 .

Electrovargol, use of, by subcutaneous or intravenous injection in typhus fever, ii. 37 .

Elimination, increase of, a means of reducing hypertension in nephritis, iv. 45; means for promoting, in chronic diseases of the labyrinth, iv. 317 ; means for promoting, in multiple neuritis, iv. 326; means for promoting, in neuralgia associated with rheumatism, iv. 337; means for promoting, in neuritis of third, fourth, and sixth nerves, iv. 308; means for promoting, in optic neuritis, iv. 306; means for promoting, in polymyositis, iv. 330 ; means for promoting, in rheumatic cases of facial paralysis, iv. 311; as means of relief from cervicoöccipital neuralgia, iv. 356; promotion of, through kidneys, important in plague, iv. 827; through skin and bowels in cerebral hemorrhage accompanying chronic interstitial nephritis, iv. 72; through skin and bowels in chronic parenchymatous nephritis, iv. 65.

Emaciation, as a symptom of tuberculous meningitis, iv. 374 .

Emanation, of radium, i. 463.

"Emanatores," employment of, in therapeutic use of radium, i. 473.

Embalming FLUIDS, occurrence of arsenic in, i. 676 .

Embolism, cerebral. See Cerebral embolism.

Embolism of Lungs. See Pulmonary embolism.

Embolism, pulmonary. See Pulmonary embolism.

Embryos, effect of X-ray therapy upon tissues of, i. 523.

Emesis, use of, in cheese poisoning, ii. 647; in meat poisoning due to $\mathrm{B}$. enteritidis, ii. 641 ; in meat poisoning due to $B$. paratyphosus, ii. 641 ; in treatment of acute gastritis, iii. 154 .

Emetics, use of, in active congestion of lung, iii. 604; in bronchial asthma, iii. 389 ; in bronchopneumonia of doubtful value, iii. 419 ; contraindicated in membranous laryngitis, iii. 343 ; in erythrophlæum poisoning, iv. 696 ; in fish poisoning, iv. 684; in plastic bronchitis, iii. 392 ; to remove bronchial secretion in acute bronchitis, iii. 364; unnecessary in cholera morbus, iii. 195. 
EmiL, method of extracting guinea worm recommended by, iv. 789 .

Emmerich AND Weibel, epidemic among trout investigated by, ii. 644 .

EMmerich's THEORY, of nature and action of cholera toxins, iv. 831.

Emollient sprays, use of, in nasal and frontal sinus headaches, iv. 603.

Emotions, as a causal factor in neuralgia, iv. 337 .

Emphysema, association of, with cardiac insufficiency, iii. 710; treatment of, iii. 731 .

Emphysema, subcutaneous, occurrence of, during thoracentesis, iii. 479.

EMPiric Metiod of DEtermining daILY FOOD REQUIREMENT, i. 604.

Emplastrum hydrargyri, use of, in stomatitis syphilitica, iii. 21.

Empyema, iii. 484; accidents during irrigation for relief of, iii. 487; associated with actinomycosis, iii. 489; bilateral form of, iii. 488; as complication of pneumonia, ii. 261; as complication of pulmonary tuberculosis, ii. 507 , iii. 489 ; in children, iii. 488; empyema necessitatis, iii. 488; encapsulated empyema, iii. 489; irrigation of pleural cavity for, iii. 487; microörganisms present in, iii. 484; pneumothorax associated with, iii. 493; prognosis of, iii. 484; resection of ribs for relief of, iii. 486; thoracotomy for relief of, iii. 485 ; treatment of, iii. 485 ; treatment in special forms of, iii. 488; tuberculosis of lungs associated with, iii. 489.

Ems water, use of, as expectorant in chronic bronchitis, iii. 373.

EMUlsion Colloids, i. 6, 28.

Encapsulated empyema. See Empyema.

ENCEPHALITIS, iv. 404; acute hemorrhagic encephalitis, iv. 404; acute hemorrhagic polioencephalitis, iv. 406; cerebral palsies of children, iv. 409; introduction to subject, iv. 404.

Encephalitis, acute hemorrhagic, iv. 404; diagnosis of, iv. 405; etiology of, iv. 404; pathology of, iv. 405; prognosis of, iv. 405 ; symptoms of, iv. 404 ; treatment of, iv. 405.

Encephalomalacia. See Cerebral softening.

Endarteritis, syphilitic. See Syphilitic endarteritis.
Endemic hematuria, iv. 783; preventive measures for, iv. 785; remedial measures for, iv. 784 .

ENDEMic nemoptysis, iv. 782 ; preventive measures for, iv. 783.

Endocarditis, acute, iii. 743 ; as a cause of cerebral embolism, iv. 400; as a complication of pneumonia, ii. 260 ; as a complication of septicopyemia, ii. 288; electrotherapy for, i. 409 ; hydrotherapy for, i. 204; malignant, iii. 746.

Endocarditis, acute, SIMPLe, iii. 743; importance of preventing, in chronic myocardial insufficiency, iii. 734; prophylaxis against, iii. 743 ; treatment of, iii. 745 .

ENDonetritis, CHronic, hydrotherapy for relief of, i. 211.

ENDOtheliomata of Brain, iv. 432.

Endotin, i. 151.

Endotoxins, i. 148.

Endurance, exercises of, iii. 700.

Enemata, use of, in acute myelitis, iv. 225; in acute nephritis, rarely beneficial, iv. 58 ; in acute pancreatitis, iii. 296 ; in bacillary dysentery for administration of opium, ii. 329 ; with caution in acute localized peritonitis, iii. 312 ; in chronic intestinal obstruction, iii. 242 ; in chronic mucous colitis, iii. 207; contraindicated in early stage of acute appendicitis, iii. 215; contraindicated in rest treatment for cardiac insufficiency, iii. 572; contraindicated in violent attacks of acute enteritis, iii. 194; in gallstone colic, iii. 269 ; graduated, in intestinal autointoxication, ii. 674 ; hypertonic alkaline salt solution by, to produce diuresis in beriberi, iv. 852 ; in intestinal amebiasis, iv. 769; in infantile diarrhea, ii. 329; iced saline solution by, to reduce temperature in Asiatic cholera, iv. 836; in later stages of mild appendicitis, iii. 216; objections to, for relief of constipation in neurasthenia, iv. 566; olive oil by, in constipation due to myocardial insufficiency, iii. 740 ; in oxyuris vermicularis, iii. 252 ; in pericarditis, iii. 515 ; to promote evacuation of bowels in gastric atony, iii. 172; in severe attacks of appendicitis, iii. 217; purgatives by, in fecal impaction, iii. 238,239 ; turpentine by, in bronchopneumonia, for relief of meteorism, iii. 
416 ; in volvulus and intussusception, iii. 241.

Enemata, high rectal, use of, after poisoning, i. 671; normal saline solution by, in Asiatic cholera, iv. 834; normal saline solution by, in cheese poisoning, ii. 647; normal saline solution by, in diabetic coma, ii. 757; normal saline solution by, in meat poisoning due to B. paratyphosus, ii. 644; protargol by, in meat poisoning due to $\mathrm{B}$. enteritidis, ii. 641 ; soap and water by, for impaction of feces in intestinal autointoxication, ii. 672 ; turpentine by, in meat poisoning due to B. botulinus, ii. 639 .

Enemata, nutrient, formulæ for, iii. 119; method of giving, i. 638; nutritive value of, i. 637; use of, in acute gastritis, iii. 157 ; in delirium tremens, ii. 602 ; in gastric crises of tabes, iv. 197; in gastric ulcer, iii. 110, 118; in hemorrhage from gastric ulcer, iii. 118; in progressive bulbar paralysis, iv. 498; in progressive paralysis of insane, iv. 493.

Enemata, saline, use of, in acute catarrhal jaundice, iii. 261; to allay thirst in starvation treatment of gastric ulcer, iii. 118; by drop method in Rocky Mountain fever, ii. 566; in enteritis of infancy, iii. 202; to relieve thirst in carcinoma of esophagus, iii. 55 .

Enemata, soapsuds, use of, in acute enteritis, iii. 193; in constipation due to chronic myocardial insufficiency, iii. 740 ; in spasmodic laryngitis, iii. 342 .

Enemata, starch, administration of laudanum by, in cholera morbus, iii. 195.

Enemata, stimulating purgative, use of, in paralytic ileus, iii. 238.

Enemata, warm saline, use of, in acute enteritis, iii. 193; to control hemorrhage in pulmonary tuberculosis, ii. 501; to control hemorrhage in typhoid fever, ii. 19 ; in influenza of gastrointestinal type, ii. 190; in membranous enteritis, iii. 208; in nephritis complicating scarlet fever, ii. 133; in septicopyemia, ii. 133; in typhoid fever, ii. 26.

ENergy ReQuirement, i. 604, 606; in diabetes mellitus, ii. 724 .

Engelmann, classification by, of influences acting on four cardinal properties of cardiac muscle, iii. 537.
ENGLAND, antituberculosis work in, ii. 440; mortality from small-pox in, before and after vaccination, ii. 93.

Entameba coli, iv. 704; characteristics of, iv. 704; distribution of, iv. 705; pathogenicity of, iv. 704 .

Entameba histolytica, iv. 704; characteristics of, iv. 704; distribution of, iv. 705; pathogenicity of, iv. 704 .

Entameba tetragena, iv. 704; characteristics of, iv. 704; distribution of, iv. 705 ; pathogenicity of, iv. 704 .

ENTERIC Diseases, hydrotherapy for relief of, i. 212.

ENTERITIS, iii. 193; acute, iii. 193; chronic, iii. 196; hydrotherapy for relief of acute, i. 213 ; in infancy, iii. 201 ; mild cases of, iii. 197; moderate cases of, iii. 199; severe cases of, iii. 200.

Enteritis, membranous, iii. 207; diet in, iii. 208; oil injections in, iii. 209; operative measures for, iii. 210 .

Enteroclysis, administration of normal saline solution by, in pyelitis, iv. 89; employment of, in renal colic, iv. 103; employment of, in sepsis complicating scarlet fever, ii. 128.

ENterocolitis, as a complication of measles, ii. 160.

Enteroptosis, iii. 243; autointoxication due to habitual constipation associated with, ii. 678 ; reflex cardiac neuroses associated with, iii. 780 .

Enuresis, iv. 125; causes of, iv. 125; diseases of remote origin as cause of, iv. 127, 130; diseases of urinary and pelvic origin as cause of, iv. 127, 130; essential enuresis, iv. 126; hygienic causes of, iv. 126, 127; idiopathic enuresis, iv. 126; treatment of, in general, iv. 121, 132; urinary changes causing, iv. 127 .

ENuResis, Nocturnal, hydrotherapy for relief of, i. 209.

Environment, effect of, on beri-beri, iv. 844.

Eosin treatment, use of, in frambœsia tropica, iv. 765 .

EPIDEMIC DROPSY, iv. 860.

Epidemic gangrenous proctitis, iv. 866.

EPIDIDYMitis, as a complication of mumps, ii. 166; electrotherapy for, i. 407: hydrotherapy for, i. 212; treatment of, ii. 171. 
Epididymitis, gonorrheal. See Gonorrheal epididymitis.

Epididymitis, recurrent, iv. 152.

Epidural injections. See Injeetion.

Epiglottectomy, employment of, in laryngeal tuberculosis, iii. 347 .

Epilepsies, the, iv. 630; diet in, iv. 646; general mode of treatment for, iv. 641; idiopathic form of, iv. 640; inheritanee of, iv. 641 ; introduction to subject of, iv. 630; major attacks of, iv. 633; marriage question in, iv. 641 ; minor attacks of, iv. 634 ; operation for relief of, iv. 639; pharmaeotherapy for, iv. 647; physical therapy for, iv. 646; prophylaxis against, iv. 641 ; psyehic equivalents to attacks of, iv. 634; symptom review of, iv. 633; tabular scheme of convulsions in, iv. 632; therapy for, in general, iv. 638 .

EpIlepsy, due to acute hemorrhagic encephalitis, iv. 405; association of, with intestinal autointoxication, ii. 682 ; as cause of nocturnal enuresis, iv. 126; due to cerebral palsies of children, iv. 411, 413; diagnosis between idiopathic, and brain tumor, iv. 437; hydrotherapy for relief of, i. 222; relation between, and asthma, iii. 382; symptoms resembling, in brain cysticercus, iv. 459; vertigoes associated with, iv. 624 .

EPILEPTIC atTACK, eonvulsive form of, iv. 633 ; major form of, iv. 633; minor form of, iv. 634; psychic equivalents for, iv. 634.

Epileptiform convulsions, due to brain tumor, measures for relief of, iv. 448; tabular scheme of, iv. 632 .

Epinephrin, absence of, in Addison's disease, i. 89; amount of, in blood of suprarenal vein, i. 68; conditions benefited by administration of, i. 96, 98, 99; as eardiovascular stimulant, i. 97 ; in control of hemorrhage, i. 97; effect produced by, on pulmonary eirculation, iii. 405; as a local hemostatic, i. 98; peculiarities of, i. 97; physiological action of, i. 90 ; preparations of, i. 100; resemblance between effects of, and effects of the hypophysis, i. 105; synonyms for, i. 100; therapeutic uses of, i. 95; unstable eharacter of, i. 68; untoward effects following use of, i. 100; use of, in disturbanees of eireulation in Addison's disease, iii. 885; use of, to enhance effect of local anesthesia, i. 99; use of, by intraperitoneal injeetion in chronic peritonitis of generalized form, iii. 323; use of, in toxemia complicating acute diffuse peritonitis, iii. 318 ; see also Adrenalin.

Epirenan. See Epinephrin.

Epistaxis, iii. 335; as a complication of glandular fever, ii. 576 ; as a complication of typhoid fever, ii. 27.

Epitheliomata, action of radium upon, i. 470; fungating, radium in treatment of, i. 482 ; of skin, superfieial, radium in treatment of, i. 481 ; superficial, X-ray therapy for relief of, i. 538 .

Epsom salts. See Magnesium sulphate.

Epstein and Koplik, pseudo-diphtheritic stomatitis described by, iii. 19 .

Epstein diet, for reduction of obesity, ii. 791.

EQuilibrium in living tissues, i. 12.

Erb-Goldflam's disease. See Myasthenia gravis.

Erections, PAINFul, treatment of, in acute urethral gonorrhea, iv. 141.

Ergot, use of, in acute myelitis, iv. 225; contraindicated in control of epistaxis, iii. 336; for control of gastric hemorrhage, iii. 112; for control of hemorrhage in pulmonary tuberculosis, ii. 500 ; in delirium tremens, ii. 605; in diabetes insipidus, ii. 605; effect produced by, on pulmonary eirculation, iii. 405; in exophthalmic goiter, iii. 899 ; in hematuria, iv. 4 ; in hematuria complicating scarlet fever, ii. 134; in hemophilia, iii. 867; in hemorrhagic form of measles, ii. 156; in neurasthenia with vasomotor dilatation, iv. 559; objections to, in anterior poliomyelitis, iv. 238; objections to, in chronic myelitis, iv. 230 ; for relief of vesical disturbance in tabes, iv. 198; in tabes, iv. 195; as vesical tonic in treatment of enuresis, iv. 134.

Ergot combined with the bromids, use of, for relief of insomnia due to chronic cerebral hyperemia, iv. $38 s$.

Ergotin, use of, in hemophilia, iii. 869; ineffectual in acute vasomotor paralysis, iii. 753; in Landry's paralysis, iv. 329; 
in neurasthenia with vasomotor dilatation, iv. 559; for relief of headache in neurasthenia, iv. 566.

Ergotism, ii. 652.

Ergotol, use of, for control of hemorrhage in hemorrhagic form of measles, ii. 156.

Erlanger, myogenic theory of cause and origin of heart beat developed by, iii. 535.

Erlanger apparatus, for estimating minimal blood pressure, iii. 557 .

ERUPTION, character of, in cow-pox, ii. 56; character of, in hemorrhagic form of varicella, ii. 113; character of, in miliary fever, ii. 578; character of, in typhoid fever, ii. 38; character of, in typhus fever, ii. 33; character of, in varicella, ii. 112; following use of antitoxin in diphtheria, ii. 271; following use of arsenobenzol in syphilis, ii. 415 ; treatment of, in small-pox, ii. 48; value of, in diagnosis between typhoid and typhus fevers, ii. 38.

Erysipelas, ii. 281; assistant causes in, ii. 281; Bier's hyperemia treatment of, ii. 285 ; characteristic lesion in, ii. 282; complications of, ii. 283; definition of, ii. 281; diagnosis of, ii. 283; diet in, ii. 283; effect of, upon other diseases, ii. 285; etiology of, ii. 281 ; incision in treatment of, ii. 285; local treatment of, ii. 284; "medical" form of, ii. 282; "migratory cases" of, ii. 283; organism causing, ii. 281; pathologic anatomy in, ii. 282; pathology of, ii. 282; predisposing causes of, ii. 281; prognosis of, ii. 283; sequelæ to, ii. 283; serum therapy in, ii. 284; small-pox complicated by, ii. 49; symptoms of, ii. 282 ; temperature in, ii. 282, 283; treatment of, ii. 283; treatment of complications in, ii. 285; vaccination complicated by, ii. 85 ; vaccine therapy for, ii. 284.

"Erythema dose" of X-ray, i. 498.

ERythema multiforme, as complication of vaccination, ii. 85 .

Erythemas, itching, radiant light and heat in treatment of, i. 459.

Erythrol tetranitrate, use of, in cardiac insufficiency, iii. 608; in chronic interstitial nephritis, iv. 70, 71; to control hemorrhage in pulmonary tuberculosis, ii. 500; in neurasthenia with high ar- terial tension, iv. 559, 565; to reduce hypertension in nephritis, iv. 47, 48.

Erythrophlatum poisoning, iv. 695; preventive measures, iv. 696; remedial measures, iv. 696.

Escalin, use of, for control of gastric hemorrhage, iii. 112; in gastric hyperacidity, iii. 97 ; in non-bleeding gastric ulcer, iii. 136.

Eserin, use of, in acute dilatation of stomach, iii. 174; in diabetes mellitus, ii. 760; for relief of corneal ulceration in smallpox, ii. 50; in syphilitic iritis, ii. 429 .

Eserin salicylate, hypodermic use of, in acute intestinal obstruction, iii. 241; injection of, into wall of intestine after enterostomy for paralytic ileus, iii. 238; use of, in chronic constipation, iii. 234; use of, in paralytic ileus, iii. 238.

Eserin sulphate, use of, in acute diffuse peritonitis, iii. 317 .

Esmarch bandage, use of, to determine site of arterial obstruction in diabetic gangrene, ii. 770.

Esophageal dilator of Sippy, iii. 49.

Esophageal stenosis, iii.. 47.

EsopHAgostomiasis, iv. 799; parasites causing, iv. 799; remedial measures in, iv. 800.

Esophagus, regional anatomy of, iii. 47.

Esophagus, carcinoma of, iii. 52 ; diet in, iii. 54; palliative treatment for, iii. 53; surgical treatment for, iii. 53 ; treatment for, in general, iii. 53; X-ray in treatment for, iii. 54,55 .

Esophagus, diseases of, iii. 47: cicatricial stenosis, iii. 48; diverticula of esophagus, iii. 60; esophageal stenosis, iii. 47; foreign bodies in esophagus, iii. 62 ; spasm of esophagus, iii. 56; ulcer of esophagus, iii. 65; Vincent's angina, iii. 65 .

Esophagus, Diverticula of, iii. 60: course, iii. 61 ; pressure diverticula, iii. 60; traction diverticula, iii. 61; traction-pressure diverticula, iii. 61; treatment, iii. 62 .

Esophagus, idiopathic dilatation of, iii. 56 ; diagnostic aids in, iii. 58; etiology of, iii. 56 ; treatment of, iii. 58 .

Esophagus, ulCER of, iii. 65: peptic ulcer, iii. 66 ; treatment, iii. 66 ; tuberculous ulcer, iii. 66 .

Estlander's operation, in empyema, iii. 486. 
Ether, use of, as cardiac stimulant in typhoid fever, ii. 24; by hypodermic injection as cardiac stimulant in cerebral anemia, iv. 382 ; by hypodermic injection in methemoglobinuria, iv. 6; by hypodermic injection in pulmonary thrombosis, iii. 411 ; by inhalation in strophanthus poisoning, iv. 695 ; internally as stimulant in cerebral anemia, iv. 382 ; by spray, for relief of neuralgic pains following cure of morphinism, ii. 624 ; by spray for small-pox, ii. 49 .

Ether and turpentine, use of, in treatment of gallstones in transit, iii. 274.

Ether anesthesia, in adenectomy, iii. 36, 37; in chronic myocardial insufficiency, iii. 743; contraindicated in retropharyngeal abscess, iii. 34; injury of kidney by, iv. 28 ; in tonsillectomy, iii. 45,46 .

Ether pneumonia. See Pneumonia.

Ethyl bromid, use of, in chronic myocardial insufficiency, iii. 743 .

Ethyl chlorid, use of, as anesthetic in lumbar puncture, iv. 370 ; as anesthet ic in thoracentesis, iii. 474; lancinating pains of tabes not relieved by, iv. 197; as spray, for relief of gastric crises in tabes, iv. 197.

Eucain, use of, in local treatment of tertiary syphilitic lesions of mucosæ and mucocutaneous junctions, ii. 427; as mouth wash in stomatitis due to hydrargyrism, ii. 385.

Eucalyptol, use of, in gangrene of lung complicating pneumonia, ii. 261; in prophylaxis of influenza, ii. 186.

Eucalyptus, efficacy of, in prevention of malaria disproved, iv. 731; use of, by inhalation of, in acute bronchitis, iii. 358 .

Eucalyptus oil, use of, as an anthelmintic in intestinal distomiasis, iv. 782; in chronic influenza or bronchiectasis complicating influenza, ii. 195; in hookworm disease, iv. 793; by inhalation in acute laryngitis, iii. 339; in scarlet fever, ii. 124.

Eucalyptus ointment, use of, to relieve itching in Rocky Mountain fever, ii. 565.

Euferrol, use of, in chlorosis, iii. 820.

Eugenics, importance of, in treatment of the neuroses, iv. 520 .

Eumydrin, use of, in gastric hyperacidity, iii. 92 .
Euphyllin, use of, to create diuresis in cardiac insufficiency with general insufficiency, iii. 677.

Euquinin, use of, in measles, ii. 151.

Europhen, use of, in leprosy, iv. 816.

Evacuatrons, copious, stage of, in Asiatic cholera, iv. 833.

EwaLd, formula for nutrient enema recommended by, iii. 119.

Ewart, Willas, inhalations of carbonic acid gas suggested by, for relief of asthma, iii. 389.

Exanthema, influence of vaccination upon, ii. 74 .

Exanthemata, hydrotherapy for relief of, i. 215 .

Excision, of bitten part in snake bite, iv. 682; of cervical lymph nodes in early stage of sleeping sickness, iv. 748; of lung, as therapeutic measure in chronic pulmonary abscess and gangrene, iii. 445; in stomatitis gangrenosa, iii. 18; of tip of uvula, in acute uvulitis, iii. 35; of ulcerating granuloma of pudenda, iv. 866.

Excitability, as cardiac function, iii. 536.

Excitement, attacks of, in progressive paralysis of the insane, iv. 493.

Excreta, Disinfection of, in small-pox, ii. 44 ; in typhoid fever, ii. 7 .

Exercise in open air, in after-treatment of pleurisy with effusion, iii. 470; importance of, in cardiac neurasthenia, iii. 775, 776; question of, in arteriosclerosis, iii. 759; value of, in hay fever, iii. 390 .

Exercise, physical, effect of, upon albumin and casts in urine, iv. 23; gradual return to, after rest cure in neurasthenia, iv. 550; influence of, on human body, i. 331 ; influence of, in psychotherapy, $i$. 596; objections to, in chlorosis, iii. $\$ 13$; question of, in tabes, iv. 213 ; regulation of, in cases of obesity associated with cardiac insufficiency, iii. 705; value of, in chronic gout, ii. 700; value of, in diabetes mellitus, ii. 763; value of, in exophthalmic goiter, iii. 897; value of, in intestinal autointoxication, ii. 676 ; value of, in reduction of obesity, ii. 506 ; value of, in tuberculosis, ii. 477,527 .

Exercises, forms of, beneficial to intestinal autointoxication, ii. 676 . 
Exercises, active and passive, use of, in paralysis due to cerebral paralysis, iv. 393.

Exercises of endurance, iii. 700.

Exercises, graduated, in treatment of occupation neuroses, iv. 661.

Exercises, gymnastic. See Gymnastic exercises.

Exercises, passive, use of, in chronic myelitis, iv. 230; in chronic myocardial degeneration, iii. 717,718 ; in spastic spinal paralysis, iv. 270.

Exercises, passive joint, use of, for relief of pain in spinal syphilis, iv. 274.

Exercises, passive resistance, use of, in multiple sclerosis, iv. 281.

Exercises, physical, classification, iii. 700; use of, in after-treatment of neuritis, iv. 288 ; in after-treatment of pleurisy with effusion, iii. 470; in chronic bronchitis, iii. 369 ; in chronic gonorrheal urethritis, with caution, iv. 153; in mild cases of chronic myocardial insufficiency, iii. 716; in muscular dystrophy, iv. 263; for paralysis due to cerebral hemorrhage in chronic interstitial hemorrhage, iv. 72; for relief of spasmodic movement of neck muscles, iv. 656; for relief of tic movements, iv. 658 ; simultaneously with carbon dioxid baths, contraindicated in chronic myocardial insufficiency, iii. 717, 721 ; in treatment of asthma, iii. 380 ; in treatment of constipation, iii. 228 .

Exercises of speed, iii. 700 .

Exercises of strength, iii. 700 .

Exertion, PHYSiCal, avoidance of, in prophylaxis of cerebral hemorrhage, iv. 391; as cause of nephritis, iv. 29.

Exhaustion, as a characteristic symptom of myasthenia gravis, iv. 504 .

Exophthalmic goiter. See Goiter.

Exotoxin, i. 148.

Expectant treatment, of acute urethral gonorrhea, iv. 148.

Expectorant mixtures, for use in acute bronchitis, iii. $360,362,365$; in chronic bronchitis, iii. 373 .

Expectorants, use of, in acute bronchitis, iii. 359 ; in bronchiectasis, iii. 396 ; in chronic bronchitis, iii. 372 ; in chronic influenza or bronchiectasis complicating influenza, ii. 195.

Expectorants, aromatic, use of, in bronchi- ectasis, iii. 396 ; to relieve profuse expectoration in chronic bronchitis, iii. $376,377$.

Expectorants, stimulating, use of, in acute bronchitis, iii. 362 ; in chronic bronchitis, iii. 372 ; in hypostatic pneumonia, iii. 407.

Expectoration, measures for relief of, when profuse in chronic bronchitis, iii. 375; in pulmonary tuberculosis, ii. 498.

Expectoration, Fetid. See Fetid expectoration.

EXPERIMENTAL METHOD OF DETERMINING TOTAL FOOD REQUIREMENT, i. 604.

Expiration, aids to, in acute bronchitis complicating emphysema, iii. 353 .

Exposure, time of, a factor in an X-ray treatment, i. 500 .

ExpUlsion time of heart, iii. 542.

Extractives, uses of, as food, i. 601.

Extrasystoles, allorrhythmia due to, iii. 566 ; auricular, iii. 568; auriculoventricular, iii. 569; clinical significance of, iii. 569 ; ventricular, iii. 567 .

Extremities, ligation of, in pulmonary edema with low blood pressure, iii. 410 .

Exudates, as a cause of pain in bladder, iv. 122 .

Exudates, pleural, cause of death from, iii. 461 ; influence of, on circulation, iii. 460 ; intrathoracic pressure of, iii. 459 , 460; mechanical effect of, iii. 460.

Exudates, putrefactive, in pneumothorax with empyema, iii. 493.

Eye headaches, iv. 601.

Exes, affections of, in diabetes mellitus, ii. 766; care of, in small-pox, ii. 50; effect of epinephrin upon, i. 92; effect of X-ray upon, i. 523; influence of poisons upon, i. 668; measures for protection of, in facial paralysis, iv. 311 .

Facial paralysis, as a symptom of basilar syphilitic meningitis, iv. 474; see also Neuritis of seventh nerve.

FACIAL SPASM, iv. 653; treatment of, iv. 654 .

FAINTNESS, significance of, during thoracentesis, iii. 478 .

FAMILY, duty of, regarding infection in scarlet fever, ii. 121.

FAMILY FORM OF CHRONIC PROGRESSIVE BULbar Paralysis, iv. 253.

FanNia, iv. 689. 
Faradic current, use of, in atrophie rhinitis, iii. 332.

Faradization, of abdominal wall for relicf of gast ric crises in tabes, iv. 197; of norve in phrenic neuritis, iv. 290; of skin of extremities in tabes, iv. 202.

Fania, AвнÉ de, pioneer in hypnotism, i. 562.

Fascioletta iliocana, iv. 781.

Fasciolor'sis BUSKI, iv. 781.

FASCIOLOPSIS FÜLLERBORNI, iv. 781.

Fasciolopsis rathouisi, iv. 781.

Fascioscapulo-humeral tYPE OF MUSCULAR DYSTROPHy, iv. 261.

FAT, digestibility of, i. 631; proportion of, in human body, ii. 780; relation of, to phosphorus poisoning, ii. 627, 628; sources of, i. 600 ; subcutaneous administration of, i. 640; value of, as a food, i. 602 .

Fat content, influence of, upon digestion of meat, i. 627.

Fат емвоlism, following intramuscular injection of mercury in syphilis, ii. 401.

Fatiguability, as a characteristic symptom of neurasthenia, iv. 567 ; presence of, in progressive bulbar paralysis, iv. 498.

FATs, in diet for diabetes, ii. 743 ; in diet for gastric hyperacidity, iii. 84; function of, in body, ii. 782; pancreatic secretion suppressed by diet of, iii. 307 ; proportion of, in diet of chronic parenchymatous nephritis, iv. 65 ; proportion of, in diet for nephritis, iv. 41; proportion of, in diet for neurasthenia, iv. 539; as a source of adipose tissue, ii. 783 ; as a source of energy and heat, ii. 782; sugar derived from, ii. 726 .

Faucitis, acute, iii. 31.

Faught and Stanton, instruments devised by, for estimating minimal blood pressure, iii. 557.

Faure, resection of rib recommended by, in opening posterior mediastinum, iii. 505.

Favus, X-ray therapy for relief of, i. 531.

Febrifuges, use of, in scarlet fever, ii. 125, 126.

Feces, method of disinfecting, in typhoid fever, ii. 7.

Fedschenho, experiments by, on guinea worm, iv. 788.
Feeblemindedness and hysteria, iv. 586.

Feeding, artificial, through esophageal bougie, iii. 62 ; by gavage, i. 639 ; by gavage in meat poisoning, ii. 639 ; method of, after intubation of larynx, ii. 278; method of, in post-diphtheritic paralysis, ii. 278; method of, in tetanus, ii. 350; methods of, i. 636 ; by nutrient enemata, i. 637 ; through nose, in parenchymatous glossitis, iii. 24; by nutrient enemata in delirium tremens, ii. 602; in progressive paralysis of the insane, iv. 493 ; by rectum, see Rectal feeding; by subcutancous injection, i. 640 ; by subeutaneous injection in gastric uleer, iii. 120; by subcutaneous injection in tetanus, ii. 250.

FeEdiNg of infaNts, precautions against tuberculosis to be observed in use of artificial foods in, ii. 815; a predisposing cause of rachitis, ii. 350 .

Feeding вотtres, necessity for cleanliness of, ii. 2 ; in stomatitis hypermycetica, ii. 13.

Feidler, association of pleurisy with tonsillar infection first noted by, iii. 465.

Fel bovis, use of, in tumors of pancreas, iii. 305.

Fellner, report by, on action of vasotonin, iv. 48.

Felon. See Whitlow.

Fenwick, characterization of gastric atony by, iii. 168 .

Ferments, use of, in depressive secretive disorders of stomach, iii. 149.

Ferratin, use of, in chlorosis, iii. 819.

Ferri-alum, use of, in acute nasopharyngitis accompanied by uvulitis, iii. 31 .

Ferric sulphate, use of, in chlorosis, iii. 818 .

Fetid expectoration, in bronchiectasis, iii. 395 ; in chronic bronchitis, iii. 376 .

Fetterolf, table prepared by, showing composition of commercial diabetic bread and flour, ii. 746 .

Fever blisters. See Herpes of lips.

Fibroids, pressure from, as a cause of pain in bladder, iv. 122.

Fibroids, uterine. See Myomata of uterus.

Fibrolysin, use of, in chronic pneumonia, iii. 422; by injection into spinal canal in tabes, iv. 198; in peritoneal adhesions, iii. 322 ; in treatment of delayed resolution in pneumonia, ii. 258. 
Filaria bancrofti, iv. 787.

Filaria diurna, iv. 789.

Filaria loA, infection with, iv. 789; treatment of, iv. 790 .

Filaria nocturna, iv. 787.

Filaria perstans, iv. 791.

Filariasis, iv. 786: dracunculus medinensis, iv. 788; filaria bancrofti, iv. 787; filaria loa, iv. 789; filaria perstans, iv. 791.

Filiforms, introduction of, into urethra, iv. 171 ; varieties of, iv. 170 .

Filix mas, use of, in destruction of filaria bancrofti, iv. 787; in endemic hematuria, iv. 784; in intestinal distomiasis, iv. 782; for tapeworms, iv. 778; in tricocephaliasis, iv. 798; in tricocephalus dispar, iii. 254.

Fillebrown conducting tube apparatus, iii. 46.

Filmaron, oil of. See Oil of filmaron.

Filtration of water, unreliable in prophylaxis of cholera, iv. 829.

Finger, on results of arsenobenzol in brain syphilis, iv. 486.

Finger and Landsteiner, contagiousness of tertiary syphilitic lesion established by, ii. 371 .

Finkelstein, Eiweiss milk recommended by, in enteritis of infancy, iii. 203.

Finsen, demonstration by; of superficial penetration of ultra-violet rays of light, i. 433 ; work by, on use of light therapy in lupus, i. 449.

Finsen light, use of, in leprosy, iv. 816; use of, in light therapy, i. 441.

Finsen's method, in treatment of tuberculosis of the skin, i. 400.

Fisch and Hill, isolation of influenza bacillus in pure culture reported by, ii. 190.

Fischer, Martin, on means of producing diuresis in beri-beri, iv. 852 .

Fischer's method, of relieving anuria, iv. $837,838$.

Fischer's solution, intravenous injection of, in yellow fever, iv. 856 .

Fish, digestibility of, i. 628.

Fish, poisonous varieties of, iv. 683 .

Fish poisoning, ii. 643, iv. 683; preventive measures against, iv. 684 ; remedial measures in, iv. 684 .

Fisher, investigations by, on association of enuresis with adenoids and hypertrophied tonsils, iv. 130.

Fissure in ano. See Fissure of anus.

Fissure of ANus, as a cause of enuresis, iv. 130; electrotherapy for relief of, i. 420.

Fistulæ, as a complication of tuberculosis, ii. 509 .

Flail JoINT, treatment of, when following anterior poliomyelitis, iv. 242.

"Flat FOOT," ii. 709.

Flatulency, treatment of, when associated with gastric atony, iii. 171.

Flaxseed poultice. See Poultice.

Fleas, measures directed against transmission of plague by, iv. 825; species of, concerned in transmission of plague, iv. 818.

FLECHSIG, opium-bromid therapy for epilepsy suggested by, iv. 646 .

Fleiner, conditions considered by, to indicate bismuth treatment in non-bleeding gastric ulcer, iii. 135; experiments by, on injection of pleural cavity with India ink, iii. 458; use of oil injections in membranous enteritis introduced by, iii. 209.

Fletcherism, iv. 538.

Flexner, Simon, period between inoculation of anterior poliomyelitis and appearance of symptoms demonstrated by, iv. 237; work by, on bacillary dysentery, ii. 316; work by, on influenza meningitis, ii. 194, 212; work by, on serum therapy in cerebrospinal meningitis, ii. 202.

Flexner and Jobling, conclusions drawn by, from analysis of 400 cases of epidemic cerebrospinal meningitis, ii. 202.

Flexner and Sweet, work by, on differences between Shiga and Flexner bacilli, ii. 317 .

Flexner serum, i. 161.

Flexner stem bacillus, ii. 317.

Flick, Dr. Lawrence, work done by, in prevention of tuberculosis, ii. 446.

Flies, dysentery transmitted by, ii. 319; small-pox transmitted by, ii. 42; tuberculosis transmitted by, ii. 455; typhoid fever transmitted by, ii. 7 .

Fint, Austin, on treatment of cholera morbus, iii. 195; use of opium in acute diffuse peritonitis advocated by, iii. 316; work on differential air pressure by, iii. 447. 
Flint's apparatus, for use of differential air pressure, iii. 446.

Flint's method, for redueing air pressure in pleural cavity, iii. 500 .

Floor maggiot, iv. 687; treatment for, iv. 688.

Fluid intake, importance of, in acute nephritis, iv. 61.

Fluids, importance of introducing, into system in blackwater fever, iv. 740; restriction of, in acute nephritis, iv. 61 , 62 ; restriction of, in arteriosclerosis, iii. 759 ; restriction of, in cardiac insufficiency, iii. 686; restriction of, in chronic interstitial nephritis, iv. 68; restriction of, in chronic myocardial insufficiency, iii. $715,716,721$; restriction of, in chronic myocardial insufficieney due to obesity, iii. 727; restriction of, in ehronic parenchymatous nephritis, iv. 66 ; restriction of, in nephritis, iv. 33; restriction of, in reduction of obesity, ii. 805 ; restriction of, for relief of constipation associated with intestinal atony and visceroptosis, iv. 566 ; use of, in gastric atony, iii. 170.

Fluorescence, as property of radiant energy, i. 436.

Fluoroscopic examination, use of, to discover foreign bodies in esophagus, iii. 64.

Flushing of bowels. See Colonic flushing. Fly, STABLE, poliomyelitis transmitted by, iv. 234.

Fly-agaric, ii. 648.

Fly blisters. See Blister.

FocAl symptoms, of tuberculous meningitis, iv. 374. -

Focke, method employed by, for standardizing digitalis preparations, iii. 583 .

Folin, investigations by, on composition. of urine, iv. 12.

Fomentations, hot, use of, in acute peritonsillitis, iii. 43 ; to kidneys in blackwater fever, iv. 740; for relief of anuria in nephrolithiasis, iv. 107; for relief of pain in pyelitis, iv. 88 ; for relief of pain in sciatica, iv. 299 ; in renal colic, iv. 102; in symptomatic parotitis, iii. 28.

Food, in acute catarrhal jaundice, iii. 261; amount of, required daily by patient with nephritis, iv. 41 ; carbohydrates as, i. 601 ; definition of, i. 599 ; extractives as, i. 601 ; golatin as, i. 601 ; infection of, a cause of food poisoning, ii. 636; infection of, a possible cause of beri-beri, iv. 848; preparation of, in tuberculosis, ii. 544; prophylactic care of, in Asiatic cholera, iv. 728 ; proteins as, i. 600; regulation of, in eases of obesity associated with eardiac insufficiency, iii. 703; salts as, i. 602; use of solid, in aftertreatment of gastric ulcer, iii. 130; uses of, in body, i. 600 ; water as, i. 602 .

FOOD POISONING, iii. 631 ; animal organisms causing, ii. 634 ; bacteria in causation of, ii. 635; bacteriology involved in, ii. 631, 634; changes in food stuffs a cause of, ii. 632 ; cheese poisoning, ii. 647; ergotism, ii. 652 ; excess in food stuffs a cause of, ii. 632 ; factors in digestive process and, ii. 631; fish poisoning, ii. 643 ; due to food of vegetable origin, ii. 647 ; due to fungi, ii. 652 ; general considerations regarding, ii. 631 ; infection of food a cause of, ii. 636; intracellular fermentation a cause of, ii. 636 ; lathyrism, ii. 652 ; meat poisoning, ii. 637; milk poisoning, ii. 645; mushroom poisoning, ii. 647 ; poisonous roots, ii. 650 ; poisonous fruits and seeds, ii. 651 ; poisonous herbage, ii. 651 ; ptomains in eausation of, ii. 635 ; special forms of, ii. 636 ; toxic substances produced in physical processes of digestion, ii. 631 ; toxins in causation of, ii. 635 ; vegetable organisms a cause of, ii. 634 .

Food preservatives, digestibility of food lessened by, ii. 634 .

Food Requirements, i. 603; of earbohydrates, i. 610 ; of fats, i. 610 ; in health, ii. 781; method of determining, i. 604 ; muscular work as influence in, i. 606 ; of proteins, i. 608 ; size as an influence in, i. 605 ; terms of expressing, i. 608 ; in tuberculosis, ii. 539.

Food stuFrs, i. 601 ; absorption of, in reetal feeding, i. 637; contamination of, a cause of poisoning, ii. 634; forms of, containing exogenous poisons, ii. 633; idiosyncrasy toward, a cause of poisoning, ii. 633; poisonous forms of, ii. 633; in relation to constipation, iii. 224; systemic poisoning caused by, ii. 632 .

Food SUPPLY, sources of, in tuberculosis, ii. 543. 
Foons, i. 599; advantages of albuminous, in chronic intestinal obstruction, iii. 242; as auxiliary articles of diet, i. 603; in causation of rachitis, ii. 815; effects of cooking upon different, i. 621; functions of, in body, ii. 781; preparation of, in diet for achylia gastrica, iii. 147; preparation of, for diet in depressive disorders of gastric secretion, iii. 147; prevention of scurvy by use of fresh, iii. 874 ; proprietary, i. 652 ; ratios of protein, fat, and carbohydrate in, i. 608; restriction of different, in diabetes mellitus, ii. 751 ; restriction of different, in reduction of obesity, ii. 800 ; use of albuminous, in chronic mucous colitis, iii. 206; use of albuminous, in diet for acute ềnteritis, iii. 194; use of artificial, in enteritis of infancy, iii. 202; use of coarse, a cause of gastric hyperacidity, iii. 78 .

Foods, Digestibility of, i. 624 ; appetite an influence on, i. 625 ; of bread, i. 629 , 630 ; of carbohydrates, i. 630 ; definition of term "digestibility," i. 624; of eggs, i. 628; of fat, i. 631; of fish, i. 628; general considerations in, i. 624 ; of meats, i. 626 ; of milk, i. 629.

Foods of Vegetable origin, POISONING BY, ii. 647: mushrooms, 647; herbage, ii. 651 ; fruits and seeds, ii. 651 ; roots, ii. 650.

Foot baths, use of cold, in cerebral hyperemia, iv. 385; use of cold, in chronic cerebral hyperemia, iv. 387; use of hot, in cerebral hemorrhage, iv. 391.

FOOT DROP, prevention of, in multiple neuritis, iv. 327,328 ; prevention of, in neuritis, 284.

Foot and mouth disease, ii. 581; definition of, ii. 581 ; production of, by inoculation, ii. 581; prophylaxis of, ii. 581; quarantine for, ii. 582; transmission of, ii. 582 ; treatment of, ii. 583 .

Foramen ovale, alcohol injection at, for relief of trigeminal neuralgia, iv. 348 .

Foramen rotundum, alcohol injection at, for relief of trigeminal neuralgia, iv. 351 .

Forced feeding. See Superalimentation.

ForchHeimer, on small doses of opium in acute appendicitis, iii. 214.

Foreign BODy, in air passages, iii. 439; in esophagus, see Esophagus.
Forlanini, production of artificial pneumothorax in tuberculosis revived by, ii. 534.

Formaldehyde, use of, in disinfection for influenza, ii. 186; for nasal douche in atrophic rhinitis, iii. 331 .

Formaldehyde gas, as disinfectant in tuberculosis, ii. 452.

Formalin, use of, for flushing colon, in intestinal autointoxication, ii. 674 ; as injection into pericardium, iii. 527 ; for irrigation of pleural cavity followed by death, iii. 487; in leprosy, iv. 816 ; in local treatment of laryngeal tuberculosis, ii. 493, iii. 347 ; use of, in mycosis of tonsils and fauces, iii. 42.

Formin. See Urotropin.

Formula, for acid expectorant mixture for use in acute bronchitis, iii. 365 ; for acid solution of quinin used by intramuscular injection in kala-azar, iv. 754; for administration of bone marrow in pernicious anemia, iii. 836; for alkaline mixture used by Sternberg in yellow fever, iv. 856 ; for amyl nitrite, iii. 606; for anodyne mixture for relief of painful urination in acute urethral gonorrhea, iv. 141; for antiseptic inhalation used in pulmonary abscess, iii. 441; for antiseptic solution used for disinfection of throat in diphtheria, ii. 272 ; for antiseptic solution used in gastric lavage, iii. 155; for application to gums in scurvy, iii. 875 ; for Baillie's or Addison's pill, iii. 286; for borax ointment, iii. 133; for buttermilk as food in enteritis of infancy, iii. 204; for butylchloral hydrate, used in neuralgia, iv. 340 ; for calcium salts used in hemophilia, iii. 862; for carbolic acid solution used in scarlet fever, ii. 120; for cathartic pills used in Lambert's treatment for opium habit, ii. 620 ; for control of restlessness in multiple neuritis due to alcoholism, iv. 323; for dinner pill, iii. 233; for dusting powder, used as prophylactic against anterior poliomyelitis, iv. 237; for Eiweiss milk, iii. 203; for expectorant mixture used in acute bronchitis, iii. 360 , 362 ; for expectorant mixture used in chronic bronchitis, iii. 373; for food in gastric ulcer, iii. 129; for fuming inhalation used in asthma, iii. 386; for gargle, used in acute tonsillitis, iii. 42; for gray 
oil of mercury used in syphilis, ii. 396; for hypnotics for hypodermic administration in alcoholism, ii. 598; for hypnotics used in Lambert's treatment of alcoholism, ii. 597; for inhalation used in acute bronchitis, iii. 358; for inhalation used in acute laryngitis, iii. 338 ; for inhalation spray used in acute laryngitis, iii. 339; for intratracheal injection used in bronchiectasis, iii. 397; for intravenous injection used in Fischer's method of relieving anuria, iv. 838; for intravenous injection used in sunstroke, iv. 678; for iodin and potassium iodid used in ozena, iii. 337; for Lambert's specific in treatment of alcoholism, ii. 598; for local use of cocain in acute rhinitis, iii. 328; for Locke's solution, iii. 534; for Loeffler's solution, iii. 39 ; for lotion used in syphilitic alopecia, ii. 429 ; for lubricant used in catheterizing urethra, iv. 158; for mercury and potassium iodid used in laryngeal tuberculosis with syphilitic infection, iii. 346 ; for mixture used to produce diuresis in beri-beri, iv. 853; for mouth wash used in lobar pneumonia, ii. 221 ; for mouth wash used as a prophylactic against bronchopneumonia, iii. 413; for mouth wash used in syphilis, ii. 369 ; for ointment used in oriental sore, iv. 756 ; for peptones, used in hemophilia, iii. 866 ; recommended by Babcock for use in lobar pncumonia, ii. 253; recommended by Cohnheim for use in chronic enteritis, iii. 200; recommended by Crocker for lotion for relief of itching in diabetes, ii. 763 ; recommended by Levy-Bing for use of biniodid of mercury by injection in syphilis, ii. 393 ; recommended by Schamberg for lotion for relief of itching in diabetes, ii. 764; for rectal injection used in Fischer's method of relieving anuria, iv. 838; for rectal injection, used in sunstroke, iv. 678; for relief of constipation in chlorosis, iii. 823; for relief of gastric hyperacidity in chlorosis, iii. 822 ; for relief of vomiting in neurasthenia, iv. 567 ; for Ringer's solution, iii. 533 ; for sandalwood oil, iv. 140 ; for sodium bromid and sodium chlorid used in hemophilia, iii. 863; for solution used in alcohol injection, iv. 342 ; for solution used in Fischer's method of relieving anuria, iv. 838 ; for solution to be in- jected into nerve in sciatica, iv. 301; for spray used in acute rhinitis, iii. 327 ; for spray used in chronic atrophic laryngitis, iii. 343 ; for spray containing cocain, used in asthma, iii. 386,387 ; for strychnin and hydrochloric acid, used in Addison's disease, iii. 884; for tonic, used in acute alcoholism, ii. 895 ; for tonic used in Keeley gold cure for alcoholism, ii. 600 ; for tonic, used in neurasthenia, iv. 559; for tonic bath, used in tabes, iv. 200 ; for use of ammonium chlorid in acute bronchitis, iii. 365 ; for use of antimony as an expectorant in acute bronchitis, iii. 360; for use of apomorphin as an expectorant in acute bronchitis, iii. 360 ; for use of arsenic in chlorosis, iii. 821 ; for use of arsenic in leukemia, iii. 847; for use of belladonna in spastic constipation, iii. 231 ; for use of benzoate of mercury in syphilis, ii. 393; for use of bichlorid of mercury by injection in syphilis, ii. 393 ; for use of bromids with arsenic in bronchial asthma, iii. 384; for use in bronchial asthma, iii. 384; for use of caffein in measles, ii. 154; for use of calomel in syphilis, ii. 397; for use of camphor in abortive treatment of acute bronchitis, iii. 354 ; for use in chronic alcoholism, ii. 595; for use in chronic catarrhal jaundice, iii. 263 ; for use of codein in acute bronchitis, iii. 361 ; for use in constipation accompanying cirrhosis of liver, iii. 281 ; for use in constipation associated with gastric atony, iii. 233; for use of copaiba in acute gonorrhea, iv. 141; for use of cream of tartar in cirrhosis of liver, iii. 286; for use of cyanid of gold and potassium in tabes, iv. 199; for use of cyanid of mercury in syphilis, ii. 394; for use in gallstone colic, iii. 268; for use of menthol by inhalation in chronic bronchitis, iii. 375; for use of menthol and eucalyptus by inhalation in bronchiectasis, iii. 396 ; for use of mercury and potassium iodid by mixed method in syphilis, ii. 410; for use of nitroglycerin, iii. 605 ; for use of opium in acute bronchitis, iii. 361 ; for use of opium to cont rol cough in chronic bronchitis, iii. 375 ; for use of potassium iodid by rectum in syphilis, ii. 409 ; for use of quinin in acute bronchitis, iii. 355; for use in scurvy, iii. 876 ; for use in secondary ane- 
mia, iii. 810; for use of sodium sulphate in chronic cholecystitis, iii. 272 ; for use in subsiding stage of acute rhinitis, iii. 328 ; for use of tartar emetic in acute laryngitis, iii. 339; for terebine as an expectorant in bronchiectasis, iii. 396.

Formula, Lincoln's. See Lincoln's formula.

Formula, Unna's. See Unna's formula.

Formulæ, for administration of mercury by mouth in syphilis, ii. 367; for aromatic expectorant mixtures for use in chronic bronchitis, iii. 377; for injections used in chronic gonorrheal urethritis, iv. 155, 156; for nutrient enema, iii. 119; for purgative used in first stage of acute bronchitis, iii. $354,355,356$; for relief of cough in chronic bronchitis, iii. 374; for relief of lancinating pains in tabes, iv. 196; for relief of nausea and vomiting in acute gastritis, iii. 156; for solutions used in Rogers' treatment of cholera, iv. 835, 836; for sprays, used in acute bronchitis, iii. 358,359 ; for use in biliousness, iii. 259; for use of gelatin internally in ulcerative colitis with hemorrhage, iii. 212; for use in plasmatic treatment of iron, iii. 821.

ForNACA, opinion of, on serum treatment of erysipelas, ii. 284.

Förster, OTFried, method of operation for contractures following the cerebral palsies of children devised by, iv. 414; series of exercises for reëducation of ataxic extremities devised by, iv. 210 .

Foucault, primary acute suppurative myositis first described by, ii. 686 .

Fowler, Kingston, modification of turpentine liniment suggested by, iii. 357 .

Fowler position, advantages of, in acute diffuse peritonitis, iii. 315 ; in pericarditis, iii. 514.

Fowler's solution of arsenic, use of, in bronchial asthma, iii. 383; in chlorosis, iii. 821 ; in combination with the bromids for epilepsy in cerebral palsies of children, iv. 413; in frambœsia tropica, iv. 765 ; in herpes of lips, iii. 6 ; in intestinal neuroses, iii. 250; in pellagra, ii. 661 ; in peristaltic unrest, iii. 250; in pernicious anemia, iii. 827 ; in syringomyelia, iv. 279; in trypanosomiasis, iv. 747.
"Fractional-dose" method for administration of an X-ray treatment, i. 500.

Fracture, delayed union of, administration of thyroid gland for, i. 77.

Fractures, massage and gymnastics in treatment of, i. 328.

Fraenkel, on cumulative effect of digitalis, iii. 585; system for reëducation of ataxic motor apparatus formulated by, i. 316 ; use of pulmonary gymnastics in chronic pneumonia advised by, iii. 422 .

Fraenkel's movements, use of, for reëducation of ataxic extremities in hereditary spinal and cerebellar ataxia, iv. 218; for reëducation of ataxic extremities in tabes, iv. 204.

Frambesia tropica, iv. 762; prophylaxis against, iv. 764; treatment of, iv. 764 .

France, antituberculosis work in, ii. 442; history of vaccination in, ii. 60 .

Free associations, as part of technique of psychoanalysis, i. 589.

Freer and Bacon, investigations by, on chemical effects of sun's rays in different places, iv. 669.

Freezing, anesthesia by, use of, in excision of chancre, ii. 378 .

Freezing point of URINe, as functional kidney test, iv. 24.

Frehier's artificial phlegmon, use of, in typhus fever, ii. 37.

Frequencies, as property of radiant energy, i. 433.

Frequency, explanation of term in electrotherapeutics, i. 364.

Fresh air, benefit from, in pulmonary edema due to nephritis, iv. 56; importance of, in bronchopneumonia, iii. 417; importance of, in chronic bronchitis, iii. 368; importance of, in pleurisy, iii. 465 ; importance of, in pyelitis, iv. 86; importance of, in tuberculosis of kidney, iv. 115.

Freud, classification of the neuroses by, iv. 519; psychoanalytic method employed by, in treatment of psychic disorders, iv. 572, 574.

Freud's system of psychotherapy, i. 583.

Freudenthal, W., fulguration recommended by, in laryngeal tuberculosis, iii. 347; instrument devised by, for employing electrical vibratory massage in atrophic rhinitis, iii. 332 . 
Freund's operation, for ehronic pulmonary emphysema, iii. 437.

Friar's balsam. See Benzoin, compound tincture of.

Friction, as a method in massage, i. 289.

Friction, wet, use of, in chlorosis, iii. 814.

Friction sparks, use of, in electrotherapeuties, i. 385.

Friediurger and Sachs, report by, of hydrophobia cured by combination of atoxyl and thioglycocholie aeid, ii. 343.

Friedlander, results of X-ray treatment for status lymphaticus summarized by, iii. 914 .

Friedreich, paramyoclonus mutiplex first described by, iv. 659 .

Friedreich's ataxia, iv. 214; definition of, iv. 214; diagnosis of, iv. 217; diagnosis between, and hereditary cerebellar ataxia, iv. 514; etiological factors, iv. 214; pathological anatomy of, iv. 217 ; symptomatology of, iv. 215 ; treatment of, iv. 218.

Friedreich's foot, iv. 216.

Frimley, scheme of graduated exercise in tuberculosis employed at, ii. 529.

Froebel, possibilities of character building in play disclosed by, ii. 592 .

Frölich, A., dystrophia adiposogenitalis described by, iii. 917 .

Frölich's SYNDrome, iv. 443.

Frontal LoBe, localizing symptoms of brain tumor in, iv. 438 .

Frontal sinuses, supfuration of, radiant heat and light in treatment of, i. 451.

Fruit, method of protecting against cholera, iv. 829 .

Fruit sugar. See Levulose, fructose.

Fuchsius, digitalis first mentioned by, iii. 575.

Fugin, fish poisoning due to, ii. 643 .

Full bath, use of, in hydrotherapy, i. 198.

FUlLer, carly description of varicella by, ii. 109.

Fumigation, as a means of administering mereury in syphilis, ii. 389 ; as a means of destroying mosquitoes, iv. 735 .

"Function transference." See Tendon grafting.

Functional kidney tests, value of, iv. 23, 25.

Functional neuroses of Bladder. See Bladder.
Functional tests, value of, in chronic myocardial insufficiency, iii. 697.

Final, poisoning due to, ii. 652.

Fungus-atrópis, ii. 648.

Funucles, electrotherapeuties in treatment of, i. 40).

Furunculosis, as a complication of typhoid fever, ii. 29 ; as a complication of vaccination, ii 86 ; X-ray therapy for relief of, i. 544 .

Gast, disturbance of, in acute hemorrhagic superior polioencephalitis, iv. 406; in cerebral diplegia type of cerebral palsies of children, iv. 411; in Friedreich's ataxia, iv. 215; in hereditary cerebellar ataxia, iv. 217 ; in spastic spinal paralysis, iv. 268 ; due to vertigo, iv. 622 .

Galactoxism. See Milk poisoning.

Galenical preparations of digitalis physiologically standardized, therapeutic value of, iii. 595.

Gall bladder, diseases of, iii. 259.

Gall BLADDER AFFEctions, as complications of typhoid fever, ii. 28.

Gallic acid, use of, in hematuria, iv. 4.

Gallistone colic, iii. 267.

Gallstones, treatment of, in transit, iii. 273.

Galvanization of spine, methods of, in tabes, iv. 202.

Galvanocautery, use of, in atrophic rhinitis, iii. 331 ; in chronic laryngitis, iii. 340 ; in chronic rhinitis, iii. 329 ; in chronic tonsillitis, iii. 45; in epistaxis, iii. 335 ; in hay fever, iii. 390 ; in pericarditis, iii. 518; in subacute rhinitis, iii. 328 ; in treatment of retention due to chronic prostatitis, iv. 168.

Gamma rays of RAdium, i. 462 ; penetrative power of, i. 465; use of, in eancer of buccal membrane, i. 484.

Gangosa, iv. 864 ; preventive measures in, iv. 865 ; remedial measures in, iv. 865 .

Gangrene, diabetic. See Diabetic gangrene; of lungs, see Pulmonary gangrene; of pock, as a complication of vaccination, ii. 87 ; of skin, as a complication of varicella, ii. 113.

Gant, physieal exercises arranged by, for use in constipation, iii. 229 .

Gargles, use of antiseptic, in phlegmonous pharyngitis, iii. 40; hot astringent, in 
edematous laryngitis, iii. 341 ; in measles, ii. 148; method of, in acute tonsillitis, iii. 41 ; in scarlet fever, ii. 125.

Garlic, use of, as an expectorant in bronchiectasis, iii. 397.

Garrod's thread test, for uric acid in gout, ii. 695 .

Gaskell, myogenic theory of cardiac contraction developed by, iii. 535 .

Gasserian operation, for relief of trigeminal neuralgia, iv. 353.

Gasterine, iii. 150.

Gastrectasis, as symptom of chlorosis, iii. 823.

Gastric analysis, value of, iii. 67,69 .

Gastric atony, iii. 168; causes of, iii. 169; diet for, iii. 170; evacuation of bowels in, iii. 172; lavage in treatment of, iii. 172; mechanical treatment of, iii. 172; medicinal treatment of, iii. 171.

Gastric CATARRH, hydrotherapy for relief of, i. 214.

GASTRIC CRISES, in tabes, iv. 191 ; treatment of, iv. 197.

Gastric dilatation, mechanotherapy for relief of, i. 352.

Gastric diseases, hydrotherapy for relief of, i. 214.

Gastric Disturbances, as a symptom of acute nephritis, iv. 63.

Gastric Function, disturbances of, caused by disease of the nervous system, iii. 185 .

Gastric hemorrhage, acute dilatation of stomach accompanying, iii. 175.

Gastric Indigestion, as a cause of enuresis, iv. 132.

Gastric irRitability, in typhoid fever, ii. 25.

Gastric lavage, use of, in acute alcoholism, ii. 589 ; in acute dilatation of stomach, iii. 174; in acute dilatation of stomach complicating pneumonia, ii. 259 ; in acute gastritis, iii. 154; in acute intestinal obstruction, iii. 241; in acute localized peritonitis, iii. 311,312 ; in alcoholic gastritis due to cirrhosis of liver, iii. 280; in appendicitis of severe type, iii. 218; in a tonic dilatation of stomach, iii. 177; in barbel cholera, ii. 644; in carcinoma of stomach, iii. 60 ; in cheese poisoning, ii. 647 ; in chronic catarrhal jaundice, iii. 263; in chronic dilatation of stomach, iii. 180; in continuous gastric hyper- secretion, iii. 106; to control gastric hemorrhage, iii. 114, 117, 122; in depressive disorders of gastric secretion, iii. 152; in enteritis of infancy, iii. 201; in gastric atony, iii. 172; in gastric hyperacidity, iii. 97, 100; in gastric hypersecretion, iii. 102; in gastric neuroses, iii. 188; in gastritis acida, iii. 101; in intestinal autoinfection, complicated with arthritis, ii. 681 ; in meat poisoning due to B. botulinus, ii. 639 ; in meat poisoning due to $\mathrm{B}$. enteritidis, ii. 641 ; in meat poisoning due to B. paratyphosus, ii. 642 ; in paralytic ileus, iii. 238 ; in pernicious anemia, of doubtful benefit, iii. 826; in poisoning by amanita phalloides, ii. 649 ; in pyloric obstruction, iii. 178; for relief of gastric disturbances in acute nephritis, iv. 63; for relief of headache due to toxemia, iv. 610 ; for relief of uremic vomiting, iv. 54; for relief of vomiting in infantile diarrhea, ii. 329 ; in secondary chronic gastritis, iii. 160.

Gastric neuroses, idiopathic, iii. 186; of motility, iii. 186; primary, iii. 186; secretory, iii. 187; sensory, iii. 187.

Gastric peristalsis. See Peristalsis.

Gastric Secretion, depressive disorders of, iii. 144; achylia gastrica and subacidity, iii. 144; dietetic treatment of, iii. 146; lavage in treatment of, iii. 152; medical treatment of, iii. 148.

Gastric SECRETION, irritative disorders of, iii. 74 .

Gastric sedatives, use of, limited in gastric disturbances in acute nephritis, iv. 64 .

Gastric siphonage, permanent, use of, in acute dilatation of stomach, iii. 174 .

Gastric tetany, iii. 143.

GASTRIC ULCER, iii. 108; after-treatment of bleeding, iii. 123; as cause of pyloric obstruction, iii. 178; as complication of chlorosis, iii. 811; diet in non-bleeding, iii. 123; drugs in treatment of bleeding, iii. 112 ; drugs in treatment of nonbleeding, iii. 132; indications for operative treatment of, iii. 70 ; lavage for control of hemorrhage in bleeding, iii. 114; lavage in treatment of non-bleeding, iii. 138; manifest hemorrhage in, iii. 109; medical treatment of bleeding, iii. 117; nutriment in bleeding, iii. 117; occult 
bleeding in, iii. 109; resection indicated in chronic indurative, iii. 177; surgieal treatment of bleeding, iii. 116; surgical treatment of non-bleeding, iii. 139; syphilitic, iii. 167.

Gastizitis acida, iii. 101.

Gastritis, ACUTE, iii. 153; alleviation of pain in, iii. 156; diet in, iii. 157; emesis in treatment of, iii. 154; evacuation of bowels in, iii. 155; lavage in treatment of, iii. 154; prophylaxis of, iii. 153.

Gastintis, alcoholic, presence of, in cirrhosis of liver, iii. 280.

Gastritis, chronic, iii. 157; causes of, iii. 158; hydrotherapy for relief of, i. 214; lavage in treatment of, iii. 160; stagnation of food in, iii. 177.

Gastritis, chronic mucous, iii. 158.

Gastritis, chronic syphilitic, iii. 166.

Gastritis, secondary chronic, iii. 159.

Gastrodiscus hominis, iv. 780; pathology of, iv. 781 .

Gastroenteritis, acute, fulminating attacks of, iii. 195.

Gastroenteroptosis, iii. 245.

Gastroenterostomy, in carcinoma of stomach, iii. 166; in continuous gastric hypersecretion, iii. 103, 105; contraindications to, in gastric ulcer, iii. 70; for gastric ulcer, iii. 116; indications for, in carcinoma of stomach, iii. 177; performance of, for pyloric obstruction due to gumma of stomach, iii. 167.

Gastrointestinal disorders, as a complication of scarlet fever, ii. 132; as a complication of tuberculosis, ii. 502; due to hydrargyrism, ii. 384; hydrotherapy for relief of, i. 212; massage and gymnastics for relief of, i. 322; mechanotherapy for relief of, i. 357 .

Gastrointestinal disturbance, accompanying chronic myocardial insufficiency, iii. 692; accompanying pyclitis, iv. 94; associated with Addison's disease, iii. 884; as predisposing cause of cholera, iv. 829 ; treatment of, when due to chronic myocardial insufficiency, iii. 738.

Gastrointestinal headache, iv. 611.

Gastrointestinal symptoms, of pernicious anemia, iii. 837; use of digitalis for, in cardiac insufficiency, 589.

Gastrointestinal tract, importance of attention to, in beri-beri, iv. 853; reflex cardiac neuroses associated with, iii. 778.

Gastiointegtinal type of influenza. See Influenza.

Gastroptosis, iii. 181; hydrotherapy for relief of, i. 214.

Gastrostomy, in peptic ulcer, iii. 66.

Gastrosuccorrhea. See Hypersecretion, eontinuous gastric.

Gatch, bed described by, useful in pericarditis, iii. 514.

Gavage, employment of, in after-treatment of diphtheria, ii. 276 ; in cerebrospinal meningitis, ii. 215; in depressive disorders of gastric digestion, iii. 153; employment of, as means of artificial feeding, i. 639 ; in meat poisoning, ii. 639 ; in post-diphtheritic paralysis, ii. 278 .

Gelatin, use of, in arteriosclerosis, iii. 766; to control epistaxis, iii. 336 ; to control gastric hemorrhage, iii. 112; to control hemorrhage, in hemorrhagic form of measles, ii. 155, 156; as food, i. 601 ; in hemophilia, iii. 863,867 ; by hypodermic injection in aneurysm, iii. 766; by hypodermic injection in hemophilia, iii. 863; by hypodermic injection in ulcerative colitis with hemorrhage, iii. 212; in pulmonary tuberculosis, ii. 501; in ulcerative colitis with hemorrhage, iii. 212.

Gelatin foods, use of, in tuberculous kidney, iv. 117 .

Gelsemin, poisoning by, i. 754 .

Gelsemium, use of, in tetanus, ii. 348 .

General Debility, mechanotherapy for relief of, i. 348.

Geneva, mortality from small-pox in, before and after vaccination, ii. 95.

Genitalia, care of, in varicella, ii. 113; disorders of, as a complication of measles, ii. 161.

Genitourinary diseases, hydrotherapy for relief of, i. 209.

Gentian, use of, in acute catarrhal jaundice, iii. 262; in depressive disorders of gastric secretion, iii. 151; as a tonic in tuberculosis, ii. 504.

Geographical tongue, iii. 27.

GePPERT, work done by, on poisoning by hydrocyanic acid, i. 741.

Gerhardt's sign, diagnostic value of, in compression bulbar paralysis, iv. 501 . 
GerhardT's test, for diacetic acid in urine, ii. 732 .

Gerlier's disease, vertigo as a symptom of, iv. 624 .

Germ LiFe, effect of radiant energy upon, i. 436.

German army, effects of vaccination and revaccination in, ii. 101 .

German measles, ii. 164; treatment of, ii. 165.

German method, for prophylactic administration of quinin, iv. 737.

German vaccination LaW, results of, ii. 100.

Germany, antituberculosis work in, ii. 441; introduction of vaccination into, ii. 60; mortality from small-pox in, before and after introduction of vaccination, ii. 93 ; passage of vaccination law in, ii. 60 ; results of vaccination law in, ii. 100 .

Gibson, on surgical treatment of visceroptosis, iii. 248.

Gibson's rule, for use in blood pressure study during treatment of pneumonia with cardiac toxemia, ii. 255.

Giddiness. See Vertigo.

Giemsa and Schaudinn, on absorption of quinin, iv. 718; experiments by, on dilution of quinin, iv. 722 .

Gigantism, iii. 917; relation between the hypophysis 'and, i. 103.

GiN, use of, dangerous in nephritis, iv. 49.

Glanders, ii. 357; artificial immunity against, ii. 357 ; immunity conferred by one attack of, ii. 357; occurrence of, ii. 357 ; precautions against infection from discharges in, ii. 358; serum treatment of, ii. 358 ; specific treatment of, ii. 358 ; transmission of, ii. 357 ; vaccine treatment of, i. 147 , ii. 358 .

Glands, enlargement of, X-ray therapy for relief of, i. 551 .

Glandular disease of Skin, X-ray therapy for relief of, i. 327.

Glandular enlargement, accompanying rachitis, ii. 818.

Glandular fever, ii. 574; adenitis accompanying, ii. 576; characteristics of, ii. 574; complications of, ii. 577; convalescence from, ii. 577; definition of, ii. 574; non-contagiousness of, ii. 575; prognosis in, ii. 577; prophylaxis against, ii. 575; treatment of, ii. 577 .
Glandular symptoms, of hypophysis tumor, iv. 442.

Glauber's salts, use of, in chronic catarrhal jaundice, iii. 264.

GLÉNard, on visceroptosis, iii. 243, 246.

Glenwood Springs, value of, in acute myelitis, iv. 228.

Gliomata of brain, iv. 433.

Guisson, early contributions by, to knowledge of rachitis, ii. 812 .

Guisson, Francis, theory of muscle irritability advanced by, i. 373 .

Globularin, use of, in diabetes insipidus, ii. 777 .

Glonoin, use of, for neuralgia associated with arteriosclerosis, iv. 336 .

Glossal SPasm, iv. 655.

Glossina palpalis, transmission of trypanosome fever and sleeping sickness by, iv. 743.

Glossina palpalis wellmani, iv. 744 .

Glossitis, acute, ii. 23; chronic, ii. 24; treatment of acute, ii. 23; treatment of chronic, ii. 25.

Glossopharyngeal Paralysis. See Neuritis of ninth nerve.

Glossopharyngo-labial paralysis. See Bulbar paralysis, progressive.

Glottis, edema of. See Edema.

Gudcose, presence of, in diabetic urine, ii. 727,728 ; as substitute for sugar in diet of diabetics, ii. 754 .

Glycerin, use of, as diluent for small-pox vaccine virus, ii. 65,66 ; as gargle in measles, ii. 148; in pernicious anemia, iii. 836 ; to prevent acidosis in diabetes mellitus, ii. 757 ; by rectum in diabetic coma, ii. 757 ; by rectum for relief of meteorism in typhoid fever, ii. 26; for relief of trichiniasis, iii. 254.

Glycerin acid, use of, to prevent acidosis in diabetes mellitus, ii. 757.

Glycerin trinitrate. See Nitroglycerin.

Glycerin suppositories, use of, in constipation due to chronic myocardial insufficiency, iii. 740 .

Glycerophosphates, use of, in tabes, iv. 195. GLycoheptonic ACID, as substitute for sugar in diabetes mellitus, ii. 750 .

Glycosuria, alimentary, ii. 726; association of, with chronic pancreatitis, iii. 297; presence of, in diabetes mellitus, ii. 722; presence of, in gout, ii. 696 ; presence of, 
in pancreatitis no contraindication to operation for, iii. 300; presence of, in tuberculous meningitis, iv. 374 ; transient, ii. 726 .

Glycuronic acid, presence of, in diabetic urine, ii. 729.

Gnathostoma siamense, infection by, iv. 800.

Goat's milk, transmission of Malta fever by, iv. 839 .

Godlee And Fowler, method of taking creosote vapor bath developed by, iii. 39.5.

GodLEwski, on effect of altitude on nervous conditions, iv. 556 .

Göerl LAMPs, use of, in light therapy, i. 443.

Gorter, iii. 891; definition of, iii. 891; drinking water a eause of, iii. 892; endemic form of, iii. 892; mortality from, iii. 894; radium in treatment of, i. 478; sporadic form of, iii. 892 ; surgical treatment of, iii. 894 ; treatment of, in general, iii. 893; varieties of, iii. 892; $\mathrm{X}$-ray therapy for, i. 551 .

Goiter, Exophthalmic, iii. 895; angina pectoris vasomotoria due to, iii. 768; balneology in treatment of, iii. 898; blood pressure raised by, iii. 555; elimatic treatment of, iii. 901; diet for, iii. 897 ; electricity in treatment of, epinephrin for diarrhea in, i. 97, iii. 897; hydrotherapy for, i. 207, iii. 898; hygienic treatment for, iii. 896 ; medicinal treatment of, iii. 899 ; mortality from, iii. 895; organotherapy for, iii. 902 ; pathology of, iii. 895; prognosis in, iii. 896; radium therapy for, i. 479 ; serum therapy for, i. 169 , iii. 902 ; thymus enlargement in, i. 118; surgieal treatment of, iii. 904 ; treatment of, in pregnancy, iii. 905; X-ray therapy for, i. 552 , iii. 899.

Gold chlorid, use of, in snake bite, iv. 682; of no value in spastic spinal paralysis, 270.

Goldberger and Anderson, investigations by, on transmission of typhus, ii. 33.

Golden sulphuret of antimony, use of, as an expectorant in pneumonia, ii. 257.

Goldscheider, division of multiple neuritis into stages by, iv. 325 ; method for reëducation of ataxic extremities elaborated by, iv. 204; reëducation therupy recommended by, in intention tremor and athetoid movenents, i. 320.

GoldsCHMIDT's POSTERIOR URETHROSCOPE, iv. 167.

Goldthwait, Painter, and Osgood, rules for recognizing sacroiliac sciatica given by, iv. 296.

Goldtiwalte, on static pains, ii. 709.

Gomenol oil, use of, by instillation in tubercular cystitis, iv. 121.

Gompertz, Louis, on effects of agar-agar in chronic constipation, iii. 236; work done by, on absorbing power of the rectum, iii. 120.

Gonococcus of NeIsser, presence of, in gonorrheal stomatitis, iii. 20.

Gonococcus INFECTIONs, electricity in treatment of, i. 406 ; radiant light and heat in treatment of, i. 449 ; vaccine treatment for, i. 146.

Gonorrhes, as a cause of reflex cardiac neuroses, iii. 781 .

Gonorrhea, ACU'TE, hydrotherapy for relief of, i. 210.

Gonorrhea, ACUte Urethral, iv. 134; abortive treatment of, iv. 137 ; cases of, suited to repressive treatment, iv. 143; choice of methods in repressive treatment, iv. 143; definition of, iv. 136; diet in, iv. 138; expectant treatment of, iv. 148; instructions to patients suffering from, iv. 142; internal medieation for, iv. 139; local treatment for, iv. 143; preventive treatment for, iv. 136; repressive treatment for, iv. 137; treatment of acute posterior urethritis complicating, iv. 146; treatment of complications in, iv. 148; treatment of, in general, iv. 147.

Gonorrhea, Forms of, iv. 134: acute posterior urethritis, iv. 146; acute urethral gonorrhea, iv. 135; chronic gonorrheal urethritis, iv. 146; gonorrheal epididymitis, iv. 167; post-gonorrheal neuroses, iv. 174; post-gonorrheal urethritis, iv. 174; urethral stricture, iv. 168.

Gonorrhea bags, iv. 138.

Gonorrheal arthritis. See Arthritis, gonocoecic.

Gonorrheal cystitis. See Cystitis. 
Gonorrheal ePIDIDYMitis, iv. 150; local applications in, iv. 151; operation for, iv. 152 ; posture in treatment of, iv. 150 ; prophylaxis against, iv. 150; treatment of declining stages, iv. 152; treatment of, in general, iv. 151; treatment of recurrence of, iv. 152; vaccine therapy for, iv. 151 .

Gonorrheal myelitis, iv. 224; treatment of, iv. 228.

Gonorrheal stomatitis. See Stomatitis, gonorrheal.

Gonorrheal URETHRItis, CHRONIC, iv. 153; definition of, iv. 135; dilatation of urethra for relief of, iv. 161; injections for, iv. 155; instillations for, iv. 159; irrigations for, iv. 157; local treatment of post-gonorrheal urethritis, iv. 154, 167; massage of prostate gland for relief of, iv. 161, 163; medicinal treatment for, iv. 154; operation for, iv. 166; periods in, iv. 136; rectal douche for, iv. 165; treatment of, in general, iv. 153; urethroscopic treatment of, iv. 166.

GoRdon sign, presence of, in traumatic hematoma, iv. 366 .

Gorgas, on destruction of the anopheles mosquito, iv. 729; extermination of yellow fever by, in Havana, iv. 857; on quinin as a prophylactic, iv. 735 .

GotTstein, tampon in treatment of atrophic rhinitis suggested by, iii. 332 .

Gottstein curette, for use in removal of adenoid vegetations, iii. 36,37 .

Goundoo, iv. 868.

Gout, ii. 692; acute form of, ii. 696 ; alcohol injurious in, ii. 698; areas of prevalence of, ii. 694; cardiovascular lesions of, ii. 695; in causation of multiple neuritis, iv. 321 ; chronic form of, ii. 696 ; climatic treatment of, i. 281 ; clinical features of, ii. 692 ; definition of, ii. 692 ; dietetic management of, ii. 699 ; drugs in treatment of acute, iii. 702; drugs in treatment of chronic, iii. 703; etiology of, ii. 693 ; exercise in treatment of, ii. 700; gouty kidney in, ii. 695; glycosuria associated with, ii. 696 ; gymnastic exercises in treatment of, i. 325; hydrotherapy for, ii. 701; influence of heredity in, ii. 694 ; irregular form of, ii. 697 ; mechanotherapy for, i. 348 ; onset of, ii. 695 ; oxaluria associated with, iv. 8 ; poor man's, ii. 698; as a predisposing cause of neuralgia, iv. 337 ; prophylactic treatment of, ii. 697; radium in treatment of, i. 471,473 ; retrocedent form of, ii. 696 ; symptoms of, ii. 695 ; treatment of, ii. 701 ; treatment of, at health resort, ii. 701 .

Governmental supervision, of dusty trades, iii. 429.

Governments, duty of, concerning precautions to be employed in manufacture of matches, ii. 630 .

Graduated bath of Von Ziemssen, i. 198.

Graham, Thomas, studies on colloids and crystalloids by, i. 4 .

Gram-Molecular solution, as basis of standardization in pharmacology, i. 10.

Gram-molecule, as unit, for standard solutions in pharmacology, i. 10.

Graminol, i. 161.

Grand mat, iv. 633.

Granville, Mortimer, reëducation of ataxic extremities in tabes first suggested by, iv. 204.

Grape sugar, as means of nourishment in acute localized peritonitis, iii. 311 .

GraUPER's METHOD, of determining chronic myocardial insufficiency, iii. 694.

Graves' disease. See Goiter, exophthalmic.

Grawitz, diet recommended by, for use in pernicious anemia, iii. 826 .

Green citrate of iron, hypodermic use of, in anemia associated with chronic myocardial insufficiency, iii. 742.

"Green DAYs," in treatment of diabetes mellitus, ii. 756 .

Grimes, pioneer in hypnotism in America, i. 563.

Ground ITCH, iv. 793.

Guaiac, ammoniated tincture of, use of, in acute peritonsillitis, iii. 43 .

Guaiac, tincture of, local use of, in abortive treatment of acute tonsillitis, iii. 41.

Guaiacol, applications of, in gonorrheal epididymitis, iv. 152; intratracheal injections of, in chronic atrophic laryngitis with involvement of trachea, iii. 343; local application of, in mumps, ii. 170,171 ; use of, to control temperature in tuberculosis of larynx, iii. 346; use of, in pneumonia, ii. 236.

Guaiacol and glycerin, applications of, in gonorrheal epididymitis, iv. 152. 
Guaiacol carbonate, use of, in arthritis deformans and chronic rheumatism, ii. 714; as expectorant, in bronchiectasis, iii. 397; as expectorant in chronic bronchitis, iii. 377 ; by inunction in scarlet fever, ii. 126.

Guaiacol iodate, use of, hypodermically, in tuberculous peritonitis, iii. 321.

Guanieri, description of small-pox organism by, ii. 41.

Guerich, removal of tonsils during attack of acute rheumatism advised by, ii. 294.

Guilhaume, Henri, summary by, of observations on effects of salt-free diet in cirrhosis of liver, iii. 285.

Guinea worm. See Dracunculus medinensis.

Guiteras, tincture of chlorid of iron recommended by, for relief of bloody vomit in yellow fever, iv. 856 .

Gumma, local treatment of, ii. 430 .

Gumma of stomach. See Stomach.

Gummata, extradural, symptoms of, iv. 272.

Gummatous infiltration OF SPINAL CORD, symptoms of, iv. 271.

Gummatous neoplasm. See Brain tumors.

GuMs, Disease of, a cause of chronic gastritis, iii. 159.

GutTstadt, on results of vaccination upon mortality from small-pox in Berlin, ii. 99.

GUY's hospital, cases of chronic pneumonia examined at autopsy in, iii. 422.

Guyon instillation, iv. 160.

Gymnastic exercises, i. 301; breathing, i. 304 ; in cardiac insufficiency, iii. 636; field, i. 301; Hertz system of, iii. 289; for increasing tone of abdominal walls, iii. 289; for muscular spasm, i. 315; for neurasthenia, i. 321 ; Oertel's, for obesity, i. 325 ; respiratory, i. 306 ; school system of, i. 300; Schott system of, i. 302 , iii. 641.

Gymnastics, of abdominal muscles for relief of constipation in neurasthenia, iv. 566 ; definition of, i. 298; in disease, i. 301 ; in health, i. 300; history of, i. 298 ; necessity for caution in use of, with arteriosclerosis, iii. 639; Oertcl's terrain cure, iii. 645; physiological action of, iii. 636; requisites to success in use of, i. 299; systems of, iii. 641 ; use of, in after-treatment of acute infectious myocarditis, iii. 751 ; use of, in after-treatment of anterior poliomyelitis, iv. 242; use of, in amytropic lateral sclerosis, iv. 259 ; use of, in chronic gastritis, iii. 162; use of, in chronic myocardial insufficiency due to long-continued rest, iii. 724 ; use of, in chronic myocardial insufficiency due to obesity, iii. 725; use of, in chronic myocardial insufficiency due to valvular lesions, iii. 734; use of, contraindicated in aneurysm, iii. 766; use of, contraindicated in severe cases of chronic myocardial insufficiency, iii. 721; use of, dangerous, in chronic myocardial insufficiency due to nephritis, iii. 731; use of, in diseases of nervous system, i. 308; use of, in goiter, iii. 894; use of, in late stages of acute endocarditis, iii. 746; use of, in paralysis following cerebral palsies of infants, iv. 413; use of, in progressive neural muscular dystrophy, iv. 266; use of, in prophylaxis of cerebral hemorrhage, iv. 391 ; value of, during adolescence in prophylaxis of the neuroses, iv. 523; vibration, iii. 660 .

Gymnastics, mechano-, iii. 640; methods and technique employed in, iii. 640, 641.

Gymnastics, pulmonary, use of, in cardiac insufficiency due to pleuritic adhesions, iii. 732 ; in chronic pneumonia, iii. 732; exercises, i. 306.

Grnecology, use of mechanical vibration in, i. 358; value of radium therapy in, $i$. 479 .

Habit choreas, iv. 664 .

HABITS of LIFE, association of, with intestinal autointoxication, ii. 675 .

HAFFKINE, vaccination against cholera introduced by, i. 126.

Haffkine's method, of inoculation against cholera, iv. 829.

Haffkine's prophylactic against plague, iv. 823.

Half bath, use of, for exophthalmic goiter, iii. 898 ; in hydrotherapy, i. 195 ; in tetany, iii. 910.

Half baths, warm, use of, for relief of lancinating pains in tabes, iv. 200.

Halitosis. See Offensive breath. 
HALL, H. J., use of occupation cure for neurasthenia by, iv. 553 .

HaLl, Dr. J. K., death from hemorrhage complicating pellagra reported by, ii. 662.

Haluducinations, in progressive paralysis of the insane, iv. 490.

Hallucinatory EXCitement, attacks of, in progressive paralysis of the insane, iv. 493.

Hallucinosis of dRUnKards, ii. 608.

Hamamelis, fluid extract of, irrigation of the colon with, in ulcerative colitis, iii. 211.

Hammock bath, use of, in hydrotherapy, i. 199.

HANNA, studies by, on vaccination done subsequently to infection with smallpox, ii. 78.

HANSEN, studies by, on transmission of leprosy by vaccination, ii. 90 .

"Hardening," advantages of, in chronic bronchitis, iii. 367 .

Hare, Francis, on apomorphin in treatment of dipsomania, ii. 601 ; on cause of delirium tremens, ii. 602; on copper sulphate in treatment of acute phosphorus poisoning, ii. 627 ; on sodium bromid in treatment of morphinism, ii. 620; on treatment of alcoholism with atropin and strychnin, ii. 597; on withdrawal of alcohol altogether in delirium tremens, ii. 607 .

HaRmonic, cases of enuresis treated by circumcision reported by, iv. 130 .

Harvey-Banting cure for obesity, ii. 790 .

HASEBROCK, views held by, on effect of gymnastics in circulatory system, iii. 637.

Hay asthma. See Hay fever.

HAY Fever, iii. 389; diet in, iii. 390; local treatment of, iii. 390 ; localities in treatment of, iii. 389 ; prophylaxis against, iii. 389 ; treatment of, during attack, iii. 390 ; treatment of, in general, iii. 389 .

Hazelin, irrigation of colon with, in operative treatment of ulcerative colitis, iii. 212.

Headache, associated with intestinal autointoxication, ii. 681 ; in dementia precox, iv. 615; diabetic, iv. 611 ; due to erythrol tetranitrate used in chronic interstitial nephritis, iv. 71; hydrotherapy for relief of, i. 222; massage and gymnastics for relief of, i. 312; measures for relief of, in brain tumor, iv. 447; due to nitroglycerin used in chronic interstitial nephritis, iv. 71 ; as a premonitory symptom of cerebral hemorrhage, iv. 389 ; as symptom of acute nephritis, iv. 63 ; as a symptom of acute cerebral meningitis, iv. 368, 369; as a symptom of basilar syphilitic meningitis, iv. 473 ; as a symptom of brain tumor, iv. 435; as a symptom of cerebral abscess, iv. 420 ; as a symptom of cerebral thrombosis, iv. 399 ; as a symptom of chlorosis, iv. 611 ; as a symptom of chronic interstitial nephritis, iv. 72; as a symptom of echinococcus cerebri, iv. 461; as a symptom of leukemia, iv. 611 ; as a symptom of neurasthenia, iv. 565 ; as a symptom of pachymeningitis interna hemorrhagica, iv. 363 ; as a symptom of syphilitic meningitis, iv. 607 ; as a symptom of toxemias and general diseases, iv. 610; as a symptom of tuberculous meningitis, iv. 374, 607 ; as a symptom of typhoid fever, ii. 27; due to uremia, iv. 54.

Headache, frontal, as a symptom of brain tumor in frontal lobe, iv. 438.

Headache, gastrointestinal, iv. 611.

Headache, LOCAlized, as a symptom of brain tumor in parietal lobe, iv. 439.

Headache mixtures, use of, in seasickness, iv. 626 .

Headaches, bone aNd Periosteal, iv. 602. Headaches, cervical sympathetic, iv. 599.

Headaches, due to high BloOd pressure, iv. 48 .

HeAdaches OF eXtracerebral NatUre, iv. 597: bone and periosteal headache, iv. 603; cervical sympathetic headache, iv. 599; ear disease headaches, iv. 603; indurative headache, iv. 603; myositis, iv. 603; nasal and frontal sinus headaches, iv. 602 ; neuralgias, iv. 597 ; neurotic muscle headache, iv. 700 ; reflex tenderness of scalp, iv. 599.

Headaches, hysterical, iv. 614.

HEADACHES OF INTRACEREBRAL NATURE, iv. 608: brain tumor, iv. 608; hydrocephalus, iv. 609

Headaches, Nephritic, iv. 610.

Headaches, neurasthenic, iv. 613. 
Headaches, post-infectious, iv. 612.

Headaches, psychogenic and psychotic, iv. 613 ; definition of, iv. 613 ; in cyclothymia, iv. 614 ; in dementia precox, iv. 615 ; in hysteria, iv. 614 ; in neurasthenia, iv. 613.

Headaches, syphiLitic, iv. 612.

Health resorts, advantages of, in cirrhosis of liver, iii. 283; advantages of, in prophylaxis of ecrebral hemorrhage, iv. 391; benefit from, in treatment of acute myelitis, iv. 228; benefit from, in treatment of cardiac neurasthenia, iii. 777; benefit from, in treatment of chronic gout, ii. 701; benefit from, in treatment of diabetes mellitus, ii. 762 ; benefit from, in treatment of obesity, ii. 789; objections to, in chronic myocardial insufficiency due to obesity, iii. 727 .

Heanley, unnamed fluke described by, iv. 781.

Heart, ehanges in, during hypnosis, i. 574; dilatation of, see Dilatation of heart; disease of, as a complication of measles, ii. 161; effect of epinephrin upon, i. 91; hypertension of, see Hypertension; necessity for attention to, in chorea minor, iv. 663 ; nerves of, iii. 538; physiological effects of cold upon, i. 190; precautions concerning, in pneumonia, ii. 225; work of, iii. 530 .

Heart, diseases of, influence of climate upon, i. 282 ; mechanotherapy for relief of, i. 350,351 .

Heart, FunCtional diseases of, iii. 772: cardiac neurasthenia, iii. 773; general cardiac neuroses, iii. 773; neuroses with well-defined symptoms, iii. 786; reflex neuroses, iii. 778; toxic neuroses, iii. 782.

Heart Block, iii. 566; treatment of, iii. 772 .

Heart lesions, congenital, see Congenital heart lesions; valvular, see Valvular heart lesions.

Heart muscle, properties of, iii. 533.

Heart sound, first, iii. 542; second, iii. 541.

Heart stimulants, hypodermic or intravenous injection of, in treatment of poisons causing heart failure, i. 672 .

Heart weight, ratio of, to body weight, iii. 545 .
HEAT, as cause of sunstroke, iv. 672; effect of tropical, upon white men, iv. 669 ; local application of, in after-treatment of anterior poliomyelitis, iv. 240 ; local application of, in renal colic, iv. 102; loss of, from body, iv. 674; loss of, from body in relation to treatment for obesity, ii. 784 .

Heat, dry, application of, in akatama, iv. 863; application of, to extremities in acute myelitis, iv. 226; in gangrenous chancre, ii. 423; in typhoid fever. as cardiac stimulant, ii. 24.

Heat stroke. See Insolation.

Heberden, early description of varicella by, ii. 109 .

Heberden's nodosities, as characteristic feature of hypertrophic arthritis, ii. 708; presence of, in chronic gout, ii. 696 .

Hebra and Kaposi, use of soluble salt of mereury in treatment of syphilis advocated by, ii. 390 .

Heidelberg frog unit, as standard for digitalis preparations, iii. 583 .

Heller, on examination of finger nails in diagnosis of oxyuris vermicularis, iii. 252.

Helmitol. See Urotropin.

Heloderma, poison of, iv. 683.

Hematemesis, treatment of, in cirrhosis of liver, iii. 288.

Hematoma, traumatic, iv. 364; diagnosis of, iv. 366 ; pathology of, iv. 364 ; symptoms of, iv. 365 ; treatment of, iv. 366 .

Hematoporphyrinuria, iv. 4 ; causes of, iv. 5 ; treatment of, iv. 6 .

Hematuria, iv. 4; as a complication of scarlet fever, ii. 134; endemic, see Endemic hematuria.

Hemianesthesia, significance of, in traumatic hematoma, iv. 365 .

Hemianopsia, significance of, in traumatic hematoma, iv. 365.

Hemicrania. See Migraine, ophthalmic.

Hemiplegia, development of, during irrigation of pleural cavity, iii. 487; as a symptom of acute hemorrhagic superior polioencephalitis, iv. 406; as a symptom of cerebral embolism, iv. 401; as a symptom of cerebral thrombosis, iv. 395; as a symptom of tuberculous meningitis, iv. 375 ; treatment of, when associated with cerebral thrombosis, iv. 399; syphilitic, see Syphilitic hemiplegia. 
Hemiplegic type of Cerebral Palsies of CHILdREN, iv. 410.

Hemisin. See Epinephrin.

Hemisphere of BRAIN, LEFT, diagnosis of tumors in, iv. 440.

Hemisphere of bRAIN, RIGHT, diagnosis of tumor in, iv. 437.

Hemmeter, formula recommended by, for antiseptic solution for use in acute gastritis, iii. 155.

Hemochromatosis, iii. 301.

Hemoglobin, importance of test for, in chronic, myocardial insufficiency, iii. 724.

Hemoglobindria, iv. 4; epidemic, iii. 860 ; intermittent, iv. 7 ; paroxysmal, iv. 6 ; due to quinin, iv. 725 ; treatment of, iv. 5 .

Hemolysin treatment, of pernicious anemia, iii. 834 .

Hemolysis test, method for, iii. 830 .

Hemopericardium, iii. 527.

Hemophilia, administration of thyroid gland for relief of, i. 77, iii. 860; anemias and, iii. 870; arthropathies and, iii. 870; coagulants of blood in treatment of, iii. 862 ; definition of, iii. 860 ; diet in, iii. 861 ; etiology of, iii. 861 ; general treatment of, iii. 861 ; local treatment of, iii. 867; medicinal treatment of, iii. 861 ; organotherapy for, iii. 866; peptones for, iii. 866 ; serum therapy for, iii. 864 , 870 ; sporadic cases of, iii. 868 ; transfusion of blood in, iii. 866; treatment of accidents in, iii. 869 ; treatment of hereditary form, iii. 868; types of, iii. 861 ; vasoconstrictors in treatment of, iii. 867,869 .

Hemophilic states, iii. 861.

Hemoplase, use of, in pernicious anemia, iii. 833 .

Hemoptan, use of, in chlorosis, iii. 819.

Hemoptysis, associated with pulmonary thrombosis, iii. 412; endemic, see Endemic hemoptysis; in influenza with complications, ii. 195.

HemorRhage, blood pressure lowered by, iii. 556; in brain, see Cerebral hemorrhage; cerebral, see Cerebral hemorrhage; in cirrhosis of liver, iii. 288; complicating pellagra, ii. 662; complicating thoracentesis, iii. 478; complicating typhoid fever, ii. 19; complicating whooping-cough, ii. 175; control of, after tonsillectomy, iii. 45; danger from, after operation for brain tumor, iv. 452; following operation for adenoid vegetations, iii. 38; following paracentesis, iii. 284; from gastric ulcer, iii. 109; of lungs, see Pulmonary hemorrhage; into pock, as complication of vaccination, ii. 87; pulmonary, see Pulmonary hemorrhage; as a symptom of pernicious anemia, iii. 837; in syphilitic gastric ulcer, iii. 167; treatment of, in hemophilia, iii. 869; use of epinephrin for control of, i. 97.

Hemorrhagic conditions, serum treatment of, i. 172.

Hemorrhagic diatheses, as a factor in cerebral hemorrhage, iv. 389 .

Hemorrhagic diseases, iii. 858: hemophilia, iii. 860 ; hemorrhagic diseases of new-born, iii. 860; purpura, iii. 858.

Hemorrhagic nephritis. See Nephritis, hemorrhagic.

Hemorrhagic pleurisy. See Pleurisy.

Hemorrhoids, electrotherapy for relief of, i. 420 ; hydrotherapy for relief of, i. 420 .

Hemostatics, use of, in hematuria, iv. 4.

Hemothorax, iii. 490.

Henpuye. See Goundoo.

"Hendon cow disease," ii. 114.

Henschen, E. S., contraindications to operation in cerebral abscess given by, iv. 423; rules given by, for operation on brain tumors, iv. 452 .

Henschen's method, of giving mercury in brain syphilis, iv. 480 .

Hepatic amebiasis. See Amebiasis, hepatic.

Hepatic distomiasis, iv. 779; preventive measures in, iv. 780 ; remedial measures in, iv. 780 ; varieties of, iv. 779 .

Hepatic hyperemia, hydrotherapy for relief of, i. 212.

Hepatoptosis, iii. 288.

Herbage, poisoning by, ii. 651 .

Hereditary Cerebellar ataxia, iv. 513; differential diagnosis of, iv. 514; etiology of, iv. 513; introduction to subject of, iv. 513 ; pathology of, iv. 513 ; prognosis in, iv. 514; symptoms of, iv. 514; treatment of, iv. 514 .

Hereditary spinal ataxia. See Friedreich's ataxia.

Heredity, in causation, of alcoholism, ii. 590 ; of arteriosclerosis, iii. 755 ; of epi- 
lepsy, iv. 641; of gastric ulcer, iii. 169; of gout, ii. 694; of laryngeal tubereulosis, iii. 344 ; of leprosy, not a factor, iv. 810; of myotonia congenita, iv. 267; of the neuroses, iv. 250 ; of rachitis, ii. 815; of syphilis, iv. 181.

Herrédo-ataxie cérébelleuse. See Hereditary cerebellar ataxia.

Heroin, use of, in acute bronchitis, iii. 361; to allay irritation in laryngeal tuberculosis, iii. 346; in bronchial asthma, iii. 388 ; in bronchit is complicating influenza, ii. 189 ; contraindicated in chronic influenza, ii. 195; to control cough in active stage of pulmonary tuberculosis, ii. 482 ; to control cough due to chronic bronchitis associated with cardiac insufficiency, iii. 731 ; to control cough in laryngeal tuberculosis, ii. 498; to control cough in pericarditis, iii. 515; as substitute for morphin in treatment of morphin habit, ii. 618; in whoopingcough, ii. 182.

Heroin and terpin hydrate, elixir of, in cough of tuberculosis, ii. 498.

HERPES OF LIPS, iii. 5.

Herpes zoster, iv. 358; definition of, iv. 358; electrotherapeuties for relief of, $i$. 417 ; late pain in, iv. 359 ; local treatment for, iv. 359 ; treatment of, as to cause, iv. 359 .

Herpetic stomatitis. See Stomatitis herpetica.

Herringham, W. H., inhalations of oxygen by Stokes' inhaler suggested by, in bronchiectasis, iii. 396 .

Hertoghe, effect of thyroid on enuresis first observed by, iv. 131.

Herxheimer reaction, significance of, in treatment of syphilis with arsenobenzol, iv. 482.

Herz, Max, on development of muscular energy, iii. 638; method of determining extent of myocardial insufficiency introduced by, iii. 693 ; system of mechanogymnastics introduced by, iii. 641.

Herz system of gymnastics, iii. 648: actively resisted movements, iii. 652 ; forced breathing, iii. 660 ; inertia movements, iii. 648; passive movements, iii. 648; resistive inertia movements, iii. 652 ; respiratory gymnasties, iii. 659 ; self- resisted movements, iii. 650; use of, in cardiae neurasthenia, iii. 776 .

HERZFEL, on preventive treatment of migraine in gastrointestinal autointoxication, ii. 682.

HETEROPHYES HETEROPHYE, iv. 781.

Hewes, rectal injection of silver nitrate advised by, in bacillary dysentery, ii. 327 ; dietary arlvised by, iii. 325 .

Hewes dietary, for use in bacillary dysentery, ii. 325.

Hexamethylamin. See Urotropin.

Hiccough, as a complication of pneumonia, ii. 260.

High candle power incandescent lights, use of, in light therapy, i. 442.

High dilution, advantages of, in quinin used for tropical malaria, iv. 722; in atoxyl used in trypanosomiasis, 745 .

High frequency current, determining characteristics of, i. 375; explanation of term in electrotherapeutics, i. 366.

High pressure stasis, blood pressure raised by, iii. 555; as indication for use of digitalis, iii. 588 .

Hill diarrhea, iv. 862; remedial measures for, iv. 863.

Hip bath, use of, in hydrotherapy, i. 200.

Hippocrates, paracentesis for diseases of pleura advocated by, iii. 455 .

Hirschfeld's diet, for reduction of obesity, ii. 794 .

HiRschlafF, morphium heil-serum introduced by, for treatment of morphinism, ii. 624 .

HirT, hypnotism and mental suggestion employed by, in treatment of norphinism, ii. 624.

Hiss, use of leukocytic extract in bronchopneumonia suggested by, iii. 415 .

Histological changes in skin due to X-rays, i. 517.

Histoplasmosis, iv. 757.

History taking, as part of technique of psychoanalysis, i. 588.

Hodgkin's disease. See Pseudoleukemia.

Hofbauer, method devised by, for compressing thorax, iii. 435; methods devised by, for prolonging expiration, iii. 436.

Hoffmans, F. A., records collected by, of operation for bronchiectasis, iii. 394 .

Hoffmann, Mr. Frederick L., statistics 
concerning decrease in death rate from tuberculosis furnished by, ii. 450 .

Hoffmann's anodyne. See Compound spirits of ether.

HoFMOCKEL, on results of operation for bronchiectasis, iii. 394 .

HollaNd, introduction of vaccination into, ii. 60 .

Holtz machine, i. 367.

HolzKNeCHT, method of measuring intensity of X-rays devised by, i. 497; method of securing conformity of deep irradiation of X-rays devised by, i. 505.

Holzknecht's radiometer, use of, for measuring intensity of X-ray, i. 468.

Home treatment, of neurasthenia and psychasthenia, iv. 561 .

Homogeneous irradiation, in X-ray therapy, i. 505.

Hook WORM DISEASE, iv. 791; manner of infection with, iv. 792; preventive measures in, iv. 794; remedial measures in, iv. 793; varieties of parasites causing, iv. 791 .

Horse chestnut, poisoning by, ii. 651 .

Horse POX, ii. 55.

Horse serum. See Serum.

Horseback riding, in prophylaxis of chronic hyperemia of brain, iv. 387 .

Hospitals, prevention of measles in, ii. 144; prevention of scarlet fever in, ii. 21.

Hospitals, sPECIal, treatment of bubonic plague in, undesirable, iv. 826.

Hospital treatment, of neurasthenia and psychasthenia, iv. 561.

Hot air baths, use of, in hydrotherapy, i. 200.

Hot applications. See Applications, hot.

Hot drink, use of, to induce sleep in arteriosclerosis, iii. 760 .

Hot springs of Arkansas, value of, in acute myelitis, iv. 228.

Hot springs of Virginia, value of, in acute myelitis, iv. 228 .

Hot water bags, use of, in acute enteritis, iii. 193; in acute fibrinous pleurisy, iii. 466 ; in acute gastritis, iii. 156 ; in appendicitis, iii. 217; in bacillary dysentery, ii. 324 ; in gonorrheal epididymitis, iv. 152 ; in multiple neuritis, iv. 326 ; for relief of backache in acute nephritis, iv. 63; for relief of pain in nephritis, iv. 88; for relief of pain in rheumatic or idio- pathic cases of facial paralysis, iv. 311 ; in renal colic, iv. 102; in syncope due to cerebral thrombosis, iv. 398.

Hot water bottle. See Hot water bags.

Hour-glass stomach, iii. 179.

House infeCtion, in tuberculosis, ii. 451.

Household, occurrence of arsenic in, i. 676.

How ARD, L. O., measures proposed by, as protection against fleas, iv. 825 .

Howe, cases reported by, of pemphigus following vaccination, ii. 91.

Howland, effect of cold air on blood pressure in bronchopneumonia demonstrated by, iii. 418 .

Hueter's treatment of erysipelas, ii. 285.

Hughes, beneficial effects of potassium nitrate in pneumonia reported by, ii. 227.

Huguenin, method of brain puncture for hydrocephalus recommended by, iv. 465 .

Human immune serum, i. 170.

Humidity, ATMospheric, i. 250; absolute, i. 251; physiological influence of, i. 263; relation between temperature of the body and, i. 262 ; relative, i. 251.

Hunt, Ramsay, theory advanced by, concerning facial nerve, iv. 311.

Huntington's chorea. See Chorea, degenerative.

Hunyadi water, use of, in acute fibrinous pleurisy, iii. 467 ; in biliousness, iii. 258 ; in cerebral hyperemia, iv. 386 ; to modify severity of pleurisy, iii. 467 ; in nephritis, iv. 82.

Hutchinson, chancres developing on vaccinations reported by, ii. 89 .

Hutchison, Robert, dietary proposed by, for use in chronic bronchitis, iii. 370.

Hydatid disease of liver, prognosis of, iii. 291.

Hydatids. See Echinococcus.

Hydrargyri chloridum mite. See Calomel.

HydrargYrism, ii. 383; buccal accidents due to, ii. 384; cutaneous accidents due to, ii. 384; gastrointestinal accidents due to, ii. 384; general intoxication due to, ii. 383; idiosyncrasy as a cause of, ii. 383 ; mercurial stomatitis dué to, ii. 384 ; prevention of, ii. 385; renal accidents due to, ii. 384 ; salivation due to, ii. 384 ; treatment of, ii. 385 .

Hydrastinin hydrochlorid, hypodermic use of, to control epistaxis, iii. 336 . 
Hydrastis canadensis, use of mixture containing, in vesical erises of tabes, iv. 197; use of, for relief of vesical disturbanee in tabes, iv. 198.

Hydrastis, fluid extract of, irrigation of colon with, in ulcerative colitis, iii. 211.

Hydration capacity of Collolds, i. 33.

Hydriodic acid, syrup of, use of, in chronic nasopharyngitis, iii. 32; in glandular enlargement accompanying rachitis, ii. 818 .

Hydrocephalus, iv. 462; acquired form of, iv. 463 ; congenital form of, iv. 462 ; diagnosis between, and brain tumor, iv. 436; idiopathic internal hydrocephalus, see Serous meningitis; introduction to subject of, iv. 462 ; rachitis as cause of, iv. 464 ; serous meningitis a form of, iv. 468 .

Hydrocephalus, acquired, iv. 463; brain puncture for, iv. 465; diagnosis of, iv. 463 ; etiology of, iv. 463; external remedies for, iv. 465; internal remedies for, iv. 465 ; lumbar puncture for, iv. 468; prognosis in, iv. 464; treatment of, iv. 464; ventricular puncture for, iv. 466.

Hydrocephalus, congenital, iv. 462; brain puncture for relief of, iv. 465; diagnosis of, iv. 462; etiology of, iv. 462; external remedies for, iv. 465 ; internal remedies for, iv. 465; lumbar puncture for relief of, iv. 468; treatment for, iv. 464; ventricular puncture in, iv. 466.

Hydrocephalus, idiopathic internal. See Meningitis, serous.

HYdROCHLORIC ACID, poisoning by, i. 732.

Hydrochloric acid, use of, in acute gastritis, iii. 157; in carcinoma of stomach, iii. 165 ; in depressive disorders of gastric secretion, iii. 148; destruction of cholera germ by, iv. 829 ; use of, in gastric hyperacidity with hypermotility, iii. 101; use of, in gastric lavage for chronic gastritis, iii. 161; pancreatic secretion increased by, iii. 307 ; in pernicious anemia, iii. 826 ; relation between, and habitual constipation, iii. 223; to relieve gastrointestinal symptomis due to chronic myocardial insufficiency, iii. 739 ; in secretory and motor gastric neuroses, iii. 188.

Hydrochloric acid, dilute, use of, in hypoacidity accompanying cirrhosis of liver, iii. 281 ; in hypochlorhydria due to chlorosis, iii. 822; in oxaluria, iv. 8 ; in tabes, iv. 213.

Hydrocyanic acid, use of, in destruction of moscquitoes, iv. 735.

Hydrocyanic acid, dilute, use of, in cirrhosis of liver, iii. $28($ ).

Hydrocyanic acid poisoning, i. 737; diagnosis of, i. 742 ; climination of poison in, i. 743 ; fatal dose in, i. 742 ; poisonous action of acid in, i. 738; post-mortem findings in, i. 743; stages in, i. 741; treatment of, i. 742 .

Hydroelectric batus. See Baths.

Hydrogen dioxid. See Hydrogen peroxid.

HydRogen ION, cholera germ susceptible to, iv. 829.

Hydrogen peroxid, use of, in acute tonsillitis, iii. 42; in amyxorrhea gastrica, iii. 101 ; in atrophic nasopharyngitis, iii. 34 ; in atrophic rhinitis, iii. 331 ; for cleansing preputial cavity in acute gonorrhea, iv. 138; contraindicated for washing out abscess cavity in diphtheria, ii. 274; after curettage for chronic tonsillitis, iii. 44 ; as disinfectant wash in balanoposthitis complicating acute gonorrhea, iv. 149 ; in gangrenous chancre, ii. 423 ; as gargle in hydrargyrism, ii. 385 ; as gargle in syphilitic angina, ii. 426; in gastric hyperacidity, iii. 95 ; in hygiene of mouth, iii. 3 ; after incision in symptomatic parotitis, iii. 28; in inflamed chancre, ii. 422; for irrigation of large intestine in intestinal amebiasis, iv. 709; for irrigation in phlegmonous pharyngitis, iii. 40; in membranous pharyngit is, iii. 39 ; in membranous rhinitis, iii. 330 ; in mercurial stomatitis, iii. 16 ; as mouth wash in mumps, ii. 170; in mycosis of tonsils and fauces, iii. 42; as spray for disinfection of mucous membranes in anterior poliomyelitis, iv. 237; as spray, in syphilis of upper respiratory tract, iii. 349 ; in stomatitis ulcerosa, iii. 15 ; in stomatitis ulcerosa chronica, iii. 16; in syphilitic onychia, ii. 429; on tampon for control of epistaxis, iii. 335; in tertiary lesions of mucous or mucocutaneous surfaces, ii. 427; in throat affections complicating scarlet fever, ii. 129, 130; in Vincent's angina, iii. 40.

Hydrogen sulphid, poisoning by, i. 634; treatment of, i. 735 . 
Hydronaphthol, use of, as mouth wash in pyorrhea alveolaris, iii. 22.

Hydronephrosis, iv. 96; treatment of, iv. 97.

HydROPERICARDIUM, iii. 525 .

HydRophilic Colloids, i. 6, 7.

HYDROPHOBIA, cauterization of wound made by rabid animal, ii. 336; complication of Pasteur treatment in, ii. 340 ; constitutional effects of inoculation in, ii. 339; constitutional measures of treatment for, ii. 336; experiments by New York Health Department in cauterization of wounds made by rabid animals, ii. 336 ; indications for antirabic treatment in, ii. 334; local effects of inoculation in, ii. 339; mortality from, affected by Pasteur treatment, ii. 342; Pasteur treatment for, ii. 334, 337; Pasteur treatment for, by mail, ii. 341 ; precautions against infection by attendants upon, ii. 343 ; prophylaxis in, ii. 335 ; treatment of developed disease, ii. 342 .

HyDROPHOBIC COLLOIDS, i. 6.

Hydrotherapeutic applications, use of, in emphysema with cardiac insufficiency, iii. 712 .

Hydrotherapeutic measures, external, uncalled for, in apyrexial heat exhaustion, iv. 679 .

Hydrotherapeutic measures, internal, use of, in apyrexial heat exhaustion, iv. 679 .

Hydrotherapy, i. 184; in acquired spinal progressive muscular dystrophy, iv. 248; in after-treatment of morphin habit, ii. 624 ; in alcoholism, ii. 594; in arteriosclerosis, iii. 760 ; in arthritis deformans and chronic rheumatism, ii. 712 ; in bacillary dysentery, ii. 324; balneology in,"i. 229 ; in bronchopneumonia, iii. 419 ; in cardiac insufficiency, iii. 615 ; in cardiac neurasthenia, iii. 777; in chlorosis, iii. 813; in chronic progressive bulbar paralysis, iv. 252 ; in chronic enlargement of spleen, iii. 889 ; in chronic gastritis, iii. 162 ; in chronic gout, ii. 701; in chronic myelitis, iv. 231; in chronic serous effusion of pleura, iii. 484 ; in constipation, iii. 230 ; in constitutional diseases, i. 206 ; to control sweating in malignant endocarditis, iv. 747 ; to control temperature in Malta fever, iv. 839 ; in convalescence from influenza, ii. 196; in delirium tremens, ii. 606; in diabetes mellitus, ii. 762 ; in diseases of circulation, i. 202; effects of cold in, i. 189; for epilepsy, iv. 646 ; in exophthalmic goiter, iii. 898; extremes of temperature in, i. 200; in gastric hyperacidity, iii. 100; in gastrointestinal diseases, ii. 212 ; in genitourinary diseases, i. 209; for gonorrheal arthritis, i. 227; in hallucinosis of drunkards, ii. 609; history of, i. 184; for insolation, iii. 678; in intoxications, i. 228; in joint disease, i. 226 ; in Keeley gold cure, ii. 600; in late stages of acute hemorrhagic encephalitis, iv. 406; in malarial fever, iv. 727; in measles, ii. 149; in membranous colitis, iii. 210 ; in meningitis, ii. 215; in metabolic diseases, ii. 206; for miliary fever, ii. 580; mode of application of, i. 193; in nervous diseases, i. 221 ; in pellagra, ii. 662 ; in pneumonia, i. 221 , ii. 231 ; in primary or idiopathic gastric neuroses, iii. 188; in progressive bulbar paralysis, iv. 499; properties of water in, i. 204; in prophylaxis of chronic pulmonary emphysema, iii. 434 ; in reduction of obesity, ii. 808 ; for relief of cardiac palpitation in neurasthenia, iv. 568; for relief of headache due to hydrocephalus, iv. 609 ; for relief of nervous excitement in acute hemorrhagic polioencephalitis, iv. 408; for relief of hysteria, i. 223; for relief of insomnia due to cerebral abscess, iv. 423; for relief of restlessness in neurasthenia, iv. 567; for relief of restlessness and insomnia in chorea minor, iv. 663; in respiratory diseases, i. 204; in Rocky Mountain fever, ii. 565; in scarlet fever, ii. 125, 127; special forms of, i. 229; in specific infectious diseases, i. 215; in spinal syphilis, iv. 274; in syphilis, ii. 365,435 ; in tabes, i. 225 , iv. 200 ; in tetanus, i. 217; in tetany, iii. 910; in toxemias, i. 228; in typhoid fever, ii. 16.

Hydrotherapy, soothing, use of, in neurasthenia and psychasthenia, iv. 546.

HydRothorAX, UNILATERAL, accompanying cardiac insufficiency, iii. 673.

Hygiene of mouth, ii. 368, iii. 2.

HYGIENIC CAUSES OF ENURESIS, iv. 126, 127.

Hygienic measures, use of, in arthritis deformans, ii. 711; in beri-beri, iv. 853; in brain tumor, iv. 447; in cerebral hem- 
orrhage, iv. 391 ; in cerebral hyperemia, iv. 384 ; in chronic cercbral hyperemia, iv. 387 ; in endemie hematuria, iv. 785 ; in German measles, ii. 165; in hydrocephalus, iv. 464 ; in intestinal amebiasis, iv. 708; in kubisagari, iv. 861; against leprosy, iv. 813, 815; in measles, ii. 145; in oriental sore, iv. 756 ; in preparation for psychotherapy, iv. 572; in progressive bulbar paralysis, iv. 499 ; in prophylaxis against the neuroses in childhood, iv. 522 ; in prophylaxis against aeute rheumatism, ii. 296; in prophylaxis against malaria, iv. 738; in prophylaxis against pneumonoconiosis, iii. 428; in prophylaxis against sunstroke, iv. 676; for relief of enuresis, iv. 132; in searlet fever, ii. 123; in syphilis, ii. 368,432 ; in treatment of alcoholism, ii. 599; in treatment of malaria, iv. 728 ; in treatment of morphin habit, ii. 624 ; in treatment of the neuroses, iv. 528; in whooping-cough, 177.

Hygienic-dietetic treatment, of tuberculosis, iii. 477.

Hymenolepis diminuta, iv. 777.

HyMeNOLEPIS NANA, iv. 777.

Hyoscin, use of, in bronchial asthma, iii. 384 ; by hypodermic injection, for relief of insomnia in cardiac insufficiency, iii. 664 ; by hypodermic injeetion for relief of restlessness in neurasthenia, iv. 567; in pneumonia of alcoholics, ii. 258; for relief of insomnia in neurasthenia, iv. 565 ; in tetany, iii. 910 ; in treatment of morphin habit, ii. 620 .

Hyoscin HYDRoBromate, use of, in paralysis agitans, iv. 666; for relief of delirium in septicemia, ii. 291 ; for relief of insomnia in typhoid fever, ii. 27.

Hyoscyamin, use of, in bronchial asthma, iii. 384 ; by hypodermic injection, for attacks of excitement in progressive paralysis of the insane, iv. 493.

Hyoscyamus, use of, in after-treatment of morphinism, ii. 624; in colon bacilluria, iv. 128; in endemic hematuria, iv. 784 ; to lessen irritability of bladder in tuberculous kidney, iv. 117; for relief of vesieal disturbance in tabes, iv. 198; in treatinent of enuresis, iv. 133.

Hyperaciditas nicotinica, iii. 76 .
HYPERACIDITY AND HYPERSECRETION OF GASTJUe JUICE, iii. 74; abuse of stimulants as cause of, iii. 76; abuse of tobacco as cause of, iii. 76 ; acute or intermittent hyperacidity, iii. $10^{2} 2$; alimentary hypersecretion, iii. 101; amyxorrhea gastrica, iii. 100; continuous hypersecretion, iii. 102; diet in treatment of, iii. 79 ; drugs in treatment of, iii. 91 ; electricity in treatment of, iii. 100; crrors in diet a cause of, iii. 76; hydrotherapy for, iii. 100 ; lavage in treatment of, iii. 97 ; overwork a cause of, iii. 75 ; summary of conditions aceompained by, iii. 100.

Hyperacidity OF stomach, iii. 75 ; in chlorosis, iii. 822 .

Hyperacidity of urine, as a cause of enuresis, iv. 128.

Hyperacusis, as a symptom of neurasthenia, iv. 568 .

Hyperalimentation. See Superalimentation.

HYPERCHLORHYDRIA, presence of, in acute eatarrhal jaundice, iii. 261 ; as a reflex neurosis, iii. 778 .

Hyperemia of brain, iv. 383 ; active cerebral hyperemia, iv. 383 ; chronic, iv. 387 ; introduetion to subject of, iv. 383 ; passive cerebral hyperemia, iv. 383 ; prognosis in, iv. 384 ; prophylaxis against, iv. 384 ; symptomatic treatment of, iv. 386 ; symptoms of, iv. 384; treatment of active hyperemia, iv. 385 ; treatment of chronic hyperemia, iv. 387 ; withdrawal of blood in, iv. 386 .

Hyperemia, production of, as effect of radiant energy, i. 43S, 445.

Hyperesthesia of tongue, iii. 4.

Hyperesthesia of special senses, as a symptom of acute cerebral meningitis, iv. 369 ; as a symptom of pachymeningitis interna hemorrhagica, iv. 363 ; as a symptom of tuberculous meningitis, iv. 374.

HyPeRglycemia in DiABETES MEllitus, explanation of, ii. 722 .

Hyperpiesis, iii. 556.

Hyperpituitarism, conditions associated with, i. 102.

Hyperpyrexia, in acute rheumatism, ii. 302.

HyPeRPYREXIAL FEVER, iv. S6S; remedial measures in, iv. 869 . 
Hypersecretion, acute gastric, iii. 102. Hypersecretion, continuous gastric, iii. 102 ; diet in, iii. 106, 107; medical treatment for, iii..106; operative treatment for, iii. 103.

HyPeRTENSION, accompanying pyelitis, iv. 94 ; in chronic interstitial nephritis, iv. 69.

Hypertension of Heart, iii. 554; in nephritis, iv. 18, 20; significance of, associated with amyloid kidney, iv. 81 ; treatment of, in nephritis, iv. 45 .

HyPERTRICHOSIS, radium in treatment of, i. 476 .

HyPERTROPHY OF CARDIAC MUSCle, effects of, iii. 545; physiology of, iii. 544 .

Hypertrophy of heart. See Cardiac hypertrophy.

Hypnosis, normal, i. 569; activity of association in, i. 569; activity of will in, i. 570; application of, i. 578; dangers of, i. 578; general sensations in, i. 573; hallucinations in, i. 572; heart during, i. 574; impulses during, i. 574; intestines during, i. 574; memory during, i. 570; motor sphere during, i. 574; reaction to, i. 571 ; secretion during, i. 574 ; sensations during, i. 572 ; stages of, i. 569 ; stomach during, i. 574; suggestibility in, i. 570; temperature of body during, i. 574 ; trophic effects of, i. 574 ; vasomotor effects of, i. 574.

Hypnotic sleep without suggestion, i. 566 .

Hypnotic suggestion, as a form of psychotherapy, iv. 570 ; principle of, iv. 571 ; for relief of enuresis, iv. 134; use of, in treatment of alcoholism, ii. 600 ; use of, in trestment of morphinism, ii. 624 .

Hypnotic suggestive therapy, i. 576.

Hypnotics, use of, in acute bronchitis, iii. 361 ; in neurasthenia, iv. 559,564 ; objections to, in arteriosclerosis, iii. 760; objections to, for relief of nocturnal dyspnea in chronic interstitial nephritis, iv. 72; for relief of insomnia due to cerebral abscess, iv. 423 ; for relief of insomnia due to chronic cerebral hyperemia, iv. 387.

Hypnotism, i. 561; awakening of patient from, i. 568; historical review of, i. 561; normal hypnosis and, i. 569; pioneers in, i. 562 ; post-hypnotic suggestion in, i. 571 ; susceptibility to, i. 563 ; technique of, i. 564; use of, in chronic alcoholism, ii. 597; use of, in delirium tremens, ii. 605; use of, in hallucinosis of drunkards, ii. 609 ; use of, in treatment of morphinism, ii. 624; utilization of, in medicine, i. 573.

HYPOCHONDRIA, associated with progressive paralysis of the insane, iv. 493.

Hypodermic injection. See Injection, hypodermic.

Hypodermoclysis, administration of normal saline solution by, for collapse in acute stage of bacillary dysentery, ii. 327; administration of normal saline solution by, in hemorrhage of pulmonary tuberculosis, ii. 502; administration of normal saline solution by, in infantile diarrhea, ii. 329 ; administration of quinin by, in malaria, iv. 721; administration of quinin by, in pernicious malaria, iv. 727; use of, in acute diffuse peritonitis, iii. 318 ; use of, in acute vasomotor paralysis, iii. 753; use of, in bilious pneumonia, ii. 258; use of, in cholera infantum, iii. 204; use of, for relief of anuria in nephrolithiasis, iv. 107; use of, for relief of hematemesis from cirrhosis of liver, iii. 288; use of, in sepsis complicating scarlet fever, ii. 128; in severe attacks of appendicitis, iii. 218.

Hypoglossus Paralysis. See Neuritis of twelfth cranial nerve.

HyPopericardium, iii. 525.

Hypophosphites, use of, in bronchial asthma, iii. 385 ; in convalescence from pneumonia, ii. 262; in laryngeal tuberculosis, iii. 348 ; in tuberculosis of mediastinal lymph glands, iii. 507.

Hypophyseal extracts, use of, in myasthenia gravis, iv. 507.

Hypophysectomy, employment of, in diseases of pituitary body, iii. 916 .

Hypophysis, diseases OF, iii. 915.

Hypophysis in organotherapeutics, i. 101; active principles contained in, i. 107; conditions benefited by administration of, i. 107, 109; contraindications to use of, i. 109; effects following extirpation of, i. 104; functions of, i. 101; hyperactivity of, i. 102, 103; hypoactivity of, i. 103; physiological effects of, i. 105; relation between acromegaly and, i. 101, 102; relation between growth and, $i$. 103 ; summary of knowledge concern- 
ing, i. 107; therapeutic uses of, i. 107; untoward effects of, i. 109.

Hypophysis, tumors of, localizing symptoms of, iv. 442; use of hypophyseal extraet followed by relief of, i. 108.

Hypopituitarism, conditions associated with, i. 103.

Hypostatic pneumonia. See Pneumonia. HYPOTENSION OF HEART, iii. 555.

HYPOTHYROIDISM, CONDITIONS OF, i. 74.

Hysteria, iv. 581; association of, with impulse of reproduction and struggle for existence, iv. 587; delusions in, iv. 584 ; exaggeration of existing conditions in, iv. 584; hallucinations in, iv. 584; narrowing of field of consciousness in, iv. 584 ; physical adjuncts to treatment of, iv. 589; psychoanalysis in treatment of, iv. 588; reëducation in treatment of, iv. 588; resemblance between, and dementia precox, iv. 583; resemblance between, and feeble-mindedness, iv. 586; symptoms resembling, in brain tumor, iv. 459.

Hysterical atTacks, accompanying chronic progressive bulbar paralysis, iv. 253.

Hysterical vertigoes, iv. 630.

ICE, transmission of typhoid fever by, ii. 3 .

Ice, use of, in acute ophthalmoplegia, iv. 508 ; in apoplectie form of vestibular vertigo, iv. 628 ; by application to head, in reaction stage of Asiatic eholera, iv. 836 ; by application to spine, in strophanthus poisoning, iv. 695; in cerebral hemorrhage, iv. 391; for control of vomiting in blackwater fever, iv. 741; for control of vomiting due to brain tumor, iv. 448; for control of vomiting in tropical relapsing fever, iv. 760; in migraine, iv. 594 ; in pachymeningitis interna hemorrhagica, iv. 264 ; in parotitis complicating typhoid fever, ii. 27; for relief of gastric crises in tabes, iv. 197 ; for relief of hematemesis in pernicious anemia, iii. 837; for relief of pain in pyelitis, iv. 88 .

Ice bag, use of, in acute appendicitis, iii. 215,217 ; in acute bronchitis, iii. 357 ; in acute catarrhal cholecystitis, iii. 266; use of, in acute cerebral meningitis, iv. 372 ; in acute hemorrhagie encephalitis, iv. 405 ; in acute infectious myocarditis, iii. 750 ; in acute myelitis, iv. 225; in acute tonsillitis, iii. 41 ; in adenitis complicating glandular fever, ii. 576; in angina Ludovici, iii. 30 ; in anterior polionyelitis, iv. 237; as application to buboes in plague, iv. 827 ; in cardiac neurasthenia, iii. 778 ; in cerebrospinal meningitis, ii. 215; in cervical adenitis complicating searlet fever, ii. 131; in chronic myocardial insufficiency, iii. 715; contraindicated in hemorrhagic form of ulcerative colitis, iii. 212 ; to control gastric hemorrhage, iii. 109, 117; to control hemorrhage in pulmonary tuberculosis, ii. 500; to control hemorrhage in typhoid fever, ii. 20; to control vomiting in acute localized peritonitis, iii. 312; in edematous laryngitis, iii. 341 ; in gonorrheal epididymitis, iv. 152; in hematemesis during cirrhosis of liver, iii. 288 ; in hemorrhagic form of measles, ii. 154; in laryngeal complications of measles, ii. 157; in mediastinitis, during early stages, iii. 504 ; in mumps, ii. 170; in pericarditis, iii. 517,520 ; in peritonsillitis, iii. 43; in phlegmonous pharyngitis, iii. 40 ; as a preventive of myocarditis, ii. 276 ; to reduce temperature in pneumonia, ii. 231, 232; for relief of eardiac symptoms in chronic progressive bulbar paralysis, iv. 253; for relief of delirium in septicemia, ii. 291; for relief of pain in acute fibrinous pleurisy, iii. 466 ; for relief of pain in acute gastritis, iii. 156; for relief of pain in acute localized peritonitis, iii. 310; for relief of pain in pneumonia, ii. 227; for relief of pain in small-pox, ii. 47; for relief of tachycardia in exophthalmic goiter, iii. 898; in renal colic, iv. 103; as a stimulant in septicemia, ii. 290; in symptomatie parotitis, iii. 28 ; in tetany, iii. 910 ; in throat affections complicating scarlet fever, ii. 130; in thyroiditis acuta and struma inflammatoria, iii. 891 .

Ice baths, uses of, in hydrotherapy, i. 201.

Ice cap, use of, in bacillary dysentery, ii. 324 ; in cerebral hyperemia, iv. 385 ; in cerebrospinal meningitis, ii. 215 ; in malarial fever, iv. 727 ; in pneumonia, ii. 226 ; for relief of headache in cercbral abseess, iv. 423; for relief of headache in typhoid fever, ii. 27; in scarlet fever, ii. 127. 
Ice collar, use of, in acute laryngitis, iii. 338; in acute nasopharyngitis, iii. 31 ; in acute uvulitis, iii. 35 ; in cervical adenitis complicating diphtheria, ii. 277 ; in membranous laryngitis, iii. 343 ; in membranous pharyngitis, iii. 39 ; in peritonsillitis, iii. 43.

Ice pack. See Pack.

Ice rub, uses of, in hydrotherapy, i. 201.

ICED DRINKS, over-use of, a cause of gastric hyperacidity, iii. 78 .

Ichthyol, use of, in atrophic rhinitis, iii. 332; in cervical adenitis complicating scarlet fever, ii. 131; in chronic mucous colitis, iii. 206 ; in erysipelas, ii. 131 ; in leprosy, iv. 816 ; as ointment in acute gout, ii. 702 ; as ointment in adenitis complicating glandular fever, ii. 576; as ointment in herpes zoster, iv. 359; as ointment, in symptomatic parotitis, iii. 28 ; in ozena, iii. 337 ; to relieve itching in varicella, ii. 112 ; after scarification in edematous laryngitis, iii. 341 ; in small-pox, ii. 49.

Iснтнуотохіsмus. See Fish poisoning.

ICTERUS GRAVIS, following paracentesis during cirrhosis of liver, iii. 285.

IDIOSYNCRASY, as cause of disagreeable effects following injection of mercury, ii. 392,403 ; as cause of hydrargyrism, ii. 383.

"Igniting Juice." See "Appetite juice."

Ileus, Paralytic, iii. 238.

Imitative CHOREA, iv. 664 .

IMMERMANN, tables by, showing yearly average of deaths from small-pox before vaccination, ii. 100 .

Immobilization, during acute stage of tuberculosis, ii. 482 ; of joints in acute endocarditis with rheumatism, iii. 746 ; of joints in arthritis deformans and chronic rheumatism, ii. 712 .

Immone Bodies. See Antibodies.

Immune sera. See Sera.

Immune therapy, malignant disease and, $i$. 175.

Iмmunity, acquired, i. 129; acquired by attack of glanders, ii. 357; acquired by attack of measles, ii. 140; acquired by attack of mumps, ii. 169; acquired by attack of varicella, ii. 110; acquired by rats to plague, iv. 820 ; active, i. 137; antibacterial, i. 129 ; antibodies in causation of, i. 130; antitoxin form of, i. 129; conferred by cowpox, ii. 57; conferred by tuberculin, ii. 511; conferred by vaccination, ii. 77; development of, after inoculation, i. 138; establishment of, in oriental sore, iv. 757 ; natural, i. 129; passive, i. 129 ; passive natural, ii. 171 ; from plague, conferred by inoculation, iv. 823 ; theories of, ii. 127.

Immunization, active, i. 137; with attenuated virus, i. 139; with bacterial extracts, i. 148; combination methods of, i. 152; with dead organisms, i. 139; with virulent living organisms, i. 139.

Immunization against anthrax, ii. 331 ; combined method of, ii. 331 ; technique of, ii. 332 .

Immunization with bacterial extracts, i. 148: tuberculin, i. 149; trichophytin, i. 152.

Immunization with dead organisms, i. 139; in prophylaxis of cholera, i. 141 ; in prophylaxis of dysentery, i. 142; in prophylaxis of plague, i. 142 ; in prophylaxis of typhoid fever, i. 142; technique of method of, i. 140; theory of method of, i. 139; in treatment of acne, i. 145; in treatment of colon bacillus infection, i. 147; in treatment of gonococcus infections, i. 146; in treatment of infections of various kinds, i. 147; in treatment of pneumococcus infections, i. 146; in treatment of staphylococcus infections, i. 145 ; in treatment of streptococcus infections, i. 146; in treatment of typhoid fever, i. 143.

Immunization against diphtheria, ii. 268.

Immunization, passive, i. 153; antitoxic sera as a means of, i. 154; bactericidal sera as a means of, i. 161; immune sera of other types as a means of, i. 168.

Immunization with virulent virus, i. 139.

Imperial drink, use of, as a diuretic and diaphoretic, iii. 352 ; in pyelitis, iv. 89 ; for relief of anuria due to nephrolithiasis, iv. 107.

IMPETIGO CONTAGIOSA, as complication of vaccination, ii. 86 .

IMPOTENCE, iv. 178; in spinal syphilis, iv. 271 ; as a symptom of diabetes mellitus, ii. 766 .

INCANDESCENT LIGHT, as source of radiant energy, i. 440.

Incandescent light baths. See Baths. 
Incision, in acute peritonsillitis with abscess, iii. 43; in angina Ludovici, iii. 30; of bites by poisonous anthropods, iv. 685 ; of buboes in plague, iv. 827; for drainage in uremia with edema of extremities, iv. 53; indications for, in abscess of urethral glands complicating acute gonorrhea, iv. 148; indications for, in periurethral abscess complicating acute gonorrhea, iv. 148; intradural, see Intradural incision; in phlegmonous pharyngitis, iii. 40; to relieve edema of extremities in acute nephritis, iv. 62 ; into substance of tongue in parenchymatous glossitis, iii. 24; in symptomatic parotitis, iii. 28 ; in thyroiditis acuta and struma inflammatoria, iii. 891 ; of uvula, in acute nasopharyngitis, iii. 31 .

Incision and sucking of wound, in curare poisoning, iv. 694 .

"INCLusion bodies," presence of, in scarlet fever, ii. 115.

INCOMPLETENESS, SENSATIONS OF, as characteristic symptom of psychasthenia, iv. 518.

INDEPENDENT IRRITABILITY OF MUSCLE CELLS, i. 372.

Indian hemp. See Cannabis indica.

INDIANS, tuberculosis among, ii. 444.

INDICANURIA, as a cause of pain in bladder, iv. 122.

INDIGO CARMIN TEST, for functional power of kidney, iv. 24.

INDIVIDUAL PROPHYLAXIS against Asiatic cholera, iv. 828 .

INDIVIDUAL PROPHYLAXIS AGAINST TUBERculosis, ii. 455; marriage a factor in, ii. 464; occupation a factor in, ii. 463; precautions to be observed in presence of tuberculosis for, ii. 465; protection of children in, ii. 458 ; protection of infants in, ii. 457; protection of youth in, ii. 462 ; rules for school-children in, ii. 460 ; rules for tuberculous subjects in, ii. 466.

InDIVIDUALIZATION, in treatment of epilepsy, iv. 644, 645.

Induced current of electricity, characteristics of, i. 374 ; as an electromechanical method, i. 389.

InDURATION OF TISSUES, mechanical effects of electrotherapeutics in, i. 395.

Indurative heAdache. See Myositis.
Inebraté Reform Association, ii. 599.

INFANCY, enteritis in, iii. 201; prophylaxis against the neuroses in, iv. 521 ; protection against tuberculosis in, ii. 456 .

INFANTILE CaCHexia in Brazil, iv. 752.

Infantile cerebral paralygis. See Cerebral palsies of children.

INFANTILE PALSY OF CEREBELLAR TYPE, iv. 512.

Infantile paralysis. See Poliomyelitis.

Infantile pseudobulbar palsy. See Pseudobulbar palsy.

INFANTILE SCURVY, iii. 876.

INFANTILE SPLENiC ANEMIA, iv. 755.

Infantilism, Pancreatic, iii. 301.

INFECTED MATERIAL, particles from, as a cause of cerebral embolism, iv. 400.

INFECTED TISSUEs, effect of radiant light and heat upon, i. 446; electrotherapeutics applied to, i. 398.

INFECTION, of the extremities, electrotherapeutics for relief of, i. 406; foci of, as cause of acute endocarditis, iii. 744; radiant light and heat in treatment of intestinal, i. 453; theory of, in causation of beri-beri, iv. 849 .

INFEctions, acute, causing nephritis, iv. 57; acute, causing neuritis of third, fourth, and sixth nerves, iv. 308; causing nephritis, iv. 57; causing neuralgia, iv. 337; chronic, as cause of nephritis, iv. 57; extension of, causing secondary sinus thrombosis, iv. 426; localized, radiant light and heat in treatment of, i. 449; localized, thermic effects of electricity upon, i. 393; simple inflammatory, radiant light and heat in treatment of, i. 455; treatment of, for prevention of multiple neuritis, iv. 325 .

InfeCtious DiarRheAs. See Diarrheas.

INFECTIOUS DISEASES, acute, in causation of acute hemorrhagic encephalitis, iv. 404; acute, in causation of multiple neuritis, iv. 322 ; acute, in causation of multiple sclerosis, iv. 280; acute dilatation of stomach accompanying, iii. 175; acute myocarditis due to, iii. 747 ; acute vasomotor paralysis in, iii. 752; in causation of acute myelitis, iv. 220; in causation of acute ophthalmoplegia, iv. 508 ; in causation of arteriosclerosis, iii. 756 ; in causation of cerebral hemorrhage, iv. 388 ; in 
causation of facial paralysis associated with disease of temporal bone, iv. 311 ; in causation of marantic sinus thrombosis, iv. 426 ; in causation of myotonia congenita, iv. 267; in causation of pyelitis, iv. 95; in causation of sciatica, iv. 298 ; in causation of serous meningitis, iv. 468 ; in causation of syringomyelia, iv. 277 ; chronic, in causation of neuritis, iv. 322 ; as complication of measles, ii. 161; hemoglobinuria associated with, iv. 4; liability to, during whooping-cough, ii. 175 ; relation between anterior poliomyelitis and, iv. 235; relation between Friedreich's ataxia and, iv. 214; transmission of, by diseased meat, ii. 637.

INFECTIOUS SINUS THROMBOSIS, iv. 427; etiology of, iv. 427 ; microörganisms present in, iv. 427; pathology of, iv. 428 ; prognosis of, iv. 429; prophylaxis against, iv. 429 ; symptoms of, iv. 428 ; surgical treatment of, iv. 429.

INFECTIOUS TYPE OF INFLAMMATION, radiant heat and light in treatment of, i. 448.

INFLAMMATION ARISING FROM TOXEMIA, radiant light and heat in treatment of, i. 454.

INFLAMMATION NOT ASSOCIATED WITH INFECTION OR TOXEMIA, radiant light and heat in treatment of, i. 455.

INFLAMMATION, SIMPLE, electrotherapeutics for relief of, i. 409 ; indications for electricity in, i. 411 ; origin of, i. 409 ; reflex muscular spasm associated with, i. 412; rules for treatment of, i. 412.

INFLAMMATION OF SOFT CEREBRAL MEMBRANES, iv. 367 : acute cerebral meningitis, iv. 368; chronic cerebral meningitis, iv. 378 ; epidemic cerebrospinal meningitis, iv. 373 ; introduction to subject, iv. 367 ; microörganisms present in, iv. 368 ; tuberculous meningitis, iv. 373 .

INFLAMMATION, TUBERCULOUS, thermic effects of electricity in, i. 393.

INFLAMMATORY CONDITIONS NOT DUE TO INFECTION, thermic effects of electricity in, i. 393.

INFLOENZA, ACUTE, ii. 184; bacteriology of, ii. 184; bronchiectasis complicating, ii. 189 ; bronchitis complicating, ii. 189; cardiocirculatory complications of, ii. 193; chronic influenza complicating, ii. 194; classification of, ii. 188; complications of, ii. 191; convalescence of, ii. 196 ; diet in, ii. 188; gastrointestinal type of, ii. 189; general treatment of, ii. 187; immunity conferred by, ii. 186; joint affections complicating, ii. 194; meningitis complicating, ii. 193; mortality from bronchopneumonia as a complication of, ii. 192; nephritis complicating, ii. 194; nervous type of, ii. 190; pneumonia complicating, ii. 191; prophylaxis in, ii. 185; rare complications of, ii. 194; respiratory type of, ii. 188; serum treatment of, ii. 186; sources of, ii. 185; specific treatment of, ii. 186; summary of subject, ii. 196; symptoms of, ii. 187; temperature in, ii. 188; treatment of, ii. 186; treatment of types of, ii. 188; types of, ii. 188.

INFLUENZA, CHRONIC, as a complication of the acute form, ii. 194; treatment of, ii. 195.

INFLUENZAL MENINGITIS, ii. 193, 212.

INFRAORBITAL FORAMEN, alcohol injection at, for relief of trigeminal neuralgia, iv. 344.

Infusion, intravenous, use of salt solution by, in acute vasomotor paralysis, iii. 753 .

Inhalation, of alkalies, for relief of laryngeal complications of measles, ii. 157; solutions available for use by, ii. 497 ; use of aromatic expectorants by, in chronic bronchitis with profuse expectoration, iii. 377.; use of drugs by, in whoopingcough, iii. 180.

Inhalation spray, method of using, in acute laryngitis, iii. 339.

Inhalations, aromatic, use of, in bronchiectasis, iii. 396; fuming, use of, in asthma, iii. 386; of oxygen, see Oxygen; for relief of bronchitis complicating tuberculosis, ii. 508; for relief of cough in chronic bronchitis, iii. 375 ; for relief of cough in pulmonary tuberculosis, ii. 497; for relief of inflammation of larynx, ii. 51; for relief of laryngotracheal cough in measles, ii. 153 ; steam, use of, for relief of expectoration in tuberculosis, iii. 498; use of, in laryngeal tuberculosis, ii. 492; use of, in pulmonary abscess and gangrene, iii. 441; use of, in respiratory type of measles, ii. 189.

Inhaler, use of, to relieve cough in chronic bronchitis, iii. 375 .

INHIBITORY HEART FIBERS, iii. 537. 
INHIBITORY NERVE FIBERS OF HEART, iii. 537.

Injection, administration of alcohol by, in diabetic neuritis or neuralgia, ii. 766 ; administration of mercury by, in syphilis, ii. 390; of mercury, by insoluble method in syphilis, ii. 394; of mercury, by soluble method in syphilis, ii. 392 ; of nose and throat, in nasal and pharyngeal complications of measles, ii. 156.

Injection, hypodermic, administration of quinin by, iv. 723; advantages of, as mode of giving medicine in cardiac asthma, iii. 737; of alcohol, in cardiac failure during influenza, ii. 193; of antianthrax serum, ii. 332 ; of arsenobenzol, in destruction of filaria bancrofti, iv. 788; of caffein, in bronchopneumonia, iii. 421; of caffein, in nephritis, iv. 50; of caffein, in toxemia complicating acute diffuse peritonitis, iii. 318 ; of caffein and camphor, iii. 410; of Calmette's serum, in snake bite, iv. 682 ; of camphor, in bronchopneumonia, iii. 421; of camphor, in cardiac failure complicating influenza, ii. 193; of camphor, in cardiac toxemia during pneumonia, ii. 253; of camphor, in pulmonary thrombosis, iii. 411; of camphor, in toxemia complicating acute diffuse peritonitis, iii. 318; of collargolum, in typhus fever, ii. 37 ; of dextrose, in diabetic coma, ii. 758; of electrovargol, in typhus fever, ii. 37 ; of ether, in cardiac failure during influenza, ii. 193; of food, i. 640 ; of food, in tetanus, ii. 350; of human serum, in chronic pancreatitis, as prophylactic against hemorrhage on operation, iii. 300; of levulose, in diabetic coma, ii. 758; method for, in use of defibrinated blood, iii. 831 ; of organic compounds of arsenic, in pernicious anemia, iii. 828 ; of oxygen, in pneumonia, ii. 237; of Rogers' hypertonic solution, in Asiatic cholera, iv. 834 ; of saline solution, in bronchopneumonia complicating influenza, ii. 192 ; of saline solution, in meat poisoning, ii. 639 ; of saline solution, in mumps, ii. 171; of saline solution, in poisoning by amanita phalloides, ii. 649; of saline solution, in typhoid fever, ii. 19; of salts of radium, i. 466; of tetanus antitoxin, ii. 344 ; of tuberculin, ii. 517.
Injection, intracerebral, of tetanus antitoxin, ii. 345 .

Injection, intralaryngeal, of guaiacol, menthol and (amphor in laryngeal tuberculosis, iii. 347; in laryngeal tuberculosis, ii. 492, 493; solution for use by, ii. 494 .

Injection into nerve trunk, for relief of pain in sciatica, iv. 300; cases benefited by, iv. 300 ; place for, iv. 301 ; results of, iv. 303 ; solutions for, iv. 300 ; technique of, iv. 303.

Injection, intramuscular, administration of quinin by, iv. 723 ; of arsenobenzol, in syphilis, ii. 416; of arsenobenzol, in syphilis of upper respiratory tract, iii. 348 ; of arsenobenzol, in tropical relapsing fever, iv. 760 ; of atoxyl, in kala-azar, iv. 754; of caffein salts, in nephritis, iv. 50; of defibrinated blood, iii. 83; of insoluble salts of mercury, ii. 394 ; of mercury, in brain syphilis, iv. 479,480 ; of mercury, in syphilitic disease of liver, iii. 265; objections to administration of mercury by, in hereditary syphilis, ii. 433 ; objections to administration of mercury by, in pregnancy, ii. 432; of quinin in acid solution, in kala-azar, iv. 754; of sodium cacodylate, in pernicious anemia, iii. 827; of soluble salts of mercury, ii. 392 ; of tetanus antitoxin, ii. 345, 346; of thiosinamin, to promote absorption in pleurisy, iii. 482 .

Injection, intraperitoneal, of epinephrin, in generalized form of chronic peritonitis, iii. 323 ; of normal saline solution, in Asiatic cholera, iv. 834; of Rogers' hypertonic solution, in Asiatic cholera, iv. 834.

Injection, intrapleural, to aid absorption, iii. 482 .

Injection, intratracheal, in bronchiectasis, iii. 397 ; in chronic atrophic laryngitis, with involvement of trachea, iii. 343 ; use of guaiacol by, iii. 397 .

Injection, intravenous, of adrenalin chlorid in pneumonia with cardiac toxemia, ii. 252; advantages of, as mode of medication in cardiac asthma, iii. 737; of antianthrax serum, ii. 332; of arsenobenzol, for destruction of filaria bancrofti, iv. 788 ; of arsenobenzol, in syphilis, ii. 415, iv. 483; of arsenobenzol, in 
syphilis of upper respiratory tract, iii. 348; of arsenobenzol, in tabes, iv. 194; of arsenobenzol, in tropical relapsing fever, iv. 759; of atoxyl, in destruction of filaria bancrofti, iv. 788; of calcium lactate, in tetany, iii. 910; of Calmette's serum, in snake bite, iv. 682 ; of defibrinated blood, iii. 831; of electrovargol, in typhus fever, ii. 37; of epinephrin, in acute diffuse peritonitis, iii. 318 ; of gelatin, in hemophilia, iii. 864; importance of, seen in septicopyemia, ii. 289; medication by means of, in malignant endocarditis, iv. 746; of saline solution, in acute diffuse peritonitis, iii. 318; of saline solution, in bronchopneumonia complicating influenza, ii. 192; of saline solution, in meat poisoning, ii. 639; of saline solution, in posthemorrhagic anemia, iii. 810; of saline solution, in sepsis complicating scarlet fever, ii. 128; of saline solution, in sunstroke, iv. 678; of saline solution, in typhoid fever, ii. 19; of Schlippe's salt, in trypanosomiasis, iv. 747 ; of sodium bicarbonate, in diabetic corna, ii. 757 ; of strophanthin, for pulmonary edema, iii. 410; of strophanthin, in toxemia complicating acute diffuse peritonitis, iii. 318 ; of strophanthus, in pneumonia with cardiac toxemia, ii. 253; of tartar emetic, in trypanosomiasis, iv. 747; of tetanus antitoxin, ii. 345 ; use of digitalis bodies by, iii. 577; use of, in Fischer's method of relieving anuria, iv. 838; use of quinin by, suggested in kala-azar, iv. 754; use of strophanthin by, iii. 600 .

Injection, rectal, administration of quinin by, iv. 721, 724; of saline solution, in Asiatic cholera, iv. 833; of saline solution, in delirium tremens, ii. 602; of saline solution, in hemorrhage during pulmonary tuberculosis, ii. 502; of saline solution, in mumps, ii. 171; of saline solution, in sunstroke, iv. 678; of sodium carbonate, in diabetic coma, ii. 757 ; use of, in enterocolitis complicating measles, ii. 161; use of, in Fischer's method of relieving anuria, iv. 838; use of small, in enteritis of infancy, iii. 203.

Injection, subcutaneous. See Injection, hypodermic.
Injection, tracheal, solution for use by, ii. 494; use of, in laryngeal tuberculosis, ii. $492,493$.

Injections, alcoholic. See Alcohol injections. Injections, epidural, use of, for relief of pain in sciatica, iv. 296, 300; subarachnoid, use of cocain by, in radicular sciatica, iv. 296; urethral, use of, in chronic gonorrheal urethritis, iv. $=155$; vaginal, use of, for relief of oxyuris vermicularis, iii. 253.

Inoculation against cholera, iv. 829; against plague, i. 142 , iv. 823 ; against rabies, see Pasteur treatment; against scarlet fever, ii. 123; against small-pox, ii. 52 .

INORGANIC GASES, poisoning by, i. 723 .

INORGANIC subSTANCES, as a food stuff, i. 600.

INosite, use of, to prevent acidosis in diabetes mellitus, ii. 757 .

INOTROPIC INFLUENCES on cardiac muscle, iii. 537 .

INSANE, PRogressive PARALYSIS of, iv. 489; differential diagnosis in, iv. 491; etiology of, iv. 489; hygienic measures in, iv. 493; pathology of, iv. 489; physical signs of, iv. 490; prognosis in, iv. 491; prophylaxis against, iv. 492; stages of, iv. 490 ; symptoms of, iv. 489 ; treatment of, iv. 492.

InsECTs, possible transmission of beri-beri by, iv. 849; transmission of leprosy by, iv. 811 ; transmission of tuberculosis by, ii. 455 .

Insolation, iv. 672; classification of, iv. 672 ; climatic relations of, i. 272 ; determination of, ii. 248; etiology of, iv. 672; hydrotherapy for relief of, i. 228; prophylaxis against, iv. 676; treatment of, iv. 677 .

INsomNIA, accompanying pernicious anemia, iii. 838; associated with mediastinal tumor, iii. 504; in chronic bronchitis, due to coughing, iii. 374 ; dietetic treatment of, in neurasthenia, iv. 564; hydrotherapy for relief of, i. 223; measures relating to, in Pasteur treatment of rabies, ii. 339; physical agents for relief of, in neurasthenia, iv. 564; relief of, in acute rheumatism, ii. 302 ; relief of, in after-cure of morphin habit, ii. 624; relief of, in brain tumor, iv. 447; relief of, in cerebral abscess, iv. 423; relief of, in 
cerebral anemia, iv. 383 ; relief of, in chorea minor, iv. 663; relief of, in delirium tromens, ii. 605$), 607$; relief of, in multiple neuritis, iv. 327 ; reliof of, in nervous type of influenza, ii. 191; relief of, in neurasthenia, iv. 5(j3); relief of, in tuberculosis, ii. 50:; rolief of, in typhoid fever, ii. 26 ; ts a symptom of ehronic cerobral hyperemia, iv. 387; due to use of eaffein in nephritis, iv. 50 .

Inspection, value of, in diagnosis of brain tumor, iv. 444.

Instillations, in chronic gonorrheal urethritis, iv. 159 ; technique of, iv. 160 ; uses of, in general, iv. 161.

Institutions, treatment of epilepsy in, iv. 645.

Instructions to PATIEnTs, for use in acute urethral gonorrhea, iv. 142.

INSTRUMENTS, aseptic preservation of, iv. 159 ; employed in instillation of urethra, iv. 159 ; lubrication of, for catheterization, iv. 158; sterilization of, for irrigation with eatheter, iv. 158.

InTENsity, as a factor in an X-ray treatment, i. 497.

INTERCostal NEURALGIA, massage and gymnastic exercises in treatment of, $i$. 310 ; treatment of, in general, iv. 357.

INTERCURRENT DISEASES, associated with chronic myocardial insufficiency, iii. 741.

INTERTRIGo, due to chronic myocardial insufficiency, iii. 740 .

Intermittent treatment, administration of mercury by, in syphilis, ii. 417 .

INTERNAL SECRETION OF KIDNEYS, iv. 13.

INTERRUPTERS, i. 368.

INTESTINAL ADHESIONS, as a cause of pain in bladder, iv. 122.

Intestinal amebiasis. See Amebiasis, intestinal.

Intestinal antisepsis, use of, in chlorosis, iii. 815 .

Intestinal antiseptics. See Antiseptics.

InTESTINAL AUTOINTOXication, ii. 664; alcoholism associated with, ii. 683; anemias associated with, ii. 680 ; causes of, ii. 665 ; circulatory disorders associated with, ii. 679 ; cutaneous disorders associated with, ii. 682 ; definition of term, ii. 664 ; diet in treatment of, ii. 668,677 , 683 ; dipsomania associated with, ii. 683 ; elimination in treatment of, ii. 671 ; ex- ercise in treatment of, ii. 676 ; habits of lifo in causation of, ii. 675 ; habitual (onstipation associated with, ii. 677; intestinal antiscotics in treatment of, ii. 674; liver disorders associated with, ii. 679; motabolic disorders associated with, ii. (ist); nervous disorders associated with, ii. 681; ocular affections assoriated with, ii. 6R2; respiratory disorders associated with, ii. 68I; treatment of, ii. 6677; treatment of conditions and symptoms associated with, ii. 675 .

Intestinal AUtOINTOXICATION, CHRONic, as a cause of seiatica, iv. 299.

InTESTINAL CIRISE, in tabes, iv. 191.

InTESTINAL DISTOMIASIS, iv. 780 ; preventive measures in, iv. 782 ; remedial measures in, iv. 782 ; species of, iv. 780 .

Intestinal elimination, value of, in treatment of exophthalmic goiter, iii. 900 .

INTESTINAL INDIGESTION, as a cause of enuresis, iv. 132.

INTESTINAL NEUROSES, iii. 249.

Intestinal obstruction, iii. 237 ; acute, iii. 238 ; chronic, iii. 242 ; fecal impaction as cause of, iii. 238; intussusception as cause of, iii. 241 ; volvulus as cause of, iii. 241.

INTESTINAL PARASITES, iii. 250: ascaris lumbricoides, iii. 250; as cause of enuresis, iv. 130 ; echinococcus disease, iii. 255 ; oxyuris vermicularis, iii. 252 ; poisoning due to presence of, in food, ii. 634 ; trichiniasis, iii. 253 ; tricocephalus dispar, iii. 255.

INTESTINAL PARESIS, use of extract from posterior lobe of hypophyseal gland for relief of, i. 109.

Intestinal peristalsis, methods for stimulating, in chronic constipation, iii. 235.

INTESTINAL schistosomiasis, iv. 785 ; preventive measures in, iv. 786 ; remedial measures in, iv. 785 .

Intestinal TENiAsis, iv. 776; preventive measures in, iv. 779 ; remedial measures in, iv. 778 ; varieties of, iv. 776.

INTESTINE, removal of fluid by, in cardiac insufficiency with general anasarca, iii. 678 .

Intestine, small, catarrh of. See Enteritis, chronic.

INTESTINEs, changes in, during hypnosis, i. 574 ; disturbances of, in measles, ii. 160; disturbances of, due to rachitis, ii. \$1S; perforation of, in typhoid fever, ii. 26; 
puncture of, for relief of meteorism in typhoid fever, ii. 26.

InTESTINES, DISEASES OF, iii. 193: acute appendicitis, iii. 213; acute enteritis, iii. 193; acute intestinal obstruction, iii. 237; appendicitis, iii. 212 ; appendicitis in pregnancy, iii. 220; appendicitis in typhoid fever, iii. 219; cholera infantum, iii. 204; chronic appendicitis, iii. 212; chronic enteritis, iii. 196; chronic intestinal obstruction, iii. 242 ; chronic mucous colitis, iii. 206; colitis, iii. 205; constipation, iii. 221; enteritis, iii. 193; enteritis in infancy, iii. 201; enterospasm, iii. 231 ; infectious diarrhea, iii. 204; intestinal obstruction, iii. 237 ; intussusception, iii. 241; membranous enteritis, iii. 207; mucous colic, see Membranous enteritis; spastic constipation, iii. 231 ; ulcerative colitis, iii. 211; volvulus, iii. 241.

Intoxication, alcoholic, see Alcoholism; bromid, see Bromid intoxication; endogenous, in causation of multiple sclerosis, iv. 280; general, following intramuscular injection of mercury for syphilis, ii. 403; hydrotherapy for relief of alcoholic, i. 228; intestinal, definition of term, ii. 664; morphin, see Morphin habit.

Intoxications, causing hemoglobinuria, iv. 4 ; causing neuralgia, iv. 337 ; causing neuritis of third, fourth, and sixth nerves, iv. 308; chronic, importance of, in etiology of arteriosclerosis, iii. 755 .

INTRAABDOMINAL SUPPORT, deficiency of, a cause of movable kidney, iv. 1.

INTRACELLULAR FERMENTS, decomposition produced by, in animal tissues and effects of, ii. 636 .

INTRACEREBRAL HEADACHE, iv. 608: brain tumor, iv. 608; hydrocephalus, iv. 609.

INTRACRANIAL DISEASE, causing neuritis of seventh nerve, iv. 310; causing optic neuritis, iv. 305 .

Intracranial gasserian operation, for relief of trigeminal neuralgia, iv. 354 .

Intradural incision, of posterior spinal nerve roots in tabes, iv. 198.

Intralaryngeal applications. See Applications.

Intramuscular injection. See Injection, intramuscular.

INTRAPELVIC DISEASE, sciatica from, iv. 298.
INTRAPELVIC NEURITIS, electrotherapeutics for relief of, i. 416.

Intraperitoneal injection. See Injection, intraperitoneal.

Intrapleural injections. See Injection, intrapleural.

INTRATHORACIC PRESSURE OF PLEURAL EXUDATES, iii. 459, 460.

Intratracheal injections. See Injection, intratracheal.

Intravenous injection. See Injection, intravenous.

INTRAVENTRICULAR HEART BLOCK, iii. 566 .

Intubation of larynx, indications for, in diphtheria, ii. 274; indications for, in laryngeal complications of measles, ii. 158; indications for, in laryngeal tuberculosis, ii. 494 ; indications for, in laryngismus stridulus complicating rachitis, ii. 820 ; indications for, in membranous laryngitis, iii. 343 ; indications for, in pneumogastric paralysis, iv. 320 ; indications for, in stenosis of larynx, iii. 349; method of performing, ii. 274; possibility of, in acute laryngitis, iii. 340 ; possibility of, for edematous laryngitis, iii. 341 ; reinsertion of tube in, after removal, ii. 275; removal of tube in. ii. 274.

InTUSSUSCEPTION, iii. 241.

INuLIN, as substitute for vegetable starch in diabetes mellitus, ii. 749 .

Inunction, administration of mercury by, in syphilis, ii. 387; administration of quinin by, iv. 724 ; employment of, in scarlet fever, ii. 124 .

INVALID BROTHS, i. 647.

INVALID's DIETARY, i. 645: arrowroot, i. 649; barley water, i. 645 ; beef juice, i. 647 ; beef pulp, i. 647 ; beef tea, i. 646 ; boiled rice, i. 649 ; bread pudding, i. 651 ; caudle, i. 649 ; chicken broth, i. 646 ; chicken jelly, i. 646 ; cocoa, i. 651 ; cocoa junket, i. 650 ; cocoa with milk, i. 651 ; coffee, i. 652; custard, baked, i. 651; custard, soft, i. 650; egg albumin water, i. 648; egg flip, i. 648; egg-nog, i. 648; invalid broths, i. 647; junket, i. 650; lemonade, i. 652; oatmeal gruel, i. 645; orangeade, i. 652 ; rennet custard, i. 650 ; rice pudding, i. 649 ; rice and egg pudding, i. 649; savory custard, i. 648 ; toast water, i. 646; vanilla ice cream, i. 651. 
Iodid of ammonium. See Ammonium iodid. Iodid of calcium. See Calcium iodid.

Iodid of iron, use of, in glandular enlargement accompanying rachitis, ii. 818 ; in mumps, ii. 170 ; syrup of, in chronic nasopharyngitis, iii. 32 .

Iodid of potash. See Potassium iodid.

Iodid of potash ointment, use of, in mumps, ii. 170 .

Iodid of rubidium. See Rubidium iodid.

Iodid of sodium. See Sodium iodid.

Iodid of sodium and chloral, use of, to relieve dyspnea due to pressure from a mediastinal tumor, iii. 503.

Iodids, use of, in actinomycosis, iii. 291; in arteriosclerosis, iii. 761; in arteriosclerotic vertigo, iv. 624 ; in atrophic rhinitis, iii. 330 ; in cardiac neurasthenia, iii. 778; in chronic cerebral meningitis of syphilitic origin, iv. 378 ; in chronic interstitial nephritis, iv. 71 ; in chronic myocardial insufficiency due to syphilis, iii. 730; in chronic ophthalmoplegia, iv. 510 ; contraindicated in pulmonary edema, iii. 411 ; in delayed resolution of pneumonia, ii. 258; in diabetes insipidus with syphilitic taint, ii. 777 ; as expectorants in chronic bronchitis, iii. 372 ; in hereditary syphilis, ii. 434; in migraine, iv. 595 ; to promote absorption after cerebral hemorrhage, iv. 393 ; to promote absorption in pleurisy, iii. 470 ; in progressive paralysis of the insane, iv. 493; in pscudobulbar paralysis, iv. 504; in radicular sciatica, iv. 295; to relieve heart block associated with syphilis, iii. 772 ; in syphilitic arthritis, ii. 306 ; in syphilitic gastritis, iii. 167; in tuberculosis of mediastinal lymph nodes, iii. 507 ; in tumor or cyst of liver, iii. 291.

Iodids, organic, use of, in syphilis, ii. 410.

Iodin, presence of, in thyroid gland, i. 78; relation of, to physiological activity of thyroid gland, i. 79.

Iodin, local use of, in abscess complicating tuberculosis, ii. 509; in atrophic rhinitis, iii. 332 ; in cervical adenitis in scarlet fever, ii. 131; in chronic glossitis, iii. 25; in chronic nasopharyngitis, iii. 32 ; in chronic rhinitis, iii. 329 ; in climatic buboes, iv. 864 ; in echinococcus disease, iii. 256; for enlargement of spleen in malaria, iv. 728 ; in goiter, iii. 893,901 ; in inflammation of labial glands, iii. 6; in laryngeal complications of tuberculosis, ii. 493, 494; in leukoplakia, iii. 26 ; in month disorders in diabetes mellitus, ii. 736; in mycosis of tonsils and uvula, iii. 42; over spine in lathyrism, iv. 693 ; in pyorrhea alveolaris, iii. 22 ; for relief of cough in tuberculosis, ii. 497 ; for relief of pain in chest in tuberculosis, ii. 506; for relief of pain in pleurisy, iii. 468 ; for relief of pharyngeal cough, ii. 495 ; in salivary deposits, iii. 29 ; in small-pox, ii. 48; in stomatitis due to hydrargyrism, ii. 38.5; in stomatitis herpetica, iii. 10; in struma inflammatoria, iii. 891 ; in symptomatic parotitis, iii. 28; in thyroiditis acuta, iii. 891 .

Iodin, use of, as disinfectant for site of puncture in thoracentesis, iii. 474 ; by hypodermic injection, in ozena, iii. 337 ; by hypodermic injection, to promote closure of pancreatic fistula, iii. 307 ; by hypodermic injection, in syphilis of upper respiratory tract, iii. 349 ; injection of, into thyroid gland, objections to, iii. 894; internally, for enlargement of lymphatic glands in measles, ii. 162; internally, in goiter, iii. 894,901 ; internally, for nephritis complicating scarlet fever, ii. 133; internally, in reduction of obesity, ii. 308; for irrigation of pleural cavity, hemiplegia following, iii. 487.

Iodin, use of, in syphilis, ii. 404; absorption of, ii. 405; action of, ii. 404; administration of, by mouth, ii. 407; dosage of different preparations of, ii. 408; elimination of, ii. 405; for gummata, ii. 430; hypodermic administration of, ii. 408; ill effects of, ii. 406 ; for mucous patches, ii. 427; for papular and tubercular tertiary lesions, ii. 426; preparations of, ii. 407; prophylaxis against iodism in, ii. 406 ; proprietary preparations of, unreliable, ii. 407,409 ; rectal administration of, ii. 40s; in syphilitic bone lesions, ii. 430; in syphilitic joint lesions, ii. 430 ; treatment of iodism, ii. 406 ; value of, ii. 404 .

Iodin and glycerin, local use of, in atrophic rhinitis, iii. 332 .

Iodin and oil of cloves, local use of, in ozena, iii. 337 . 
Iodin prepared with gelatin, use of, in atrophic rhinitis with goiter, iii. 330 .

Iodin-vasogen, use of, in local treatment of gummata, ii. 430 .

Iodipin, use of, as expectorant in chronic bronchitis, iii. 372 ; in syphilis, ii. 410.

IoD I SM, ii. 406; caused by the action of hydrochloric acid upon iodids, iii. 167.

Iodoform, use of, as dressing for sores in frambœsia tropica, iv. 765; as dusting powder for gangrenous chancre, ii. 423; as dusting powder for mucous patches on genitalia, ii. 427 ; in frambœsia tropica, iv. 764 ; by insufflation, in laryngeal tuberculosis, iii. 347 ; by insufflation in syphilis of upper respiratory tract, iii. 349 ; in local treatment of laryngeal tuberculosis, ii. 493; as suppositories for rectal chancre, ii. 424 ; in tropical phagedenic ulcer, iv. 868 .

Iodoform gauze tampon, use of, for control of epistaxis, iii. 335.

Iodolena, hypodermic injection of, in atrophic rhinitis, iii. 330 .

Iodopetrogen, use of, in chronic parotitis, iii. 28 ; in goiter, iii. 894 .

IoDOTHYRIN, i. 82 ; use of, in athyrea and hypothyrea, iii. 907.

Iodovasogen, use of, in goiter, iii. 894.

IONIC CONCENTRATION, as basis of standardization in pharmacology, i. 11.

Ionization, process of, i. 396.

Ipecac, use of, in after-treatment of liver abscess, iv. 711; in bacillary dysentery, ii. 326 ; on cultures of amebæ followed by lethal action, iv. 706; in dysentery, iv. 708; as emetic in acute alcoholism, ii. 589; as emetic in acute bronchitis, iii. 364 ; as expectorant in chronic bronchitis, iii. 372; in hepatic abscess, iv. 711; in prophylaxis of amebiasis, iv. 710 ; in sprue, iv. 862 ; in ulcerative colitis due to Balantidium coli, iv. 762 .

Ipecac, syrup of, use of, in acute laryngitis of children, iii. 340 ; in spasmodic laryngitis, iii. 342 ; in stomatitis herpetica, iii. 9.

Ipecac and cinchonin, use of, in acute laryngitis of young children, iii. 339 .

Ipecac and squills, use of, in pill form for control of cough in chronic bronchitis, iii. 375 .
Ipecac treatment in dysentery, liver abscess limited by, iii. 290.

Ipecacuanha, use of, in bronchial asthma, iii. 389 ; to induce emesis in acute gastritis, iii. 154; in plastic bronchitis, iii. 392.

Ipecacuanha wine, use of, as spray to control profuse expectoration in chronic bronchitis, iii. 378; in stomatitis herpetica, iii. 9 .

IPOH AKER, iv. 694.

IRELAND, antituberculosis work in, ii. 441.

Irish-Roman baths, use of, in hydrotherapy, i. 201 .

Iron, use of, in Addison's disease, iii. 882; in after-treatment for chronic opium poisoning, ii. 620; in after-treatment of herpes zoster, iv. 359 ; in amyloid kidney, iv. 81; in amyxorrhea gastrica, iii. 101 ; in anemia associated with chronic myocardial insufficiency, iii. 742; in anemia of tuberculosis, ii. 507 ; in bronchial asthma, iii. 385; in bronchoadenitis, iii. 398 ; in chlorosis, iii. $812,815,816$; in chlorosis, baths and,' iii. 820; in chlorosis, mineral springs and, iii. 820; in convalescence from diphtheria, ii. 275; in convalescence from glandular fever, ii. 577; in convalescence from influenza, ii. 196; in convalescence from pneumonia, ii. 262; in epidemic dropsy, iv. 860; formulæ for, in chlorosis, iii. 818; in hay fever, iii. 390 ; in hookworm disease, iv. 794; by hypodermic injection in chlorosis, iii. 820 ; with intestinal antiseptics in chlorosis, iii. 820 ; in nephritis, iv. 289 ; in neuritis, iv. 289 ; in occupation pareses, iv. 332 ; in optic neuritis without apparent cause, iv. 306 ; in organic compounds, in chlorosis, iii. 819; in post-diphtheritic paralysis, ii. 278 ; in rachitis, ii. 818; to relieve anemia in acute nephritis, iv. 63 ; in secondary anemias, iii. 810 ; in tabes, iv. 213 ; in treatment of enuresis, iv. 133.

Iron and arsenic, use of, hypodermically, in convalescence from acute endocarditis, iii. 746 .

Iron and strychnin, use of, in neurasthenia, iv. 558 .

Iron chlorid, use of, to control epistaxis, objectionable, iii. 335 . 
Iron compounds, organic, use of, in chlorosis, iii. 819.

Iron, iodid of. See Iodid of iron.

Iron persulphate, use of, in acute nasopharyngitis accompanied with uvulitis, iii. 31.

Iron, salts of, use of, in neuralgic headache, iv. 598 .

Iron somatose, use of, in chlorosis, iii. 819.

Iron, syrup of lodid of, use of, in chronic rhinitis of children, iii. 328 ; in tuberculosis of mediastinal glands, iii. 507.

Iron, tincture of chlorid of, use of, in acute tonsillitis, iii. 41 ; in membranous laryngitis, iii. 343 ; in membranous pharyngitis, iii. 840; in pneumonia, ii. 234; for relief of bloody vomit in yellow fever, iv. 856 ; in scarlet fever, ii. 125 ; in stomatitis ulcerosa, eombined with potassium chlorate, iii. 16; in subsiding stage of acute rhinitis, iii. 328 ; in throat affections complicating scarlet fever, ii. 130.

Iron waters, action of, i. 236 ; classification of, i. 233.

IroN wiRe, introduction of, in aneurysm, iii. 767.

Irrigation, in acute nasopharyngitis, iii. 31; in acute rhinitis, iii. 327 ; of adenoid vegetations, iii. 38; of bladder, to lessen vesical irritability in tuberculous kidney, iv. 117; of bladder, for relief of endemic hematuria, iv. 785; of colon, see Colonic flushing; in diverticula of esophagus, iii. 62 ; hot, in acute peritonsillitis, iii. 43; hot, in acute uvulitis, iii. 35 ; hot, in phlegmonous pharyngitis, iii. 40; with hydrogen peroxid in membranous pharyngitis, iii. 39 ; of intestines, in bacillary dysentery, ii. 327 ; of nasopharynx, in diphtheria, ii. 272 ; of nasopharynx in diphtheria, a cause of otitis media, ii. 277 ; of pleural cavity, for empyema, iii. 487; of rectum, in tricocephalus, iv. 789 ; of the tissues in septicopyemia, ii. 290; of urethra, in chronic gonorrheal urethritis, iv. 157; of urethra, in connection with massage of prostate, iv. 164; of vagina, in diabetic vulvitis, ii. 765 .

Irrigating solutions, asepsis of, iv. 159.

IRRITANTs, prohibition of, in food or drink in chancre of lips, mouth, or tongue, ii. 425; use of, direet, as emetics, çontraindicated in acute gastritis, iii. 154.
Irritating drugs, employment of, to cause "abscess fixation" in pneumonia, ii. 246.

Imitation, sYMrтoms of, in acute cerebral moningitis, iv. 369; in brain tumor, iv. 436.

IRITIS, SYPHLLTIC, local treatment of, ii. 429.

Isolation, indieations for, in neurasthenia, iv. 535; in nembranous pharyngitis, iii. 39 ; necessity for, in anterior poliomyelitis, iv. 237.

Italians, predisposition of, to rachitis, ii. 815.

ITALY, introduction of vaccination into, ii. 59 ; trial of antianthrax serum in, ii. 332 .

Iтching, in acute nephritis with uremia, iv. 63 ; as a symptom of diabetes mellitus, ii. 764 .

ITCHING ERYTHEMAS, radiant light and heat in treatment of, i. 459.

Izal, use of, by intratracheal injection, for bronchiectasis, iii. 397 .

Jaboulay, excision of skull for relief of trigeminal neuralgia by, iv. 354 .

Jacket, plaster, use of, in Friedreich's ataxia, iv. 218 ; in sacroiliac sciatica, iv. 297.

Jacket, wooden, use of, in Friedreich's ataxia, iv. 218.

JACKSONIAN EPILEPSY, iv. 633.

JaIl fever. See Typhus fever.

JAKOWLEFF, on cholera "carriers," iv. 828.

Jalap, use of, in cirrhosis of liver, iii. 286; in nephritis, iv. 33.

Jalap, compound powder, use of, in pleurisy, iii. 469.

Jalap, rhubarb, and calomel, use of, in cerebral hyperemia, iv. 386 .

Jalap powder, use of, in wood alcohol poisoning, ii. 610 .

JANET, classification of phobias by, iv. 518; views held by, on hypnosis, iv. 570 .

JANET AND BerNheim, hypnotic suggestion employed by, in treatment of psychic disorders, iv. 570.

JAPAN, history of vaecination in, ii. 102.

JaPANese flood fever. See Shima mushi.

Japanese hand-stove, use of, in typhoid fever, ii. $23,28$.

Japanese river fever. See Shima mushi.

JAUNDice, iii. 260; acute catarrhal, iii. 260 ; associated with gallstones in 
transit, iii. 274; chronic eatarrhal, iii. 262.

Jaurez, Prof., theory held by, regarding delirium tremens, ii. 602.

Jefferson, President, interest taken in vaecination by, ii. 62 .

JENDRÁSSIK's PHENOMENON, iv. 187.

JENNER, discovery of vaceination by, i. 125, ii. 57; inoculation experiments by, ii. 92.

Jennings, Oscar, method employed by, for treatment of morphinism, ii. 623 .

JEQUIRITY SEED, poisonous elements in, of a proteid character, ii. 635 .

JESSEN AND RzewUski, compression apparatus employed by, in X-ray treatment of mediastinal tumor, iii. 502 .

Jesty, BenJamin, first inoculation of cowpox for prevention of small-pox by, ii. 57.

JEWISH RACE, liability to diabetes among, ii. 719 .

Jock-strap, iv. 138, 151.

JoHn of GadDesden, red light treatment of small-pox suggested by, ii. 51 .

Johnson, JAMEs, on elothing as a means of protection against plague, iv. 825 .

Joint movements, use of, in massage, i. 292.

Joint Puncture. See Puncture.

JoINTs, affections of, as complication of influenza, ii. 194; electrotherapeutics for relief of simple inflammation of, $i$. 412; electrotherapeutics for relief of toxic inflammation of, i. 408; hydrotherapy for relief of disturbances of, $i$. 226 ; local treatment for syphilitic lesions of, ii. 430; radiant light and heat in, for relief of toxic inflammation of, i. 455; use of thyroid gland in affections of, i. 76 .

Jones, Carmalt, results published by, of tuberculin treatment in bronchoadenitis, iii. 399.

JossErAND, experiments by, on transmission of tuberculosis by vaccination, ii. 90 .

JoUKOFFSKY, vaccination by, of healthy children from syphilitic children without transmission of syphilis, ii. 88.

JUGULAR VEIN, treatment of, in operation for thrombosis of lateral sinus, iv. 430 .

JUNG, association experiment employed by, in psychotherapy, iv. 579; on resemblance between hysteria and dementia precox, 584.
JuNkET, as article of invalid dietary, i. 650 .

JUNIPER BERRIES, use of, dangerous in nephritis, iv. 49 .

JÜRGENSEN, use of alcohol in pneumonia advocated by, ii. 230 .

JüsGEN, results of use of Eichhorst's formula for eream of tartar in cirrhosis of liver with ascites reported by, iii. 286 .

JUVENILE FORM OF MUSCULAR DYSTROPHY, iv. 261 .

Kafindo. See Onyalai.

KALA-AZAR, iv. 753; microörganism causing, iv. 753; preventive measures in, iv. 755 ; remedial measures in, iv. 753 .

Kamala, use of, as substitute for male fern, iv. 778 .

Kaolin, use of, in gastric hyperacidity, iii. 97.

KAPsAmmer, phloridzin injection test modified by, iv. 25 .

Karrell's diet, for reduction of obesity, ii. 795.

Katayama disease, iv. 786.

Katzenstein's METHOD, of determining extent of chronic myocardial insufficiency, iii. 694 .

Kaufmann, formula for nutrient enema recommended by, iii. $\mathbf{1 1 9 .}$

KaUsch, W., on ventricular puncture for relief of hydrocephalus, iv. 466.

KEAN, Dr., results of antityphoid vaccination in the U. S. Army reported by, ii. 4.

Kedani mite, iv. 692.

Keeley gold cure, ii. 600 .

Keen, W. W., on operative treatment of traumatic hematoma, iv. 366.

KEHR, HaNs, on operation for gallstones, iii. 276.

KEITH's NODE, iii. 540.

KElaiditis, claim by, for making antitoxic serum for whooping-cough, ii. 183.

KELLAR, investigations by, on causation of rachitis, ii. 815 .

Kelligrew, atrophic rhinitis treated with vibratory massage by, iii. 332 .

KellogG, rules given by, for abdominal massage, i. 323.

Kellogg's incandescent light baths, iii. 378 .

Kelly, H. A., on appendieitis in typhoid fever, iii. 219; eases of appendicitis classified by, with reference to operation, iii. 218 . 
Kesolds, radium in treatment of, i. 476 ; $\mathrm{X}$-ray therapy for relief of, i. 536.

Kemp's ReCtal, irrigatols, ii. 674.

KenNeby, carly report on small-pox inoc'ulation by, ii. 52 .

Keratosis buccalis. See Leukoplakia.

KERION OF sCALP, X-ray therapy for relief of, i. 533.

Kermes mineral, use of, as an expeetorant in pneumonia, ii. 257.

Kernig's sign, presence of, in acute cerebral meningitis, iv. 369 ; in delirium tremens, ii. 606; in traumatic hematoma, iv. 365 ; in tuberculous meningitis, iv. 374 .

Ketonuria, carbohydrates in treatment of, ii. 756.

Keyes instillator, iv. 160.

KIDD, case reported by, of mediastinal tumor relieved by simple incision and drainage, iii. 501.

Kidd, Benjamin, statistics given by, regarding production in tropies, iv. 670 .

KIDNey, dceapsulation of, in acute nephritis, iv. 61 ; decapsulation of, in toxemia of pregnaney, iv. 83; internal secretion of, iv. 13; passive congestion of, a cause of nephritis, iv. 29; substances excreted with difficulty by, iv. 59, 60 .

Kidney, amyloid disease of. See Amyloid disease of kidney.

KidNey, Contracted, association of, with chronic myocardial insufficiency, iii. 731; digitalis contraindicated in eardiac insufficiency due to, iii. 588; effect of strophanthin upon, iii. 579, 600 ; use of digitalis for gastrointestinal symptoms dependent upon, iii. 590.

Kidney, floating. See Kidney, movable.

KIDNEY, GOUTY, ii. 695.

Kidney, movable, iv. 1; eauses of, iv. 1; mechanical vibration in treatment of, i. 358 ; treatment of, iv. 3 .

Kidney of PREgnancy, iv. 82.

Kidney, tuberculous, iv. 112; complications in, iv. 117; cystoscopic examination in diagnosis of, iv. 114; diet for, iv. 116; early signs of, iv. 113; hygienic measures in treatment of, iv. 115; modes of infection in, iv. 112; operation for, iv. 114; prophylaxis against, iv. 118; symptomatic treatment of, iv. 117; treatment of, in general, iv. 112; tuber- rulin in diagnosis of, iv. 114; tubereulin in treatment of, iv. 116; urine findings in diagnosis of, iv. 113; X-ray in diagnosis of, iv. 114.

KidDEy ImLITANTs, avoidance of, in diseases that predispose to nephritis, iv. 59.

Kidney tests, functional, value of, iv. 23.

KidNeys, effect of caffein series upon, iii. 604 ; effects of $\mathrm{X}$-rays upon, i. 519; importance of attention to, in yellow fever, iv. 856; local treatment for syphilitic lesions of, ii. 431 ; measures for stimulating action of, in multiple neuritis due to lead poisoning, iv. 325; normal physiology of, iv. 10; pathological physiology of, iv. 14.

Kidneys, disorders of, due to arsenobenzol in treatment of syphilis, ii. 415; as a eomplication of diphtheria, ii. 279; as a complication of measles, ii. 161; due to hydrargyrism, ii. 385 ; hydrotherapy for, i. 209; influence of climate upon, i. 283.

Kienboeck, method employed by, for $\mathrm{X}$-ray treatment of mediastinal tumors, iii. 502.

Kienboeck and Adamson, modification by, of system of X-ray therapy for relief of tinea and favus, i. 532.

Kienboeck's quantimeter, for measuring intensity of X-ray, i. 479.

KilianI, on results of alcohol injection for trigeminal neuralgia, iv. $344,345,353$; on results of operation for trigeminal neuralgia, iv. 355 .

KilmarNock, mortality from small-pox in, before and after vaceination, ii. 96 .

Kilmer, method suggested by, for controlling paroxysms of whooping-cough, ii. 178.

KInK-COUGH. See Whooping-cough.

Kinnicut, ease of idiopathic dilatation of esophagus described by, iii. 57 .

KLEBS-LOEFFLer BACILLUS, presence of, in membranous rhinitis, iii. 329 ; in stomatitis diphtheritiea, iii. 20; in stomatitis gangrenosa, iii. 17, 18; in Vincent's angina, iii. 40.

KLEIN, post mortem evidence of poisoning from canned fish reported by, ii. 644 .

Klein and Durham, investigations by, showing difficulty in detecting microorganisms eausing food poisoning, ii. 636 . 
Kneading, use of, in massage, i. 290.

Knee-chest position, in acute dilatation of stomach, iii. 174; for administration of enemata in intestinal amebiasis, iv. 709.

KneE Jerk. See Tendon reflex.

KNOPF, on breathing exercises in chronic bronchitis, iii. 369 ; window tent devised by, for use in tuberculosis, ii. 480 .

KNopf's SCHEDUle of RULES, for protection of chíldren against tuberculosis, ii. 460 .

Koch, Robert, discovery of tuberculin by, i. 126 , ii. $509,510$.

Koch's bacillin emulsion, ii. 512, 513.

Koch's new tuberculin, ii. 512.

Koch's old tuberculin, ii. 512.

KolLE, vaccination by means of cultures killed by heat or antiseptics introduced by, i. 126.

Kolle's method, of inoculation against cholera, iv. 830.

Kollman dilator, iv. 162.

Kophoscoliosis, chronic myocardial insufficiency due to, iii. 712; treatment of, iii. 732 .

KöRNER, mortality after operation for infectious thrombosis reported by, iv. 430 ; statistics of mortality from operation for cerebral abscess published by, iv. 424 .

KoRSAKOw'S PSYCHOSIS, ii. 609; in multiple neuritis, iv. 323 .

Kousso, use of, as substitute for male fern, iv. 778 .

Koveski, on composition of sweat in nephritis, iv. 38.

Krameria, use of, for colonic flushing in colitis, iii. 205.

Kraske-Riedel's treatment of erysipelas, ii. 285.

Kraus's antitoxic serum, results from, in Asiatic cholera, iv. 832.

Kreatotoxismus. See Meat poisoning.

Kreuse, work by, on bacillus of dysentery, ii. 316.

Kromaier lamp, as source of radiant energy, i. 441.

KroneCKer, experiment by, on dog's heart, iii. 535 .

"Kropfherz," iii. 892.

Kryofin, use of, for relief of pain in neuritis, iv. 286.

KubISAGari, iv. 860; remedial measures in, iv. 861 .
KuliabKo, experiment by, on human heart, iii. 537.

Kussmaul, on chronic dilatation of stomach, iii. 176; congenital type of gastroptosis first clearly described by, iii. 181; report by, of syphilis transmitted by lancet used in vaccination, ii. 88 ; stomach tube introduced into medicine by, iii. 67 ; views held by, on use of lavage in gastric hyperacidity, iii. 97.

Kussmaul's BREathing, presence of, in diabetic coma, ii. 732 .

Labarraque's solution, use of, in colonic flushing for intestinal amebiasis, iv. 710; by rectum in ulcerative colitis due to Balantidium coli, iv. 762 .

LABIA MAJORA, swelling of, as a complication of mumps, ii. 166.

LABIAL GLANDS, INFLAMMATION OF, iii. 6.

Labioglossolaryngeal paralysis. See Bulbar paralysis, chronic progressive.

LABYRINTHINE NYSTAGMUS, iv. 603.

LABYRINTHINE VERTIGOES, iv. 624.

LACERTILIA, treatment of poisoning by, iv. 683.

LACQUer POISONING, iv. 693.

Lactate of lime, use of, in convalescence from pneumonia, ii. 262.

Lactic acid, use of, in local treatment, of laryngeal tuberculosis, ii. 493, iii. 347; of syphilis of upper respiratory tract, iii. 349.

LACTONE, use of, as a food in acute rheumatism, ii. 298.

Lactophenin, use of, to control sweating in acute endocarditis, iii. 747 .

LACTOSE, presence of, in diabetic urine, ii. 728; use of, in diet of pericarditis, iii. 316; use of, as food in bacillary dysentery, ii. 325 ; use of, as substitute for vegetable starch in diet of diabetics, ii. 749 .

LAËNNEC, influence of, upon diseases of pleura, iii. 456.

LAËNNEC'S CIRRHOSIS. See Cirrhosis of liver. LAFontaIne, as pioneer in hypnotism, i. 562.

LAGOCHILASCARIS MINOR, infection by, iv. 800.

Lake mixture, for use in local treatment of laryngeal complications of tuberculosis, ii. 494 , iii. 347 .

Lakton. See Glycoheptonic acid. 
Lamalou, value of treatment at, in acute myelit is, iv. 228.

Lambert, Sir Alexander, ergot in delirium tremens recommended by, ii. 605; hyoscin, apomorphin, and strychnin in combination recommended by, as hypnotie in delirium tremens, ii. 606 ; method of treating chronic alcoholism employed by, ii. 597 ; method of treating morphinism employed by, ii. 621 ; milk in typhoid fever forbidden by, ii. 13 .

LANCEREAUX, use of chloral in delirium tremens recommended by, ii. 606 .

LANCINATING Pains, as diagnostic sign of tabes, iv. 188; intestinal antiseptics for relief of, iv. 198; operative measures for relief of, iv. 198; treatment of, iv. 196, 198.

LAND, elimatic relations of, i. 249 .

LANDMANN'S TUBERCULOLE, i. 151.

LANDOUZY AND DÉJERINE, fascioscapulohumeral type of muscular dystrophy first noted by, iv. 261.

LANDRY'S PARALYSIS, iv. 328.

Lane, W. Arbuthnot, on choice of operation in constipation from obstruction, iii. 237.

L'ANÉmie séreuse, iollowing paracentesis in eirrhosis of liver, iii. 285.

LANG, use of metallic mercury in syphilis advocated by, ii. 396 .

Lang's formula, for gray oil of mercury to be used in injection, ii. 396 .

Lanolin, use of, as diluent for small-pox vaccine virus, ii. 66 ; as a protective in employment of counterirritation, ii. 159; in stomatitis syphilitica, iii. 21.

Laparotomy, exploratory, employment of, in carcinoma of stomach, iii. 163; in membranous enteritis, iii. 210 ; in syphilitic gumma of stomach, iii. 167.

LARKSPUR, poisoning by, ii. 652 .

LARV E AND PLANTS, treatment of stings from, iv. 686.

LARYNGEAL COMPLICATIONS, IN TUBERCULosis, ii. 490; local treatment of, ii. 492.

LARYNGEAL CRISES, in tabes, iv. 181 ; treatment of, iv. 197.

LARYNGEAL STENOSIS, operative measures for, iii. 349 .

Laryngeal spray, method of using, in chronic atrophic laryngitis, iii. 343.
Laryngectomy, employment of, in laryngeal tuberculosis, iii. 347.

Iaryngismus stridulus. See Laryngitis, spasmodic:

LARYNGitis, as a complicistion of measles, ii. 158 ; as a complication of small-pox, ii. 51; as a complication of tuberculosis, ii. 490; electrotherapeutics for relief of, i. 409 ; influence of climate in treatment of tuberculous, i. 409.

LARYNGITIS, ACUTE, iii. 338; internal treatment of, iii. 338; local treatment of, iii. 339; prophylactic treatment of, iii. 338 .

Laryngitis, chronic, iii. 340.

LARYNGITIS, CHRONIC ATrOPHIC, iii. 343.

LARYNGITIS, EDEMATOUS, iii. 341.

Laryngitis, membranous, iii. 342.

Laryngitis sicca. See Laryngitis, chronic atrophic.

LARYNGITIS, SPASMODIC, iii. 341 ; as a complication of rachitis, ii. 820 .

LARYNX, intubation of, see Intubation; involvement of, in diphtheria, ii. 490; involvement of, in tuberculosis, ii. 490.

LARYNX, DISEASES OF, iii. 338: acute laryngitis, iii. 338; chronic atrophic laryngitis, iii. 343; chronic laryngitis, iii. 340 ; edematous laryngitis, iii. 341 ; membranous laryngitis, iii. 342 ; spasmodic laryngit is, iii. 341 ; syphilis of upper respiratory tract, iii. 348 ; tuberculosis of larynx, iii. 344 .

LARYNX, tUBerculosis of, iii. 344 ; climatic treatment of, iii. 344; hygienic treatment of, iii. 344 ; local treatment of, iii. 347; operative measures for relief of, iii. 347 ; prophylactic treatment of, iii. 344; specific remedies for, iii. 345 .

Lassar's paste, application of, to vaccinated arm, ii. 73.

"Latent period," in an X-ray treatment, i. $500,511$.

Lateral sinUs, thrombosis of, operation for, iv. 729 .

Latham, cases of tuberculosis considered suitable for sanatorium treatment by, ii. 470 ; results reported by, on oral administration of tuberculin, ii. 517.

LATHAM, earliest writer on diseases of pericardium, iii. 511.

Latham, A. C., on vaccine therapy in chronic bronchitis, iii. 271 . 
LATHYRISM, ii. 652, iv. 693; treatment of, ii. 653.

Lathyrus sativus, iv. 693.

Laudanum, use of, in gallstone colic, iii. 268.

Laughing gas. See Nitrous oxid gas.

LAUNDRY, care of, in tuberculosis, ii. 467.

Lavage, of blood, in methemoglobinuria, iv. 5; of colon, see Colonic flushing; gastric, see Gastric lavage; internal, for relief of chronic gastritis, iii. 161; internal, for relief of uremic vomiting, iv. 54; of stomach, see Gastric lavage.

Laveran and Catrin, studies by, on bacteriology of mumps, ii. 167.

Lawson, Dr. Arnold, investigations by, on value of radium therapy in ophthalmology, i. 480.

Laxatives, use of, in acute bronchitis, iii. 359 ; in acute cerebral meningitis, iv. 372 ; in neuritis, iv. 289 ; in pericarditis, iii. 515; saline, see Cathartic, saline; sweetened, contraindicated in diabetes mellitus, ii. 736; in treatment of tricocephalus dispar, iii. 255.

LEAD, influence of, in producing arteriosclerosis, iii. 756 .

Lead and opium wash, use of, for atriplicism, iv. 693.

Lead acetate, use of, in abscess and gangrene of lung, iii. 440; in hematuria, iv. 4 ; for relief of edema of glottis in acute nephritis, iv. 63.

LEAD POISONING, i. 701; fatal dose in, i. 704; frequency of, i. 701; headache as a symptom of, iv. 610 ; influence of, in causation of gout, ii. 694 ; post-mortem findings in, i. 704; symptoms of, i. 702; treatment of, i. 704; treatment of multiple neuritis due to, iv. 324 .

Lecithin, use of, for relief of insomnia in neurasthenia, iv. 565 .

Leeches, use of, in acute bronchitis, iii. 357; in acute hemorrhagic encephalitis, iv. 405 ; in acute stage of cerebral palsies of children, iv. 412; in angina Ludovici, iii. 30 ; in anterior poliomyelitis, iv. 237; in apoplectic form of vestibular vertigo, iv. 628 ; in cerebral hyperemia, iv. 386 ; in facial paralysis due to rheumatism, iv. 311 ; in marantic sinus thrombosis, iv. 427 ; in neuritis of third, fourth, and sixth nerves, iv. 308 ; in pachymeningitis interna hemorrhagica, iv. 364 ; in paren- chymatous glossitis, iii. 24; in pericarditis, iii. 518; in pneumonia, ii. 256, 257 ; in pneumonia together with oxygen, ii. 237; for relief of headache in acute cerebral meningitis, iv. 372; for relief of headache due to brain tumor, iv. 448; for relief of headache due to cerebral thrombosis, iv. 399; for relief of pain in cerebral abscess, iv. 423; for relief of pain in sciatica, iv. 299.

LEECHES, injuries from, iv. 800; preventive measures for, iv. 801; remedial measures for, iv. 801; species causing infection, iv. 800 .

LEGRAIN, use of convalescent serum by, in treatment of typhus fever, ii. 37.

Leichtenstern, cases of collapse and sudden death from thoracentesis classified by, iii. 480 ; epidemic of psittacosis reported by, ii. 584 .

LeIshmaNia DONOVANI, parasite causing kala-azar, iv. 753.

LEISHMANIA INFANTUM, parasite causing infantile splenic anemia, iv. 755 .

Leishmaniases, iv. 753: infantile splenic anemia, iv. 755; kala-azar, iv. 753; oriental sore, iv. 756 .

Leiter coil, use of, in acute laryngitis, iii. 338 ; in acute tonsillitis, iii. 41 ; in pericarditis, iii. 517 ; to reduce temperature in pneumonia, ii. 232.

Leiter's tubing. See Leiter coil.

LELAND, Dr., method of tonsillectomy recommended by, iii. 44 .

LEMONADE, as article of invalid dietary, $i$. 652 ; use of, in delirium tremens, ii. 602 , 603; use of, for relief of anuria due to nephrolithiasis, iv. 107.

LENHARTZ FORMULA, for diet in gastric ulcer, iii. 123.

LENHARTZ METHOD, of feeding in gastric ulcer, iii. 121.

Leprosy, iv. 809; as a complication of vaccination, see Vaccination; definition of, iv. 809 ; etiology of, iv. 810 ; microorganism in, iv. 809, 811; prognosis in, iv. 809 ; prophylaxis against, iv. 812 ; surgical treatment for, iv. 816 ; transmission of, iv. 811 ; transmission of, by vaccination, iv. 812 ; treatment of, iv. 813.

Leptomeningitis, acute Cerebral. See Meningitis, acute cerebral. 
Leptus aUtUMNalis, iv. 692.

LEUBE, formula for nutrient enema recommended by, iii. 119.

Leukzmia, iii. 844; headache as a symptom of, iv. 611 ; lymphatie form of, iii. 845; myelogenetic form of, iii. 846; purin bodies produced in, by $\mathrm{X}$-ray therapy, iii. 851 ; radium treatment of, iii. 853; technique of $\mathrm{X}$-ray therapy for. i. 549; treatment of, in general, iii. 846 ; treatment of, by mixed toxins, iii. 847 ; tuberculin in treatment of, 847 ; uric acid increased by $\mathrm{X}$-ray treatment of, iii. 851; X-ray therapy for, i. 545, iii. 84.

LEUKEMIA, LYMPHATIC, iii. 845; acute type of, iii. 845; chronic type of, iii. 845; clinical picture of, iii. 845; treatment of, iii. 846; X-ray therapy for, iii. 852.

LeUKeMia, Myelogenetic, iii. 846; clinical picture of, iii. 846 ; treatment of, iii. 846 ; $\mathrm{X}$-ray therapy for, iii. 852.

Leukocyte extract, production of passive natural immunity by, i. 173.

Leukocytic extract, use of, in bronchopneumonia, iii. 415; in pneumonia, iii. 244 ; value of, in cerebrospinal meningit is not determined, ii. 201.

Leukodescent lamp, employment of, in acute uvulitis, iii. 35 .

LEUKOPENIA, produced by X-ray treatment of leukemia, iii. 850 .

LEUKOPLAKIA, iii. 25; syphilis associated with, iii. 25 ; treatment of, iii. 26.

LEURIAUX, claim for making an antitoxic serum for whooping-cough made by, ii. 183.

LEVADITI AND LANDSTEINER, dusting powder recommended by, for disinfection of mucous membranes against anterior poliomyelitis, iv. 237.

Levulose, subcutaneous injection of, in diabetic coma, ii. 758 ; use of, to prevent acidosis in diabetes mellitus, ii. 757; use of, as substitute for vegetable starch in diabetes mellitus, ii. 749 .

Levulose Fructose, presence of, in diabetic urine, ii. 728 .

LEVULOSURIA, conditions governing, ii. 728.

LÉVY AND BAUDOUIN, directions by, for alcohol injection for relief of trigeminal neuralgia, iv. 346,349 .

IevY-Bing, direct parasitic action of mercury established by, ii. 381 .
LEwIs, toxic symptoms following therapeutic use of phosphorus reported by, ii. 627 .

IEYDEN, mixture suggested by, for rectal feeding in tetanus, ii. 350 .

LEYDEN AND Möbius, type of muscular dystrophy noted by, iv. 260.

LEYDEN AND RENVERS, use of hard rubber cannulas by, in carcinomatous stricture of esophagus, iii. 54.

LiCHeN planus, treatment of, by X-ray, i. 528.

Liddef, Dúncan, ehicken-pox first distinguished from small-pox by, ii. 109.

LIEBEN's TEST, for acetone in urine, ii. 733.

LIЕвенмеister, on use of cold wet pack in typhoid, ii. 18.

Ligation of extremities, for control of gastric hemorrhage, iii. 112; for control of hemorrhage in pulmonary tuberculosis, ii. 501 ; in snake bite, iv. 682 .

Ligation of limb, for bites of arthropods, iv. 685 ; in treatment of poisoning, i. 671.

Ligation above wound, in curare poisoning, iv. 694 .

Light, protection from, as a therapeutic measure in varicella, ii. 112; use of, in treatment of diabetes insipidus, ii. 777 .

Light bath cabinets, use of, in light therapy, i. 442 .

Light therapy, i. 432; antagonism between Röntgen rays and, i. 447; apparatus employed in, i. 441; conditions benefited by, i. 448; in dermatology, i. 458; indications for, i. 448; in phlebitis, i. 458; physical properties of radiant energy in, i. 332; physiological effects of, i. 444; post-operative uses of, i. 457; sources of radiant energies in, i. 438; uses of, i. 448.

LightNing pains. See Lancinating pains. Lime water, use of, in addition to milk in cirrhosis of liver, iii. 281 ; in lavage for chronic gastritis, iii. 160; locally, in atriplicism, iv. 693.

Lime water, atomized, use of, in plastic bronchitis, iii. 392 .

IAMNATIS CEYLONICA, iv, 800.

LimNatis NilOtica, iv. 800 .

Lincoln formula, for use in acute rhinitis, iii. 327 .

Ling, Peter, first important contribution to use of gymnastics in circulatory dis- 
eases made by, iii. 636; medical gymnastics introduced by, i. 298.

Liniment, of ether, chloroform, and menthol, for relief of neuralgic pain following withdrawal of morphin, ii. 624; turpentine, see Turpentine.

Liniments, use of, in chronic bronchitis, iii. 373.

Liniments, stimulating, use of, in acute bronchitis, iii. 356; for relief of pain in sciatica, iv. 299.

LiNk, operation by, for pancreatic calculi, iii. 302 .

Linseed poultices. See Poultice.

Lipoids, i. 19.

Lips, Diseases of, iii. 4: herpes, iii. 5; rhagades of commissure of lips, iii. 4; treatment, iii. 5 .

Liquor ammonii acetatis, use of, in nephritis, iv. 49 .

Liquor bismuthi et ammonii citratis, use of, in nervous diarrhea, iii. 250.

Liquor calcis, use of, as nasal douche in rnembranous rhinitis, iii. 330 .

Liquor ferri et ammonii acetatis, use of, in chronic parenchymatous nephritis, iv. 65 ; in nephritis, iv. 49.

Liquor hydrargyri nitratis, use of, for application to verumontanum in postgonorrheal neuroses, iv. 174.

Liquor magnesia citrate, use of, in nephritis, iv. 32 .

Liquor potassæ, use of, in acute urethral gonorrhea, iv. 139.

Liquor potassii arsenitis, use of, in nervous type of influenza, ii. 191.

Liquor potassii citratis, use of, in stomatitis catarrhalis, iii. 7 ; in stomatitis herpetica, iii. 10.

Liquor trinitrini. See Nitroglycerin.

Liтнемia, ii. 697.

Lithiasis, treatment of, iv. 108.

Lithium carbonate, use of, in diabetes mellitus, ii. 760 .

Lithium citrate, use of, in diabetes mellitus, ii. 760 .

LitTLE'S DiseAsE, iv. 411.

LIVER, action of radium upon, i. 470; disturbances of, associated with intestinal autointoxication, ii. 679 ; effects of X-ray upon, i. 519; importance of attention to, in treatment of chronic myocardial insufficiency, iii. 717.
Liver, DISEAsEs of, iii. 258: abscess of liver, iii. 290; acute catarrhal jaundice, biliousness, iii. 258; cholecystitis and cholelithiasis, iii. 265; chronic catarrhal jaundice, iii. 262; cirrhosis of liver, iii. 280; congenital syphilis of liver, iii. 280 ; diseases of bile passages, iii. 258; diseases of gall bladder, iii. 258; jaundice, iii. 260 ; prolapse of liver, -iii. 288 ; relapsing jaundice, iii. 264; syphilitic disease of liver, iii. 264.

LIVER ABSCESS, as a complication of influenza, iii. 190; exploratory puncture in diagnosis of, iv. 711; irrigation of, with sterile bihydrochlorate of quinin, iv. 711 .

LIVER FLUKES, iv. 779.

"Liver pills," use of, in biliousness, iii. 260 .

LIVING MATTER, constitution of, i. 3.

Llewellyn's solution of compound wine of iodin, use of, in chronic nasopharyngitis, iii. 32 .

Lobar pneumonia. See Pneumonia.

Lobelia, use of, in acute bronchitis, iii. 363 .

Local injury, removal of, in occupation pareses, iv. 332 .

LocALITY, in treatment of hay fever, iii. 389.

Localized baths, use of, in hydrotherapy, $i$. 200.

Locke's solution, formula for, iii. 534.

Loco weEds, poisoning by, ii. 652 .

Locomotion, DifFiculty of, in tabes, iv. 189.

Locomotor ataxia. See Tabes.

Loeffler AND Frosch, work by, on prophylactic serum for foot and mouth disease, ii. 583.

Loeffler's serum, use of, in membranous pharyngitis, iii. 39 .

Loeffler's solution, use of, in phlegmonous pharyngitis, iii. 40 .

Lolium temulentum. See Darnel.

LoNG, plan suggested by, for suppression of cholera in the Philippines, iv. 828 .

LOOMIS SANATORIUM, experiments performed in, concerning dosage of tuberculin, ii. 522 ; experiments performed in, showing condition of gastrointestinal tract in tuberculosis, ii. 559; menus for meals served in, ii. 552; menus for meals in two-hour diet employed in, ii. 554; percentage of patients having otitis 
media at, ii. 509; records of tuberculin therapy kept in, ii. 518; statistics of laryngeal complications in tuberculosis kept in, ii. 490 ; test diet for tuberculous patients employed in, ii. 502 .

Lord, on empyema associated with actinomycosis, iii. 489.

LоTT, hyoscin first used by, in treatment of morphinism, ii. 620.

LöWENFELD, method of hypnotization suggested by, i. 566 .

Lozenges of ammonium chlorid, iii. 365 .

Lubricants, sterilization of, for use in catheterization, iv. 158.

Lubrication, method of, for catheterization, iv. 158.

Ludwig's angina. See Angina ludovici

LUDWIG's THEORY, of renal secretion, iv. 10.

Lues. See Syphilis.

Lugol's solution, use of, in Vincent's angina, iii. 40.

LUGOL's TEST, for acetone in urine, ii. 733.

Lumbar plexus, neuritis of, iv. 293.

Lumbar pressure, value of, in diagnosis between hydrocephalus and brain tumor, iv. 436 .

Lumbar puncture. See Puncture.

Lumboabdominal neuralgia. See Neuralgia.

Lunar caustic, objections to use of, in chancre, ii. 422; see also Caustic.

Lung, EDEMA oF, hydrotherapy applied for relief of, i. 205.

LUNG, HYPEREMIA OF, hydrotherapy applied to, i. 205.

Lungs, abscess of, see Pulmonary abscess; active congestion of, iii. 407; changes in during hypnosis, i. 574; effects of hot baths upon, i. 192; embolism of, see Pulmonary embolism; gangrene of, see Pulmonary gangrene; hypostatic congestion of, iii. 407 ; indurative processes in, associated with cardiac insufficiency, iii. 712 ; passive congestion of, iii. 406 ; thrombosis of, see Pulmonary thrombosis; tuberculosis of, see Tuberculosis of lungs.

LuNGS, CirCulatory disturbances of, iii. 403: active congestion, iii. 405; bronchopneumonia, iii. 412; chronic pneumonia, iii. 421 ; chronic pulmonary emphysema, iii. 430; general considerations affecting subject, iii. 403; hypostatic congestion, iii. 407; passive congestion, iii. 406; pneumonoconiosis, iii. 423; pulmonary abscess, iii. 438; pulmonary edema, iii. 407; pulmonary gangrene, iii. 438; pulmonary thrombosis and embolism, iii. 411; tumors of lung, iii. 439.

LUNG, тUMOHs of, iii. 445; differential air pressure in treatment of, iii. 445; results of operation for malignant forms of, iii. 447; X-ray in diagnosis of, iii. 445.

Lupinus, poisoning by, ii. 651 .

Lupulin, use of, for relief of painful erections and chordee in acute urethral gonorrhea, iv. 141 .

Lupus, as a complication of vaccination, ii. 90 .

Lupus ERYTHEMATOSUs, therapeutic use of radium for, ii. 474; X-ray therapy for relief of, i. 535.

LUPUS VULGARIS, radium in treatment of, i. 474 ; X-ray therapy for relief of, i. 533.

LÜTHJE, H., on causation of gout, ii. 693.

LYMPHANGiTIS, complicating acute gonorrhea, iv. 149.

Lymphatic enlargement, X-ray therapy for relief of, i. 551 .

LyMPHATIC GLANDS, disease of, associated with pneumogastric paralysis, iv. 319; effects of X-ray upon, i. 518; enlargement of, as a complication of measles, ii. 161 ; treatment of enlargement of, ii 162.

Lymphatic LeUkemia. See Leukemia, lymphatic.

LYMPHATIC AND BLOOD DISEASES, value of $\mathrm{X}$-ray therapy in treatment of, i. 544 .

Iymphosarcoma, X-ray therapy for relief of, i. 549.

Lymphosarcoma of mediastinum, iii. 501.

LyOPHILIC COLLOIDS, i. 6.

LyopHOBIC ColloIDs, i. 6.

Lysins, action of, i. 131.

Lysol, injections of, in solution, in chancre with phimosis, ii. 423; irrigation of bowels with a solution of, in chronic stage of bacillary dysentery, ii. 325 ; use of, as disinfectant in tuberculosis, ii. 452, 453 ; use of, as a lotion in pustular or ulcerative syphiloderms, ii. 426.

MaCewen, statisties of mortality from operation for cerebral abscess published by, iv. 424; statistics of mortality from operation for infectious sinus thrombosis published by, iv. 430 . 
Macewen's treatment of aneurysm, iii. 767 .

MACGILCHRIST, on absorption of quinin, iv. 718; experiments by, on solubility of quinin salts, iv. 722 .

Mackenzie, on Nauheim baths, iii. 617.

Mackenzie, William, modification of cardiolysis suggested by, iii. 509 .

MAGDEBERG EXPERIMENTS, on frequency of syphilis, ii. 370,373 .

Magnesia, preparations of, used in gastric hyperacidity, iii. 95; use of, in acute gastritis with sodium bicarbonate, iii. 155; use of, in chronic constipation, iii. 234; use of liquid, as mouth wash in salivary deposits, iii. 29; use of, in stomatitis herpetica, iii. 9 .

Magnesia, calcined, use of, in acute nephritis, iv. 58; in gastric hyperacidity, iii. 95 ; in nephritis, iv. 32.

Magnesia, calcined, and sodium bicarbonate, use of, in habitual constipation, iii. 226.

Magnesia, milk of, manner of taking, iv. 33; use of, following calomel in membranous laryngitis, iii. 342; use of, as mouth wash in diabetes mellitus, ii. 736; use of, in nephritis, iv. 32 ; use of, in spasmodic laryngitis, iii. 342 .

Magnesia oxidum. See Magnesia, calcined.

Magnesium citrate, use of, to empty gastrointestinal tract in pneumonia, ii. 227; to expel tape worms, iv. 778 .

Magnesium perhydrol, use of, in amyxorrhea gastrica, iii. 101; in diabetes mellitus, ii. 760; in gastric hyperacidity, iii. 95.

Magnesium salicylate, use of, in peristaltic unrest, iii. 250.

Magnesium sulphate, use of, in acute alcoholism, ii. 589; in acute fibrinous pleurisy, iii. 467 ; in acute gastritis, iii. 155 ; in acute nephritis, iv. 58 ; in acute peritonsillitis, iii. 43 ; in bacillary dysentery, ii. 326 ; in biliousness, iii. 258, 259; in chronic alcoholism, ii. 595; in chronic catarrhal jaundice, iii. 264; in chronic mucous colitis, iii. 207; for constipation associated with gastric hyperacidity, iii. 94; for constipation in Rocky Mountain fever, ii. 566; contraindicated in acute diffuse peritonitis, iii. 317 ; in edematous laryngitis, iii. 341 ; to modify severity of pleurisy, iii. 467; in prevention of lead poison, iv. 325 ; by rectum in diabetic coma, ii. 757; in saturated solution for gonorrheal epididymitis, iv. 152; by subdural injection in tetanus, ii. 352 ; in tetanus, ii. 349 ; in wood alcohol poisoning, ii. 610 .

Magnesium sulphate and anthelmintics, in hookworm disease, iv. 793; in intestinal distomiasis, iv. 782 .

Magnus-Levy, on diabetic bread, ii. 747.

Maisonneuve urethrotome, use of, in operation for resilient urethral stricture, iv. 172.

Malaria, as a cause of neuralgia, iv. 337 ; hydrotherapy for relief of, i. 220; not associated with cirrhosis of liver, iii. 282.

MALARIA, TROPICAL, iv. 712; atypical forms of, iv. 714 ; cachexia due to, iv. 727 ; chronic form of, iv. 727; clinical types of, iv. 714 ; complications of, iv. 715 ; definition of, iv. 712; destruction of mosquitoes as a means of preventing, iv. 731; drugs in prophylaxis against, iv. 735 ; parasites causing, iv. 712 ; pathology of, iv. 716; pernicious form of, iv. 727; preventive measures against, iv. 729; quartan form of, iv. 715; quinin in prophylaxis against, iv. 735; quinin in treatment of, iv. 717 ; remedial measures in, iv. 716; specific treatment of, iv. 717; splenic enlargement in, iv. 728; symptomatic treatment of, iv. 725; symptoms of, iv. 713 ; surgical interference in, iv. 728; tertian form of, iv. 715 ; transmission of, by mosquitoes, iv. 729.

Malarial parasites. See Parasites.

"Malarial sores," iv. 729.

"Malarial" ulcers, iv. 867.

Malbranc, importance of position in acute dilatation of stomach first noted by, iii. 174.

Male fern. See Filix mas.

Malignant BRAIN TUMORS, iv. 434; varieties of, iv. 434.

Malignant Changes IN SKIN, following X-ray treatment, i. 516."

Malignant Disease, immune therapy in, i. 175; value of radium therapy in, i. 481.

Malignant disease of peritoneum, iii. 323.

Malignant Disease of SKIN, value of $\mathrm{X}$-ray therapy in treatment of, i. 537. 
Malignant pustule, ii. 330.

Malnutrition, as a cause of marantic: sinus thrombosis, iv. 426.

Malt, use of, in convalescence from pneumonia, ii. 262; in laryngeal tuberculosis, iii. 348 .

Malt extract, use of, in rest cure for neurasthenia, iv. 558.

MaltTa FeVEr, iv. 839 ; prophylaxis against, iv. 839 ; treatment of, iv. 840 ; vaccine treatment in, i. 147.

Maltose, presence of, in diabetic urine, ii. 728.

Mammary Gland, swelling of, as a complication of mumps, ii. 166; use of, in organotherapy, i. 116.

MAN, relation of, to spread of plague, iv. 819.

Manganese, use of, in chlorosis, iii. 821.

Mania, hydrotherapy for relief of, i. 226.

MaNic-depressive psychoses, differentiation of, from hysteria, iv. 585.

Mannite, use of, to prevent acidosis in diabetes mellitus, ii. 757 .

Mannitol hexanitrate, use of, in cardiac insufficiency, iii. 610 .

Manson, on general treatment of trypanosomiasis, iv. 748 ; on insects carrying beri-beri, iv. 849 ; method of extracting guinea worm recommended by, iv. 789 .

MaNUal LABOR, HARD, as an etiological factor in arteriosclerosis, iii. 754 .

MaNUfactured aRTicles, occurrence of arsenic in, i. 676.

Maragliano, 5 per cent solution of phenol recommended by, for use in tetanus, ii. 349 .

Maragliano's anti-tuberculosis serum, i. 168 , ii. 525 .

Marantic sinus thrombosis, iv. 426; prognosis in, iv. 427; prophylaxis against, iv. 427 ; symptoms of, iv. 426 ; treatment of, iv. 427.

Marchant, Gerard, fixation of liver for relief of prolapse first performed by, iii. 289.

Marchiafava and Bignami, observations by, on time between administration of quinin and its action, iv. 719 .

MARColini, transmission of syphilis by means of vaccination reported by, ii. 88 .

MAREY, means of estimating blood pressure first devised by, iii. 556 .
Marie, Pienre, hereditary cerebellar ataxia first described by, iv. 216, 513.

Marte and Guillais, hypothesis advanced by, regarding pathogenesis of syphilis, iv. 184.

Marine climates, tuberculous children benefited by, ii. 473 .

Marine Hospital, San Francisco, use of ipecac at, in prophylaxis of hepatic amebiasis, iv. 710 .

Marine Hospital Service, rules issued by, for disinfection in tuberculosis, ii. 454.

Marine searchlight, use of, in light therapy, i. 441.

Marital prophylaxis, against syphilis, ii. 376.

Marmoreck's antistreptococcic serum, use of, in scarlet fever, ii. 128.

Marmoreck's antituberculosis serum, i. 168 , ii. 525 .

Marriage, question of, in cardiac insufficiency, iii. 680; in diabetes mellitus, ii. 769 ; in epilepsy, iv. 641 ; in muscular dystrophy, iv. 262; among neurotics, iv. 521 ; in syphilis, iv. 479 ; in tuberculosis, ii. 464.

Martin, use of pilocarpin in mumps advised by, ii. 172 .

Masing and Strasburger method, of estimating minimal blood pressure, iii. 557.

Mask, metal, employment of, for inhalations, iii. 441.

MASSACHUSETTS, antituberculosis work in, ii. 445.

Massachusetts General Hospital, cases treated with digipuratum at, iii. 597; coöperative occupational method of treating neurasthenia and psychasthenia at, iv. 554; disinfection of typhoid urine and stools at, ii. 8; mortality from typhoid fever at, ii. 15, 19; results of administration of urotropin in pneumonia at, ii. 223; results of antityphoid vaccination at, ii. 5 ; rules for prevention of typhoid extension in, ii. 7; study of influenza at, ii. 188; technique of antityphoid vaccination at, ii. 6 .

Massage, i. 289; contraindications to use of, i. 322 ; effects of, on abdominal viscera, i. 297; effects of, on blood, i. 294; effects of, on circulation. i. 294; effects of, on muscular system, i. 29s; effects of, on nervous system, i. 296; effects of, on 
respiratory system, i. 296 ; friction movements in, i. 289 ; history of, i. 287 ; indications for use of vibratory, i. 356; joint movements in, i. 292; kneading movements in, i. 290; movements in, i. 289; percussion movements in, i. 292; physiological effects of, i. 294; requisites to success of, i. 294 ; stroking movements in, i. 289 ; technique of, i. 288 ; vibration movements, i. 291 ; vibratory method of, i. 353 .

Massage, use of, in acute myelitis, iv. 227; in after-treatment of anterior poliomyelitis, iv. 239; in after-treatment of morphin habit, ii. 624; in alcoholism, ii. 594; in apyrexial heat exhaustion, iv. 679; in arthritis associated with intestinal autointoxication, ii. 681 ; in arthritis deformans, ii. 712; in asthenic bulbar paralysis, iv. 256; in beri-beri, iv. 854 ; in brachial neuralgia, iv. 357 ; in cardiac neurasthenia, iii. 778; with caution for phlebitis complicating typhoid fever, ii. 28 ; for cerebral hemorrhage due to chronic interstitial nephritis, iv. 72; in chronic enlargement of spleen, iii. 889 ; in chronic gastritis, iii. 161; in chronic gout, ii. 700; in chronic interstitial nephritis, iv. 31 ; in chronic myelitis, iv. 230, 231; in chronic progressive bulbar paralysis, iv. 252 ; in chronic pulmonary emphysema, iii. 434 ; in chronic rheumatism, ii. 712; in constipation, iii. 227 ; contraindicated in acute intestinal obstruction, iii. 240 ; contraindicated in early stage of multiple neuritis, iv. 327 : contraindicated in pulmonary thrombosis, iii. 411; contraindicated in recent cases of neuritis, iv. 284; in convalescence from dengue, iv. 860 ; in convalescence from influenza, ii. 196; in convalescence from Landry's paralysis, iv. 329 ; in facial paralysis, iv. 312 ; following injection of defibrinated blood, iii. 831; in gastric atony, iii. 173; in hallucinosis of drunkards, ii. 609; in hysteria, i. 322; in Korsakow's psychosis, ii. 609; in membranous enteritis, iii. 210; in multiple neuritis, iv. 328 ; in muscular weakness accompanying rachitis, ii. 818 ; in nervous type of influenza, ii. 191; for neuralgic headache, iv. 599; in neurasthenia, iv. 546; in neuritis, iv. 287; for obstetric paralysis, iv. 292 ; in occupation neuroses, iv. 661 ; in occupation pareses, iv. 332 ; for ozena, iii. 337 ; in paralysis agitans, iv. 666; for paralysis due to cerebral hemorrhage, iv. 393 ; for paralysis in cerebral palsies of children, iv. 413 ; in paralysis following cerebrospinal meningitis, ii. 216; in progressive bulbar paralysis, iv. 499 ; in progressive neural muscular dystrophy, iv. 266; in reduction of obesity, ii. 807 ; for relief of backache in acute nephritis, iv. 63; for relief of headache in neurasthenia, iv. 565 ; for relief of myositis, iv. 605; for relief of pain in spinal syphilis, iv. 274; for relief of post-infectious headache, iv. 612 ; during rest cure for neurasthenia, iv. 540, 544; in severe cases of chronic enteritis, iii. 200; in spastic spinal paralysis, iv. 270 ; in spinal progressive muscular atrophy of acquired form, iv. 247; in syphilitic arthritis, ii. 306; in tetany, iii. 910.

Massage, abdominal, i. 293; use of, in chronic gastritis, iii. 162 ; in depressive gastric neuroses, iii. 188; in membranous enteritis, iii. 209 ; objections to, in irritative gastric neuroses, iii. 188; for relief of constipation in neurasthenia, iv. 566 .

Massage of prostate gland, in chronic gonorrheal urethritis, iv. 161 ; contraindications to use of, in chronic gonorrheal urethritis, iv. 165; in irritable stricture of urethra, iv. 173; in post-gonorrheal neuroses, iv. 175; in relapsing prostatitis, iv. 168; technique of, in chronic gonorrheal urethritis, iv. 163.

Massage and gymnastics in pathological conditions, i. 301 ; in diseases of circulation, i. 302 ; in diseases of the nervous system, i. 308; in diseases of the respiratory system, i. 304 ; in gastrointestinal disorders, i. 322 ; in metabolic disorders, i. 325 ; in sprains, fractures, and postoperative treatment, i. 328 ; in tabes dorsalis, i. 316.

"Massive-dose" method, for administration of an X-ray treatment, i. 500.

MAstication, imperfect, a cause of gastric hyperacidity, iii. 78 .

Masticatory SPASM, iv. 655.

MAstitis, as complication of typhoid fever, ii. 28 . 
MAstoiditis, radiant heat and light in treatment of, i. 451.

Masturbation, as cause of myocardial insufficiency, iii. 723 ; ats at cause of reflex cardiac neuroses, iii. 781 .

Match factonies, chronie phosphorus poisoning in, ii. 628 ; means of preventing phosphorus poisoning in, ii, 629 .

Matches, phosphorus, suicide by means of, ii. 626 .

Matrues, summary by, of knowledge concerning local application of heat for relief of renal colic, iii. 103 .

Mauriac, passage of iodin through system demonstrated by, ii. 405 .

Maw's improved inhaler, for use in laryngeal complications of tuberculosis, ii. 492.

Mayo, William, investigations by, showing chronic appendicitis and cholecystitis to be the cause of gastric disease, iii. 180.

MCBride, directions by, for atropin and strychnin treatment of alcoholism, ii. 595, 596.

McCall Anderson's dusting powder, use of, in acute catarrhal jaundice, iii. 262.

McCaskey's method, of applying adhesive plaster in visceroptosis, iii. 247.

McPhedran, death following use of turpeth mineral for spasmodic laryngitis reported by, iii. 342 .

McVicar, C. S., on use of oxygen inhalations to relieve cyanosis in acute dyspnea, iii. 364 .

Meals, arrangement of, in treatment of obesity, ii. 797; menus for, in treatment of tuberculosis, ii. 548 .

Meara's apparatus, for administration of normal saline solution in pyelitis, iv. 89 .

Measles, ii. 138; admission of light in, ii. 147; antipyretics in, ii. 150 ; asthenic form of, ii. 153; bacteriology of, ii. 138; bronchitis complicating, ii. 153; bronchopneumonia complicating, ii. 158; care of mouth in, ii. 148; cleanliness important in, ii. 147, 150; complications of, ii. 156; contagiousness of, during period of eruption, ii. 143; contagiousness of, during period of invasion, ii. 141 ; cough as a symptom of, ii. 152; diagnosis between, and typhus fever, ii. 38 ; diet in, ii. 148; enterocolitis compli- cating, ii. 160; eruption in, ii. 142; eruption in hernorrhagie form of, ii. 154; exaggerated form of, ii. 149; fresh air ireatment of, ii, 146, 159; genital disorders complicating, ii. 161 ; hemorrhagic form of, ii. 154; hydrotherapy in, ii. 149, 152; hygienic treatment of, ii. 145; immunity conferred by, ii. 140; intestinal disorders complicating, ii. 160; laryngeal disorders complicating, ii. 157; lymph glands enlarged in, ii. 161; mortality in asthenic form of, ii. 154; mortality from bronchopneumonia in, ii. 158; mouth diseases complicating, ii. 157 ; mucous membranes and their diseases complicating, ii. 156; nervous symptoms of, ii. 152; nose affections complicating, ii. 152,156 ; non-contagiousness of, during period of desquamation, ii. 143; non-contagiousness of, during period of incubation, ii. 141; other infectious diseases complicating, ii. 161; pharyngeal affections complicating, ii. 156; physician's duty as regards infection in, ii. 139; as a predisposing cause of rachitis, ii. 814; prevention of, in hospitals, ii. 144; prevention of, in schools, ii. 144; prophylaxis of, ii. 139; prophylaxis of, in private practice, ii. 145; rare complications of, ii. 161; rules applying to prophylaxis of, ii. 141; sequelæ to, ii. 162 ; sickroom in, ii. 146 ; symptomatic treatment in, ii. 148; temperature in, ii. 142,149 ; treatment of, ii. 145; ventilation during, ii. 146 .

Measles, German, ii. 164; treatment of, ii. 165.

Meat, use of, contraindicated in chronic cerebral hyperemia, iv. 387 ; in diabetes mellitus, ii. 744 .

Meat extractives, avoidance of, in chronic parenchymatous nephritis, iv. 65 ; objections to, in nephritis, 43.

Meat polsoning, ii. 637; antitoxin of, see Antitoxin; by B. anthracis, ii. 330 ; by B. botulinus, ii. 638 ; by $\mathrm{B}$. breslaviensis, ii. 641 ; by B. enteritidis, ii. 640 ; by B. friedebergensis, ii. 642 ; by B. paratyphosus, ii. 642 ; by B. pneumoniæ, ii. 642 ; by $B$. proteus vulgaris, ii. 642 ; by $B$. suisepticus, ii. 642 .

MEATs, cooking of, i. 622 ; in diet of achylia gastrica, iii. 146; in diet of depressive 
disorders of gastric secretion, iii. 146; in diet of gastric hyperacidity, iii. 86; in diet for nephritis, iv. 43,65 ; digestibility of, i. 626; effects of, on blood pressure, iv. 43 ; influence of fat content upon, i. 627 ; objections to use of smoked, in nephritis, iv. 43.

MECHANICAL APPLIANCES, for aiding expiration in cardiac insufficiency due to pulmonary conditions, iii. 711 .

Mechanical Percussion, in MechanoTHERAPY, i. 353 ; apparatus for, i. 354; indications for, i. 356.

Mechanogymnastics, use of, in cardiac disease, iii. 640.

Mechanotherapy, i. 331; active movements in, i. 336 ; in atonic dilatation of stomach, iii. 177; conditions benefited by, i. 348; in chronic dilatation of stomach, iii. 180; in chronic gout, ii. 700; in chronic pulmonary emphysema, iii. 434; contraindications to use of, in spastic constipation, i. 231; in convalescence from lobar pneumonia, i. 350 ; difference between gymnastics and, i. 350; for epilepsy, iv. 647; for gastric atony, iii. 172; in habitual constipation, iii. 227 ; to increase tone of abdominal walls in prolapse of liver, iii. 289 ; lancinating pains in tabes not relieved by, iv. 197; of little value in visceroptosis, iii. 247; for movable kidney, iv. 3 ; for movable spleen, iii. 887; of no value in myotonia congenita, iv. 268; object of, i. 331 ; passive movements in, i. 345 ; principles of, i. 331 ; in reduction of obesity, ii. 805 ; use of suspension in, i. 359; Zander system of, i. 333.

Mediastinal LYMPH GLANDS, TUBERCULOSIS OF, iii. 505; prophylaxis against, ii. 505 ; treatment of, ii. 506 .

Mediastinal tumors, iii. 499 : carcinomata, iii. 502; curative treatment, iii. 499; dermoid cysts, iii. 500; lymphosarcomata, iii. 501; palliative treatment, iii. 501; resection of ribs for relief of tumors, iii. 503; teratomata, iii. 500; X-ray in treatment, iii. 502.

Mediastinitis, iii. 504.

Mediastinopericarditis, indURAtive, iii. 508 ; operation for, iii. 509.

Mediastindm, disfases of, iii. 499: mediastinitis, iii. 504; mediastinopericarditis, iii. 508; tuberculosis of mediastinal lymph glands, iii. 505; tumors of mediastinum, iii. 499.

Mediastinum, posterior method of opening, in suppurative mediastinitis, iii. 505.

Medical climatology, i. 259.

"Medical" erysipelas, ii. 282.

MEDICAL PROFESSION, influence of, toward proper use of alcohol, ii. 592; rôle of, in future control of tropics, iv. 667.

Medical supervision, of dusty trades, iii. 429.

Medicated vapor, methods of applying in acute laryngitis, iii. 338; use of, in acute bronchitis, 358.

Medina worm, iv. 788 .

Medinal, use of, for restraint of delirium in pneumonia, ii. 228.

Medulla oblongata, localizing symptoms of tumor in, iv. 443.

MFHnert, effect of vaccination on whooping cough in young infants reported by, ii. 74 .

Meininger, use of mercurial inunction to promote absorption in pleurisy advocated by, iii. 470 .

Meltzer, suggestions by, on causes of acute dilatation of stomach in pneumonia, ii. 259.

Meltzer-Auer apparatus, for use of differential air pressure, iii. 446, 447.

Membranes surRounding Cells, i. 55; cellular, i. 53 , 55 ; osmotic, i. 53 ; precipitation, i. 54; theories of, i. 56.

Membranous croup. See Laryngitis, membranous.

Membranous enteritis. See Enteritis, membranous.

Membranous laryngitis. See Laryngitis.

Membranous pharyngitis. See Pharyngitis.

Memory, utilization of increased power of, due to hypnosis, i. 577.

MENDELSOHN-GRAUPNER METHOD, of determining extent of myocardial insufficiency, iii. 693.

MÉniÈre's disease. See Vestibular vertigoes.

Meningeal headaches, iv. 606 .

Meningitis, as a complication of acute rheumatism, ii. 304 ; as a complication of erysipelas, ii. 285; as a complication of influenza, ii. 193; as a complication of 
mumps, ii. 171 ; as a complication of pneumonia, ii. 259; diagnosis between, and typhus fever, ii. 39 .

Meningitis, acute ceremiral, iv. 368 ; diagnosis of, iv. 369 ; prognosis of, iv, 371 ; prophylaxis against, iv. 371 ; specific remedies for, iv. 372 ; symptoms of, iv. 368 ; treatment of, iv. 372.

Meningitis, Basilar syphiLitic, iv. 473

Meningitis, cerebrospinal. See Cerebrospinal meningitis.

Meningitis, chronic cerebral, iv. 378.

Meningitis, epidemic cerebrospinal, iv. 373.

Meningitis, influenzal. See Influenzal meningitis.

Meningitis, pneumococcic, ii. 213.

Meningitis, serous, iv. 468; definition of, iv. 468; differential diagnosis of, iv. 469; etiology of, iv. 468; introduction to subject of, iv. 468; prognosis, iv. 469; symptoms of, iv. 468 ; treatment of, iv. 469 ; varieties of, iv. 468 .

Meningitis, tuberculous, iv. 373 ; diagnosis of, iv. 375; operative treatment of, iv. 377 ; pathological anatomy of, iv. 373 ; prophylaxis of, iv. 377 ; symptoms of, iv. 373 ; treatment of symptoms, iv. 377.

Menopause, acute myelitis during, iv. 224; as a cause of reflex cardiac neuroses, iii. 781 ; prevention of the neuroses at, iv. 524 ; use of ovary for relief of disturbances at, i. 114.

Menorrhagia, hydrotherapy for relief of, i. 211 .

Menstruation, Disorders of, extract of ovary for relief of, i. 114; extract of thyroid for relief of, i. 76 ; in tuberculosis, ii. 507.

Mental apathy, as symptom of diphtheria, ii. 270.

Mental Deficiency, presence of, in hereditary cerebellar ataxia, iv. 217 ; as a symptom of basilar syphilitic meningitis, iv. 473 ; as a symptom of cerebral palsies of children, iv. 410, 411; as a symptom of pseudobulbar paralysis, iv. 504 .

Mental disturbances, as a symptom of brain cysticercus, iv. 459 ; as a symptom of brain syphilis, iv. 476 ; as a symptom of parasites of brain, iv. 459 .

Mental fatigue, smple, hygienic treatment of, iv. 572 .
Mental foramen, alcohol injection at, for relicf of trigeminal neuralgia, iv. 346.

Menthol, use of, in atrophic rhinitis, iii. 331; in chronic pharyngitis, iii. 33 ; for disinfeetion of mouth in measles, ii. 148; by inhalation in chronic bronchitis, iii. 375; by intratrucheal injection, iii. 343,397 ; as local anesthetic in tonsillectomy, iii. 45; for relief of cough in pulmonary tuberculosis, ii. 497; for relief of itching in diabetes mellitus, ii. 764 ; for relief of itehing in small-pox, ii. 49; for relief of itching in varicella, ii. 112; as spray in acute tonsillitis, iii. 41 ; as spray in adenoid vegetations, iii. 38 ; as spray in ehronic atrophic laryngitis, iii. 343 ; as spray in hay fever, iii. 391 ; as spray in hypertrophy of pharyngeal tonsil, iii. 35 .

Menthol, alcoholic solution of, for relief of tender toes in typhoid fever, ii. 29.

Menthol and camphor, use of, to clear nasal passages of secretions, iii. 334 ; by inhalation in acute laryngitis, iii. 339; in lozenges in acute laryngitis, iii. 339 ; as spray after local applications in atrophic rhinitis, iii. 332.

Menthol and chloroform, inhalations of, in acute rhinitis, iii. 328 .

Menthol lozenges, use of, in acute tonsillitis, iii. 42.

Menthol ointment, use of, in acute catarrhal jaundice, iii. 262; during attack of hay fever, iii. 390 .

Menthol pills, use of, in peristaltic unrest, iii. 250 .

Mentholated lozenges, use of, in acute nasopharyngitis, iii. 31 ; to relieve cough in pulmonary tuberculosis, ii. 497.

Menzer's antistreptococcic serum, use of, in scarlet fever, ii. 128.

Meralgia paresthetica. See Neuritis, external cutaneous.

Mercolint aprons, administration of mercury by, in syphilis, ii. 390 .

Mercurial baths. See Bath.

Mercurial dusting powders, use of, in secondary syphilis of mucosæ and mucocutaneous surfaces, ii. 426.

Mercurial flannels, administration of mercury by, in syphilis, ii. 390.

Mercurial injections, use of, in acute cerebral meningitis, iv. 372 ; in acute hemorrhagic inferior polioencephalitis, iv. 40s; 
in brain syphilis, iv. 480 ; in congenital syphilis of liver, iii. 265; in Landry's paralysis, iv. 329; in optic neuritis without apparent cause, iv. 306 ; to promote absorption in pleurisy, iii. 470 ; to relieve heart block associated with syphilis, iii. 772; in spinal syphilis, iv. 274 ; in syphilitic arteritis, iii. 763 ; in tabes, iv. 194; in tabetic amaurosis, iv. 199; in tuberculous peritonitis, iii. 321 .

Mercurial ointments, use of, in chancre of general integument, ii. 425 ; in chancre of lips, ii. 425 ; in chancre of rectum, ii. 424 ; in gummata, ii. 430 ; in hereditary syphilis, ii. 434; in macular and papular syphiloderms, ii. 425; in palmar and plantar syphilids, ii. 430 ; in papular or tubercular tertiary lesions of syphilis, ii. 426 ; as a prophylactic measure in syphilis, ii. 373 ; in pustular and ulcerative syphiloderms, ii. 426; in syphilitic arthritis, ii. 306; in syphilitic condylomata, ii. 428; in syphilitic lesions of bones and joints, ii. 430; in syphilitic onychia, ii. 429 .

Mercurial poisoning, i. 692; by external application of mercury, i. 695; fatal dose in, i. 695; frequency of, i. 692 ; poisonous action of drug in, i. 693 ; post-mortem changes in, i. 696 ; treatment of, i. 694.

Mercurial purgative, use of, in acute bronchitis, iii. 354.

Mercurial stomatitis. See Stomatitis.

Mercurialism, Chronic, hydrotherapy for relief of, i. 228.

Mercurials, use of, in aneurysm, iii. 766; externally in thyroiditis acuta and struma inflammatoria, iii. 891 ; in trypanosomiasis, iv. 747.

Mercuric chlorid. See Mercury bichlorid.

Mercury, use of, in acute myelitis, iv. 226; in aneurysm of cerebral arteries with syphilitic history, iv. 457; in apoplectic form of vestibular vertigo, with syphilitic origin, iv. 628; in brain syphilis, iv. 479 ; in chronic cerebral meningitis of syphilitic origin, iv. 378 ; in disease of eighth nerve, iv. 315,316 ; in frambœsia tropica, iv. 764; in hydrocephalus, iv. 465 ; indications for, in tabes, iv. 212; in laryngeal tuberculosis complicated with syphilis, iii. 346 ; in leprosy, iv. 816 ; in localized neuritis, iv. 289; by mouth, contraindicated in syphilitic gastritis, iii. 167; in multiple sclerosis, iv. 281; nephritis due to, iv. 27,57 ; objections to, for vesical irrigation in cystitis complicating acute myelitis, iv. 227; in optic neuritis, iv. 305 ; in optic neuritis without apparent cause, iv. 306 ; in pneumogastric paralysis, iv. 319 ; in radicular sciatica, iv. 295; in syphilis of pancreas, iii. 302 ; in syphilis of upper respiratory tract, iii. 348 ; in syphilitic disease of liver, iii. 265; in tumors or cysts of liver, iii. 291.

Mercury, ammoniated, use of, in exulcerated chancre, ii. 423.

Mercury with chalk, use of, in congenital syphilis of liver, iii. 265; in congenital syphilis of upper respiratory tract, iii. 349 ; in hereditary syphilis, ii. 434 ; in syphilis, ii. 386 .

Mercury, gray oil of, use of, in syphilis, by intramuscular injection, ii. 396 ; by rectal injection, ii. 387 .

Mercury, injection of insoluble salts of, ii. 394; as calomel, ii. 396; early use of method, ii. 394; as gray oil of, ii. 396; injection fluid in, ii. 397; injection implement for, ii. 398; injection site for, ii. 398; objections to, ii. 402 ; preparations used for, ii. 396; as salicylate, ii. 397; sterilization of reedle in, ii. 400 ; technique of, ii. 401 ; therry of, ii. 395.

Mercury, metallic, use of, in syphilis, ii. 396.

Mercury in syphilis, ii. 380; absorption of, ii. 382 ; action of, ii. 381 ; administration of, by mouth, ii. 385 ; administration of, in general, ii. 388; continuous intermittent treatment with, ii. 418; continuous treatment with, ii. 417 ; cutaneous administration of, ii. 387 ; danger of, in diabetes associated with syphilis, ii. 768; elimination of, ii. 382 ; in hereditary syphilis, ii. 433; hydrargyrism from excessive use of, ii. 383 ; injection method of administering, ii. 390; insoluble injections of, ii. 394; inunction method of administering, ii. 387; inunction method desirable in infants, ii. 434; "mixed" form of treatment with, ii. 385, 410; in pregnancy, ii. 431 ; preparations of, suitable for use, ii. 385 ; rectal administration of, ii. 387 ; soluble injections of, ii. 392. 
Mercury, yellow oxid of, use of, in cure of eyes in small-pox, ii. 50; as ointment, in rhagades of lip commissures, iii. 5.

Mercury, white precipitate of, use of, in mercurial plasters, ii. 390.

Mercury benzoate, use of, in syphilis, ii. 394.

Mercury bichlorid, use of, in chancre of general integument, ii. 425; as cleansing solution in atrophic rhinitis, iii. 331; after curettage for chronic tonsillitis, iii. 44; as dressing for oriental sore, iv. 756; as dressing for sores in frambosia tropica, iv. 765 ; in echinococcus disease, iii. 256 ; in extraction of guinea worm, iv. 789 ; in exulcerated chanere, ii. 422 ; in gangrenous chancre, ii. 423 ; as gargle in chancre of mouth and tongue, ii. 425; in gonorrheal stomatitis, iii. 21 ; in gummata, ii. 430 ; by hypodermic injection in cerebral thrombosis due to syphilitic endarteritis, iv. 399 ; by hypodermic injection in papula or tubercular tertiary cutaneous lesions of syphilis, ii. 426 ; by hypodermic injection in syphilis, ii. 393 ; by hypodermic injection in tabes, iv. 194; by instillation in chronic gonorrheal urethritis, iv. 160 ; by instillation in tubercular cystitis, iv. $\cdot 121$; by intramuscular injeetion in brain syphilis, iv. 479 ; by intravenous injection in trypanosomiasis, iv. 747; by irrigation for abscess of cervical glands, ii. 247; by irrigation for chancre of vagina or uterine neck, ii. 425 ; by irrigation for mucous patches on general integument, ii. 427; for irrigation of intestines in chronic stage of bacillary dysentery, ii. 328; for irrigation of pleural cavity followed by poisoning, iii. 487; as lotion in craw-craw, iv. 692; in mucous patches, ii. 427 ; in mycosis of tonsils and fauces, iii. 42 ; objections to, as disinfectant for tuberculous sputum, ii. 453 ; in papular and tubercular tertiary syphilitic lesions, ii. 426 ; in pustular and ulcerative syphiloderms, ii. 426; in small-pox, ii. 49; as spray in ozena of syphilitic origin, iii. 337 ; as spray in syphilis of upper respiratory tract, iii. 349 ; in stomatitis diphtheritica, iii. 20 ; in stomatitis hypermycetica, iii. 13 ; in stomatitis syphilitica, iii. 21 ; in syphilitic alopecia, ii. 429 ; in syphilitic onychia, ii. 429; in tertiary syphilitic lesions of mucous or mucocutanceus surfaces, ii. 427; in throat affections complicating scarlet fever, ii. 130.

Mercury biniodid, use of, in scarlet fever, ii. 124 ; in syphilis by hypordermic injection, ii. 393 ; in syphilis, by mouth, ii. 357 .

Mercury cacodylate, use of, in syphilis, ii. 394.

Mercury chlorid. See Calomel.

Mercury cyanid, use of, by hypodermic injection in syphilis of upper respiratory tract, iii. 349; in syphilis, ii. 394.

Mercury lactate, use of, in syphilis, ii. 394.

Mercury oleate, use of, in administration of mercurial plasters, ii. 390 ; in administration of mercury by inunction, ii. 388; in chancre of general integument, ii. 425 ; in macular and papular syphiloderms, ii. 425.

Mercury peptonate, use of, in syphilis, ii. 394.

Mercury perchlorid, use of, in secondary anemias, iii. 810.

Mercury protoiodid, use of, in syphilis, ii. 386.

Mercury salicylate, use of, by hypodermic injection in tabes, iv. 194; in syphilis, ii. 397 ; in syphilitic disease of liver, iii. 265.

Mercury succinamid, use of, in brain syphilis, iv. 480; in cerebral thrombosis due to syphilitic endarteritis, iv. 399.

Mercury tannate, use of, in hydrargyrism affecting kidneys, ii. 385 ; in syphilis, ii. 387.

Mercury vapor lamps, as source of radiant energy, i. 440.

Mesmer, as pioneer in hypnotism, i. 562.

Metabolic Disorders, associated with intestinal autointoxication, ii. 680 .

Metabolism, effects of boracic acid upon, i. 713 ; effects of epinephrin upon, i. 93; effects of radiant energy upon, i. 445; influence of age upon, i. 605; massage and gymnastics in treatment of disorders of, i. 325; radiant light and heat in treatment of, when impaired, i. 435.

Metabolism, disturbances of, chronic myocardial insufficiency due to, iii. 702 ; treatment of, iii. 723 .

Metallic ferments, use of, in treatment of pneumonia, ii. 246. 
Metallic poisoning, in causation of multiple sclerosis, iv. 280.

Metchnikoff, experiments by, on causation of arterial sclerosis, ii. 679 .

MeтchniкоFF AND Roux, demonstration by, that anthropoid apes are susceptible to syphilis, ii. 362 ; experiments by, on prophylactic measures in syphilis, ii. 373 .

METCHNIKOFF's THEORY OF IMMUNITY, i. 128.

Meteorism, in intestinal neuroses, iii. 250; in typhoid fever, ii. 26.

Meteorological climatology, i. 247.

Methemoglobinuria, iv. 4; treatment of, iv. 5 .

Methyl alcohol. See Wood alcohol.

Methyl chlorids, local use of, for neuralgic headache, iv. 598.

Methyl salicylate, local application of, in mumps, ii. 170.

Methylene blue, use of, in destruction of filaria bancrofti, iv. 787; in endemic hematuria, iv. 784; for irrigation of bowels in chronic stage of bacillary dysentery, ii. 228; locally in oriental sore, iv. 756; locally in Vincent's angina, iii. 40 ; to relieve sciatic pain in diabetes mellitus, ii. 766 ; as a substitute for quinin, iv. 726.

Metritis, chronic, hydrotherapy for relief of, i. 211.

Meyer, Adolph, analysis by, of factors essential to psychotherapy, iv. 574; on use of terms in psychotherapy, iv. 574.

Meyer, Willy, apparatus for use of differential air pressure devised by, iii. 446 .

Meyer apparatus, for use of differential air pressure, iii. $446,447$.

Meyer-Ruppel antistreptococcus serum, i. 163.

Mezereon ointment, use of, in syphilitic iritis, ii. 429.

Miana fever, iv. 759.

Michaelis, results obtained by, on use of arsenobenzol in syphilis, iv. 485 .

Michigan, antituberculosis work in, ii. 446.

Micrococcus CatarRHaLis INFECTION, vaccine treatment in, i. 147.

Micrococcus melitensis, iv. 839.

Micrococcus rheumaticus, iii. 743.

MicroörganisM, causing gangosa, iv. 865; causing Malta fever, iv. 839; present in leprosy, iv. 809, 811.
Microörganisms, present in acute cerebral meningitis, iv. 368 ; in acute peritonsillitis, iii. 43 ; in acute rhinitis, iii. 328 ; in bronchopneumonia, iii. 415; in cerebral abscess, iv. 419 ; in cystitis, iv. 129 ; in cholecystitis and cholelithiasis, iii. 265; in empyema, iii. 484 ; in infectious sinus thrombosis, iv. 427; in membranous pharyngitis, iii. 38 ; in pyelitis, iv. 84,89 ; in rheumatism, iii. 744 .

MIDDLE EAR, importance of protecting, in influenza, ii. 189; suppuration of, causing optic neuritis, iv. 306.

Migraine, iv. 589; abortive attacks of, iv. 590; administration of thyroid gland for relief of, i. 76; association of, with periodic ocular paralysis, iv. 309; constitutional treatment of, iv. 594; equivalents for, iv. 595; groups of, iv. 589; hydrotherapy for relief of, i. 222; as a symptom of neurasthenia, iv. 565; treatment of, during attack, iv. 592; varieties of, iv. 590 .

Migraine, ophthalmic, iv. 589.

Migraine, ophthalmoplegic, iv. 589.

Migraine, symptomatic, iv. 589.

"Migrating cases," in erysipelas, ii. 283.

Mikulicz, operation introduced by, for rèlief of idiopathic dilatation of esophagus, iii. 59.

Mikulicz's Disease, X-ray therapy for relief of, i. 551.

MILK, in diet for acute nephritis, iv. 60; in diet of chronic interstitial nephritis, iv. 68 ; in diet of chronic parenchymatous nephritis, iv. 65 ; in diet of diabetes mellitus, ii. 41 ; in diet for depressive disorders of gastric secretion, iii. 147; in diet for gastric hyperacidity, iii. 83; in diet for nephritis, iv. 41 ; in diet for pyelitis, iv. 87; in diet for typhoid fever, ii. 12 ; digestibility of, i. 629 ; as food in chronic enteritis of moderate severity, iii. 199; ice-cold, as food in cholera infantum, iii. 205; idiosyncrasies to, i. 629 ; as infants' food, iii. 201 ; long continued use of boiled, a cause of rachitis, ii. 816 ; long continued use of condensed, a cause of rachitis, ii. 817; long continued use of dried, a cause of rachitis, ii. 817; long continued use of sterilized, a cause of rachitis, ii. 816; objections to, in diet of acute dysentery, 
ii. 328 ; objections to, in diet of acute enteritis, iii. 193; objections to, in diet of chronic dysentery, ii. 328; transmission of dysentery by, ii. 319 ; transmission of foot and mouth disease by, ii. 582; transmission of scarlet fever by, ii. 16; transmission of typhoid fever by, ii. 23; in treatment of uremia, iv. 52 ; use of hot, for relief of cough in pulmonary tuberculosis, ii. 497.

"Milk of bismuth," use of, in enteritis of infancy, iii. 203.

Milk of magnesia. See Magnesia.

MiLK AND EGGS, in diet of gastric ulcer, iii. 129.

MilK AND MILK PRoducts, poisoning by, ii. 645 .

Milk and water, hot, use of, as gargle in acute tonsillitis, iii. 42.

MilK DIET, in acute infectious myocarditis, iii. 750; in autotoxic methemoglobinemia, iii. 879; in bronchial asthma, iii. 382; in chronic myocardial insufficiency, iii. 716,721 ; in chronic myocardial insufficiency due to obesity, iii. 727 ; in continuous gastric hypersecretion, iii. 106; in delirium tremens, ii. 603; in diphtheria, ii. 276; in gastric hyperacidity, iii. 100; in gastric ulcer of pylorus, iii. 181; in hill diarrhea, iv. 863; indications for, in neurasthenia, iv. 537 ; in influenza with gastrointestinal symptoms, ii. 190; in nephritis, iv. 43 ; in nephritis complicating scarlet fever, ii. 133, 134; in nonbleeding gastric ulcer, iii. 127, 128; objections to, in cirrhosis of liver, iii. 281; in pericarditis, iii. 516; in pneumonia, ii. 229; recommended by Dubois for neurasthenia, iv. 545; in scarlet fever as preventive of nephritis, ii. 133; in smallpox, ii. 46 ; in sprue, iv. 862 ; in treatment of ascites in cirrhosis of liver, iii. 285 ; in whooping-cough, ii. 179 ; in wood alcohol poisoning, ii. 610 .

Milk sugar. See Lactose.

Miliary fever, ii. 578; characteristics of, ii. 578; convalescence from, ii. 578; definition of, iii. 578; eruption in, ii. 578; mortality from, ii. 579; prophylaxis against, ii. 579; quarantine in, ii. 579; treatment of, ii. 579 .

Mills, investigations by, on action of radium upon liver, i. 470.
Muton, case reporterl by, of removal of large part of sternum for suppurative mediastinitis, iii. 505.

MIND hLINDNEss, as localizing symptom of tumor in left cerebral hemisphere, iv. 440.

Mineral acids, poisoning by, i. 723.

Mineral acids, action of, on glandular secretion in severe infections, ii. 25; use of, to lessen bronchial secretion in acute bronchitis, iii. 365; use of, in oxaluria, iv. 8 ; use of, to relieve profuse expectoration in chronic bronchitis, iii. 376.

Mineral ions, use of, in hemophilia, iii. 862.

Mineral oils, use of, in chronic constipation, iii. 235.

Mineral polsons, i. 673,674 : ammonia, i. 745; antimony, i. 688; arsenic, i. 674; bismuth subnitrite, ii. 721; borax and boracic acid, i. 710 ; bromin, i. 733 ; carbon monoxid, i. 735 ; caustic alkalies, i. 743; caustic potash, i. 744; caustic soda, i. 744; chlorin, i. 733; copper, i. 697; hydrochloric acid, i. 732; hydrocyanic acid, i. 737; hydrogen sulphid, i. 735; inorganic gases, i. 723; lead, i. 701; mercury, i. 692; mineral acids, i. 723 ; nitric acid, i. 630 ; nitrites, i. 732 ; nitrous acid, i. 732; potassium chlorate, i. 720; silver, i. 708 ; sulphuric acid, i. 724 ; sulphurous acid and the sulphates, i. 726 ; tin, i. 701 ; varieties of, i. 673 ; zinc, i. 707 .

Mineral springs, benefit from, in treatment of acute myelitis, iv. 228; benefit from thermal, in tabes, iv. 201; percentage of iron in, iii. 820.

Mineral waters, action of, i. 235 ; classification of, i. 223; composition of different, i. 231 ; presence of radioactivity in, $i$. 472; principles of, i. 229; resultant action of, ii. 236 ; temperature of, i. 231 ; use of, in chronic cholecystitis and cholelithiasis, iii. 271; use of, in chronic constipation, iii. 234; use of, in chronic gastritis, iii. 161; use of, in cirrhosis of liver, iii. 283; use of, for hyperacidity in chlorosis, iii. S23; use of, to promote evacuation of bowels in gastric atony, iii. 172.

Miners' Phthisis, causes of, iii. 425.

Minim, Prof., minim light for use in light therapy devised by, i. 442 .

Minim light, use of, in light therapy, i. 442. 
Mistura ferri et ammonii acetatis. See Basham's mixture.

Mitchell, J. K., schedule for dietary during rest cure arranged by, iv. 541

Mitchell, S. Weir, advocacy of massage by, i. 287; rest cure for neurasthenia established by, iv. 533 .

Mitten friction, use of, to reduce temperature in pneumonia, ii. 233.

"Mixed treatment," administration of mercury and iodin together by, in syphilis, ii. 407; use of, in brain syphilis, iv. 481 ; use of, in hereditary syphilis, ii. 434; use of, during pregnancy, ii. 432; use of, in syphilis of upper respiratory tract, iii. 348 .

Modalities from static machine, i. 374.

Möвıus, work cure for neurasthenia introduced by, iv. 552 .

MонN, disinfection of sick room as means of curtailing whooping-cough advocated by, ii. 179 .

Moist alkaline atmosphere, benefit of, in membranous pharyngitis, iii. 39 .

Moisture, excessive, injurious effects of, in tropics combined with excessive heat, iv. 669 .

MolDs, as cause of pellagra, ii. 657.

Monkeys, reaction of, to vaccination and small-pox, ii. 93; ulcerative colitis due to Balantidium coli observed among, iv. 761.

Monsel's solution, use of, to control epistaxis objectionable, iii. 335 .

Montague, Lady Mary Wortley, inoculation for small-pox introduced by, ii. 52 .

Montenuis, on indications for treatment in enteroptosis, iii. 246.

Moore's treatment of anelirysm, iii. 767.

MORAL PERVERSION, accompanying chlorosis, iii. 824.

Morawitz AND ZahN, on use of iron in chlorosis, iii. 816.

Morbid anatomy, of prenatal type of cerebral palsies of children, iv. 409 .

Morbus maculosus, iii. 860 .

Moritz and Dietren, method employed by for determining extent of chronic myocardial insufficiency by Röntgen rays, iii. 677 .

“"Morning diarrhea," iii. 250.

Morphia. See Morphin.
Morphin, use of, to abort attacks of pulmonary edema in chronic interstitial nephritis, iv. 72; in acute catarrhal cholecystitis, iii. 267 ; in acute enteritis, iii. 194; in acute gastritis, iii. 156 ; in acute gout, ii. 703; in acute inferior hemorrhagic poliomyelitis, iv. 408; in acute or intermittent gastric hypersecretion, iii. 102 ; to allay hunger in starvation treatment of gastric ulcer, iii. 118; in apoplectic form of vestibular vertigo, iv. 629 ; in appendicitis, iii. 214, 217, 218 ; in bacillary dysentery, ii. 327 ; in bronchial asthma, iii. 388; in bronchopneumonia with cyanosis due to bronchial obstruction, undesirable, iii. 454; in carcinoma of liver, iii. 291 ; in cardiac asthma due to chronic myocardial insufficiency, iii.737; in cholera infantum, iii. 204 ; in chronic intestinal obstruction, iii. 242 ; in chronic progressive bulbar paralysis, iv. 253; to control cough during active stage of tuberculosis, ii. 482 ; to control cough due to chronic bronchitis associated with cardiac insuffciency, iii. 731; to control cough in pericarditis, iii. 515; to control cough in progressive bulbar paralysis, iv. 499 ; to control diarrhea in Addison's disease, iii. 885; to control drooling in chronic progressive bulbar paralysis, iv. 253; to control hematemesis during cirrhosis of liver, iii. 288; to control hemorrhage in pulmonary tuberculosis, ii. 500; to control hemorrhage during thoracentesis, iii. 479; to control hemorrhage in typhoid fever, ii. 19; to control restlessness in cerebral hyperemia, iv. 386 ; to control spasmodic cough in chronic progressive bulbar paralysis, iv. 253 ; to control uremic vomiting, iv. 54; to control vomiting in acute localized peritonitis, iii. 312 ; contraindicated in bronchopneumonia complicating influenza, ii. 192; contraindicated for cough of pulmonary tuberculosis, ii. 498; contraindicated for nervous symptoms complicating measles, ii. 152 ; contraindicated in plastic bronchitis, iii. 392; contraindicated for relief of insomnia in neurasthenia, iv. 565; in eclampsia of pregnancy, iv. 83; for edema of glottis in acute nephritis, iv. 63 ; in enterocolitis complicating measles, 
ii. 161 ; in gallstone colic, iii. 267 ; in hydrophobia, ii. 342 ; indispensable in cholera morbus, iii. 195; in intubation of larynx, ii. 275; in laryngeal tuberculosis, iii. 346,348 ; in inalarial fever for control of vomiting, iv. 727 ; in membranous enteritis, iii. 208; in migraine, iv. 593 ; in myocardial insufficiency of a severe type, iii. 721 ; in periearditis as cardiac stimulant, iii. 520 ; in pernicious malaria of a choleraic type, iii. 727 ; in pneumonia of alcoholics, ii. 258; in pneumothorax with empyema, iii. 493 ; in pulmonary edema, iii. 409; in pulmonary edema complicating pneumonia, ii. 259 ; in pulmonary edema occurring during thoracentesis, iii. 480 ; in pulmonary thrombosis, iii. 411 ; for relief of convulsions due to brain tumor, iv. 448; for relicf of crises in tabes, iv. 197; for relief of dyspnea in chronic pulmonary dyspnea, iii. 438; for relief of dyspnea in tuberculosis, ii. 506; for relief of gastric irritability in typhoid fever, ii. 25; for relief of headache in pachymeningitis, iv. 364 ; for relief of hiccough complicating pneumonia, ii. 260; for relief of insomnia due to brain tumor, iv. 447 ; for relief of insomnia in cardiac insufficiency, iii. 664 ; for relief of insomnia due to chronic cerebral hyperemia, iv. 387 ; for relief of insomnia in influenza, ii. 192; for relief of insomnia in typhoid fever, ii. 26 ; for relief of lancinating pains in tabes, iv. 197; for relief of nocturnal dyspnea in chronic nephritis, iv. 72 ; for relief of pain and dyspnea in acute congestion of lungs, iii. 406; for relief of pain in acute diffuse peritonitis, iii. 315 ; for relief of pain in acute localized peritonitis, iii. 311 ; for relief of pain in acute myelitis, iv. 226; for relief of pain in acute pancreatitis, iii. 296 ; for relief of pain in acute peritonsillitis, iii. 43; for relief of pain in acute polymyositis, ii. 685; for relief of acute pulmonary edema in nephritis, iv. 55; for relief of pain in acute rheumatism, ii. 297 ; for relief of pain in angina pectoris, iii. 768 ; for relief of pain in arthropathies due to hemophilia, iii. 870; for relief of pain in carcinoma of stomach, iii. 166; for relief of pain in cerebrospinal meningitis, ii.
215 ; for relief of pain in chest in tubereulosis, ii. 506; for relief of pain in esophagus due to chemicals swallowed, iii. 65; for relief of pain in joints and limbs in tropieal rolapsing fever, iv. 760; for reliof of pain in Malta fever, iv. 839 ; for relief of pain in mediastinal tumor, iii. 504; for relief of pain in muscarin poisoning, ii. 648; for relief of pain in neuralgia, iv. 340 ; for relief of pain in neuritis, iv. 286; for relief of pain in pleurisy, iii. 467, 468; for relief of pain in pneumonia, ii. 227 ; for relief of pain in pneumothorax acutissimus, iii. 491; for relief of pain in pyelitis, iv. 88 ; for relief of pain in renal colic, iv. 104; for relief of pain in Roeky Mountain fever, ii. 565; for relief of pain in septicemia, ii. 291 ; for relief of pain in small-pox, i. 47; for relief of pain in strangulation of bowels, iii. 240 ; for relief of pain in tumors of pancreas, iii. 305; for relief of restlessness in acute ophthalmoplegia, iv. 509; for relief of restlessness in black water fever, iv. 741 ; for relief of restlessness in cerebral hyperemia, iv. 392 ; for relief of vomiting due to brain tumor, iv. 448; for relief of vomiting in cerebral abscess, iv. 423 ; in respiratory type of influenza, ii. 189; for restraint of delirium in pneumonia, ii. 228 ; in spastic constipation, iii. 231; in spasmodic laryngitis, iii. 342 ; in suppositories for relief of pain in pyelitis, iv. 88 ; in tetanus, ii. 348 ; in tetany, iii. 910 ; in treatment of morphinism, ii. 61S, 623; in tuberculous kidney, iv. 117 ; in ulcerative colitis, iii. 212 ; in volvulus and intussusception, iii. 241.

Morphin and apomorphin, use of, in bronchitis due to chronic myocardial insufficiency, iii. 738 .

Morphin and atropin, use of, to control gastric hemorrhage, iii. 109; in renal colic, iv. 105; to sustain strength during thoracentesis, iii. 478; in treatment of nephritis complicating scarlet fever, ii. 133.

Morphin and calomel, use of, in acute nephritis, iii. 195.

Morphin and chloral, use of, for hallucinatory excitement in progressive paralysis of the insane, iv. 493. 
Morphin, digitalis, and strychnin, use of, in delirium tremens, ii. 607.

Morphin and hyoscin, use of, for relief of insomnia in typhoid fever, iii. 27.

MoRPHIN HABIT, ii. 617; danger of acquiring from use of morphin in asthma, iii. 388; danger of acquiring from use of morphin to relieve cough in chronic bronchitis, iii. 375 ; in the East, ii. 614; Hirschlaff's heil-scrum for cure of, ii. 624; hydrotherapy for cure of, i. 228; hypnotism in treatment of, ii. 624; Jenning's method of treating, ii. 623; Lambert's method of treating, ii. 621 ; methods of acquiring, ii. 616; Town's specific treatment for, ii. 621 ; treatment of, in general, ii. 617.

Morphinism. See Morphin habit.

MorRis, R. S., results of operation for dermoid cyst of mediastinum collected by, iii. 501.

Morrow, Dr. Howard, formula recommended by, for use in acute catarrhal jaundice, iii. 262.

Mortality, from acute diffuse peritonitis, iii. 314; from acute glossitis, iii. 23; from appendicitis in pregnancy, iii. 220; from Asiatic cholera under serum therapy, iv. 832; from Asiatic cholera with and without inoculation, iv. 830; from asthenic form of measles, ii. 154; from brain syphilis, iv. 477; from brain tumors after operation, iv. 452 ; from bronchopneumonia complicating influenza, ii. 192 ; from bronchopneumonia complicating measles, ii. 158; from bronchopneumonia complicating whooping-cough, ii. 175; from cerebral abscess, iv. 421 ; from cerebrospinal meningitis, ii. 202; from cirrhosis of liver, iii. 287; from delirium tremens, ii. 607; from dengue, iv. 858; from diabetes mellitus, ii. 718; from disease of pituitary body, iii. 917; effect of serum treatment upon, from anthrax, ii. 332 ; effect of serum treatment upon, from cerebrospinal meningitis, ii. 202; effect of serum treatment upon, from dysentery, ii. 321; effect of tuberculin upon, from tuberculosis, ii. 523; effect of vaccination upon in small-pox, ii. 93; from epidemic dropsy, iv. 860 ; from exophthalmic goiter, iii. 895, 905; from gastric hemorrhage, iii. 117; following gastroenter- ostomy for gastric ulcer, iii. 141; from glandular fever, ii. 577; from goiter, iii. 894; from hydatid disease of liver, iii. 291; from influenza meningitis, ii. 212; from Ludwig's angina, iii. 30; from miliary fever, ii. 579; from occupations, iii. $424,425,426,427$; following operation for chronic pancreatitis, iii. 300; following operation for infectious sinus thrombosis, iv. 430; following operation for pancreatic calculi, iii. 301; following operation for pancreatic eysts, iii. 302; following operation for tuberculous peritonitis, iii. 319; from paroxysmal hemoglobinuria, iv. 7; from plague with and without inoculation, iv. 823; from pneumonia, ii. 218, 234; from psittacosis among parrots, ii. 585; from pulmonary abscess and gangrene under medical treatment, iii. 441; from pulmonary abscess and gangrene under surgical treatment, iii. 442; from shima mushi, iv. 692; from small-pox before vaccination, ii. 54, 93; from small-pox since vaccination, ii. 93 ; from small-pox in the vaccinated and unvaccinated, ii. 91; from small-pox with and without vaccination scars, ii. 92 ; from stomatitis gangrenosa, iii. 16; from streptococcus and pneumococcus meningitis, ii. 213; from tetanus, ii. 350 ; from tetradon poisoning, iv. 684; from trichiniasis, iii. 254; from tuberculosis, ii. 450; from tuberculosis treated with tuberculin, ii. 523; from tuberculous meningitis, ii. 212; from typhoid fever, ii. 15, 19; from typhus fever, ii. 37; from uremia in Asiatic cholera, iv. 837; from uremia associated with nephrolithiasis, iv. 107; from verruga peruviana, iv. 863; from Vincent's angina, iii. 40; from Weil's disease, ii. 571.

Morton, on production of artificial pneumothorax in tuberculosis, ii. 533.

Morvan's disease, iv. 278.

Moser's antistreptococcic serum, use of, in scarlet fever, ii. 129.

Moser's scarlatinal serum, i. 164.

Mosquitoes, in transmission of filaria, iv. 787 ; in transmission of malarial fever, iv. 729 .

Motor DIsturbances, as symptom of poison, i. 668; of stomach, see Stomach. 
Motor dynamo, i. 369.

Motor mritative phexomena, value of, in regional diagnosis of brain tumor, iv. 437.

Motor paralysis, value of, in regional diagnosis of brain tumor, iv. 438.

Motor sphere, changes in, during hypnosis, i. 573.

Motoring, as a therapeutic measure in cardiac insufficiency, iii. 666 .

Motschutkowsky, suspension as therapeutic agent in tabes first recommended by, iv. 203.

Mountain resorts, value of, in neurasthenia, iv. 556.

Mountain sickness, physiology of, i. 270, 272.

Mouth, Care of, in cholera infantum, iii. 205; in diabetes mellitus, ii. 736 ; in measles, ii. 148; in meat poisoning, ii. 639 ; in pellagra, ii. 662 ; in pernicious anemia, iv. 825 ; in pneumonia, ii. 221; in progressive paralysis of the insane, iv. 494; as prophylactic measure against bronchopneumonia, iii. 413; in scurvy, iii. 875 ; in syphilis, ii. 368 ; in syphilis of upper respiratory tract, iii. 349 ; in varicella, ii. 113.

Mouth, diseases of, iii. 1: bacteria present in mouth, iii. 11; Baelz's disease, see Inflammation of labial glands; Bednar's aphthæ, iii. 11; classification, iii. 2; diseases of lips, iii. 4 ; disturbances of sensation, iii. 3; disturbances of taste, iii. 4; gonorrheal stomatitis, iii. 20; herpes of lips, iii. 5; inflammation of labial glands, iii. 6 ; mercurial stomatitis, iii. 16; pyorrhea alveolaris, iii. 22 ; offensive breath, iii. 3 ; septic infection of oral origin, iii. 2 ; stomatitis, iii. 6 ; stomatitis catarrhalis, iii. 6 ; stomatitis crouposa, iii. 19; stomatitis gangrenosa, iii. 19 ; stomatitis herpetica, iii. 8 ; stomatitis hypermycetica, iii. 11; stomatitis syphilitica, iii. 21 ; stomatitis ulcerosa, iii. 13; stomatitis ulcerosa chronica, iii. 16.

Mouth breathing, evils of, in chronic bronchitis, iii. 368 .

Mouth wash, for use, in lobar pneumonia, ii. 222 ; in mumps, ii. 170 ; in Rocky Mountain fever, ii. 565; in stomatitis due to hydrargyrism, ii. 385 ; in syphilis, ii. 269.
Mouth washes, antiseptic, use of, in pyorrhea alveolaris, iii. 22 ; in stomatitis syphilitica, 21.

MoviMents, involuntary, as a characteristic symptom of cerebral diplegia type of cerebral palsies of children, iv. 411.

Movements, passive, use of, in beri-beri, iv. 854; during convalescence from Landry's paralysis, iv. 329 ; to relieve spasticity in syringomyelia, iv. 279 ; in treatment of obstetric paralysis, iv. 292.

Movements, tic, iv. 653.

Mucocutaneous junctions, treatment of secondary syphilitic lesions of, ii. 426; treatment of tertiary syphilitic lesions of, ii. 427 .

Mucos $x$, treatment of secondary syphilitic lesions of, ii. 426 ; treatment of tertiary syphilitic lesions of, ii. 427.

Mucous membranes of throat, swelling of, in mumps, ii. 171.

Mucous patches, local treatment of, ii. 427.

Mucus, increased secretion of, in gastric ulcer, iii. 136; by stomach from use of silver nitrate, iii. 137.

Muller, A., method employed by, for determining extent of chronic myocardial insufficieney, iii. 696.

Multiple neuritis. See Neuritis.

Multiple sclerosis, iv. 279; etiology of, iv. 280 ; frequeney of, iv. 279 ; symptoms of, iv. 280; treatment of, iv. 280.

Multivalent vaccines, i. 140.

Mummery, appendicostomy in ulcerative colitis with hemorrhage advised by, iii. 212 ; cases of appendicostomy in membranous enteritis collected by, iii. 210; on ehoice of operation in constipation due to obstruetion, iii. 237; formula recommended by, for rectal injection in membranous injection, iii. 20s; on postmortem conditions in membranous enteritis, iii. 20s; protargol and argyrol recommended by, for use by enemata in chronic mucous colitis, iii. 207.

Mumps, ii. 166; complications in, ii. 166; definition of, ii. 166; diet in, ii. 169; epidemics of, ii. 168; epididymitis complicating, ii. 166, 171; general considerations on, ii. 166; history of, ii. 167; immunity conferred by, ii. 169; incubation period in, ii. 168; infectiousness of, 
ii. 168; inflammation of mammary gland complicating, ii. 166; inflammation of ovaries complicating, ii. 166; operative measures in, ii. 171 ; orchitis complicating, ii. 166, 171; organism found in, ii. 157; prophylaxis of, ii. 169; ptyalism complicating, ii. 171 ; suppuration complicating, ii. 171; susceptibility to, ii. 167 ; synonyms for, ii. 166 ; transmission of, ii. 168; treatment of, ii. 169.

MURPhy, on appendicitis in typhoid fever, iii. 219; on mortality following operation for acute diffuse peritonitis, iii. 313 ; production of artificial pneumothorax in tuberculosis advocated by, ii. 534 .

Murphy drip method, administration of hot saline by, in acute diffuse peritonitis, iii. 317,318 ; administration of hot saline by, in appendicitis, iii. 218; administration of normal saline solution by, in acute localized peritonitis, iii. 312 ; administration of normal saline solution by, in blackwater fever, iv. 740; administration of normal saline solution by, in pyelitis, iv. 89; administration of normal saline solution by, for relief of anuria in nephrolithiasis, iv. 107; administration of normal saline solution by, in uremia without edema, iv. 62 ; administration of nourishment by, in gastric ulcer, iii. 120; administration of sodium bicarbonate by, in diabetic coma, ii. 757.

Murrell, on polyarticular character of gonococcic arthritis in children, ii. 306.

Murrell and Hicks, on bacillus found in gonococcic arthritis, ii. 306; bacillus obtained by, from gleety discharges, ii. 295.

Muscarin, as a cause of food poisoning, ii. 635.

Muscle cELls, independent irritability of, i. 372 .

Muscles, effect of cold baths upon, i. 190; effect of hot baths upon, i. 192; electrotherapeutics for relief of reflex spasm of, i. 412 .

Muscles of NECK, spasm of, iv. 655; treatment of, iv. 656 .

Muscles, syphilis of, ii. 685; treatment of, ii. 686 .

Muscle-cutting, employment of, in spasm of neck muscles, iv. 657 .
Muscle-group isolation, employment of, in spasm of neck muscles, iv. 657.

Muscular Cramps, ii. 689.

Muscular Dystrophy, iv. 260; clinical types of, iv. 261; definition of, iv. 260; progressive neural type of, iv. 265; treatment of, iv. 262.

Muscular dystrophy, NEURAL PKOGREsSIVE, iv. 265; treatment of, iv. 266.

Muscular REsponse to Electrical NERVOUS IRRITATION, i. 271.

Muscular rheumatism, ii. 688; hydrotherapy for relief of, i. 209.

Muscular RIGIDITY, as a symptom of tuberculous meningitis, iv. 374 .

Muscular spasms, lOcalized, iv. 652: chorea minor, iv. 662 ; definition of, iv. 652 ; occupation neuroses, iv. 660 ; paralysis agitans, iv. 665; paramyoclonus multiplex, iv. 659 ; tic, iv. 652 .

Muscular system, effect of massage upon, i. 298.

Muscular WeAKNess, accompanying rachitis, ii. 818.

Muscular work, influence of, on food requirement, i. 606.

Musculature, of ventricles, iii. 531; weakness in, a leading symptom of myasthenia gravis, iv. 505.

Mushroom FAMILY, poisoning by members of, ii. 647 .

Musk, use of, for relief of hiccough complicating pneumonia, ii. 260.

Mussel poisoning, ii. 645.

Musser, use of nux vomica in asthma for long time in small doses suggested by, iii. 384.

Mustard and water, use of, as emetic in acute bronchitis, iii. 365 ; in acute opium poisoning, ii. 614 .

Mustard foot bath, hot, use of, in disease of eighth cranial nerve beginning in labyrinth, iv. 316 ; in rheumatic cases of facial paralysis, iv. 311.

Mustard foot baths, use of, in pulmonary edema complicating pneumonia, ii. 259.

Mustard leaves, use of, in acute bronchitis, iii. 356 ; in chronic bronchitis, iii. 373 ; for relief of pain in sciatica, iv. 299 .

Mustard pack, use of, to relieve cyanosis and dyspnea in bronchopneumonia, iii. 419. 
Mustard plasters, use of, in cerebral anemia, iv. 382 ; in edematous laryngitis, iii. 34 ; to relieve pain in chest accompanying tuberculosis, ii. 506; over trachea and bronchi for relief of cough in pulmonary tuberculosis, ii. 497.

Mustard poultices. See Poultices.

Mutron, meat poisoning due to, ii. 638 .

Myaldia. See Muscular rheumatism.

Myasthenia gastirica, iii. 168.

Myasthenia gravis, iv. 498, 504; differential diagnosis of, iv. 506; etiology of, iv. 504 ; introduction to subject of, iv. 504; pathology of, iv. 504; prognosis of, iv. 506 ; symptoms of, iv. 505 ; treatment of, iv. 507 ; types of, iv. 506.

Myasthenia gravis pseudoparalytica. See Bulbar paralysis, asthenic.

Myasthenic reaction, iv. 505.

Mrcetismus, ii. 647.

Mycosis Fungoides, X-ray therapy for relief of, i. 530.

Mycosis of TONSILS AND FAUCES, iii. 42.

Myelitis, ACUTE, iv. 218; as a cause of optic neuritis, iv. 306; classification of, iv. 219 ; course of, iv. 224; definition of, iv. 218; diagnosis of, iv. 224; etiology of, iv. 219; gonorrheal form of, iv. 224, 228; hydrotherapy for relief of, i. 223; incomplete forms of, iv. 221; at menopause, iv. 224; pathology of, iv. 221 ; prognosis of, iv. 224 ; in puerperal fever, iv. 224 ; symptoms of, iv. 222; treatment of, iv. 224.

Myelitis, acute bulbar. See Acute hemorrhagic inferior polioencephalitis.

Myelitis, Chronic, iv. 228; etiology of, iv. 229; hydrotherapy for relief of, i. 224 ; symptoms of, iv. 229 ; treatment of, iv. 230 .

Myelitis, Funicular, iv. 231; etiology of, iv. 231 ; pathology of, iv. 231 ; symptomatology of, iv. 232; treatment of, iv. 232.

Myelitis, traumatic, electrotherapeutics for relief of, i. 429.

Myiasis, cutaneous, iv. 686; preventive measures in, iv. 688 ; remedial measures in, iv. 688; species of flies causing, iv. 687.

Myiasis, intestinal, iv. 689 ; preventive measures in, iv. 690; remedial measures in, iv. 689; species of flies causing, iv. 689.

Myiasis of Natural orifices of Body, iv. 688 ; preventive measures in, iv. 689 ; remedial measures in, iv. 689; species of flies causing, iv. 688 .

Mrocardial insurficiency, chronic, aneurysm associated with, iii. 766 ; estimation of extent of, iii. 693; functional tests for, iii.693; Graupner method for, iii.694; Herz "Selbst-Hemmung" method for, iii. 693; Katzenstein's method for, iii. 694; Mendelsohn-Gräupner method for, iii. 693; methods for, dependent upon blood pressure changes, iii. 694; Moritz and Dietlen methorl for, iii. 697; Plesch's method for, iii. 696; plethysmographic method of A. Muller for, iii. 696 ; principles involved in, iii. 693; relation of maximal and minimal blood pressures to, iii. 695; Röntgen method of, see Moritz and Dietlen; value of functional tests for, iii. 697.

Myocardial insufficiency, CHRonic, phoPHYLAXIS OF, iii. 698; from use of alcohol, iii. 707; in chronic myocarditis, iii. 707; in chronic nephritis, iii. 709; from concretio pericardii, iii. 708 ; in disturbances of general metabolism, iii. 702; in kyphoscoliosis, iii. 712; in long-continued rest, iii. 702; in obesity, iii. 703; from overst imulation, iii. 698; in pulmonary conditions, iii. 709; from valvular lesions, iii. 712 .

MYocaRdial INSUFFICIENCY, CHRONic, SYMPTOMATIC TREATMENT OF, iii. 736; of bronchitis, iii. 738; of cardiac asthma, iii. 737; of constipation, iii. 739; of cutaneous symptoms, iii. 740; of gastrointestinal disturbances, iii. 738; of nervous symptoms, iii. 740 .

MYocardial iNSUFFICIENCY, CHRONIC, TREATMENT OF, iii. 714; due to alcohol, iii. 728; due to arteriosclerosis, iii. 728 ; due to chronic myocarditis, iii. 72s; due to chronic nephritis, iii. 730 ; due to concretio pericardii, iii. 730 ; due to congenital heart lesion, iii. 735; of doubtful diagnosis, iii. 722; due to kyphosis, iii. 732; due to long-continued rest, iii. 724 ; due to metabolic disturbances, iii. 723 ; in mild cases, iii. 715 ; in moderate cases, iii. 716 ; in the obese, iii. 724 ; due to overstimulation, iii. 723; due to pulmonary conditions, iii. 731 ; in severe cases, iii. 718; due to syphilis of myocardium, iii. 729 ; due to valvular lesions, iii. 732 .

M YOCARDIAL SYPHILIS, cardiac insufficiency 
from, iii. 708; chronic myocardial insufficiency due to, iii. 729 .

Myocarditis, as a complication of diphtheria, ii. 276.

Myocarditis, aCUTE, iii. 747.

Myocarditis, aCUTE SIMPle, iii. 747; after-treatment of, iii. 751; diet in, iii. 750; frequency of, iii. 748; infectious diseases associated with, iii. 747 ; medicinal treatment of, iii. 750 ; prophylaxis against, iii. 748; treatment of, iii. 749.

Mrocarditis, ACute infectious. See Myocarditis, acute simple.

Myocarditis, ACUTE PRIMARY, iii. 747.

MYocarditis, chronic, chronic myocardial insufficiency due to, iii. 707; treatment of, iii. 728.

MYocaRditis, SEPTIC, iii. 747.

Mrocardium, anatomical structure of, iii. 531 ; deficient nutrition of, a cause of cardiac insufficiency, iii. 586; dilatation of, due to repeated strain, iii. 548 ; recognition of functional insufficiency of, iii. 691; strain causing dilatation of normal, iii. 546; strain causing dilatation of, when weakened from any cause, iii. 548 .

Myocardium, diseases of, iii. 530; alcohol in causation of, iii. 687; anatomical and physiological considerations relating to, iii. 530; diet in, iii. 683 ; digitalis therapy in, iii. 575; diseases of arteries, iii. 723; drug treatment of, iii. 575; endocarditis, iii. 743; functional diseases of heart, iii. 772; general treatment of, iii. 571; gymnastics for, iii. 636 ; hydrotherapy for, iii. 615 ; marriage a question in, iii. 680 ; myocardial insufficiency, iii. 690 ; myocarditis, iii. 747 ; occupation a question in, iii. 678; pathological physiology of cardiac dilatation, iii. 546; physiology of cardiac hypertrophy, iii. 544 ; recreation a question in, iii. 682 ; sleep in, iii. 663 ; tobacco in causation of, iii. 688 ; travel a benefit in, iii. 665 ; treatment of, in general, iii. 571; treatment of general anesthesia and serous effusion, iii. 672; vasomotor paralysis, iii. 751 ; venesection in treatment of, iii. 667 .

MYOGENIC THEORY, of cardiac contractions, iii. 535.

Myomata of uterus, X-ray therapy for relief of, i. 555 .
Myositis, various Forms of, ii. 684: acute polymyositis, ii. 684 ; muscular cramps, ii. 689; muscular rheumatism, ii. 688; myositis fibrosa, ii. 687; myositis ossificans, ii. 687 ; primary suppurative myositis, ii. 686; secondary suppurative myositis, ii. 687; syphilis of muscles, ii. 685; tuberculous myositis, ii. 687.

Myositis, Chronic, iv. 604; treatment of, iv. 605 .

Myotonia congenita, iv. 266.

Myrrh, tincture of, use of, in chronic glossitis, iii. 25.

Myrtol, use of, as an expectorant in bronchiectasis, iii. 397.

Myтilotoxin, as a cause of food poisoning, ii. 635 .

Myтilotoxismus. See Mussel poisoning.

NAEgeli, classification of anemias by, iii. 806.

NAVI, radium in treatment of, i. 476.

NÄGELE, method suggested by, for cutting short paroxysm of whooping-cough, ii. 178.

NAGEOTTE, work on pathogenesis of tabes first done by, iv. 183.

NANCY SCHOOL OF PSYCHOTHERAPY, iv. 570.

NANSEN, etiology of scurvy demonstrated by, iii. 874 .

Naphthalin, use of, as an antiseptic in intestinal autointoxication, ii. 675 ; in destruction of tsetse fly, iv. 750 .

Naphthalin tetrachlorid, use of, in leukemia, iii. 847 .

Narcotic Drugs, symptoms due to misuse of, in whooping-cough, ii. 175.

Narcotics, use of, in irritative gastric neuroses, iii. 188; in marantic sinus thrombosis with convulsions and delirium, iv. 427 ; for relief of insomnia due to cerebral abscess, iv. 423; for relief of late pain in herpes zoster, iv. 359; for relief of pain in aneurysm, iii. 766 ; for relief of pudendohemorrhoidal neuralgia, iii. 357; for relief of restlessness due to cerebral anemia, iv. 392.

Nasal douche. See Douche.

Nasal and frontal sinus headaches, iv. 602; treatment of, iv. 603.

Nasal passages, method of clearing, iii. 334; removal of abnormalities from, for relief of hay fever, iii. 390 . 
NASAL POLYPI, removal of, as a preventive of bronchitis, iii. 352 .

Nasal sinuses, acute suppurative inFLAMMATION OF, iii. 333.

Nasal tampon, anterior method of, iii. 335; posterior method of, iii. 335 .

NASAL TUBE, feeding by means of, in ecrebral hemorrhage with unconsciousness, iv. 392 .

Nasopharyegrts, acute, iii. 31 ; atrophic, iii. 34 ; chronic, iii. 32.

Nastin, hypodermic injection of, in leprosy, iv. 815 .

Nasturtium officinale. See Watercress.

National Committee of Mental HyGIENE, recognition by, of psychic prophylaxis, iv. 569 .

Nauneim, value of treatment at, in acute myelitis, iv. 228 .

Nauheim baths, artificial, iii. 628; cases suited for, iii. 623; composition of, iii. 629 ; duration of, iii. 632 ; frequency of, iii. 632 ; general rules for use of, iii. 631; graduation of, iii. 632 ; indications and contraindications for use of, iii. 622; methods of producing carbon dioxid for, 629; mismanagement for, iii. 616; natural, iii. 627 ; natural versus artificial, iii. 625; physiological action of, iii. 618; strength of, iii. 632 ; technique of, iii. 630 ; use of, in exophthalmic goiter, iii. 898; use of, in gynecological affections, i. 211; use of, for relief of dyspnea in chronic interstitial nephritis, iv. 72.

NAUnyn's “starvation DaYs," ii. 753.

NAUSEA AND vomiting accompanying renal colic, iv. 106; as symptoms of brain tumor, iv. 435.

Neapolitan method of vaccination, ii. 60.

Neapolitan ointment, use of, in administration of mercury by inunction, ii. 388; use of, in hereditary syphilis, ii. 434 .

Nebulizer, use of, in acute peritonsillitis, iii. 43 .

Necator americands, iv. 792.

Necrosis of Jaw, due to phosphorus poisoning, ii. 628.

“Negative phase," i. 138.

NEGRE, work on vaccination by, ii. 60 .

Negroes, predisposition of, to rachitis, ii. 815.

Neisser, contagiousness of tertiary syphi- lit ic lesions established by, ii. 371 ; report by, on use of arsenobenzol in syphilis, iv. 452 .

Nematode infectione, iv. 791: Cochin China diarrhea, iv. 796; esophagostomiasis, iv. 799 ; gnathostoma siamense, iv. 800; hookworm disease, iv. 791; leeches, iv. 800 ; strongylosis, iv. 798; tricocephaliasis, iv. 796.

Nematodes of docbtfll pathogenesis, iv. 800 .

Nephrectomy, indications for, in movable kidney, iv. 3 ; in nephrolithiasis, iv. 110; in pyelitis, iv. 92 ; in tuberculous kilney, iv. 114 .

Nephritic neadaches. See Headaches.

NePHRITIS, iv. 10; blood pressure raised by, iii. 554, iv. 18; care of skin in treatment of, iv. 32 ; as a complication of German measles, ii. 165; as a complication of glandular fever, ii. 576; as a complication of influenza, ii. 194; as a complication of lobar pneumonia, ii. 261; as a complication of Rocky Mountain fever, ii. 566; as a complication of scarlet fever, ii. 132 ; as a complication of Weil's disease, ii. 572 ; composition of urine in, iv. 12 ; diet for, iv. 39,52 ; diuretics in treatment of, iv. 49 ; edema due to, iv. 15; effect of diuretics in experimental, iv. 51; electrotherapeutics for relief of, i. 424 ; elimination through skin and bowels in treatment of, iv. 32 ; functional kidney tests and their value in diagnosis of, iv. 43 ; heart hypertrophy in, iv. 20; hemorrhagic form of, iv. 64; influence of elimate upon, iv. 29; internal secretion of kidney and, iv. 13; normal physiology in relation to, iv. 10; pathological physiology of kidney in relation to, iv. 18; principles of treatment for, iv. 26; prophylaxis against, iv. 27 ; rest in treatment of, iv. 31 ; restriction of fluids in treatment of, iv. 33 ; restriction of sodium chlorid in treatment of, iv. 35 ; special treat ment for, iv. 56 ; sweating in treatment of, iv. 37 ; theories of renal secretion in relation to, iv. 10; treatment of hypertension in, iv: 45 ; treatment of uremia in, iv. 51 ; urea in relation to, iv. 12 ; uremia in, iv. 20 ; vasodilators in treatment of, iv. 47 ; venesection in treatment of, iv. 47 . 
NePhritis, ACUTE, iv. 56; active treatment for, iv. 57; anemia in, iv. 63; anuria in, iv. 62 ; backache in, iv. 63 ; catharsis in treatment of, iv. 58 ; decapsulation of kidney for, iv. 16 ; diet for, iv. 59 ; dyspnea in, iv. 63; edema in, iv. 62; edema of glottis complicating, iv. 63; gastric disturbance in, iv. 63; headache in, iv. 63; hydrotherapy for, i. 209; itching of skin in, iv. 63 ; prophylaxis against, iv. 56 ; sweating for, iv. 61 ; treatment of, iv. 61 ; uremia in, iv. 62 ; use of diuretics in, iv. 58.

NePhritis, Chronic, hydrotherapy for relief of, i. 210; myocardial insufficiency due to, iii. 709; prognosis in, iii. 731; treatment of, iii. 730 .

Nephritis, Chronic INTERstitial, iv. 67; association of, with hypertension of arteries and cerebral hemorrhage, iv. 388 ; blood pressure in, iv. 67 ; cerebral hemorrhage in, iv. 72 ; diet in, iv. 68 ; dyspnea in, iv. 71; headache due to, iv. 72 ; hypertension in, iv. 69 ; restriction of sodium chlorid in, iv. 68; summary of subject, iv. 73 ; vasodilators, iv. 70 ; venesection in, iv. 70.

Nephritis, Chronic parenchymatous, iv. 64; diet for, iv. 65; elimination in treatment of, iv. 65 ; radiant light and heat in treatment of, i. 455 ; use of diuretics in treatment of, iv. 65 .

Nephrolithiasis, iv. 100; character of pain in, iv. 100; chill and fever in, iv. 106; collapse in, iv. 106; drugs for relief of pain in, iv. 104; enteroclysis for relief of pain in, iv. 103; gravity of, iv. 100; local application of cold for relief of pain in, iv. 103; local application of heat for relief of pain in, iv. 102; nausea and vomiting in, iv. 106; operation for, iv. 110; posture for relief of pain in, iv. 104; suppression of urine in, iv. 106; treatment to dislodge stones in, iv. 110; treatment of the lithiasis, iv. 108; treatment of renal colic, iv. 101; treatment of symptoms other than pain in, iv. 106; warm drinks for relief of pain in, iv. 104.

Nephrolithotomy, indications for, in nephrolithiasis, iv. 110.

Nephrorrhaphy, employment of, for movable kidney, iv. 3 .
Nephrotomy, indications for, in pyelitis, iv. 92 ; indications for, in tuberculous kidney, iv. 114; for relief of hematuria, iv. 4; for relief of urethral stricture complicated by pyelonephritis, iv. 173 .

Nephrotoxic substances, iv. 14.

"Nephrotyphus," ii. 24.

Neptune girdle bath, use of, in hydrotherapy, i. 198.

Nerve anastomosis, indications for, in neuritis, iv. 289.

Nerve deafness and vertigo, as a symptom of basilar syphilitic meningitis, iv. 474.

Nerve section, employment of, in spasm of neck muscles, iv. 657.

Nerve stimulation, local, effect of, i. 272.

Nerve stretching, for relief of intercostal neuralgia, iv. 357 ; for relief of pain in leprosy, iv. 816; for relief of pain in sciatica, iv. 304.

Nerve stretching, passive, for relief of pain in syringomyelia, iv. 278.

Nerve suture, indications for, in obstetric paralysis due to trauma, iv. 292 ; in paralysis of hypoglossal nerve due to trauma, iv. 321 .

NeRve SYPHILIS virus, iv. 472.

Nerve trunk, injection into, for relief of pain in sciatica, iv. 300 ; sciatica from damage to, iv. 298.

Nerves, disturbances of, in tabes, iv. 190.

Nerves, Divided, treatment of, iv. 282.

Nerves of Heart, iii. 537: accelerator fibers, iii. 538; afferent fibers, iii. 539; conducting paths, iii. 539; inhibitory fibers, iii. 537.

Nervous asthma. See Asthma.

Nervous deafness. See Disease of eighth nerve.

Nervous DYSPEPSIA, iii. 182; association of, with gastroenteroptosis, iii. 245 .

Nervous influences, as etiological factor in arteriosclerosis, iii. 754 .

Nervous strain, influence of, in causation of alcoholism, ii. 590 .

Nervous symptoms, in chlorosis, iii. 823; treatment of, when due to chronic myocardial insufficiency, iii. 740.

Nervous SYSTEM, action of camphor upon, iii. 613 ; disease of, due to use of arsenobenzol in syphilis, iii. 415; disturbances of, in Addison's disease, iii. 885; dis- 
turbances of, due to gastric diseases, iii. 123; effeet of caffein series upon, iii. 6()$^{2}$; effect of X-rays upon, i. 522; exertion of central, in neurasthenia, iv. 5.50 ; influence of exercise upon, i. 33:3; mechanotherapy in functional disorders of, i. 352 ; protection and reconstruction of, in treatment of the neuroses, iv. 533; rolation between irritability of, and asthma, iii. 382 ; vibratory massage in functional disorlers of, i. 358.

Nervous system, Diseases of, effect of massage upon, i. 296; hydrotherapy for relief of, i. 221 ; influence of climate upon, i. 283; massage and gymnastics for relief of, i. 308; X-ray therapy for, i. 554 .

Nervous type of influenza, ii. 190.

Nervousness, measures for relief of, in cerebral anemia, iv. 383.

Nervus vestibularis, relation of, to vertigo, iv. 619 .

Nestlé's Food, use of, in summer diarrhea, iii. 202.

Neuralgia, iv. 334; accompanying chlorosis, iii. 824 ; as a complication of acute rheumatism, ii. 304; definition of, iv. 334; diabetic, see Diabetic neuralgia; hydrotherapy for relief of, i. 310 ; intercostal, i. 310 ; local causes of, iv. 335 ; massage and gymnastics in treatment of, i. 310 ; measures for relief of pain in, iv. 338 ; occipital, i. 310 ; sciatic, i. 311 ; systemic causes of, iv. 336 ; treatment of, as to cause, iv. 335 ; treatment of cervicoöccipital, iv. 356 ; treatment of crural, iv. 357 ; treatment of lumboabdominal, iv. 357; use of thyroid gland for relief of, i. 76; X-ray therapy for relief of, i. 554 .

Neuralgia, brachial, i. 310 ; treatment of, iv. 356 .

Neuralgia, trigeminal, iv. 354; alcohol injections for relief of, at cranial foramina, iv. 346; alcohol injections for relief of, at superficial foramina, iv. 344 ; cutting operations for, iv. 341 ; injections in treatment of, iv. 342 ; local causes of, iv. 335 ; measures for relief of pain, iv. 338 ; summary of results from treatment of, iv. 355 ; surgical operation for, iv. 353 ; treatment of, as to cause, iv. 335 .
Neuralgias, thes, iv. 334 ; brachial neuralgia, iv. 356 ; cervicoüceipital neuralgia, iv. 35); coceygorlynia, iv. 358; crural neuralgia, iv. 357; herpes zoster, iv. 3is. injections in treatment of, iv. 342 ; intoreostal neuralgia, iv. 357 ; local causes of, iv. 3335; lumboabdominal neuralgia, iv. 357 ; neuralgia in general, iv. 334 ; pudendohemorrhoidal neuralgia, iv. 357 ; relief of pain in, iv. 338 ; systemic causes of, iv. 336 ; treatment of, as to cause, iv. 335 ; trigeminal neuralgia, iv. 354.

Neurasthenia, associated with disease of kidney, iv. 81 ; associated with gastroptosis, iii. 181 ; associated with intestinal autointoxication, ii. 682; cardiac, see Cardiac ncurasthenia; hydrotherapy for relief of, i. 224; massage and gymnastics in treatment of, i. 320 ; sexual, see Sexual neurasthenia.

Neurasthenia and psychasthenia, iv. 516 ; exertion of central nervous system in treatment of, iv. 550; introduction to subject of, iv. 516; phobias a feature of, iv. 518; preliminary therapeutic reflections in, iv. 524; prophylaxis against, iv. 519; protection and reconstruction of nervous system in treatment of, iv. 518; psychotherapy for, iv. 569; supplementary therapeutic procedures for use in, iv. 555; symptomatic treatment of, 562 ; therapeutic principles generally accepted in, iv. 532 .

Neurasthenic headaches. See Headache. Neurasthenic vertigoes, iv. 630.

NEURIN, as a cause of food poisoning, ii. 635. Neuritides, as local expression of constitutional disease, i. 210.

Neuritis, due to administration of arsenic in syphilis, ii. 384 ; of anterior crural nerve, iv. 293; of brachial plexus, iv. 290; over cranium, ii. 417; diabetic, see Diabetic neuritis; effects of electrical stimulation upon, i. 372; electrotherapy for, i. 444; external cutaneous, iv. 293; herpes zoster a form of, i. 417; due to hydrargyrism in syphilis, ii. 354; hydrotherapy for, i. 225; intrapelvic form of, i. 416 ; of nerves of arm, iv. 290; of ninth nerve, iv. 318 ; of obdurator nerve, iv. 293 ; optic, see Optic neuritis; phrenic, iv. 289; sciatic, see Sciatica; of tenth nerve, 
iv. 319 ; of third, fourth, and sixth nerves, iv. 308; tic douloureux a form of, i. 417; trigeminal, iv. 309; of twelfth nerve, iv. 321 ; X-ray therapy for, i. 554 .

Neuritis, brachial, i. 416; electrotherapeutics for relief of, i. 416; massage and gymnastics for relief of, i. 310; as symptom of diabetes mellitus, ii. 766 .

Neuritis, multiple, iv. 321 ; alcoholism as cause of, iv. 322 ; arsenical poisoning as cause of, iv. 323 ; convalescence from, iv. 328 ; diabetes as cause of, iv. 325 ; infections as cause of, iv. 325; lead poisoning as cause of, iv. 324 ; treatment of causes for, iv. 321; treatment of, irrespective of causes, iv. 325.

Neuritis of Lumbar plexus, iv. 293: anterior crural neuritis, iv. 293; external cutaneous neuritis, iv. 293; obdurator neuritis, iv. 293; sciatic neuritis, iv. 294 ; sciatica, i. 416.

Neuritis of seventh Nerve, iv. 310; cases of, due to disease of temporal bone, iv. 310 ; cases of, due to intracranial disease, iv. 310; congenital form of, iv. 314 ; idiopathic cases of, iv. 311 ; operation for, iv. 313; rheumatic cases of, iv. 311.

Neuritis of SPINAL Nerves, iv. 289: neuritis of brachial plexus, iv. 290; neuritis of lumbar plexus, iv. 293; obstetric paralysis, iv. 291; phrenic neuritis, iv. 289.

Neuritis, treatment of, in general, iv. 282: baths, iv. 288; change of scene, iv. 289 ; counterirritation, iv. 286 ; drugs, iv. 289; electricity, iv. 287; exercise, iv. 288; late operation, iv. 288; massage, iv. 287; relief of pain, iv. 285; removal of cause, iv. 282 ; rest, iv. 284 ; sleep, iv. 286.

NeUrogenic THEORY, of cardiac contractions, iii. 534.

Neurolysis, indications for, in facial paralysis, iv. 313; in injured nerves, iv. 283; in neuritis, iv. 289 .

Neuronidia, use of, to secure sleep in neuritis, iv. 286.

"Neuro-récidives," iv. 478.

Neuroses, THE, iv. 516; associated with certain conscious abnormal factors, iv. 573 ; associated with certain unconscious abnormal factors, iv. 573 ; elec- trotherapeutics for relief of functional, i. 431 ; headache of external cerebral origin, iv. 597; headache of intracranial and extracerebral origin, iv. 605; headache as symptom of toxemia and general diseases, iv. 610; hysteria and, iv. 581; idiopathic gastric, iii. 186; intestinal, iii. 249; migraines, the, iv. 589; neurasthenia and psychasthenia, iv. 613; of occupation, see Occupation neuroses; primary gastric, iii. 186; psychogenic and psychotic headache, iv. 613; psychotherapy in treatment of, iv. 569; treatment of the epilepsies, iv. 160; treatment of the vertigoes, iv. 615 .

Neurotic Muscle headache, iv. 600; treatment of, iv. 602.

NeUrotic STATES, angina pectoris vasomotoria due to, iii. 768 .

"Neurovascular training," i. 224.

Neutralon, use of, in gastric hyperacidity, iii. 97.

"New cacodyle." See Arrhenal.

New Jersey, antituberculosis work in, ii. 446.

New York City, antituberculosis work in, ii. 447; means of house disinfection in, ii. 452 .

New York City Health Department, experiments performed by, on effects of cauterization for rabies, ii. 336; Pasteur antirabic virus sent by mail from, ii. 341 ; preparation of antirabic virus by, ii. 338 ; record by, of immunization with diphtheria antitoxin, ii. 268.

Newlin, Dr. Arthur, formula proposed by, for use of camphor in acute bronchitis, iii. 354 .

Newsholme, investigations by, on decreased death rate from phthisis, ii. 439 , 441.

Nicotin, effects of, in tobacco smoking, iii. 782; use of, in tetanus, ii. 248; vertigo due to use of, iv. 624 .

Nicotin POIsoning, i. 761; associated with angina pectoris vasomotoria, iii. 768 ; fatal dose in, i. 762; headache as a symptom of, iv. 610; hydrotherapy for chronic form of, i. 228; occurrence of, i. 761 ; treatment of, i. 763 .

Nicotinism. See Nicotin poisoning.

Night sweats, in tuberculosis, ii. 505.

Night Walking, in epilepsy, iv. 636. 
Nitrate of silver. See Silver nitrate.

Nitrates, use of, as diureties in acute nephritis, iv. 58.

Nitric acid, fuming, results of, in treatment of bite by rabid animal, ii. 336 .

Nitric acid polsoning, i. 730 .

Nitrite of amyl. See Amyl nitrite.

Nitrites, poisoning by, i. 732.

Nitrites, use of, in arterioselerosis, iii. 762 ; effect produced by, on pulmonary circulation, iii. 405; limited in cardiae neurasthenia, iii. 778 ; during paroxysm of asthına, iii. 385 ; in pulmonary edema with high blood pressure, iii. 410; to relieve vascular pain in ergotism, ii. 652 ; value of, in angina pectoris, iii. 768.

Nitrogen elimination, delay of, in nephritis due to uremia, iv. 21 ; through bowels in nephritis, iv. 32 .

Nitrogen injection treatment, ii. 534; method employed in, ii. 536 ; possibilities of, in bronchiectasis, iii. 394 .

Nitrogen starvation, as cause of beriberi, iv. 846 .

Nitrogenous foods. See Proteid foods.

Nitroglycerin, use of, following amyl nitrite in angina pectoris, iii. 769 ; in cardiac insufficiency, iii. 606; in cardiac insufficiency in repeated doses, iii. 607; in chronic interstitial nephritis, iv. 70; to control hemorrhage from pulmonary tuberculosis, ii. 500; dangerous in pneumonia, ii. 248; in diabetic coma, ii. 757; effect produced by, on blood pressure in cardiac insufficiency, iii. 606; effect produced by, on pulmonary circulation, iii. 405; in first stage of migraine, iv. 592 ; by hypodermic injection to control epistaxis, iii. 336 ; by hypodermic injection in diphtheria, ii. 277; by hypodermic injection for relief of heart failure in beri-beri, iv. 854; in neurasthenia with high arterial tension, iv. 559 ; during paroxysm of asthma, iii. 385 ; in pulmonary edema with high blood pressure, iii. 410; to reduce hypertension in nephritis, iv. 47; for relief of dyspnea in tubereulosis, ii. 506; in scarlet fever with cardiac weakness, ii. 127.

Nitroglycerin, digitalis, and strophanthus, use of, in cerebral thrombosis, iv. 398.

Nitroglycerin and the nitrites, use of, in cardiace insufficiency, iii. 605: amyl nitritc, iii. 610; (rythrol tetrunitrate, iii. 608; mannitol hexanitrate, iii. 610; physiological action, iii. 606; potassium nitrite's, iii. 607 ; sodium nitrite, iii. 607 ; therapeutic dosage of various nitrites, iii. 612 .

Nitrohydrochloric acid, use of, in hypoacidity acconpanying cirrhosis of liver, iii. 281.

Nitrohydrochloric acid, dilute, use of, in oxaluria, iv. 8.

Nitromuriatic acid, use of, in acute catarrhat jaundice, iii. 262.

Nitrous ACID, poisoning by, i. 732 .

Nitrous oxid gas, use of, as anesthetic, in adenectomy, iii. 36,37 ; contraindicated in chronic myocardial insufficiency, iii. 743 ; in operation for tuberculous peritonitis, iii. 320 .

Nocard, isolation by, of bacillus supposed to be cause of psittacosis, ii. 584 .

Noguchi's bUtrric acid test, value of, in diagnosis of brain syphilis, iv. 476 .

Noma. See Stomatitis gangrenosa.

Non-diphtheritic croup. See Croup.

Non-electrolytes, i. 7; comparative activity of, in living cells, i. 55; comparatively slight effects of, on gelatin and fibrin in acid solution, i. 3i, 33; definition of, i. 7 ; relation between electricity and, i. 7 ; relation between the laws of osmotic pressure and, i. 54 .

NonNe, report by, of cerebellar atrophy in three brothers, iv. 513.

NonNe's GLOBULin test, value of, in diagnosis of brain syphilis, iv. 477 .

Normal saline solution, use of, in anuria with normal kidneys, iv. 3 ; for colonic flushing in colitis, iii. 205; contraindicated in toxic form of anuria, iv. 3 ; by epidural injection, for relief of enuresis, iv. 134; hot, by reetal injection in ulcerative colitis of hemorrhagic form, iii. 212; by hypodermic injeetion in anemia due to hemophilia, iii. 870 ; by hypodermic injection in gastric tetany, iii. 143; by hypodermic injection in phlegmonous pharyngitis, iii. 40; by hypodermic injection in tetany, iii. 909 ; for injection into nerve trunk, iv. 301 ; by intravenous injection in blackwater fever, iv. 740; by intravenous injection in insolation. 
iv. 678 ; by intravenous injection in methemoglobinuria, iv. 5 ; for irrigation of adenoid vegetations, iii. 38 ; for irrigation in appendicitis, iii. 218; for irrigation of nasal passages in acute inflammation, iii. 334; methods for, in pyelitis, iv. 89 ; as nasal douche, in membranous rhinitis, iii. 330 ; by rectal injections in Asiatic cholera, iv. 834; by rectal injection in gastric tetany, iii. 143; by rectal injection in insolation, iv. 668; by rectal injection in membranous enteritis, iii. 208; by rectal injection for suppression of urine in nephrolithiasis, iv. 107; by rectal injection for suppression of urine in renal colic, iv. 106; as spray in chronic rhinitis, iii. 329; as spray or irrigation in acute laryngitis, iii. 339 ; by transfusion in cerebral anemia, iv. 382 ; by transfusion in funicular myelitis, iv. 232.

Normal serum, therapeutic use of, i. 171: in alimentation, i. 173; in hemorrhagic conditions, i. 172 ; in suppurative processes, i. 171 ; in toxemias of pregnancy, i. 172.

Normal solutions, as basis of standardization in pharmacology, i. 11.

Norway, introduction of vaccination into, ii. 60 .

Nose, affections of, complicating diphtheria, ii. 272 ; affections of, complicating measles, ii. 156; affections of, complicating scarlet fever, ii. 129; importance of attention to, in prophylaxis of acute cerebral meningitis, iv. 371 ; irrigation of, in diphtheria, ii. 271,272 ; otitis media caused by irrigation of, ii. 277; relation between abnormalities of, and asthma, iii. 382 .

Nose, Diseases of, iii. 325: acute rhinitis, iii. 326 ; acute suppurative inflammation of sinuses, iii. 333; atrophic rhinitis, iii. 330; chronic rhinitis, iii. 328; epistaxis, iii. 335; membranous rhinitis, iii. 329; ozena, iii. 336 ; rhinoscleroma, iii. 332.

Nose AND THRoAT, eare of, a means of preventing acute rheumatism, ii. 296.

Nose-bleed. See Epistaxis.

Nothnagel, table by, showing mortality from small-pox, after vaccination, ii. 100.

Notification, value of, in control of tuberculosis, ii. 447.
Novaspirin, use of, in bronchial asthma, iii. 388.

Novocain, injection of, into spinal canal in tabes, iv. 198.

Nucleus, large Cell, iv. 620.

Nucleus, triangular, iv. 620,621 .

Nurse, duty of, in acute rheumatism, ii. 296 ; in pneumonia, ii. 222 ; in prevention of small-pox, ii. 44; in scarlet fever, ii. 119; requirements in, for nursing the neuroses, iv. 531.

Nursing of INFant, possibilities of infection through, in syphilis, ii. 432; in tuberculosis, ii. 457.

NURSING HOMES, treatment of neurasthenia and psychasthenia in, iv. 562 .

Nutrition, changes in, due to pneumonia, ii. 228; measures for increasing, in prolapse of liver, iii. 289.

NUTRITION AND DIETETICS, i. 599: artificial feeding, i. 636; effects of over-feeding, i. 635; effects of starvation, i. 634; foods, i. 600 ; invalid's dietary, i. 645 ; a purinfree diet, i. 644; a salt-free diet, i. 644; proprietary foods, i. 652 .

NuTs, use of, as food in diabetes mellitus, ii. 745 .

Nux vomica, use of, in acute catarrhal jaundice, iii. 260, 262; in Addison's disease, iii. 884; in bronchial asthma, iii. 384 ; in carcinoma of stomach, iii. 165 ; in gastric atony, iii..172; in hemophilia, iii. 869 ; for hyperacidity in chlorosis, iii. 823; for relief of insomnia caused by coughing in acute bronchitis, iii. 374; as tonic in tuberculosis, ii. 504; in treatment of enuresis with atony of sphincter, iv. 133.

Nux vomica, tincture of, use of, in aftertreatment of acute gastritis, iii. 157; for relief of insomnia in neurasthenia, iv. 565; as tonic in stomatitis ulcerosa, iii. 5 .

Nux vomica and pepsin, use of, as a tonic in stomatitis catarrhalis, iii. 7 .

Nystagmus, associated with vestibular vertigoes, iv. 626 ; in Friedreich's disease, iv. 216; in hereditary cerebellar ataxia, iv. 217; in multiple sclerosis, iv. 280; as a symptom of cerebral thrombosis, iv. 393; as a symptom of pachymeningitis interna hemorrhagica, iv. 363.

Nystagmus, labyrinthine, iv. 603. 
Oatmeal diet, value of, in after-tratment of diabetic coma, ii. 758 .

Oatmeal girole, as anticle of invalid dictary, i. (645).

Oatmeal treatment of diabetes mellitus, ii. 753.

Ordurator nerve, neuritis of, iv. 293.

Obese, THE, cardiac insufficiency of, iii. 7033 .

Oвеsiтy, chronic myocardial insufficiency due to, iii. 707; danger from, in chronic interstitial nephritis, iv. 68; definition of, ii. 779; as etiological factor in arteriosclerosis, iii. 754; functions of different foods in relation to, ii. 782 ; hereditary in causation of, ii. 785 ; hydrotherapy applied to relief of, i. 207 ; influence of alcohol upon, iii. 706; injurious influence of, on chronic bronchitis, iii. 370 ; massage and gymnastics in treatment of, i. 325; mechanotherapy for relief of, i. 348; necessity for caution in reduction of, when associated with chronic myocardial insufficiency, iii. 726; physiology of, ii. 781 ; prophylaxis against, ii. 786 ; secretion of sexual organs in relation to, ii. 786 ; sedentary life in causation of, ii. 785 ; treatment of chronic myocardial insufficiency due to, iii. 724 ; treatment of, in general, ii. 787; treatment of, after reduction, ii. 810 .

Obesity, Reduction of, ii. 779; alcohol in, ii. 805; arrangement of meals in, ii. 797; Bouchard's method for, ii. 794; calisthenics in, ii. 807; causes of, stated by von Noorden, ii. 784; choice of cases for, ii. 787; comparison of diets for, ii. 796; by diet, in cardiac insufficiency, iii. 685 ; dietetics in, ii. 790; Epstein diet for, ii. 791 ; exercise in, ii. 806 ; foods allowable in, ii. 800; foods to be avoided or restricted in, ii. 800 ; functions of different foods in relation to, ii. 782 ; general considerations in, ii. 788; general principles of diet in, ii. 796; Harvey-Banting cure in, ii. 790; health resorts for, ii. 789 , 809 ; heat loss in relation to, ii. 784 ; Hirschfeld's diet for, ii. 794; hydrotherapy in, ii. 808; Karrell's diet for, ii. 795; limitation of fluids in, ii. 805; massage in, ii. 807 ; mechanotherapy in, ii. 805 ; medicinal treatment in, ii. $80 s$; menus for meals in, ii. 799; Oertel cure in, ii. 792 ; passive mechanical exercises in, ii. 807; Robin's diet in, ii. 793; Srehweninger system in, ii. 793; Spa trontment in, ii. $8(0) 9$; thyroid gland in, ii. $786,8(59)$; von Noorden's system for, ii. 794 .

OBstethic palalysis, iv. 291; treatment of, iv. 292.

Occipital bath, use of, in hydrotherapy, i. 227.

Occipital, LOBE, localizing symptoms of brain tumor in, iv. 441.

OCCipital Neuralgias, massage and gymnastics in treatment of, i. 310 .

Occlusion by metal bands, use of, in treatment of aneurysm of abdominal aorta, iii. 767 .

Occupation, in causation of acquired spinal progressive muscular atrophy, iv. 246 ; choice of, in tuberculosis, ii. 463 ; influence of, in causation of gout, ii. 694; question of, in arteriosclerosis, iii. 759 ; question of, in cardiac insufficiency, iii. 698; question of, in treatment of the neuroses, iv. 529; therapeutic use of, in psychotherapy, i. 596.

Occupation cure, value of, in neurasthenia and psychasthenia, iv. 552 .

Occupation Neuroses, iv. 660 ; as a cause of headache, iv. 601; definition of, iv. 660 ; hydropathy for relief of, i. 225 ; massage and gymnastics in treatment of, i. 313 ; treatment of, in general, iv. 660 ; treatment of muscles involved in, iv. 661 .

Occupation pareses, iv. 330; definition of, iv. 330 ; essential causes of, iv. 330 ; indications for treatment of, iv. 331.

Occupational methods, coöperative, in treatment of neurasthenia and psychasthenia, iv. 554 .

Occupational therapy, value of, in neurasthenia and psychasthenia, iv. 553 .

Occupations favoring transmission of syphilis, ii. 372 , 375 ; having high percentage of respiratory diseases, iii. 424 , 425

OchsNer, views held by, on treatment of acute localized peritonitis, iii. 311 .

Ocular afFections, associated with intestinal autoinfection, ii. 682 .

Ocular paralysis, periodic, iv. 309.

OCUlar tyPE of Myasthenia gravis, iv. 506. 
Ocular vertigoes, iv. 629.

O'Dwyer treatment of thermic fever, $i$. 229.

Oertel cure, for obesity, ii. 792.

Oertel exercises, i. 304; use of, in asthma, iii. 382 .

Oertel's terrain cure, iii. 645 ; cases suited to, iii. 647,704 ; disadvantages of, iii. 646 ; indications and contraindications for, iii. 647 ; principle involved in, iii. 645 ; technique employed in, iii. 647 ; use, of in chronic myocardial insufficiency due to obesity, iii. 726 .

Esophagostomum apiostomum, iv. 799.

CESOPHAGOSTOMUM BRUMPTI, iv. 799.

Esophagostomum stephaNostomum, iv. 799.

Oeynhausen, value of treatment at, in acute myelitis, iv. 228.

OfFENSIVE BREATH, iii. 3.

Онм's LAw, explanation of, i. 364.

Oidium albicans, iii. 11.

Oil of camphor, use of, as cardiac stimulant in cerebral anemia, iv. 382.

Oil of Cashew, use of, in leprosy, iv. 816.

Oil of filmaron, use of, as substitute for male fern, iv. 778 .

Oil of sandalwood. See Sandalwood oil.

Oil of sylvestrian pine, use of, in gangrene of lung complicating pneumonia, ii. 261.

Oil of turpentine. See Turpentine.

Oil silk jacket, use of, in bronchopneumonia, iii. 416.

Oil treatment, in non-bleeding gastric ulcer, iii. 137.

Ointments, use of antiseptics by, in smallpox, ii. 50; use of mercury by, in congenital syphilis of upper respiratory tract, iii. 349; use of mercury by, in parotitis, iii. 28; use of salicylic acid by, for vaccinated arm, ii. 73 .

Oleum juniperi, use of, by inhalation in acute laryngitis, iii. 339 .

Oleum phosphoratus, use of, in rachitis, ii. $818,819$.

Oleum picis, local use of, in chronic atrophic laryngitis, iii. 343 ; use of, in atrophic rhinitis, iii. 331 .

Oleum pine, use of, by inhalation, in acute laryngitis, iii. 339 .

Oleum terebinthinæ. See Turpentine, oil of.
OlfaCtORY NeRVe, paralysis of, in basilar syphilitic meningitis, iv. 474 .

Olive oil, use of, in chronic cholecystitis and cholelithiasis, iii. 273; use of, in chronic constipation, iii. 234 ; in chronic intestinal obstruction, iii. 242 ; in continuous gastric hypersecretion, iii. 106; for hyperacidity in chlorosis, iii. 823 ; in idiopathic dilatation of the esophagus, iii. 58 ; in membranous enteritis, iii. 209; by rectal injection in fecal impaction, iii. 238, 239; by rectal injection in membranous enteritis, iii. 208; to stimulate peristalsis in chronic constipation, iii. 235 ; in treatment of gallstones in transit, iii. 274.

Oliver, mixture suggested by, for use in respiratory diseases due to dust inhalation, iii. 430.

ONDREJOVICH's TEST, for diacetic acid in urine, ii. 733.

ONyalai, iv. 861.

ONYchia, syphilitic, local treatment of. ii. 429.

Open air, importance of, during active stage of tuberculosis, ii. 479,480 .

Open-air treatment, in bronchopneumonia, iii. 417; in bronchopneumonia complicating diphtheria, ii. 278 ; in chronic serous effusion of pleura, iii. 484; in measles, ii. 146, 159; in pleurisy, iii. 465 ; in pneumonia, ii. 224 ; in septicopyemia, ii. 290.

Operation, for abscess or infarct of spleen, iii. 888; for abscess of lung complicating pneumonia, ii. 261 ; in actinomycosis, ii. 360 ; in acute intestinal obstruction, iii. 241 ; for adenoid vegetations, iii. 37 ; for adhesive pericarditis, iii. 528; in aftertreatment of facial paralysis, iv. 313; for aneurysm, iii. 766; for aneurysm of cerebral arteries, iv. 458; in angina Ludovici, iii. 28, 30; in arthritis deformans, ii. 714; in bacillary dysentery at chronic stage, ii. 328; in Banti's disease, iii. 890; upon brain, for cerebral palsies of children, iv. 413, 414; in carcinoma of stomach, ii. 162; in cervical adenitis complicating diphtheria, ii. 277 ; in chancre of general integument, ii. 425; in chancre with phimosis, ii. 424; for chronic catarrhal jaundice, iii. 264; for chronic enlargement of spleen, iii. 889; 
in chronic intestinal obstruction, iii. 243; in chronic nasopharyngitis, iii. 33; in chronic pharyngitis, iii. 33; in cirrhosis of liver with adhesions, iii. 322; conditions demanding, in tropical malaria, iv. 728; in constipation due to obstruction, iii. 237; for eontructures following cerebral palsies of children, iv. 414 ; contraindic:ted in acute dilatation of stomach, ii. 174; contraindicated in eerebral abseess, iv. 423; contraindicated for infectious sinus thrombosis, iv. 430; for cysticereus of brain, iv. 461; dangers of, in exophthalmic goiter complicating diabetes mellitus, ii. 767 ; for echinococcus disease, iii. 255; Estlander's, see Estlander; in exophthalmic goiter, iii. 904; for gangrenous or phagedenic chanere, ii. 423; for gastric ulcer of bleeding type, ii. 116; for gastric ulcer of non-bleeding type, ii. 139; for gastric ulcer of syphilitic origin, iii. 167; in goiter, iii. 894; for hydronephrosis, iv. 98; in hypertrophy of pharyngeal tonsil, iii. 36 ; indications for, in cerebral abscess, iv. 423; indications for, in epilepsies, iv. 639; indieations for, in gallstones, iii. 274, 275, 279; indications for, in gonorrheal epididymitis, iv. 152; indications for, in neuritis, iv. 288; indications for, in prolapse of liver, iii. 289 ; indications for, in traumatic hematoma, iv. 366 ; in infectious sinus thrombosis, iv. 429 ; in intussusception, iii. 241 ; in leprosy, iv. 816 ; in mediastinal tumors, iii. 499; in mediastinitis, iii. 504; for mediastinopericarditis, iii. 508; for movable kidney, iv. 3 ; for movable spleen, iii. 888; for necrosis of jaw in phosphorus poisoning, ii. 630 ; in nephrolithiasis, iv. 110; in obstetric paralysis, iv. 292 ; in pachymeningitis interna hemorrhagica, iv. 364 ; palliative, for relief of brain tumor, iv. 451; in paralytic ileus, iii. 238; for peritoneal adhesions, iii. 322; for pleurisy with effusion, see Thoracentesis; for pleurisy with effusion complicating pneumonia, iii. 261; in pyelitis, iv. 92 ; for rectal chanere, ii. 425 ; for relief of continuous gastric hypersecretion, ii. 103; for relief of contractures due to beri-beri, iv. 854 ; for relief of goundoo, iv. 868; for relief of hemorrhage in typhoid fever, ii. 21 ; for relief of neuritis of seventh nerve due to suppurative otitis, iv. 311; for relief of optic neuritis due to suppuration of middle (ar, iv. 305), 306; for relief of trigeminal neuralgia, iv. 341,3533 ; in retropharyngeal abscess, iii. 34; for rupture of spleen, iii. 888; Sehede's, see Schede; in sciatica, iv. 304 ; in somatic teniasis, iv. 776; for spasmorlic movement of neck muscles, iv. 656; in stomatitis gangrenosa, iii. 19; in syringomyelia, iv. 279 ; in tabes, iv. 198 ; for traumatic hematoma, iv. 366 ; in tuberculosis of mediastinal lymph nodes, iii. 506, 508; in tubereulous kidney, iv. 114; in tuberculous meningitis, iv. 377 ; in tumors or cysts of liver, iii. 291 ; for visceroptosis, iii. 248; in volvulus, iii. 242.

Operative measures. See Operation.

Operation for brain tumor, iv. 449 ; dangers of, iv. 452 ; exploratory, iv. 450 ; indications for, iv. 449; meningitis following, iv. 452 ; mortality from, iv. 452 ; palliative, iv. 450 ; radical, iv. 451 ; rules for, iv. 452 ; trephining with extirpation of tumor, iv. 451; tumors suited for, iv. 449.

Ophidism, iv. 681; effects of, iv. 681; prophylaxis against, iv. 683; remedial measures for, iv. 682; specific treatment for, iv. 682 .

Ophthalmic BRANCH OF FIFTh NERVE, alcohol injection of, for trigeminal neuralgia, iv. 353.

Ophthalmic'migraine, iv. 589.

OphThalmology, value of radium therapy in treatment of, i. 479.

Ophthalmoplegia, iv. 507; acute, iv. 507; chronic, iv. 509; introduction to subject of, iv. 507; as a symptom of acute hemorrhagic superior polioencephalitis, iv. 406.

Ophthalmoplegia, acute, iv. 507; differential diagnosis in, iv. 508; prognosis of, iv. 508; symptoms of, iv. 508; treatment of, iv. 508.

Ophthalmoplegia, ChroNic, iv. 509; differential diagnosis in, iv. 509; pathology of, iv. 509 ; prognosis of, iv. 509 ; symptoms, iv. 509; treatment of, iv. 510.

Ophthalmoplegia, ChroNic Progressive, iv. 257; congenital variety of, iv. 258; elinical phenomena of, iv. $25 \pi$; treatment of, iv. 258; varieties of, iv. 257. 
OPHTHALMOSCOPIC EXAMINATION, results of, in basilar syphilitic meningitis, iv. 473.

Opiates, use of, in barbel cholera, ii. 644; in carcinoma of liver, iii. 291; in chronic cholecystitis and cholelithiasis, iii. 273; dangerous in the aged, iii. 195; for relief of cough in progressive bulbar paralysis, iv. 499 ; for relief of pain in acute fibrinous pleurisy, iii. 467 ; for relief of pain in neuralgia, iv. 340 ; in treatment of gallstones in transit, iii. 273.

OpIsthORCHIS NOVERCA, iv. 779; pathology of, iv. 780 .

Opisthotonos, as a sign of traumatic hematoma, iv. 365 .

Opium, use of, in acute enteritis, iii. 194; for attacks of depression in progressive paralysis, iv. 493; in chronic enteritis, iii. 199; contraindicated in acute bronchitis as a soporific, iii. 361 ; contraindicated in bronchiectasis, iii. 395 ; contraindicated in neurasthenia, iv. 559; contraindicated in plague, iv. 827 ; to control cough during active stage of tuberculosis, ii. 482 ; to control cough in acute bronchitis, iii. 361 ; to control cough in cerebral hyperemia, iv. 385 ; to control cough in chronic bronchitis, iii. 375; to control cough in laryngeal complications of tuberculosis, ii. 491, 498; to control cough in measles, ii. 153; to control diarrhea in Addison's disease, iii. 885; to control diarrhea complicating bronchopneumonia, a necessity, iii. 421; to control diarrhea in trichiniasis, iii. 254 ; to control diarrhea in typhoid fever, ii. 26; in diabetes insipidus, ii. 777 ; in diabetes mellitus, ii. 759 ; in diabetic gangrene, ii. 770; in enteritis of infancy, iii. 203; in enterocolitis complicating measles, ii. 160; in gallstone colic, iii. 268 ; in gastrointestinal type of influenza, ii. 190; in hemorrhagic form of ulcerative colitis, iii. 212 ; by hypodermic injection in carcinoma of stomach, iii. 166; in influenza, ii. 190; in laryngeal tuberculosis, iii. 346 ; to lessen irritability of bladder in tuberculous kidney, iv. 117; locally, in atriplicism, iv. 693; for relief of insomnia due to brain tumor, iv. 447; for relief of insomnia due to dyspnea in acute nephritis, iv. 63 ; for relief of insom- nia in multiple neuritis, iv. 327 ; for relief of insomnia in typhoid fever, ii. 26; for relief of lancinating pains in tabes, iv. 196; for relief of meat poisoning by B. enteritidis, ii. 641 ; for relief of neuralgia, accompanied by restlessness, iv. 336 ; for relief of nocturnal dyspnea in chronic interstitial nephritis, iv. 72; for relief of pain in acute diffuse peritonitis, iii. 315; for relief of pain in acute gout, ii. 703; for relief of pain in acute localized peritonitis, iii. 311 ; for relief of pain in dengue, iv. 860 ; for relief of pain in infantile diarrhea, ii. 329 ; for relief of pain in mediastinal tumor, iii. 503; for relief of pain in muscles in trichiniasis, iii. 234; for relief of pain in neuralgia, iv. 340 ; for relief of pain in neuritis, iv. 286 ; for relief of pain in pericarditis, iii. 520; in scarlet fever, ii. 127; in severe attacks of appendicitis, iii. 218; in spastic constipation, iii. 231; in strangulation of intestines, iii. 240; as a substitute for quinin, iv. 726; by suppositories, in colitis, iii. 205; by suppositories for relief of pain in acute gastritis, iii. 156; by suppositories for relief of pain of nervous character in anus, perineum and rectum, iv. 358 ; in tuberculosis of intestines, ii. 505; in ulcerative colitis, iii. 211; in volvulus and intussusception, iii. 241.

Opium and ipecac, use of, for relief of pain in pneumonia, ii. 227.

Opium and tannic acid, use of, to control diarrhea due to chronic myocardial insufficiency, iii. 739 .

Opium-bromid therapy, results of, in epilepsy, iv. 648.

OPIUM Eaters, ii. 616.

Opium навiт. See Morphin habit.

OpIUM POIsoning, ii. 614; acute form of, ii. 614 ; chronic form of, ii. 616 ; effects of chronic form of, on Eastern races, ii. 614; opium eating and, ii. 616; opium smoking and, ii. 616; treatment of acute form of, ii. 614; treatment of chronic form of, ii. 617 .

OPIUM SMOKING, iii. 616.

Opotherapy. See Organotherapeutics.

OPPENHEIM, on brachial neuralgia, iv. 356; definition of occupation pareses by, iv. 330 ; description by, of myasthenia gra- 
vis, iv. 498; on distinctive features of compression bulbar paralysis, iv. 501; results obtained by, on use of arsenobenzol in syphilis, iv. 485 ; on use of calomel in large doses in acute apoplectic bulbar paralyses, iv. 501 .

Oppenueim's classification, of muscle group spasins, iv. 655.

OPPENHEM reflex, in multiple sclerosis, iv. 280; in spastic spinal paralysis, iv. 268; in traumatic hematoma, iv. 366.

Oppenheim sign. See Oppenheim reflex.

OPSONIC INDEx, method of estimating, developed by Wright, i. 140.

Opsonins, nature of, i. 131.

Optic Aтrophy, iv. 307 ; presence of, in progressive paralysis of the insane, iv. 490.

Optic chiasm, disturbances of, as localizing symptoms of tumor in neighborhood of it, iv. 442.

Optic Nerve, changes in, during multiple sclerosis, iv. 280; diseases of, iv. 306 ; paralysis of, as a symptom of basilar syphilitic meningitis, iv. 473.

Optic Neuritis, iv. 305; without apparent cause, iv. 306; due to arsenobenzol used in syphilis, ii. 415; due to myelitis, iv. 306 ; due to organic intracranial disease, iv. 305; due to suppuration of middle ear, ii. 306; due to toxic conditions, ii. 305 ; due to wood alcohol poisoning, ii. 610.

Orangeade, as article of invalid diet, i. 652.

Orchitis, as a complication of mumps, ii. 166 ; as a complication of typhoid fever, ii. 27; hydrotherapy for relief of, i. 212; treatment of, in mumps, ii. 171 .

Orexin hydrochlorate, use of, in depressive disorders of gastric secretion, iii. 151.

Organic extracts, use of, in diabetes insipidus, ii. 778; in diabetes mellitus, ii. 760 ; in hemophilia, iii. 866 ; locally, iii. 867 ; in reduction of obesity, ii. 809 .

Organotherapeutics, i. 66 ; in acquired spinal progressive muscular atrophy, iv. 248; in Addison's disease, iii. 882; in asthenic bulbar paralysis, iv. 256; in cirrhosis of liver, iii. 286 ; classification of, i. 69 ; in deficiency of pancreatic juice, iii. 293, 299; in diabetes mellitus, 760 ; in syphilis, iii. 436 ; in epilepsy, iv. 640 ; in exophthalmic goiter, iii. 901 ; hypophysis in, i. 107; limitations of, i. 67; mammary gland in, i. 110; in multiple selerosis, iv. 281 ; in muscular dystrophy, iv. 264; pancreas in, i. 110 ; parathyroids in, i. 84; in pernicious anemia, iii. 836 ; principles underlying successful use of, i. 67 ; in rachitis, ii. 818; sexual glands in, i. 113; suprarenal glands in, i. 88; for tetany, iii. 910 ; thymus gland in, i. 117; thyroid gland in, i. 70 .

Organotherapy. S'ce Organotherapeutics.

Oriental sork, iv. 756; microörganism causing, iv. 756 ; preventive measures in, iv. 757 ; remedial measures in, iv. 756.

ORNITHORHYNCHUS PARADOXUS, treatment of poisoning by, iv. 684, 685.

Orthoform, use of, in acute nasopharyngitis, iii. 31 ; by insufflation, in phlegmonous pharyngitis, iii. 40; for relief of pain in laryngeal tuberculosis, iii. 347: in smallpox accompanied by dysphagia, ii. 46 .

Orthoform lozenges, use of, in acute tonsillitis, iii. 42.

Orthopedic treatment, unnecessary in early stages of rachitis, ii. 819 .

ORTHOPNEA, treatment of, in chronic myocardial insufficiency, iii. $718,721$.

OrTNER, on use of oxygen inhalations to relieve cyanosis in acute bronchitis, iii. 363.

Osmic acid injections, use of, in trigeminal neuralgia, iv. 342 .

OsMotic Cells, i. 54.

Osmotic membranes, i. 55.

Osmotic pressure, laws of, i. 54.

Osteomalacia, administration of epinephrin in, i. 96.

Otis urethrotome, use of, in operation for resilient urethral stricture, iv. 172.

Otitic Cerebral abscesses, iv. 421.

OTITIs, as a complication of scarlet fever, ii. 130 ; as a complication of typhoid fever, ii. 27.

OTITIS MEDIA, as a complication of diphtheria, ii. 277; as a complication of tuberculosis, ii. 509; organism causing, ii. 278; prevention of, in measles, ii. 156; radiant light and heat in treatment of. acute, i. 450; radiant light and heat in treatment of chronic, i. 451; surgical treatment of chronic, as prophylactic measure against cerebral abscess, iv. 422 ; treatment of, in general, ii. $27 \mathrm{~S}$. 
Otitis media, PURUlent, as a cause of infectious sinus thrombosis, iv. 428; as a cause of neuritis of seventh nerve, iv. 310.

Otitis, suppurative. See Otitis, purulent. Oudin, Dr., investigations by, on value of radium therapy in gynecology, i. 479.

"Our Lady at Lourdes," individualization of charity at, iv. 644 .

Ovarian extract, use of, in myasthenia gravis, iv. 507.

Ovaries, influence of X-ray therapy upon, i. 555; swelling of, as a complication of mumps, ii. 166 ; technique for use of $\mathrm{X}$-ray in disease of, i. 556.

Ovaritis, electrotherapeutics for relief of, i. 419 .

Ovary, in organotherapy, i. 114; disorders of menstruation relieved by, i. 114; disturbances of menopause relieved by, $\mathrm{i}$. 114; preparations of, i. 116.

Over-EATING, a cause of chronic gastritis, iii. 159.

Over-EXertion, as a cause of pulmonary edema in nephritis, iv. 56; danger from, in angina pectoris, iii. $769,770,771$; danger from, in multiple sclerosis, iv. 280 ; danger from, in syringomyelia, iv. 278; necessity for avoiding, in chronic interstitial nephritis, iv. 71.

Over-stimulation, chronic myocardial insufficiency due to, iii. 698; treatment of, iii. 723 .

Overwork, as a cause of hyperacidity of stomach, iii. 75 .

Oxalic acid diathesis, iv. 7.

Oxaluria, iv. 7 ; associated with intestinal autointoxication, iv. 7 ; treatment of, iv. 8.

"Oxidation temperature," ii. 485.

Oxygen, hypodermic injection of, in pneumonia, ii. 237; in pneumonia with asphyxia, ii. 237 ; to relieve cyanosis in acute bronchitis, iii. 364 .

Oxygen, inhalation of, in bronchiectasis, iii. 396 ; in bronchopneumonia, iii. 420 ; in bronchopneumonia complicating measles, ii. 159; in chronic bronchitis, iii. 378; in diabetic coma, ii. 757 ; in eclampsia of pregnancy, iv. 83 ; in Landry's paralysis, iv. 329; in lung complications of Rocky Mountain fever, ii. 566; in methemoglobinuria, iv. 5; negative results from, in polycythemia with splenomegaly, iii. 878; in phrenic neuritis, iv. 289 ; in pneumonia, ii. 236 ; in pulmonary edema, iii. 409; for relief of cyanosis in acute bronchitis, iii. 363; for relief of dyspnea and cyanosis due to myocardial insufficiency, iii. 736; for relief of dyspnea due to pressure of mediastinal tumor, iii. 503; for relief of dyspnea in tuberculosis, ii. 506; for relief of dyspnea in tuberculosis of mediastinal lymph glands, iii. 508; for relief of edema of glottis in acute nephritis, iv. 63 ; for relief of pulmonary edema in acute interstitial nephritis, iv. 72 ; as remedy for poisons causing heart failure, i. 672 ; in tetanus, ii. 350 ; useless in pernicious anemia, iii. 826 .

Oxygen, intrapleural injection of, to stimulate absorption of exudate, iii. 482.

Oxygen baths. See Baths.

OXYURIS Vermicularis, iii. 252.

Oysters, transmission of typhoid fever by, ii. 3 .

Ozena, iii. 336.

Pachymeningitis, electrotherapeutics for relief of, i. 429 .

Pachymeningitis externa, iv. 362.

Pachymeningitis interna hemorrhagica, iv. 362 ; diagnosis between, and brain tumor, iv. 437; pathology of, iv. 363 ; prognosis of, iv. 363 ; prophylaxis against, iv. 364 ; symptoms of, iv. 363 ; treatment of, iv. 364 .

Pack, blanket, use of, to reduce temperature in pneumonia, ii. 233.

Pack, cold, i. 196; in after-treatment of morphinism, ii. 624 ; in delirium tremens, ii. 606 ; to reduce temperature in pneumonia, ii. 231; to reduce temperature in Rocky Mountain fever, ii. 565; to reduce temperature in scarlet fever, ii. 126; to reduce temperature in tuberculosis of mediastinal lymph glands, iii. 508; in typhoid fever, ii. 18.

Pack, cold wet, use of, in tabes, iv. 201.

Pack, cool, use of, in hyperpyrexial fever, iv. 869 .

Pack, hot, use of, in after-treatment of morphinism, ii. 624; in delirium tremens, ii. 602; in nephritis complicating Rocky Mountain fever, ii. 566; in nephritis 
complicating scarlet fever, ii. 126; in optic neuritis without apparent cause, iv. 306 ; for relief of anuria in nephrolithiasis, iv. 107; for relief of headuche in hydrocephalus, iv. 609: in treatment of nephritis at home, iv. 39 .

Pack, hot air, use of, in home treatment of nephritis, iv. 39.

Pack, hot blanket, i. 197.

Pack, hot dry, use of, in acute polymyositis. ii. 685 .

Pack, ice, i. 201; use of, for insolation, iv. 678 ; use of, for relief of pain in plague, iv. 827; uses of, in hydrotherapy, i. 201.

Pack, lukewarm, use of, in tetany, iii. 910.

Pack, mustard. See Mustard pack.

Pack, warm, use of, in anterior poliomyelitis, iv. 238; in apyrexial heat exhaustion, iv. 679 ; in scarlet fever, ii. 126.

Pack, wet, i. 196; use of, in neurasthenia, iv. 547.

Pack, wet sheet, use of, for relief of cardiac palpitation in neurasthenia, iv. 568 .

PAIN, character of, in spinal syphilis, iv. 271; relief of, a cause of morphinism, ii. 616 ; significance of, in rheumatic cases of facial paralysis, iv. 311 ; significance of, in thoracentesis, iii. 478 .

Pain sense in pleura, iii. 462.

Painful points, presence of, in myositis, iv. 603, 604.

Palatine nerve, alcohol injection into, for relief of neuralgia in, iv. 346.

Palpation, value of, in diagnosis of brain tumor, iv. 444.

Palpitatio cordis, as a symptom of neurasthenia, iv. 567.

Palpitation, attacks of, in cardiac neurasthenia, iii. 778 .

Palpitation of heart. See Palpitatio cordis.

Paltauf, status lymphaticus first described by, iii. 911 .

PancoAst, on technique of X-ray therapy in leukemia, i. 548.

Pancreas, bullet wounds of, iii. 306; injuries to, iii. 305: medical treatment of tumors in, iii. 305; in organotherapy, i. 110; penetrating wounds of, iii. 306 ; relation between removal of, and diabetes mellitus, ii. 720 ; rupture of, iii. 305 ; surgical treatment of, iii. 294; surgical treatment of tumors in, iii. 304 ; syphilis of, iii. 301; transplantation of, i. 112; tuberculosis of, iii. 301 ; tumors of, iii. 3()$; 3$.

Panchras, Distase's of, iii. 293: acute pancreatitis, iii. 294; chronic pancreatitis, iii. 296; general consulerations on subjeet, iii. 293; hemochromatosis, iii. 301; injuries to pancreas, iii. 305; opotherapy for, iii. 293, 299; pancreatic calculi, iii. $30^{\circ}$; pancreatic cysts, iii. 302 ; pancreatic fistula, iii. 306; pancreatic infantilism, iii. 301; surgical treatment of pancreas in general, iii. 294; syphilis of pancreas, iii. 301; tuberculosis of pancreas, iii. 301; tumors of pancreas, iii. 303.

Pancreas, preparations of, administration of, by mouth, i. 110; administration of, by subcutaneous and intravenous injection, i. 111; use of, in nutrient enemata, iii. 119; use of, in tumors of pancreas, iii. 30\%).

Pancreatic calculi, iii. 302.

Pancreatic cysts, iii. 302 ; treatment for, iii. 303.

Pancreatic extract, use of, in diabetes mellitus, ii. 760 .

Pancreatic fistula, iii. 306.

Pancreatic infantilism, iii. 301.

Pancreatitis, acute, iii. 294; dietetic treatment of, iii. 296; early stage of, iii. 295 ; late stage of, iii. 296 ; surgical treatment of, iii. 295, 296; symptomatic treatment of, iii. 296.

Pancreatitis, chronic, iii. 296; diet in, iii. 298; medical treatment of, iii. 293; opotherapy for, iii. 299; surgical treatment of, iii. 299; treatment of, in general, iii. 298.

Pantopon, use of, in diabetes mellitus, ii. 759.

Papilledema. See Optic neuritis.

Pappenheim, division of severe anemias by, iii. 805 ; varieties of leukemia defined by, 844 .

Paquelin cautery, use of, in gangrenous or phagedenic chancre, ii. 423 ; in pericarditis, iii. 51S; for relief of headache in neurasthenia, iv. 566; for relief of late pain in herpes zoster, iv. 3.59; for relief of pain in sciatica, iv. 299; in stomatitis gangrenosa, iii. 1S.

Paracentesis, of abdomen, iii. 674; choice of site for puncture in, iii. 523; in hydronephrosis, iv. 98 ; in hydroperi- 
cardium, iii. 526; indications for performance of, in unilateral hydrothorax, iii. 674; local anesthesia in, iii. 525; method of performing, in cirrhosis of liver, iii. 284; in pericarditis, with effusion, iii 522 ; for pyopericardium, iii. 525.

Paradysentery group of Bacilli, ii. 317.

Paraffin, rectal injection of, in chronic constipation, iii. 235.

Parafuchsin, use of, in trypanosomiasis, iv. 747.

Paragonimus westermanil, iv. 782.

Paraldehyde, use of, in delirium tremens, ii. 607 ; in pneumonia of alcoholics, ii. 258; for relief of insomnia in acute bronchitis, iii. 362 ; for relief of insomnia in neurasthenia, iv. 559 ; in tetanus, ii. 348.

Paralysis, acute ascending, see Landry's paralysis; following acute hemorrhagic polioencephalitis, treatment of, iv. 409; due to basilar syphilitic meningitis, iv. 473; of bladder, see Bladder; following brain syphilis, treatment of, iv. 488; due to cerebral palsies of children, iv. 412 ; due to cerebral thrombosis, iv. 396; due to cerebral thrombosis, prognosis in, iv. 396 ; due to cerebral thrombosis, treatment for, iv. 399; cerebrobulbar glossopharyngo-labial, see Cerebrobulbar glossopharyngo-labial paralysis; of diaphragm, see Neuritis, phrenic; general epileptic attacks as a manifestation of, iv. 639; facial, see Neuritis of seventh nerve; glossopharyngeal, see Neuritis of ninth nerve; hypoglossal, see Neuritis of twelfth nerve; infantile, see Poliomyelitis; due to Korsakow's psychosis, ii. 609; labioglossolaryngeal, see Bulbar paralysis, chronic progressive; Landry's, see Landry; obstetric, see Obstetric paralysis; periodic ocular, see Ocular paralysis; pneumogastric, see Neuritis of tenth nerve; progressive bulbar, see Bulbar paralysis, progressive; pseudobulbar, see Pseudobulbar paralysis; spinal accessory, see Neuritis of eleventh cranial nerve; as a symptom of cerebral embolism, iv. 400; as a symptom of cerebral thrombosis, iv. 396; symptoms of, in acute cerebral meningitis, iv. 369 .
Paralysis, acute vasomotor, iii. 751; acute infectious diseases associated with, iii. 751 ; treatment of, iii. 752 .

Paralysis agitans, iv. 665; administration of parathyroid glands for relief of, i. 87 ; treatment of, iv. 666 .

Paralysis, arsenical, i. 681; hydrotherapy for relief of, i. 225.

Paralysis, MOTOR, as a symptom of traumatic hematoma, iv. 365; value of, in regional diagnosis of brain tumor, iv. 438.

Paralysis, sensory, value of, in regional diagnosis of brain tumor, iv. 438.

Paralysis, spastic spinal, iv. 268; definition of, iv. 268; diagnosis of, iv. 269; pathogenesis of, iv. 269; pathology of, iv. 269; symptoms of, iv. 268; treatment of, iv. 270.

Paramyoclonus multiplex, iv. 659 ; treatment of, iv. 660 .

Paranephritic abscess. See Abscess.

Paranoid ideas, in epilepsy, iv. 636.

Paraplegia, as a symptom of acute myelitis, iv. 223.

Pararrhythmias, iii. 564.

Parasites, of brain. See Brain, parasites of.

Parasites, causing Cochin-China diarrhea, iv. 796; causing esophagostomiasis, iv. 799; causing filariasis, iv. 787, 788, 789, 791; causing hepatic distomiasis, iv. 779; causing hookworm disease, iv. 791; causing infantile splenic anemia, iv. 755; causing intestinal distomiasis, iv. 780; causing intestinal teniasis, iv. 776 ; causing kala-azar, iv. 753; causing schistosomiasis, iv. 783, 785, 786; causing somatic teniasis, iv. 775; causing tropical relapsing fever, iv. 758; causing trypanosomiasis, iv. 742; influence of protozoon upon white man in tropics, iv. 668; intestinal, see Intestinal parasites; malarial, iv. 712; presence of malarial in blackwater fever, iv. 738 .

Parasitic diseases of Skin, radium therapy for relief of, i. 475.

Parathyroid glands, diseases of, iii. 909; effects of deficiency of secretion by, i. 84; effects following removal of, mistaken for effects following removal of thyroid gland, i. 70, 71; experimental use of, i. 85; functions of, i. 84; method of administering, i. 87; in organotherapy, i. 
84; supposed relation between disturbance of, and rachitis, ii. 813 ; therapeutic uses of, i. 85.

Paregoric, use of, to control cough in acute bronchitis, iii. 375; in gallstone colic, iii. 268; in typhoid fever, ii. 26.

Paresis, general, diagnosis between, and brain tumor, iv. 436 .

Paresis of stomach, following operations, iii. 173.

Paresthesia, in diagnosis of tabes, iv. 189.

Parietal lobe, diagnosis of brain tumor in, iv. 439 .

PARIS school of PSYCHOTHERAPY, iv. 570.

Parotitis, acute, iii. 26; chronic, iii. 28; as a complication of typhoid fever, ii. 27 ; epidemica, see Mumps; symptomatic, iii. 26 ; treatment of symptomatic, iii. 28.

Paroxysmal hemoglobinuria, iv. 6 .

Parrots, association of, with psittacosis, ii. 594 .

Parry's disease. See Exophthalmic goiter.

Parturition, cerebral hemorrhage associated with, iv. 388; danger from, in eardiac insufficiency, iii. 681; as a factor in causation of cerebral palsies of children, iv. 409 .

Passive mechanical exercise, in treatment of obesity, ii. 807 .

Passive movements, in treatment of cardiac insufficiency, iii. 573.

Pässler, research by, on treatment of acute vasomotor paralysis, iii. 751, 752.

Pässler AND Rolly, researches conducted by, on acute vasomotor paralysis, iii. 752.

Pässler And Romberg, investigations by, showing reason for failure of circulation in infectious diseases, iii. 420.

Pasteur, work on immunization by, i. 125.

Pasteur Institute of Paris, results of Pasteur treatment of, ii. 341.

Pasteur treatment of hydrophobia, ii. 337; complications in, ii. 340 ; duration of, iii. 339; indications for, ii. 334 ; invention of, ii 337 ; local and constitutional effects of, ii. 339 ; mortality from rabies affected by, ii. 342 ; preparation of antirabic virus used in, ii. 338 ; results of, ii. 341 ; technique of administration of, ii. 337 : use of, by mail, ii. 341 .
Pasteurization of Milk, as a protection against tuberculosis, ii. 457; risks associated with, ii. 646.

Pasteur's method, of immunization for anthrax, iii. 3331 .

Pateleson, Malicus, method employed by, for gauging effects of exereise on tuberculous pationts, ii. 528; scientific principle of exercise in tuberculosis discovered by, ii. 527; system of graduated labor developed by, ii. 528, 529; system of rest during active stage of pulmonary tuberculosis introducel by, ii. 483.

Pathological tissues, different susceptibility of, to rocntgenization, i. 510 .

Pavlov, work done by, in digestion, i. 625, 626.

Pearce, investigations by, on cause of renal edema, iv. 17.

Pediculus vestimentoizum, transmission of typhus fever by, ii. 32.

Pellagra, ii. 657; causation of, ii. 657 ; diet in, ii. 661 ; drugs in, ii. 659 ; fulminating form of, ii. 660; hemorrhage complicating, ii. 662; hydrotherapy for, ii. 662; institutions for care of, ii. 662; prophylaxis of, ii. 657 ; treatment of, ii. 659 .

Pelletierin, use of, as substitute for male fern, iv. 778.

Pelvic cellulitis, electrotherapeutics for relief of, i. 406.

Pelvic congestion, radiant light and heat in treatment of, i. 453.

Pelvic inflammation, electrotherapeutics for relief of, i. 418.

Pelvic organs, diseases of, a cause of enuresis, iv. $127,128$.

Pemphigus, as a complication of vaccination, ii. 91 .

Pendulum movements. See Herz system of gymnastics.

Penetration, as a factor in X-ray therapy, i. 494; increase of, in deep therapy, $i$. 506 ; measurement of, i. 496 ; as property of radiant energy, i. 433.

Pengewar compass, use of, in hemophilia, iii. 867 .

Pennies, lodgment of, in esophagus, iii. 63.

Pennsylvania, antitubertulosis work in, ii. 446.

Pentose, presence of, in diabetic urine, ii. 72 S. 
Pentosuria, conditions governing, ii. 729.

Penzoldt, on benefits of ice bag in acute localized peritonitis, iii. 311 .

Peppermint, use of, for flatulency in gastric atony, iii. 172.

Pepsin, use of, after meals, in hill diarrhea, iv. 863.

Pepsin and nux vomica, use of, as a tonic in stomatitis catarrhalis, iii. 7 .

Peptic asthma. See Asthma.

Peptones, use of, in hemophilia, iii. 866; in nutrient enemata for gastric ulcer, iii. 118.

Peptonized milk, use of, unsatisfactory, in gastric ulcer, iii. 127.

Percussion, use of, in diagnosis of brain tumor, iv. 444; in massage, i. 292.

Perforating ulcer, in tabes, iv. 192.

Perforation of gastric UlCer, immediate operations required by, iii. 143.

Perforation of intestines, occurrence of, in typhoid fever, ii. 22; operation for, in syphilitic gastric ulcer, iii. 167 .

Pericardial synechis, association of, with angina pectoris, iii. 768 .

Pericarditis, iii. 513; after-treatment of, iii. 521 ; as a complication of pneumonia, ii. 261 ; drugs in treatment of, iii. 518; electrotherapy for relief of, i. 409; former treatment of, iii. 511; hydrotherapy for relief of, iii. 204; local treatment of, iii. 517; prophylaxis of, iii. 513 ; rheumatism as cause of, iii. 513; stimulants in treatment of, iii. 520; treatment of, in general, iii. 514.

Pericarditis, adhesive, iii. 527; operation for, iii. 529.

Pericardium, diseases of, iii. 511: adhesive pericardium, iii. 527; hemopericardium, iii. 527; history of, iii. 511; hydropericardium, iii. 525; pericarditis, iii. 511 ; pericarditis with effusion, iii. 521 ; pneumopericardium, iii. 527 ; pyopericardium, iii. 526.

Perineal section, employment of, for relief of complications in paralysis of bladder, iv. 123; for relief of impassable urethral stricture with retention, iv. 172; for relief of irritable stricture of the urethra, iv. 173; for relief of urethral section complicated by periurethritis, iv. 173; for relief of urethral stricture complicated by acute pyelonephritis, iv. 173 .
Periodic alcoholism, ii. 591.

Periodic ocular paralysis. See Ocular paralysis.

Periodicity, explanation of term in electrotherapeutics, i. 364, 365.

Periostitis, as a complication of typhoid fever, ii. 27.

Peripheral nerves, diseases of, iv. 382: neuritis of brachial plexus, iv. 290; neuritis of spinal nerves, iv. 289; phrenic neuritis, iv. 288; treatment of neuritis in general, iv. 282.

Peripheral resistance, influence of, upon pulse, iii. 560 .

Peristalsis, gastric, recent knowledge concerning, iii. 68.

Peristaltic unrest, iii. 250.

Peritoneal adhesions, iii. 322.

Peritoneum, diseases of, iii. 309: acute diffuse peritonitis, iii. 313 ; acute localized peritonitis, iii. 309 ; chronic peritonitis, iii. 323; malignant disease of peritoneum, iii. 323 ; peritoneal adhesions, iii. 322 ; tuberculous peritonitis, iii. 319.

Peritonitis, administration of epinephrin for relief of, i. 98; administration of extract from posterior lobe of hypophysis for, i. 109; chronic, iii. 323; as a complication of pyelitis, iv. 94; hydrotherapy for relief of general diffuse, i. 214; progressive septic, see Peritonitis, acute diffuse.

Peritonitis, acute diffuse, iii. 313; care - of bowels in, iii. 317; diet in, iii. 317; etiology of, iii. 313 ; medical treatment of, iii. 315 ; mortality from, iii. 313 ; prophylaxis against, iii. 314; surgical indications in, iii. 314 ; temperature in, iii. 318 ; toxemia in, iii. 318 ; treatment of, in general, iii. 314; vomiting in, iii. 318 .

Peritonitis, acute general. See Peritonitis, acute diffuse.

Peritonitis, acute localized, iii. 309; care of bowels in, iii. 312 ; diet in, iii. 311; etiology of, iii. 309; general measures of treatment in, iii. 312; medical treatment of, iii. 310; prophylaxis against, iii. 309; summary of treatment of, iii. 313 ; surgical treatment of, iii. 310 ; treatment of, in general, iii. 310; vomiting in, iii. 312 .

Peritonitis, tuberculous, iii. 319 ; autoserotherapy for, iii. 320 ; constipation in, 
iii. 321 ; electricity combined with $\mathrm{X}$-ray in treatment of, i. 403 ; measures of treatment for, in general, iii. 320 ; medical treatment for, iii. 320 ; radiant light and heat in treatment of, i. 452; surgical indications and contraindications in, iii. 320 ; surgical versus medical results in, iii. 319 ; treatment of effusion in, iii. 321 ; tuberculin in treatment of, iii. 320.

Periuretiral abscess. See Abscess.

Perlsucht tuberculin, i. 151.

Permanganate irrigation, use of, in acute posterior urethritis, iv. 147.

Permanganate ointment, use of, in oriental sore, iv. 756.

Permanganate solution, cholera germs destroyed by, iv. 829 .

Pernet, observations by, on pemphigus following vaccination, ii. 91 .

Pernicious malaria. See Malaria.

Personal idiosyncrasy, theory of, as explanation of injury from X-ray therapy, i. 513.

Personal precautions, against trypanosomiasis, iv. 750 .

Perspiration, stimulation of, in treatment of intestinal autointoxication, ii. 672 .

Persuasion, as a form of psychotherapy, iv. 571.

Perthez, early work on X-ray filter by, i. 504.

Pertussis. See Whooping-cough.

Pes cavus. See Friedreich's foot.

Petit mal, iv. 634.

Petrolatum, external use of, in small-pox, ii. 50.

Pfahler, early work by, on X-ray filter, i. 504; favorable results obtained by, in $\mathrm{X}$-ray treatment of secondary mediastinal carcinoma, iii. 502; method employed by, for X-ray treatment of mediastinal tumors, iii. 502; statistics collected by, on results of X-ray therapy in exophthalmic goiter, i. 552.

Pfeiffer, discovery of influenza bacillus by, ii. 184; glandular fever first described by, ii. 574 .

Pfeiffer's theory, of nature and action of cholera toxins, iv. 831.

Pflueger-Arndt law, i. 308.

Phagedena, tropical sloughing. See Tropical sloughing phagedena.

Phallin, ii. 649.
Phanemgenetic anemiag. See Anemias. Pinamacology, standard solutions and comparative methods in, i.9.

Pharmacotherapy, use of, in epilepsy, iv. 6.17.

Pharyngeal cirises, in tabeg, iv. 191.

Pharyagal tonsil, Hyertrolhy of, iii. 35.

Pinaryaltis, acute, iii. 31 ; chronic, iii. 3.3 ; electrotherajeutics for relief of, i. 409 ; membranous, iii. 38 ; phlegmonous, iii. 40.

Pharynx, disorders of, a complication of measles, ii. 156; importance of attention to, in prophylaxis of acute cerebral meningitis, iv. 371 ; irrigation of, in diphtheria, ii. 271 ; otitis media caused by irrigation of, ii. 277 .

Pharynx, Diseases of, iii. 31 : acute nasopharyngitis, iii. 31 ; acute uvulit is, iii. 35 ; adenoid vegetations, iii. 37; atrophic nasopharyngitis, iii. 34 ; chronic nasopharyngitis, iii. 32 ; chronic pharyngitis, iii. 32 ; hypertrophy of pharyngeal tonsil, iii. 35; membranous pharyngitis, iii. 38; phlegmonous pharyngitis, iii. 40 ; Vincent's angina, iii. 34 .

Phenacetin, use of, in acute nasopharyngitis, iii. 31 ; in acute rheumatism, ii. 301 ; in aural vertigo, iv. 316 ; to deplete circulation in cerebral hyperemia, iv. 386 ; in erysipelas, ii. 284; in gastrointestinal type of influenza, ii. 190; in influenza, ii. 187; in measles, ii. 152; in migraine, iv. 592 ; to reduce temperature in acute cercbral meningitis, iv. 372 ; to reduce temperature in bronchopneumonia, iii. 418; to reduce temperature in dengue, iv. 859 ; for relief of cardiac palpitation in neurasthenia, iv. 568 ; for relief of cervicoöccipital neuralgia, iv. 356 ; for relief of diabetic neuralgia and neuritis, ii. 766 ; for relief of headache due to cerebral thrombosis, iv. 399 ; for relief of headache in chronic interstitial nephritis, iv. 72 ; for relief of headache in neurasthenia, iv. 566; for relief of headache in pachymeningitis interna hemorrhagica, iv. 364 ; for relief of headache due to sunstroke, iv. 679; for relief of pain in acute myelitis, iv. 226; for relief of pain in neuralgia, iv. 339 ; for relief of pain in neuritis, iv. 285; for relief of pain in 
pyelitis, iv. 88; for relief of pain in Rocky Mountain fever, ii. 564; for relief of pain in septicemia, ii. 291; for relief of pain in small-pox, ii. 47 ; in scarlet fever, ii. 126; in varicella, ii. 111; in Weil's disease, ii. 572.

Phenacetin and salol, use of, to reduce fever in anterior poliomyelitis, iv. 237.

Phenol, local use of, to relieve itching in diabetes mellitus, ii. 764; local use of, in Rocky Mountain fever, ii. 565; objections to, as disinfectant for tuberculous sputum, ii. 453; subcutaneous use of, in tetanus, ii. 349 ; use of, as disinfectant for site of puncture in thoracentesis, iii. 474.

Phenolphthalein, use of, in chronic constipation, iii. 234 ; combined with rhubarb, in diabetes mellitus, ii. 736; in nephritis, iv. 33 ; for relief of constipation in neurasthenia, iv. 566 ; in uremia, iv. 53.

Phenolsulphonates, the, use of, as antiseptics in intestinal autointoxication, ii. 675.

Phenyl salicylate, use of, as an antiseptic in intestinal autointoxication, ii. 675.

Philip, Dr. R. W., work by, on prevention of tuberculosis, ii. 441.

Philippines, THe, history of vaccination in, ii. 104 ; results of prophylaxis against beri-beri in, iv. 851 .

Philoff, investigation by, on persistence of cholera vibrios, iii. 830 .

Phimosis, as a cause of enuresis, iv. 130.

Phipps, JAMes, first inoculation for smallpox performed upon, ii. 58 .

Phlebitis, as a complication of typhoid fever, ii. 28 ; treatment of, by radiant light and heat, i. 458.

Phlebitis of iliac veins, as a cause of pain in bladder, iv. 122 .

Phlebosclerosis, association of, with arteriosclerosis, iii. 762 .

Phlegmonous Pharyngitis. See Pharyngitis.

Phloridzin, glycosuria produced by injection of, ii. 724 ; injection of, as test for functional power of kidney, iv. 25.

Phobias, as a characteristic phenomenon in psychasthenia, iv. 518 .

Phosphates, use of, in tuberculosis of mediastinal lymph glands, iii. 507.

Phosphaturia, iv. 8; treatment of, iv. 9.
"Phosphorisme," ii. 628.

Phosphorus, use of, in after-treatment for morphinism, ii. 623 ; in beri-beri, iv. 854 ; in chronic atrophic laryngitis, iii. 343; in chronic myelitis, iv. 230; in laryngeal tuberculosis, iii. 346; for neuralgia due to anemia, iv. 336 ; in neurasthenia, iv. 559 ; in rachitis, ii. 817 .

Phosphorus poisoning, ii. 626; acute form of, ii. 627 ; chronic form of, ii. 628 ; history of, ii. 626 ; operative measures in treatment of chronic form of, ii. 630; prophylaxis against, ii. 629 ; symptoms of chronic, ii. 628 ; treatment of acute, ii. 627 ; treatment of chronic, ii. 630 .

Phrenic neuritis. See Neuritis.

Phrenocardia, iii. 788.

Phrenolysin, iv. 683.

Physaloptera mordens, infection by, iv. 800.

Physical exercise, forms of, classified, iii. 700 ; in open air, use of, in biliousness, iii. 260 ; in treatment of constipation, iii. 228.

Physical therapy, value of, in epilepsy, iv. 646.

Physical training, of adolescent as a prophylactic measure against the neuroses, iii. 523.

Physician, attitude essential for, in employment of psychotherapy, iv. 572; duty of, in cardiac neurasthenia, iii. 775; duty of, in diabetes mellitus, ii. 734, 738; duty of, in prescribing drugs producing toxic effects, iv. 5; duty of, as regards infection in measles, ii. 139, 145; duty of, as regards infection in scarlet fever, ii. 120 ; duty of, as regards infection in small-pox, ii. 44; requirements in, for treatment of the neuroses, iv. 530 .

Physiochemical therapy, principles in, i. 1: absorption and secretion of dissolved substances, i. 45; colloids as constituents of living tissues, i. 4 ; crystalloids as constituents of living tissues, i. 7 ; distribution, i. 18; effect of colloids upon crystalloids, i. 34 ; effect of external conditions upon the colloidal state, i. 28; equilibrium in living tissues, i. 12; fusion, i. 16; general constitution of living matter, i. 3 ; inequalities in distribution due to inequalities in adsorption, i. 22; inequalities in distribution due to in- 
equalities in solubility, i. 18; inequalities in distribution due to interference from "membranes," i. 24; inequalities in distribution clue to specifie cell differences, i. 24; osmosis and question of cellular membranes, i. 53; pharmacological importance of theory of eleetrolytic dissociation, i. 26 ; rôle of water in therapeutic procelures, i. 35 ; standard solutions and comparative methods in pharmacology, i. 9; survey and application of principles, i. 35 ; water as a constituent of living tissues, i. 9.

Physiological effects of light therapy, i. 444; antiseptic, i. 446; local, i. 445; upon infection in tissues, i. 446; upon metabolism, i. 445.

Physiological influence of atmospheric HUMIDITY, i. 263.

Physiological reaction to external TEMPERATURE, i. 260.

Physiological and medical climatology, i. 259 ; dust and atmospheric impurities as a factor in, i. 274; physiological influence of atmospheric humidity in, i. 263 ; physiological influence of diminished barometric pressure in, i. 268; physiological reaction to external temperature in, i. 260; psychology of, i. 275; ventilation and, i. 265.

Physiology of obesity, ii. 781.

Physostigma venenosum, poisoning by, iv. 695; remedial measures, iv. 696.

Physostigmin, use of, in chronic constipation, iii. 234 .

Physostigmin salicylate, use of, in asthenic bulbar paralysis, iv. 256.

Phytolacce decandra. See Pokeroot.

Pia mater, indications of a tumor in, iv. 445.

Picric acid, use of, as a substitute for quinin, iv. 726 .

"Pied Bot," in tabes, iv. 191; in Friedreich's disease, iv. 216.

Pigment IN skin, as a protection against direct sunlight, iv. 669 .

Pilocarpin, use of, in acute bronchitis, iii. 361 ; in chronic atrophic laryngitis, iii. 343 ; as a diaphoretic in pleurisy, iii. 470 ; in edematous laryngitis due to a foreign body, iii. 341; as expectorant in chronic bronchitis, iii. 372 ; by hypodermic injection in acute catarrhal jaundice, iii. 262; by hypolermie injection in disease of (eighth nerve, iv. 315, 316; by hypodermic: iujection in solution for snake bite, iv. 652; in nephritis complicating searlet fever, ii. 1333; in nervous deafness and aural vertigo, iv. $315,316,317$; in orchitis and epididymitis in mumps, ii. 172; in plastic bronchitis, iii. 391 ; in pneumonia, ii. 236; rarely indicated in nephritis, iv. 39 ; for relief of anuria in nephrolithiasis, iv. 107 ; in tetanus, ii. 230 ; in uremia, iv. 53 ; in wood alcohol poisoning, ii. 610 .

Pilocarpin injections, association of, with pulmonary edema, iii. 408.

Pilocarpin nitrate, use of, hypodermically, in bronchial asthma, iii. 388.

Pine-needle extract, use of, as a cutancous stimulant in tabes, iv. 200.

Pineal gland, disease of, iii. 918.

Pinworms. See Oxyuris vermicularis.

Pipe sMoking, effects of, iii. 783.

Piperazin, non-efficacy of, in nephrolithiasis, iv. 110.

Pipes, use of, for smoking, in cardiac insufficiency, iii. 689.

Pituitary body. See Hypophysis.

Pituitary extract, use of, in diabetes insipidus, ii. 778; as diuretic in nephritis, iv. 50 ; for relief of dystrophia adiposogenitalis, iii. 917.

Pituitary substance, effect produced by, on pulmonary circulation, iii. 405.

Pituitrin, i. 107; use of, as diuretic in nephritis, iv. 51 ; use of, in hematuria, iv. 4.

Pitchblende, as a source of radium, i. 461 ; workers in, affected by, i. 472 .

Places, infected, as possible cause of beri-beri, iv. 849 .

Plague, iv. 816; drug treatment of, iv. 821; extermination of rats as protection against, iv. 821; factors influencing seasonal prevalence of, iv. 819 ; inoculation for, iv. 823; measures yielding temporary protection against, iv. 823 ; microörganism causing, iv. 816 ; mortality from, under carbolic acid treatment, iv. 827 ; mortality from, with and without inoculation, iv. 823 ; prophylaxis against, iv. 816 ; prophylaxis against pneumonic, iv. 826 ; protective measures now in vogue that should be abolished, iv. $\$ 25$; recrudescence of, iv. 
820 ; relation of animals other than rats to spread of, iv. 818; relation of epizootic form in rats to spread of epidemic form in human beings, iv. 818; relation of man to spread of, iv. 819; relation of rats to spread of, iv. 817; relation of unsanitary conditions to spread of, iv. 819; serum treatment for, iv. 826 ; species of fleas concerned in, iv. 818; species of rodents concerned in, iv. 818 ; vaccine therapy for, iv. 823.

Plan of treatment, importance of, in tabes, iv. 211.

Plasmatic treatment of chlorosis, iii. 821.

Plasmodium falciparum, iv. 713.

Plasmodium malarie, iv. 713.

Plasmodium vivax, iv. 712 .

Plasmotherapy, in chlorosis, iii. 822; for pernicious anemia, 833 .

Plaster, adhesive, application of zinc oxid by means of, in herpes zoster, iv. 359; method of applying, in viceroptosis, iii. 247; use of, to support prolapsed liver, iii. 289 .

Plaster, menthol, use of, to relieve neuralgias in chlorosis, iii. 824 .

Plaster, mercurial. See Mercurial plaster.

Plaster, mustard. See Mustard plaster.

Plaster jacket. See Jacket.

Plasters, simple, use of, to relieve backache in acute nephritis, iv. 63.

PlatT, early inoculation with cowpox for prevention against small-pox, ii. 57 .

Plesch's method, of determining extent of chronic myocardial insufficiency, iii. 696.

Plethysmographic method of determining extent of chronic myocardial insufficiency, iii. 696.

Pleura, echinococcus cysts of, iii. 493; pain sense in, iii. 462; tumors of, iii. 493.

Pleura, diseases of, iii. 455; absorption of exudate in, iii. 457; absorption of fibrin in, iii. 458; absorption of fluid in, iii. 458; absorption of gases in, iii. 459; cause of death from pleural exudate in, iii. 461; chronic serous effusion, iii. 483; chylothorax, iii. 489; clinical pathology of, iii. 457; empyema, iii. 484; fibrinous pleurisy, iii. 466; hemothorax, iii. 490; history of, iii. 455 ; influence of exudate upon circulation in, iii. 460 ; intratho- racic pressure of exudate in, iii. 459,460 ; mechanical effect of exudate in, iii. 460; pain sense of pleura in, iii. 462 ; permeability of mediastinum affected by exudate in, iii. 459; pneumothorax, iii. 491; serofibrinous pleurisy, iii. 467; thoracentesis for, see Thoracentesis; tumors of pleura, iii. 493 .

Pleural effusion, accompanying tuberculosis, ii. 507.

Pleurisy, blood pressure lowered in, iii. 556; clinical course of, iii. 463; dry, see Pleurisy, fibrinous; with effusion, as a complication of pneumonia, ii. 261; end result after absorption in, iii. 464; hemorrhagic, iii. 490; hydrotherapy for relief of, i. 205; indications for treatment in, iii. 465; mechanotherapy for relief of, i. 350 ; onset of, iii. 463 ; pain in, iii. 462 ; prophylaxis against, ii. 464 ; treatment of, iii. 464.

Pleurisy, fibrinous, iii. 466; acute form of, iii. 466; autoserotherapy for, iii. 483; empyema following, see Empyema; indications for operation for large effusions with slow absorption in, iii. 471; indications for operation in moderate effusions with slow absorption in, iii. 472; indications for operation to save life in, iii. 471 ; measures to check secretion and aid absorption of effusion in, iii. 468; nature of, iii. 466; operative measures for, see Thoracentesis; pain in acute form of, iii. 466; serofibrinous form of, iii. 467; sudden death during or following operation for, iii. 480; symptomatic treatment of, iii. 467; treatment of infection in, iii. 467 ; transudates and exudates in, iii. 468.

Pleuritic adhesions, chronic myocardial insufficiency due to, iii. 732.

Plimmer's salt. See Antimony sodiotartrate.

Plumbism, chronic, blood pressure raised by, iii. 555; hydrotherapy for relief of, i. 228.

Plunge bath, cold, i. 199.

Pneumococci, autolyzed, use of, in pneumonia, ii. 243.

Pneumococcus, history of, ii. 220.

Pneumococcus infections, vaccine treatment in, i. 146.

Pneumococcus meningitis, ii. 213. 
Pneumococcus serum, use of, in meningitis complicating pneumonia, ii. 259.

Pneumococcus vaccine, use of, in mixed infection, iii. 243; in pneumonia, ii. 240; results of, ii. $241,242$.

Pneumogastric paralysis. See Neuritis of tenth nerve.

Pneumonia, lolbar, ii. 217; abscess of lung complicating, ii. 266; adrenalin in treatment of, ii. 252 ; afebrile form of, ii. 258 ; in alcoholics, ii. 258; antipneumococcus serum in treatment of, ii. 238 ; autolyzed pneumococci in treatment of, ii. 243; bacteriology of, ii. 217 ; bilious type of, ii. 258; blood pressure in cardiac toxemias in, ii. 254; bradyeardia complicating, ii. 260; bronchorrhea complicating, ii. 261 ; care of body in, ii. 226 ; "carriers" of, ii. 222; chemoimm nuity in treatment of, ii. 245; in childhood, ii. 220; chill as an initial symptom, ii. 226; cleanliness important in prophylaxis of, ii. 223; as a complication of influenza, ii. 191 ; as a complication of whoopingcough, ii. 175; complications and sequelæ of, ii. 257; convalescence from, ii. 262; correction of predisposing conditions in prophylaxis of, ii. 222; cough and expectoration as a symptom of, ii. 225, 257; crisis in, ii. 257; definition of, ii. 217; delayed resolution in, ii. 257; delirium as a symptom of, ii. 228; diabetic type of, ii. 258; diagnosis between, and typhoid fever, ii. 39 ; diet in, ii. 228 ; dilatation of stomach complicating, ii. 229 ; disinfection in, ii. 222; division of cases in, ii. 219; education of public a factor in prophylaxis of, ii. 223; empyema complicating, ii. 261; endocarditis complicating, ii. 260; epidemic of, due to $\mathrm{B}$. pneumoniæ in infected bacon, ii. 643 ; examination of patient in, ii. 221 ; gastrointestinal tract in, ii. 227 ; irritating drugs in treatment of, to cause abscess, ii. 246; isolation of cases in, ii. 220; leukocytic extract in treatment of, ii. 244; medicinal treatment of, ii. 233; meningitis complicating, ii. 259; metallic ferments in treatment of, ii. 246; mortality from, ii. 218; nephritis complicating, ii. 259; nurse's duty in, ii. 222 ; nutritive changes in, ii. 228 ; in old age, ii. 220; open-air treatment of, ii.
224 ; operative treatment indicated in, ii. 261; oral cleanliness important in prophylaxis of, ii. 221 ; oxygen in treatment of, ii. 236; pain in, ii. 226; pericarditis complicating, ii. 261; pleurisy with effusion complicating, ii. 261; pneumococcus vaccine in treatment of, ii. 240; position of patient in, ii. 225; prevention of second attack of, ii. 222 ; prophylaxis in, ii. 220 ; public health in regard to, ii. 222 ; in public institutions, ii. 221; pulmonary edema complicating, ii. 259; recurrence of, ii. 220 ; requisites of treatment for, ii. 219; specific treatment of, ii. 237 ; statistics of, ii. 218; tachycardia complicating, ii. 229 ; treatment of complications and sequelie, ii. 2.57 ; treatment of, in general, ii. 223; treatment of sputum in, ii. 221 ; venesection in treatment of, ii. 225; veratrum in treatment of, ii. 248.

Pneumonia, afebrile, ii. 258.

Pneumonia, aspiration, danger of, in acute apoplectic bulbar paralysis, iv. 50; danger of, in meat poisoning, ii. 639 .

Pnedmonia, chronic, iii. 421.

Pneumonia, ether, prevention of, ii. 222.

Pnedmonia, hypostatic, in progressive paralysis of the insane, iv. 494; precautions against, in cerebral hemorrhage, iv. 392.

Pneumonia, secondary, prevention of, ii. 222.

Pneumonia, unresolved, X-ray therapy for relief of, i. 553.

"Pneumonia bags," ii. 232.

Pneumonia Plague, prophylaxis against, iv. 826.

Pneumonoconiosis, iii. 423; definition of, iii. 423; etiology of, iii. 424 ; general hygiene in treatment of, iii. 425 ; government supervision of dusty trades a prophylactic measure against, iii. 429; medical supervision of dusty trades a prophylactic measure against, iii. 429 ; prophylaxis in, iii. 428 ; protection from dust a means of preventing, iii. 428 .

Pneumopericardium, iii. 527.

Pneumotherapeutic applications, use of, in emphysema associated with cardiac insufficiency, iii. 711 .

Pneumothorax, iii. 491 ; accidental form of, iii. 491; acutissimus, iii. 491; empyema associated with, iii. 493 ; serous effusion 
associated with, iii. 491; significance of, in thoracentesis, iii. 479; spontaneous form of, iii. 492; suffocative form of, see Acutissimus; traumatic form of, iii. 492; tuberculous form of, iii. 492.

Pneumothorax, artificial, production of, in tuberculosis, ii. 533; apparatus employed in, for injection of gas, ii. 536; methods of production of, ii. 534; method of injecting gas or air in, ii. 536; selection of cases for, ii. 535 .

Pneumotomy, as a therapeutic measure in chronic abscess and gangrene of lung, iii. 445.

Pock diseases of animals, ii. 55: ape-pox, ii. 56; cow-pox, ii. 56; groups of, ii. 56; horse-pox, ii. 55 ; sheep-pox, ii. 55.

Podagra, hydrotherapy for relief of, i. 208.

Podophyllin, use of, in chronic constipation, iii. 234.

Poison CUP, ii. 648.

Poison test, african. See African poison test.

Poisoning By Food. See Food.

Poisonous fruits AND seeds, ii. 651.

Poisonous Reptiles, Fish, Plants, aNd ARTHROPODS, DISEASES DUE TO, iv. 681: atriplicism, iv. 693 ; due to batrachia, iv. 683 ; due to biting and stinging arthropods, iv. 685; due to chiggers, iv. 690; craw-craw, iv. 691; due to lacertillia, iv. 683; due to larvæ, iv. 686; lathyrism, iv. 693; myiasis, iv. 686; myiasis of natural orifices of body, iv. 688; nature poisons used for murder, suicide, and abortion, iv. 697; ophidism, iv. 681; due to plant and animal poisons, iv. 694; due to plants, iv. 686; due to poisonous fish, iv. 683 ; due to poisonous mammals, iv. 684 ; shima mushi, iv. 692.

Poisonous Roots, poisoning caused by eating, ii. 650 .

Porsons, action of, i. 662; animal, i. 674; autogenous, as a cause of toxic anemias, iii. 809 ; bacterial, i. 674 ; classification of, i. 673 ; definition of term, i. 662 ; diagnosis of, i. 669; disposition of, by animal body, i. 666; endogenous, in causation of multiple neuritis, iv. 321 ; exogenous, in causation of multiple neuritis, iv. 231; extraneous, as a cause of toxic anemia, iii. 809 ; mineral, i. 673,674 ; symptomatology of, i. 667 ; synthetic, i. 674 ; treatment for, i. 670 ; variations in effects of, ii. 665; vegetable, i. 674, 746 .

Poisons, Conditions influencing the EFFECTS OF, i. 665: avenue of administration, i. 666; condition of recipient, i. 666 ; physical state of poison, i. 665 ; size of dose, i. 665.

Poisons, NATIVE, USED FOR MURder, SUICIDE, AND PRODUCTION OF ABORTION, iv. 697 ; preventive measures in, iv. 698; remedial measures in, iv. 697.

Poкeroot, poisoning by, ii. 650 .

Polioncephalitis, acUte hemorrhagic, iv. 406; diagnosis of, iv. 407; inferior form of, iv. 407; pathology of, iv. 407; prognosis of, iv. 407; superior form of, iv. 406 ; treatment of, iv. 408.

Poliomyelitis, iv. 233; causes of, iv. 233; classification of, iv. 235; course of, iv. 235; definition of, iv. 233; morbid process in, iv. 233; muscular atrophy in, iv. 235; prognosis in, iv. 236; prophylaxis, iv. 237; symptoms of, iv. 235 ; transmission of, iv. 234; treatment of attack of, iv. 237; treatment of early consequences in, iv. 238; treatment of, in general, iv. 236; treatment of remote effects of, iv. 241.

Poliomyelitis, ACUTE, electrotherapeutics for relief of, i. 430; relation between, and acute hemorrhagic inferior polioencephalitis, iv. 407.

Pollantin, i. 160; use of, in hay fever, iii. 391.

Polonium, discovery of, i. 460.

Polycythemia, X-ray therapy for relief of, i. 551.

Polycythemia chronica, blood pressure raised by, iii. 555 .

Polycythemia rubra megalosplenica. See Polycythemia with splenomegaly.

Polycythemia with splenomegaly, iii. 877; blood examination in, iii. 878; historical notes on, iii. 877; pathogenesis of, iii. 878; symptomatology of, iii. 877; synonyms for, iii. 877 ; treatment of, iii. 878.

Polycythemia, true idiopathic. See Polycythemia with splenomegaly.

Polymyositis, iv. 329 ; acute, ii. 684; hemorrhagica, iv. 330 .

Polypi, Nasal, as a cause of headache, iv. 603. 
Polypi, rectal, as a cause of enuresis, iv. 130.

Polyuria, as a cause of enuresis, iv. 127.

Polyuria, rixperimental, as test for functional power of kidney, iv. 24 .

Polyvalent serum, use of, in treatment of pneumonia, ii. 240.

Polyvalent vaccines, i. 140.

Pomegranate root, use of, as substitute for male fern, iv. 778 .

Pons And medulla, diseases of, iv. 496: acute apoplectic bulbar paralysis, iv. 500; compression bulbar paralysis, iv. 501; myasthenia gravis, iv. 504; ophthalmoplegia, iv. 507 ; progressive bulbar paralysis, iv. 496; pseudobulbar paralysis, iv. 502 .

Pork, meat poisoning due to, ii. 638 ; trichiniasis acquired from raw, iii. 253.

Port SaID, extermination of dengue and malaria in, iv. 859.

Portal cirriosis. See Cirrhosis of liver.

Portugal, introduction of vaccination into, ii. 60 .

Posen, mortality from small-pox in, before and after vaccination, ii. 95 .

Position of Body. See Posture.

Position, Wolfenden. See Wolfenden.

Post-diphtheritic paralysis, ii. 278.

Post-gonorrheal neuroses, iv. 174.

Post-gonorrheal uréthritis, treatment of, iv. 167.

Post-hemorrhagic anemias, iii. 808.

Post-hypnotic suggestion, i. 574.

Post-infectious headaches. See Headaches.

Post-operative treatment, massage and gymnastics in, i. 328 .

Post-operative uses of radiant light and heat, i. 457.

Post-Typhoid psychoses, as a complication of typhoid fever, ii. 29.

Postural treatment, of cerebral anemia, iv. 382 ; of chronic bronchitis with profuse secretion, iii. 376; of movable kidney, iv. 3 ; in movable spleen, iii. 887 ; in neuritis, iv. 284 ; for relief of insomnia in cerebral anemia, iv. 383 ; of syncope due to cerebral thrombosis, iv. 398 ; in tabes, iv. 203.

Posture, in acute appendicitis, iii. 214, 217; in acute diffuse peritonitis, iii. 315 ; in acute dilatation of stomach, iii. 174; in acute gonorrheal epididymitis, iv. 150; benefit from, in chronic bronchitis with profuse expectoration, iii. 376 ; in cardiac insufficiency, iii. 571; in causation of neuritis of brachial plexus or nerves of arm, iv. 291; in cerebral hemorrhage, iv. 391 ; change of, as prophylactic measure against bronchopneumonia, iii. 413; choice of, in severe cases of chronic myocardial insufficiency, iii. 718 ; faintness during thoracentesis relieved by recumbent, iii. 478; importance of, in bronchiectasis, iii. 393; importance of changing, in hypostatic congestion of lungs, iii. 407 ; importance of, in diverticula of esophagus, iii. 62 ; importance of, in gastric hemorrhage, iii. 109, 117; importance of, in multiple neuritis, iv. 327 ; influence of, in angina pectoris, iii. 769 ; influence of, on expectoration in abscess of lung, iii. 440; influence of, in heart block, iii. 772 ; for injection into nerve trunk, iv. 303; necessity for change of, in acute bronchitis, iii. 353 ; necessity for change of, in bronchopneumonia, iii. 417 ; necessity for change of, in cerebral hemorrhage, iv. 392 ; in phrenic nephritis, iv. 289; in pulmonary edema, iii. 408; in relief of dyspnea due to chronic myocardial insufficiency, iii. 736; in sciatica, iv. 299; in severe cases of chronic myocardial insufficiency, iii. 721; in tonsillectomy, iii. 46.

Potassium acetate, use of, in acute urethral gonorrhea, iv. 139; in colon bacilluria, iv. 128; in nephritis, iv. 49; for relief of anuria due to nephrolithiasis, iv. 107.

Potassium bicarbonate, use of, for relief of anuria due to nephrolithiasis, iv. 107.

Potassium bichromate, use of, to remove bronchial sccretion in acute bronchitis, iii. 365 .

Potassium bromid, use of, in acute alcoholism, ii. 589; to prevent recurrence of spasmodic laryngitis. iii. 342 ; in spasmodic croup of children, iii. 340 ; in tetanus, ii. 348 .

Potassium bromid and potassium iodid, use of, for relief of spasmodic twitching due to brain tumor, iv. 448 .

Potassium chlorate, poisoning by, i. 720 .

Potassium chlorate, use of, in acute glossitis, iii. 24; with alum, as gargle in 
chronic pharyngitis, iii. 33 ; in care of teeth and gums during mercurial treatment of brain syphilis, iv. 480; as gargle, in foot and mouth disease, ii. 583; as gargle in syphilitic angina, ii. 426 ; in mercurial stomatitis, iii. 16; in scurvy, iii. 375 ; in stomatitis ulcerosa, iii. 15 ; in stomatitis ulcerosa chronica, iii. 16.

Potassium chlorate lozenges, use of, in acute tonsillitis, iii. 42 .

Potassium citrate, use of, in acute urethral gonorrhea, iv. 139; in colon bacilluria, iv. 128; for relief of anuria, due to nephrolithiasis, iv. 107.

Potassium iodid, use of, in actinomycosis, ii. 560; in acute bronchitis, during late stages, iii. 365 ; in acute inferior hemorrhagic polioencephalitis, iv. 408 ; in acute myelitis, iv. 226; in after-treatment of herpes zoster, iv. 359 ; in aneurysm, iii. 765 ; in angina pectoris, iii. 770 ; in arteriosclerosis, iii. 761; in brain syphilis, iv. 480; in bronchial asthma, iii. 383 ; in bronchitis complicating influenza, ii. 189 ; in cerebral thrombosis due to syphilitic endarteritis, iv. 399; cervicoöcipital neuralgia, iv. 356 ; in chronic disease of labyrinth, iv. 317 ; in chronic laryngitis, iii. 340; in chronic nasopharyngitis, iii. 32 ; in chronic parotitis, iii. 28 ; in chronic pulmonary emphysema, iii. 437; in cirrhosis of liver, iii. 282,286 ; in congenital syphilis of liver, iii. 265; contraindicated in edematous laryngitis, iii. 341 ; contraindicated in tabetic amaurosis, iv. 199; in disease of eighth nerve, iv. 315,316 ; in empyema associated with actinomycosis, iii. 489 ; as an expectorant in bronchiectasis, iv. 397; in frambœsia tropica, iv. 764; in glandular enlargement accompanying rachitis, ii. 818 ; in hydrocephalus, iv. 465 ; in kubisagari, iv. 861 ; in multiple neuritis due to arsenical poisoning, iv. 323 ; in multiple neuritis due to lead poisoning, iv. 325 ; in mumps, ii. 170 ; for neuralgia, iv. 337 ; for neuralgia due to arteriosclerosis, iv. 336 ; in optic neuritis, iv. 305 ; in optic neuritis without apparent cause, iv. 306 ; in optic neuritis due to metallic poisoning, iv. 306 ; in optic neuritis due to poisoning with wood alcohol, ii. 610 ; of no value in spastic spinal paralysis, iv. 270 ; in plas- tic bronchitis, iii. 391; in pneumogastric paralysis, iv. 319; for relief of headache due to brain tumor, iv. 448; in spinal syphilis, iv. 273,274 ; in symptomatic parotitis, iii. 28; in syphilis, ii. 408 ; in syphilis of pancreas, iii. 302 ; in syphilitic arteritis, iii. 763 ; in syphilitic disease of liver, iii. 265; in tabes, iv. 195; in ulcerating granuloma of pudenda, iv. 866 .

Potassium nitrate, use of, in pneumonia, ii. 227.

Potassium nitrite, use of, in cardiac insufficiency, iii. 607.

Potassium permanganate, use of, as an antidote to organic poisons, iv. 696, 697; as antiseptic wash after passage of urethral sound, iv. 169; as cleansing solution in atrophic rhinitis, iii. 331 ; for disinfection of drinking wells as protection against cholera, iv. 830; for destruction of cholera toxins in bowels, iv. 836; for dressing of oriental sore, iv. 756; use of, for flushing colon in intestinal autointoxication, ii. 674 ; in foot and mouth disease, ii. 583; for gastric lavage in acute opium poisoning, ii. 614; for gastric lavage in meat poisoning, ii. 689 ; for gastric lavage in phosphorus poisoning, ii. 627; by injection around wound in curare poisoning, iv. 694; by injection in chancre with phimosis, ii. 423; by injection in chronic gonorrheal urethritis, iv. 155; by injection as preventive of gonorrheal infection, iv. 137, 144; by injection in snake bite, iv. 682 ; for irrigation in acute posterior urethritis, iv. 147; for irrigation after passage of urethral sound, iv. 162; for irrigation of bladder in acute cystitis, iv. 120 ; for irrigation in carcinomatous stricture of esophagus, iii. 55; for irrigation in diverticula of esophagus, iii. 62 ; for irrigation of large intestine in intestinal amebiasis, iv. 709; in mucous patches, ii. 427 ; for relief of throat symptoms in small-pox, ii. 47; as spray, in acute tonsillitis, iii. 42; as spray, in $\mathrm{c} \mathrm{h} \mathrm{r} \mathrm{o} \mathrm{i} \mathrm{c}$ rhinitis, iii. 329 ; in stomatitis gangrenosa, iii. 18; in stomatitis herpetica, iii. 10; in stomatitis hypermycetica, iii. 13; in stomatitis ulcerosa, iii. 16; in syphilis of upper respiratory tract, iii. 349 ; for tertiary syphilitic lesions of mucous or mucocutaneous surfaces, ii. 427 . 
Potassium permanganate $\mathrm{t} r$ e a $\mathrm{t} m$ e $\mathrm{n} t$ of small-pox, ii. 51.

Potassium picric-nitrate, use of, in destruction of filaria bancrofti, iv. 787 .

Potassium tartrate acid, use of, for relief of anuria due to nephrolithiasis, iv. 107.

Potato, common, poisoning by, ii. 650.

Pottencier, value of tuberculin in laryngeal tuberculosis demonstrated by, iii. 345 .

PotTery, respiratory diseases due to manufacture of, iii. 425 .

Pouchet, bacillus suisepticus described by, ii. 642 .

Poultice, compound soap, use of, in delayed resolution in pneumonia, iii. 257.

Poultice, flaxseed, use of, in gastric hyperacidity, iii. 100; in gonorrheal epididymitis, iv. 152; in non-bleeding gastric ulcer, iii. 133; in parotitis complicating typhoid fever, ii. 27 ; in perforation in typhoid fever, ii. 23; for relief of boils accompanying diabetes mellitus, ii. 675; for relief of plulebitis complicating typhoid fever, ii. 28.

Poultice, tobacco, use of, in gonorrheal epididymitis, iv. 151.

Poultices, use of, in anuria, iv. 3 ; in bacillary dysentery, ii. 324 ; in bronchopneumonia, complicating measles, ii. 159 ; in chronic bronchitis, iii. 373 ; in rheumatic or idiopathic cases of facial paralysis, iv. 311 ; in spasmodic laryngitis, iii. 342 ; in thyroiditis acuta and struma inflammatoria, iii. 891.

Poultices, hot, use of, in chronic pancreatitis, iii. 298; in multiple neuritis, iv. 326 ; for relief of pain in acute gastritis, iii. 156; in renal colic, iv. 102 .

Poultices, linseed, use of, in acute bronchitis, iii. 356.

Poultices, mud, use of, in gastric hyperacidity, iii. 100.

Poultices, mustard, use of, in active congestion of lungs, iii. 406 ; in acute bronchitis, iii. 356 ; to relieve cyanosis and dyspnea in bronchopneumonia, iii. 419; to relieve pain in pulmonary thrombosis, iii. 411 .

Powell, mixture recommended by, for relief of dyspnea due to pressure of a mediastinal tumor, iii. 503; on tapping as means of relief for dyspnea in mediastinal tumor, iii. 503.
Poyton and Paine, isolation by, of microeoceus rheumaticus, iii. 743 .

Precipltatuon membiranes, i. j-

Precinitias, nature of, i. 1:31.

Pregnancy, appendicitis complicating, iii. 220 ; chorea associated with, iv. 6633; danger from, in cardiac insufficiency, iii. 681; diabetes in, ii. 768 ; etiology of nephritis in, iv. 29; inoculation for plague in, iv. 824 ; kidney of, iv. 82 ; treatment of, when associated with tetany, iii. 910; treatment for, in exophthalmic goiter, iii. 905 ; -treatment of syphilis in, ii. 431; use of quinin in, for tropical malaria, iv. 725; vomiting of, associated with intestinal autointoxication, ii. 683.

Pregnant uterus, pressure from, a cause of pain in bladder, iv. 123.

Premarital neurasthenia, iv. 524.

Prepared chalk and talcum, use of, internally, in non-bleeding gastric ulcer, iii. 136.

Prepuce, elongated or adherent, as a cause of enuresis, iv. 130.

Presbyterian Hospital of New York, results in, attending the use of urotropin for prevention of pericarditis in pneumonia, ii. 223.

Prescription. See Formula.

Pressure, properties of, in hydrotherapy, i. 193.

Pressure symptoms, in diagnosis of brain tumor in frontal lobe, iv. 439 ; of hypophysis tumor, iv. 442.

Price's distilled glycerin, use of, for intratracheal injections in bronchiectasis, iii. 397 .

Priessnitz applications, use of, in bronchopneumonia, iii. 419 ; in bronchopneumonia complicating measles, ii. 159; in laryngeal complications of measles, ii. 167 ; in laryngotracheal cough of measles, ii. 152 ; to relieve cyanosis and dyspnea in bronchopneumonia, iii. 419; to relieve pain and cough in bronchopneumonia, iii. 421.

Priessnitz compresses, action of, iii. 357; in acute bronchitis, iii. 357 ; in acute intestinal obstruction, iii. 241 ; in bronchitis due to myocardial insufficiency. iii. 738 ; in chronic cholecystitis, iii. 270 ; in gallstone colic, iii. 269 ; in gastric hyper- 
acidity, iii. 100; in membranous enteritis, iii. 209; for relief of gastrectasis in chlorosis, iii. 823; in severe cases of chronic enteritis, iii. 200; in ulcerative colitis, iii. 211.

Primary lateral sclerosis. See Paralysis, spastic spinal.

Primary myelogenous polycythemia megalosplenica. See Polycythemia with splenomegaly.

Primary sinus thrombosis. See Marantic sinus thrombosis.

Prison fever. See Typhus fever.

Proctitis, epidemic gangrenous. See Epidemic gangrenous proctitis.

Proctoclysis, use of, in septicopyemia, ii. 290.

Prodigiosus toxins in treatment of tumors, i. 174 .

Progressive bulbar Paralysis. See Bulbar paralysis.

Progressive muscular atrophies, iv. 243: acquired spinal form, iv. 245; amyotrophic lateral sclerosis, iv. 258; asthenic bulbar paralysis, iv. 254; chronic progressive bulbar paralysis, iv. 249; chronic progressive ophthalmoplegia, iv. 257; definition of, iv. 243; morbid process in, iv. 243; varieties of, iv. 243.

Progressive muscular atrophy, aCQUIRED SPINAL, iv. 245; course of, iv. 246; etiology of, iv. 246; family type of, iv. 248 ; treatment of, iv. 247.

Prolapse of liver, iii. 288.

Prophylactic irradiation, in X-ray therapy following excision for deep cancer, $\mathrm{i}$. 541 .

Proprietary foods, i. 652; composition of, i. 653 ; economic aspects of, i. 659 ; longcontinued use of, a cause of rickets, ii. 817.

Proprietary peptone foods, objections to use of, in nutrient enemata, for gastric ulcer, iii. 118 .

Proprietary preparations, objections to use of, in acute laryngitis, iii. 339 .

Prostate gland, massage of. See Massage.

Prostatectomy, indications for, in chronic gonorrheal urethritis, iv. 166.

Prostatic abscess. See Abscess.

Prostatic disease, as a symptom of diabetes mellitus, ii. 766 .
Prostatic enlargement, X-ray therapy for relief of, i. 552 .

Prostatitis, acute form of, complicating acute gonorrhea, iv. 149; as a cause of pain in bladder, iv. 122; electricity together with $\mathrm{X}$-ray in treatment of tuberculous, i. 402 ; electrotherapeutics for relief of, i. 421; electrotherapeutics for relief of gonorrheal, i. 407; hydrotherapy for relief of, i. 211 ; treatment of relapsing, iv. 167.

Prostatorrhea, iv. 176.

Protargol, use of, by enemata, in chronic mucous colitis, iii. 207; in gonorrheal stomatitis, iii. 21 ; by injection in chronic gonorrheal urethritis, iv. 155; by injection, in expectant treatinent of acute gonorrhea, iv. 148; by injection, as preventive of gonorrheal infection, iv. 137, 144; by instillation in acute posterior urethritis, iv. 146; in pellagra, ii. 662 ; rules for, by patient in prevention of gonorrheal infection, iv. 145; in smallpox for care of eyes, ii. 50 .

Protargol ointment, use of, in tropical phagedenic ulcer, iv. 868 .

Protection, necessity for, in deep X-ray therapy, i. 506.

Protective dressing, use of, in acute urethral gonorrhea, iv. 138.

Proteid food, prohibition of, in yellow fever, iv. 856 .

Proteids, form of taking, in nephritis, iv. 42 ; proportion of, in diet for nephritis, iv. 42 ; proportion of, in diet for neurasthenia, iv. 539; restriction of, in acute nephritis, iv. 59 ; restriction of, in acute nephritis with anuria, iv. 62 ; restriction of, in chronic interstitial nephritis, iv. 68 ; restriction of, in chronic parenchymatous nephritis, iv. 65; restriction of, in kidney of pregnancy, iv. 82 ; restriction of, in nephritis, iv. 44; restriction of, in uremia, iv. 51 .

Protein diet, pancreatic secretion little influenced by, iii. 307.

Protein requirement, i. 608; injurious effect of excess of, i. 610 ; method of reckoning, for a diet of definite energy value, $i$. 613 ; variations in, i. 609.

Proteins, as antidote to corrosive poisons, i. 671 ; in diet for gastric hyperacidity, iii. 82 ; excess of, in diet injurious, i. 610 ; 
function of, in body, ii. 782 ; necessity for, in diet for malarial cachexia, iv. 728; proportion of, in diet for neurasthenia, iv. 539 ; sources of, i. 600 ; subcutaneous administration of, i. 640; sugar derived from, ii. 725; value of, as food, i. 600 .

Protozon, diseases due to, iv. 703: amebiasis, iv. 703; balantidium coli, iv. 760; blackwater fever, iv. 738; frambosia tropica, iv. 762; histoplasmosis, iv. 757; infantile eachexia in Brazil, iv. 752; Leishmaniasis, iv. 753; tropieal malaria, iv. 712 ; tropical relapsing fever, iv. 757 ; trypanosomiasis, iv. 742.

Prudential Insurance Company, statisties concerning death from tuberculosis furnished by, ii. 450 .

Pruritus, radium in treatment of, i. 475; treatment of, in diabetes, ii. 765 ; treatment of, in varicella, ii. 113; X-ray therapy for relief of, i. 554 .

Prossia, introduction of vaccination into, ii. 60 .

Pseudo-angina pectoris, iii. 767.

Pseudobulbar palsy, iv. 497.

Pseudobulbar Palsy, infantile, iv. 503.

Pseudobulbar paraìsis, iv. 502: differential diagnosis of, iv. 504; etiology of, iv. 503; introduction to subject of, iv. 502 ; pathology of, iv. 503; symptoms of, iv. 503; treatment of, iv. 504.

Pseudo-diphtheritic stomatitis. See Stomatitis.

PSEUdOHYPERTROPhIC TYPE OF MUSCULAR DYSTROPHY, iv. 261.

Pseudoleukemia, iii. 854; X-ray therapy for relief of, i. 550 .

Pseudo-rachitic osteoporosis, ii. 813.

Pseudo-rickets, ii. 813.

Pseudotabes. See Tabetic form of spinal syphilis.

Pseudotrichinosis. See Polymyositis, acute.

Psitracosis, ii. 584; definition of, ii. 584; diet in, ii. 586; etiology of, ii. 584; parrots in causation of, ii. 584 ; prognosis in, ii. 586 ; prophylaxis against, ii. 584; treatment of, ii. 586 .

Psoriasis, association of, with intestinal autointoxication, ii. 683; as a complication of vaceination, ii. 92 ; radiant light and heat in treatment of, i. 458 ; radium in treatment of, i. 474; X-ray in treatment of, i. 527.

Psomasis lingues. See Leukoplakia.

Psychalgias, measures for relief of, in neurasthenia, iv. 565.

Psychasthexia, associated with intestinal antointoxication, ii. 682.

Psychic equivalexts, to attacks of epilepsy, iv. 634.

Psychic Influence, in gastric neuroses, iii. 187 ; in treatment of whooping-cough, ii. 178 .

Psychic propilylaxis, iv, 570).

Psychic treatment, use of, in brachial neuralgia, iv. 357; in bronchial asthma, iii. 385 ; in the neuroses, iv. 524 ; in pudendohemorrhoidal neuralgia, iv. 357 .

Psychoanalysis, i. 588; dream analysis in, i. 593; free associations in, i. 589; historytaking in, i. 588; word time association in, i. 589.

Psychoanalytic method of psychotherapy, iv. 572 ; association experiment in, iv. 579 ; dream analysis in, iv. 578 ; fundamental idea of, iv. 574; indications and contraindications for, iv. 578; objections to, iv. 580 .

Psychogenic headaches. See Headaches.

Psychological mechanisms, use of, in psychotheiapy, i. 583: complexes, i. 585; dream mechanism, i. 586 ; repression, i. 584.

Psychology, of climate, i. 275.

Psychotherapy, i. 558; application of hypnosis in, i. 578; choice of method in, i. 594 ; complexes in, i. 585; danger attending use of hypnosis in, i. 59S; dream mechanism in, i. 586; Dubois' method of reëducation in, i. 579; Freud's system of, i. 583; historical comments upon, i. 558,561 ; hypnotic sleep without suggestion in, i. 576; hypnotic suggestive therapy, i. 576; hypnotism in, i. 561; in neurasthenia and psychasthenia, iv. 569 ; normal hypnotism in, i. 569; осcupation as secondary agent in, i. 596; post-hypnotic suggestion in, i. 594; psychologieal mechanism in, i. 583 ; reëducation in, i. 579; for relief of cardiac palpitation in neurasthenia, iv. 568; for relief of chorea electrica, iv. 664; for relief of vomiting in neurasthenia, iv. 567 ; repression in, i. 5S4; secondary 
agents in, i. 596; simple suggestion in conscious state in, i. 559 ; in spasmodic movements of tic, iv. 659; suggestion therapy in, i. 559 ; technique of psychoanalysis employed in, i. 558; use of stimulating, in psychasthenia, iv. 551; utilization of hypnotism in medicine, i. 575; utilization of increased memory power during hypnosis in, i. 577. See also Neurasthenia and Psychasthenia.

Psychotherapy in FUNCTIONAL NeURoses, iv. 569; Bernheim's method of, iv. 570; conditions of environment important in, iv. 574; definition of, iv. 569; Dubois' method of, iv. 571; forms of, iv. 570; Freud's method of, iv. 572, 574; Janet's method of, iv. 570; limitations to, iv. 569; Meyer's analysis of factors essential to, iv. 574; Nancy school of, iv. 570; Paris school of, iv. 570; physician's attitude toward, iv. 572; preliminary conditions of, iv. 572; prophylactic function of, iv. 570; types of neuroses benefited by, iv. 573; Wundt school of, iv. 579.

Psychotic headaches. See Headache.

Psychrometer, i. 252.

Promains, poisonous action of, ii. 635 .

Ptyalism, as a complication of mumps, ii. 171.

Public health, relation of, to pneumonia, ii. 222.

Public prophylaxis against syphilis, ii. 374.

Pudendohemorrhoidal neuralgia, treatment of, iv. 357.

Puerperal fever, acute myelitis during, iv. 224.

Puerperium, the, prevention of the neuroses at, iv. 524 .

Pulmonary abscess and gangrene, iii. 438; etiology 'of, iii. 439; indications for operation in acute, iii. 442 ; indications for operation in chronic, iii. 444; medical treatment of, iii. 440; operation for acute, iii. 443; operation for chronic, iii. 444; prophylaxis against, iii. 439; surgical treatment of acute, iii. 441.

Pulmonary circulation, effect of drugs upon, iii. 404.

Pulmonary conditions, chronic myocardial insufficiency due to, iii. 709 ; treatment of, iii. 731.

Pulmonary edema, after-treatment of, iii.
410; in chronic interstitial nephritis, iv. 72 ; as a complication of pneumonia, ii. 259; as a complication of Rocky Mountain fever, ii. 565; etiology of, iii. 407; occurrence of, during thoracentesis, iii. 479 ; as symptom of nephritis, iv. 54; treatment of, iii. 408.

Pulmonary embolism, iii. 411; death from, in chlorosis, iii. 811.

Pulmonary emphysema, chronic, iii. 430; aerotherapy for, iii. 434; climate in treatment of, iii. 433; definition of, iii. 430; forms of, iii. 430; hydrotherapy for, iii. 434; massage for, iii. 434; mechanotherapy for, iii. 434; operation for, iii. 436 ; pathogenesis of, iii. 431 ; pathological conditions associated with, iii. 432 ; prophylaxis against, iii. 433 ; treatment of, iii. 434 ; treatment of complications in, iii. 437.

Pulmonary Gangrene, as a complication of pneumonia, ii. 261; see also Lung, abscess and gangrene of.

Pulmonary gymnastics. See Gymnastics.

Pulmonary hemorrhage, epinephrin for relief of, i. 99; extract of hypophysis for relief of, i. 109; in pulmonary tuberculosis, ii. 499 ; relation of, to exercise in pulmonary tuberculosis, ii. 529; treatment of, in pulmonary tuberculosis, ii. 500.

Pulmonary pressure, conditions affecting, iii. 403.

Pulmonary stenosis, chronic myocardial insufficiency due to, iii. 735 .

PUlmonary thrombosis AND EMBOLISM, iii. 411.

Pulmonary tubbrculosis. See Tuberculosis of lungs.

Pulsatory current, explanation of term in electrotherapeutics, i. 366.

Pulsatory Oudin current, as an electromechanical method, i. 388.

Pulse, the, iii. 559; catacrotic waves of, iii. 560 ; collapsing, iii. 561 ; curve of, iii. 560 ; influence of digitalis upon, iii. 578, 580; influence of peripheral resistance upon, iii. 560; influence of poisons upon, i. 668; pressure of, an indication of digitalis action, iii. 580; rate of, as indicator of digitalis action, iii. 581; weakness of, during thoracentesis important, iii. 478.

Pulse pressure, iii. 553. 
Pulsus alternans, iii. 566.

Pulsus mregularis perpetues, iii. 570.

Pulsus inregularis respiratorius, iii. 565.

Pulsus paradoxus, iii. 566.

Pulvis ipecac et opil. See Dover's powder.

Pumpkin seeds, use of, followed by a purge in intestinal myiasis, iv. 689 ; as substitute for male fern, iv. 778, 779.

P u n c t u re, of brain, see Brain puncture; deep, in muscular rheumatism, ii. 689; of joint, in arthritis deformans and chronic rheumatism, ii. 712; of skin, for relief of general anasarca in cardiac insufficiency, iii. 676 ; in uremia with edema of extremities, iv. 53 ; of vein, in cardiac insufficiency, iii. 670 .

Puncture, lumbar, use of, in acute attacks of hydrocephalus, iv. 465 ; in acute hemorrhagic inferior poliomyelitis, iv. 408; in acute ophthalmoplegia with increasing intracranial pressure, iv. 509; appearance of fluid withdrawn by, iv. 370 ; bacteriologic examination of fluid withdrawn by, iv. 370 ; in cerebrospinal meningitis, ii. 201; in chronic cerebral meningitis with hydrocephalus, iv. 378 ; in cysticercus of brain, iv. 461 ; in diagnosis of acute cerebral meningitis, iv. 369 ; in diagnosis of meningitis complicating influenza, ii. 194; in diagnosis of meningitis complicating pneumonia, ii. 259; in diagnosis of traumatic hematoma, iv. 366 ; in disease of brain and spinal cord, iv. 371 ; objections to, in syringomyelia, iv. 279; pressure of, iv. 370 ; for relief of brain tumor, iv. 450; for relief of headache in hydrocephalus, iv. 609; for relief of hydrocephalus, iv. 468; for relief of nervous deafness and aural vertigo, iv. 818 ; technique of, ii. 202 , iv. 369 ; in tuberculous meningitis, iv. 377 .

Pupils, changes in, as a symptom of pachymeningitis hemorrhagica, iv. 363 ; inequalities of presence of, in progressive paralysis of the insane, iv. 490.

Purgation, necessity for caution in use of, for chronic myocardial insufficiency of moderate severity, iii. 716; necessity for caution in use of, in pleurisy, iii. 469 ; use of, contraindicated during rest treatment for cardiac insufficiency, iii. 572: use of, dangerous, in pericarditis with effusion, iii. 522 .
Purgatives, abuse of, a cause of chronic gastritis, iii. 159; objections to use of drastic, in gastric atony, iii. 172; objections to use of, for relief of constipation in neurasthenia, iv. 566; use of, in beginning of enteritis in infancy, iii. 201 ; use of, contraindicated in chronic cholecystitis and cholelithiasis, iii. 273; use of, contraindicated in gallstone colic, iii. 269; use of, contraindicated in treatment of gallstones in transit, iii. 274; use of, limited in cirrhosis of liver, iii. 286.

Punin lionies, in causation of gout, ii. 693; production of, in X-ray treatment of leukemia, iii. 851.

Purin-free diet, i. 644.

PURPura, as a complication of vaccination, ii. 85 .

Punpuras, iii. 858; classification of, iii. 858; clinical picture of, iii. 858; definition of, iii. 858 ; serum therapy for, iii. 859 ; treatment of, in general, iii. 859 .

Purton, idiopathic dilatation of esophagus first described by, iii. 56 .

Purulent exudates, absorption of, iii. 459.

Pyelitis, associated with acute myelitis, iv. 227; cardiac hypertrophy complicating, iv. 194; care of bowels in, iv. 89 ; as a cause of enuresis, iv. 129; as a complication of tuberculous kidney, iv. 117; complications in, iv. 94; cystitis complicating, iv. 94; diet in, iv. 87; diuretics in, iv. 89; gastrointestinal disturbances in, iv. 94; hygienic measures for, iv. 86 ; hypertension complicating, iv. 94; local treatment for, iv. 88; operation for, iv. 92 ; organisms causing, iv. 84 ; peritonitis complicating, iv. 94; prophylaxis against, iv. 95; retention of urine associated with, iv. 94 ; routes of infection in, iv. 84; suppression of urine complicating, iv. 94 ; symptomatology of, iv. 85 ; treatment of, in general, iv. 86 ; urinary antiseptics in treatment of, iv. 89,90 ; vaccine therapy for, iv. 91.

Pyelonephritis, as a complication of acute gonorrhea, iv. 150; as complication of tuberculous kidney, iv. 117; electrotherapeutics for relief of, i. 405.

Pyelotomy, indications for, in nephrolithiasis, iv. 10; in pyelitis, iv. 92.

Pyemia, definition of term, ii. 286. 
Pyloric obstruction, as cause of gastric dilatation, iii. 178; caused by gumma, iii. 167; operative measures for relief of, iii. 179; in syphilitic gastrie ulcer, iii. 167.

Pylorospasm, iii. 103; as cause of pyloric obstruetion, iii. 177.

Pyocyanase, therapeutic use of, i. 173.

Pyogenic infection, electrotherapeuties for relief of, i. 403.

Pyopericardium, iii. 526.

Pyorrhea alveolaris, iii. 22; association of, with intestinal autointoxication, ii. 679 ; danger of, in diabetes mellitus, ii. 736 ; presence of, in pernicious anemia, iii. 837.

Pyosalpinx, electrotherapeutics for relief of, i. 408.

Pyothorax. See Empyema.

Pyramidon, use of, to control sweating in malignant endocarditis, iii. 747 ; in measles, ii. 151 ; in migraine, iv. 592; for relief of headache in neurasthenia, iv. 566; for relief of lancinating pain in tabes, iv. 196; to relieve pain in diabetic neuralgia or neuritis, ii. 766 ; for relief of pain in neuralgia, iv. 339 ; for relief of pain in neuritis, iv. 286 ; for relief of pain in syringomyelia, iv. 278 .

Pyrethrum powder, use of, in destruction of mosquitoes by fumigation, iv. 735 .

Pyridin BASES, presence of, in tobaceo smoke not injurious, iii. 783.

Pyrogallol, use of, in leprosy, iv. 816.

Qualitative Dietary, in neurasthenia, iv. 538.

QuAntitative Dietary, in neurasthenia, iv. 538 .

QUARANTINe, failure of, as regards eholera, iv. 828 ; importance of, in plague overestimated, iv. 825 ; measures for, in membranous pharyngitis, iii. 38 , 39; measures for, in sleeping sickness and trypanosome fever, iv. 749 ; period of, in anterior poliomyelitis, iv. 237; period of, in diphtheria, ii. 267; period of, in foot and mouth disease, ii. 582; period of, in miliary fever, ii. 579 ; period of, in scarlet fever, ii. 579 ; period of, in smallpox, ii. 43.

QUINCKe, work by, on differentiation of varieties of meningitis, iv. 469 .
Quincke's lumbar puncture. See Puncture, lumbar.

Quinin, use of, for acute laryngitis in malarious districts, iii. 338; in acute myelitis, iv. 225; after arsenic in intermittent hemoglobinuria, iv. 7 ; in after-treatment of morphin habit, ii. 620 ; in blackwater fever, iv. 741, 742; in bronchitis complicating influenza, ii. 189 ; in bronchopneumonia, iii. 415; in ehronic disease of labyrinth, iv. 317 ; contraindicated in acute inflammation of nasal sinuses, iii. 334; for control of cough in measles, ii. 151 ; in convalescence from influenza, ii. 196; in exophthalmic goiter, iii. 899 ; in funicular myelitis, iv. 232 ; in influenza, ii. 187 ; in influenza of nervous type, ii. 191; for irrigation of abscess cavity in hepatic abscess, iv. 711; for irrigation of large intestine in intestinal amebiasis, iv. 709; in kala-azar, iv. 754 ; in measles, ii. 151 ; in miliary fever, ii. 580; in neuralgic headache, iv. 598; in pericarditis, iii. 520 ; in phlegmonous pharyngitis, iii. 40 ; in pleurisy, iii. 467; in pneumonia, ii. 233; in prophylaxis of influenza, ii. 186 ; by reetum, in ulcerative colitis due to Balantidium coli, iv. 761 ; to reduce temperature in acute bronehitis, iii. 355 ; to reduce temperature in epidemic dropsy, iv. 360 ; to reduce temperature in tuberculosis of larynx, iii. 346; in Rocky Mountain fever, ii. 565 ; in scarlatinal arthritis, ii. 305 ; in shima mushi, iv. 692 ; in stomatitis gangrenosa, iii. 18; in tabes, iv. 213 ; in trypanosomiasis associated with malaria, iii. 748; vertigo due to, iv. 624 ; in whooping-cough, ii. 181 ; in whoopingcough sometimes followed by disagreeable symptoms, ii. 175 .

Quinin, use of, in tropical malaria, iv. 717; absorption of, iv. 717; as acetyl salicylate of, iv. 718; action of, on cause of disease, iv. 719; as bihydrochlorate of, iv. 718; as bisulphate of, iv. 718; in children, iv. 728; choice of preparations of, iv. 718; in chronic form, iv. 727; contraindications to, iv. 724; in convalescence, iv. 729 ; dose of, iv. 721; effects of, on patient, iv. 724; as ethylcarbonate, iv. 718; excretion of, iv. 718; hypodermic administration of, iv. 723; hypodermoclysis as 
a mode of administering, iv. 722 ; intramuscular injection of, iv. 723 ; in latent cases, iv. 736; method of administering, iv. 721; method of administering as prophylactic, iv. 737 ; methods of administering in special conditions, iv. 727 ; as nucleinate, iv. 738; oral administration of, iv. 721 ; in prophylaxis, iv. 735 ; reetal injection of, iv. 724 ; substitutes for, iv. 726; as sulphate, iv. 718; as tannate, iv. 738; time for administration of, iv. 719 .

Quinin and sodium hypochlorid, use of, by rectum in intestinal schistosomiasis, iv. 785 .

Quinin hydrochlorate, use of, hypodermically in pneumonia, ii. 234 .

Quinin hydrochlorid, use of, in intestinal autointoxication in prevention of complications, ii. 682 .

Quinin hydrochlorid and urea, use of, for relief of pain in neuritis, iv. 285.

Quinin hydrochlorosulphate, use of, hypodermically in pneumonia, ii. 234.

Quinin tannate, use of, in enterocolitis complicating measles, ii. 161; in measles, ii. 151 .

Quinin and urea hydrochlorid, use of, hypodermically in pneumonia, ii. 233; method of administering, ii. 234, 235.

Quinzy. See Acute peritonsillitis.

RABBITs, use of, for small-pox virus, ii. 67 . Rabies. See Hydrophobia.

RACE, as an etiological factor in the neuroses, iv. 520 .

Rachits, 812; blood changes in, ii. 818; craniotabes in, ii. 817; deficiency of calcium a cause of, ii. 813 ; deformities occasioned by, ii. 818; dietetic treatment of, ii. 816 ; etiology of, ii. 814 ; geographical distribution of, ii. 812 ; glandular enlargement accompanying, ii. 818; heredity in causation of, ii. 815 ; history of, ii. 812 ; intestinal disorders complicating, ii. 818; medicinal treatment of, ii. 817 ; muscular weakness accompanying, ii. 818; nervous complications of, ii. 818; pathogenesis of, ii. 812; prophylaxis against, ii. 815; respiratory disorders accompanying, ii. 818; syphilis associated with, ii. 814 ; treatment in early cases of, ii. 817 ; treatment in florid form of, ii. 817 ; treatment of, in general, ii. 816 .

RADIANT ENERGY, artificial scurces of, $i$. 439; indications for therapeutic use of, i. 448 ; natural sources of, i. 435; summary of effect produced by, i. 448.

Radiant energy, pIIYsical properteg ()P, i. 432: color, i. 436; effect on germ life, i. 436; fluorescence, i. 436; frequencies, i. 432 ; penetration, i. 433 ; polarization, i. 436; production of hyperemia, i. 438; reflection, i. 434; refraction, i. 433; tanning of skin, i. 437.

Radiant light and heat, in treatment of erysipelas, i. 454 .

Radicular sciatica, iv. 295.

Radiferous mud, i. 467.

Radioactivity and radioactive substances, i. 460 .

Radiotherapy. See X-ray therapy.

Radium, action of, on process of inflammation, i. 469; Alpha rays of, i. 465; analgesic action of, i. 475; chemical action and physical properties of, i. 461 ; "cross method" of applying, i. 460; discovery of, i. 460; dosage of the emanation of, i. 466; dosage of the radiation of, i. 465; emanation of, i. 463; Gamma rays of, i. $462,465,484$; histological changes induced by, i. 468; injection of salts of, i. 467 ; ions of, i. 467 ; methods of applying, i. 463; position of, as a therapeutic agent, i. 487; preparation of, i. 461 ; radiation of, i. 462 ; sear tissue following use of, i. 471; therapeutic action of, in dermatology, i. 473 ; therapeutic action of,-in general, i. 471 ; therapeutic use of, in goiter, i. 478; therapeutic use of, in gout, rheumatism, and allied affections, i. 471; therapeutic use of, in gynecology, i. 479; therapeutic use of, in malignant disease, i. 451; therapeutic use of, in ophthalmology, i. 479 ; use of, in therapeutics, i. 468.

Radium baths, use of, in syringomyelia, iv. 279.

Radium ions, use of, i. 467.

Radium rays, use of, in syringomyelia, iv. 279.

Radium salts, use of, by subcutaneous injection, i. 466 .

Radium therapy, i. 460 : value of, in arthritis deformans, ii. 713 ; in carcinoma of 
stomach, iii. 164 ; in dermatology, i. 473 ; in goiter, i.478; in gout, rheumatism, and allied affections, i. 471 ; in gynecology, i. 479 ; in leukemia, iii. 853 ; in malignant disease, i. 481; in ophthalmology, i. 479.

Railways, plague disseminated by, iv. 826 .

RAIN, hygienic importance of, i. 252.

RAt Bite Disease, iv. 684.

RAT PLAGUE, epizoötic, relation of, to human epidemic, iv. 818.

Rathana, use of, in hemophilia, iii. 867.

RATs, extermination of, as protection against plague, iv. 821 ; method of destroying, iv. 824; transmission of plague by, iv. 817 .

RAw, Nathan, on use of human type of tuberculin in bronchoadenitis, iii. 399 .

"Reaction," significance of term in psychotherapy, iv. 574.

Reaction stage, of Asiatic cholera, iv. 836.

ReCiprocal RELATIONSHIP BETWEeN SECRETING ORgans, i. 39.

Recreation, nature of, in cardiac insufficiency, iii. 682.

Rectal chancre. See Chancre.

Rectal Crises, in tabes, iv. 191.

Rectal douche. See Douche.

Rectal feeding, i. 636 ; in acute pancreatitis, iii. 296; in carcinoma of esophagus, iii. 55 ; in chronic progressive bulbar paralysis, iv. 251; in continuous gastric hypersecretion, iii. 106; in gastric ulcer, iii. 118,120 ; in glossopharyngeal paralysis, iv. 318; in hematemesis during cirrhosis of liver, iii. 288; in influenza with gastrointestinal symptoms, ii. 190; method of, i. 636 ; in neurasthenia with persistent vomiting, iv. 567 ; in nonbleeding rectal ulcer, iii. 126 ; in parenchymatous glossitis, iii. 24 ; in stomatitis gangrenosa, iii. 18; in tetanus, ii. 350 ; in whooping-cough, ii. 180.

ReCtal POlyPi. See Polypi.

Rectified spirits, use of, in laryngeal tuberculosis, iii. 346.

Rectum, administration of mercury by, in syphilis, ii. 387; feeding by, see Rectal feeding.

RED BLOOD CORPUSCles, increase of, at high altitudes, i. 271.

Red gum lozenges, use of, in acute uvulitis, iii. 35 ; in chronic pharyngitis, iii. 35 .

Red light treatment, of small-pox, ii. 51 .
Reduvind BUG, carrier of infantile cachexia in Brazil, iv. 752.

Reëducation therapy, i. 579; in ataxia, i. 316 , iv. 204; in conditions in general, i. 320 ; Dubois' method for, i. 579; Freud's method for, i. 583; in psychic treatment of disease, iv. 570.

Reëducational gymnastic movements, use of, in ataxia, i. 316; contraindications to, i. 320 ; other conditions benefited by, i. 320.

"Referred pain," iii. 462.

Reflection, as property of radiant energy, i. 434.

Reflex angina pectoris, iii. 767.

Reflex asthma. See Asthma.

Reflex neuroses. See Neuroses.

REFlex NeURoses of Bladder. See Bladder.

Reflex vertigoes, iv. 623.

Refraction, as property of radiant energy, i. 433.

REFractory PERIOD, of heart's systole, iii. 533.

Regional diagnosis, in brain tumor, iv. 437.

Regulin, use of, in chronic constipation, iii. 236.

Reichmann's disease. See Hypersecretion, continuous gastric.

Reid's method, of applying leeches in pneumonia, ii. 257.

REIss, analysis by, of gastrointestinal symptoms in influenza, ii. 189.

Relapsing prostatitis. See Prostatitis.

REMLINGER, on treatment for hydrophobia, ii. 335.

Renal Calculi. See Calculi.

ReNal COlic, iv. 100; treatment of, iv. 101.

Renal derangements. See Kidney.

Renal disease, balneology for relief of, i. 241.

Renal edema, iv. 15.

Renal secretion, theories of, iv. 10.

Renal tuberculosis. See Kidney, tuberculous.

Renal URemia, iv. 20.

ReNAULT, work on small-pox organism by, ii. 41.

Rennet custard, as article of invalid dietary, i. 650.

Repression, as a psychological mechanism, i. 584 . 
Repressive treatment of acute gonorrhea, iv. 137, 143; cases suitable for, iv. 143; choice of method for, iv. 143; organic silver salts in, iv. 144; variation in treatment of, iv. 146.

Resection, advantages of, in gastric ulcer, iii. 142; of external cutaneous nerve, for relief of neuritis, iv. 293; indications for, in carcinoma of stomach, iii. 177; indications for, in chronic indurated ulcer of stomach wall, iii. 177; of sternum, for relief of dyspnea due to pressure by mediastinal tumor, iii. 503; of testicular nerve, for relief of pudendohemorrhoidal neuralgia, iv. 357; of urethra, for relief of urethral stricture, iv. 168, 173.

Resection of ribs, in bronchiectasis, iii. 394; in empyema, iii. 486; as palliative measure in mediastinal tumors, iii. 503; for relief of dyspnea due to pressure of mediastinal tumors, iii. 503; for relief of intercostal neuralgia, iv. 357 ; as a therapeutic measure in chronic abscess and gangrene of lung, iii. 445.

RESERVE POWER IN HYPERTROPHIC HEART, iii. 546 .

Residence, question of, in cardiac insufficiency, iii. 680.

Resistance, explanation of term in electrotherapeutics, i. 365.

"Resistance powers" of body, i. 279.

Resonator, i. 369.

Resorcin, use of, in atrophic rhinitis, iii. 332; in gastric atony, iii. 172 ; in gastric lavage for chronic gastritis, iii. 161 ; in irrigation of bowels in chronic stage of bacillary dysentery, ii. 328 ; in leprosy, iv. 816.

Resorcinol, use of, to relieve itehing in varicella, ii. 112.

Respiration, action of strychnin upon, iii. 615; artificial, see Artificial respiration; disturbance of, in tuberculous meningitis, iv. 374 ; effect of caffein series upon, iii. 602; influence of poisons upon, i. 668; methods of improving mechanics of, iii. 434.

Respirator, use of, as protection against acute bronchitis, iii. 352 ; as protection against dust in certain occupations, iii. 429.
Resiriratory appairatus, effect of massage upon, i. 296.

Respiratory exercises, i. 306; method of using, i. 304; use of, in chronic pulmonary emphysema, iii. 43.5.

Respiratomy organs, reflex cardiac neuroses caused by, iii. 781 .

Respiratory stasis, as indication for use of digitalis, iii. 587.

REsPiRATORY SYSTEM, DISÉAsEs OF, climate in treatment of, i. 281; hydrotherapy for relief of, i. 204; massage and gymnastics for relief of, i. 304; Spa treatment for, i. 239 ; X-ray therapy for relief of, $\mathrm{i}$. 553.

RESPIRATORY TRACT, UPPER, relation of general therapeutics to special treatment of, iii. 325 .

Respiratory tyPE OF INFLUENZA, ii. 188.

Rest, as a therapeutic measure, in active stage of tuberculosis, ii. 479, 482; in acute appendicitis, iii. 214; in acute diffuse peritonitis, iii. 315 ; in acute hemorrhagic encephalitis, iv. 405; in acute hemorrhagic inferior polioencephalitis, iv. 408; in acute localized peritonitis, iii. 310 ; in acute rheumatism, ii. 296 ; in acute urethral gonorrhea, iv. 138 ; to affected area in neuritis, iv. 284; in bacillary dysentery, ii. 324 ; in brachial neuralgia, iv. 357 ; in cardiac complications of acute rheumatism, ii. 303 ; in cardiac insufficieney, iii. 571 ; in cardiac neurasthenia, iii. 776 ; in chronic cholecystitis and cholelithiasis, iii. 270; in chronic opium poisoning, ii. 619; in "colds" during tubereulosis, ii. 508; in continuous gastric hypersecretion, iii. 106; in convalescence from pneumonia, ii. 262 ; in diphtheria, ii. 275 ; in disease of eighth eranial nerve beginning within labyrinth, iv. 316; in exophthalmic goiter, iii. 895; in "fatiguability" of neurasthenia, iv. 567; in gangrenous or phagedenic chancre, ii. 423; in gastric hemorrhage, iii. 109; in hematemesis accompanying cirrhosis of liver, iii. 28s; during and after hemorrhage from lungs in tuberculosis, ii. 528, 529; in Korsakow's psychosis, ii. 609; in laryngeal complications of tuberculosis, ii. 491 ; in multiple neuritis, iv. 326 ; in muscarin poisoning, ii. 647 ; in neuralgia, 
iii. 357 ; in neuralgia due to systemic causes, iv. 336 ; in neuritis of third, fourth, and sixth nerves, iv. 308 ; in occupation pareses, iv. 332 ; in optic neuritis, iv. 305 ; in pellagra, ii. 662 ; in pernicious anemia, iii. 825 ; in poisoning by amanita phalloides, ii. 649 ; in polymyositis, iv. 330 ; in pyelitis, iv. 86 ; in sciatica, iv. 297, 299; in tropical gangrenous phagedena, iv. 868 ; in tuberculosis of mediastinal lymph nodes, iii. 506; for voice in acute laryngitis, iii. 338; for voice in chronic laryngitis with chorditis nodosa, iii. 340 .

Rest, absolute, as a therapeutic measure, in acute tuberculosis, iii. 745; in acute cerebral meningitis, iv. 372 ; in acute myelitis, iv. 224; in acute myocarditis, iii. 749; in acute nephritis, iv. 31,57 ; in aneurysm, iii. 764; in apoplectic form of vestibular vertigo, iv. 628 ; in asthenic bulbar paralysis, iv. 256 ; in beri-beri, iv. 853; in blackwater fever, iv. 740 ; in cardiac insufficiency, iii. 571 ; in cerebral embolism, iv. 402; in cerebral hemorrhage, iv. 391 ; in cerebral hemorrhage accompanying chronic interstitial nephritis, iv. 72; in chronic paren chymatous nephritis, iv. 31 ; in chronic myocardial insufficiency due to overstimulation, iii. 723; indications for, in neurasthenia, iv. 534; in Landry's paralysis, iv. 329 ; in malignant endocarditis, iii. 746 ; in myasthenia gravis, iv. 507; in myocardial insufficiency, iii. $715,716,718$; in obstetric paralysis, iv. 292 ; in pericarditis, iii. 514,515 ; in phrenic neuritis, iv. 289 ; in post-hemorrhagic anemias, iii. 809; in prophylaxis of acute endocarditis, iii. 744 ; in pulmonary thrombosis, iii. 411 ; in relief of hemorrhage complicating thoracentesis, iv. 479 ; for relief of occupation neuroses, iv. 660 .

Rest, artificially induced, in spasmodic movements of tic, iv. 659 .

Rest in bed, as a therapeutic measure in acute cystitis, iv. 120; in chlorosis, iii. 812 ; in chorea minor, iv. 662 ; in chronic pancreatitis, iii. 298; indications for, in neurasthenia, iv. 534; in intestinal disorders, iii. 193; necessity for, in pleurisy, iii. 465 ; in pulmonary abscess and gan- grene, iii. 440; in severe cases of chronic enteritis, iii. 200.

Rest, LONG-CONTINUED, a cause of chronic myocardial insufficiency, iii. 702; treatment of, iii. 724 .

Rest after meals, in treatment of movable spleen, iii. 887.

Rest, relative, as a therapeutic measure, in ehronic myocardial insufficiency, due to obesity, iii. 725; indications for, in chronic parenchymatous nephritis, iv. 31 ; indications for, in neurasthenia, iv. 533.

Rest cure, in alimentary hypersecretion, iii. 102 ; in atonic dilatation of stomach, iii. 177; essential features of, iv. 540, 544; in gastric atony, iii. 169; for neuralgia, iv. 336 ; for relief of nervousness due to cerebral anemia, iv. 383 ; for relief of post-infectious headaches, iv. 612 ; for relief of tachycardia, iii. 787; in treatment of non-bleeding gastric ulcer, iii. 123.

Restlessness, as a characteristic symptom of neurasthenia, iv. 567; control of, in uremia, iv. 54; measures for relief of, in chorea minor, iv. 663.

Resiraint, method of applying, in delirium tremens, ii. 604.

Retention of URine, due to chronic prostatitis, iv. 168; precautions against, in progressive paralysis of the insane, iv. 494.

Retention theory, of renal uremia, iv. 21, 22.

Retinitis, accompanying diabetes mellitus, ii. 767.

Retropharyngeal abscess, iii. 34 .

Revaccination and its value, ii. 75 .

RhaGAdES OF LIP COMMISSURES, iii. 4 ; treatment of, iii. 5 .

Rheumatic Fever, as a common cause of pericarditis, iii. 511 .

Rheumatism, causing facial paralysis, iv. 311 ; chronic (so-called), ii. 707; gonorrheal, see Arthritis, gonococcic; massage and gymnastics in treatment of, i. 326; muscular, see Muscular rheumatism; as a predisposing cause of neuralgia, iv. 336 ; radium therapy for, i. 471 ; scarlatinal, see Arthritis, acute scarlatinal; Spa treatment for, i. 241; syphilitic, see Arthritis, syphilitic; use of thyroid gland for relief of, i. 76 . 
Rheumatism, ACUTe, ii. 292; alkaline treatment of, ii. 301; anemia complieating, ii. 303; cardiac complications of, ii. 302; eausative agents in, ii. 292 ; choreat complicating, ii. 304; clothing of patient. in, ii. 299; course of, ii. 292; definition of, ii. 292 ; development of, ii. 292 ; dict in, ii. 298; drugs in treatment of, ii. 298; evidence of infectious nature of, ii. 294; hygienic measures in, prophylaxis of, ii. 296; hyperpyretic rases of, ii. 302; immobilization of joints in, ii. 297, 306; indications for direct treatment of, ii. 296 ; infectious nature of, ii. 292; insomnia complicating, ii. 302; meningit is complicating, ii. 304 ; neuralgia complicating, ii. 304 ; nurse's duty in, ii. 296 ; organisms associated with, ii. 293; portals of infection in, ii. 292; prophylaxis in, ii. 294; removal of adenoids as a prophylactic measure in, ii. 296 ; removal of tonsils as a prophylactic measure in, ii. 296 ; salicylates and similar drugs in treatment of, ii. 299; serum therapy a failure in, ii. 301 ; symptoms due to drugs administered in, ii. 301; symptomatic treatment of, ii. 302 ; synonyms for, ii. 292 ; tonsils in relation to, ii. 294 ; treatment of, ii. 296; treatment of, during convalescence, ii. 301 ; vaccine therapy a failure in, ii. 301.

Rheumatoid arthritis, electrotherapeutics for relief of, i. 414; hydrotherapy for relief of, i. 226.

Rheumatoid diseases, influence of climate upon, i. 281.

Rhinitis, acute, iii. 326; atrophic, iii. 330 ; chronic, iii. 328 ; membranous, iii. 329 ; vasomotor, see Hay fever.

Rhinorrhea, paroxysmal. See Hay fever.

Rhinosclerene, use of, suggested in rhinoscleroma, iii. 332 .

Rhododendron, poisoning by, ii. 652 .

Rhubarb, use of, in chronic constipation, iii. 233 ; in nephritis, iv. 33 ; to promote evacuation of bowels in gastric atony, iii. 172 .

Rhubarb and phenolphthalein, use of, in diabetes mellitus, ii. 736 .

Rhus aromatica. See Rhus toxicodendron.

Rhus toxicodendron, use of, fer enuresis, iv. $122,133$.

Rhus vernicifera, iv. 693.

RhythMicity OF HEART MUSCle, iii. 533.
Rice, relation between preparation of grain of, and causation of beri-beri, iv. 846 ; use of boiled, as art icle of invalid dietary, i. 4.49.

Rice bran, infusions of, use of, in beri-beri, iv. 85.4 .

Rice AND EGi pUDdisg, as article of invalid dietary, i. 649.

Rice puding, as article of invalid dietary, i. 649 .

Richlielo Simisis, benefit from, in acute myelitis, iv. 22s; in tabes, iv. 201.

Rickets. See Rachitis.

RickETTs, work by, on transmission of typhus fever, ii. 32.

RidiNG on HORSEBACK, question of, in cardiac insufficiency, iii. 682 .

Riegel's pulse, iii. 566.

Rigg's disease. See Pyorrhea alveolaris.

RINGER's SOlution, formula for, iii. 533.

"Ringworm of tongue," iii. 24.

Riolan, paracentesis of pericardium first suggested by, iii. 522 .

Riva-Rocci aPPARATUS, for estimating blood pressure, iii. 557 .

Riviera, THE, climate of, for laryngeal tuberculosis, iii. 345 .

Roberts, Sir William, investigations by, on results of use of opium in India, ii. 614.

Robertson, spread of infection in frambosia tropica prevented by, iv. 763 .

Robin, Albert, results obtained by, in treatment of pneumonia by means of metallic ferments, ii. 246.

Robin's diet, for reduction of obesity, ii. 793.

Robinson, Dr. Samuel, apparatus devised by, for injection of gas in pneumothorax, ii. 536; on surgical treatment of bronchiectasis, iii. 394.

Robinson's apparatus, for use of differential air pressure, iii. 446.

Rochelle salts, use of, in acute alcoholism, ii. 589 ; in cerebral hyperemia, iv. 386 ; in chronic alcoholism, ii. 595; in membranous rhinitis, iii. 330 .

Rocky Mountain spotted Fever, ii. 562; bronchitis complicating, ii. 565; cerebrospinal symptoms in, ii. 566; constipation in, ii. 565; convalescence from, ii. 567 ; diet in, ii. 566; general and local treatment of, ii. 564; geographical dis- 
tribution of, ii. 562 ; heart failure in, ii. 565 , 566; hydrotherapy in, ii. 565; immunity produced by, ii. 562 ; incubation period of, ii. 564; introduction to subject of, ii. 562; lung complications of, ii. 566; nephritis complicating, ii. 566 ; organism supposed to be cause of, ii. 562; prophylaxis against, ii. 563; serum therapy in, ii. 567; summary of treatment for, ii. 567; transmission of, ii. 562.

RODENT ULCER, radium in treatment of, $i$. 481.

Rodents, species of, concerned in transmission of plague, iv. 818 .

Rogers, investigations by, on post-choleraic uremia, iv. 837.

Rogers' treatment of Asiatic cholera, iv. 833; destruction of cholera toxins by, iv. 835 ; diet in, iv. 838 ; intraperitoneal injections in, iv. 834 ; intravenous injection in, iv. 834 ; potassium permanganate in, iv. 835 ; rectal injection in, iv. 834; solution for use in, iv. 835, 836; in stage of preliminary diarrhea and collapse, iv. 833; in stage of reaction, iv. 836; subcutaneous injection in, iv. 834; technique of, iv. 834; temperature of injection in relation to temperature of body in, iv. 835; uremia during stage of reaction relieved by, iv. 837 .

Rogers' treatment of exophthalmic goiter, iii. 903.

RoLleston, on length of time necessary for antisyphilitic treatment in syphilitic disease of liver, iii. 265; use of thyroid extract in dipsomania suggested by, ii. 601.

RomberG, researches conducted by, on acute vasomotor paralysis, iii. 751 .

ROMBERG'S SIGN, in diagnosis of Friedreich's ataxia, iv. 215; in diagnosis of tabes, iv. 188.

Römer serum, preparation and results of, in treatment of pneumonia, ii. 239.

Röntgen Carcinoma, i. 516.

RöNTGEN EXANTHEM, i. 514.

Röntgen irradiation. See X-ray therapy.

RöNTGEN METHOD, of determining extent of chronic myocardial insufficiency, iii. 697.

RÖNTGEN RAY, antagonism of radiant light and heat to, i. 447; therapeutic use of, in conjunction with electricity, i. 451; see also X-ray.

Rose Fever. See Hay fever.

Rose's METHOD, of applying adhesive plaster in visceroptosis, iii. 247.

Rosenbach, A., on action of diuretics in fibrinous pleurisy, iii. 469 ; on action of iodids on transudates and exudates in pleurisy, iii. 470.

Rosenbach's tuberculin, i. 151.

Rosenburg, A., intratracheal injection first employed by, iii. 397 .

Rosenow, recent report by, on results of autolyzed pneumococci in treatment of pneumonia, ii. 243.

Ross's THEORY, as to cause of beri-beri, iv. 848.

Rossbach, mechanism for compressing thorax devised by, iii. 435 .

ROUND SHOULDER, as a cause of neuritis of ulnar nerve, iv. 291.

Roussel LAW, effect of, in prevention of tuberculosis in France, ii. 442.

ROUX-BERGER, cases of cardiolysis collected by, iii. 509 .

ROUX-WEIGERT THEORY OF BALANCE, iv. 182.

Rovsing, views held by, on enteroptosis, iii. 244.

Royal Victoria Hospital, treatment of pernicious anemia at, iii. 825 .

Rubber bag dilator, devised by Sippy for relief of idiopathic dilatation of esophagus, iii. 59.

Rubella. See German measles.

Rubella morbillosa, ii. 164.

Rubella scarlatinosa, ii. 165.

Rubidium iodid, use of, in syphilis, ii. 409.

Rubinat water, use of, in biliousness, iii. 258.

RugGles, formula employed by, for lubricant used in catheterizing urethra, iv. 158.

RUHRÄH, report by, on use of thyroid gland for relief of enuresis, iv. 131.

Rum and milk, use of, as expectorant in chronic bronchitis, iii. 373 .

RURAL RETREAT, treatment of neurasthenia in, iv. 562.

Russian bath, uses of, in hydrotherapy, ii. 206.

RUSSIAN EMPIRE, mortality from smallpox in, before and after vaccination, ii. 97. 
Saboureaud, system of X-ray therapy developed by, for relief of tinea and favus, i. 531.

Saboureaud and Noire cimromoradiomETER, for measuring intensity of X-ray, i. 498.

Saccharin, as substitute for sugar in diabetes mellitus, ii. 749 .

Saccharomyces albicans, iii. 11.

Sacchanose, presence of, in diabetic urine, ii. 728 .

Sacco, Luigr, early work in vaccination by, ii. 58,59 .

Sacquepee, Dr., on hypodermic administration of oxygen in acute respiratory conditions, iii. 364.

Sacroiliac disease, electrotherapeutics for relief of, i. 413.

Sacroiliac sciatica, iv. 296; treatment of, iv. 297.

"SADDle NOSE," method for relief of, iii. 349.

SAHLI, conditions included under respiratory stasis by, iii. 588; importance of infectious edema of lungs insisted upon by, iii. 407; varieties of cardiae stasis given by, iii. 587 .

Sahli's lavage of blood, iv. 5 .

Sahli's method, of giving morphin in acute appendicitis, iii. 214.

SalamandRin, iv. 683.

SAlembeni, serum therapy for Asiatic cholera tested by, iv. 832 .

Salicin, use of, in acute rheumatism, ii. 300; in pericarditis, iii. 519; in scarlet fever, ii. 124.

Salicyl compounds, irritation of kidney caused by, iv. $28,57$.

Salicylate of mercury. See Mereury.

Sallcylate of physostigmin. See Physostigmin salicylate.

Salicylates, use of, in acute attacks of hydrocephalus, iv. 465 ; in acute endocarditis, iii. 745 ; in acute myelitis, iv. 225 ; in acute peritonsillitis due to rheumatism, iii. 43 ; in beri-beri to produce diuresis, iv. 853 ; in chorea minor, iv. 662 ; in chronic disease of labyrinth, iv. 317 ; in chronic myocardial insufficiency due to valvular lesions, iii. 734 ; to deplete circulation in cerebral hyperemia, iv. 386 ; in diabetes mellitus, ii. 760 ; in influenzal type of nasal and frontal sinus headaclues, iv. 603; for irrigation of bladder in eystitis complicating acute myclitis, iv. 227; in leprosy, iv. 816; in migraine, iv. 592; objections to, in aural vertigo, iv. 316 ; in pericarditis, iii. 519 ; in pleurisy, iii. 467 ; in pyelitis with alkaline urine, iv. 90 ; to reduce temperature in acute cerebral meningitis, iv. 372 ; for relief of headache - due to sunstroke, iv. 679 ; for relief of pain in acute polymyositis, ii. 685; for relief of pain in arthropathies due to hemophilia, iii. 870; in Rocky Mountain fever, ii. 564; in tetany, iii. 910.

Salicylic acid, local use of, in alcoholic solution for craw-craw, iv. 692; in leukoplakia, iii. 26 ; in small-pox, ii. 49 ; in scarlet fever, ii. 29; for vaccinated arm, ii. 73 .

Salicylic acid, use of, in chronic enteritis, iii. 199 ; in gastric atony, iii. 172; by lavage in chronic gastritis, iii. 161; in pleurisy, iii. 467,468 ; by rectal injection in chronic mucous colitis, iii. 207.

Salicylic acid and related drugs, use of, in acute rheumatism, ii. 299; disagreeable symptoms following, ii. 301 ; in measles, ii. 151.

Salicylic acid ointment, use of, in disbetic vulvitis, ii. 765 ; in ulcerating granuloma of pudenda, iv. 866; in varicella, ii. 113.

Saline cathartics. See Cathartics.

Saline diuretics, physiological action of, i. 43.

Saline infusions, use of, by hypodermic injection in malignant endocarditis, iii. 746 ; in pneumonia in connection with inhalations of oxygen, ii. 237.

Saline solution, normal, see Normal saline solution; intravenous injection of, see Injection, intravenous; rectal injection of, see Injection, rectal; subcutaneous injections of, see Injection, hypodermic.

Salines, effervescing, use of, contraindicated in nephritis, iv. 33.

Salipyrin, use of, in acute rheumatism, ii. 300; in acute scarlatinal arthritis, ii. 305 ; in measles, ii. 151 ; for relief of pain in acute polymyositis, ii. 685 ; for relief of pain in neuralgia, iv. 339 .

Salisbury method of Diet, use of, in neurasthenia, iv. 538 .

Salivary Calculi, iii. 29; treatment of, 30. 
"Salivary COLic," iii. 28.

Salivary deposits, iii. 29.

Salivary glands, diseases of, iii. 26: angina Ludovici, iii. 30; chronic parotitis, iii. 28; parotitis, iii. 26; salivary calculi, iii. 29; salivary deposits, iii. 29; symptomatic parotitis, iii. 26.

Salivation. See Stomatitis, mercurial.

Salol, use of, in acute rheumatism, ii. 300; contraindicated in acute urethral gonorrhea, iv. 139; to create intestinal antisepsis in chlorosis, iii. 715 ; in diarrhea complicating septicemia, ii. 291 ; in enterocolitis complicating measles, ii. 161; followed by irritation of kidney, iv. 28,57 ; in glandular fever, ii. 576 ; in infantile diarrhea, ii. 329 ; in intestinal amebiasis, iv. 709 ; in intestinal autointoxication, ii. 675 ; in pyelitis complicating acute myelitis, iv. 227; for relief of pain in Rocky Mountain fever, ii. 564; suggested in distomiasis, iv. 780; in Weil's disease, ii. 572.

Salol, and acid acetyl salicylate, use of, in acute nasopharyngitis, iii. 31 .

Salophen, use of, in herpes zoster, iv. 359 ; for neuralgia associated with gout, iv. 337 ; for relief of headache due to cerebral thrombosis, 399 .

SALPINGITIS, electrotherapeutics for relief of, i. 407; hydrotherapy for relief of, i. 211.

SalpinX-uterine-ovarian Disease, hydrotherapy for relief of, i. 211.

Salpyrin, use of, for relicf of pain in neuritis, iv. 286.

Salt. See Sodium chlorid.

Salt-Free diet, in ascites due to cirrhosis of liver, iii. 285; in epilepsy, iv. 648; in gastric hyperacidity, iii. 90; in scarlet fever, as preventive of nephritis, ii. 133; in tuberculous peritonitis with effusion, iii. 321 .

Salt-poor diet, i. 640 ; in acute nephritis, iv. 61 ; in angina pectoris, iii. 770 ; in nephritis, iv. 36 ; value of, in arteriosclerosis, iii. 758; value of, in diabetes insipidus, ii. 776 .

Salt rub, use of, in pellagra, ii. 662.

Salt waters, common action of, i. 235; classification of, i. 232.

SAlts, uses of, as food, i. 602 ; behavior of inorganic, in uremia due to nephritis, iv. 22 ; inorganic, contained in urine, iv. 13.
Salvarsan. See Arsenobenzol.

SAMBoN, theory of pellagra as a protozoal disease held by, ii. 658 .

Sanatorium, Adirondack Cottage. See Adirondack Cottage Sanatorium.

Sanatorium treatment, for alcoholism, ii. 594; for cardiac neurasthenia, iii. 777; for morphin habit, ii. 617; for neurasthenia and psychasthenia, iv. 560; objections to, in reduction of obesity, ii. 789, 809 ; objections to, in tabes, iv. 212; objections to, in tuberculosis complicated with diabetes, ii. 767; for tuberculosis, ii. 594.

Sandalwood oil, use of, in acute urethral gonorrhea, iv. 140.

Sanguinaria, tincture of, use of, to relieve cough during convalescence from pneumonia, ii. 257.

Santonin, use of, in ascariasis, iii. 251; for destruction of tricocephalus dispar, iii. 255; in tricocephaliasis, iv. 798.

Santonin and castor oil, use of, in intestinal myiasis, iv. 689 .

SAPONIN, poisonous action of, ii. 650, 651.

SARcoma of LUNG, favorable results of operation for, iii. 448.

Sarcomata, action of radium upon, i. 470; of brain, iv. 433; value of radium therapy for, i. 485 ; value of X-ray therapy for, i. 543.

SARCOPHAGIDE, iv. $688,689$.

Sargent system, for cultivation of certain groups of muscles, i. 333.

SaUerbruch, differential air pressure established on therapeutic basis by, iii. 446.

Sauerbruch's negative pressure apparatus, use of, in teratomata of mediastinum, iii. 500; in traumatic hemothorax, iii. 490.

Sauvages, on headache, iv. 596, 597.

Savory custard, as article of invalid diet, i. 648.

SAXONY, history of vaccination in, ii. 102.

SAYRE, suspension apparatus devised by, $i$. 359.

Sayre suspension apparatus, use of, in syphilis, iv. 203.

Scalp, reflex tenderness of, iv. 599.

ScANNing SPEECH, in multiple sclerosis, iv. 280. 
Scar tissue, eleetrolysis in treatment of, $i$. 397 ; radium in treatment of, i. 476 .

Scamenzio, use of insoluble sialts of mercury in treatment of syphilis advaneed by, ii. 390,394 .

Scarification, employment of, for edema of glottis in acute nephritis, iv. 6.3 ; in edematous laryngitis, iii. 341.

Scarlatina, hydrotherapy for relief of, $i$. 216.

Scarlatinal rheumatism. See Arthritis, acute scarlatinal.

Scarlet Fever, ii. 114; arthritis complicating, ii. 132 ; cardiac weakness complicating, ii. 127 ; cause of, ii. 114 ; cerebral symptoms in, ii. 127 ; cervical adenitis complicating, ii. 131; diet in, ii. 123; disinfection in, ii. 120; dissemination of, ii. 116; diureties in, ii. 125; drugs in prevention of, ii. 123, 125; febrifuges in, 125; gargles in, ii. 125; gastrointestinal disorders complicating, ii. 132; hydrotherapy in, ii. 125; hygienic measures in, ii. 123; inunctions in, ii. 124; isolation in, ii. 118; medical treatment of, ii. 124 ; mode of entrance of germ of, ii. 117; nervous symptoms in, ii. 127 ; nephritis complicating, ii. 132; nurse's duty as regards infection in, ii. 119; opcrative measures in, ii. 131, 132; otitis complicating, ii. 130 ; period of greatest infection in, ii. 117; persistence of virulence of, ii. 117; prevention of, in hospitals, ii. 121; prevention of, in schools, ii. 122, 125; preventive drugs in, ii. 123 ; preventive inoculation for, ii. 123 ; preventive treatment for, ii. 118; quarantine for, ii. 118, 121; respiratory complications and sequelæ, ii. 134; "return cases" of, ii. 117; sepsis complicating, ii. 128; serum treatment of, ii. 128 ; sick room in, ii. 118; sloughing of tonsils in, ii. 130 ; source of germs in, ii. 115; temperature in, ii. 125; throat affections complicating, .ii. 129; treatment of attack, ii. 123; treatment of complications and sequelæ, ii. 129 ; treatment of convalescence in, ii. 128 .

Scars of vaccination, ii. 74 ; value of, as sign of immunity, ii. 98 .

SснӓғFER, effect of hypophysis preparations as diuretic in nephritis demonstrated by, iv. 50; epidemic of miliary fever re- portud by, ii. 579; evidence collecterl by, to (munulative reffect of digitalis, iii. 5 5.).

Schamber, ointment recommended by, for itching in diabetes mellitus, ii. 764.

Schavding and Hofrmas, discovery of spirochata pallida by, ii. 362 , iv. 472 .

Schede's operation, employment of, in ('mpy'ma, iii. 486.

Scheich infiltration, use of, as an anesthetic in performance of paracentesis, iii. 522 .

Scuevisk, on reaction of the Japanese to antipyretics, iv. 692 .

Schistosomasis, iv. 783 ; endemic hematuria, iv. 783 ; intestinal schistosomiasis, iv. 785; katayama disease, iv. 786 .

Schistosomum h.matomicm, iv. 783.

Schistosomem japonicem, iv. $7 \mathrm{S6}$.

Schistosomum maxsoni, iv. 78.5.

Schizotrypanum CRUZI, iv. 752.

Schlayer axd Hodinger, investigations by, on causes of renal edema, iv. 16 .

Schlippe's salt, use of, in trypanosomiasis, iv. 747 .

ScHLösser, alcoholic injections into nerve trunk introduced by, iv. 300 ; method of treating trigeminal neuralgia by alcohol injections introduced by, iv. 342 .

Schlösser's deep alcohol injections, iv. 353.

Schmidt, Adolph, on functional constipation, iii. 223; test diet prepared by, for use in chronic enteritis, iii. 198; test proposed by, for estimating state of intestinal tract in tuberculosis, ii. 502; work by, on intestinal complications in tuberculosis, ii. 557 .

Schmidt, M., discovery of radium by, i. 460 .

Schmiedeberg, influence of, on pharmacology of digitalis, iii. 577.

School CHILdREN, protection of, against tuberculosis, ii. 459.

School Education, importance of, as factor in prophylaxis of the neuroses, iv. 422.

Schools, duty of, to teach injurious effects of alcohol, ii. 592; establishment of, for tuberculous children, ii. 460; prevention of measles in, ii. 144; prevention of scarlet fever in, ii. 122 ; prevention of tuberculosis in, ii. 453; prophylaxis of the neuroses in, iv. 523 .

Schorstein, Dr. Gustave, on strychnin for relief of insomnia in chronic bronchitis, iii. 374 . 
Schott gymnastic exercises, i. 302; movements of arm in, iii. 643; movements of ankle joint in, iii. 644; movements of elbow joint in, iii. 643; movements at finger joints in, iii. 644; movements at hips in, iii. 644; movements at knee in, iii. 644 ; movements of trunk in, iii. 644; movements at wrist in, iii. 644 ; resistive exercises in, iii. 641 ; self-resistive exercises in, iii. 645 .

Schott treatment, use of, in bronchial asthma, iii. 382.

SCHRoETTER's TUBEs, passage of, in laryngeal stenosis, iii. 349 .

Schrotr's DRY DIET, use of, in pleurisy, iii. 468.

Schultze Eye, iv. 278.

Schurupow's antitoxic serum, results from use of, in Asiatic cholera, iv. 832.

Schwab ANd Allison, method of operation devised by, for relief of athetosis and contractures following cerebral palsies of children, iv. 415.

Schweninger system, the, reduction of obesity by, ii. 793 .

Sciatic Neuritis. See Sciatica.

Sciatic Pain, as symptom of diabetes mellitus, ii. 766.

Sciatica, i. 416, iv. 294; due to constitutional states, iv. 298; due to damage to nerve trunk, iv. 298; definition of, iv. 294; electrotherapy for relief of, i. 416 ; injections into nerve trunk for relief of, iv. 300 ; due to intrapelvic disease, iv. 298; massage and gymnastics for relief of, i. 311 ; radicular form of, iv. 295; sacroiliac form of, iv. 296; surgical treatment for, iv. 304; symptomatic treatment for, iv. 299.

Sclavo, antianthrax serum produced by, ii. 331 ; results of serum on mortality of anthrax reported by, ii. 332 .

Sclerosis of AORTA, association of, with angina pectoris vera, iii. 767 .

Sclerosis of cerebellum. See Cerebellum.

Sclierosis of coronary arteries. See Coronary sclerosis.

Sclerosis, multiple. See Multiple sclerosis.

Sclerosis, primary lateral. See Paralysis, spastic spinal.

Scopolamin, use of, in delirium tremens, ii. 606.
Scotch douche, use of, in neurasthenia and psychasthenia, iv. 551 .

Scotland, antituberculosis work in, ii. 440.

Scotomata, as an accompaniment of ophthalmic migraine, iv. 590, 591.

Scrofuloderma, X-ray therapy for relief of, i. 535 .

Scurvy, iii. 874; care of mouth in, iii. 875 ; definition of, iii. 874 ; diet for, iii. 875 ; etiology of, iii. 874 ; infantile, iii. 876 ; prophylaxis against, iii. 874 ; treatment of, iii. 874; treatment of infantile form of, iii. 876 .

Sea air, benefits of, in bronchoadenitis, iii. 398 ; influence of, in neurasthenia, iv. 556.

Sea-bathing, in bronchiectasis, iii. 393; contraindicated, in parenchymatous nephritis with weak heart, iv. 30 ; contraindicated in pyelitis, iv. 87 ; in tuberculosis of mediastinal lymph nodes, iii. 506; use of, in light therapy, i. 439 ; value of, in diabetes mellitus, ii. 762 ; value of, in rachitis, ii. 816 .

Sea-sickness, as a form of labyrinthine vertigo, iv. 624 .

Sea side, change to, in cholera infantum, iii. 205.

Sea voyages, value of, in treatment of tuberculosis, ii. 473.

SEARs, case of collapse and sudden death after thoracentesis reported by, iii. 481 .

Seasons, relation of, to spread of plague, iv. 819 .

"Second therapy," use of, in an X-ray treatment, i. 501.

Secondary agents in psychotherapy, i. 596. SECONDARY CUTANEOUS SYMPTOMS OF SYPHILIS. See Syphilis.

Secondary elaboration, as a part of dream mechanism in psychotherapy, i. 587.

Secondary pneumonia. See Pneumonia.

Secondary sinus thrombosis. See Infectious sinus thrombosis.

Section of posterior spinal nerve roots, for relief of contractures due to multiple sclerosis, iv. 281 ; for relief of late pain in herpes zoster, iv. 360 .

Secretin, use of, in diabetes mellitus, ii. 761 . Secretion, changes in, due to hypnosis, i. 574 .

Sedatives, use of, in cerebral hyperemia, accompanied by restlessness, iv. 368; 
in ehronic mucous colitis, iii. 207; in gastroptosis, to improve the nervous condition, iii. 182; in irritative gastric neuroses, iii. 188; in membranous enteritis, iii. 210; in neurasthenia and psychasthenia, iv. 558; to prevent recurrence of spasmodic laryngitis, iii. 342 ; for relief of insomnia due to brain tumor, iv. 447; for relief of insomnia due to sunstroke, iv. 679 ; for relief of pain due to bites of arthropods, - iv. 685 ; for relief of restlessness due to cerebral hemorrhage, iv. 392 .

Sedatives, gastric, of no benefit in uremic vomiting, iv. 54 .

SEDENTARY LIFE, relation of, to obesity, ii. 785.

Sehriwald and Reichert, experiments by, on intratracheal injections, iii. 397.

Seidlitz powder, use of, in acute gastritis, iii. 155 ; in acute nephritis, iv. 58 .

Seigert's rule, in prophylaxis of measles, ii. 144.

Seiler's solution, use of, as spray in acute bronehitis, iii. 358; during attack of hay fever, iii. 390 ; in chronic bronchitis, iii. 373 ; in ehronic laryngitis, iii. 340 ; in chronic rhinitis, iii. 329 ; for control of profuse expectoration in ehronic bronchitis, iii. 378 ; for irrigation in acute laryngitis, iii. 339 ; in ozena, iii. 336.

Seiler's solution, use of, as mouth wash, in mumps, ii. 170.

Selective ACtion, in radium, i. 468; in $\mathrm{X}$-ray, i. 502, 509; of X-ray upon eancer cells, i. 537.

Self-antagonizing method, of determining extent of chronic myocardial insufficieney. See Herz method.

Sermi, separation of poisonous alkaloids from food first effected by, ii. 634 .

Seminal vesiculitis, eomplieating acute urethritis, iv. 149.

Senator, formula by, for food in gastric ulcer, iii. 129.

Senega, use of, in aeute bronchitis, iii. 362 .

SENN, Nicholas, radiotherapy first employed by, in leukemia, iii. 848 .

Senna, use of, in cerebral hyperemia, iv. 386 ; in ehronic constipation, iii. 232 ; in chronic intestinal obstruction, iii. 242; in nephritis, iv. 33 ; for relief of trichiniasis, iii. 254 .
Sensation, abnormalities of, as result of poison, i. 6fj8; dclayerl, in diagnosis of tabes, iv. 189; disturbances of, in tongue, iii. 3.

Sensitized vaccines, i. 152.

"Sensitized virus," i. 138.

Sensory misturibances, absenee of, in Friedreich's ataxia, iv. 216.

Sensory iritative phenomena, value of, in regional diagnosis of brain tumor, iv. 438.

Sensory paralysis, value of, in regional diagnosis of brain tumor, iv. 438 .

Sepsin, as a cause of food poisoning, ii. 635 .

SEPsis, as a complication of searlet fever, ii. 128; after operation for brain tumor, iv. 452 .

SePtic Foci, importance of attention to, in prophylaxis of acute cerebral meningitis, iv. 372 .

Septic infection of oral origin, iii. 2.

Septicemia, as a complieation of vaceination, ii. 91 ; definition of term, ii. 256 .

Septicopyemia, ii. 286; definition of, ii. 286; diagnosis of, ii. 288 ; diet in, ii. 290 ; etiology of, ii. 287; gastric irritability in, ii. 291 ; organisms eausing, ii. 287 ; pathology of, ii. 287; prognosis of, ii. 2s8; prophylaxis of, ii. 289 ; specific treatment of, ii. 289 ; surgieal treatment of, ii. 259 ; symptoms of, ii. 287 ; symptomatic treatment of, ii. 290 ; treatment of, in general, ii. 289 .

Sera, method of administration of, i. 176 .

Sera, immune, antianthrax, ii. 331 ; antidiphtheritic, ii. 179; antiinfluenza, ii. 194, 213; antimeningitic, ii. 202; antipneumococeic, ii. 235; antirabic, ii. 239; antistreptococcic, ii. 183, 639; antitoxic, i. 153 , ii. 83,639 ; antityphoid, ii. 7 ; bactericidal, i. 154; Moser's, ii. 129; polyvalent, ii. 240 ; Römer's, ii. 37 ; types of, in general, i. 168.

Serodiagnosis, value of, in brain syphilis, iv. 476.

Serovaccination, i. 152.

Serous EFfusion, accompanying pneumothorax, iii. 492.

Serous effusion, chronic, iii. 483 ; aftertreatment of, iii. 483 ; diet in, iii. 484 ; hydrotherapy for relief of, iii. 484 ; outdoor life in treatment of, iii. 484.

Serous meningitis. See Meningitis. 
Serum, antimeningitic, use of, in disease of eighth cranial nerve, iv. 315.

Serum, antistreptococcus, use of, in malignant endocarditis, iii. 746.

"Sérum antivenimeux," i. 160.

Serum, artificial, transfusion of, in funicular myelitis, iv. 232; use of, as a coagulant in hemophilia, iii. 863.

Serum, horse's, use of, to control epistaxis, iii. 336 ; to control hemorrhage in hemorrhagic measles, ii. 155; in trypanosomiasis, iv. 748; in tuberculous kidney, iv. 117 .

Serum, human, subcutaneous injection of, in chronic pancreatitis, as prophylactic against hemorrhage at operation, iii. 300 .

Serum, human, immune, i. 170.

Serum, streptococcus, use of, in typhus fever, ii. 37.

Serum, Trunecek's. See Trunecek's serum.

Serum anaphylaxis, definition of, i. 132; occurrence of, after intraspinal injections, i. 136; other forms of, in man, i. 137; production of, i. 133.

Serum disease, i. 133; treatment of, i. 136.

Serum hypersusceptibility, i. 132.

Serum therapy, i. 153 ; in acute myelitis, iv. 228 ; in acute rheumatism, ii. 301 ; in anthrax, ii. 331; antitoxic sera in, i. 154; in Asiatic cholera, iv. 832; autoserotherapy in, i. 170; in bacillary dysentery, ii. 321 ; bactericidal sera in, i. 161; in blackwater fever, iv. 741 ; in cerebrospinal meningitis, ii. 202; in chlorosis, iii. 822 ; in chronic bronchitis, iii. 372 ; for control of gastric hemorrhage, iii. 113; diseases benefited by, i. 154; in erysipelas, ii. 284; in exophthalmic goiter, iii. 902 ; in glanders, ii. 358 ; impossibility of, in epilepsy, iv. 648 ; in influenza, ii. 186; in leprosy, iv. 814; in membranous pharyngitis, iii. 38; in multiple sclerosis, iv. 281; in pernicious anemia, iii. 832 ; in phlegmonous pharyngitis, iii. 40; in plague, iv. 826 ; in pneumococcus meningitis, ii. 213 ; in pneumonia, ii. 238 ; results of, i. 178; in Rocky Mountain fever, ii. 567; in scarlet fever, ii. 128; in septicopyemia, ii. 290 ; in small-pox, ii. 52 ; in streptococcus meningitis, ii. 213 ; in syphilis, ii. 437 ; in tabes, iv. 196; in tropical relapsing fever, iv. 759 ; in trypanosomiasis, iv. 748 ; in tuberculosis, ii. 525 ; types of immune sera employed in, i. 168; use of normal serum in, i. 171; use of types of immune sera in, i. 168.

Serum therapy in epidemic cerebrospinal meningitis, ii. 202; in chronic cases, ii. 209; frequency of administration of, ii. 207 ; in fulminating cases, ii. 208 ; indications for use of, ii. 206; manner of obtaining serum for, ii. 203; nature of serum for, ii. 202; precautions to be observed in, ii. 200; in protracted cases, ii. 208; in relapses, ii. 209; in resistant cases, ii. 209; size of dose in, ii. 207; summary of subject of, ii. 211 ; technique of administration of, ii. 204.

Serum therapy in hemophilia, iii. 864; kind of serum employed in, iii. 865 ; intravenous method of using, iii. 865; local use of, iii. 866,867 ; mode of administration of, iii. 865; subcutaneous method of using, iii. 863.

Serum therapy in purpuras, iii. 859: in acute purpura, iii. 859 ; in chronic purpura, iii. 860; in post-typhoidal purpura hemorrhagica, iii. 860.

Sex glands in organotherapy, i. 113; dosage of, i. 115; experimental work on therapeutics of, i. 113; preparations of, i. 115; reciprocal relation between other organs and, i. 114; relation between the hypophysis and, i. 102, 108; therapeutic use of, i. 114; use of corpus luteum, i. 115; use of ovary, i. 114; use of testicle, i. 115 .

SeX REPREssion, as a factor in functional neuroses, iv. $575,577,578$.

Sexual coefficient, iv. 178.

Sexual contact, transmission of leprosy by, iv. 811 .

Sexual excesses, as a cause of reflex cardiac neuroses, iii. 781 .

Sexual hygiene, in acute gonorrhea, iv. 138; in chronic gonorrheal urethritis, iv. 154.

Sexual instinct, as a factor in psychasthenia, iv. 575 .

Sexual neurasthenia, as a cause of pain in bladder, iv. 120.

Sexual organs, effects of X-rays upon, i. 521 ; reflex cardiac neuroses associated with, iii. 787; relation between secretion of, and body weight, ii. 786; relation of, to diseases of pituitary body, iii. 916; relation of, to phrenocardia, iii. 788 . 
Sexual relations, in spread of ulcerating granuloma of pudenda, iv. 866 .

SEYDEL, on results of operation for sarcoma and carcinoma of lung, iii. 448.

SHEEP-POX, ii. 55.

Sheet, wet, use of, in chlorosis, iii. 814 .

Sheet bath, uses of, in hydrotherapy, i. 195.

SheLL-FIsII, poisoning by, ii. 645 .

Shibayama, on calusation of beri-beri, iv. 847.

Shiga, determination by, of causal agent in epidemic dysentery, ii. 316 ; preparation of immune serum by, for use in epidemic dysentery, ii. 321 ; preparation of plague vaccine by, for immediate use, iv. 824 .

Shiga bacillus, ii. 317.

Shima mushi, iv. 692.

Ship Fever. See Typhus fever.

Sноск, blood pressure lowered by, iii. 556; danger from, after operation for brain tumor, iv. 452.

"Sick headache." See Migraine.

Side-chain theory, i. 128.

Sieber-Schoumow, B. piseicidus agilis isolated by, from fish, ii. 644 .

Siegel's inhaler, use of, in acute bronchitis, iii. 363 .

Siegrist's method, of applying hot fomentations in sciatica, iv. 299.

"Siguatera," ii. 633.

Silk ThREAD, use of, as guide in passing esophageal dilators, iii. 49, 59, 62 .

Silver, organic salts of, use of, in acute gonorrhea, iv. 137, 144 ; in chronic gonorrheal urethritis, iv. 155 .

Silver, poisoning By, i. 708.

Silver City, climate of, for laryngeal tuberculosis, iii. 345 .

Silver nitrate, use of, in abortive treatment of acute tonsillitis, iii. 41 ; in acute nasopharyngitis, iii. 31 ; in acute uvulitis, iii. 35; as antiseptic wash after passage of urethral sound, iv. 169; as application to verumontanum in postgonorrheal neuroses, iv. 174; in Bednar's aphthæ, iii. 11; in cauterization of bitc by rabid animal, ii. 336 ; in chronic laryngitis, iii. 340 ; in chronic myelitis, iv. 230 ; in chronic nasopharyngitis, iii. 32 ; in chronic pharyngitis, iii. 33 ; in chronic rhinitis, iii. 329 ; in chronic stage of bacillary dysentery, ii. 327 ; in continu- ous gastric hypersecretion, iii. 106; in diabetic vulvitis, ii. 765; by enemata in chronic mucous colitis, iii. 207; in foot and mouth disease, ii. 58.3 ; in gonorrheal stomatitis, iii. 21; by injeetion into kidney, in pyolitis, iv. 91 ; by injection to promot " closure of pancreatic fistula, iii. 307 ; by instillation in acutr. posterior urethritis, iv. 149; by instillation after passage of urethral sound, iv. 1652, 169; by instillation in (hronic gonorrheal urethritis, iv. 160, 161; by instillation in post-gonorrheal neuroses, iv. 174; by instillation for relief of irritable stricture of urethra, iv. 173; for irrigation of bladder in acute cystitis, iv. 120; for irrigation of bladder in eystitis complieating acute myelitis, iv. 227 ; for irrigation of bladder in endemic hematuria, iv. 785; for irrigation of colon, in ulcerative colitis, iii. 211; for irrigation of urethra after passage of urethral sound, iv. 162 ; by irrigation in tuberculosis of intestines, ii. 505; by intralaryngeal application in chronic laryngitis, iii. 341 ; in lavage for chronic gastritis, iii. 160; in membranous rhinitis, iii. 330 ; in mercurial stomatitis, ii. 385 ; in mueous patches, ii. 457 ; in multiple sclerosis, iv. 281; in mycosis of tonsils and fauces, iii. 42; in non-bleeding gastric ulcer, iii. 136 ; in oriental sore, iv. 756 ; in pharyngeal eough, ii. 495; in phlegmonous pharyngitis, iii. 40 ; by rectal injection in acute stage of bacillary dysentery, ii. 327 ; to relieve hematemesis in pernicious anemia, iii. 837 ; in rhagades of lip commissures, iii. 5; in stomatitis catarrhalis, iii. 7 ; in stomatitis crouposa, iii. 19; in stomatit is syphilitica, iii. 21 ; in stomatitis ulcerosa chroniea, iii. 16; in syphilis of upper respiratory tract, iii. 349 ; in syphilitic onychia, ii. 429 ; in tabes, iv. 195 ; in tertiary syphilitic lesions of mucous and mucoeutaneous surfaces, ii. $42 \bar{i}$; in throat affections complicating scarlet fever, ii. 130; for ulceration of cornea in small-pox, ii. 50; in urethroscopic treatment of ehronic gonorrheal urethritis, iv. 166 ; without value in spastic spinal paralysis, iv. 270; in Vincent's angina, iii. 40. 
Silver nitrate, fused, use of, in chronic nasopharyngitis, iii. 32 ; in chronic tonsillitis, iii. 44.

Silver nitrate, solid stick of, use of, to control epistaxis, iii. 335 ; in stomatitis ulcerosa, iii. 15.

Simaruba, use of, in bacillary dysentery, ii. 327.

Sims' Position, use of, for administration of enemata in intestinal amebiasis, iv. 709.

Simulidm Reptans, as cause of pellagra, ii. 658.

Sinapisms, use of, in acute hemorrhagic inferior polioencephalitis, iv. 408 ; in cerebral anemia, iv. 382 ; in hydrocephalus, iv. 465 ; as stimulant in cerebral hyperemia, iv. 387 ; as a stimulant in marantic thrombosis, iv. 427.

"Singer's NODEs," iii. 340.

SinoAURicular heART BLOCK, iii. 566 .

Sinusoidal current, determining characteristics of, i. 375 ; use of, as electromechanical method, i. 388.

Sinus, frontal, irrigation of, in acute suppurative inflammation, iii. 335.

Sinds, Nasal. See Nasal sinuses.

Sinus thrombosis, iv. 426 ; infectious sinus thrombosis, iv. 427 ; introduction to subject of, iv. 426 ; marantic sinus thrombosis, iv. 426 ; types of, iv. 426 .

Siphonage, use of, in thoracentesis, iii. 478.

Sippy, Bertram W., method introduced by, for dilating cicatricial stenosis of esophagus, iii. 49 ; rubber-bag dilator invented by, iii. 59 ; use of method in carcinomatous stenosis of esophagus, iii. 54 .

Siriasis. See Insolation.

Sitotoxismus, ii. 652.

"Situation," definition of, in psychotherapy, iv. 574.

Sitz bath, use of, in chlorosis, iii. 814; in chronic cerebral hyperemia, iv. 387 ; in hydropathy, i. 200.

Sitz baths, cool, use of, for relief of urogenital symptoms in tabes, iv. 201.

Sitz bath, hot, use of, for relief of impassable urethral stricture with retention, iv. 172; for relief of painful urination in acute urethral gonorrhea, iv. 142.

“ 606." See Arsenobenzol.

Size, influence of, on food requirement, $i$. 605.
Skeletal system, effect of massage upon, i. 297.

SkIN, administration of mercury by, in syphilis, ii. 387; effect of cold upon, i. 189; effect of heat upon, i. 192; effect of radiant energy upon, i. 437; effect of $\mathrm{X}$-rays upon, i. 514, 517; elimination through, in cardiac insufficiency with general anasarca, iii. 678; elimination through, in chronic interstitial nephritis, iv. 69; elimination through, as prophylactic measure against pulmonary edema in nephritis, iv. 56; elimination through, in uremia, iv. 53; importance of care of, in nephritis, iv. 32; puncture of, see Puncture.

Skin disease, X-Ray therapy For, when due to fungi, i. 536; when originating in hair follicles, i. 530 .

Skin, Disturbances of, administration of thyroid gland for, i. 76; due to arsenobenzol used in syphilis, ii. 415; as a complication of diphtheria, ii. 279; due to hydrargyrism, ii. 384 ; influence of climate upon, i. 282.

Skin Lesions, as result of poison, i. 669.

Skin grafting, employment of, in tropical phagedenic ulcer, iv. 868 .

Skin pustules, protection of, in plague, iv. 827.

SKIPPING THE ROPE, injury done by, in cardiac insufficiency, iii. 682 .

Slaked lime, inhalation of vapor from, in membranous laryngitis, iii. 343 .

Sleep, importance of, in arteriosclerosis, iii. 760; importance of, in cardiac insufficiency, iii. 663; importance of, in cardiac neurasthenia, iii. 776 ; importance of, in neuritis, iv. 286; methods of securing, in delirium tremens, ii. 607.

SLEEPING SICKNESS, iv. 742.

Sleeplessness. See Insomnia.

SLOw PULSE, as a symptom of brain tumor, iv. 435.

Sluder tonsillotome, iii. 46.

Small-Pox, ii. 40; control of itching in, ii. 48 ; definition of, ii. 40 ; delirium in, ii. 48; diet in, ii. 46; disposal of dead in, ii. 45; edema of glottis complicating, ii. 51; eruption in, ii. 48; erysipelas complicating, ii. 49; eyes and their care in, ii. 50 ; history of, ii. 40 ; immunity from, ii. 41 ; incubation period of, ii. 43 ; infec- 
tive period in, ii. 41 ; inflammation of larynx complicating, ii. 51; inflammation of tongue complicating, ii. 51: inoculation for, ii. 52 ; insomnia in, ii. 48 ; organism causing, ii. 41 ; pain as a symptom of, ii. 47; physician's duty in, ii. 44 ; potassium permanganate treatment of, ii. 51; prophylaxis in, ii. 43 ; quarantine in, ii. 43 ; red light treatment of, ii. 51 ; scrum treatment of, ii. 52 ; suppuration in, ii. 48 ; susceptibility to, ii. 41 ; synonyms for, ii. 40 ; temperature in, ii. 47 ; throat disturbances in, ii. 47; tracheotomy in, ii. 51 ; transmission of, ii. 42 ; treatment of, ii. 45 ; vaccination for, ii. 43, 45; see also Vaccination.

Smali-Pox hospital, ii. 43.

Small-pox virus, diluents for, ii. 65; preparation of, ii. 62,63 ; preservation of, ii. 65; use of rabbit for, ii. 67 .

Smith, Eustace, mouth wash for use in mumps suggested by, ii. 171.

Smith, Dr. James, experiments by, on inoculation for small-pox, ii. 92.

Smith, Nathan, early employment of hydrotherapy by, in general infections, ii. 16; on fan bath in typhoid fever, ii. 18 .

Sмiтн, R. H., views held by, on enteroptosis based on X-ray investigation, iii. 244.

SNAKE Venom, antitoxin against, i. 159; effect of, upon kidneys, iv. 14, 15.

SNAKes, Poisonous, varieties of, iv. 681 .

Soamin, use of, in kala-azar, iv. 754 ; in pellagra, ii. 660 ; as substitute for atoxyl in trypanosomiasis, iv. 746.

Social customs, influence of, in causation of alcoholism, ii. 590 .

Soda, washing, external use of, for itching in diabetes mellitus, ii. 764 .

Sodii nitris. See Sodium nitrite.

Sodium acetate, use of, as diuretic in hydropericardium, iii. 525; in nephritis, iv. 49.

Sodium aminophenylarsenate. See Soamin.

Sodium arsenate, use of, in preventive treatment of boils in diabetes mellitus, ii. 765 .

Soda benzoate, use of, in asthenic form of measles, ii. 153; in cholera infantum, iii. 205; in destruction of filaria bancrofti, iv. 787; by hypodermic injection in toxemia complicating acute diffuse peritonitis, iii. 318 .
Sodium biborate, use of, as douche for nose or throat, iii. 32 ; in gast ric hyperacidity, iii. 95.

Sodium bicarbonate, use of, in acidosis during diabetes mellitus, ii. 756,770 ; in acute rheumatism, ii. 299, 301; in acute peritonsillitis, iii. 43; in acute tonsillit is, iii. 42 ; in acute urethral gonorrhea, iv. 139; in chronic gast ritis, iii. 160; in cirrhosis of liver, in milk, ii. 281 ; in constipation ussociated with gastric constipation, iii. 95; in diabetic coma, ii. 757 ; for disinfection of mouth in measles, 148 ; for disinfection of mouth in smallpox, ii. 50 ; by gastric lavage in acute alcoholism, ii. 589 ; by gastric lavage in acute gastritis, iii. 154 ; by gast ric lavage in gastric hypersecretion, ii. 98 ; by intravenous injection in diabetic patients under influence of a general anesthesia, ii. 770; by irrigation in acute peritonsillitis, iii. 43; as nasal douche in membranous rhinitis, iii. 330; objections to, in gastric hyperacidity, iii. 93; pancreatic secretion diminished by, iii. 307 ; in pneumonia, ii. 234 ; for relief of painful erections and chordee in acute urethral gonorrhea, iv. 141; in stomatitis hypermycetica, iii. 13 ; in treatment of morphin habit, ii. 624 .

Sodium bicarbonate and calomel, use of, in stomatitis catarrhalis, iii. 7 .

Sodium bicarbonate and nux vomica, use of, in acute alcoholism, ii. $5 \$ 9$.

Sodium bicarbonate and salicylic acid, use of, in pericarditis, iii. 519.

Sodium borate, use of, in reduction of obesity, ii. 809; as spray in acute glossitis, iii. 24; in stomatitis herpetica, iii. 10.

Sodium bromid, use of, in apoplectic form of vestibular vertigo, iv. 629 ; in enuresis associated with night terrors, iv. 133 ; in measles, ii. 152; to prevent recurrence of spasmodic laryngitis, iii. 342 ; for relief of headache due to erysipelas, ii. 284 ; for relief of insomnia due to brain tumor, iv. 447 ; for relief of laryngismus stridulus in rachit is, ii. $\$ 20$; in treatment of morphin habit, ii. 620; in varicella, ii. 113.

Sodium bromid and chloral hydrate, use of, for relief of headache accompanied by convulsions or delirium in cerebral thrombosis, iv. 399 . 
Sodium bromid, elixir of, use of, for relief of headache due to toxemia, iv. 610 .

Sodium cacodylate, use of, by hypodermic injection in acquired spinal progressive muscular atrophy, iv. 248; by hypodermic injection in multiple sclerosis, iv. 281; by hypodermic injection in neurasthenia, iv. 559 ; by hypodermic injection in syringomyelia, iv. 279 ; by intramuscular injection in pernicious anemia, iii. 827 ; for neuralgia due to anemia, iv. 336 ; in pellagra, ii. 660 .

SODIUM, CHLORID, restriction of, in diet, in acute nephritis, iv. 61 ; restriction of, in diet, in chronic interstitial nephritis, iv. 68; restriction of, in diet. in chronic parenchymatous nephritis with edema, iv. 66; restriction of, in diet in nephritis, iv. 35 ; retention of, in body a cause of edema, iv. 18; solution of, see Saline solution.

Sodium chlorid, use of, as cutaneous stimulant in tabes, iv. 200; by gastric lavage, iii. 152 ; in pellagra, ii. 659 .

Sodium chlorid waters, use of, in depressive disorders of gastric secretion, iii. 151.

Sodium citrate, use of, in acidosis during diabetes mellitus, ii. 756 ; in acute rheumatism, ii. 301; for control of vomiting in whooping-cough, ii. 180; in diabetic coma, ii. 757 ; in gastric hyperacidity, iii. 95 .

Sodium HYPOCHLORITE. See Labarraque solution.

Sodium hyposulphate, use of, combined with glycerin, in geographical tongue, iii. 25 .

Sodium iodid, use of, in aneurysm of cerebral arteries, iv. 457; in arteriosclerosis, iii. 761; in arthritis deformans and chronic rheumatism, ii. 714 ; in compression bulbar paralysis due to aneurysm, iv. 502; in glandular enlargement accompanying rachitis, ii. 818 ; in goiter, iii. 894 ; in hydrocephalus, iv. 465 ; in syphilis, ii. 409.

Sodium nitrate, use of, to reduce hypertension in nephritis, iv. 47,48 .

Sodium nitrite, use of, in angina pectoris, iii. 769 ; in cardiac insufficiency, iii. 606, 607 ; in chronic interstitial nephritis, iv. 70,71 ; in control of hemorrhage from pulmonary tuberculosis, ii. 500 ; in neu- ralgia due to arteriosclerosis, iv. 336 ; in neurasthenia with high arterial tension, iv. 559; for relief of dyspnea in chronic interstitial nephritis, iv. 72 .

Sodium phosphate, use of, in acute nephritis, iv. 58 ; in chronic cholecystitis and cholelithiasis, iii. 272 ; in constipation associated with gastric hyperacidity, iii. 94 ; in exophthalmic goiter, iii. 901 ; in influenza, ii. 187 ; in intestinal autointoxication, ii. 672 ; as a laxative in fibrinous pleurisy, iii. 469 ; in nephritis, iv. 32 ; in prevention of boils in diabetes mellitus, ii. 765 ; in Weil's disease, ii. 572.

Sodium salicylate, use of, in acute tonsillitis of rheumatic tendency, iii. 41 ; in asthenic form of measles, ii. 153; in bacillary dysentery, ii. 326 ; for cervicooccipital neuralgia, iv. 356; as diuretic in multiple neuritis due to gout or rheumatism, iv. 326; in enuresis associated with chorea, iv. 133; in herpes zoster, iv. 359 ; in influenza, ii. 187; in Landry's paralysis, iv. 329 ; for neuralgia associated with gout, iv. 337 ; for neuralgia associated with rheumatism, iv. 337; neuritis with a rheumatic or gouty element, iv. 289 ; in occupation pareses, iv. 332 ; by rectum, in acute rheumatism, ii. 306 ; to reduce temperature in pleurisy, iii. 468 ; in shima mushi, iv. 692 ; in treatment of gallstones in transit, iii. 274 ; in Weil's disease, ii. 572.

Sodium sulphate, use of, in acute diffuse peritonitis, iii. 317 ; in acute gastritis, iii. 155; after calomel in yellow fever, iv: 855 ; in chronic cholecystitis and cholelithiasis, iii. 272 ; in constipation associated with gastric hyperacidity, iii. 94; in nephritis, iv. 58 ; in prevention of lead poisoning, iv. 325 ; in treatment of gallstones, in transit, iii. 274.

Sodium SUlphite, preservative action of, $i$. 729.

Softening of brain. See Cerebral softening.

SolL, occurrence of arsenic in, i. 675 ; relation of, to climate, i. 256.

Soja, use of, in diabetes mellitus, ii. 748 .

SolAR RAYs, relation of, to insolation, iv. 674.

Solarium sun bath, i. 439. 
Solrtude, objections to, for patient with brain tumor, iv. 447.

Sollier, PaUl, on suffering following treatment for morphinism, ii. 625.

Solution, for use in alcohol injections, iv. 343.

Solutions, for injection into nerve trunk in sciatica, iv. 300 ; use of antiseptie, for irrigation of pleural cavity, iii. 487; use of eleansing, in atrophic rhinitis, iii. 331.

Somatic teniasis, iv. 775 ; preventive measures in, iv. 776 ; remedial measures in, iv. 776 ; varieties of, iv. 775 .

Sompolence, as a symptom of pachymeningitis interna hemorrhagica, iv. 363.

Soporifics. See Hypnotics.

Sore ARM AND UlCERATION, as a complication of vaccination, ii. 86 .

Soul buindness. See Mind blindness.

Sounds, passage of, for relief of dysphagia in tuberculosis of mediastinal lymph glands, iii. 508 .

Soups, in diet for gastric ulcer, iii. 129.

Source of LIGHT, distance of, in an X-ray treatment, i. 502.

Southey's tubes, use of, to relieve edema of extremities in acute nephritis, iv. 62 .

Spa treatment, choice of Spa, i. 237; in chronic bronchitis, iii. 370; climate of Spas, i. 240; in diabetes mellitus, ii. 763; in gout, ii. 701; indications for, i. 237; in neurasthenia and psychasthenia, iv. 557; objections to, in cardiac insufficiency, iii. 617 ; objections to, in chronic myocardial insufficiency due to obesity, iii. 727 ; principles of, i. 230 ; in reduction of obesity, ii. 809; the Spa itself, i. 240 .

SpaIN, introduction of vaccination into, ii. 60 .

Sparganum baxteri, iv. 775.

SPARgaNUM MANSONI, iv. 775.

Sparganum proliferum, iv. 775.

Sparks, friction, i. 385; indications for use of i. 393; physical effects of, i. 385; static, i. 383 ; use of, in electrotherapeutics, i. 383.

Spartein, use of, as cardiac stimulant in beri-beri, iv. 853 ; in nephritis complicating scarlet fever, ii. 134; in pneumonia with cardiac toxemia, ii. 254 ; in treatment of morphinism, ii. 624 .

SPASM, electrotherapeutics for relief of, at external abdominal ring, i. 421 ; at internal abdominal ring, i. 421 ; at sigmoid flexure, i. 421 ; of sphincters, i. 421.

Spasmonic: harsiatis. See Laryngitis, spasmodic.

Spasmonc Tic, hydrotherapy for relief of, $\mathrm{i}$. 206 .

Spasmonic Twitchess, due to brain tumor, measures for relicf of, iv. 448.

Spasmophula, relation of, to rachitis, ii. 819.

Spastic constilation, iii. 231.

Spastic paraplegia, electrotherapeutics for relief of, i. 430.

Spastic spinal paralysis. See Paralysis.

Spasticity, massage and gymnasties in treatment of, i. 315.

Specific infectious diseases, hydrotherapy for relief of, i. 215.

Specific serum reaction of syphills, discovery of, ii. 362.

Specifics, for use in laryngeal tuberculosis, iii. 345 .

Speech, disturbance of, from effect of poisons, i. 668; in Friedreich's disease, iv. 216; in hereditary cerebellar ataxia, iv. 217 ; in progressive bulbar paralysis, iv. 496; in progressive paralysis of the insane, iv. 490.

Speed, exercises of, iii. 700.

Spermatorrhea, iv. 176.

Spermin sodium chlorid, hypodermic use of, in myasthenia gravis, iv. 507 .

SpHincter, ATONY OF, as a cause of enuresis, iv. 128 .

SPHinCter PARALYsis, in progressive paralysis of the insane, iv. 490.

Sphygmogram, iii. 561; determination of pulse form by, iii. 561 ; inferences to be drawn from, iii. 561 .

Sphygmogram, the absolute, iii. 562.

Sphygmomanometer, use of, in treatment of cardiovascular disease by electricity, i. 425 .

Sphygmotonometer of v. RecklingHAUSEN, for estimating minimal blood pressure, iii. 558 .

Spina bifida occulta, as a cause of enuresis, iv. 126 .

Spinal accessory paralysis. Sce Neurit is of eleventh cranial nerve.

SPINAL column, treatment of deformities of, following anterior poliomyelitis, iv. 242. 
SPINAL CORD, DISEASES OF, iv. 180: acute myelitis, iv. 218; chronic myelitis, iv. 228; Friedreich's disease, iv. 214; funicular myelitis, iv. 231; hereditary cerebellar ataxia, iv. 214, 216; infantile paralysis, see Poliomyelitis; multiple sclerosis, iv. 279; muscular dystrophy, iv. 260; myotonia congenita, iv. 266; poliomyelitis, iv. 232; progressive muscular atrophy of central origin, iv. 243; spastic spinal paralysis, iv. 268; spinal syphilis, iv. 270; syringomyelia, iv. 275; tabes, iv. 180.

SpINAL CORD, DISEASES of, elecitrotherapeutics for relief of, i. 428.

SPINAL CURVATURE, association of, with rickets, ii. $816,818$.

SpINAL SYPhilis, iv. 270; diagnosis of, iv. 272 ; pathology of, iv. 274; prognosis of, iv. 273; symptomatology of, iv. 270; treatment of, iv. 273.

SPINAL TyPe OF MYASTHENIA GRAvis, iv. 506.

Spinal vibration. See Vibration to spine.

Spine, galvanization of. See Galvanization of spine.

Spirits of chloroform, use of, combined with turpentine, as spray in acute bronchitis, iii. 359 .

Spirits of nitrous ether. See Hoffman's anodyne.

Spiritus glycerylis nitratis, iii. 606 .

SPIRoCh ETA ABORIGinalis, iv. 865.

SPIRocheta dutToni, cause of tropical relapsing fever, iv. 758.

Spirochata Pallida, discovery of, ii. 362 ; value of, in diagnosis of syphilis, iv. 472.

Spirochetal fever. See Tropical relapsing fever.

Splanchnic stasis, as indication for use of digitalis, iii. 588 .

SpleEN, effect of X-rays upon, i. 519; electrotherapeutics for hypertrophy of, i. 423; enlargement of, in malaria, iv. 728; extirpation of, in treatment of leukemia, iii. 848; operation for rupture of, in malaria, iv. 728; X-ray therapy for enlargement of, i. 551.

Spleen, diseases of, iii. 887: abscess of spleen, iii. 888; Banti's disease, iii. 889; chronic enlargement of spleen, iii. 889; infarct of spleen, iii. 888; movable spleen, iii. 887; rupture of spleen, iii. 888.
Spleen, movable, iii. 887; medical treatment of, iii. 887; surgical treatment of, iii. 888 .

Splenectomy, performance of, in polycythemia with splenomegaly, iii. 878 .

Splints, use of, to overcome contractures following cerebral hemorrhage, iv. 393.

Spondylitis, ii. 710.

Sponge bath, uses of, in hydrotherapy, i. 196.

Spontaneous pneumothorax. See Pneumothorax.

Spot PAINS. See Lancinating pains.

SpotTed Fever, serum therapy in, i. 170.

Sprains, electrotherapeutics in treatment of, i. 410; massage and gymnastics for relief of, i. 328.

Spraying of nose and throat, in diphtheria, i. 272; in laryngeal tuberculosis, i. 492; limited value of, in whooping-cough, $i$. 181 ; in measles, i. 156; for relief of "colds" accompanying tuberculosis, i. 508.

Sprays, use of, in acute bronchitis, iii. 358 ; in chronic bronchitis, iii. 373,375 ; to control profuse expectoration in chronic bronchitis, iii. 378 .

Spriggs, E. I., diet scheme suggested by, for use in constipation, iii. 226.

SPring Catarrh of EYE, radium therapy in treatment of, i. 480 .

Sprue, iv. 861 ; remedial measures in, iv. 862.

SpUrious vaccination, as complication of vaccination, ii. 80 .

SPUTUM, treatment of, in pneumonia, ii. 221 ; treatment of, in whooping-cough, ii. $186,187$.

Squills, use of, as expectorant in acute bronchitis, iii. 362 .

SquirRels, relation of, to spread of plague, iv. 819,820 .

Stagnation of Food, in chronic dilatation of stomach, iii. 176; due to chronic gastritis, iii. 177; due to muscular insufficiency, iii. 177.

Standardization oF Digitalis PREParaTIONs, iii. 581; methods of, iii. 582; objects of, iii. 582 .

Staphylococcus infection, electrotherapeutics for relief of, i. 399; radiant light and heat in treatment of, i. 449 ; vaccine treatment for, i. 145 . 
Staphylococcus meningitis, ii. 213.

Stanca, in diet of achylia gastrica, iii. 147; in diet of depressive disorders of gast ric: secretion. iii. 147.

Starling and 'TubBy, experiments by, on absorption of pleural fluid by blood, iii. 458 .

Starvation, i. 634; in acute appendicitis, iii. 214,217 ; in acute diffuse peritonitis, iii. 317 ; in acute gastritis, iii. 157; in acute localized peritonitis, iii. 311,312 ; in acute stage of bacillary dysentery, ii. 325: in after-treatment of gastric ulcer, iii. 123; in chronic stage of bacillary dysentery, ii. 328 ; effects of complete, i. 634 ; effects of partial, i. 635 ; in enterit is of infancy, iii. 202 ; in gallstone colic, iii. 269 ; in gastric hemorrhage, iii. 110 , 117,120 ; in hematemesis from cirrhosis of liver, iii. 288; in hematemesis of pernicious anemia, iii. 837 ; indications for, in neurasthenia, iv. 537; for relief of anorexia in neurasthenia, iv. 566; in treatment of biliousness, iii. 258; in treatment of yellow fever, iv. 855 ; in ulcerative colitis, iii. 212.

Stasis, cardiac, see Cardiac stasis; respiratory, see Respiratory stasis; vasomotor, see Vasomotor stasis.

Static allments, ii. 709.

Static brush discharge, as an electromechanical method, i. 388.

Static current, determining characteristics of, i. 374 ; in mechanical application of electricity, i. 377.

Static dilatation of heart, iii. 547.

Static electricity, use of, in tabes, iv. 202.

Static induced current, use of, in electrotherapeutics, i. 382.

Static machines, i. 367.

Static spark, use of, in electrotherapeutics, i. 383 .

Static spray, as an electromechanical method, i. 387.

Static wave current in electrotherapeutics, i. 379: duration of treatment, i. 382 ; electrodes, i. 381 ; grounding, i. 380 ; insulated platform, i. 381 ; rate of discharge, i. 380 ; size of terminal balls, i. 380 ; spark-gap, i. 380 ; speed control, i. 381 .

Statistics, of pulmonary disease among Sheffield metal workers, iii. 424 .
States lymphaters, danger of surgery in, iii. 912; operation for, iii. 912 ; relation betweren death following injection of diphtheria antitoxin and, i. 134; relation between enlargement of ovaries and, $i$. 117; relation between excessive activity of thymus gland and, i. 118; X-ray treatment for, iii. 913.

ST. BARTHOLомЕW's Hosiral, formula for acid expectorant mixture used at, ii. 36.5 .

Steam, use of, as disinfectant in tuberculosis, ii. 4533 ; by inhalation in acute bronchitis, iii. 358 ; by inhalation in bronchopneumonia, iii. 419.

Steam baths, use of, in hydrotherapy, i. 200.

Steel Grinding, mortality from, iii. 124.

Stegomyia fasciata, yellow fever transmitted by, iv. 857.

Stenosis, of esophagus, see Esophageal stenosis; of larynx, see Laryngeal stenosis.

Sterilization of mucous membranes, importance of, in prophylaxis of anterior poliomyelitis, iv. 237 .

SternberG, method of treating yellow fever recommended by, iv. 856 .

Stevenson, Sir Thomas, epidemic of malignant edema due to 13 . proteus vulgaris infecting sardines described by, ii. 644.

STICKER, statistics on leprosy pub!ished by, iv. 811 .

Strles, number of eases of trichiniasis reported by, iii. 254 .

Still's disease, ii. 710 .

STILler, "asthenia universalis congenita" first deseribed by, iii. 181 ; views held by, on enteroptosis, iii. 243.

Stimulants, abuse of, a cause of gastric hyperacidity, iii. 76 ; objections to, in cerebral anemia, iv. $3 \$ 5$; objections to, in hemorrhage during pulmonary tubereulosis, ii. 501.

Stimulants, use of, in cerebrospinal meningitis, ii. 215; in depressive gastric neuroses, iii. 18S; in fish poisoning, iv. 684 ; before giving cold bath in measles, ii. 149 ; indications for, in cerebral hyperemia, iv. 387 ; in marantic thrombosis, iv. 427 ; in methemoglobinuria, iv. 6 ; in syncope due to cerebral thrombosis, iv. 398. 
Stimulants, alcoholic, use of, in acute diarrhea of the aged, iii. 195; in acute enteritis, iii. 194; in acute myelitis, iv. 226; in cerebrospinal meningitis, ii. 216; contraindicated in chronic hyperemia of brain, iv. 387 ; in enteritis of infancy, iii. 203; in pneumonia with cardiac toxemia, ii. 254; in stomatitis diphtheritica, iii. 20 ; in stomatitis gangrenosa, iii. 18.

Stimulants, cardiac, use of, in acute myelitis, iv. 226 ; in after-treatment of cerebral thrombosis, iv. 398; in beri-beri, iv. 853 ; in collapse associated with renal colic, iv. 106; in combination with epinephrin to relieve disturbances of eirculation in Addison's disease, iii. 885; contraindicated in cardiac palpitation in neurasthenia, iv. 568; in muscarin poisoning, ii. 648 ; in pachymeningitis interna hemorrhagica, iv. 364 ; in pericarditis, iii. 520; to prevent collapse in snake polsoning, iv. 682; in pulmonary edema complicating pneumonia, ii. 259; in pulmonary edema with low blood pressure, iii. 410; for relief of cardiac symptoms in chronic progressive bulbar paralysis, iv. 253.

Stimulants, cardiovascular, epinephrin, i. 97 ; use of, in pleurisy, iii. 469; use of, to relieve pain and dyspnea in pneumothorax acutissimus, iii. 491.

Stimulants, diffusible, use of, in pneumonia of alcoholics, ii. 258; in pneumonia with cardiac toxemia, ii. 254.

Stimulants, ethereal, use of, in crisis of pneumonia, ii. 157.

Stimulants, respiratory, use of, in bronchopneumonia, iii. 420.

Stimulants, vasomotor, use of, in collapse associated with renal colic, iv. 106.

Stimulation, objections to, in gastric ulcer, iii. 111.

Stimulus production, as cardiac function, iii. $535,536$.

St. John Long's liniment, iii. 357.

Stock vaccines, i. 140.

Stockton, on use of opium in acute diffuse peritonitis, iii. 316.

Stoker's inhaler, use of, for administration of oxvgen in bronehiectasis, iii. 396.

STokes, on training of patients with cardiac disease, iii. 645 .
StöLtzener, hypothesis advanced by, as to causation of rickets, ii. 814; investigation by, on relation between rickets and suprarenal glands, ii. 813 .

Sтомасн, changes in, due to hypnosis, i. 574; as an excretory organ, iii. 161; fibroushyperplastic infiltration of, due to syphilis, iii. 168; functional disorders of, iii. 185; gumma of, iii. 167; hydrotherapy for relief of atony of, i. 214; hydrotherapy for relief of dilatation of, i. 214; hydrotherapy for relief of ulcer in, i. 215; length of time that food remains in, $i$. 631 ; motor neuroses of, iii. 186; rupture of, due to use of stomach pump, i. 671; secretory neuroses of, iii. 187; sensory neuroses of, iii. 187.

Stomach, aCute dilatation of, iii. 173; treatment of, iii. 174; types of, iii. 174.

Stomach, Carcinoma of, iii. 162 ; alleviation of suffering in, iii. 166; diet in, iii. 164 ; drugs in treatment of, iii. 165; gastroenterostomy for relief of, iii. 166; lavage in treatment of, iii. 165; operative treatment for, iii. 162; pyloric obstruction due to, iii. 178.

Stomach, Chronic dilatation of, iii. 175; atonic dilatation, iii. 177; diet in, iii. 180; diseases outside stomach as cause of, iii. 179; hour-glass contraction, iii. 179; lavage in treatment of, iii. 180; mechanotherapy for, iii. 180; medical treatment for, iii. 180; operative treatment for, iii. 180; pyloric obstruction as cause of, iii. 178; stagnation of food in, iii. 176.

Stomach, diseases of, iii. 67: acute dilatation of stomach, iii. 173, 175; acute gastritis, iii. 153; carcinoma, iii. 62; chronic dilatation of stomach, iii. 73; chronic gastritis, iii. 157; classification, iii. 73; depressive disorders of gastric secretion, iii. 68 , 144; gastric analysis and its value in, iii. 67 ; gastric atony, iii. 168; gastric tetany, iii. 143; gastric ulcer, iii. 108; gastroptosis, iii. 181; hour-glass contraction of stomach, iii. 179; hyperacidity, iii. 75 ; hyperacidity and hypersecretion, iii. 74; idiopathic gastric neuroses, iii. 186; introduction to subject, iii. 67 ; irritative disorders of gastric secretion, iii. 74; motor disturbances of stomach, iii. 168; necessity for cautior. in operative measures, iii. 189; net:- 
rosis of stomach, iii. 182 ; primary gastric neuroses, iii. 186 ; pyloric obstruce tion, iii. 17s; syphilis of stomach, iii. 16ifi.

Stomacil, motor Disolzders of, iii. Ilis: acute dilatation, iii. 173; atony; iii. 16s; chronic dilatation, iii. 175.

Stomach, sypulas of, iii. 166: chronic gastritis, iii. 166; gastric uleer, iii. 167 ; fibrous-hyperplastic infltration, iii. 167; gumma, iii. 167.

"Stomach cough," ii. 495.

Stomach pump. See Stomach tube.

Stomach tube, administration of food by, in meat poisoning, ii. 439 ; evacuation of stomach by, in acute dilatation, iii. 174; evacuation of stomach by, in acute opium poisoning, ii. 614; evacuation of stomach by, in acute phosphorus poisoning, ii. 627; evacuation of stomach by, in fish poisoning, iv. 684 ; evacuation of stomach by, in meat poisoning, iii. 639 , 641; use of, after poison, i. 670; use of, contraindicated in asthenic bulbar paralysis, iv. 256.

Stomach washing. See Gastric lavage.

Stomachics, use of, in ulcerative colitis, iii. 211.

Stomatitis, iii. 6 .

Stomatitis aphthosa, as a complication of measles, ii. 157.

Stomatitis Catarrhalis, iii. 6 ; association of, with thrush, iii. 11 ; treatment of, iii. 7.

Stomatitis crouposa, iii. 19.

Stomatitis Diphtheritica, iii. 19 ; treatment of, iii. 20.

Stomatitis gangrenosa, iii. 16 ; as a complication of measles, iii. 157; treatment of, iii. 18.

Stomatitis, gonorrheal, iii. 20 ; treatment of, iii. 21.

Stomatitis Herpetica, iii. ${ }^{\circ} 8$; treatment of, iii. 9.

Stomatitis Hypomycetica, iii. 11 ; treatment of, iii. 12.

Stomatitis, mercurial, iii. 16; due to hydrargyrism, ii. 384 ; treatment of, ii. 385.

Stomatitis, pseudo-diphtheritic, iii. 19.

Stomatitis syphilitica, iii. 21.

Stomatitis ulcerosa, iii. 13 ; as a complication of measles, ii. 157 ; treatment of, iii. 15.

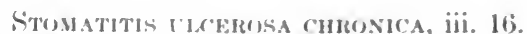

Sंтuњs, imlications for cxamining, in asthula, iii. 3\$1.

Storm binder, iii. '24s.

Stovain, use of, by epidural injection in raclicular sciaticat, iv. 29t; by injertion inta) spiual ("anal in tabes, iv. 195; by subarachnoil injection in ralioular sciatica, iv. 2ut;.

Stramonium leaves, burning and inhalation of, in chronic pulmonary emphysema, iii. 438 .

Strapping, as a therapeutic measure in gonorrheal epididymitis, iv. 152; for relicf of sacroiliac sciatica, iv. 297.

Strapping the chest, for relief of pain accompanying tuberculosis, iii. 506.

Strapping the side, for reliof of pain in acute fibrinous pleurisy, iii. 466; for relief of pain in pnoumonia, ii. 227.

Strassburger, on significance of retarded bowel action in intestinal autointoxication, ii. 671 .

STrauss, investigations by, on sweat in nephritis, iv. 38 .

Strengti, exercises of, iii. 700.

Streptococcus infection, electrotherapeuties for relief of, i. 399 ; radiant light and heat in treatment of, i. 449 ; vaccine therapy for, i. 146.

Streptococcus mexingitis, ii. 213.

Streptococcus scarlatinæ, ii. 114.

Streptococcus serum. See Serum.

STREPTOCOCCUS TOXINS IN TREATMENT OF TUMORs, i. 174.

Stretching of peripheral nerves, objections to, in tabes, iv. 203.

Stricture of urethra. See Urethral stricture.

Stroking, use of, in massage, i. 289.

Strong, on inoculation for plague with living avirulent cultures, iv. $\$ 24$.

Stroxg axd Teague, on use of arsenophenylglycin in trypanosomiasis, iv. 746 .

Stroxgaxoff, method of treating eclampsia in pregnancy employed by, iv. \$3.

Strongyloides stercoralis, iv. 796.

Strongrlosis, iv. 798; parasites causing, iv. 798 ; remedial measures in, iv. 799.

Strongrlus gibsoni, iv. 798.

Strongrles subtilis, iv. 798.

Strontium bromid, use of, for relief of hiccough in pneumonia, ii. 260. 
Strophanthin, influence of, as diuretic, iii. 579 ; influence of, on heart, iii. 578; influence of, on renal cases, iii. 577; technique of injecting, iii. 600; therapy of, iii. 600 ; toxic effect of, iii. 584 ; use of, in collapse associated with renal colic, iv. 106 ; use of, in toxemia complicating acute diffuse peritonitis, iii. 318 .

Strophanthin, intravenous injection of, in attacks of cardiac asthma, iii. 737; in chronic myocardial insufficiency due to nephritis, iii. 730 ; a means of studying digitalis bodies and their action, iii. 577 ; in pericarditis with acute heart failure, iii. 520; in pulmonary edema with low blood pressure, iii. 410; in severe cases of chronic myocardial insufficiency, iii. 718,719 .

Strophanthus, poisoning by, iv. 695 .

Strophanthus, iii. 599; use of, indications for, iii. 600; in acute myelitis, iv. 226; in acute vasomotor paralysis, iii. 753 ; in cerebrospinal meningitis, ii. 216; in cardiac failure in influenza, ii. 193; as cardiac stimulant in beri-beri, iv. 853; as cardiac stimulant in later stages of cerebral thrombosis, iv. 398; as cardiac stimulant in plague, iv. 827 ; in diphtheria, ii. 277; in epidemic dropsy, iv. 860 ; in enteritis of infancy, iii. 203; in pneumonia with cardiac toxemia, ii. 252 .

Struma inflammatoria, iii. 891.

Strychnia. See Strychnin.

Strychnin, use of, in acute opium poisoning, ii. 615 ; in acute rheumatism, ii. 300 ; in acute vasomotor paralysis, ineffectual, iii. 753; in after-treatment of anterior poliomyelitis, iv. 241 ; in after-treatment of herpes zoster, iv. 359 ; in asthenic bulbar paralysis, iv. 256 ; in barbel cholera, ii. 644; in bronchopneumonia complicatiug influenza, ii. 192; in cardiac failure complicating influenza, ii. 193; in cardiac insufficiency, iii. 614; as cardiac stimulant in beri-beri, iv. 853,854 ; as cardiac stimulant in blackwater fever, iv. 741; as cardiac stimulant in bronchopneumonia, iii. 420; as cardiac stimulant in cerebral embolism, iv. 402; as cardiac stimulant in cerebral thrombosis, iv. 398; as cardiac stimulant in malarial fever, iv. 727; as cardiac stimulant in Rocky Mountain fever, ii. 565,
566 ; in cerebrospinal meningitis, ii. 216; in cervical adenitis complicating scarlet fever, ii. 131; in cheese poisoning, ii. 647; in chronic myelitis, iv. 230; in chronic ophthalmoplegia, iv. 510 ; in chronic opium poisoning, ii. 193; in chronic progressive bulbar paralysis, iv. 252; in collapse during acute stage of bacillary dysentery, ii. 644 ; in convalescence from scarlet fever, ii. 128; in convalescence from small-pox, ii. 47 ; in delirium tremens, ii. 606, 607; in diabetes insipidus, ii. 777 ; in diphtheria, ii. 277 ; in enteritis of infancy, iii. 203; in facial paralysis, iv. 313 ; in funicular myelitis, iv. 232; in gastric atony, iii. 172; in hay fever, iii. 390 ; in hemophilia, iii. 869 ; by hypodermic injection of, in acquired spinal progressive muscular atrophy, iv. 247 ; by hypodermic injection in acute alcoholism, ii. 589; by hypodermic injection, in chronic bronchitis, iii. 378; by hypodermic injection, in fish poisoning, iv. 684 ; by hypodermic injection, in optic atrophy, iv. 307 ; by hypodermic injection, in progressive bulbar paralysis, iv. 499; by hypodermic injection, for relief of cyanosis in acute bronchitis, iii. 363 ; in hypostatic congestion of lung, iii. 407; in Landry's paralysis, iv. 329 ; in multiple neuritis, iv. 326 ; in myasthenia gravis, iv. 507; in neurasthenia, iv. 558; in neurasthenia with vasomotor relaxation, iv. 559 ; in neuritis, iv. 289 ; in nervous type of influenza, ii. 191; objections to, in multiple sclerosis, iv. 281; in occupation pareses, iv. 332; in optic neuritis without apparent cause, iv. 306; in phlegmonous pharyngitis, iii. 40; in phrenic neuritis, iv. 289; in pneumonia with cardiac toxemia, ii. 251 ; in poisoning by amanita phalloides, ii. 649 ; in post-diphtheritic paralysis, ii. 278; to prevent collapse in snake poisoning, iv. 683; as prophylactic measure in cardiac asthma due to chronic myocardial insufficiency, iii. 737 ; in pulmonary thrombosis, iii. 411 ; to raise blood pressure, ii. 255; for relief of dyspnea in tuberculosis, ii. 506; for relief of gastric stasis in chlorosis, iii. 823; for relief of insomnia caused by coughing in chronic bronchitis, iii. 374 ; in scarlet fever with 
cardiac weakness, ii. 127 ; in septicemia, ii. 291 ; to stimulate atonic stomach, iii. 180; in stomatitis diphtheritica, iii. 20; in stomatitis gangrenosa, iii. 18; in syringomyclia, iv. 279 ; in tabes, iv. 195, 213 ; as tonic in tuberculosis, ii. 504; in toxemia complicating acute diffuse peritonitis, iii. 318 ; in treatment of enuresis with atony of sphincter, iv. 133; in typhoid fever, ii. 47; in wood alcohol poisoning, ii. 610 .

Strychnin and apomorphin, use of, in acute alcoholism, ii. 589.

Strychnin and atropin, use of, in chronic alcoholism, ii. 595; to counteract symptoms due to pilocarpin, iv. 317 ; by hypodermic injection in curare poisoning, iv. 694 .

Strychnin and capsicum, use of, in acute alcoholism, ii. 589.

Strychnin and digitalis, use of, in cardiac failure complicating delirium tremens, ii. 606,607 ; in meat poisoning, ii. 640.

Strychnin poisoning, i. 746; diagnosis of, i. 749 ; elimination of poison in, i. 750 ; poisonous action of drug in, i. 747; poisonous dose in, i. 748 ; post-mortem appearances in, i. 750; time required for action of poison in, i. 748; treatment of, i. 749 .

Strychnin sulphate, injection of, in tabetic amaurosis, iv. 199.

Strychnos Poisoning, iv. 694.

Stupes, use of, in spasmodic laryngitis, iii. 342.

Stypticin, use of, hypodermically, to control epistaxis, iii. 336.

Styptics, objections to use of, in control of gastric hemorrhage, iii. 112.

Subarachnoid injections. See Injection, subarachnoid.

Subcutaneous injection. See Injection, hypodermic.

Subcutaneous method, for injection of defibrinated blood, iii. 831 .

Subinvolution, electrotherapeutics for relief of, i. 419.

"Submeried Tonsil," importance of removing, in chronic myocardial insufficiency due to valvular lesions as a prevention of endocarditis, iii. 734 .

Substance sensibilisatrice, i. 128.
Substances which form complex insoluble colloids, uke of, to control hemorrhage in hemophilia, iii. 8633 .

Substitution therapy, mininistration of thyroid gland an instance of, i. 70.

Sucking of wound, in snake bite, iv. 652.

SUCTION-PUMP ACTION OF H:Alt, iii. 513.

Sudorifics, use of, in anuria, iv. 3.

Sugar, as cause of diarrhea in bottle-fed infants, iii. 202 ; in dict for acute nephritis, iv. 60; in dict for chronic parenchymatous nephritis, iv. 6.5 ; in diet for gastric ulcer, iii. 129; sources of, in body, ii. 725 ; subcutaneous administration of, i. 640 ; substitutes for, in diabetes mellitus, ii. 749 .

Sugar, milk. See Milk sugar.

Suggestion, hypnotic. See Hypnotic suggestion.

Suggestion therapy, i. 559; autosuggestion in, i. 560; definition of, i. 559; hypnotism as a means of, i. 560; post-hypnotic suggestion and, i. 574; simple suggestion as a means of, i. 559 .

Surcide, danger of, in acute hallucinosis of drunkards, ii. 608 ; following hematochyluria, iv. 787; phosphorus taken for purpose of, ii. 626.

Sulphemoglobinemia, iii. 879 .

Sulphid of soda and antimony. See Schlippe's salt.

Sulphites, preservative action of, i. 729.

Sulphonal, use of, in after-treatment of morphinism, ii. 624; in cerebrospinal meningitis, ii. 215; combined with an analgesic, for relief of lancinating pains in tabes, iv. 196; contraindicated in cardiac insufficiency, iii. 664; in delirium tremens, ii. 606,607 ; in destruction of mosquitoes by fumigation, iv. 735 ; hematoporphyrinuria due to, iv. 5 ; to induce sleep in acute bronchitis, iii. 361 ; for relief of insomnia associated with mediastinal tumors, iii. 504; for relief of insomnia due to brain tumor, iv. 447 ; for relief of insomnia in neurasthenia, iv. 559 .

Sulphonethylmethane, use of, in nervous type of influenza, ii. 191.

Sulphur, use of, in chronic constipation, iii. 234 ; to promote evacuation of bowels in gastric atony, iii. 172 .

Sulphur dioxid, fumigation with, as protection against plague, iv. 825 . 
Sulphur ointment, use of, in craw-craw, iv. 692.

Sulphur waters, action of, i. 236 ; classification of, i. 234.

SulphuRIC ACID, POISONING BY, i. 724; symptoms of, i. 724; treatment of, i. 726 .

SulPhurous aCID AND THE SUlPhites, POISONING BY, i. 726: effects of sodium sulphite on animals, i. 727; effects of the sulphite on stomach and digestion, i. 730; poisonous effects of the gas, i. 727 ; properties and uses, i. 726.

Sun, as source of radiant energy, i. 438.

SuniJGHT, TROPICAL, effects of, upon the white man, iv. 669.

Sunstroke. See Insolation.

Superacidity of stomach. See Hyperacidity.

Superalimentation, effects of, i. 635; in neurasthenia, iv. 538; in treatment of movable spleen, iii. 887 ; in tuberculosis, ii. 478,538 .

Superficial Foramina, alcohol injection at, iv. 344 .

Suppositories, administration of caffein by, in nephritis, iv. 50; administration of calomel by, in chancre of rectum, ii. 424 ; administration of mercury by, in syphilis, ii. 387 ; administration of opium by, in bacillary dysentery, ii. 327 ; objections to, for relief of constipation in neurasthenia, iv. 566; use of, in chronic constipation, iii. 235.

Supracapsulin. See Epinephrin.

SUPRAORBITAL BRANCH, alcohol injection of, for relief of trigeminal neuralgia, iv. 344 .

Suprarenal extract, use of, necessity for caution in, in acute rhinitis, iii. 328; in neurasthenia with vasomotor relaxation, iv. 559; on tampon to control epistaxis, iii. 335 .

SUPRARENAL GLAND, relation between secretion of, and rickets, ii. 813; use of, in acute rhinitis, iii. 327 .

Suprarenal glands in organotherapy, i. 88; conditions benefited by use of, i. 96,98 , 99 ; conditions characterized by insufficiency of, i. 96 ; effects of administration of, i. 90; effects of disease in, i. 88; effects of extirpation of, in animals, i. 89; experimental use of, i. 95; function of, i. 94; preparations of, i. 100; untoward effects of, i. 100.
Suprarenalin. See Epinephrin.

Suprarenin. See Epinephrin.

Surgery, coöperation between X-ray therapy and, i. 538, 541, 545; in treatment of the neuroses, iv. 526 .

Surgical measures. See Surgical operation.

SURgical operation, danger of, in diabetic patients, ii. 769 ; in status lymphaticus, iii. 912.

Surgical PROPHYlaXIs, against cerebral abscess, iv. 422.

Surgical tonsil, iii. 46.

Suspension, contraindicated in syringomyelia, iv. 278; as a therapeutic agent in tabes, iv. 203; uses of, in mechanotherapy, i. 359.

Suspension colloids, i. 6, 28.

Suspensory, use of, in acute gonorrhea, as a preventive of epididymitis, iv. 150; necessity for, in acute stage of gonorrhea, iv. 138; for neuralgia affecting spermatic cord, iv. 357 ; for pruritus of scrotum in diabetes mellitus, ii. 765 .

Sutherland, G. A., on benefits of cod liver oil in chronic bronchitis, iii. 371 .

SutTon AND Fewster, inoculation of immune individual with small-pox by, ii. 57 .

Svapnia, use of, for neuralgia accompanied by restlessness, iv. 336 .

Sweating, use of, in acute nephritis, iv. 61; for eclampsia of pregnancy, iv. 83 ; in kidney of pregnancy, iv. 82 ; method of producing, in nephritis, iv. 38 ; in miliary fever, ii. 578; in nephritis, iv. 37 ; to reduce hypertension in chronic interstitial nephritis, iv. 69 ; for relief of anuria in acute nephritis, iv. 62 ; for relief of dyspnea in acute nephritis, iv. 63; for relief of dyspnea in chronic interstitial nephritis, iv. 72.

Sweating, EXCEssive, due to chronic myocardial insufficiency, iii. 740.

Sweats, Night, treatment of, in tuberculous kidney, iv. 117.

Sweden, introduction of vaccination into, ii. 60; mortality from small-pox in, before and after vaccination, ii. 94, 106.

Swedish movements, use of, in prophylaxis of chronic cerebral hyperemia, iv. 387 ; for relief of cerebral hyperemia, iv. 384 ; in rest cure for neurasthenia, iv. 540 .

Sweeping AND Dusting, rules for, in tuberculosis, ii. 467. 
Swimming, injurious effects of, in arteriosclerosis, iii. 761 .

Swinburne's urethroscope, use of, in treatment of verumontanum, iv. 167.

SwITzerLaNd, introduction of vaceination into, ii. 60.

Sycosis simplex, X-ray therapy for relief of, i. 530.

Symbiotic bacteria, rôle of, in amebiasis, iv. 706.

Sympathomimetic drugs, i. 69.

Sympathy and encouragement, employment of, in neurasthenia and psychasthenia, iv. 549.

Syncope, due to cerebral thrombosis, iv. 398.

Synovitis, electrotherapeutics for relief of, i. 413.

Synthetic POISONS, i. 674.

Syphilids, palmar and plantar, local treatment of, ii. 429 .

Syphilis, ii. 361 ; abortive treatment of, ii. 376 ; administration of mercury by mouth in, ii. 385 ; alcohol in, ii. 369 ; arsenic in treatment of, ii. 411 ; arsenobenzol in treatment of, ii. 412; atoxyl in treatment of, ii. 412 ; auxiliary medication in, ii. 435; as a cause of acquired spinal progressive muscular atrophy, iv. 246 ; as cause of acute nephritis, iv. 27; as a cause of amyloid diseases of kidney, iv. 81 ; as cause of chronic cerebral meningitis, iv. 378; as cause of chronic myelitis, iv. 229; as a cause of dementia paralytica, iv. 489 ; as a cause of general paresis, iv. 489; as a cause of neuralgia, iv. 337; as cause of neuritis of third, fourth, and sixth nerves, iv. 308; as cause of paroxysmal hemoglobinuria, iv. 6 ; as cause of pneumogastric paralysis, iv. 319 ; as cause of trigeminal neuritis, iv. 309; classifications of patients in, ii. 366 ; as a complication of vaccination, ii. 87; constitutional treatment of, ii. 416; contagiousness of lesions in, ii. 371 ; contagiousness of normal secretions in, ii. 371; continuous mercurial treatment of, ii. 417; continuous intermit tent treatment for, ii. 418; cutaneous administration of mercury in, ii. 387 ; diabetes associated with, ii. 768 ; divisions of treatment for, ii. 363; educational prophylaxis against, ii. 375 ; as a factor in cerchral hemorrhage, iv. 389; factors predingosing to infection from, ii. 370 ; hydrotherapy for relief of, i. 216; influence of other maladies upon, ii. 370; injection methed of ardministering mercury in, ii. 3990; intermittent mercurial treatment for, ii. 417; inunction method of administering mercury in, ii. 387; iodids in treatment of, ii. 404 ; local treatment of lesions in, ii. 421 ; marital prophylaxis against, ii. 376 ; mercurial baths in treatment of, ii. 388, 433; mercurial preparations suitable for use in, ii. 385; ; mercury in cure of, see Mercury; nervous hygiene in, of myocardium, see Myocardial syphilis; of muscles, see Muscles; nervous hygiene in, ii. 366; oral hygiene in, ii. 368; organotherapy for, ii. 436; of pancreas, iii. 301 ; personal hygiene in, ii. 365; prophylaxis against brain tumor in, iv. 446; prophylaxis against, ingeneral, ii. 370 ; public prophylaxis against, ii. 374 ; question of eure in, ii. 420 ; rachitis associated with, ii. 814 ; rectal administration of mercury in, ii. 387 ; registration and examination as means of prophylaxis against, ii. 375 ; resemblance of, to frambœsia tropica, iv. 762 ; secondary cutaneous symptoms of, ii. 425; serum therapy for, ii. 436; specific medication for, ii. 380; of spinal blood-vessels, iv. 272; of stomach, see Stomach; tobacco smoking injurious in, ii. 369 ; tonies in treatment of, ii. 435 ; treatment of hereditary, ii. 432 ; treatment of, in pregnaney, ii. 432 ; treatment of secondary cutaneous symptoms of, ii. 425; treatment of tertiary cutaneous symptoms of, ii. 426; vaccination for small-pox a means of transmitting, ii. 372 ; vaccine therapy for, ii. 437; Wassermann's reaction as test for, ii. $379,420$.

SyPhilis, hereditary, treatment of, ii. 432; arsenobenzol in, ii. 433; hygienic measures in, ii. 433; injeetions unsuitable in, ii. 434; inunctions best suited for, ii. 434 ; iodids in, ii. 434 ; mereury in, ii. 434 ; mixed treatment in, ii. 434 .

Syphilis, hygiene of, ii. 364 ; alcohol injurious to, ii. 369 ; importance of, ii. 365 ; influence of other diseases, local and general, upon, ii. 370; mouth hygiene in, ii..36s; nervous hygiene in, 
ii. 366 ; physical hygiene in, ii. 365 ; sexual hygiene in, ii. 368 ; tobacco injurious in, ii. 369 .

SyPHILIS OF UPPER RESPIRATORY TRACT, iii. 348; general treatment of, iii. 348 ; local treatment of, iii. 349.

Syphilitic ARTERITIs, iii. 763: treatment of, iii. 763 .

SyPhilitic arthritis, ii. 306.

Syphilitic disease of liver, iii. 264.

Syphilitic endarteritis, cerebral thrombosis due to, iv. 395,475 ; prognosis of, iv. 397 ; treatment of, iv. 398.

Syphilitic headache. See Headache.

Syphilitic hemiplegia, iv. 475.

Syphilitic hypertrophic PAPULE, local treatment of, ii. 428.

Syphilitic lesions, local treatment of, ii. 421 ; of chancre, ii. 422 ; of secondary cutaneous symptoms, ii. 425 ; of secondary mucous symptoms, ii. 426 ; of special secondary and tertiary lesions, ii. 427; of tertiary cutaneous symptoms, ii. 426 ; of tertiary mucous symptoms, ii. 427.

Syphilitic Rheumatism. See Arthritis, syphilitic.

Syphilitic spinal Paralysis, iv. 271.

Syphilitic stomatitis. See Stomatitis syphilitica.

Syphilitic TUBercle, local treatment of, ii. 428 .

Syphiloderms, local treatment of, ii. 425 .

Syphiloma. See Brain tumor.

Syringe, hypodermic, for use in intramuscular injection of mercury in syphilis, ii. 399 .

Syringes, asepsis of, iv. 159.

Syringomyelia, iv. 275; definition of, iv. 275 ; etiology of, iv. 276 ; morbid anatomy of, iv. 275; operative measures for, iv. 279 ; symptoms of, iv. 277 ; treatment of, iv. 278; X-ray therapy for relief of, $i$. 554.

Syrup of iodid of iron, use of, in hookworm disease, iv. 794 .

Syzygium jambulanum, use of, in diabetes mellitus, ii. 760 .

TABES, iv. 180; analgesia as symptom of, iv. 188; anesthesia as symptom of, iv. 188; Argyll-Robertson pupil in diagnosis of, iv. 187; arthropathies in, iv. 191; causal therapy for, iv. 193 ; causes of, iv.
180; climatic influence in, iv. 213; course of disease in, iv. 184; definition of, iv. 180; delayed sensation as symptom of, iv. 189; diagnosis of, iv. 184; diet in, iv. 213 ; electricity in treatment of, iv. 201 ; lancinating pains in, iv. 188; lesions in, iv. 182 ; massage in treatment for, iv. 203; medicinal springs of benefit in, iv. 201; operative treatment for, iv. 198; paresthesia as symptom of, iv. 189; plan of treatment important in, iv. 211; reëducation of ataxic extremities in, iv. 204; Romberg's sign in diagnosis of, iv. 188; suspension in treatment of, iv. 203; symptomatic treatment of, iv. 196 ; symptomatology of, iv. 186 ; tendon reflex in diagnosis of, iv. 187; treatment against morbid process in, iv. 195 ; treatment of, in general, iv. 192, 200; treatment of special symptoms in, iv. 199; trophic disturbances in, iv. 191.

Tabetic amaurosis, iv. 199.

TABETIC FORM OF SPINAL SYPhILIS, iv. 272. TABLE, showing action of carbon dioxid baths upon blood pressure and pulse, iii. 619, 620; showing amount of albumen contained in different foods, iv. 40 , 41; showing amount of fluid removed from case of mitral insufficiency, iii. 675; showing amount of salt in articles of average diet, iv. 36 ; showing chemicals used for irrigation of urethra and their various strengths, iv. 159; showing death rate from consumption in certain occupations, iii. 425; showing death rate following operation for liver abscess, iv. 711; showing death rate from respiratory diseases other than phthisis in certain occupations, iii. 427; showing dietary in pericarditis, iii. 516; showing dose of thymol according to age in hookworm disease, iv. 794; showing effect of erythrol tetranitrate upon blood pressure, iii. 610; showing effect of nitrites on blood pressure, iii. 611; showing effect of nitroglycerin on blood pressure, iii. 607, 608; showing effect of potassium nitrite on blood pressure, iii. 609 ; showing effect of sodium nitrite on blood pressure, iii. 609; showing events of cardiac cycle, iii. 543; showing latent malarial infection at different ages in different districts, iv. 736; showing loss 
of body heat by radiation and conduction, iv. 674; showing reeord of series of Nauheim baths, iii. 6333; showing results of operation for pulmonary abscess and gangrene, iii. 442; showing results of $\mathrm{X}$-ray therapy in leukemia, iii. 849, 850; showing series of Nuuheim baths, iii. 632 ; showing similarity and differenees in the types of museular dystrophy, iv. 262; showing solubility and alkaloidal value of the most important salts of quinin, iv. 719 ; showing tropieal possessions of western nations, iv. 668; showing typical amyl nitrite effect, iii. 612,613 .

TÁhe cérébrale, iv. 374.

TACHYCARDIA, as a complication of exophthalmic goiter, iii. 898 ; as a complication of pneumonia, ii. 260; essential paroxysmal, iii. 786; treatment of essential paroxysmal, iii. 787 .

Tenia africana, iv. 776; mode of infection by, iv. 777.

TENIA BREMNERI, iv. 777.

T ENIA HOMINIS, iv. 777.

Tenia Philippina, iv. 777.

Tampon, use of, in arrest of epistaxis, iii. 335 ; in atrophic rhinitis, iii. 332 .

TANKs, asepsis of, iv. 159.

Tannalbin, use of, to control diarrhea due to ehronic myocardial insufficiency, iii. 739.

Tannic acid, use of, in acute nasopharyngitis accompanying uvulitis, iii. 31 ; in curare poisoning, iv. 694 ; in diarrhea, ii. 162; in diarrhea of Addison's disease, iii. 885; as gargle in measles, ii. 148; in hematuria, iv. 4 ; for irrigation of bowels in bacillary dysentery, ii. 327 ; for irrigation of colon in colitis, iii. 205; for irrigation of colon in ulcerative colitis, iii. 211,212 ; in pulmonary abscess, iii. 441 ; by rectal injection in chronic mucous colitis, iii. 207.

Tannic acid and glycerin, use of, in pyorrhea alveolaris, iii. 22 .

Tannigen, use of, to control diarrhea due to chronic myocardial insufficiency, iii. 739.

Tannin, use of, as an antidote to musearin poisoning, ii. 648; in ergotism, ii. 652 ; to neutralize morphin in the stomaeh, ii. 615; in stomatitis catarrhalis, iii. 7 .

Tannogallic acid, use of, on tampon to control epistaxis, iii. 335 .
TAPEWORMS, LARVAL, iv. 775.

Tapping, necessity for, in generalized form of chronic peritonitis, iii. 323; precautions to be taken in performance of, iii. 476; for reliof of edema in acute nephritis, iv. 62 ; for relicef of effusion in malignant disease of peritoneum, iii. 323; for relief of pleural ceffusion following a pulmonary infarct, iii. 412 ; use of, contraindicated in tuberculous peritonitis, with effusion, iii. 321 .

Tar, use of, as expectorant in chronic bronchitis, iii. 377 .

Tarbagan disease, iv. 821 .

TARDIEU, poisoning from match heads reported by, ii. 627 .

Tartar emetic, use of, in active congestion of lungs, iii. 406; in acute laryngitis, iii. 339; in bronchopneumonia, iii. 419 ; by intravenous injection in trypanosomiasis, iv. 747.

Taste, disturbances of, iii. 4.

TaWARA SYSTEM OF MUSCle Fibers, iii. 540.

TAylor, A. E., on autointoxication due to gastrointestinal infeetion, ii. 664 .

TEA, as a cause of reflex cardiac neuroses, iii. 786; in diet for ehronic parenchymatous nephritis, iv. 66 ; in diet for gastric hyperacidity, iii. 91 ; in diet for nephritis, iv. 44, 59; over-indulgence in, a cause of chronie gastritis, iii. 159 ; physical effeets of, iii. 603 ; use of strong green, in acute opium poisoning, ii. 615.

TEa AND COFFEe, excessive use of, a cause of angina pectoris vasomotoria, iii. 768 ; excessive use of, injurious in arteriosclerosis, iii. 759; prohibition of, in aneurysm of cerebral arteries, iv. 457; prohibition of, in asthma, iii. 391 ; prohibition of, in palpitation of heart due to neurasthenia, iv. 567 ; prohibition of, in progressive paralysis of the insane, iv. 493; prohibition of, for relief of tachycardia, iii. 787 ; use of, in cardiac insufficieney, iii. 686 .

Tebean, i. 151.

Teeth, care of, a factor in prevention of acute rheumatism, ii. 296 ; care of, a necessity in cure of tuberculosis, ii. 490; disease of, a cause of chronic gastritis, iii. 159; method of cleaning, in infants and young children, iii. 2 ; necessity for attention to, in seurvy, iii. $\$ 75$; necessity 
for attention to, in pernicious anemia, iii. 825,837 ; necessity for attention to, in stomatitis ulcerosa, iii. 15; sharpedged, a cause of leukoplakia, iii. 26.

TeEth AND Gums, care of, during mercurial treatment of syphilis, iv. 480.

Teixera de Mattos, buttermilk as food in enteritis of infancy first recommended by, iii. 204.

Temperature, for baths given in soothing hydrotherapy for neurasthenia, iv. 546; for baths given in stimulating hydrotherapy for neurasthenia, iv. 551; for baths from therapeutic standpoint, iv. 546 ; as a climatic factor, i. 247 ; of injection employed in Fischer's method of relieving anuria, iv. 838; of injection employed in Rogers' treatment of Asiatic cholera, iv. 835; of mineral waters, i. 231; physiological reaction of human beings to external, i. 246 ; relation of external, to wind, ii. 254; of room important in acute bronchitis, iii. 352 .

Temperature of BODY, importance of, during stage of reaction in Asiatic cholera, iv. 836; influence of poisons upon, i. 667 ; local reduction of, beneficial in acute localized peritonitis, iii. 311; relation of, to treatment by injections in Asiatic cholera, iv. 835; variations in, during hypnosis, i. 574; variations in, during residence in opposite extremes of climate, i. 261.

Temporal bone, disease of, causing neuritis of seventh nerve, iv. 310 .

Temporal lobe, diagnosis of tumor in, iv. 439.

Temulin, poisonous action of, ii. 650 .

Tenderness on pressure, as a symptom of tumor in frontal lobe, iv. 439; as a symptom of tumor in parietal lobe, iv. 439.

Tender toes, in typhoid fever, ii. 29.

Tendon grafting, performance of, to correct deformities following anterior poliomyelitis, iv. 242.

Tendon Reflex, absence of, a diagnostic sign of Friedreich's ataxia, iv. 216; absence of, as diagnostic sign of tabes, iv. 187; changes in, during tuberculous meningitis, iv. 374; exaggeration of, in hereditary cerebellar ataxia, iv. 217; exaggeration of, in multiple sclerosis, iv. 280; exaggeration of, in spastic spinal paralysis, iv. 268; exaggeration of, in traumatic hematoma, iv. 366.

Tendon reflexes, changes in, in acute hemorrhagic supcrior polioencephalitis, iv. 406; changes in, during progressive paralysis of the insane, iv. 490; decrease of, in progressive bulbar paralysis, iv. 497; exaggeration of, in cerebral diplegia type of cerebral palsies of children, iv. 410.

Tendon transplantation, for relief of anterior crural or obdurator neuritis, iv. 294.

Tenesmus, treatment of, in bacillary dysentery, ii. 327 .

Teniasis, iv. 775; intestinal, iv. 776 ; somatic, iv. 775 .

Tenosynovitis, electrotherapeutics for relief of, i. 414.

Tenotomy, indications for performance of, in neuritis, iv. 289 ; for relief of contractures acquired during beri-beri, iv. 854; for relief of contractures in multiple sclerosis, iv. 281; for relief of deformity following anterior poliomyelitis, iv. 240 .

Teratomata of mediastinum, iii. 500.

Terebene, use of, as expectorant in bronchiectasis, iii. 396; as expectorant in chronic bronchitis, iii. 377; for relief of gangrene of lung complicating pneumonia, ii. 261 ; in treatment of bronchorrhea complicating pneumonia, ii. 261.

Terrain cure, iii. 645.

Tesla apparatus, i. 368.

Test diet of Schmidt, iii. 198.

Testicles, elevation of, in acute gonorrhea as preventive of epididymitis, iv. 150; hydrotherapy for disease of, i. 212; uses of, in organotherapy, i. 115.

Tetanus, ii. 344; antitoxin in treatment of, ii. 344; Bacelli's method of treating, ii. 349 ; cases of, treated with antitoxin and chloretone, ii. 354 ; cases of, treated with antitoxin and sedatives, ii. 351 ; cases of, treated with subdural injection of magnesium sulphate, ii. 348; as a complication of vaccination for small-pox, ii. 90; danger of, from use of gelatin for control of hemorrhage, ii. 155; dose of antitoxin for, ii. 346, 347; drugs in treatment for, ii. 347; feeding in, ii. 350 ; general measures of treatment for, ii. 350 ; method of injecting 
antitoxin for, ii. 345; mortality from, ii. 350 ; nursing in, ii. 350 ; prevention of, ii. 347 ; results of antitoxin treatment for, ii. 350; rules for use of antitoxin in, ii. 350 ; single and multiple injections of antitoxin in, ii. 346 ; symptomatic treatment of, ii. 349 ; treatment of actual cases of, ii. 351 ; treatment of infectred area in, ii. 347.

Tetanolysin, i. 158.

Tetanospasmin, i. 158.

Tetanus antitoxin, i. 158, ii. 344 ; administration of, i. 159 ; administration of, in doubtful cases, ii. 351 ; curative dose of, ii. 346 ; method of injecting, ii. 345 ; mortality affected by, ii. 350 ; preventive dose of, ii. 347 ; production of, i. 158 ; rules for use of, ii. 350 ; secretion of, i. 158; single or multiple injections of, ii. 346 ; standardization of, i. 159 ; uses of, ii. 344.

Tetany, iii. 909; as complication of rachitis, ii. 819; due to loss of parathyroid glands, i. 84; gastric, see Gastric tetany; treatment of, i. 85 .

Teufel bandage, iii. 248.

Thallin sulphate, use of, by instillation in chronic gonorrheal urethritis, iv. 160; in tubercular cystitis, iv. 121.

Theobald Smith phenomenon, i. 132.

Theobromin, physiological action of, iii. 605; therapeutic aetion of, iii. 605; use of, in angina pectoris, iii. 770 ; use of, in chronic myocardial insufficiency due to nephritis, iii. 730 ; use of, to induce diuresis in cardiac insufficiency, iii. 677; use of, for relief of insomnia in neurasthenia, iv. 565 ; use of, in severe cases of chronic myocardial insufficiency, iii. 722 .

Theobromin and sodium benzoate, use of, to induce diuresis in cardiac insufficiency with general anasarca, iii. 677.

Theobromin-sodiosalicylate. See Diuretin. Theocin, use of, in diabetes insipidus, ii. 777 ; in nephritis, iv. 49,50 ; in nephritis complicating scarlet fever, ii. 134; for relief of anuria in nephrolithiasis, iv. 107. See also Sodium acetate.

Theophyllin, physiological action of, iii. 605; therapeutic action of, iii. 605 ; use of, in acute nephritis, iv. 58; use of, in angina pectoris, iii. 770; use of, in ehronic myocardial insufficiency due to nephritis, iii.
730; use of, to create diuresis in eardiac insufficiency with gencral anasarea, iii. 677; use of, in severe cases of chronic myocardial insufficirncy, iii. 722 .

Theophyllin and sodium acetate, use of, to creaste diuresis in cardiar: insufficiency with gencral ansusarca, iii. 677.

'Tuerapeté prixciples, generally accepted in the neuroses, iv. 53:2.

Therapeutic procedures, supplementary, for use in nourasthenia and psychasthenia, iv. 55i).

Therapeutic: TEst, value of, in diagnosis of brain syphilis, iv. 477.

Thermal waters, simple, i. 232.

THERnic EFFEcts of Electurucity, therapeutic indications for, i. 393.

Thenuc frver. See Insolation.

Thermocautery, use of, over spine in lathyrism, iv. 693.

Thermophor, use of, in chronic pancreatitis, iii. 298.

Thiersch, avulsion method of. See Avulsion method.

Thiosinamin, intramuscular injection of, to stimulate absorption of pleural exudate, iii. 482 .

Thompson, report by, on reduction in mortality in typhoid fever treated with Brand baths, i. 219.

Thompstone and Bennetr, hyperpyrexial fever first deseribed by, iv. 86.8 .

Thomsen's Disease. See Myotonia congenita.

Thomson, on use of earbolic acid in plague, iv. 827 .

Thoracentesis in pleurisy with effusion, iii. 469 ; amount of fluid withdrawn in, iii. 477; apparatus for exploration in, iii. 475; aspiration of fluid in, iii. 475 ; choice of instruments for, iii. 474 ; choice of time for, iii. 472; collapse and sudden death during or after, iii. 4\$0; contraindications to, iii. 472 ; dangerous symptoms during, iii. 47s; history of, iii. 455; indications for, in large effusions with slow absorption, iii. 471 ; indieations for, in moderate effusions with slow absorption, iii. 472 ; indications for, to save life, iii. 471; intrapleural injections during, to aid absorption, iii. 4\$2; location of puncture in, iii. 473 ; method of evacuation in, iii. 475; pneumothorax caused 
by, iii. 491 ; position of patient in, iii. 473; precautions against collapse in, iii. 481; precautions in tapping in, iii. 476; preparations for, iii. 474; rate for withdrawal of fluid in, iii. 477; untoward symptoms during, iii. 477; uselessness of, in empyema, iii. 485.

Thoracentesis in pneumothorax acutissimus, iii. 491.

Thoracotomy, performance of, for relief of empyema accompanying tuberculosis, ii. 507; for relief of empyema, iii. 485; for relief of suppurative mediastinal tumor, iii. 504.

"Three valerianates" pill, use of, in neurasthenia, iv. 558.

ThroAt AFFECTIONS, as a complication of scarlet fever, ii. 129.

Thrombosis, as a cause of cerebral hemorrhage, iv. 389; cerebral, see Cerebral thrombosis; diagnosis of, from embolism, iv. 397; of lungs, see Pulmonary thrombosis; venous, as a complication of chlorosis, iii. 811.

Thrush. See Stomatitis hypermycetica.

Thymectomy, employment of, in status lymphaticus, iii. 912 .

Thymol, use of, as an anthelmintic in Cochin China diarrhea, iv. 797; as an anthelmintic in expulsion of esophagostomum apiostomum, iv. 800; as an anthelmintic in destruction of filaria bancrofti, iv. 787; as an anthelmintic in hookworm disease, iv. 793; as an anthelmintic in intestinal distomiasis, iv. 782; as an anthelmintic in tricocephaliasis, iv. 798; as an antiseptic in intestinal autointoxication, ii. 675 ; in atrophic rhinitis, iii. 331 ; in chronic atrophic laryngitis, iii. 343; for gastric lavage in chronic gastritis, iii. 161 ; local use of, for irrigating bowels in chronic stage of bacillary dysentery, ii. 327 ; local use of, for relief of itching complicating diabetes mellitus, ii. 764; local use of, as a spray for throat in scarlet fever, ii. 129 ; use of, in stomatitis gangrenosa, iii. 18.

Thymus GLAND, conditions associated with disturbance of, i. 118; diseases of, iii. 911; extirpation of, suggested for relief of asthenic bulbar paralysis, iv. 256; functions of, i. 117; supposed relation between disturbance of, and rachitis, ii.
813; X-ray therapy for enlargement of, i. 552 .

Thymus gland, conditions benefited by administration of, i. 118; in organotherapy, i. 117; therapeutic uses of, ii. 118; use of, in exophthalmic goiter, iii. 902; use of extract of, in treatment of arthritis deformans and chronic rheumatism, ii. 713 .

Thyradin, i. 82 .

Thyreopriva. See Cachexia strumipriva.

THYROID GLAND, amount of secretion in, $i$. 67 ; congestion of, iii. 891 ; effects of deficiency of secretion of, in adults, i. 72; effects of deficiency of secretion of, in child, i. 71; functions of, i. 70; hypersecretion of, i. 74; method of removing, in tetany, iii. 910; relation of, to growth and development, i. 71 ; removal of, followed by hypertrophy of hypophysis, $i$. 103; stability of secretion from, i. 68; supposed relation between disturbance of, and rachitis, ii. 813; transplantation of, in athyrea and hypothyrea, iii. 906; X-ray therapy for relief of enlargement in, i. 552 .

Thyroid gland, as a therapeutic measure, i. 70,74 ; administration of, an instance of substitution therapy, i. 70; in arthritis deformans and chronic rheumatism, ii. 713; in athyrea and hypothyrea, iii. 906; basis for, i. 70; chemistry of, i. 78; conditions benefited by, i. 76; contraindications to, i. 83; in dipsomania, ii. 601; in exophthalmic goiter, iii. 902; indications for, i. 74; influence of, on diabetes mellitus, i. 766; in kidney of pregnancy, iv. 83; methods of administrating, i. 77; preparations of, i. 80 ; in reduction of obesity, ii. 786, 809; untoward effects following, i. 83.

Thyroid gland, desiccated, use of, for relief of enuresis, iv. 131.

Thyroid GLAND, Diseases of, iii. 891: athyrea, iii. 906; congestion of thyroid gland, iii. 891; goiter, iii. 891; hypothyrea, iii. 906; struma inflammatoria, iii. 891; thyroiditis acuta, iii. 891 .

Thyroid insufficiency, as a cause of dipsomania, ii. 601.

Thyroid preparations, use of, in goiter, iii. 894. 
Thyroidin, use of, in athyrea and hypothyrea, iii. 907.

Thyroidin-Merck, i. 81.

Thyroidin-Notkin, i. 81.

Thyroiditis acUta, iii. 891.

Thyrotomy, employment of, in laryngeal tubereulosis, iii. 347.

Tic, iv. 653; aleohol injections for relief of, iv. 654,659 ; artificially indueed rest for relief of, iv. 659; cause of movements in, iv. 657; definition of, iv. 653; exereises for relief of, iv. 656, 658; psychotherapy for relief of, iv. 659 ; surgical treatment of, iv. 656 ; tic eonvulsif, iv. 653 ; tie douloureux, iv. 653 ; treatment of, in general, iv. 657; type of movement in, iv. 653 .

Tic Douloureux, iv. 653 ; electrotherapeuties for relief of, i. 417 .

Tick fever. See Tropical relapsing fever.

Ticks, as earriers of African relapsing fever, iv. 759 .

Tigerstedt and Bergman, presence of "renin" in kidney extracts first reported by, iv. 13.

Tight LACing, as cause of movable kidney, iv. 2.

Time relation of Cardiac SYstole and Diastole, iii. 543.

Tin, poisoning by, i. 701.

TineA, X-ray therapy for relief of, i. 531

Tinea tonsurans, as complication of vaccination, ii. 85.

Tissues, Living, difference in suseeptibility of, to roentgenization, i. 509; distribution among, i. 18; inequalities of distribution among, due to cell differences, i. 24 ; inequalities of distribution among, due to inequalities of absorption, i. 22; inequalities of distribution among, due to inequalities in solubility, i. 18; inequalities of distribution among, due to interference from "membranes," $i$. 24 ; irrigation of, see Irrigation.

Trtus, Dr. E. C., ventilation of light bath suggested by, i. 443.

TOAst water, as article of invalid dietary, i. 646 .

Tовасco, abuse of, a eause of ehronic bronchitis, iii. 369 ; abuse of, a cause of chronic gastritis, iii. 158; abuse of, a cause of chronic rhinitis, iii. 328 ; abuse of, a cause of hyperacidity of stomach, iii. 76 ; cffect of, upon gastric secretion, iii. 76 ; as an indirect cause of occupation neuroses, iv. 660; influence of, in calusation of arteriosclerosis, iii. 756; injurious influener: of, in ceretoral hyperemia, iv. 385; moderation in use of, in chronic parenchymatous nephritis, iv. 66 ; morleration in use of, in nephritis, iv. 44; moderation in use of, in spastic spinal paralysis, iv. 270; prohibition of, in angina pectoris, iii. 770; prohibition of, in arterioselerosis, iii. 758; prohibition of, in ehronic laryngitis, iii. 340; prohibition of, in chronic myocardial insufficiency, iii. 723 ; prohibition of, in chronic pharyngitis, iii. 33 ; prohibition of, in exophthalmic goiter, iii. 897; prohibition of, for relief of palpitation of heart in neurasthenia, iv. 567 ; prohibition of, in tachycardia, iii. 787; therapeutic use of, in asthma, iii. 386 .

Tobacco angina, iii. 768 .

Tobacco heart, iii. 782 ; etiology of, iii. 782 ; groups of, iii. 782 ; treatment of, iii. 784 .

Tobacco poultice. See Poultice.

ToBacco sMoking, amount of nicotin present in, iii 782 ; cardiac neuroses due to, iii. 781 ; as a eause of cough in pulmonary tuberculosis, ii. 496 ; influence of, in causation of gout, ii. 697 ; injurious effeets of, in syphilis, ii. 369 ; leukoplakia eaused by, iii. 25; physical effects of, iii. 782; prohibition of, in chanere of lips, mouth, or tongue a neeessity, ii. 425; reasons for enjoyment of, iii. 783.

Todd's serum, use of, against scorpion venom, iv. 685.

Tokay wine, use of, in pneumonia with severe asthenia, ii. 254.

Tongue, disturbances of sensation in, iii. 3 ; inflammation of, as complication of small-pox, ii. 51.

Tongue, diseases of, iii. 23: acute glossitis, iii. 23; chronic glossitis, iii. 24 ; geographical tongue, iii. 24; leukoplakia, iii. 25 ; ringworm of tongue, iii. 24.

Tonic treatment, use of, in tabes, iv. 195.

Tonics, use of, in acquired spinal progressive muscular atrophy, iv. 248 ; in acute laryngitis, iii. 340 ; in atriplieism, iv. 693 ; in atrophic rhinitis, iii. 331 ; in 
chronic mucous colitis, iii. 207 ; in convalescence from acute ophthalmoplegia, iv. 509; in convalescence from bronchopneumonia, iii. 421 ; in convalescence from dengue, iv. 860; in gastroptosis to improve the nervous condition, iii. 182; after Lambert's treatment for alcoholism, ii. 599; in lathyrism, iv. 693 ; in membranous enteritis, iii. 210 ; in membranous rhinitis, iii. 330 ; in multiple neuritis, iv. 326 ; in neurasthenia, iv. 544,558 ; in neuritis, iv. 289 ; in optic neuritis without apparent cause, iv. 306 ; in ozena, iii. 336; for relief of anorexia in neurasthenia, iv. 566; after spasmodic laryngitis, iii. 342 ; in syphilis, ii. 435 ; in syringomyelia, iv. 279 ; in tropical phagedenic ulcer, iv. 868; in ulcerative colitis, iii. 211.

Tonics, bitter, use of, in cirrhosis of liver, iii. 281; use of solutions of, in gastric lavage for depressive disorders of gastric secretion, iii. 152 .

Tonicity, as cardiac function, iii. 536 .

Tonsillar infection, as cause of acute nephritis, iv. $27,57$.

Tonsillectomy, following acute peritonsillitis, iii. 44; complete, technique of, iii. 46; indications for, in chronic tonsillitis, iii. 44, 45; indications for complete, iii. 44, 45 .

Tonsillitis, CHRONIc, iii. 44 ; methods of technique in operation for, iii. 46 ; selection of treatment for, iii. 45; treatment of, when operation is contraindicated, iii. 45 .

Tonsillitis, Follicular, diagnosis between Vincent's angina and, iii. 40.

Tonsillitis, suppurative, electrotherapeutics for relief of, i. 405 .

Tonsillotomy, indications for, in chronic tonsillitis, iii. 45.

Tonsils, care of, in pneumonia, ii. 221; as portal of infection for rheumatism, iii. 513; relation between acute rheumatism and, ii. 294; removal of, as prophylactic measure against acute endocarditis, ii. 296 , iii. 744 ; removal of, as a prophylactic measure against bronchitis, iii. 352 ; removal of, as prophylactic measure against nephritis and acute articular rheumatism, iv. 57 ; removal of, as prophylactic measure against tuberculosis of mediastinal lymph nodes, iii. 506; sloughing of, as a complication of scarlet fever, ii. 130 .

TONSILS, HYPERTROPHIED, association of, with enuresis, iv. 130; removal of, for relief of spasmodic laryngitis, iii. 342; removal of, in treatment of chronic rhinitis, iii. 328 .

Tonsils, Diseases of, iii. 41: acute peritonsillitis, iii. 43; acute tonsillitis, iii. 41; chronic tonsillitis, iii. 44; mycosis of tonsils and fauces, iii. 42; quinzy, see Acute peritonsillitis.

Torrington, Conn., results of antityphoid inoculation in, ii. 5.

Toulouse AND Richter, modified bromid therapy introduced by, for relief of epilepsy, iv. 648.

Toussaint, report by, on immunization in anthrax, ii. 331.

Town's specific treatment, for cure of alcoholism, ii. 597; for cure of morphinism, ii. 621 .

Toxalbumins, obtained from diphtheria antitoxin, ii. 635.

Toxemins, associated with acute diffuse peritonitis, iii. 318; headache as a symptom of, iv. 610; hydrotherapy for relief of, i. 228 ; serum treatment of, in pregnancy, i. 172.

Toxic action of Digitalis, iii. 584.

Toxic anemias. See Anemia.

Toxic asthma. See Asthma.

Toxic conditions, causing optic neuritis, iv. 305 .

TOXIC HeMoglobinuria, iv. 4.

Toxic PSYCHOSEs, massage in treatment of, i. 321 .

Toxic sUBSTANCES, produced during physiological process of digestion, ii. 632 .

Toxic vertigoes, iv. 624 .

Toxicology, i. 662 : action of poisons, i. 662 ; animal poisons, i. 674 ; bacterial poisons, i. 674 ; definition of term poison, i. 662 ; diagnosis of poisoning, i. 669 ; disposition of poisons by animal body, i. 666 ; introduction to toxicology, i. 662; mineral poisons, i. 673,674 ; synthetic poisons, i. 674 ; variations in effects of poisons, $i$. 667; vegetable poisons, i. 674, 746 .

Toxin CONCEntration, importance of, i. 52. Toxins, as a cause of food poisoning, ii. 635 ; causing chronic myelitis, iv. 229; con- 
cerned in causation of acute myelitis, iv . 220 ; destruction of, in bowels, in cholera, iv. 835; difficulty of destroying, ii. 636; endogenous, as possible cause of nephritis, iv. 28; nature and action of, in Asiatic cholera, iv. 831.

Toxins, use of mixed, in treatment of leukemia, iii. 847.

Tracheotomy, performance of, in acute parenchymatous glossitis, ii. 24 ; in diphtheria, ii. 279 ; in edema of glottis in acute nephritis, iv. 63 ; in edema of glottis complicating small-pox, ii. 51; indications for, in acute laryngitis, iii. 340 ; indications for, in chronic progressive bulbar paralysis, iv. 253 ; indications for, in edematous laryngitis, iii. 341; indications for, in leprosy, iv. 816; indications for, in membranous laryngitis, iii. 343 ; indications for, in pneumogastric paralysis, iv. 320 ; in laryngeal complications of measles, ii. 158; in laryngeal stenosis, iii. 349 ; in laryngeal tuberculosis, ii. 494 , iii. 347 ; in laryngismus stridulus complicating rachitis, ii. 820 ; in mumps, ii. 171 ; in progressive bulbar paralysis, iv. 500; for relief of dyspnea due to pressure by mediastinal tumors, iii. 503; for relief of dyspnea in tuberculosis of the mediastinal lymph glands, iii. 508; technique of, ii. 279.

Trade winds, i. 254.

Trades in.Jurious to health, iii. 424.

TraIN sickness, as a form of labyrinthine vertigo, iv. 624 .

Transfusion of blood, in chronic pancreatitis, as prophylactic against hemorrhage at operation, iii. 300 ; combined with venesection, in snake bite, iv. 682 ; in hemophilia, iii. 866; in treatment of poisons, i. 673 .

Transfusion of blood in pernicious anemia, iii. 829; arm-to-arm, iii. 830; dangers of, iii. 832; hemolysis test for use in, iii. 830; injection of defibrinated blood and, iii. 831 ; value of, iii. 832 .

Transfusion of normal saline solution, in cerebral anemia, iv. 382 ; combined with venesection, in snake bite, iv. 682 ; contraindicated in uremia with edema, iv. 62 ; in uremia without edema, iv. 62; after venesection for kidney of preg- nancy, iv. 82 ; after venesection in uremis, iv. 53.

Transplantation, in Addison's disease, iii. 853 ; in athyrea and hypothyrea, iii. 907; of muscles in neuritis, iv. 259; of muscles for relief of contractures due to beri-beri, iv. 8.54; of nerves, for rolief of contractures due to beri-beri, iv. 854.

Transudates and ExcDatrs, difference between, in fibrinous pleurisy, iii. 468.

Trauma, in causation of acute myelitis iv. 221 ; of brain syphilis, iv. 479 ; of brain tumor, iv. 446.

Tradmatic cereiral abscesses, iv. 421.

Traumatic hematoma. See Hematoma.

Traumatic pneumothorax. See Pneumothorax.

Travel, objections to, in cardiac neurasthenia, iii. 777; therapeutic value of, in cardiac insufficiency, iii. 665; therapeutic value of, in treatment of neurasthenia and psychasthenia, iv. 55.5.

Trendelenburg position, advantages of, in bronchiectasis, iii. 394 .

Trephining, employment of, to open bony cavities in myiasis, iv. 659 ; in traumatic hematoma, iv. 366.

Trephining of sternum, in suppurative mediastinit is, iii. 504 .

Trephining operation with extirpation, for relief of brain tumor, iv. 451 .

Trichina spiralis, poisoning from, in food, ii. 634 .

Trichiniasis, iii. 253; prophylaxis against, iii. 253 ; treatment of, iii. 254 .

Trichloracetic acid, local use of, in chronic rhinitis, iii. 329 ; to control epistaxis, iii. 335 ; in stomatitis herpetica, iii. 10 ; in syphilis of upper respiratory tract, iii. 349 ; in Vincent's angina, iii. 40.

Trichostrongrlus instabilis, iv. 795.

Trichostrongrlus probolurus, iv. 795.

Trichostrongylus vitrixus, iv. 799.

Tricocephaliasis, iv. 797 ; parasite causing, iv. 797 ; preventive measures in, iv. 798 ; remedial measures in, iv. 795 .

Tricocephalt's dispar, iii. 255, iv. 797 .

Tricocephalus Trichicris, iv. 797 .

Tridontophorus DImisutus, infection by, iv. 800 .

Trigeminal neuralgia. See Neuralgia.

Trigeminal neuritis. See Neuritis. 
Trigeminus Nerve, disturbances of, in basilar syphilitic meningitis, iv. 474 .

Trimethylamin, as a cause of food poisoning, ii. 635 .

Trional, use of, in cerebrospinal irritation, ii. 215; combined with an analgesic, for relief of lightning pains in tabes, iv. 196; contraindicated in cardiac insufficiency, iii. 664; in delirium of pneumonia, ii. 228 ; in delirium tremens, ii. 606; hematoporphyrinuria due to, iv. 5 ; to induce sleep in acute bronchitis, iii. 362; for relief of insomnia associated with mediastinal tumors, iii. 504; for relief of insomnia during influenza, ii. 192; for relief of insomnia in neurasthenia, iv. 559, 565; for relief of insomnia during Pasteur treatment for rabies, ii. 339 ; for relief of insomnia in septicopyemia, ii. 391 ; for relief of insomnia due to sunstroke, iv. 679 ; for relief of painful erections and chordee in acuto urethral gonorrhea, iv. 141; in tuberculosis of lungs, iii. 346 .

Trocar, puncture of skin with, for removal of fluid in general anasarca due to cardiac insufficiency, iii. 676 .

Trophic diśturbances, of tabes, iv. 191.

TrOPHIC EFFECTS, due to hypnosis, i. 574.

Tropical cestodes, trematodes, and NEMATODES, diseases of, iv. 775: distomiasis, iv. 780; endemic hemoptysis, iv. 782 ; filariasis, iv. 786 ; nematode infection, iv. 791; schistosomiasis, iv. 783; teniasis, iv. 775 .

Tropical Fevers, undifferentiated, iv. 869.

Tropical plants, poisonous, iv. 686.

Tropical relapsing Fevers, iv. 757; parasites causing, iv. 758 ; preventive measures in, iv. 760; remedial measures in, iv. 759; transmission of, iv. 759 .

Tropical SLOUghing phagedena, iv. 866; remedial measures in, iv. 868 ; varieties of, iv. 867.

Tropics, relation of, to English-speaking races, iv. 667 ; as affording room for expansion, iv. 668; injurious effects of excessive heat in, iv. 669 ; injurious effects of sunlight in, iv. 669 ; value of production in, iv. 670 .

Tropins. See Opsonins.
Trousseau's sign, presence of, in rachitis, ii. 819.

TrudeaU, opinion expressed by, on results of tuberculin therapy, ii. 524; statistics by, showing effect of tuberculin therapy on length of life, ii. 523; on use of tuberculin in laryngeal tuberculosis, iii. 345 .

Trunecek's serum, use of, in arteriosclerosis, iii. 762 .

Trychophytin, i. 152.

Trypan-blue, use of, in trypanosomiasis, iv. 747.

Trypan-red, use of, in trypanosomiasis, iv. 747.

Trypanosoma gambiense, iv. 742.

Trypanosoma rhodesiense, iv. 743.

Trypanosome Fever, iv. 742.

Trypanosomiasis, iv. 742; antimony compound in treatment of, iv. 747 ; arsenical compound in treatment of, iv. 745; benzidin dyes in treatment of, iv. 747 ; basic triphenyl, in treatment of, iv. 747; convalescence from, iv. 748; frequency of, iv. 742; general treatment of, iv. 748 ; mercurials in treatment of, iv. 747 ; microörganisms causing, iv. 742 ; pathology of, iv. 743; preventive measures for, iv. 748; remedial measures for, iv. 744; sleeping sickness and, iv. 742; surgical treatment of, iv. 748; symptoms of, iv. 743 ; trypanosome fever, iv. 742.

Trypsogen, use of, in diabetes mellitus, ii. 762.

TSETSE FLY, transmission of trypanosome fever and sleeping sickness by, iv. 743; recognition of, iv. 750 .

TSETSE FLY AREAS, clearance of, as preventive measure against trypanosomiasis, iv. 749.

Tuberculatomata of brain, iv. 432.

Tuberculin, i. 149; in Addison's disease, iii. 883 ; in bronchoadenitis, iii. 399 ; dose of, i. 151; immunization by means of, i. 149 ; introduction of, by Koch, i. 126; in leukemia, iii. 847 ; objections to use of, in tuberculous meningitis, iv. 377 ; preparation of, i. 150 ; in treatment of tuberculosis, i. 149; in tuberculosis of mediastinal lymph glands, iii. 507; in tuberculous cystitis, iv. 121; in tuberculous laryngitis, iii. 345 ; in tuberculous peritonitis, iii. 320 ; value of, in tuberculosis of kidney, iv. 116. 
Tuberculin, new, ii. 512.

Tuberculin, old, ii. 512.

Tuberculin purum, i. 151.

TUBbrculin TEST, employment of, in diagnosis of tubereulous kidney, iv. 114.

Tuberculin therapy, ii. 510; dosage in, ii. 520; duration of treatment with, ii. 522; effects of treatment with, ii. 523; final dose in, ii. 522; history of, ii. 510; method of administering dose in, ii. 517; method of diluting dose in, ii. 513; prophylactic mortality influenced by, ii. 523 ; reaction from tuberculin injection, ii. 522 ; reeords of results obtained by, ii. 524; selection of patients for use of, ii. 516; site for injection in, ii. 518; theory of action in, ii. 511; use of, ii. 523; varieties of tuberculin employed in, ii. 511.

Tuberculocidin, i. 151.

Tuberculoplasmin, i. 151.

Tuberculosis in animals, laws controlling, in Denmark, ii. 442.

Tuberculosis of BladDER, treatment of, by electricity conjoined with X-ray, i. 402.

TUBerculosis of KIDNEY, treatment of, by electricity conjoined with X-ray, i. 402.

Tuberculosis of LarynX, iii. 344.

Tuberculosis of lungs. See Tuberculosis, pulmonary.

Tuberculosis of mediastinal glands. See Mediastinal glands.

Tuberculosis of mucous membranes, $\mathrm{X}$ ray therapy for, i. 535 .

Tuberculosis of pancreas, iii. 30 .

Tuberculosis, pulmonary, ii. 438; abscesses complicating, ii. 509; absolute rest in treatment of, i. 482 ; advice to discharged patients in, i. 559; alcohol in diet of, i. 558; alcohol in night sweats of, i. 502; alcohol as tonic in, i. 504; amusements in treatment of, i. 488; anemia in, i. 507; anorexia in, i. 504; anti-acid diet in, i. 557; anti-constipation diet in, i. 555; antituberculosis societies and their work, i. 451; antituberculosis work in different countries, i. 440; artificial pneumothorax in treatment of, ii. 535; autoinoculation in, ii. 525; bronchial conditions accompanying, ii. 507; care of teeth in, ii. 490; climate in treatment of, ii. 472 ; clothing in, ii. 489 ; as a complication of vaccination for small-pox, ii.
90 ; concentrated diet in, ii. 556; constipation accompanying, ii. $50 \pi$; cough in, ii. 495; diabetes associated with, ii. 767 ; diarrhea accompanying, ii. 505; diet in acute, advaneed, and complicated cases of, ii. 551 ; diet during acute stage of, ii. $4 \$ 1$; diet for ambulant cases, ii. 538; diet during gastrointestinal disturbances in, ii. 503; diet in general in, ii. 478, 53s; diet in intestinal form of, ii. 50.5; disinfection in, ii. 452; drug treatment of, ii. 509; dyspnea accompanying, ii. 506; (empyema accompanying, ii. 507; epinephrin in, i. 96; examination of stools in, ii. 513; excrcise in treatment of, ii. 477, 527; expectoration in, ii. 498; fistula complicating, ii. 509; food requirements in, ii. 539; gastrointestinal disturbances accompanying, ii. 502; hemorrhage accompanying, ii. 499; hemorrhage in relation to exercise in, ii. 527; high percentage of, in certain trades, iii. 424; history of work against, ii. 438 ; house infection in, ii. 451 ; hydrotherapy for relief of, i. 27 ; individual prophylaxis against, ii. 455; influence of, in causation of amyloid disease of kidney, iv. $\$ 1$; influence of, in causation of arteriosclerosis, iii. 756; insomnia in, ii. 506; intestinal form of, ii. 505; laryngeal complications in, ii. 490; liquid diet in, ii. 55.5; marriage as a question in, ii. 464; menstrual disturbances in, ii. 507; menus for meals in, ii. 547 ; night sweats in, ii. 505 ; number and arrangement of meals in, ii. 546; nursing of importance in active stage of, ii. 482; open air in treatment of, ii. 479 ; otitis media complicating, ii. 509; pain in chest accompanying, ii. 506; pleural effusion aecompanying, ii. 507; pneumothorax accompanying, ii. 507; preeautions to be observed in presence of, ii. 465; preparation of food in, ii. 544; prophylaxis against, in general, ii. 438, 451; prophylaxis against brain tumor in, iv. 446; protection of child against, ii. 45S; protection of infant against, ii. 456; protection of youth against, ii. 462, 4\$2; pulmonary stenosis associated with, iii. 735 ; rest in treatment of, ii. 482 ; routine in treatment of, ii. 487 ; sanatorium treatment for, ii. 477 ; selection of cases for sanatorium treatment in, ii. 469 ; se- 
lection of cases for tuberculin therapy, ii. 516; semi-liquid diet in, ii. 554; serum therapy in, ii. 525; sources of food supply in, ii. 543; symptomatic treatment of, ii. 494; system of graduated exercise for use in, ii. 529; temperature during active stage of, ii. 479,483 ; temperature as a guide to effect of graduated exercise in, ii. 528; test diet in, ii. 502; treatment of, in general, ii. 468; tuberculin therapy in, ii. 510; "two-hour diet" for advanced cases of, ii. 552 ; vaccine therapy for control of hemorrhage in, ii. 499; variety of diet essential in, ii. 566; X-ray therapy for, ii. 553.

Tuberculosis, renal. See Kidney, tuberculous.

TUBerculosis of sKIN, action of radium upon, i. 469; electrolysis combined with $\mathrm{X}$-ray for relief of, i. 400; value of X-ray treatment in, i. 533.

Tuberculosis verrucosa cutis, X-ray therapy for relief of, i. 534 .

Tuberculous cystitis. See Cystitis, tuberculous.

Tuberculous infections, electricity in treatment of, i. 399; radiant light and heat in treatment of, i. 449, 452 .

Tuberculous Kidney. See Kidney, tuberculous.

Tuberculous meningitis. See Meningitis, tuberculous.

Tuberculous myositis. See Myositis.

Tuberculous peritonitis. See Peritonitis.

Tuberculous pneumothorax. See Pneumothorax.

Tufnell's diet for aneurysm, iii. 765 .

Tulase, i. 151.

Tumor, TUBERculous, diagnosis between tuberculous meningitis and, iv. 375 .

Tumors of Lung. See Lung, tumors of.

Tumors of mediastinum. See Mediastinal tumors.

TUNGsten lamp, as source of radiant energy, i. 440.

Tunneled catheters, use of, in urethral stricture, iv. 170.

Tunneled sounds, use of, in urethral stricture, iv. 170.

Turbinectomy, total, employment of, in hay fever, iii. 390 .

Turkish bath, therapeutic use of, in acute alcoholism, ii. 389; in arthritis deformans, ii. 713 ; in chronic bronchitis, iii. 368 ; as prophylaxis against acute bronchitis, iii. 352 ; in syphilis, ii. 389 ; in treatment of morphin habit, ii. 624 .

Turpentine, use of, as anthelmintic in hookworm disease, iv. 798; dangerous in nephritis, iv. 49; as expectorant in chronic bronchitis, iii. 377 ; by injection, to destroy larvæ in myiasis, iv. 689; irritation of kidney due to, iv. 57 ; locally, in acute bronchitis, iii. 356 ; by rectal injection, in cirrhosis of liver with melena, iii. 288; in scarlet fever for prevention of nephritis, ii. 133; as spray in acute bronchitis, iii. 359; as stimulant in cerebral hyperemia, iv. 387 ; in typhoid fever, ii. 26.

Turpentine, oil of, use of, in acute phosphorus poisoning, ii. 628 ; by inhalation in acute laryngitis, iii. 339 ; in pulmonary abscess, iii. 441.

Turpentine enemata. See Enemata.

Turpentine liniment, use of, in acute bronchitis, iii. 356.

Turpentine stupes, use of, in acute bronchitis, iii. 356; in bronchial asthma, iii. 389 ; in bronchopneumonia for relief of meteorism, iii. 416 ; for relief of meteorism in acute diffuse peritonitis, iii. 317 ; for relief of pain, iii. 156; in typhoid fever, ii. 26.

Turpeth mineral, use of, in acute laryngitis of children, iii. 340 ; in spasmodic laryngitis, iii. 342 .

Tuttle's apparatus, use of, as rectal douche in chronic gonorrheal urethritis, iv. 165 .

TYPHOID CARRIERs, antityphoid vaccination of, ii. 6 ; number of, ii. 2 ; treatment of, ii. 9.

TyphoId FEVER, ii. 1; alcohol in treatment of, ii. 14, 23; appendicitis in, iii. 219; circulatory disturbances in, ii. 23; diagnosis between, and tuberculous meningitis, iv. 375; diagnosis between, and typhus fever, ii. 38 ; diarrhea as symptom of, ii. 25; diet in, ii. 11; disinfection of excreta in, ii. 7; epistaxis as a symptom of, ii. 27 ; eruption in, ii. 38 ; furunculosis complicating, ii. 29; gall-bladder affections complicating, ii. 28; gastric irritability complicating, ii. 25; general care of, ii. 9 ; genitourinary disturbances 
complicating, ii. 24; headache as a symptom of, ii. 27; hemorrhage complicating, ii. 19 ; hydrotherapy for, i. 219 , ii. 16 ; insomnia complicating, ii. 26 ; lung affections complicating, ii. 24 ; management of bowels in, ii. 28 ; mastit is complicating, ii. 27; meteorism complicating, ii. 26; milk contaminated by infected water a cause of, ii. 646 ; mortality in, ii. $15,19,23$; operative measures in, ii. 21 ; otitis complicating, ii. 27 ; oysters contaminated by sewage a cause of, ii. 645; parotitis complicating, ii. 27 ; perforation of intestines complicating, ii. 22 ; periostitis complicating, ii. 28 ; phlebitis complieating, ii. 27; post-typhoid psychosis, as a sequel to, ii. 29 ; prevention of extension of, ii. 7 ; prophylactic inoculation against, i. 142; prophylaxis against, in general, ii. 1; resemblance between trichinosis and, ii. 254; septic eruption in, mistaken for typhus fever, ii. 39 ; serum therapy for, ii. 7 ; symptomatic parotitis complicating, ii. 27 ; tender toes as sequel to, ii. 29; therapeutic inoculation in, i. 143 ; treatment of convalescence from, ii. 29 ; treatment of, in general, ii. 9; typhoid spine as a sequel to, ii. 29 ; vaccination for, ii. 3 ; vehicles of transmission for, ii. 1.

Typhoid spine, ii. 29.

Typhus Fever, ii. 31 ; convalescent serum used in treatment of, ii. 37 ; definition of, ii. 31 ; diet in, ii. 37 ; diagnosis between, and typhoid fever, ii. 38 ; eruption in, ii. 33 ; history of, ii. 31 ; incubation period in, ii. 33 ; mortality from, ii. 37 ; symptoms of, ii. 33 ; transmission of, ii. 32 ; treatment of, ii. 36.

Tyrotoxicon, as a cause of food poisoning, ii. 635 ; milk poisoning due to, ii. 647 .

Tyrotoxisuus. See Cheese poisoning.

Ulcer of esophagus. See Esophagus.

Ulcerating granuloma of PUDENDa, iv. 865; remedial measures in, iv. 866 .

Ulcerative colitis. See Colitis.

Uluyue. See Goundoo.

"Unconscious symbolization," significance of term in psychasthenia, iv. $\mathbf{5 7 5}$.

Unguentum Credé, use of, in acute cerebral meningitis, iv. 372 ; in Ludwig's angina, iii. 30 .
Unguentum tartrate of antimony, use of, in hydrocephalus, iv. 46.5.

UNIFoRмITY, as a factor in an X-ray treatment, i. 502.

UNited STATEs, antituberculosis work in, ii. 443 .

United States Army, results of antityphoid inoculation in, ii. 4.

Unna's formula, for ointment for use in erysiplas, ii. 334 .

UNIVEISAL CONGENITAL ASTHENIA, ii. 709.

UNSANITARY CONDITIONS, relation of, to spread of plague, iv. 819.

Uranium nitrate, use of, in diabetes mellitus, ii. 760 .

UraniUm Nitrate, chronic nephritis caused by, iv. 15 .

UrEA, effect of cold baths upon amount of, excreted in typhoid fever, i. 190; origin and significance of, iv. 12.

Urea and quinin hydrochlorate, use of, in tonsillectomy, iii. 445 .

Uremia, in acute nephritis, iv. 62 ; bydrotherapy for relief of, i. 210; importance of, during reaction stage of Asiatic cholera, iv. 836; method of treating, in nephritis, iv. 51 ; in nephritis, iv. 20 ; due to suppression of urine associated with renal colic, iv. 107; due to suppression of urine complicating tuberculous kidney, iv. 117.

Uremic dysprea. See Dyspnea.

Uremic vomiting. See Vomiting, uremic.

Ureteral catheterization, use of, in nephrolithiasis, iv. 110.

Ureterolithotomy, indications for, in nephrolithiasis, iv. 110.

Urethan, use of, in tetanus, ii. 348.

Urethra, dilatation of, see Dilatation; local treatment of anterior, in acute gonorrhea, iv. 14s; local treatment of, contraindicated in gonorrheal epididymitis, iv. 152; local treatment of posterior, iv. 148; resection of, see Resection; stricture of, see Urethral stricture.

URETHRAL CARUNCLES, electrotherapeuties for relief of, i. 420.

UREThral DISEASE, hydrotherapy for, i. 210.

Urethral injections. See Injections.

Urethral meatus, congestion of, in females, electrotherapeutics for relief of, i. 421 . 
Urethral sound, passage of, at intervals, in urethral stricture, iv. 169.

URETHRAL STRICTURE, iv. 168 ; curative treatment of, iv. 168; introduction of filiforms in treatment of, iv. 171; prophylaxis against, iv. 168; treatment of impassable, with retention, iv. 172; treatment of impassable, without retention, iv. 172; treatment of indurated or nodular, iv. 173; treatment of irritable, iv. 173; treatment of, admitting only a filiform, iv. 170; treatment of, complicated by acute pyelonephritis, iv. 173; treatment of, complicated by false passage, iv. 173; treatment of, complicated by fistula, iv. 173; treatment of, complicated by periurethritis, iv. 173; treatment of, of large caliber, iv. 169; treatment of resilient, iv. 172; treatment of, with retention of urine, iv. 171 ; treatment of, of small caliber, iv. 170; treatment of traumatic, iv. 172; varieties of, iv. 168.

URETHRAL WARTS, urethroscopic treatment of, iv. 166.

Urethritis, as a cause of enuresis, iv. 129; definition of acute, iv. 136; definition of chronic, iv. 136; post-gonorrheal, see Post-gonorrheal urethritis; acute posterior, complicating acute gonorrhea, iv. 149; treatment of acute posterior, iv. 149.

URETHRORRHEA, iv. 176.

Urethroscopic treatment, of chronic gonorrheal urethritis, iv. 166.

Urethrotomy, emplovment of, for relief of impassable urethral stricture without retention of urine, iv. 172.

Urethrotomy, external, performance of, for relief of indurated fistula associated with resilient or impassable stricture, iv. 173.

URIC ACID, increase of, caused by X-ray treatment of leukemia, iii. 851 .

URINARY ANALYSIS, importance of, in treatment of enuresis, iv. 127.

Urinary antiseptics. See Antiseptics.

URINARY BLADDER, effects of epinephrin upon, i. 92.

URINARY CASTS, significance of, as functional kidney test, iv. 24 .

URINARY ORGANS, DISEASES OF, as cause of enuresis, iv. 127, 128.

URINATION, PAINFUL, in acute urethral gonorrhea, iv. 141.
Urine, characteristics of, in diabetes mellitus, ii. 727; effect of cold baths upon, i. 190; incontinence of, in tabes, iv. 198; method of disinfecting, in typhoid fever, ii. 7 ; necessity for frequent examination of, in asthma, iii. 381 ; necessity for frequent examination of, in chronic myocardial insufficiency, iii. 716, 721; necessity for frequent examination of, in diabetes mellitus, ii. 731 ; necessity for frequent examination of, after German measles, ii. 165; necessity for frequent examination of, in membranous pharyngitis, ii. 439; necessity for frequent examination of, in pneumonia, ii. 227; reaction of, in pyelitis, iv. 90 ; relation between amount of, and amount of water imbibed, i. 40; typhoid fever transmitted by, ii. 7, 24 .

URINE, RETENTION OF, due to paralysis of bladder, iv. 124; as a symptom of pyelitis, iv. 94; in tabes, iv. 198; treatment of, when due to stricture, iv. 171.

UriNe, SUPPRESSION OF, in pyelitis, iv. 94; in renal colic, iv. 106; in tuberculous kidney, iv. 117.

Urotropin, use of, in acute catarrhal cholecystitis, iii. 267 ; in acute myelitis, iv. 225; in cerebrospinal meningitis, ii. 201, 216 ; in chronic cystitis, iv. 120; in chronic gout, ii. 704; in colon bacilluria, iv. 128; contraindicated in acute urethral gonorrhea, iv. 139; before dilatation of urethra, iv. 162 ; in endemic hematuria, iv. 784; in hematuria, iv. 4 ; in herpes zoster, iv. 359 ; in intestinal autointoxication, ii. 675 ; in irritable stricture of urethra, iv. 173; in Landry's paralysis, iv. 329 ; in meningitis complicating influenza, ii. 194; in meningitis complicating pneumonia, ii. 289; before passage of urethral sound, iv. 169; in pneumonia, for prevention of complications, ii. 223 ; in prevention of infection of bladder by spontaneous pyogenic organisms, iv. 120 ; in prophylaxis against anterior poliomyelitis, iv. 237; in prophylaxis against influenza, ii. 186; in pyelitis, iv. 90 ; in pyelitis complicating acute myelitis, iv. 227; for relief of vesical disturbance in tabes, iv. 198; in retention of urine from paralysis of bladder, iv. 125; in scarlet fever for prevention of neph- 
ritis, ii. 133; in typhoid fever, ii. 2.5; in whooping-cough, ii. 181.

Urotropin, and stock vaccines, use of, in acute rhinitis, iii. $32 \mathrm{~S}$.

UrTICARIa, as a complication of vaccination, ii. 84 ; radiant light and heat in treatment of, i. 459 .

UTERINE displacements, as a cause of pain in bladder, iv. 123.

UTERINE HEMORRHAGE, extract of posterior lobe of the hypophysis for relief of, ii. 109.

Uterine neck, chancre of. See Chanere.

UTERUS, effects of epinephrin upon, i. 92.

Uvula, removal of elongated, for relief of spasmodic laryngitis, iii. 342 ; swelling of, in mumps, ii. 171.

Uvulitis, ACUTE, iii. 35.

Vaccinal Lichen, as complication of vaccination, ii. 84.

VaCcINal miliaria, as complication of vaccination, ii. 84 .

VACCINATION FOR BACILLARY DYSENTERY, ii. 322 .

VACCINATION For SMALl-POX, ii. 54; accidents complicating, ii. 78 ; antivaccination agitation and, ii. 104; ape-pox and, ii. 56 ; Blue Book on, ii. 59 ; choice of instruments for, ii. 68; clinical history of, ii. 70; complications of, due to vaccine virus, ii. 80 ; complications of, ii. 78 ; constitutional complications in, ii. 87; contraindications to, ii. 73 ; cow-pox and, ii. 56 ; definition of, ii. 54 ; diluents for virus in, ii. 65; discovery of, by Jenner, ii. 58 ; drug eruptions mistaken for, ii. 92; eczema complicating, ii. 91 ; erysipelas complicating, ii. 85; erythema complicating, ii. 85; furunculosis complicating, ii. 86; gangrene of pock in, ii. 87; generalized gangrenous vaccinia complicating, ii. 83 ; generalized hemorrhagic vaccinia complicating, ii. 82 ; generalized vaccine erythema complicating, ii. 83; generalized vaccinia complicating, ii. 80 ; generalized vaccinia from autoinfection complicating, ii. 82; German vaccination law and its results, ii. 100; hemorrhage into pock in, ii. 87 ; history of, ii. 87; history of, in Cuba, ii. 103; history of, in France, ii. 60; history of, in Japan, ii. 102; history of, in the Phil- ippines, ii. 104; history of, in Saxony, ii. 102; horse-pox and, ii. 55; immunity conferred by, ii. 77; impetigo contagiosa complicating, ii. 86 ; indications for, ii. 73; influence of, on exanthema, ii. 74 ; infected vesicles in, and their treatment, ii. 72 ; insusceptibility to, ii. 77 ; introduction of, into America, ii. 61 ; introduction of, into European countries, ii. 59 ; leprosy transmitted by, ii. 90; lupus complicating, ii. 90 ; mortality from small-pox before discovery of, ii. 54, 94; mortality from small-pox since discovery of, ii. 94; Neapolitan method of, ii. 60 ; pemphigus complicating, ii. 91 ; pock diseases of animals and, ii. 54; preparation of vaccine virus for, ii. 62; protection afforded by, ii. 94 ; protection of vaccine vesicle after, ii. 69 ; psoriasis complicating, ii. 92 ; purpura complicating, ii. 85 ; rabbit virus used in, ii. 67 ; revaccination and the value of, ii. 75 ; ruptured vesicles and their treatment, ii. 72 ; scars caused by, ii. 74 ; septicemia complicating, ii. 91 ; sheep-pox and, ii. 55; skin affections complicating, ii. 86 ; sore arm complicating, ii. 86 ; spurious vaccination and, ii. 80 ; summary of subject of, ii. 107 ; syphilis transmitted by, ii. 87,372 ; technique of, ii. 67 ; tetanus transmitted by, ii. 90 ; tinea tonsurans complicating, ii. 85; tuberculosis transmitted by, ii. 90; urticaria complicating, ii. $\mathrm{S4}$; vaccinal iichen complicating, ii. 84 ; vaccinal miliaria complicating, ii. $\$ 4$; value of, ii. 92 ; whooping-cough in relation to, ii. 74 , 179.

VACCINATION FOR SYPHILIS, ii. 437.

VACCINATION FOR TYPHOID FEVER, i. 142, ii. 3 ; treatment of typhoid fever by, i. 143.

Vaccine erythema, geNeralized, as a complication of vaccination, ii. 83 .

Vaccine therapy, i. 137; in acne, i. 145; in acute bronchitis, iii. 354 ; in acute laryngitis, iii. 339 ; in acute myelitis of gonorrheal origin, iv. 228 ; in acute peritonsillitis, iii. 43 ; in acute rheumatism, ii. 301 ; in anthrax, ii. 221 ; in bronchiectasis, iii. 393 ; in bronchitis, complicating pulmonary tuberculosis, ii. $50 \mathrm{~s}$; in bronchopneumonia, iii. 415; in cerebrospinal 
meningitis, ii. 201, 204; in chronic bronchitis, iii. 371 ; in colon bacilluria, iv. 128,129 ; in colon bacillus infection, i. 147 ; to control hemorrhage in pulmonary tuberculosis, ii. 499 ; in diabetes mellitus for relief of boils, ii. 765 ; in erysipelas, ii. 284; in gangosa, iv. 865 ; in glanders, ii. 358 ; in gonococcus infection, i. 146; in gonorrheal epididymitis, iv. 151; immunity conferred by, i. 139; in influenza, i. 147, ii. 189; in leprosy, iv. 814 ; in malignant endocarditis, iii. 746 ; in Malta fever, iv. 839; in membranous pharyngitis, iii. 38 ; in multiple sclerosis, iv. 281 ; in phlegmonous pharyngitis, iii. 40 ; for plague, iv. 823 ; in pneumonia, ii. 239, 240; in pneumococcus infection, i. 146; in polymyositis, iv. 330 ; for post-gonorrheal urethritis, iv. 167 ; in prophylaxis of acute rhinitis, iii. 326 ; in prophylaxis of cholera, i. 141; in prophylaxis against dysentery, i. 142; in prophylaxis against plague, i. 142; in prophylaxis against typhoid fever, i. 142 ; in pyelitis, iv. 91 ; to relieve expectoration due to mixed infection in tuberculosis, ii. 498; in septicopyemia, ii. 290; in small-pox, i. 178; in staphyloccus infection, i. 145; in streptococcus infection, i. 146; technique for, i. 140; theory of method in, i. 139; in typhoid fever, i. 143; in Vincent's angina, iii. 40.

Vaccine and serum therapy, i. 125: active immunization, i. 137; bacterial products, treatment with, i. 173 ; conclusions regarding, i. 178; historical account of, i. 125; immune therapy and malignant disease, i. 175; methods of administering sera and vaccines, i. 176 ; nature of immunity, i. 129; passive immunization, i. 153; passive natural immunity, i. 171; serum therapy, i. 153; theories of immunity, i. 127; vaccine therapy, i. 137.

Vaccines, autogenous, in acute inflammation of nasal sinuses, iii. 334 ; in acute rhinitis, iii. 328 ; in furunculosis complicating typhoid fever, ii. 29 ; in pyelitis, iv. 92 ; in rhinoscleroma, iii. 332 .

Vaccines, bacterial, recovery following administration of, in glanders, ii. 358 .

Vaccines, stock, use of, in acute rhinitis, iii. 328 ; in pyelitis, iv. 92 .
VACCINIA, GeNgralized, from autoinoculation, as a complication of vaccination, ii. 82 ; as a complication of vaccination, ii. 80; gangrenous, as a complication of vaccination, ii. 83 ; hemorrhagic, as a complication of vaccination, ii. 82 .

Vacuum tube wave current, use of, in electrotherapeutics, i. 382.

Vagina, Chancre of. See Chancre.

VAGUS NERVE, involvement of, in progressive bulbar paralysis, iv. 497 .

Valentin and Desoteaux, first work on vaccination in France done by, ii. 60 .

Valerian, use of, in diabetes insipidus, ii. 777 ; in neurasthenia, iv. 558 ; in peristaltic unrest, iii. 250 ; in pneumonia with cardiac toxemia, ii. 254; for relief of cardiac palpitation in neurasthenia iv. 568 .

Valerianate of ammonia, use of, for relief of headache in neurasthenia, iv. 565; for relief of insomnia in neurasthenia, iv. 566.

Valerianates, use of, for relief of restlessness in neurasthenia, iv. 567.

Valerianic acid, salts of, use of, in neurasthenia, iv. 558.

Validol, use of, in flatulency due to gastric atony, iii. 172.

Vallée's antituberculosis serum, i. 168.

Vallet's pill, use of, in chlorosis, iii. 818.

VALSALVA's TREATMENT OF ANEURYSM, iii. 765.

Valvotomy, employment of, in constipation due to obstruction, iii. 237.

VAlvUlar heart Lesions, chronic myocardial insufficiency due to, iii. 712 ; treatment of, iii. 732 .

Van Gehuchten, operation by, for trigeminal neuralgia, iv. 355 .

Van Giesen, on iron metabolism, iii. 817.

Vanilla iCE CREAM, as article of invalid diet, i. 651.

Vaporoles, iii. 742 .

VAQUEZ, polycythemia with splenomegaly first described by, iii. 877 .

Vaquez' Disease. See Polycythemia with splenomegaly.

VARICELLA, ii. 109; care of mouth in, ii. 113; control of itching in, ii. 112; convalescence from, ii. 113; definition of, ii. 109; diet in, ii. 113; eruption in, ii. 112; hemorrhagic eruption in, ii. 113; history of, 
ii. 109; immunity conferred by attack of, ii. 110; incubation period of, ì. 110; itching in, ii. 112; prophylaxis of, ii. 111; susceptibility to, ii. 110; synonyms for, ii. 109 ; transmission of, ii. 110 ; treatment of, ii. 111.

Varicose ulcers, radiant light and heat in treatment of, i. 459.

Vas deferens, ligation of, for relief of chronic gonorrheal urethritis, iv. 166.

Vascular crises of Pal, blood pressure raised by, iii. 555 .

Vascular insufficiency. See Paralysis, acute vasomotor.

Vascular lesions, as a cause of cerebral palsies of children, iv. 410.

Vasoconstrictors, use of, in hemophilia, iii. 867 ; to relieve disturbances of circulation in Addison's disease, iii. 885; in sporadic cases, iii. 869.

Vasodilators, use of, in chronic interstitial nephritis, iv. 70; in migraine, iv. 592; to reduce hypertension in nephritis, iv. 47 ; for relief of neuralgia due to arteriosclerosis, iv. 336 ; for relief of uremic dyspnea, iv. 55 ; in uremia, iv. 53.

VAsomotor aNGiNa PECTORIS, iii. 767.

VASOMOTOR DISTURBANCES, as symptom of poison, i. 667.

VASOMOTOR EFFECTS, due to hypnosis, i. 574.

VASOMOTOR FAILURE, administration of epinephrin for relief of, i. 98 .

VASOMOTOR PARALYSIS, administration of epinephrin for relief of, i. 98.

Vasomotor paralysis, acute. See Paralysis, acute vasomotor.

VAsomotor Rhinitis. See Hay fever.

VASOMOTOR STASIS, as indication for use of digitalis, iii. 588.

VASOMOTOR SYSTEM, action of strychnin upon, iii. 615.

Vasotonin, use of, by injection, in neurasthenia accompanying beginning arteriosclerosis, iv. 559 ; to reduce hypertension in nephritis, iv. $47,48$.

VAUGHaN, isolation of tyrotoxicon by, ii. 647.

Vaughan and McClymonds, work by, on bacteria present in chcese poisoning, ii. 647.

Vegetable cathartics. See Cathartics.

Vegetable organisus, as a cause of food poisoning, ii. 634 .
Vegetable polsons, i. 674, 746: aconitin, i. 764 ; atropin, 752 ; brucin, i. 751 ; cocain, i. 763; colchicum, i. 758; coniin, i. 759; digitalin, i. 757; gelsemin, i. 754; nicotin, i. 761 ; strychnin, i. 746 ; veretrin, i. 756.

Vegetable starch, substitutes for, in diabetes mellitus, ii. 748 .

Vegetables, avoidance of sharp, in diet for chronie parenchymatous nephritis, iv. 65 ; cooking of, i. 623 ; in diet of gastric hyperacidity, iii. 87,89 ; irritation of kidneys by sharp, a cause of acute nephritis, iv. 57, 59, 65; use of, in anemia accompanying rachit is, iii. $\$ 1 S$; use of, in diabetes mellit us, ii. 744 ; varieties of, to be avoided in nephritis, iv. 44,57 , $59,65$.

Vegetation, influence of, upon climate, i. 258.

Vein puncture, employment of, in cardiac insufficiency, iii. 670.

Venereal buboes, X-ray therapy for relief of, i. 544

Venesection, employment of, in active congestion, iii. 406; in acute hemorrhagic inferior polioencephalitis, iv. $40 \mathrm{~s}$; in aneurysm, iii. 765 ; in attacks of cardiac asthma, iii. 737; in aural vertigo, iv. 316 ; in beri-beri, iv. 854; in cerebral hemorrhage, iv. 391 ; in chronic bronchitis, with cyanosis, iii. $3 \bar{\imath} S$; in chronic interstitial nephritis, iv. 70 ; contraindications to, in brain tumor, iv. 449; for elimination of toxic substances in intestinal autointoxication, ii. 672; in gastric hemorrhage, iii. 112; in kidney of pregnancy, iv. 82 ; in pneumonia, ii. 255; in pneumonia complicated with edema of the lungs, ii. 259; in pneumonia in connection with inhalation of oxygen, ii. 237; in polycythemia with splenomegaly, iii. 878 ; in pulmonary edema. iii. 410 ; to reduce hypertension in nephritis, iv. 47 ; for relief of cerebral hyperemia, iv. 386 ; for relief of cyanosis in acute bronchitis, iii. 363 ; for relief of edema of glottis in acute nephritis, iv. 63; for relief of epileptiform convulsions due to brain tumor, iv. 448 ; for relief of pulmonary edema in chronic interstitial nephritis, iv. 72; for relief of uremic dy'spnea, iv. 55; in severe cases of chronic 
myocardial insufficiency, iii. 718; in treatment of bite by rabid animal, ii. 336 ; in uremia, iv. 53 ; in uremia due to nephrolithiasis, iv. 107.

Venesection, employment of, in cardiac insufficiency, iii. 667; amount of blood withdrawn in, iii. 671; contraindications to, iii. 669 ; effects of, iii. 669 ; indications for, iii. 669; physiological action of, iii. 667 ; technique of, iii. 669 ; technique of vein puncture in, iii. 670 .

Venesection combined with sweatings, employment of, in pachymeningitis interna hemorrhagica, iv. 364 .

Ventilation, hygienic and therapeutic value of, i. 265.

Ventricle, Left, insufficiency of, iii. 549.

Ventricle, Right, insufficiency of, iii. 549.

Ventricles, drainage of, in acute cerebral meningitis, iv. 372 ; for relief of pressure in tuberculous meningitis, iv. 377.

Ventricular extrasystoles, iii. 567.

Ventricular puncture, in hydrocephalus, iv. 466; for relief of brain tumors, iv. 451; for relief of cysticercus of fourth ventricle, iv. 461 ; for relief of headache in hydrocephalus, iv. 609.

Ventricular tapping, employment of, in chronic cerebral meningitis with hydrocephalus, iv. 378.

VER DU CAYOR, iv. 687; treatment of bite by, iv. 688 .

VER MACAQUE, iv. 687; treatment of bite by, iv. 688 .

Veratris, poisoning by, j. 756.

Veratrum viride, use of, in cerebral hemorrhage, iv. 391 ; in pneumonia, ii. 248 ; in pneumonia with abnormally high blood pressure, ii. 255.

Verchère, Dr., investigations by, on value of radium therapy in gynecology, i. 479.

Vermifuges, use of, in ulcerative colitis due to Balantidium coli, iv. 762 .

Veronal, use of, in acute hemorrhagic inferior polioencephalitis with extreme motor excitement, iv. 408; in delirium of pneumonia, ii. 228; in delirium tremens, ii. 606 ; in multiple sclerosis, iv. 281 ; for relief of insomnia in acute bronchitis, iii. 361 ; for relief of insomnia in acute rheumatism, ii. 302 ; for relief of insomnia due to brain tumor, iv. 447 ; for relief of insomnia in cardiac insufficiency, iii. 664 ; for relief of insomnia associated with mediastinal tumors, iii. 504; for relief of insomnia in neurasthenia, iv. 559, 565 ; for relief of insomnia in septicopyemia, ii. 291 ; for relief of painful erections and chordee in acute urethral gonorrhea, iv. 141; in scarlet fever, ii. 127; to secure sleep in neuritis, iv. 286 ; in tuberculosis of larynx, iii. 346.

Veronal, sodium salt of, use of, by rectum, for relief of headache in seasickness, iv. 626.

Verruga Peruviana, iv. 863; remedial measures in, iv. 864 .

Vertigo, as a premonitory symptom of cerebral hemorrhage, iv. 389 ; as a symptom of basilar syphilitic meningitis, iv. 473 ; as a symptom of brain tumor, iv. 435; as a symptom in pellagra, iv. 624 .

Vertigo, AURAL, iv. 314; treatment of, iv. 315.

Vertigo DUE TO BRAIN TUMOR, measures for relief of, iv. 448 .

Vertigoes, the, iv. 615; anatomical considerations affecting, iv. 617; aural form of, iv. 629 ; clinical forms of, iv. 623 ; definition of, iv. 615 ; ear labyrinth in relation to, iv. 617 ; etymology of word, iv. 615; hysterical form of, iv. 630; impaired cerebral circulation a cause of, iv. 623 ; labyrinthine form of, iv. 624 ; neurasthenic form of, iv. 630; ocular form of, iv. 629; reflex form of, iv. 623 ; symptoms of, iv. 621 ; vestibular form of, iv. 626.

VERUMONTANUM, congestion of, a cause of pain in bladder, iv. 122; treatment of, through urethroscope in post-gonorrheal neuroses, iv. 174.

Vesical calculus. See Calculus.

Vesical crises, in tabes, iv. 191; treatment of, iv. 197.

Vesicants, application of, over heart in beri-beri, iv. 854 ; use of, in chronic myelitis, iv. 231.

VESICULITIS, as a cause of pain in bladder, iv. 122; electrotherapeutics for relief of, i. 407,423 ; seminal, see Seminal vesiculitis.

Vestibular vertigoes, iv. 626; treatment of, iv. 628.

Veterinary Medicine, value of immunization in, i. 139. 
Vibration, use of, in massage, i. 291; for occipital neuralgia, i. 310; for relief of pain in syringomyelia, iv. 278; for relief of paralysis agitans, i. 320 .

Vibration to spine, employment of, in spastic spinal paralysis, iv. 270.

Vibratory massage, i. 353; use of, in at rophic rhinitıs, iii. 332; in cardiac insufficiency, iii. 660; use of, in constipation, iii. 230; contraindications to, i. 357; indications for, i. 356; in post-gonorrheal neuroses, iv. 175; see also Massage.

Vichy Celestins, use of, in acute urethral gonorhea, iv. 139.

Victoria Park Hospital, formula used at, for administration of terebine in chronic bronchitis, iii. 377 ; method of giving terebene at, as expectorant in bronchiectasis, iii. 396.

Vidius, distinction between chicken-pox and small-pox first made by, ii. 109.

Viexsors, theory held by, that syphilis is transmitted only when lymph is con= taminated with blood, ii. 88 .

Vincent's angina. See Stomatitis ulcerosa.

VinceNt's FUSIForm bacillus, presence of, in stomatitis ulcerosa, iii. 14.

Vinegar, sponging with, to control night sweats, iv. 117.

Violet ray, use of, to relieve pain in syringomyelia, iv. $27 \mathrm{~s}$.

Vipond, cases of glandular fever reported by, ii. 576 .

Virchow, leukemia first described by, iii. 844 .

Virginia Hot Springs, benefit of, in tabes, iv. 201.

Virus, attenuated, immunization with, i. 139.

Virus, virulent living, immunization with, i. 139.

Viscera, Malignant disease of, radium in treatment of, i. 486.

Visceral CONGESTION, electrotherapeutics for relief of, i. 423.

Visceroptosis, iii. 243; associated with autointoxication due to habitual constipation, ii. 688 ; causes of, iii. 243 ; diet in, iii. 247; medical treatment of, iii. 246; methods of bandaging for relief of, iii. 247 ; surgical treatment for, iii. 248.

Visual disturbances, as localizing symptom in brain tumor of occipital lobe, iv. 441 ; as localizing symptom of tumor of hypophysis, iv. 442 .
Vocation, Rescmption of, after treatment for cardiac insufficiency, iii. 662.

Vogi, fractional method of hypnotization arlvocated by, i. 567 .

Volce, rest for, essential, in acute laryngitis, iii. 338; in chronic laryngitis associated with chorditis nodosa, iii. 340; in laryngeal tuberculosis, iii. 344.

Volckeir axd Josepir, indigo carmin test developed by, iv. 24.

Voltagie, explanation of term, i. 364.

Volvulus, iii. 241.

Vomising, absence of, in acute dilatation of stomach, iii. 174; accompanying renal colic, iv. 106; measures for relief of, in acute localized peritonitis, iii. 312; as a symptom of basilar syphilitic meningitis, iv. 473 ; as a symptom of cerebral abscess, iv. 420 ; as a symptom of neurasthenia. iv. 566.

Vomiting dUe to brain tUMor, measures for relief of, iv. 448.

VoMitiNG OF PREGNANCY, administration of epinephrin in, i. 97.

Vomiting AND PLrging, as symptom of poison, i. 667.

Vomiting, uremic, measures for relief of, iv. 54.

von Behring, discovery of diphtheria antitoxin by, i. 127, 128.

voN B6KAY, on lumbar puncture for relief of hydrocephalus, iv. 468.

Von Bokay's method of lumbar puncture, in hydrocephalus, iv. 468 ; in serous meningitis, iv. 469.

von Eiselsberg, cases of mediastinal tumor collected by, iii. 500 .

von Ermegen, epidemic of meat poisoning due to $\mathrm{B}$. botulinus reported by, ii. 638.

von Gresic, E., on aspirin and novaspirin in asthma, iii. 3Ss.

voN KoncyNski, on effect of condiments upon gastric mucosa, iii. $14 \mathrm{~S}$.

voN LeUbe, definition of nervous dy'spepsia by, iii. 183 .

von Noordex, effect of calcium carbonate on elimination of phosphates in urine demonstrated by, iv. 9 ; levulose recommended by, in diabetic coma, ii. 749 ; lists of diabetic food prepared by, ii. 735 ; modification of Gerhardt's test for acetone bodies suggested by, ii. 733; oat- 
meal treatment of diabetes mellitus introduced by, ii. 753; on over-rapid reduction of weight, ii. 789 ; on physical therapy in treatment of obesity, ii. 806; principle of dietetics in treatment of membranous enteritis first noted by, iii. 208; table by, showing how butter and oil can be added to vegetables in a diabetic diet, ii. 743; on use of iron in chlorosis, iii. 816.

von Noorden diet, for use in membranous enteritis, iii. 208.

von NoORden's “GREen days," ii. 753.

von Noorden's system, for reduction of obesity, ii. 794 .

VON RECKLINGHAUSEN'S SPHYGMOTOMETER. See Sphygmotometer.

von Ruck's "watery extract of tubercle bacilli," i. 151.

von Ziemssen, graduated bath as given by, i. 198.

Vulvitis, Diabetic. See Diabetic vulvitis.

Vulvovaginitis, as a cause of enuresis, iv. 130.

Wagner, C., polymyositis first described by, ii. 684 .

Wall plates, i. 369.

WALTER, tables prepared by, for estimating dosage of X-ray, i. 499.

Walter's penetrometer, i. 496.

WaLther, OtTo, method of using exercise in tuberculosis employed by, ii. 527 .

WANNER, method recommended by, for examining sputum for albumin, in chronic bronchitis, iii. 710 .

Warm full bath, use of, in hydrotherapy, i. 199.

Warmth, benefit from, in brachial neuralgia, iv. 357 ; benefit from, in neuralgia in general, iv. 357 ; benefit from, in neuralgia of rheumatic origin, iv. 336 ; importance of, in acute nephritis, iv. 58; necessity for, in pernicious anemia, iii. 825 ; necessity for, in treatment of neuritis, iv. 284; value of, in multiple neuritis, iv. 326.

WARTS, electrolysis in treatment of, i. 397.

WassermanN, discovery by, of specific serum reaction in syphilis, ii. 362 .

Wassermann and Kolle antimeningococcus serum, i. 162.

WASSERMANN REACTION, arsenobenzol and, iv. 483,488 ; in diagnosis of cerebral tumor, iv. 669; in diagnosis of syphilis of stomach, iii. 166; in diagnosis of tabes, iv. 185; as test for syphilis, ii. 379 ; value of, in brain syphilis, iv. $47 \dot{6}$; value of, in progressive paralysis of the insane, iv. 491; value of, in question of marriage for syphilitic patient, ii. 420 ; value of, in spinal syphilis, iv. 273.

Wassermann test. See Wassermann reaction.

Wasting disease, chronic, blood pressure lowered by, iii. 555 .

WATER, climatic relations of, i. 249; as constituent of living matter, i. 39; as food, i. 662; in hydrotherapy, i. 187; loss of, from body a cause of sunstroke, iv. 676 ; relation between quantity of, in body and secretion of gastric body, i. 626 ; retention of, in body a cause of edema, iv. 16; use of, in removal of toxins from body, i. 49; use of, as a solvent, i. 9 ; use of, as a therapeutic agent, i. 35.

WATER, DRINKING, bacillary dysentery transmitted by, ii. 319 ; in causation of endemic goiter, iii. 892 ; in diet of gastric atony, iii. 170 ; in diet of gastric hyperacidity, iii. 91; filtration of, unreliable as protection against cholera, iv. 829; importance of, in plague, iv. 827 ; methods of destroying cholera germs in, iv. 829; systemic poison due to, ii. 634; typhoid fever transmitted by, ii. 23; use of, as food, i. 602.

Water, drinking, therapeutic value of, i. 35 ; in acute rheumatism, ii. 298; in alcoholic gastritis, iii. 281; in bacillary dysentery, ii. 325 ; in chronic cholecystitis and cholelithiasis, iii. 273; in delirium tremens, ii. 602 ; in eclampsia, iv. 83 ; in epilepsy, iv. 648 ; in gastrointestinal type of influenza, ii. 90 ; in infantile diarrhea, ii. 328 ; in kidney of pregnancy, iv. 82 ; in nephritis complicating scarlet fever, ii. 125; in nephrolithiasis, iv. 109; in pneumonia, ii. 227 ; in pyelitis, iv. 88 ; in Rocky Mountain fever, ii. 364; in scarlet fever, ii. 125; in septicopyemia, ii. 290 ; in tuberculosis of kidney, iv. 116; in typhoid fever, ii. 14.

Water hemlock. See Cicuta roots.

WATERCREss, poisoning by, ii. 651 .

Waterhouse, Dr. Benjamin, early work 
in vaccination by, ii. 61 ; experiments by, on inoculation for small-pox, ii. 92 .

Watering places, advantages of, in chronic: bronchitis, iii. 366 .

Waters, alkaline, see Alkaline waters; earbonated, objections to, in gastric atony, iii. 171 ; medicated, of no value in treatment of nephritis, iv. 35; saline, use of, in acute nephritis, iv. 58.

Watson, Sir Thomas, views of, on diseases of pericardium, iii. 511 .

WaTt, explanation of term in electrotherapeusis, i. 365.

Wechselmans, report by, on use of salvarsan in brain syphilis, iv. 484 .

WehnetT's AND Benoist's instruments, for measuring penetrating power of $\mathrm{X}$ ray, i. 496.

Weigert, cytoryctes variolæ first described by, ii. 41.

Weight of Body. See Body weight.

WeIL, types of hemophilia recognized by, iii. 865 .

WeIL's disease, ii. 570; convalescence from, ii. 573 ; diet in, ii. 570 ; general treatment of, ii. 570; medication in, ii. 572; muscular pains in, ii. 572; nephritis eomplicating, ii. 572 ; prognosis in, ii. 570 ; prophylaxis against, ii. 570; treatment of, in general, ii. 570 .

Weir Mitchell, use of cod liver oil in rest cure by, iv. 558 .

Weir Mitchell-Playfair cure, use of, in reflex cardiac neuroses associated with neuroses, iii. 780 .

Weir Mitchell rest cure, establishment of, iv. 539 ; meehanotherapy in connection with, i. 352 ; use of, in psychotherapy, i. 281 .

Welander's MERCOLINT APRONS, ii. 390.

Welch and Cohnheim, theory of, concerning pulmonary edema, iii. 408.

Welch and Schamberg, incision of eonjunctiva in small-pox advised by, ii. 50; investigation by, on revaccination, ii. 76 ; prognostic value of vaecination scars investigated by, ii. 75 ; silver nitrate in care of eyes in small-pox advised by, ii. 50 ; on treatment of eruption in small-pox, ii. $48,50$.

Wellmanius Wellmani, iv. $7 S 1$.

WERNICKE, acute hemorrhagic superior polioencephalitis first described by, iv. 406 .
West, cases of glandular fever reported by, ii. 574, 575; mortality from glandular fever, in cases reported by, ii. 577; on use of calomel in treatment of glandular fever, ii. 536.

West, Samuel, on culture of pneumococei in chronic bronchitis, iii. 3i1.

Westphal's sigs. See Tendon reflex, absence of.

"Wet brain," occurrence of, in delirium tremens, ii. 608.

Wet cups. See Cups.

WET Nurses, syphilis communieated by or to, ii. 374 .

"Whey cure," use of, in chronic bronchitis, iii. 370 .

Whipworm. See Tricocephalus dispar.

Whiskey, use of, as a cardiar stimulant in eurare poisoning, iv. 694; as a cardiac stimulant in strophanthus poisoning, iv. 695.

White, case of mumps six days after birth reported by, ii. 167; methorl of measuring tolerance to tubereulin proposed by, ii. 520 .

White, Hale, method of arlministering castor oil employed by, iii. 206; on prognosis in cirrhosis of liver after appearance of ascites, iii. 283; régime suggested by, for use in constipation, iii. 227 ; requisites for corset as an abdominal support in prolapse of liver, iii. 259; on unnecessary operations for constipation, iii. 237 ; on use of mineral oils by mouth in chronic eonstipation, iii. 235.

White BLOOD CORPUSCLEs, increase of, at high altitudes, i. 271.

White precipitate ointment, use of, in hereditary syphilis, ii. 434; in macular and papular syphiloderms, ii. 425; in pustular and uleerative syphiloderms, ii. 426; in syphilitic onychia, ii. 427; in uncomplicated chancre, ii. 422.

WhitLA, formula given by, for use in gallstone colic, iii. 268 .

WHitLows, electrotherapeutics for relief of, i. 404.

WhoopiNG-COеgh, ii. 173; administration of drugs in, ii. 1s2; antitoxie serum for, ii. 183; breathing compressed air in, ii. 179 ; as a complication of measles, ii. 161; complications in, ii. 173; definition of, ii. 173; diet for, ii. 179; disinfeetion of pa- 
tient's room in, ii. 179; drugs beneficial in, ii. 180, 181; etiology of, ii. 173; external use of drugs in, ii. 180; history of, ii. 173 ; hygienic measures in, ii. 177; hypodermic use of drugs in, ii. 180, 181; immunity conferred by, ii. 175; incubation period in, ii. 175; inhalation of drugs in, ii. 180; internal use of drugs in, ii. 181; mechanical measures for relief of cough in, ii. 178; misuse of drugs for, ii. 175; mortality from, ii. 175; mortality from bronchopneumonia complicating, ii. 175; organism causing, ii. 174; predisposing cause of rachitis, ii. 814; prophylaxis in, ii. 176; psychic treatment of, ii. 178; rectal feeding in, ii. 180; stages in, ii. 175 ; symptoms due to misuse of drugs in, ii. 175; synonyms for, ii. 173; transmission of, ii. 174; treatment of, ii. 177; vaccination for small-pox and its influence upon, ii. 74,179 ; vaccines and their possibilities in treatment of, ii. 183.

Wickham, Prof. Louis, work done by, on radium, i. 461, 483.

Widal and Javel, on elimination of sodium chlorid in nephritis, iv. 35 .

WigGers, investigations by, on effect of drugs on pulmonary pressure, iii. 445 .

Wilder, R. M., work by, on transmission of typhus fever, ii. 32 .

WILEy, Dr., work done by, on effects of boracic preparations on metabolism, i. 714 .

Williams, LeONARD, report by, on use of thyroid gland for relief of enuresis, iv. 131.

Williamson, method of preparing milk suggested by, for use in diabetes mellitus, ii. 743 .

Willis, Thomas, sweet character of diabetic urine first noted by, ii. 717; whooping-cough first recognized by, in England, ii. 173.

Willson, work cure combined with forced feeding employed by, in neurasthenia, iv. 553 .

Winckel's disease. See Hemoglobinuria, epidemic.

WINDS, i. 253 ; causes of, i. 253 ; relation between temperature and, i. 254.

Wine, benefit from, during attack of angina pectoris, iii. 769 .

Wintergreen oil, use of, in acute urethral gonorrhea, iv. 141; to relieve pain in chest accompanying tuberculosis, ii. 506 .

Winternitz, alternate half and whole cold baths as recommended by, i. 198; combination compress used by, i. 198; effect of baths on nervous system first noted by, iv. 557; general bath as given by, i. 195; pioneer work by, on physiological properties of water, i. 188.

Withering, William, early use of digitalis by, iii. 555 .

Witthauer, indications for use of vibration massage given by, i. 356 .

Wolfenden position, advantages of, in laryngeal tuberculosis, iii. 347.

Wood, Dr. Alexander, hypodermic needle invented by, ii. 617 .

Wood AlCOHOL POISONING, ACUTE, ii. 609; treatment of, ii. 610.

Wood Naphtha. See Wood alcohol.

Wood TICK, transmission of Rocky Mountain fever by, ii. $562,563$.

Woodruff, Major, exposure to direct sun rays believed to be injurious by, ii. 459 .

Woodward, Dr., first communication regarding inoculation for small-pox made by, ii. 52 .

Woolsorters' disease, ii. 330.

WoRD BLINDNEss, as localizing symptom of tumor in left cerebral hemisphere, iv. 440.

WORD DEAFNESS, as localizing symptom of tumor in left cerebral hemisphere, iv. 440.

Word-time associations, as part of technique of psychoanalysis, i. 589.

Work cure, value of, in neurasthenia, iv. 552 .

WORKSHOPs, disinfection of, in tuberculosis, ii. 453.

WoRRY, as a cause of functional neuroses, iv. 573.

Wright, Sir Almroth, active immunization introduced by, i. 126; bacterial vaccines introduced by, i. 139 ; formation of immune bodies in subcutaneous tissue supported by, i. 140; immunization ágainst chronic infections introduced by, i. 126; method of finding opsonic index introduced by, i. 140; theories of, concerning autoinoculation in tuberculosis, ii. 525; typhoid fever inoculation introduced by, i. 126, 142, 143; use of calcium preparations for control of hemorrhage introduced by, ii. 
155; work done by, severely criticized, i. 179 .

Wright's THEORY, of causation of beriberi, iv. 849 .

Wrist-Drop, prevention of, in multiple neuritis, iv. 327; in neuritis, iv. 284.

WUNDT SCHOOL OF PSYCHOTHERAPY, iv. 579.

WürTzEN, on red light treatment of smallpox, ii. 51.

WyLIE, manner of artificial introduction of air into stomach explained by, iii. 779 .

Wylie's mixture, for use in membranous enteritis, iii. 210.

Wruan, improvement of instruments used in paracentesis by, iii. 457 .

Wyss, investigation by, of epidemic among fish, ii. 644 .

Xanthoma diabeticorum, as a symptom of diabetes mellitus, ii. 765 .

Xeroform, use of, as dusting powder in gangrenous chancre, ii. 423 ; in mucous patches upon genitalia, ii. 427 ; in papular or tubercular tertiary lesions of syphilis, ii. 426; use of vaginal tampons impregnated with, in chancre of vagina or uterine neck, ii. 425.

X-RAY BURN, i. 515.

"X-ray Cancer," i. 516.

X-ray filter, i. 503; construction of, i. 504; discovery of, i. 504; use of, in deep therapy, i. 506; use of, in treatment of cancer, i. 541; use of, in treatment of ovaries, i. 556.

$\mathrm{X}$-ray therapy. See X-rays, therapeutic use of.

X-RAY TUBe, i. 492.

X-RAY UlCER, i. 515.

X-RAYs, action of, on cancer cells, i. 537; antipruritic effects of, i. 537; biological effects of, i. 508, 536; cumulative effects of, i. 512; curative effects of, i. 512; "early reaction" of, i. 512; effects of, on bacterial life, i. 517, 525; effects of, on deeper structures of body, i. 517; effects of, on long bones, iii. 826; effects of, on skin, i. 514; effects of, on tissues of young children and embryos, i. 523; influence of, upon ovaries, i. 555; "late reaction" of, i. 512, 516; physical characteristics of, i. 491 ; physical effects of, i. 492; production of, i. 490; radiation in, i. 493; reaction of, i. 516; selective action of, i. 509; toxenias caused by, i. 513.

X-rays, diagnostic use of, in carcinoma of stomach, iii. 163; in foreign bodies present in esophagus, iii. 163; in hourglass st omach, iii. 179; in lung tumor, iii. 445; in salivary calculi, iii. 30; in tubereulous kidney, iv. 14.

$\mathrm{X}$-rays, therapeutic use of, in arthritis deformans and chronic rheumatism, ii. 713; in asthenic bulbar paralysis, iv. 256 ; in benign growths, i. 535; in carcinoma of esophagus, iii. 54, 5.5; in chronic bronchitis, iii. 378; in chronic influenza, ii. 195; coöperation between surgery and, i. 541, 545; dangers in, iii. 85.3 ; in delayed resolution of pneumonia, ii. 258 ; in dermatology, i. 526; in destruction of filaria bancrofti, iv. 757 ; to determine indications for operation in pulmonary abscess and gangrene, iii. 443 ; to determine site of arterial obstruction in diabetic gangrene, ii. $\mathbf{7 0 0}$; in diabetes mellitus complicating thyroid disease, ii. 767 ; distance of sources of light in, i. 502; dosage in, i. 497, iii. 853 ; factors in, i. 494 ; in funicular myelitis connected with leukemia, iv. 232; in glandular enlargements, i. 5.51; in glandular skin diseases, i. 527: in goiter, iii. 594 , 899; guide to apportionment of dose in, i. 509; histological changes in skin due to, i. 517; intensity as factor in, i. 467; in internal tuberculosis, i. 553; latent period in, i. 511; in leprosy, iv. 816 ; in leukemia, iii. 848 ; in lymphoid and blood diseases, i. 544; in malignant growths of skin, i. 537; mode of action of, iii. 848; in mediastinal tumor, iii. 502; in nephrolithiasis, iv. 10s; in nervous conditions, i. 554; objects of, i. 505; objections to, in laryngeal tuberculosis, iii. 347 ; in oriental sore, iv. 756 ; in ovarian disease, i. 55.5; penetration as a factor in, i. 494 ; in pernicious anemia, iii. $\$ 26$; physical principles in, i. 489 ; in polyeythemia with splenomegaly, iii. Sis; to prevent acidosis in diabetes mellitus, ii. 757; production of X-ray in, i. 490; prophylactic irradiation in, i. 541 ; protection of patient and operation in, $i$. 506 ; in pruritus accompanying diabetes mellitus, ii. 765 ; in pseudoleukemia, iii. 
854; purin bodies produced by, iii. 851 ; quality of radiation in, i. 493; quantity of radiation in, i. 494; reaction of X-ray in, i. 511; regulation of quantity and quality in, i. 494 ; requirements in deep therapy, i. 505; in respiratory disorders, i. 553 ; selective power of $\mathrm{X}$-rays in, $\mathrm{i}$. 509 ; in skin diseases arising in hair follicles, i. 530; in skin diseases due to fungi, i. 536; in special conditions, i. 525; for status lymphaticus, iii. 913 ; in superficial epitheliomata, i. 538; in syringomyelia, iv. 279; tables showing results of, iii. 849,850 ; technique of, in deeper therapy, i. 503; technique of, in general, i. 503 , iii. 852 ; theory of personal idiosyncrasy in, i. 513; therapeutic effects of, iii. 852 ; time of exposure in, i. 500 ; toxemias due to, i. 513 ; in tuberculosis, i. 533; in tuberculosis of skin, i. 533; in tuberculous peritonitis, iii. 321 ; in ulcerating granuloma of pudenda, iv. 866; uniformity of deep irradiation in, i. 505; uniformity as a factor in, i. 502; uric acid increased by, iii. 851; X-ray filter in, i. 503.

$\mathrm{X}$-rays and electricity, therapeutic use of, i. 399 ; in abscess of colon, mesentery, and liver, i. 405 ; in acne, i. 406 ; in appendicitis, i. 405; in carbuncles, i. 404 ; in infections, i. 399; in pulmonary tuberculosis, i. 400; in tuberculosis of bladder, i. 402; in tuberculosis of kidney, i. 402; in tuberculosis of skin, i. 400; in tuberculous adenitis, i. 401; in tuberculous arthritis, i. 403; in tuberculous infections, i. 451 ; in tuberculous peritonitis, i. 403 ; in tuberculous prostatitis, i. 402.

YAKInoff, Dr., experiments by, on technique of salvarsan injection, iv. 487.

YAMs, certain species of, dangerous as food, ii. 651.

YAws. See Frambœia tropica.

Yeast, internal use of, in diabetes mellitus, ii. 761 ; to relieve boils complicating diabetes mellitus, ii. 675 ; local use of, to relieve pruritis vulvæ in diabetes mellitus, ii. 765.

YeLLOW FEVER, iv. 855; hydrotherapy for, i. 218; methods of exterminating, iv. 857; preventive measures in, iv. 856; remedial measures in, iv. 855 ; Sternberg's method of treatment for, iv. 856 ; symptoms of, iv. 855; transmission of, iv. 857.

Yeo, Dr. Burney, cod liver oil as spray in hay fever recommended by, iii. 391 ; formula for expectorant mixture suggested by, iii. 373; salicin recommended by, in acute rheumatism, ii. 300 .

Yeo inhaler, use of, for creosote inhalations in bronchiectasis, iii. 396 ; in plastic bronchitis, iii. 392.

Xeo mask, inhalations by means of, to relieve cough in pulmonary tuberculosis, ii. 497.

Yersin's anti-plague serum, i. 167.

Yersin-Roux anti-plague serum, iv. 826.

Yohimbin, use of, in impotence accompanying diabetes mellitus, ii. 766 .

Yоштн, protection against tuberculosis in, ii. 462 .

Z

Zander apparatus. See Zander machine.

Zander machine, i. 333; use of, in treatment of obesity, ii. 807 .

Zander system of mechanotherapy, i. 333; active apparatus in, i. 334 ; arm movements in, i. 335; balancing movements in, i. 345 ; indications for use of, iii. 641 ; leg movements in, i. 339 ; passive movements in, i. 345; therapeutic uses of, i. 348 ; trunk movements in, i. 343 ; vibratory massage in, i. 355.

Zeists, ii. 657.

Ziemann's method, for prophylactic administration of quinin, iv. 737.

Ziemssen, on value of high altitudes in neurasthenia, iv. 556.

Ziemssen and Zenker, cases of idiopathic dilatation of esophagus described by, iii. 56.

Zinc, POISONING BY, i. 707; treatment of, i. 708 .

Zinc, use of, as spray in chronic laryngitis, iii. 340.

Zinc chlorid, intralaryngeal application of, in chronic laryngitis, iii. 341.

Zinc oxid, use of, as dusting powder, in diabetic vulvitis, ii. 765 .

Zinc oxid adhesive plaster, use of, in herpes zoster, iv. 359 .

Zinc oxid ointment, use of, in diabetic vulvitis, ii. 765; in oriental sore, iv.756; as protection against injury from counter- 
irritation, ii. 159 ; in stomatitis herpetica, iii. 10.

Zinc permanganate, injections of, in chronic gonorrheal urethritis, iv. 155.

Zinc preparations, use of, in neurasthenia, iv. 558 .

Zinc sulphate, use of, in aqueous solution for herpes of lips, iii. 6 ; by injection in chronic gonorrheal urethritis, iv. 155; in chronic atrophic laryngitis, iii. 343; in continuous gastric hypersecretion, iii. 106; in lavage for chronic gastritis, iii. 160 ; in lavage for gastric hyperacidity, iii. 98,99 ; to unload stomach in acute opium poisoning, ii. 614.

Zinc sulphocarbolate, use of, in chronic nasopharyngitis, iii. 33.

Zinc valerianate, use of, in peristaltic unrest, iii. 2, 250 .

Zlatogonoff, on cholera "carriers," iv. 828.

ZWEIG, diet scheme suggested by, for use in constipation, iii. 226; internal use of hydrastis and hamamelis advised by, in ulcerative colitis with hemorrhage, iii. 212. 
-

$-$

,

. 


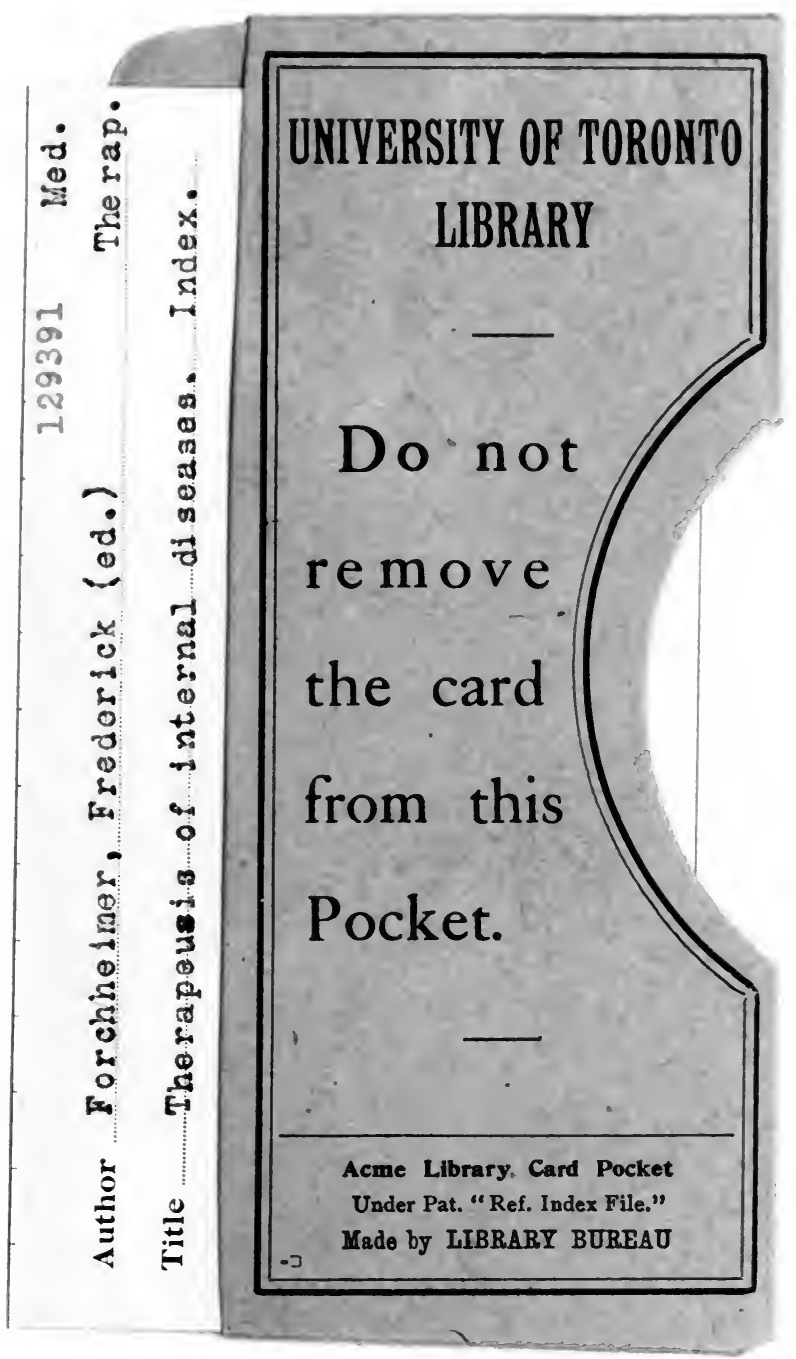


